\title{
Streptococcal collagen-like protein 1, Scl1, modulates group a Streptococcus adhesion, biofilm formation and virulence
}

\author{
Beth Alexandra Bachert
}

Follow this and additional works at: https://researchrepository.wvu.edu/etd

\section{Recommended Citation}

Bachert, Beth Alexandra, "Streptococcal collagen-like protein 1, Scl1, modulates group a Streptococcus adhesion, biofilm formation and virulence" (2016). Graduate Theses, Dissertations, and Problem Reports. 5137.

https://researchrepository.wvu.edu/etd/5137

This Dissertation is protected by copyright and/or related rights. It has been brought to you by the The Research Repository @ WVU with permission from the rights-holder(s). You are free to use this Dissertation in any way that is permitted by the copyright and related rights legislation that applies to your use. For other uses you must obtain permission from the rights-holder(s) directly, unless additional rights are indicated by a Creative Commons license in the record and/ or on the work itself. This Dissertation has been accepted for inclusion in WVU Graduate Theses, Dissertations, and Problem Reports collection by an authorized administrator of The Research Repository @ WVU.

For more information, please contact researchrepository@mail.wvu.edu. 
STREPTOCOCCAL COLLAGEN-LIKE PROTEIN 1, SCL1, MODULATES GROUP A STREPTOCOCCUS ADHESION, BIOFILM FORMATION AND VIRULENCE

\author{
Beth Alexandra Bachert \\ Dissertation submitted to the School of Medicine \\ at West Virginia University \\ in partial fulfillment of the requirements \\ for the degree of \\ Doctor of Philosophy \\ In \\ Immunology and Microbial Pathogenesis
}
Slawomir Lukomski, Ph.D., Committee Chairperson
Thomas Elliott, Ph.D.
Christopher F. Cuff, Ph.D.
Karen H. Martin, Ph.D.
P. Rocco LaSala, M.D.
Department of Microbiology, Immunology, and Cell Biology
Morgantown, West Virginia
2016

Keywords: group A Streptococcus, collagen-like proteins, Scl1, Scl2, biofilm, host colonization, M3 type

Copyright 2016 Beth Bachert 


\section{ABSTRACT \\ Streptococcal collagen-like protein 1, Scl1, modulates group A Streptococcus adhesion, biofilm formation and virulence}

\section{Beth A. Bachert}

Background: The collagens comprise a large family of versatile proteins found in all three domains of life. The streptococcal collagen-like protein 1, Scl1, of group A Streptococcus (GAS) binds extracellular matrix components (ECM), cellular fibronectin and laminin, via the surface-exposed globular domain. GAS strains express Scl1 and form biofilm in vitro, except for M3-type strains that are particularly invasive to humans. Hypothesis: Lack of Scl1 adhesin in M3 GAS results in decreased adherence and biofilm formation, and increased virulence. Results and Discussion: First crystal structure of the globular domain revealed a unique six-helical bundle fold, consisting of three pairs of alpha helices connected by variable loops. ECM binding by Scl1 promotes the formation of stable tissue microcolonies, which was demonstrated in vitro during infection of wounded human skin equivalents. A conserved nonsense mutation was identified in the scl1 allele of the M3-type strains (sc/1.3) that truncates the coding sequence, presumably resulting in a secreted Scl1 variant. Absence of Scl1 on the surface of M3-type GAS was demonstrated experimentally, as well as diminished expression of the scl1 transcript in $\mathrm{M} 3$ strains relative to other $\mathrm{M}$ types. Therefore, M3-type strains have reduced biofilm capacity on ECM coatings relative to other M-types. Constructed full-length recombinant Scl1.3 protein displayed binding capacity to cellular fibronectin and laminin, and M3 strains complemented with functional Scl1.3 adhesin displayed increased biofilm formation. The isoallelic M3 strain, carrying a rare "carrier" allele encoding cell-associated Scl1.3 variant, showed decreased pathology in mice, compared to the invasive M3 strain. Similarly, scl1 inactivation in biofilm-capable M28- and M41-type GAS led to increased lesion size during subcutaneous infection. Conclusions: The studies presented here demonstrate the importance of surface Scl1 in modulating biofilm formation and virulence of GAS, and provide insight into the structure and function of Scl proteins. 


\section{Preface}

The Ph.D. Thesis presented here represents a subset of research performed by Beth Bachert that is thematically focused on the importance of the major surface adhesin, the streptococcal collagen-like protein 1 (Scl1), in the pathogenesis of group A Streptococcus. The chapters herein correspond to published peer-reviewed research papers and are grouped into two parts that address: Part I) the role of Scl1 in adherence, biofilm formation, and virulence, and Part II) structural analysis of the Scl proteins. Additional published data are presented in Part III that resulted from Author's projects, focused on utilization of collagen-like genes as epidemiological and detection markers, which were carried out under a two-year Graduate Fellowship in Nanotechnology Sensing Advances in Field and Environment (NanoSAFE).

Research included in Part I demonstrates that Scl1 significantly contributes to biofilm formation in multiple M-types, except for invasive M3-type strains that were naturally diminished in the capacity to form biofilm (Chapter 1 ). Non-invasive M28- and M41-type strains produced significant biomass and biofilm structure associated with extracellular glycocalyx. Biofilm phenotype was significantly reduced in scl1 isogenic mutants but was gained following heterologous complementation of Lactococcus lactis with Scl1. A unique scl1.3 allele is reported that is conserved in M3 strains, which contains a null mutation in the collagen-like region, resulting in a truncated secreted protein. Research in Chapter 2 assesses the effect of this null mutation on adherence and biofilm formation. The absence of Scl1 on the surface of M3-type GAS was demonstrated, as well as diminished sc/1-transcript level in comparison to other M-types. Complementation of Scl1-deficient M3 GAS with full-length Scl1.3 surface protein conferred biofilm formation by these strains. The M3 wild-type strain lacking surface Scl1 did not form tissue microcolonies in an in vitro pseudo-organ skin equivalents, whereas Scl1-expressing M41 strain formed glycocalyx-embedded microcolonies. Inactivation of scl1 in M28 and M41 GAS resulted in increased virulence in mice. Research in Chapter 3 focuses on M3 strains harboring a rare carrier allele of $s c / 1.3$, which restored the open reading frame and the expression of full-length Scl1.3 protein. These strains were attenuated for virulence and had increased host cell adherence compared to the wild-type strain. 
Part II focuses on crystallization and structural characterization of Scl proteins. The globular non-collagenous domain of Scl2 from M3-type GAS was crystallized (Chapter 4), and revealed a six-helical bundle fold that is rare in bacterial proteins (Chapter 5).

Overall, studies presented in Parts I and II demonstrate the importance of Scl1 in adherence and biofilm formation, while revealing an inverse correlation with invasiveness. Importantly, our studies provide new insights into the structure-function relationship of $\mathrm{Scl}$ proteins.

Additional data presented in Part III, describe interdisciplinary projects aimed at development of bacterial models for the microfluidic assessment of epidemiology of group A streptococcal strains (Chapter 6) and the detection of the select agents, Burkholderia pseudomallei and B. mallei, by targeting collagen-like genes (Chapter 7). 


\section{DEDICATION}

This work is dedicated to my fiancé Eric Witt, who has been a constant source of love, support, and encouragement over the last 5 years. Your strength, kindness, thoughtfulness, and lighthearted sense of humor has had a tremendous impact on my life and been an inspiration to me. I also dedicate this work to my parents Yvonne and Danny Bachert, who have taught me the value of education, hard work, and perseverance. Without their unwavering faith and support, this work would not have been possible. 


\section{ACKNOWLEDGMENTS}

I would like to thank my mentor Dr. Slawomir Lukomski for his constant support and guidance, and his encouragement not only in advancing our research, but also in developing my skills as an independent researcher and presenter. I would like to thank my committee members, Dr. Christopher Cuff, Dr. Karen Martin, Dr. Thomas Elliott, and Dr. Rocco LaSala, for their advice and guidance throughout this project which has helped improve the experimental design and quality of the data. I thank Dr. Joan Olson, Dr. Nyles Charon, Dr. Lisa Salati, and Dr. Jennifer Franko for critical readings of various documents including manuscripts, proposals, and applications throughout my time here. I thank Dr. Lisa Holland and Dr. Jeremy Dawson for their support and collaboration on our NanoSAFE projects. I thank Dr. Rita Berisio, Flavia Squeglia, and Daniela Marasco for their continued contribution to ongoing projects in our laboratory and their insights into structural aspects of our work. I thank Dr. Meenal Elliott and Dr. Kathy Brundage for help troubleshooting and designing experiments involving flow cytometry, and Dr. Linqing Zhang, Dr. Karen Martin, and Dr. Amanda Ammer for assistance with microscope imaging experiments. I thank Dr. Mariette Barbier and Dr. Heath Damron for helpful discussions on qPCR data analysis. 


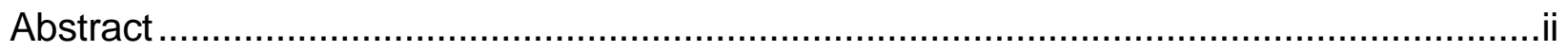

Preface

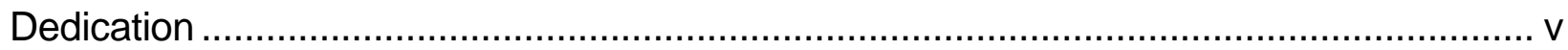

Acknowledgments .............................................................................................

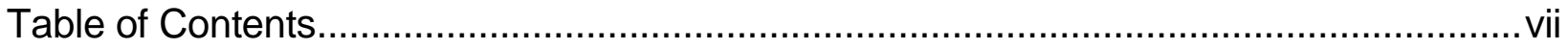

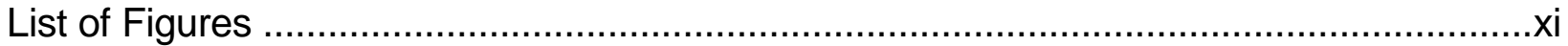

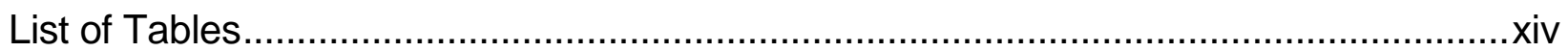

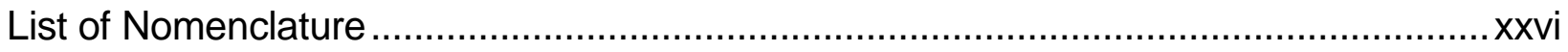

General Introduction: Collagen-like proteins of pathogenic streptococci .......................... 1

Part I. Role of Scl1 in adherence, biofilm, and virulence ...................................... 14

Chapter 1: The streptococcal collagen-like protein-1 (Scl1) is a significant determinant for biofilm formation by group A Streptococcus ......................................................... 15

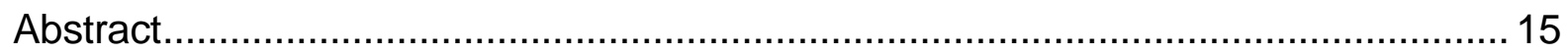

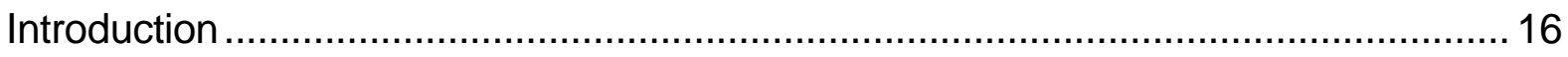

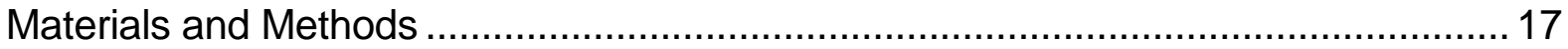

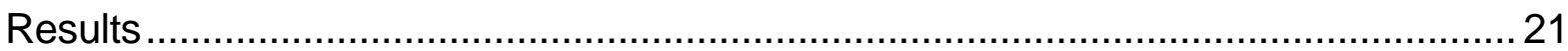

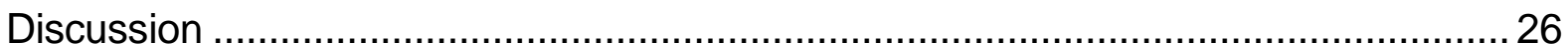

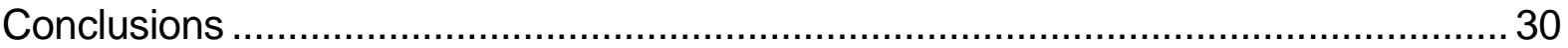

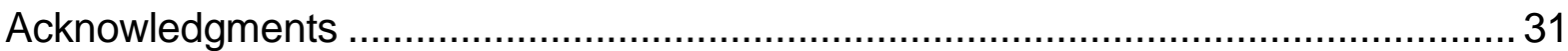

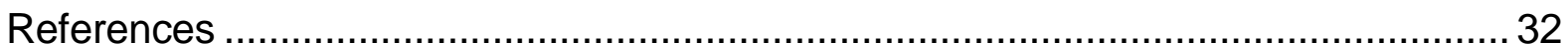

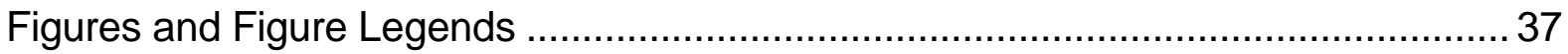

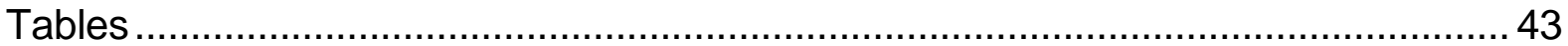

Chapter 2: Unique footprint in the scl1.3 locus affects adhesion and biofilm formation of the invasive M3-type group A Streptococcus.......................................................... 45

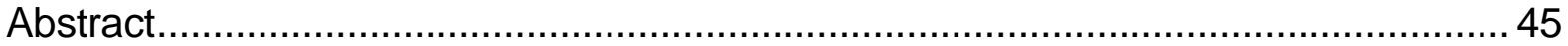

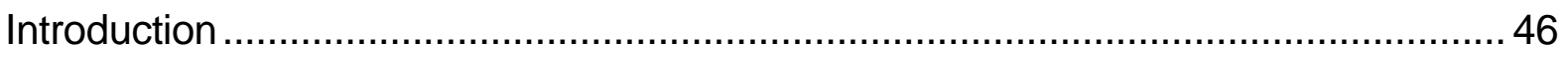

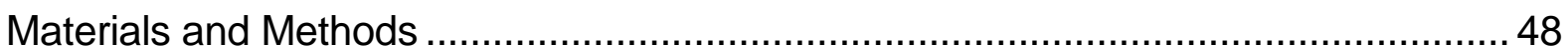

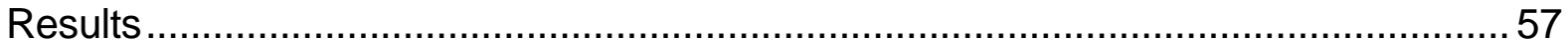




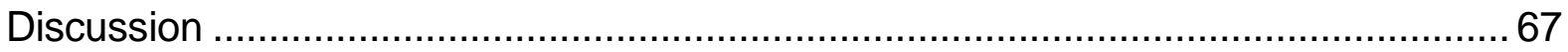

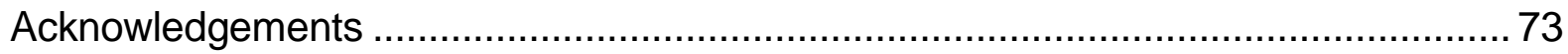

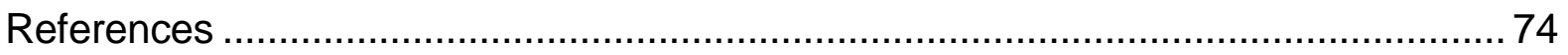

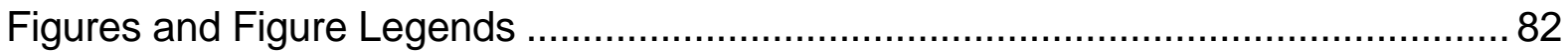

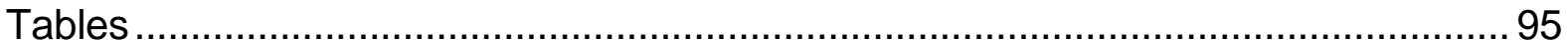

Chapter 3: Natural variant of collagen-like protein $A$ in serotype M3 group $A$ Streptococcus increases adherence and decreases invasive potential ....................... 96

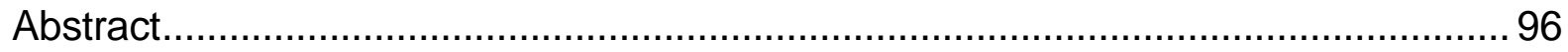

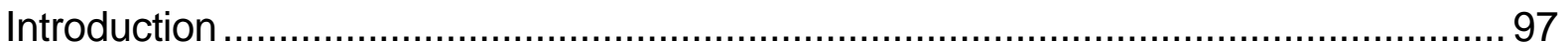

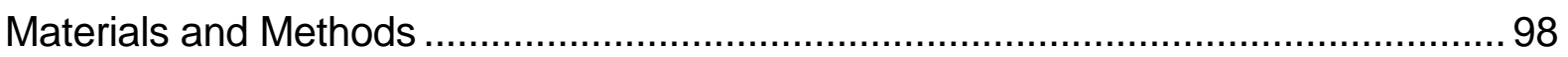

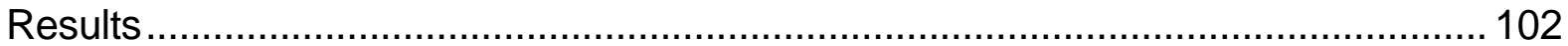

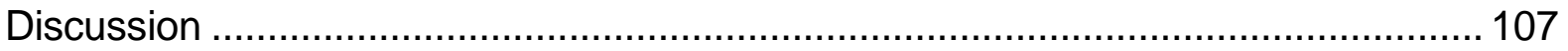

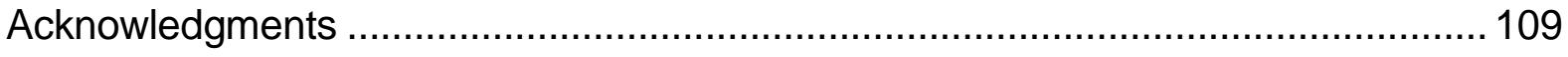

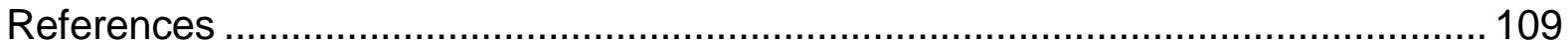

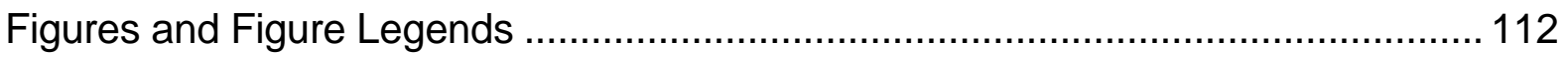

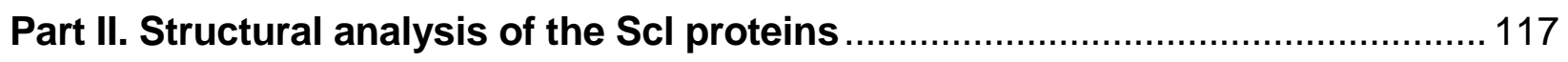

Chapter 4: Crystallization and preliminary $X$-ray crystallographic analysis of the variable domain of Scl2.3, a streptococcal collagen-like protein from invasive M3-type Streptococcus pyogenes................................................................................. 118

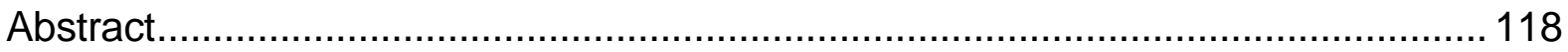

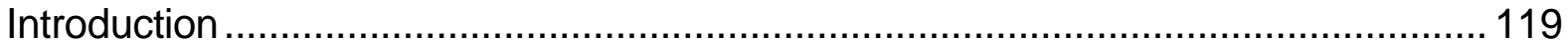

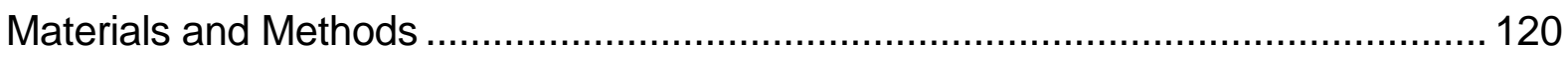

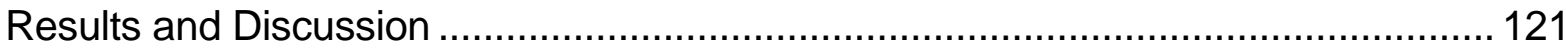

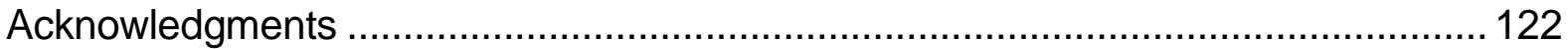

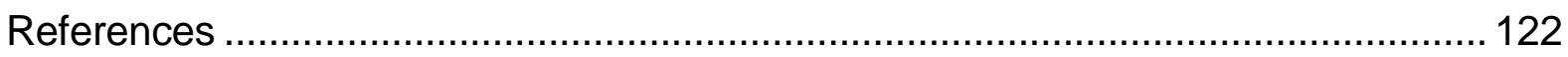

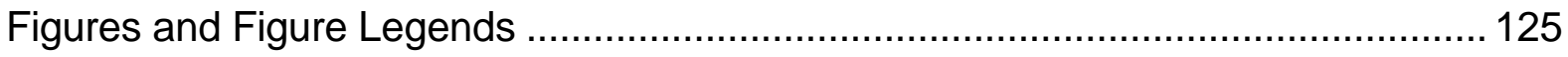

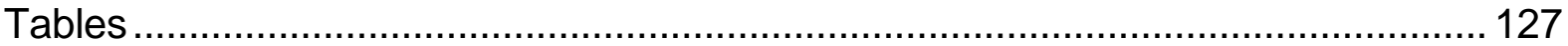

Chapter 5. The crystal structure of the streptococcal collagen-like protein 2 globular domain from invasive M3-type group A Streptococcus shows significant similarity to immunomodulatory HIV protein gp41 


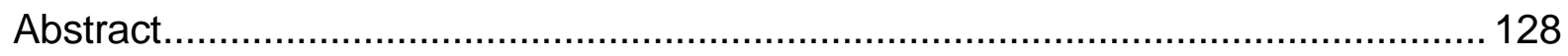

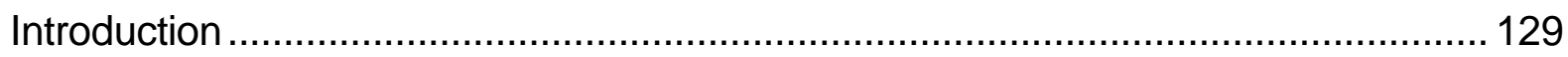

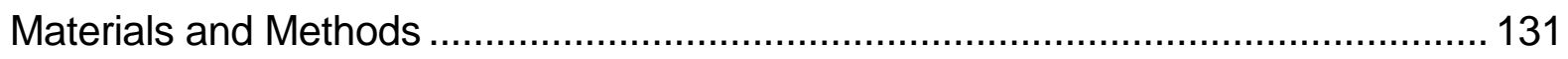

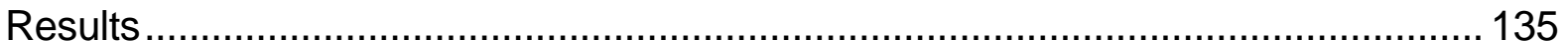

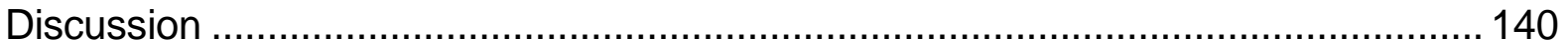

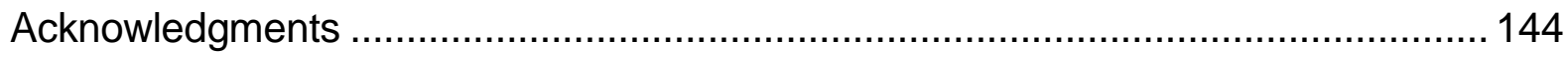

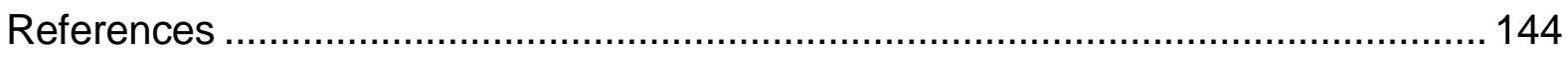

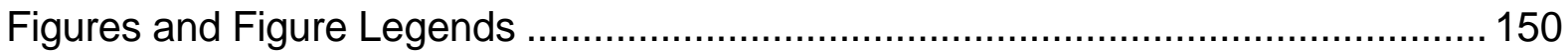

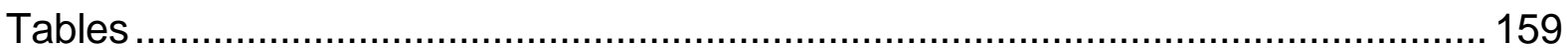

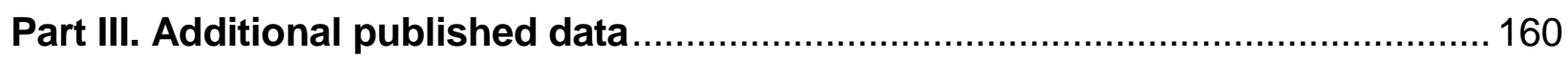

Chapter 6. Reversible Phospholipid Nanogels for Deoxyribonucleic Acid Fragment Size Determinations up to 1,500 Base Pairs and Integrated Sample Stacking.................. 161

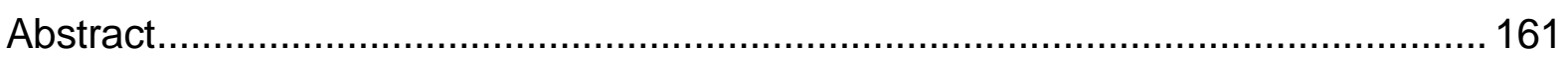

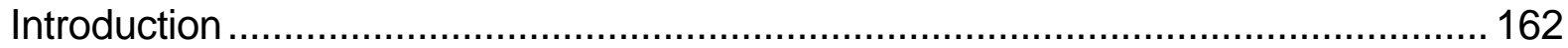

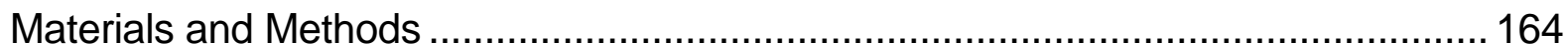

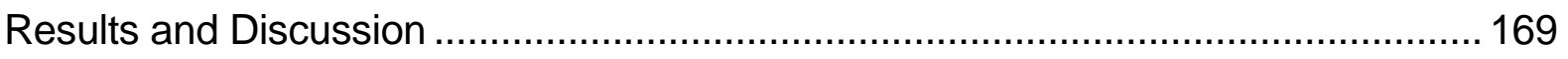

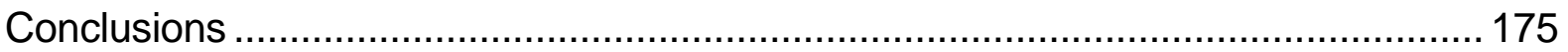

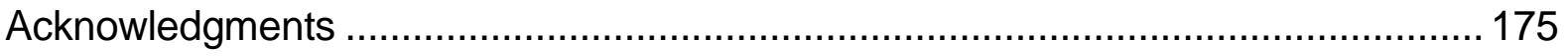

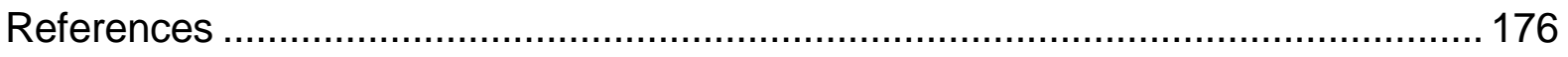

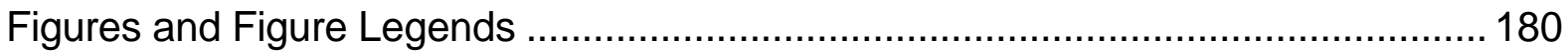

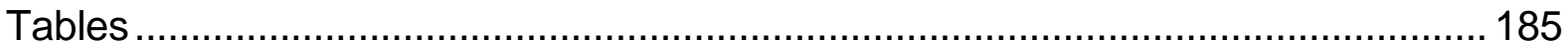

Chapter 7. A Unique Set of the Burkholderia Collagen-like Proteins Provides Insight into Pathogenesis, Genome Evolution and Niche Adaptation, and Infection Detection..... 189

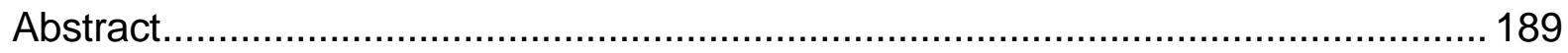

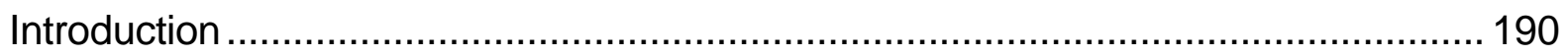

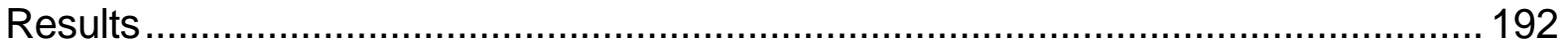

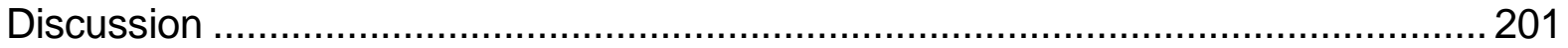

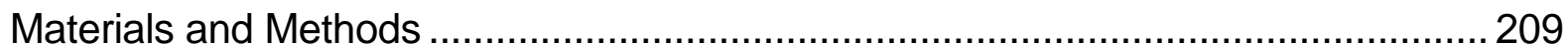

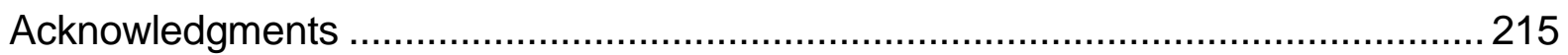

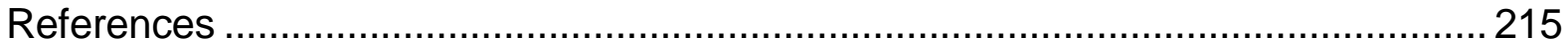




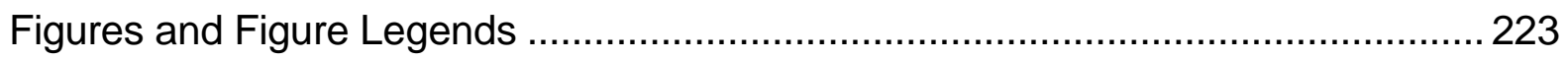

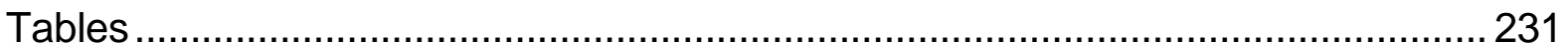

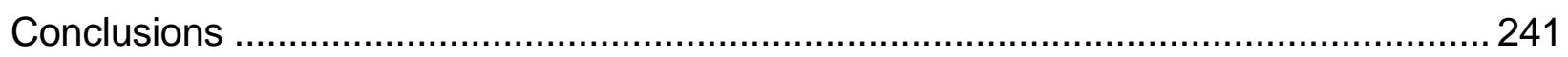

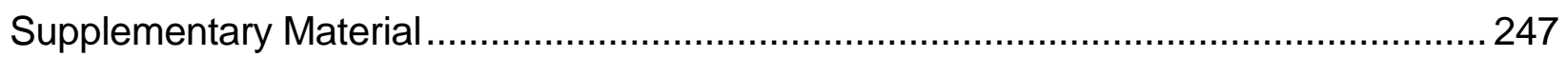

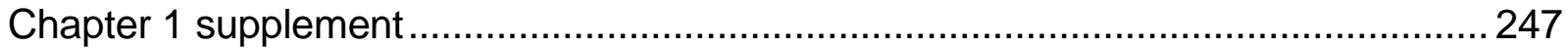

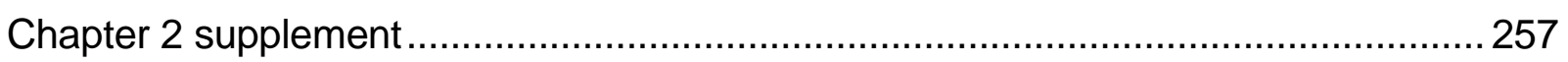

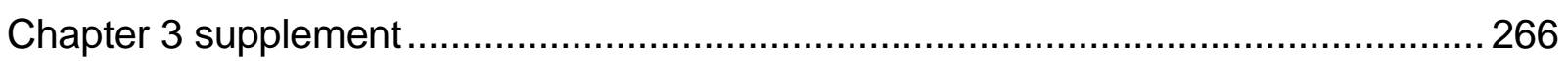

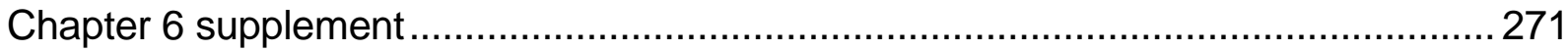

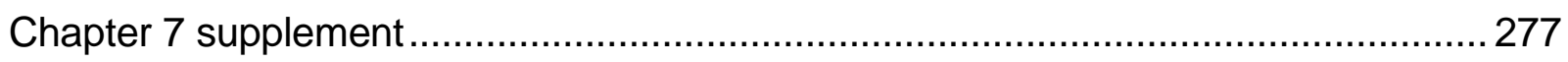

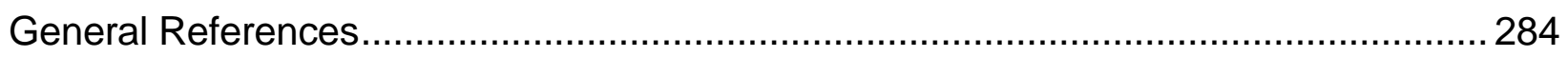




\section{LIST OF FIGURES}

\section{General Introduction}

Figure 1. Sequence similarity and structure of Scl proteins in streptococci ................... 4

Figure 2. Dichotomous nature of ligand binding by Scl1 in human tissue and blood 10 Chapter 1. The streptococcal collagen-like protein-1 (Scl1) is a significant determinant for biofilm formation by group A Streptococcus

Figure 1. Variation in biofilm formation among GAS strains .................................... 37

Figure 2. Field emission scanning electron microscopy of GAS biofilms ..................... 38

Figure 3. Production of bacterial-associated extracellular matrix ................................. 39

Figure 4. Biofilm formation by wild type and sc/1-inactivated isogenic mutants ............ 40

Figure 5. Scl1 expression in L. lactis promotes biofilm formation................................ 41

Figure 6. Scl1-mediated model of GAS biofilm (not to scale) .................................... 42

Chapter 2. Unique footprint in the scl1.3 locus affects adhesion and biofilm formation of the invasive M3-type group A Streptococcus

Figure 1. M3-type GAS strains harbor unique polymorphisms in the scl1.3 locus ........ 82

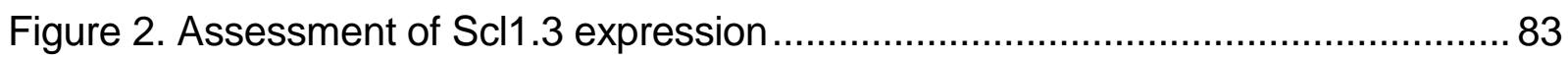

Figure 3. Characterization of the scl2.3 locus in M3-type GAS ................................... 85

Figure 4. In vitro skin equivalent model of GAS infection ........................................... 86

Figure 5. In vitro biofilm formation and hypothetical model of microcolony formation by invasive M3-type GAS

Figure 6. Construction and binding characterization of recombinant full-length Scl1.3FL and Scl2.3 proteins.

Figure 7. Homologous complementation of M3-type GAS with full-length surfaceexpressed Scl1.3 protein confers biofilm formation 90

Figure 8. Heterologous complementation of sc/1-deficient mutant of M41-type GAS with full-length Scl1.3 restores biofilm formation

Figure 9. Skin pathology of SKH1 hairless mice infected subcutaneously with wild-type and sc/1-inactivated mutants of M28- and M41-type GAS .93 
Figure 10. Model of Scl1-mediated GAS adhesion, biofilm formation, and host colonization.

Chapter 3. Natural variant of collagen-like protein A in serotype M3 group A Streptococcus increases adherence and decreases invasive potential

Figure 1. SclA differs in GAS serotype M3 invasive and carrier strains and is expressed

Figure 2. Strain with $s c / A^{\text {Carrier }}$ has reduced virulence compared to the parental invasive strain 113

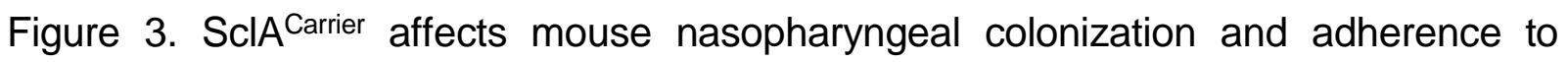
cultured epithelial cells 114

Figure 4. Recombinant full-length $\mathrm{SclA}$ ( $\mathrm{rScl} \mathrm{A}^{\mathrm{M} 3-\mathrm{FL}}$ ) binds the human extracellular matrix proteins cellular fibronectin ( $\mathrm{CFn}$ ) and laminin $(\mathrm{Lm})$. 115

Figure 5. Model summarizing the effect of invasive ( $\left.s c / A^{\text {Invasive }}\right)$ or carrier ( $\left.s c / A^{\text {Carrier }}\right) s c l A$ alleles in serotype M3 GAS 116

Chapter 4. Crystallization and preliminary X-ray crystallographic analysis of the variable domain of Scl2.3, a streptococcal collagen-like protein from invasive M3-type Streptococcus pyogenes

Figure 1. Image of typical rScl2.3-V crystals 125

Figure 2. Diffraction pattern of a rScl2.3-V native crystal. 126

Chapter 5. The crystal structure of the streptococcal collagen-like protein 2 globular domain from invasive M3-type group A Streptococcus shows significant similarity to immunomodulatory HIV protein gp41

Figure 1. Expression of Scl2.3 surface protein by the invasive M3-type strain MGAS 315 150

Figure 2. Structural characterization of Scl2.3-V in solution .................................. 151

Figure 3. Cartoon representation of the crystal structure of Scl2.3-V trimer .............. 152

Figure 4. Main interactions stabilizing the six-helix bundle of Scl2.3-V ...................... 153

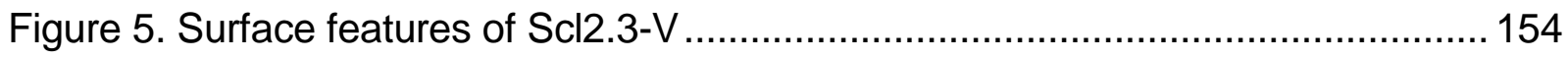

Figure 6. Multiple sequence alignment analysis of Scl2-V region variants.................. 155

Figure 7. Scl2.3-V structural alignment with the HIV protein gp41 ........................... 156 
Figure 8. Modeling and molecular dynamics analyses

Chapter 6. Reversible phospholipid nanogels for deoxyribonucleic acid fragment size determinations up to 1,500 base pairs and integrated sample stacking

Figure 1. Separations obtained at different phospholipid concentration and run temperature 180

Figure 2. Separation of PCR amplicon obtained with the phospholipid nanogel optimized for DNA up to $1,500 \mathrm{bp}$ in length 182

Figure 3. DNA stacking with phospholipid additives 184

Chapter 7. A unique set of the Burkholderia collagen-like proteins provides insight into pathogenesis, genome evolution and niche adaptation, and infection detection

Figure 1. Identification and characterization of bucl genes in B. pseudomallei reference strain K96243 223

Figure 2. Chromosomal rearrangements and deletions involving bucl loci ................ 224

Figure 3. Thermal stability of the Bucl collagen regions .......................................... 225

Figure 4. Characterization of Burkholderia collagen-like proteins ............................. 226

Figure 5. Phylogenetic analysis of B. pseudomallei, B. mallei, and B. thailandensis strains by bucl-locus typing..... 227

Figure 6. Phylogenetic analysis of B. pseudomallei, B. mallei, and B. thailandensis strains using individual bucl 3 and bucl 4 genes 228

Figure 7. Distribution of bucl genes among Burkholderia spp. select agents by PCR 229 Figure 8. Detection of B. pseudomallei and B. mallei by qPCR 230

\section{Conclusions}

Figure 1. Hypothesis model

Figure 2. Model of Scl1-mediated GAS adhesion, biofilm formation, and host colonization 


\section{LIST OF TABLES}

\section{Introduction}

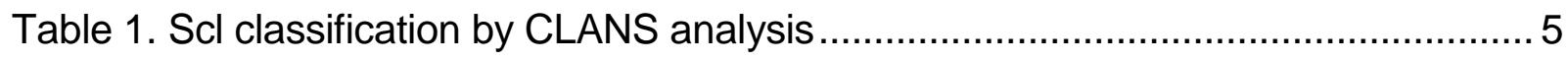

Chapter 1. The streptococcal collagen-like protein-1 (Scl1) is a significant determinant for biofilm formation by group A Streptococcus.

Table 1. Cell surface hydrophobicity of GAS strains............................................... 43

Table 2. Cell surface hydrophobicity of Lactococcus strains ...................................... 44

Chapter 2. Unique footprint in the scl1.3 locus affects adhesion and biofilm formation of the invasive M3-type group A Streptococcus

Table 1. Constructs used in this study 95

Chapter 4. Crystallization and preliminary X-ray crystallographic analysis of the variable domain of Scl2.3, a streptococcal collagen-like protein from invasive M3-type Streptococcus pyogenes

Table 1. Data collection statistics 127

Chapter 5. The crystal structure of the streptococcal collagen-like protein 2 globular domain from invasive M3-type group A Streptococcus shows significant similarity to immunomodulatory HIV protein gp41

Table 1. Data collection and refinement statistics 159

Chapter 6. Reversible Phospholipid Nanogels for Deoxyribonucleic Acid Fragment Size Determinations up to 1,500 Base Pairs and Integrated Sample Stacking ......

Table 1. Effect of concentration on resolution. 185

Table 2. Effect of [DMPC]/[DHPC] ratio on resolution at $23^{\circ} \mathrm{C}$ 186

Table 3. Performance of $2.5 \%$ phospholipid for Aspergillus biomarkers . 187

Table 4. Performance of $2.5 \%$ phospholipid for Streptococcus biomarkers 188

Chapter 7. A Unique Set of the Burkholderia Collagen-like Proteins Provides Insight into Pathogenesis, Genome Evolution and Niche Adaptation, and Infection Detection

Table 1. Assessment of genomic plasticity of B. pseudomallei and B. mallei using biomarkers. 231

Table 2. Characterization of Bucl proteins in Burkholderia. 232 


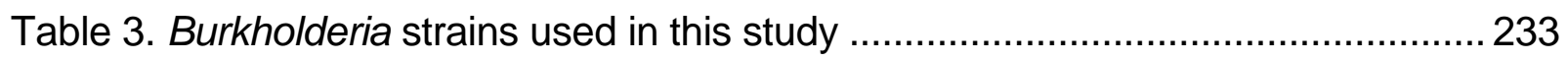
Table 4. Distribution of all buclgenes in Burkholderia spp. as assessed by bioinformatics

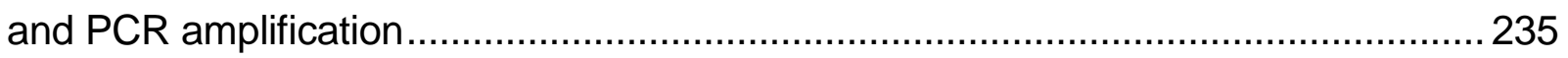

Table 5. Primers and probe used for bucl amplification.......................................... 237

Table 6. Genomic DNA collection ...................................................................... 238 


\section{LIST OF NOMENCLATURE}

1. Scl, streptococcal collagen-like protein

2. $\mathrm{rScl}$, recombinant streptococcal collagen-like protein

3. Scl1.3, Scl1 from M3-type strain

4. Scl2.3, Scl2 from M3-type strain

5. GAS, group A Streptococcus

6. cFn, cellular fibronectin

7. ECM, extracellular matrix

8. EDA/cFn, EDA-containing cellular fibronectin

9. Lm, laminin

10. Bucl, Burkholderia collagen-like protein 


\section{GENERAL INTRODUCTION \\ COLLAGEN-LIKE PROTEINS OF PATHOGENIC STREPTOCOCCI: BIOLOGY, STRUCTURE AND FUNCTION}

\section{Summary}

The collagen domain, which is defined by the presence of the Gly-X-Y triplet repeats, is amongst the most versatile and widespread known structures found in proteins from organisms representing all three domains of life. The streptococcal collagen-like (Scl) proteins are widely present in pathogenic streptococci, including Streptococcus pyogenes, S. agalactiae, S. pneumoniae, and S. equi. Experiments and bioinformatic analyses support that all Scl proteins are homotrimeric and cell wall-anchored. These proteins contain the rod-shaped collagenous domain proximal to cell surface, as well as a variety of outermost non-collagenous domains that generally lack predicted functions but can be grouped into one of six clusters based on sequence similarity. The wellcharacterized Scl1 proteins of $S$. pyogenes show a dichotomous switch in ligand binding modalities between human tissue and blood environments. Scl1 adhesin specifically recognizes the wound microenvironment, resists killing by neutrophil extracellular traps, promotes adhesion and biofilm formation, and modulates S. pyogenes virulence. In blood, binding modalities include components of the complement and coagulation-fibrinolytic systems, as well as plasma lipoproteins. In all, the Scl proteins signify a large family of structurally related surface proteins, which contribute to the ability of streptococci to colonize and cause diseases in humans and animals.

\section{Overview of collagens and collagen-like proteins}

A common structure among diverse proteins

Collagens are ubiquitous in nature. The common feature of the collagen module is a triplehelical structure consisting of three polyproline-II-like helices supercoiled in a righthanded direction around a central axis (1). The tight packing of the polypeptide chains requires a glycine every third residue, defining the Gly-X-Y repeat motif, where proline and hydroxyproline often occupy the $X$ and $Y$ positions of human collagen, respectively. Current knowledge on collagen structure is a culmination of fiber diffraction studies, 
modeling, and crystallographic studies on collagen mimetic peptides. Since a history of the structural dissection of collagen is out of the scope of this review, we direct the reader to several excellent reviews (2-5). The collagens emerged as essential group of modular proteins of metazoans (6). It is a versatile structure, appearing in human extracellular matrix proteins, host defense proteins, and anchoring fibrils (5). The collagen domain is also present in important proteins in invertebrates, such as the exoskeleton collagens of sponges (7), holdfast structure of the mussel byssus (8), and basement membrane and cuticle collagens of the nematode Caenorhabditis elegans (9). There has been a large number of 18,874 collagen-like proteins (CLPs) annotated in bacteria, 695 in viruses, and 157 in archaea (search conducted on 9/24/16 in Uniprot database). The name streptococcal collagen-like proteins, Scl, in S. pyogenes was coined (10), which was followed by Bacillus proteins $\mathrm{Bcl}(11,12)$, pneumococcal protein $\mathrm{Pcl}(13)$, Lcl of Legionella pneumophila (14), and Bucl proteins of Burkholderia spp. (15). However, only a small proportion of predicted bacterial CLPs have been investigated thus far.

\section{Origin of the collagenous domain in bacteria}

The origin of the collagenous domain in prokaryotes is still unknown. However, the composition of the collagen domain in human and bacterial collagens differs considerably. In human collagens, proline and hydroxyproline residues are found preferentially in the $X$ and $Y$ positions, respectively, with frequencies of $27 \%$ and $38 \%$ (3). The abundance of hydroxyprolines in mammalian collagens is a major contributor to structural stability of the triple helix $(16,17)$ but prokaryotes lack the prolyl hydroxylase enzyme to perform this post-translational modification. Therefore, in bacteria more than $30 \%$ of proline residues are found in the $X$ position but only $5 \%$ in the $Y$ position (18). Despite the lack of hydroxyproline residues, bacterial CLPs have been shown to form stable triple helices, with thermal stabilities similar to human collagens, in the range of $35^{\circ}-39^{\circ} \mathrm{C}$, (19-23). These CLPs rely on other mechanisms of helix stabilization, including hydration-mediated hydrogen bonding networks, electrostatic interactions between side chains, and the presence of specific stabilizing tripeptide repeats $(19,24)$. Proper folding of the triple helix is necessary for collagen functionality. 
Horizontal transfer of collagenous sequences from eukaryotes to prokaryotes has been proposed (25). However, recent studies have shown that collagen-like repeats arose independently via separate repeat amplification (26). Low-complexity repeats of the collagen triple helix could emerge by spontaneous mutations and amplify via simple repeat expansion, supporting the hypothesis that eukaryotic and prokaryotic collagens may have emerged by convergent evolution. Here, we present clustering analysis, which identified common domains in Scl proteins from different streptococcal species and subspecies, suggesting that horizontal scl-gene transfer is possible between bacteria that share the same human and animal hosts.

\section{Classification of Scl proteins}

CLuster ANalysis of Sequences (CLANS)

CLANS analysis was performed to obtain a sequence-based classification of Scl proteins. This analysis groups protein sequences based on all-against-all pairwise sequence similarities without phylogenetic reconstruction (27). CLANS identified six distinct clusters (1-6) of Scl proteins across pathogenic streptococci and two clusters (7-8) with phage proteins that will not be discussed here (Figure 1A). All Scl proteins share a distinct set of conserved features and a similar domain organization (Fig. 1B). They contain a signal peptide (not shown), an $\mathrm{N}$-terminal non-collagenous sequence-variable (V) domain, a central collagen-like $(\mathrm{CL})$ domain, and a cell-wall associated domain containing the LPXTG anchor motif (Gram-positive anchor). The CL domains of different Scl proteins are comprised of varying types of Gly-X-Y triplet repeats, and exhibit significant length variation due to the expansion and contraction of these repeats. 

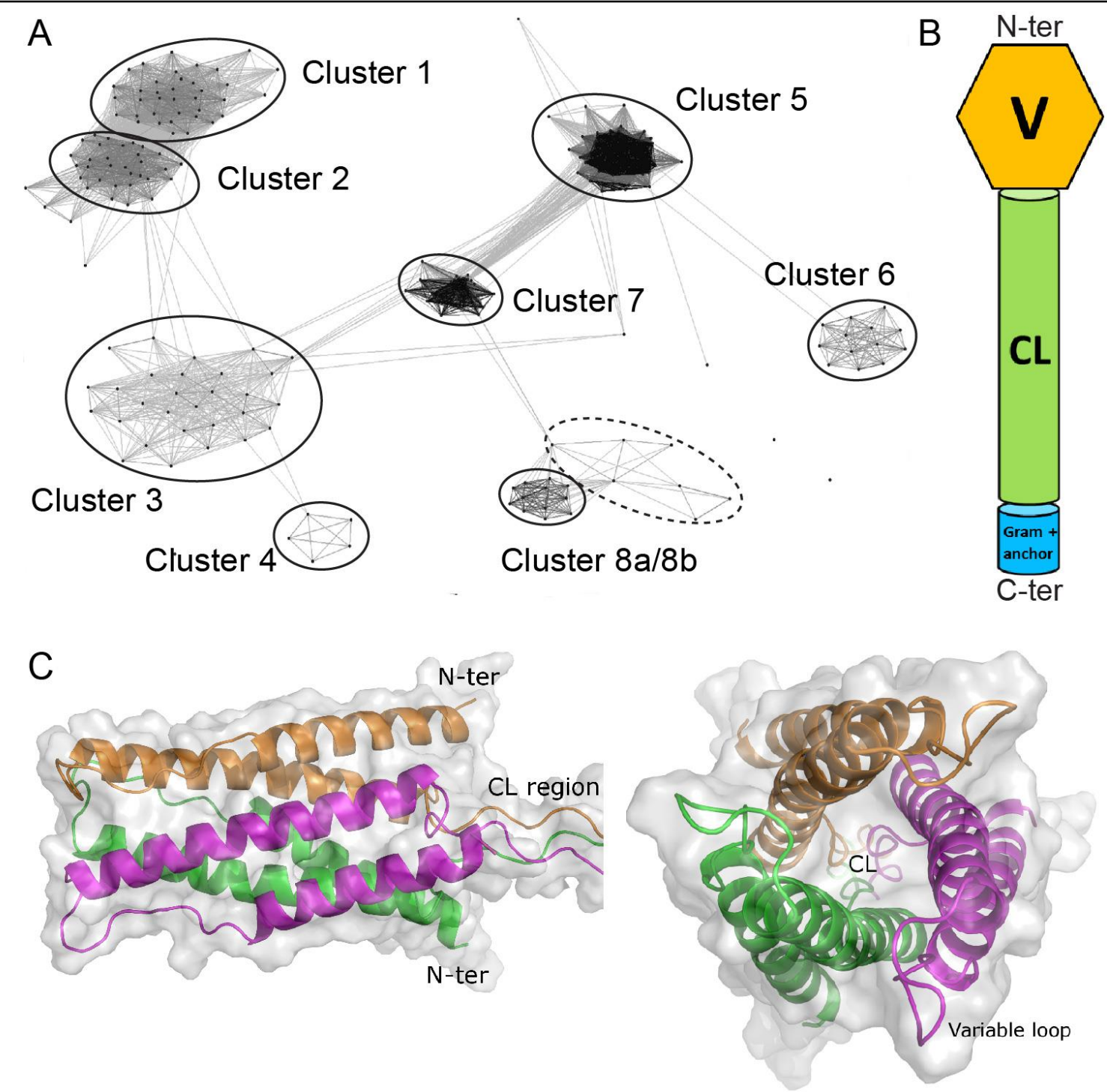

Figure 1. Sequence similarity and structure of Scl proteins in streptococci.

A. Clustering of Scl proteins using CLuster Analysis of Sequences (CLANS). CLANS clusters and visualizes groups of protein sequences based on all-against-all pairwise sequence similarities (e-value cutoff for analysis was $10^{-13}$ ) without phylogenetic reconstruction. Each point on the plot represents a Scl amino acid sequence, while the connecting lines represent BLAST high-scoring segment pairs. High confidence clusters are indicated by circles, while a single low confidence cluster is indicated by a dashed circle. A list of Scl proteins identified in each cluster is provided in Table 1. clusters 7 and $8 \mathrm{a} / 8 \mathrm{~b}$ consist of phage-associated proteins and are not discussed in this review.

B. Cartoon representation of domain organization shared by mature Scl proteins. The Scl proteins consist of an N-terminal noncollagenous variable domain $(\mathrm{V})$, a collagen-like domain $(\mathrm{CL})$ containing repeats of the classical Gly-X-Y motif, and a C-terminally located cell wall-associated Gram-positive anchor with LPXTG motif.

C. Ribbon and surface models of the homotrimeric Scl2-protein globular domain. Left. Side view of the sixhelix bundle, containing three pairs of antiparallel helices, colored green, orange and magenta, and the point of CL-region attachment. Right. Top view, showing the location of exposed hypervariable loop regions between each alpha helix pair and the arrangement of the three external helices wrapped around three internal helices. 
Scl proteins of S. pyogenes (clusters 1,2)

The Scl1 and Scl2 proteins (also known as SclA and SclB) of S. pyogenes are the first bacterial CLPs to be reported and studied (10,28-31). Both proteins are homotrimeric and contain the globular $V$ domain projected away from the cell surface by the rod-shaped CL domain. The $\mathrm{V}$ region sequences differ significantly both between and within Scl1 and Scl2 variants; for each Scl protein, variants are conserved in strains of the same M-type but vary between M-types. The scl1 and scl2 genes have been found in all S. pyogenes strains tested and are co-expressed in the exponential phase of growth, although their expression is regulated differently. Transcription of the $s c / 1$ gene is positively regulated by the multiple gene regulator of $S$. pyogenes, Mga $(28,30,32,33)$. Scl2 expression depends on phase variation associated with CAAAA repeats located downstream of the start codon $(28,29,31)$.

Scl proteins in streptococci pathogenic to animals (clusters 3, 4 and 6)

The Scl3, 4 and 6 proteins are found in S. zooepidemicus, a commensal organism found in domesticated animals rarely transmitting to humans, and $S$. equi, a causative agent of the serious disease strangles in horses (34). CLANS classified several Scl3 proteins, originally denoted $\mathrm{ScIC}$ for group $\mathrm{C}$ Streptococcus (35), as well as the related proteins ScID-I and SclZ.1-5, 7 and 12 (36), as belonging to the same cluster 3 , whereas SclF (37) formed an independent cluster 4. ScIC was shown to be expressed during strangles infection, and immunization with the recombinant ScIC protein partially protects against infection in both mouse and horse models (38-40). An effective multicomponent vaccine for strangles was developed that include recombinant

Table 1. Scl classification by CLANS analysis

\begin{tabular}{|c|c|c|}
\hline $\begin{array}{l}\text { Cluster } \\
\text { No. }\end{array}$ & $\begin{array}{l}\text { Proteins } \\
\text { identified }\end{array}$ & Organisms $^{*}$ \\
\hline 1 & Scl1 (SclA) & S. pyogenes (GAS) \\
\hline 2 & Scl2 (SclB) & S. pyogenes (GAS) \\
\hline 3 & $\begin{array}{l}\text { SclC, D, E, G, } \\
\text { H, I } \\
\text { SclZ.1-5, 7, } 12\end{array}$ & $\begin{array}{l}\text { S. equi, } \\
\text { S. zooepidemicus (GCS) }\end{array}$ \\
\hline 4 & SclF & $\begin{array}{l}\text { S. equi, } \\
\text { S. zooepidemicus (GCS) }\end{array}$ \\
\hline 5 & PclA & $\begin{array}{l}\text { S. pneumoniae, } \\
\text { S. agalactiae (GBS) }\end{array}$ \\
\hline 6 & $\begin{array}{l}\text { SclZ.6, 9, } 10 \\
\text { FneC, E, F }\end{array}$ & $\begin{array}{l}\text { S. equi, } \\
\text { S. zooepidemicus (GCS) }\end{array}$ \\
\hline 7 & $\begin{array}{l}\text { Phage minor } \\
\text { structural } \\
\text { protein }\end{array}$ & $\begin{array}{l}\text { S. pneumoniae, } \\
\text { S. agalactiae (GBS) }\end{array}$ \\
\hline $8 a / b$ & $\begin{array}{l}\text { Phage- } \\
\text { associated } \\
\text { hyaluronidase }\end{array}$ & $\begin{array}{l}\text { S. pyogenes (GAS), } \\
\text { S. equi and } \\
\text { S. dysgalactiae (GCS) }\end{array}$ \\
\hline
\end{tabular}


SclC (40). Several cluster 3 proteins share signature sequences with cluster 2 proteins (35), suggesting some Scl3 variants are related to Scl2 and could emerge via interspecies horizontal gene transfer. A second group of proteins found in both $S$. equi and $S$. zooepidemicus, classified in cluster 6, included the SclZ.6, 9, and 10 proteins (36), as well as $\mathrm{FneC}, \mathrm{E}$, and $\mathrm{F}$ that were annotated as fibronectin-binding proteins (41).

Scl proteins in S. pneumoniae and S. agalactiae (cluster 5)

The pneumococcal CLP, PclA (13), is a large surface protein (265 kDa) that contains predicted G5 and FIVAR domains, though FIVAR was predicted with low confidence. The G5 domain is associated with binding to $\mathrm{N}$-acetylglucosamine and biofilm formation for a variety of proteins found in streptococcal and staphylococcal species (42), as well as the mycobacterial protein, RpfB, which also contains a triple helix motif $(43,44)$. The FIVAR domain is found in surface-associated proteins SasC of Staphylococcus aureus and Embp of $S$. epidermidis that promote fibronectin binding and biofilm formation $(45,46)$. The $S$. agalactiae CLPS also contain the G5 domain, but lack the FIVAR domain. The presence of $p c / A$ gene was correlated with pneumococcal adherence (13) and also with resistance to penicillin and trimethoprim-sulfamethoxazole (47).

The non-collagenous domain in streptococcal CLPS

The best structurally characterized streptococcal collagens are the two CLPs, Scl1 and Scl2, of $S$. pyogenes. The proteins form stable triple-helical structures when expressed as recombinant $(\mathrm{rScl})$ polypeptides $(20,24)$. The non-collagenous $\mathrm{V}$ region constitutes trimerization domain that augments proper collagen assembly to avoid the misfolding of the triple helix due to its repeating structure. However, the $V$ domain of Scls is not necessary for triple helix formation in vivo, since the CL region of Scl1 can be expressed without the $\mathrm{V}$ region as recombinant protein in a folded triple helical state; still, this $\mathrm{rScl}$ CL construct could not re-fold after thermal denaturation in vitro $(20,48)$. These results suggest that $\mathrm{V}$ domains of Scls present both structural and ligand-binding functions reviewed below.

Recently, the crystal structure of the V domain of Scl2 from M3-type S. pyogenes was reported $(49,50)$. The $V$ domain folds into a six-helical bundle, with three pairs of 
antiparallel alpha helices each connected by a variable loop region. Three of the helices are wound in a left-handed super-helix forming the inner core, which is further wrapped by three external alpha helices antiparallel to the internal helices (Fig. 1C). The six-helix bundle forms an elongated cylinder measuring about $30 \AA$ in diameter and $60 \AA$ in height. This fold is consistent with previous secondary structure predictions that deduced a helixloop-helix motif in the primary amino acid sequence $(30,51)$ and is predicted to be conserved among Scl1 and Scl2 proteins. Indeed, hydrophobic residues located at regular positions of Scl sequences were shown to play a central role in the stabilization of the inner core of the 6-helix bundle fold. In addition, the variable loops adopt a welldefined polyproline II conformation (50).

\section{Scl proteins in pathogenesis: dichotomy of Scl1-ligand binding in human tissue and blood}

The Scl1 variants of $S$. pyogenes bind a wide range of host ligands. The nature of Scl1binding modalities between tissue and blood environments is highly significant, as described below (Fig. 2). In contrast, the Scl2 proteins failed to bind the majority of ligands and their role in pathogenesis is less understood.

Binding modalities in tissue to extracellular matrix and cell receptors

Scl1 selectively binds cellular fibronectin (cFn), but not plasma fibronectin ( $p F n)$, and laminin (Lm) (Caswell et al., 2010). Cellular fibronectin is deposited by a variety of cells as an insoluble crosslinked protein in tissues and humans express over $20 \mathrm{cFn}$ isoforms $(52,53)$. Both $\mathrm{pFn}$ and $\mathrm{cFn}$ are encoded by a single fibronectin gene and contain a conserved structure, consisting of three regions of repeats, type I, II, and III. cFn differs from $p F n$ via the inclusion by alternative splicing of extra domains $A$ (EDA) and $B$ (EDB), as well as varying numbers of the variable $V$ domain (53). Scl1 proteins specifically bind cFn via recognition of the type III repeat, EDA (54). EDA/cFn isoforms are found in low levels in normal adult tissue but are upregulated in wounded tissue $(55,56)$, where the EDA domain interacts with keratinocyte integrin receptors (57) and is important in the wound healing processes (58). S. pyogenes strains may express multiple fibronectinbinding proteins, including Sfbl/PrtF1, PrtF2, SOF, FbaB, SfbX, and Shr, reviewed in (59) 
that bind the type I and type II repeats; thus, Scl1 binds cFn via unique mechanism different from other Fn-binding proteins of $S$. pyogenes

Laminin binding was observed for the same Scl1 variants that bind EDA/cFn isoforms (60). The molecular basis for recognizing both ligands is undetermined due to a lack of sequence similarity between EDA and Lm chains $\alpha, \beta$, or $\gamma$. At least 15 laminin heterotrimers have been identified in human tissues and binding studies with individual laminins represent a technical challenge. Two Lm-binding proteins, Lbp and Shr, in $S$. pyogenes have been reported in addition to Scl1 (61-63). Given the localization of some laminins to basement membranes, Lm binding by $S$. pyogenes likely represents a relevant pathogenesis trait.

The Scl1.41 variant, expressed by M41-type strains, directly binds human collagen receptors $\alpha_{2} \beta_{1}$ and $\alpha_{11} \beta_{1}$ integrins through the GLPGER motif in the Scl1-collagenous domain $(64,65)$. A similar binding motif GF/LOGER (O represents hydroxyproline), as well as derived sequence motifs GR/AOGER and GASGER, were identified in human collagens as integrin-binding sites (66). Other Scl-integrin binding motifs are found in Scl2-CL regions, such as RGD and KGD sequences. In addition, the GAPGER and GKPGER motifs are found in ScIZ/Scl3 proteins (36).

\section{Significance of ligand-binding modalities in tissue}

S. pyogenes forms biofilms, or microcolonies, in infected tissue $(67,68)$. In vitro assays showed enhanced biofilms formed by strains of multiple M-types on ECM coatings, including Fn and collagens type I and IV $(69,70)$. Scl1 plays an important role in biofilm formation, as isogenic sc/1-inactivated mutants had significantly reduced overall biofilm biomass and decreased biofilm thickness (71). In addition, enhanced Scl1-mediated biofilms were observed on simple cFn and Lm coatings, as well as on complex extracellular matrix deposited by human dermal fibroblasts (54). Furthermore, inhibition experiments employing EDA-derived peptide and anti-EDA mAb showed significantly reduced adherence of $S$. pyogenes cells to fibroblast-derived matrix, indicating the Scl1$\mathrm{EDA} / \mathrm{cFn}$ interaction supports bacterial adherence

The M3-type S. pyogenes strains, which are particularly invasive to humans, lack Scl1 protein due to a null mutation within the CL-region coding sequence (10), and form 
insignificant biofilms (71). Restoration of full-length Scl1 on the surface of M3-type S. pyogenes restored biofilm formation on $\mathrm{cFn}$ and Lm coatings (72). Also, biofilm-capable Scl1-expressing M41-type strain produced glycocalyx-embedded tissue microcolonies in an in vitro skin equivalent infection model, while biofilm-poor Scl1-lacking M3 strain did not. Rare M3 strains containing the "scl1.3 carrier allele", with restored open reading frame, were less invasive in a mouse model of necrotizing fasciitis than typical M3 strains lacking Scl1 (73). Scl1-deficient mutants of M28- and M41-type S. pyogenes as well as M3-type S. pyogenes naturally lacking Scl1, displayed increased invasiveness in mice (72). Altogether, these results support the concept that $S$. pyogenes adherence to ECM and stable biofilm formation that are conferred by surface-attached Scl1, promote localized infection over invasive spread.

Scl1 binding to the $\alpha_{2} \beta_{1}$ integrin promoted fibroblast adhesion and spreading, and induced intracellular signaling typical of integrin pathway, thus, mimicking the functional role of human collagen. Direct binding of Scl1 to the $\alpha_{2} \beta_{1}$ integrins promotes $S$. pyogenes internalization by epithelial cells, resulting in increased intracellular pools as well as increased re-emergence of bacteria (74). The $\alpha_{2} \beta_{1}$ and $\alpha_{11} \beta_{1}$ integrins are expressed by fibroblasts, endothelial, and epithelial cells $(75,76)$, indicating Scl1 has the potential to adhere to and invade a variety of cell types in the human host. Additionally, the RGD and KGD sequences that are cryptic within the collagen triple helix, become available for binding during tissue remodeling (77).

A recent study reported a novel role for Scl1 of M1 S. pyogenes in resistance to neutrophil extracellular traps (NETs). The Scl1-deficient mutant produced smaller skin lesions in mice in vivo, compared to the parental strain, which was associated with increased killing by NETs in vitro. Neutrophils incubated with Scl1-deficient mutant in vitro displayed increased NET formation than with wild-type strain, indicating Scl1 inhibited NET production by neutrophils. Moreover, Scl1-deficient S. pyogenes had increased sensitivity to cathelicidin killing in vitro (78). Additional studies would confirm if Scl1 variants from divergent M-types also harbor this function. 


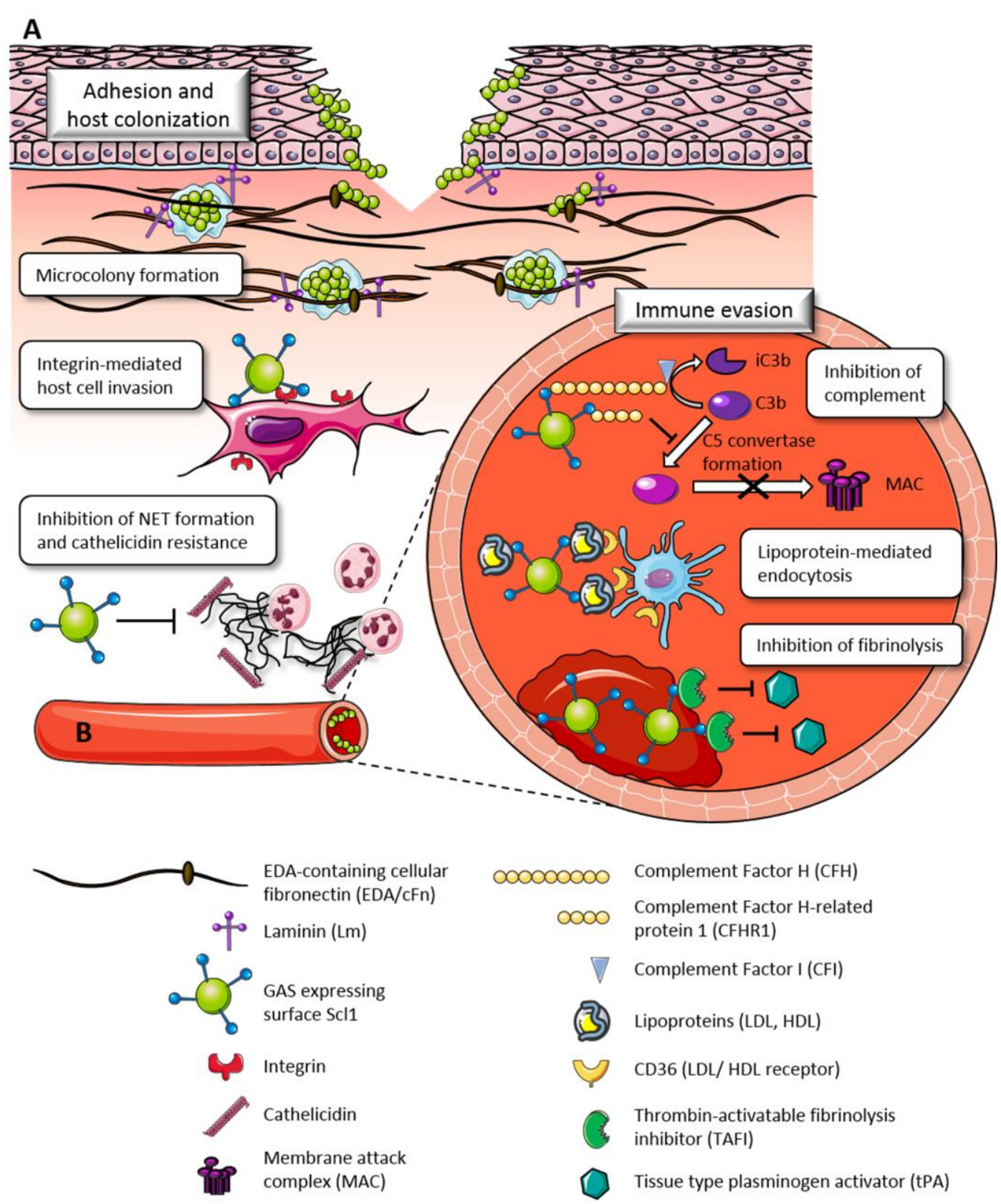

Figure 2. Dichotomous nature of ligand binding by Scl1 in human tissue and blood.

A. Binding modalities in tissue. S. pyogenes accesses human tissue via portal of entry (skin, pharyngeal mucosa) represented here as a breach of skin epidermis extending into the underlying dermis. The globular domain of Scl1 surface adhesin selectively recognizes the EDA-containing isoforms of cellular fibronectin (EDA/cFn) and laminin (Lm). Scl1-mediated tissue microcolonies, embedded in glycocalyx, are formed and stabilized by interactions between Scl1 and the surrounding wound microenvironment. The collagenous domain of Scl1 directly binds human collagen receptors, integrins $\alpha_{2} \beta_{1}$ and $\alpha_{11} \beta_{1}$ on the host cell surface, promoting pathogen internalization, survival and reemergence. Scl1 decreases the formation of extracellular traps (NETs) produced by infiltrating neutrophils and killing by NET-associated cathelicidin. B. Binding modalities in blood. During dissemination to the blood and deeper tissue, Scl1 contributes immune evasion and survival of $S$. pyogenes via several mechanisms. Binding to complement factor $\mathrm{H}(\mathrm{CFH})$ mediates cleavage of $\mathrm{C} 3 \mathrm{~b}$ by factor $\mathrm{I}$, thus, preventing $\mathrm{S}$. pyogenes opsonization, while binding to factor $\mathrm{H}$ related protein 1 (CFHR1) prevents the formation of the C5 convertase and assembly of the membrane attack complex (MAC). Scl1-mediated adsorption of plasma lipoproteins LDL/HDL on the $S$. pyogenes surface may prevent immune recognition and/or promote LDL/HDL receptor-mediated endocytosis, intracellular survival and reemergence. Scl1 binding to and activation of the thrombin-activatable fibrinolysis inhibitor (TAFI) prevents clot breakdown, providing a protective niche for streptococci. 


\section{Binding modalities in blood}

The Scl1 proteins from M6- and M55-type S. pyogenes bind the complement factor $\mathrm{H}$ (CFH) and complement factor H-related protein 1 (CFHR1) via Scl1's globular $\mathrm{V}$ domain (79). The CFH protein is composed of 20 short consensus repeats (SCRs) that facilitate ligand binding and co-factor function for complement factor I (CFI)-mediated C3b degradation; the C-terminal SCRs 18-20 are involved in binding to cell surfaces, while SCRs 1-4 harbors the co-factor function (80). The CFHR1 protein comprises five SCRs, of which the C-terminal SCRs 3-5 share 99\% sequence identity, and therefore surfacebinding function, with the SCRs $18-20$ of CFH. CFHR1 inhibits the C5 convertase and the formation of the membrane attack complex (81). Using recombinant fragments of the $\mathrm{CFH}$ proteins, the Scl1 binding site was mapped in the C-terminal SCRs 19-20 of CFH and SCR 4 of CFHR1 (82). Notably, other known CFH-binding proteins of S. pyogenes, such as certain $\mathrm{M}$ and $\mathrm{M}$-like proteins and $\mathrm{Fba}$, bind to SCR7 of $\mathrm{CFH}$, as well as complement factor H-like (CFHL) protein (82). Borrelia burgdorferi also expresses surface proteins with similar CFH-binding characteristics via the C-terminus $(83,84)$.

Recombinant Scl1 proteins derived from diverse M-types bind the apolipoprotein ApoB100 of low density lipoprotein (LDL) (51). Binding of rScl1 constructs to LDL was facilitated by the Scl1-globular $V$ domain with binding affinities of $K_{D}$ values in the nanomolar range. Importantly, LDL from human plasma was absorbed by the wild-type cells of $S$. pyogenes but not by the scl1-inactivated mutant cells (51). A similar binding with ApoAl apolipoprotein and high density lipoprotein (HDL) was demonstrated for rScl1 construct derived from M41-type S. pyogenes (85). HDL binding was inhibited by low concentration of the nonionic detergent Tween20, suggesting hydrophobic interaction to both rScl1 protein and S. pyogenes cells.

Some recombinant Scl proteins also bind thrombin-activatable fibrinolysis inhibitor (TAFI) with $K_{\mathrm{D}}$ values in the nanomolar range (86) and the binding site was mapped to residues 205-232 within the TAFI protein (87). TAFI is a zinc-dependent procarboxypeptidase, which acts as an important fibrinolysis regulator and inflammatory mediator upon activation by thrombin, thrombin-thrombomodulin complex, or plasmin. 


\section{Significance of ligand-binding modalities in blood}

Diverse pathogens express surface proteins that bind complement regulatory proteins, including Neisseria gonorrhoeae, Borrelia burgdorferi, Yersinia enterocolitica, and several streptococcal species (88). CFH binding to the cell surface prevents C3b deposition and phagocytosis, as well as downstream complement-mediated cell lysis. In vitro, rScl1bound $\mathrm{CFH}$ retained its co-factor function, mediating the proteolytic breakdown of $\mathrm{C} 3 \mathrm{~b}$ by CFI (79). Similarly, CFHR1 binding to rScl1 inhibited the formation of the membrane attack complex in vitro. Both CFH and CFHR1 bound to M6-S. pyogenes cells. However, $\mathrm{CFH}$ binding by M5 $S$. pyogenes did not contribute significantly to phagocytosis resistance or virulence (89). Since M6 protein, was the first bacterial CFH-binding protein reported (90), CFH binding by Scl1, in addition to M6 protein, might be necessary for antiphagocytic phenotype observed for M6 S. pyogenes.

Plasma lipoproteins are being increasingly recognized as innate immune components. For example, HDL and LDL neutralize LPS endotoxin (91) and Staphylococcus aureus a-toxin (92), and HDL is known to downregulate host adhesion molecules and inflammatory cytokines (93). Elevated lipoprotein levels may be protective against bacterial infections and sepsis in humans (94), and LDL-deficient mice showed increased susceptibility to infections with Gram-negative bacteria and Candida albicans $(95,96)$. Interaction between Yersinia pestis and ApoB-containing lipoproteins, mediated by the pH6 antigen, prevented binding of the bacteria to macrophages in vitro (97). In contrast, LDL and HDL could act as opsonins to increase S. pyogenes phagocytosis and killing via a CD36-mediated endocytosis $(98,99)$. Notably, half of $S$. pyogenes strains express serum opacity factor, which binds and disrupts the HDL structure (100), which could protect $S$. pyogenes from lipoprotein-mediated opsonization. Clearly, the Scl1lipoprotein interactions may have multiple functions during infection and in vivo studies are required to determine the effects of these interactions on the host.

Scl-recruited TAFI to the $S$. pyogenes cell surface was cleaved and activated by plasmin and thrombin-thrombomodulin (86). TAFI functions by removing exposed Cterminal lysine residues from fibrin during blood clot formation, thereby preventing recognition and cleavage of these residues by the tissue-type plasminogen activator, ultimately inhibiting fibrinolysis, or the breakdown of clots (101). Additionally, it can 
regulate inflammation by cleaving the $\mathrm{C}$-terminal residues of bradykinin, osteopontin, and the chemoattractants C3a, C5a, and chemerin (102). Therefore, binding and prolonged activation of plasmin on the surface of $S$. pyogenes could contribute significantly to TAFI activation. Additionally, activation of TAFI on the $S$. pyogenes cell surface induced inflammation via modulation of the kallikrein/kinin system (103). Since this interaction maintains the formation of clots, it may also represent a mechanism for bacteria to remain associated with the fibrin clot and evade recognition by immune defenses.

\section{Final Remarks}

i) Modular collagens evolved in higher eukaryotes as members of extracellular matrix to support tissue structure and provide an essential network for cell function. Bacterial collagens likely emerged by convergent evolution via simple Gly-X-Y-repeat amplification; sequence similarity amongst Scl proteins suggests horizontal gene transfer between closely related species.

ii) Scl proteins are homotrimeric and surface attached, and have several modalities of sensing host surroundings. The amino-terminal globular "sensing" domain is variable in primary sequence but displays a conserved structure.

iii) Scl1 proteins have adapted binding modalities in tissue and blood to mediate $S$. pyogenes colonization and immune evasion.

iv) Scl1 down-modulates virulence by fostering the formation of stable tissue microcolonies, thus, promoting a focused nidus of infection.

v) The majority of Scl proteins remain uncharacterized and may exert similar functions to those described for Scl proteins of $S$. pyogenes that are important in pathogenesis. 


\section{PART I}

ROLE OF SCL1 IN ADHERENCE, BIOFILM, AND VIRULENCE 


\title{
CHAPTER 1
}

\section{THE STREPTOCOCCAL COLLAGEN-LIKE PROTEIN-1 (SCL1) IS A SIGNIFICANT DETERMINANT FOR BIOFILM FORMATION BY GROUP A STREPTOCOCCUS}

Heaven A Oliver-Kozup, Meenal Elliott, Beth A Bachert, Karen H Martin, Sean D Reid, Diane E Schwegler-Berry, Brett J Green, and Slawomir Lukomski

Published in BMC Microbiology 2011 11:262

\begin{abstract}
Background: Group A Streptococcus (GAS) is a human-specific pathogen responsible for a number of diseases characterized by a wide range of clinical manifestations. During host colonization GAS-cell aggregates or microcolonies are observed in tissues. GAS biofilm, which is an in vitro equivalent of tissue microcolony, has only recently been studied and little is known about the specific surface determinants that aid biofilm formation. In this study, we demonstrate that surface-associated streptococcal collagenlike protein-1 (Scl1) plays an important role in GAS biofilm formation.

Results: Biofilm formation by M1-, M3-, M28-, and M41-type GAS strains, representing an intraspecies breadth, were analyzed spectrophotometrically following crystal violet staining, and characterized using confocal and field emission scanning electron microscopy. The M41-type strain formed the most robust biofilm under static conditions, followed by M28- and M1-type strains, while the M3-type strains analyzed here did not form biofilm under the same experimental conditions. Differences in architecture and cellsurface morphology were observed in biofilms formed by the M1- and M41-wild-type strains, accompanied by varying amounts of deposited extracellular matrix and differences in cell-to-cell junctions within each biofilm. Importantly, all Scl1-negative mutants examined showed significantly decreased ability to form biofilm in vitro. Furthermore, the Scl1 protein expressed on the surface of a heterologous host, Lactococcus lactis, was sufficient to induce biofilm formation by this organism.
\end{abstract}

Conclusions: Overall, this work (i) identifies variations in biofilm formation capacity among pathogenically different GAS strains, (ii) identifies GAS surface properties that may aid in biofilm stability and, (iii) establishes that the Scl1 surface protein is an important determinant of GAS biofilm, which is sufficient to enable biofilm formation in the 
heterologous host Lactococcus. In summary, the GAS surface adhesin Scl1 may have an important role in biofilm-associated pathogenicity.

\section{INTRODUCTION}

Microbial biofilm formation is an important virulence mechanism, which allows immune evasion and survival against antibiotic treatments $(1,2)$. Many bacterial nosocomial infections are associated with biofilms formed on contaminated medical devices. Dispersal of biofilm has also been proposed to augment infection spread (3-8). For group A Streptococcus (GAS), biofilm research is an emerging field and little is known about the specific surface determinants that aid in biofilm formation. GAS is characteristically associated with significant human morbidity and it is responsible for the clinically common superficial throat and skin infections, such as pharyngitis and impetigo, as well as invasive soft tissue and blood infections like necrotizing fasciitis and toxic shock syndrome (9). Although GAS biofilm has not been associated with implanted medical devices, tissue microcolonies of GAS encased in an extracellular matrix were demonstrated in human clinical specimens (10). Studies reported to date support the involvement of GAS surface components in biofilm formation, including the $M$ and $M$-like proteins, hyaluronic acid capsule, pili and lipoteichoic acid (11-13). As shown by Cho and Caparon (11), multiple genes are upregulated during biofilm formation and development, including the streptococcal collagen-like protein-1 (Scl1).

The scl1 gene encoding the Scl1 protein has been found in every GAS strain investigated and its transcription is positively regulated by Mga (14-18), indicating that Scl1 is co-expressed with a number of proven virulence factors. Structurally, the extracellular portion of Scl1 protein extends from the GAS surface as a homotrimeric molecule composed of distinct domains that include the most outward $\mathrm{N}$-terminal variable (V) region and the adjacent collagen-like $(\mathrm{CL})$ region composed of repeating GlyXaaYaa (GXY) sequence. The linker $(\mathrm{L})$ region is close to the cell surface and contains a series of conserved direct repeats. The Scl1 protein can bind selected human extracellular matrix components (19) and cellular integrin receptors (20-22), as well as plasma components (23-27). 
In this study, we investigated the importance of Scl1 in GAS biofilm using defined isogenic wild-type and sc/1-inactivated mutant strains of GAS. We report that (i) the pathogenically diverse M41-, M28-, M3- and M1-type GAS wild-type strains have varying capacities to produce biofilm on an abiotic surface; (ii) Scl1 plays an important role during the main stages of biofilm formation with Scl1-negative mutants having an abrogated capacity for adhesion, microcolony formation and biofilm maturation; and (iii) variations in surface morphology as well as in extracellular matrix associated with bacterial cells suggest two distinct but plausible mechanisms that potentially stabilize bacterial microcolonies. We additionally show that expression of Scl1 in Lactococcus lactis is sufficient to support a biofilm phenotype. Overall, this work reveals a significant role for the Scl1 protein as a cell-surface component during GAS biofilm formation among pathogenically varying strains.

\section{MATERIALS AND METHODS}

\section{GAS strains and growth conditions}

The wild-type GAS strains M41- MGAS6183, M1 - MGAS5005, and M28-type MGAS6143, as well as their sc/1-inactivated isogenic mutants and complemented M41 $\Delta s c / 1$ mutant have been previously described $(22,27,28)$. In addition, a set of the wild-type M3-type GAS strains MGAS158, MGAS274, MGAS315, MGAS335, MGAS1313, and MGAS2079 was also used. GAS cultures were routinely grown on brain-heart infusion agar (BD Biosciences) and in Todd-Hewitt broth (BD Biosciences) supplemented with $0.2 \%$ yeast extract (THY medium) at $37^{\circ} \mathrm{C}$ in an atmosphere of $5 \% \mathrm{CO}_{2}-20 \% \mathrm{O}_{2}$. Logarithmic phase cultures harvested at the optical density $(600 \mathrm{~nm})$ of about $0.5\left(\mathrm{OD}_{600} \sim 0.5\right)$ were used to prepare GAS inocula for biofilm analysis. Colony counts were verified by plating on tryptic soy agar with $5 \%$ sheep's blood (Remel). Lactococcus lactis subsp. cremoris strain MG1363 (provided by Dr. Anton Steen) were grown using M17 broth or agar media (Oxoid) supplemented with $0.5 \mathrm{M}$ sucrose and $0.5 \%$ glucose (SGM17 media) at $30^{\circ} \mathrm{C}$ in an atmosphere of $5 \% \mathrm{CO}_{2}-20 \% \mathrm{O}_{2}$. 


\section{Heterologous Scl1 expression in Lactococcus lactis}

Lactococcus transformation: To obtain electrocompetent cells, $500 \mathrm{ml}$ of SGM17 broth supplemented with $2 \%$ glycine was inoculated with an overnight culture and grown until $\mathrm{OD}_{600} \sim 0.4$ was reached. Cells were harvested and washed twice with ice-cold solution A $(0.5 \mathrm{M}$ sucrose, $10 \%$ glycerol); cells were then re-suspended in solution A (1/1000 of original culture volume) and stored at $-80^{\circ} \mathrm{C}(29)$. For transformation, cells were thawed on ice and mixed with $1 \mu \mathrm{l}$ of DNA of the Scl1.41-expressing plasmid pSL230 or pJRS525vector(22); and transferred to a cold 1-mm electrode-gap cuvette. Cells were pulsed with $2.0 \mathrm{kV}$ at $25 \mu \mathrm{F}$ and 400 ohms. Immediately following, suspensions were mixed with $1 \mathrm{ml}$ outgrowth medium (SGM17 broth supplemented with $20 \mathrm{mM} \mathrm{MgCl}_{2}$ and $2 \mathrm{mM} \mathrm{CaCl}$ ) and incubated for $2.5 \mathrm{~h}$ before plating on SGM17 agar supplemented with spectinomycin (30).

Molecular characterization of transformants: The pSL230 was detected in Lactococcus lactis MG1363 transformants by PCR amplification directly from bacterial colonies with scl1.41-gene specific primers 232up (5'-CTCCACAAAGAGTGATCAGTC) and 232rev (5'-TTAGTTGTTTTCTTTGCGTTT); pSL230 plasmid DNA was used as a positive control. PCR samples were analyzed on 1\% agarose gel in Tris-acetate-EDTA buffer and stained with ethidium bromide. Inocula from colonies of L. lactis MG1363, as well as colonies harboring either pJRS525 vector or pSL230 construct were used in subsequent experiments.

Western blot analysis: Cell-wall extracts were prepared as previously described (22). Briefly, cells grown to OD $600 \sim 0.4$ were harvested, washed with TES (10 mM Tris, $1 \mathrm{mM}$ EDTA, 25\% Sucrose), re-suspended in TES-LMR (TES containing $1 \mathrm{mg} / \mathrm{ml}$ hen egg lysozyme, $0.1 \mathrm{mg} / \mathrm{ml}$ mutanolysin, $0.1 \mathrm{mg} / \mathrm{ml}$ RNAseA and $1 \mathrm{mM} \mathrm{PMSF}$ ) and incubated at $37^{\circ} \mathrm{C}$ for $1 \mathrm{~h}$. After centrifugation at $2500 \mathrm{~g}$ for $10 \mathrm{~min}$, the supernatants were precipitated with ice-cold TCA ( $16 \%$ final) at $-20^{\circ} \mathrm{C}$ overnight. Precipitates were rinsed thoroughly with ice-cold acetone and dissolved in 1x sample buffer at $250 \mu \mathrm{l}$ per unit $\mathrm{OD}_{600}$. Samples were subjected to $10 \%$ SDS-PAGE, transferred to nitrocellulose, and probed with anti-P176 antiserum followed by goat anti-rabbit-HRP and detected employing chemiluminescent substrate (Pierce). 
Flow cytometry: Bacterial cells were grown to mid-log phase $\left(\mathrm{OD}_{600} \sim 0.4\right)$, washed once with filtered DPBS containing $1 \%$ FBS and re-suspended in the same buffer. Five million cells were incubated with 1:400 dilution of primary reagents, either rabbit pre-bleed (control) or rabbit anti-P176 antiserum for 30 min on ice, washed with DPBS-FBS and then incubated with 1:200 dilution of second reagent donkey anti-rabbit-APC (Jackson ImmunoResearch) for 30 min on ice. After a final wash and re-suspension in DPBS-FBS, flow cytometric data were acquired with FACSCaliber (BD Biosciences) and analyzed employing FCS Express (De Novo Software).

\section{Analysis of biofilm formation}

Crystal violet staining assay: Biofilm formation was tested using tissue culture treated polystyrene 24-well plates. $1.5 \mathrm{ml}$ of logarithmic-phase GAS or Lactococcus cultures were seeded without dilution into wells and incubated at $37^{\circ} \mathrm{C}$ for GAS and $30^{\circ} \mathrm{C}$ for Lactococcus in an atmosphere of $5 \% \mathrm{CO}_{2}-20 \% \mathrm{O}_{2}$ according to indicated time points upon which medium was aspirated. Wells were washed with PBS and $500 \mu \mathrm{l}$ of $1 \%$ crystal violet was added to each well, and incubated at room temperature for $30 \mathrm{~min}$. Dye was then aspirated, wells were washed with PBS, and stain was solubilized with $500 \mu$ of $100 \%$ ethanol. Spectrophotometric readings at $\mathrm{OD}_{600}$ were recorded for each sample per time point. Samples were analyzed in triplicate in at least three experiments.

Confocal Laser Scanning Microscopy (CLSM): To visualize GAS and L. lactis strains by CLSM, bacterial cells were transformed with a GFP-encoding plasmid, pSB027 (31). 15-mm glass cover slips were placed into 24 -well tissue culture plate wells. Logarithmicphase bacterial cultures were inoculated without dilution and grown for $24 \mathrm{~h}$. Cover slips were rinsed with PBS and fixed with 3\% paraformaldehyde at room temperature for 30 min. Biofilms present on cover slips were washed with PBS and mounted onto slides using Prolong Gold mounting media (Invitrogen). Confocal images were acquired using a 63x/1.40 Plan-Apochromat objective and a Zeiss LSM 510 laser scanning confocal on an Axiolmager Z1 microscope. An orthogonal view of the Z-stacks was used to display and measure biofilm thickness using Zeiss LSM software. Ten representative images 
within a single experiment were used to calculate the average vertical thickness measured in micrometers.

To visualize extracellular matrix associated with GAS cells, $24 \mathrm{~h}$ biofilm samples were reacted with $100 \mu \mathrm{g}$ of tetramethyl rhodamine isothiocyanate- (TRITC)-conjugated concanavalin A (TRITC-ConA) (Invitrogen) for $30 \mathrm{~min}$ at room temperature in the dark prior to mounting with Prolong Gold medium. An average of ten microscopic views within each sample was reviewed using the $63 x / 1.40$ objective, as described above.

Field emission scanning electron microscopy (FESEM): GAS biofilm samples were grown for $24 \mathrm{~h}$ on glass cover slips, washed with PBS, and fixed with $3 \%$ paraformaldehyde for $2 \mathrm{~h}$ and post-fixed in osmium tetroxide. Samples were next dehydrated in an ethanol gradient, dried using hexamethyldisalizane, mounted onto aluminum stubs and sputter-coated with gold/palladium. The samples were then imaged on a Hitachi S-4800 field emission scanning electron microscope.

\section{Quantitation of hydrophobicity}

A modified hexadecane method $(12,32,33)$ was used to determine the cell hydrophobicity. Briefly, $5 \mathrm{ml}$ of the logarithmic-phase GAS or Lactococcus cultures (OD600 0.5) were pelleted, washed and re-suspended in $5 \mathrm{ml}$ of PBS. One $\mathrm{ml}$ of hexadecane was added, vortexed for $1 \mathrm{~min}$ and incubated for $10 \mathrm{~min}$ at $30^{\circ} \mathrm{C}$. Mixtures were then vortexed for an additional $1 \mathrm{~min}$ and allowed to stand for $2 \mathrm{~min}$ for phase separation at room temperature. The absorbance of the lower aqueous phase was read at $\mathrm{OD}_{600}$ and compared against the PBS control. Actual hydrophobicity value was calculated using the following equation: Actual Value $=\left[1-\left(A / A_{\circ}\right)\right] \times 100$, where $A$ is $O_{600}$ value after hexadecane treatment and $A_{\circ}$ is $O_{600}$ prior to hexadecane treatment.

\section{Statistical analysis}

Statistical significance was determined using a two-tailed paired Student's $t$-test. The results were considered statistically significant with $P \leq 0.05\left({ }^{*}\right)$ and $P \leq 0.001\left(^{* *}\right)$. 


\section{RESULTS}

\section{Wild-type GAS strains have heterogeneous capacity for biofilm formation on abiotic surfaces}

Biofilm formation was compared between M41-, M28-, M3- and M1-type GAS strains representing distinct epidemiological traits (Figure 1). To assess biofilm formation after $24 \mathrm{~h}$, we used spectrophotometric measurements recorded following crystal violet staining (Figure 1a). Both the M41- and M28-type strains produced more biomass as compared with M1 strain. Furthermore, the M3-type strain produced the lowest absorbance values in a crystal violet assay, indicative of lower cell biomass, as compared with the other wild-type strains. These experiments confirm previous observations $(1,34)$ that GAS strains have varying capacity to form biofilm in vitro.

The failure of M3-type strain MGAS315 to produce substantial cellular biomass in the above assay was intriguing because sequence analysis of the $s c / 1.3$ allele found in MGAS315 revealed the presence of a TAA stop codon in the $11^{\text {th }}$ GXY repeat of the Scl1.3-CL region containing a total of $25 \mathrm{GXY}$ triplets (35). This premature stop codon results in a truncated Scl1.3 variant composed of 102 amino acids $(\sim 11.4 \mathrm{kDa})$, which does not contain the cell wall-membrane (WM) associated region, thus, preventing it from anchoring to the bacterial cell surface (Figure 1b). This prompted us to investigate the biofilm formation by five additional M3-type strains, all harboring the same sc/1.3 allele. Five additional M3-type strains, MGAS335, MGAS1313, MGAS2079, MGAS274 and MGAS158, all harboring the same scl1.3 allele (35) also produced poor biofilm under static conditions, as measured by crystal violet staining. Confocal laser scanning microscopy (CLSM) of three representative strains (MGAS315, MGAS2079, and MGAS158) corroborated results obtained from the crystal violet assay, indicating that these M3-type strains lack the ability to form appreciable biofilm structure. Our data suggest that the lack of capacity for biofilm-formation among M3-type GAS strains examined here might be correlated, at least in part, with lack of surface-attached Scl1.3 protein. 


\section{Microscopic evaluation reveals differences in biofilm surface morphology}

We next conducted microscopic analysis of the biofilms formed by the wild-type (WT) M41-, M28-, and M1-type GAS strains. First, we examined the overall structural characteristics of biofilms formed after $24 \mathrm{~h}$ using CLSM (Figure $4 \mathrm{~d}$-f); Additional file 1: Figure S1 a-f). The average biofilm thickness (see Methods section) differed among all three strains with M1 producing considerably thinner biofilm (mean value of $9 \mu \mathrm{m}$ ) compared to M28 $(12 \mu \mathrm{m})$ and M41 $(15 \mu \mathrm{m})$, a result consistent with lower spectrophotometric absorbance values (Figure 1a). In addition to measured differences in biofilm thickness, closer examination of the $X-Y$ orthogonal Z-stack views, representing biofilm cross-sections, revealed architectural differences among the M41, M28, and M1 biofilms. The M1 biofilm, although the thinnest, seems to consist of densely-packed cells that form continuous layers, while the M28 and especially M41 biofilms seem to be less dense but exhibit more elevated supracellular assembly. We therefore used field emission scanning electron microscopy (FESEM) to define more accurately these supracellular differences observed by CLSM between the biofilms produced by the WT M1 and M41 GAS (Figure 2). FESEM exposed notable architectural differences between biofilms formed by these two strains. The M41 (Figure 2, panel a) biofilm was characterized by more diverse surface architecture with the evidence of depressions or crypts, whereas the M1 biofilm (panel b) seems to lack such pronounced surface characteristics. At higher magnification, the M41 cells have a studded cell surface morphology with protrusions linking both sister cells and cells in adjacent chains (panel c). In contrast, the M1 cells had a relatively smoother appearance likely due to the rich bacterial-associated extracellular matrix (BAEM) surrounding these cells and covering their surface (panel d). BAEM material, which was clearly seen at higher resolution between the M1-type cells, was not as evident between cells of the M41-type GAS.

\section{GAS biofilms differ in production of bacterial-associated extracellular matrix}

The production of BAEM has been shown to be an integral component in the structural integrity of a biofilm, imparting protection from dehydration, host immune attack, and antibiotic sensitivity $(36,37)$. GAS cells encased in a glycocalyx were first identified by Akiyama et al. in skin biopsies obtained from impetigo patients. We therefore compared 
the production of BAEM within biofilms employing GFP-expressing GAS strains of the M1 and M41 type (Figure 3). Cells were grown to form biofilms on glass cover slips for $24 \mathrm{~h}$ and stained with TRITC-concanavalin A (ConA), a fluorescently-labeled lectin that binds to the extracellular polysaccharides in biofilms (38). Fluorescent microscopy was performed to compare matrix production (red staining) by GAS strains (green). Visual screening of both biofilms indicated that the M41-type strain formed a more dispersed extracellular matrix as compared to the M1 strain, which had a dense, more closely associated matrix. In addition, averages of at least 10 fields of ConA stained matrix by CLSM support our FESEM observations that more BAEM is deposited within the biofilm by the M1 GAS cells as compared to M41 GAS. This is in agreement with the report from Akiyama et al that showed a substantial FITC-ConA stained matrix associated with T1type GAS microcolonies in vivo and in vitro (10).

\section{Scl1 protein significantly contributes to biofilm formation by GAS}

Variations in GAS pathogenicity and capacity to form biofilm are driven by specific proteins and components present on the cell surface or are secreted by the organism. It has been shown that deletion of the $\mathrm{M}$ and $\mathrm{M}$-like surface proteins or capsule, as well as increased expression of the secreted SpeB protease decreases biofilm formation dramatically for some strains of GAS $(12,39,40)$. Therefore, we investigated the role of Scl1 in biofilm formation by comparing biofilms formed by GAS WT and sc/1-inactivated $(\Delta s c / 1)$ mutant strains (Figure 4; Additional file 1: Figure S1 a-f). Bacterial biomass was evaluated spectrophotometrically following crystal violet staining at 1, 6, 12, and $24 \mathrm{~h}$ time points, representing different stages of biofilm formation, and absorbance values rendered for the WT and $\Delta s c / 1$ isogenic mutant strains were compared. The M41 $\Delta s c / 1$ mutant showed a $29-35 \%$ decrease in biofilm formation (the $\mathrm{OD}_{600}$ value obtained for the WT strain at each time point was considered $100 \%$ ), which was sustained throughout all time points. This reduction was statistically significant at initial adherence $(1 \mathrm{~h})$, as well as during biofilm development (6-12 h) and at maturation (24 h) (Figure 4a; $P \leq 0.05$ at 1 and $12 \mathrm{~h}, P \leq 0.001$ at 6 and $24 \mathrm{~h}$ ). Complementation of Scl1.41 expression in the $\mathrm{M} 41 \Delta s c / 1$ mutant $(\mathrm{M} 41 \mathrm{C}$ ) restored its ability to form biofilm to WT levels. Similarly, the $\mathrm{M} 28 \Delta \mathrm{sc} / 1$ mutant had a significantly decreased capacity for biofilm formation in the range 
of $29-44 \%$ as compared to WT strain (Figure $4 \mathrm{~b} ; P \leq 0.05$ at 1 and $6 \mathrm{~h}, P \leq 0.001$ at 3,12 and $24 \mathrm{~h}$ ). Likewise, there was a statistically significant decrease in M1 $\Delta s c / 1$ biofilm biomass by $\sim 42-75 \%$ compared to the WT strain (Figure 4c; $P \leq 0.001$ at $1-24 \mathrm{~h}$ ). CLSM analysis of corresponding 24-h biofilms of these strains confirmed our crystal violet staining results at $24 \mathrm{~h}$. The $\Delta s c / 1$ mutants had substantially decreased average biofilm thickness by more than $50 \%$ (mean values) as compared to the parental WT organisms (Figure 4d-f). While these low average biofilm thickness values measured for the $\mathrm{M} 1 \Delta s c / 41(6 \mu \mathrm{M})$ and $\mathrm{M} 28 \Delta s c / 1(5 \mu \mathrm{M})$ correspond to residual biofilms made by those mutants (Figure S1a-d), by comparison, the M1 $\Delta s c / 1(4 \mu \mathrm{M})$ was shown not to produce a continuous biofilm layer under these conditions (Figure S1e-f). Our data support the hypothesis that the Scl1 protein plays an important functional role during GAS biofilm formation and that Scl1 contribution varies among GAS strains with different genetic backgrounds.

\section{Scl1 expression affects surface hydrophobicity}

The surface hydrophobicity of GAS has been shown to influence the adherence to abiotic surfaces. The presence of pili (13) , M and M-like proteins, and lipoteichoic acid contributes to cell surface hydrophobic properties $(12,41)$, which in turn may influence biofilm formation by GAS. Here, we have investigated the contribution of Scl1 to surface hydrophobicity of M41-, M28-, and M1-type GAS strains using a modified hexadecane binding assay $(12,33,42)$. As shown in Table 1, the M28-type GAS strain MGAS6143 gave the highest actual hydrophobicity value of $94.3 \pm 0.73$, followed by the M41-type strain MGAS6183 (92.6 \pm 0.86$)$. In contrast, the overall surface hydrophobicity of the M1type GAS strain MGAS5005 (80.3 \pm 0.89$)$ was significantly lower compared to both M28 and M41 strains ( $P \leq 0.001$ for each comparison). Inactivation of $s c / 1.41$ in M41-type GAS resulted in a modest, although statistically significant, reduction in the hydrophobicity index $(100 \%$ for WT vs. $92 \%$ for mutant, $P \leq 0.001)$. In-trans complementation of the Scl1.41 expression in M41 $\Delta s c / 1-C$ restored the hydrophobic phenotype of the cells to WT level (hydrophobicity index $\sim 105 \%$ ). In comparison, the contribution of the Scl1.1 and Scl1.28 proteins to surface hydrophobicity is more substantial, as evidenced by a $\sim 21 \%$ and $\sim 22 \%$ reduction of the hydrophobicity indices of the mutants as compared to the 
corresponding WT strains, respectively ( $P \leq 0.001$ for both). Thus, the Scl1-mediated GAS-cell surface hydrophobicity reported here may contribute to the ability of this organism to form biofilm, as suggested for other cell surface components $(12,41)$.

\section{Scl1 is sufficient to support biofilm formation in Lactococcus lactis}

To assess whether Scl1 expression is sufficient to confer the ability for biofilm formation, we chose to express this protein in a heterologous $L$. lactis system $(43,44)$. The wild-type L. lactis strain MG1363 was transformed with plasmid pSL230 encoding the Scl1.41 protein (22) or with the shuttle vector pJRS525 alone. As shown in Figure 5a, PCR amplification of the scl1.41 gene employing specific primers yielded no product from the WT L. lactis MG1363 (lane 1) and the MG1363::pJRS525 transformant (lane 2). A product of the expected size of $1.4 \mathrm{~kb}$ was amplified from the pSL230 plasmid DNA control (lane 4,) as well as was amplified from the MG1363::pSL230 transformant (lane 3). Surface expression of Scl1.41 was confirmed by immunoblot analysis of cell-wall extracts prepared from L. lactis WT, and the MG1363::pJRS525 and MG1363::pSL230 transformants, as well as MGAS6163 (WT M41 GAS). As shown in Figure 5b, rabbit antiserum raised against purified recombinant Scl1.41 protein P176 lacking the WM region detected the corresponding immunogen (lane 1), and the homologous full length protein in cell-wall extracts of MGAS6183 (lane 5) as well as MG1363::pSL230 L. lactis transformant (lane 4). This band was absent in cell-wall extracts prepared from the WT L. lactis MG1363 (lane 2) and MG1363::pJRS525 transformant (lane 3). Expression of Scl1.41 at the cell surface was further established by flow cytometry. Rabbit anti-p176 antibodies stained Scl1.41 MG1363::pSL230 transformant, confirming the expression of Scl1.41 protein at the cell surface in the heterologous host $L$. lactis (Figure $5 \mathrm{c}$, red trace). This protein was absent at the surface of WT MG1363 (black trace) and MG1363::pJRS525 transformant (green trace).

The capacity of $L$. lactis expressing Scl1.41 to form biofilm was evaluated spectrophotometrically following crystal violet staining. As shown in Figure $5 d$, the MG1363::pSL230 transformant demonstrated a significant increase in biofilm-associated biomass at $24 \mathrm{~h}$, as compared to wild type L. lactis or L. lactis-containing pJRS525 vector $(P \leq 0.001)$. Crystal violet stained wells were photographed for visual representation of 
biofilm formation prior to spectrophotometric assay. Biofilm thickness and architecture were evaluated by CLSM (Figure 5e; Additional file 1: Figure S2 a-c). The MG1363::pSL230 transformant produced a substantially thicker biofilm (14 $\mu \mathrm{m})$ as compared to both MG1363 WT $(6 \mu \mathrm{m})$ and the vector-only transformant MG1363::pJRS525 (6 $\mu \mathrm{m})$. The MG1363::pSL230 cells formed highly aggregated structures, thus, acquiring a phenotype consistent with biofilm formation. As shown in Table 2, the MG1363::pSL230 transformant, expressing Scl1.41 surface protein, had significantly enhanced cell surface hydrophobicity (hydrophobicity index of $\sim 137 \%$ vs. $100 \%$ WT,$P \leq 0.001$ ) with an actual value of $82.0 \pm 2.6$, when compared to the MG1363 WT $(59.7 \pm 7.2)$ and the vector-only MGAS1363::pJRS525 control (56.6 \pm 5.5$)$. These data suggest a direct relationship between Scl1 expression and cell surface hydrophobicity and establish their involvement in the microorganism's ability to form biofilm in vitro.

\section{DISCUSSION}

Group A Streptococcus strains vary because of the vast number of M-protein types, and this variation is associated with varying frequency of isolation and exacerbation of disease $(45,46)$. The M41-, M28-, M3-, and M1-type strains selected for the current study represent a significant intraspecies diversity among clinical isolates of GAS. M41 GAS was a major causative agent of superficial skin infections (47-49), and strain MGAS6183, harboring the Scl1.41 protein, has been studied extensively $(19,21,22)$. M28-type GAS (strain MGAS6143) has historically been associated with puerperal fever and currently is responsible for extensive human infections world-wide (50). M1T1 GAS, represented by strain MGAS5005, is a globally disseminated clone responsible for both pharyngitis and invasive infections (51-53). The M3-type strains of GAS cause a disproportionally large number of invasive GAS infections that are responsible for traumatic morbidity and death $(54,55)$.

Initial studies by Lembke et al. that characterized biofilm formation among various M types of GAS typically included several strains of the same M type $(1,34)$. These studies reported a significant strain-to-strain variation in ability to form biofilms within each $M$ type. Studies that followed compared biofilm formation by defined isogenic WT and mutant 
strains to assess the contribution of specific GAS surface components responsible for a biofilm phenotype, including $\mathrm{M}$ and $\mathrm{M}$-like proteins, hyaluronic acid capsule, lipoteichoic acid, and pili $(12,13)$. In the current study, we have assessed the role and contribution of the surface protein Scl1 in the ability to support biofilm formation by GAS strains of four distinct $\mathrm{M}$ types.

Recent advances in molecular mega- and pathogenomics has enabled the characterization of numerous M3-type strains with a single nucleotide resolution $(56,57)$. Interestingly, all five M3-type strains MGAS158, 274, 315, 335, and 1313 that were originally used for scl1-gene sequencing (14), plus an additional strain MGAS2079 (not reported) harbor the same sc/1.3 allele containing a null mutation that would result in secretion of a truncated Scl1.3-protein variant. Here, we demonstrate that these GAS strains do not form biofilm on an abiotic surface. Recently, bioinformatic screening of the sequences of $\sim 250$ invasive M3-type strains isolated globally, has led to the detection of this single nucleotide polymorphism that results in disruption of Scl1.3 protein (Steve Beres and Jim Musser, personal communication). Lembke et al. reported heterogeneous biofilm formation among four M3-type GAS strains examined over a 24, 48, and 72-hour period (34). Biofilm was detected for one strain at a $48 \mathrm{~h}$ time point, on a fibrinogen-coated surface; however, it is not known whether this clinical isolate forms biofilm on abiotic surface, whether it expresses the truncated or full-length Scl1.3 protein, and whether it produces an unknown fibrinogen-binding protein, which could augment the attachment and biofilm formation. Therefore, additional studies are necessary to define the contributions of other biofilm-formation determinants in M3-type strains.

Inasmuch as, variation in biofilm formation among GAS isolates of the same Mtype has been established, the molecular basis of this phenotypic variation is not known. Several GAS surface-associated and secreted components were shown to contribute to variation in biofilm $(12,13,39)$. In addition, transcription regulators, such as Mga, CovR, and Srv are likely to play substantial roles in GAS biofilm formation $(11,39)$ due to their transcriptional regulation of numerous genes. Therefore, it is logical to assume that the combination of genomic/proteomic make up, allelic polymorphisms, and transcription regulation all contribute to this phenomenon. In addition, discrepancies between in vitro data obtained with laboratory-stored strains and microcolony formation in vivo likely exist 
and add yet another unknown to the complexity of GAS biofilm/microcolony formation and its role in pathogenesis. Despite this complexity, the analyses involving isogenic strains of the same genetic background provide valuable information that allows assessment of the role and contribution of a given GAS component to biofilm formation.

The M1 MGAS5005 strain was shown to form biofilm in vitro and in experimental animals $(8,39,58)$, and the present study demonstrates a significant role of Scl1.1 in this process. Likewise, the MGAS6183 strain, representing M41-type isolates often associated with pyoderma, produced a more robust biofilm biomass under the same experimental conditions and Scl1.41-deficient mutant was found to be an important determinant in this process. Similarly, Scl1.28 protein significantly contributes to a robust biofilm made by the M28-type strain MGAS6143. However, a recent study reported that another surface protein, designated AspA, found in M28-type GAS significantly contributed to biofilm formation (59). The $\triangle a s p A$ isogenic mutant showed $60 \%$ reduction in biofilm formation. The strain MGAS6180, which they used, expresses the same Scl1.28 variant present in the MGAS6143 strain we used; our Scl1.28 mutant showed $\sim 44 \%$ reduction in $24 \mathrm{~h}$ biofilm. We propose that several surface proteins contribute to biofilm formation by M28-type strains including proteins AspA and Scl1.28, and potentially, proteins $\mathrm{F} 1 / \mathrm{Sfbl}$ and $\mathrm{F} 2$ that are also present in these strains (22). This redundancy is likely responsible for the observed residual biofilms produced by the AspA- and Scl1.28deficient mutants.

The observed heterogeneity in biofilm architecture of different GAS strains was previously observed by Lembke et al. (34) and was also documented in the current study using FESEM. In addition, here we report the differences in GAS-cell surface morphology and within cell-to-cell junctions in biofilms formed by M1- and M41-type strains. The structural and genetic determination of these differences is not known since M41 genome has not been sequenced, but may be associated with the presence of additional surface proteins, such as the F2 protein (60) encoded by prtf2 gene found in this strain (22). Even more striking was an observed difference in the amount of the extracellular material associated with each strain, referred to as BAEM (bacteria-associated extracellular matrix). It has been shown that extracellular matrix, also called glycocalyx, is produced by biofilm-forming bacteria. DNA, lipids, proteins (39), polysaccharides and dead cell 
debris (61) were identified in this matrix and for gram-positive bacteria, teichoic acids have also been detected $(62,63)$. The exopolysaccharide component of the glycocalyx is detected using carbohydrate-binding lectins, such as concanavalin A (ConA) (10). Both FESEM analysis and ConA staining detected more BAEM associated with M1 biofilm compared to M41, which produced larger biofilm. These observations suggest that GAS biofilm is stabilized differently by different strains and that higher BAEM production does not necessarily pre-determine larger biofilm mass. Consequently, a combination of biofilm features rather than biofilm size alone may be more relevant to pathogenicity of a given GAS strain.

Diminished adherence and biofilm formation could be associated with changes in cell surface hydrophobicity (64) of the scl1 mutants. Indeed, the lack of Scl1 resulted in both decreased hydrophobicity and the ability to form biofilm, albeit in a somewhat disproportionate manner. A decrease in the hydrophobicity index by only $\sim 8 \%$, as compared to the wild type-strain, was measured for the $M 41 \Delta s c / 1$ mutant and this modest decrease was accompanied by a rather large reduction in biofilm formation capacity after $24 \mathrm{~h}$ by $30 \%$. Greater decrease in cell-surface hydrophobicity was measured for the $\mathrm{M} 1 \Delta s c / 1$ ( 21\%) and M28 $\Delta s c / 1$ ( 22\%) mutants, which was accompanied by a significant loss in biofilm formation after $24 \mathrm{~h}$ by both isogenic strains by $~ 55 \%$ and $\sim 41 \%$ ( $P \leq 0.001$ for each comparison), respectively. In addition, heterologous expression of Scl.41 in $L$. lactis increased hydrophobicity index of this organism to $~ 137 \%$ of the WT level, which was accompanied by significant increase in its ability to form biofilm. Similar observations have been reported for the $\mathrm{M}$ and $\mathrm{M}$-like protein mutants that typically, but not always, exhibit concurrent loss of both biological features (12). For example, isogenic $\Delta$ Mrp49 mutant had a non-significant drop in hydrophobicity ( 2\%) but significantly lower biofilm formation after $48 \mathrm{~h}$ by $\sim 30 \%$, whereas $\Delta \mathrm{Emm} 1$ mutant lost $\sim 78 \%$ hydrophobicity and $\sim 44 \%$ biofilm formation capacity. In summary: (i) here we report that the Scl1 adhesin is also a hydrophobin with varying contribution to the overall surface hydrophobicity among GAS strains representing different $M$ types and (ii) Scl1-associated surface hydrophobicity is likely to contribute to Scl1-mediated biofilm formation.

To test whether Scl1 alone could support biofilm formation, we used a heterologous $L$. lactis strain, which provides an expression system for membrane-bound 
proteins of gram- positive bacteria with LPXTG cell-wall anchoring motifs $(30,44,65,66)$, including the group A streptococcal M6 protein $(43,67)$. In a recent study by Maddocks et al. (59) it was shown that heterologous expression of AspA GAS surface protein was able to induce a biofilm phenotype in L. lactis MG1363. We were also able to achieve a gainof-function derivative of the L. lactis WT MG1363 strain, (MG1363::pSL230), displaying an altered phenotype associated with biofilm formation, as compared to wild-type parental and vector-only controls. These data support our current model that Scl1 protein is an important determinant of GAS biofilm formation.

As shown by crystal violet staining and CLSM, biofilm formation by the Scl1negative mutants was compromised during the initial stage of adherence, as well as microcolony stabilization and maturation. Consequently, their capacity for biofilm formation as compared to the respective WT controls was greatly reduced. This comparison identifies for the first time that the Scl1 protein contributes significantly to biofilm assembly and stability. Based on these observations, as well as previous work by us and others, we propose the following model of Scl1 contribution to GAS tissue microcolony formation (Figure 6). First, the Scl1 hydrophobin (current study) initiates bacterial adhesion to animate surfaces within the host (64). Next, the Scl1 adhesin anchors the outside edge of growing microcolony in tissue by direct binding to tissue extracellular matrix components, cellular fibronectin and laminin (19). Microcolony development is stabilized by Scl1-Scl1 scaffolding resulting from Scl1's capacity to form head-to-head dimers (68) between molecules located on adjacent chains. This model will be tested experimentally in future studies.

\section{CONCLUSIONS}

In the present work, using pathogenically differing GAS strains, we have demonstrated three concepts. First, we have confirmed previous observations that biofilm formation is an innate property of GAS strains. The M41-type strain used formed a more robust biofilm when compared to M28-type strain as well as M1-type strain. Importantly, the highly invasive M3-type strains devoid of the surface-associated Scl1 also lack the ability to form biofilm. Secondly, the absence of surface-associated Scl1 decreases GAS-cell hydrophobicity suggesting that Scl1 plays a role on the GAS surface as a hydrophobin. 
Thirdly, we have established that the Scl1 protein is a significant determinant for GAS biofilm formation. This concept was further tested by the heterologous expression of Scl1 in Lactococcus, an organism found in dairy fermentation environments, enabling it to form biofilm. Altogether, these data underscore the importance of Scl1 in biofilm-associated regulation of GAS pathogenicity. Recently published work has shown that the recombinant Scl1 binds to the extracellular matrix components, cellular fibronectin and laminin (19). Our current research provides a foundation warranting additional investigation as to whether direct Scl1-ECM binding may promote GAS biofilm as a bridging mechanism within host tissues.

\section{ACKNOWLEDGMENTS}

We would like to thank Dr. Steen for providing the Lactococcus lactis subsp. cremoris strain MG1363. This work was supported in part by National Institutes and Health Grant Al50666 and by a research grant (RFDG) from the West Virginia University Research and Graduate Education (to S. L.). H. Oliver-Kozup was supported by a grant from the West Virginia Graduate Student Fellowship in Science, Technology, Engineering and Mathematics (STEM). Confocal microscopy experiments were performed in the West Virginia University Microscope Imaging Facility, which is supported in part by the Mary Babb Randolph Cancer Center and NIH grant P20 RR016440. We would like to acknowledge the assistance of the West Virginia University Flow Cytometry core facility which was supported in part by a grant P30 RR032138 from the National Institutes of Health. The findings and conclusions in this report are those of the authors and do not necessarily represent the views of the National Institute of Occupational Safety and Health. 


\section{REFERENCES}

1. Conley, J., Olson, M. E., Cook, L. S., Ceri, H., Phan, V., and Davies, H. D. (2003) Biofilm formation by group a streptococci: is there a relationship with treatment failure? J Clin Microbiol 41, 4043-4048

2. Ogawa, T., Terao, Y., Okuni, H., Ninomiya, K., Sakata, H., Ikebe, K., Maeda, Y., and Kawabata, S. (2011) Biofilm formation or internalization into epithelial cells enable Streptococcus pyogenes to evade antibiotic eradication in patients with pharyngitis. Microb Pathog 51, 58-68

3. Boles, B. R., Thoendel, M., and Singh, P. K. (2005) Genetic variation in biofilms and the insurance effects of diversity. Microbiology 151, 2816-2818

4. Lauderdale, K. J., Malone, C. L., Boles, B. R., Morcuende, J., and Horswill, A. R. (2010) Biofilm dispersal of community-associated methicillin-resistant Staphylococcus aureus on orthopedic implant material. J Orthop Res 28, 55-61

5. Kaplan, J. B., Meyenhofer, M. F., and Fine, D. H. (2003) Biofilm growth and detachment of Actinobacillus actinomycetemcomitans. J Bacteriol 185, 1399-1404

6. Raad, II, Fang, X., Keutgen, X. M., Jiang, Y., Sherertz, R., and Hachem, R. (2008) The role of chelators in preventing biofilm formation and catheter-related bloodstream infections. Curr Opin Infect Dis 21, 385-392

7. Wang, R., Khan, B. A., Cheung, G. Y., Bach, T. H., Jameson-Lee, M., Kong, K. F., Queck, S. Y., and Otto, M. (2011) Staphylococcus epidermidis surfactant peptides promote biofilm maturation and dissemination of biofilm-associated infection in mice. J Clin Invest 121, 238-248

8. Connolly, K. L., Roberts, A. L., Holder, R. C., and Reid, S. D. (2011) Dispersal of Group A streptococcal biofilms by the cysteine protease SpeB leads to increased disease severity in a murine model. PLOS ONE 6, e18984

9. Cunningham, M. W. (2000) Pathogenesis of group A streptococcal infections. Clin Microbiol Rev 13, 470-511

10. Akiyama, H., Morizane, S., Yamasaki, O., Oono, T., and Iwatsuki, K. (2003) Assessment of Streptococcus pyogenes microcolony formation in infected skin by confocal laser scanning microscopy. J Dermatol Sci 32, 193-199

11. Cho, K., and Caparon, M. (2005) Patterns of virulence gene expression differ between biofilm and tissue communities of Streptococcus pyogenes. Mol Microbiol 57, 1545-1556

12. Courtney, H. S., Ofek, I., Penfound, T., Nizet, V., Pence, M. A., Kreikemeyer, B., Podbielski, A., Hasty, D. L., et al. (2009) Relationship between expression of the family of $\mathrm{M}$ proteins and lipoteichoic acid to hydrophobicity and biofilm formation in Streptococcus pyogenes. PLoS ONE 4, e4166

13. Manetti, A., Zingaretti, C., Falugi, F., Capo, S., Bombaci, M., Bagnoli, F., Gambellini, G., Bensi, G., et al. (2007) Streptococcus pyogenes pili promote pharyngeal cell adhesion and biofilm formation. Mol Microbiol 64, 968-983

14. Lukomski, S., Nakashima, K., Abdi, I., Cipriano, V. J., Ireland, R. M., Reid, S. D., Adams, G. G., and Musser, J. M. (2000) Identification and characterization of the scl gene encoding a group A Streptococcus extracellular protein virulence factor with similarity to human collagen. Infect Immun 68, 6542-6553

15. Lukomski, S., Nakashima, K., Abdi, I., Cipriano, V. J., Shelvin, B. J., Graviss, E. A., and Musser, J. M. (2001) Identification and characterization of a second 
extracellular collagen-like protein made by group A Streptococcus: control of production at the level of translation. Infect Immun 69, 1729-1738

16. Rasmussen, M., Eden, A., and Bjorck, L. (2000) SclA, a novel collagen-like surface protein of Streptococcus pyogenes. Infect Immun 68, 6370-6377

17. Almengor, A. C., and Mclver, K. S. (2004) Transcriptional activation of sclA by Mga requires a distal binding site in Streptococcus pyogenes. J. Bacteriol. 186, 78477857

18. Almengor, A. C., Walters, M. S., and Mclver, K. S. (2006) Mga is sufficient to activate transcription in vitro of sof-sfb $X$ and other Mga-regulated virulence genes in the group A Streptococcus. J. Bacteriol. 188, 2038-2047

19. Caswell, C. C., Oliver-Kozup, H., Han, R., Lukomska, E., and Lukomski, S. (2010) Scl1, the multifunctional adhesin of group A Streptococcus, selectively binds cellular fibronectin and laminin, and mediates pathogen internalization by human cells. FEMS Microbiol Lett 303, 61-68

20. Humtsoe, J. O., Kim, J. K., Xu, Y., Keene, D. R., Hook, M., Lukomski, S., and Wary, K. K. (2005) A streptococcal collagen-like protein interacts with the a2b1 integrin and induces intracellular signaling. J. Biol. Chem. 280, 13848-13857

21. Caswell, C. C., Barczyk, M., Keene, D. R., Lukomska, E., Gullberg, D. E., and Lukomski, S. (2008) Identification of the first prokaryotic collagen sequence motif that mediates binding to human collagen receptors, integrins a2b1 and a11b1. $J$ Biol Chem 283, 36168-36175

22. Caswell, C. C., Lukomska, E., Seo, N. S., Hook, M., and Lukomski, S. (2007) Scl1dependent internalization of group A Streptococcus via direct interactions with the alpha2beta(1) integrin enhances pathogen survival and re-emergence. $\mathrm{Mol}$ Microbio/ 64, 1319-1331

23. Gao, Y., Liang, C., Zhao, R., Lukomski, S., and Han, R. (2010) The Scl1 of M41type group A Streptococcus binds the high-density lipoprotein. FEMS Microbiol Lett 309, 55-61

24. Pahlman, L. I., Marx, P. F., Morgelin, M., Lukomski, S., Meijers, J. C. M., and Herwald, H. (2007) Thrombin-activatable Fibrinolysis Inhibitor Binds to Streptococcus pyogenes by Interacting with Collagen-like Proteins A and B. J. Biol. Chem. 282, 24873-24881

25. Caswell, C., Han, R., Hovis, K., Ciborowski, P., Keene, D., Marconi, R., and Lukomski, S. (2008) The Scl1 protein of M6-type group A Streptococcus binds the human complement regulatory protein, factor $\mathrm{H}$, and inhibits the alternative pathway of complement. Mol Microbio/ 67, 584-596

26. Reuter, M., Caswell, C. C., Lukomski, S., and Zipfel, P. F. (2010) Binding of the human complement regulators CFHR1 and factor $\mathrm{H}$ by streptococcal collagen-like protein 1 (Scl1) via their conserved $C$ termini allows control of the complement cascade at multiple levels. J Biol Chem 285, 38473-38485

27. Han, R., Caswell, C. C., Lukomska, E., Keene, D. R., Pawlowski, M., Bujnicki, J. M., Kim, J. K., and Lukomski, S. (2006) Binding of the low-density lipoprotein by streptococcal collagen-like protein Scl1 of Streptococcus pyogenes. Mol Microbiol 61(2), 351-367

28. Lukomski, S., Hoe, N. P., Abdi, I., Rurangirwa, J., Kordari, P., Liu, M., Dou, S. J., Adams, G. G., et al. (2000) Nonpolar inactivation of the hypervariable 
streptococcal inhibitor of complement gene (sic) in serotype M1 Streptococcus pyogenes significantly decreases mouse mucosal colonization. Infect Immun 68, 535-542

29. Holo, H., and Nes, I. F. (1989) High-Frequency Transformation, by Electroporation, of Lactococcus lactis subsp. cremoris Grown with Glycine in Osmotically Stabilized Media. Appl Environ Microbiol 55, 3119-3123

30. Que, Y. A., Haefliger, J. A., Francioli, P., and Moreillon, P. (2000) Expression of Staphylococcus aureus clumping factor A in Lactococcus lactis subsp. cremoris using a new shuttle vector. Infect Immun 68, 3516-3522

31. Cramer, T., Yamanishi, Y., Clausen, B. E., Forster, I., Pawlinski, R., Mackman, N., Haase, V. H., Jaenisch, R., et al. (2003) HIF-1alpha is essential for myeloid cellmediated inflammation. Cell 112, 645-657

32. Grivet, M., Morrier, J. J., Benay, G., and Barsotti, O. (2000) Effect of hydrophobicity on in vitro streptococcal adhesion to dental alloys. J Mater Sci Mater Med 11, 637642

33. Pan, W. H., Li, P. L., and Liu, Z. (2006) The correlation between surface hydrophobicity and adherence of Bifidobacterium strains from centenarians' faeces. Anaerobe 12, 148-152

34. Lembke, C., Podbielski, A., Hidalgo-Grass, C., Jonas, L., Hanski, E., and Kreikemeyer, B. (2006) Characterization of Biofilm Formation by Clinically Relevant Serotypes of Group A Streptococci. Appl. Environ. Microbiol. 72, 28642875

35. Lukomski, S., Sreevatsan, S., Amberg, C., Reichardt, W., Woischnik, M., Podbielski, A., and Musser, J. M. (1997) Inactivation of Streptococcus pyogenes extracellular cysteine protease significantly decreases mouse lethality of serotype M3 and M49 strains. J. Clin. Invest. 99, 2574-2580

36. Donlan, R. M. (2002) Biofilms: microbial life on surfaces. Emerg Infect Dis 8, 881890

37. Kania, R. E., Lamers, G. E., Vonk, M. J., Huy, P. T., Hiemstra, P. S., Bloemberg, G. V., and Grote, J. J. (2007) Demonstration of bacterial cells and glycocalyx in biofilms on human tonsils. Arch Otolaryngol Head Neck Surg 133, 115-121

38. Maeyama, R., Mizunoe, Y., Anderson, J. M., Tanaka, M., and Matsuda, T. (2004) Confocal imaging of biofilm formation process using fluoroprobed Escherichia coli and fluoro-stained exopolysaccharide. J Biomed Mater Res A 70, 274-282

39. Doern, C. D., Roberts, A. L., Hong, W., Nelson, J., Lukomski, S., Swords, W. E., and Reid, S. D. (2009) Biofilm formation by group A Streptococcus: a role for the streptococcal regulator of virulence (Srv) and streptococcal cysteine protease (SpeB). Microbiology 155, 46-52

40. Roberts, A. L., Connolly, K. L., Doern, C. D., Holder, R. C., and Reid, S. D. (2010) Loss of the group A Streptococcus regulator Srv decreases biofilm formation in vivo in an otitis media model of infection. Infect Immun 78, 4800-4808

41. Wadstrom, T., Schmidt, K. H., Kuhnemund, O., Havlicek, J., and Kohler, W. (1984) Comparative Studies on Surface Hydrophobicity of Streptococcal Strains of Group-a, Group-B, Group-C, Group-D and Group-G. J. Gen. Microbiol. 130, 657664 
42. Grivetti, L. E., and Ogle, B. M. (2000) Value of traditional foods in meeting macroand micronutrient needs: the wild plant connection. Nutr Res Rev 13, 31-46

43. Piard, J. C., Hautefort, I., Fischetti, V. A., Ehrlich, S. D., Fons, M., and Gruss, A. (1997) Cell wall anchoring of the Streptococcus pyogenes M6 protein in various lactic acid bacteria. J. Bacteriol. 179, 3068-3072

44. Linares, D. M., Kok, J., and Poolman, B. (2010) Genome Sequences of Lactococcus lactis MG1363 (Revised) and NZ9000 and Comparative Physiological Studies. J. Bacteriol. 192, 5806-5812

45. Whatmore, A. M., Kapur, V., Sullivan, D. J., Musser, J. M., and Kehoe, M. A. (1994) Non-congruent relationships between variation in emm gene sequences and the population genetic structure of group A streptococci. Mol. Microbiol. 14, 619-631

46. Bessen, D. E., Sotir, C. M., Readdy, T. L., and Hollingshead, S. K. (1996) Genetic corelates of throat and skin isolates of group A streptococci. J. Infect. Dis. 173, 896-900

47. Anthony, B. F. (2000) Streptococcal pyoderma. in Streptococcal infections (Stevens, D. L., and Kaplan, E. L. eds.), Oxford University Press, New York, N. Y. pp 144-151

48. Anthony, B. F., Perlman, L. V., and Wannamaker, L. W. (1967) Skin Infections And Acute Nephritis In American Indian Children. Pediatrics 39, 263-279

49. Top, F. H., Jr., Wannamaker, L. W., Maxted, W. R., and Anthony, B. F. (1967) M antigens among group A streptococci isolated from skin lesions. J Exp Med 126, 667-685

50. Green, N. M., Beres, S. B., Graviss, E. A., Allison, J. E., McGeer, A. J., VuopioVarkila, J., LeFebvre, R. B., and Musser, J. M. (2005) Genetic Diversity among Type emm28 Group A Streptococcus Strains Causing Invasive Infections and Pharyngitis. $J$ Clin Microbiol 43, 4083-4091

51. Aziz, R., and Kotb, M. (2008) Rise and persistence of global M1T1 clone of Streptococcus pyogenes. Emerg Infect Dis 14, 1511-1517

52. Aziz, R. K., Edwards, R. A., Taylor, W. W., Low, D. E., McGeer, A., and Kotb, M. (2005) Mosaic Prophages with Horizontally Acquired Genes Account for the Emergence and Diversification of the Globally Disseminated M1T1 Clone of Streptococcus pyogenes. J. Bacteriol. 187, 3311-3318

53. Musser, J. M., Kapur, V., Szeto, J., Pan, X., Swanson, D. S., and Martin, D. R. (1995) Genetic diversity and relationships among Streptococcus pyogenes strains expressing serotype M1 protein: recent intercontinental spread of a subclone causing episodes of invasive disease. Infect. Immun. 63, 994-1003

54. Kaul, R., McGeer, A., Low, D. E., Green, K., Schwartz, B., Study, O. G. A. S., and Simor, A. E. (1997) Population-based surveillance for group A streptococcal necrotizing fasciitis: clinical features, prognostic indicators, and microbiologic analysis of seventy-seven cases. Am. J. Med. 103, 18-24

55. Sharkawy, A., Low, D. E., Saginur, R., Gregson, D., Schwartz, B., Jessamine, P., Green, K., and McGeer, A. (2002) Severe group a streptococcal soft-tissue infections in Ontario: 1992-1996. Clin Infect Dis 34, 454-460

56. Beres, S. B., Sylva, G. L., Barbian, K. D., Lei, B., Hoff, J. S., Mammarella, N. D., Liu, M.-Y., Smoot, J. C., et al. (2002) Genome sequence of a serotype M3 strain 
of group A Streptococcus: Phage-encoded toxins, the high-virulence phenotype, and clone emergence. Proc. Natl. Acad. Sci. USA 99, 10078-10083

57. Beres, S. B., Sylva, G. L., Sturdevant, D. E., Granville, C. N., Liu, M., Ricklefs, S. M., Whitney, A. R., Parkins, L. D., et al. (2004) Genome-wide molecular dissection of serotype M3 group A Streptococcus strains causing two epidemics of invasive infections. Proc. Natl. Acad. Sci. USA 101, 11833-11838

58. Roberts, A. L., Connolly, K. L., Doern, C. D., Holder, R. C., and Reid, S. D. (2010) Loss of the group A Streptococcus regulator Srv decreases biofilm formation in vivo in an otitis media model of infection. Infect Immun 78, 4800-4808

59. Maddocks, S. E., Wright, C. J., Nobbs, A. H., Brittan, J. L., Franklin, L., Stromberg, N., Kadioglu, A., Jepson, M. A., et al. (2011) Streptococcus pyogenes antigen I/IIfamily polypeptide AspA shows differential ligand-binding properties and mediates biofilm formation. Mol Microbiol 81, 1034-1049

60. Jaffe, J., Natanson-Yaron, S., Caparon, M. G., and Hanski, E. (1996) Protein F2, a novel fibronectin-binding protein from Streptococcus pyogenes, possesses two domains. Mol Microbiol 21, 373-384

61. Branda, S. S., Gonzalez-Pastor, J. E., Dervyn, E., Ehrlich, S. D., Losick, R., and Kolter, R. (2004) Genes involved in formation of structured multicellular communities by Bacillus subtilis. J. Bacteriol. 186, 3970-3979

62. Gotz, F. (2002) Staphylococcus and biofilms. Mol. Microbiol. 43, 1367-1378

63. Nadell, C. D., Xavier, J. B., and Foster, K. R. (2009) The sociobiology of biofilms. FEMS Microbiol. Rev. 33, 206-224

64. Courtney, H. S., Dale, J. B., and Hasty, D. L. (2000) Strategies for preventing group A streptococcal adhesion and infection. in Handbook of bacterial adhesion: principles, methods, and applications (An, Y. H., and Friedman, R. J. eds.), Humana Press, Inc., Totowa, N. J. pp 553-579

65. Luo, H. L., Wan, K., and Wang, H. H. (2005) High-frequency conjugation system facilitates biofilm formation and pAM beta 1 transmission by Lactococcus lactis. Appl Environ Microb 71, 2970-2978

66. Gerber, S. D., and Solioz, M. (2007) Efficient transformation of Lactococcus lactis IL1403 and generation of knock-out mutants by homologous recombination. $J$ Basic Microb 47, 281-286

67. Piard, J. C., JimenezDiaz, R., Fischetti, V. A., Ehrlich, S. D., and Gruss, A. (1997) The M6 protein of Streptococcus pyogenes and its potential as a tool to anchor biologically active molecules at the surface of lactic acid, bacteria. Streptococci and the Host 418, 545-550

68. Xu, Y., Keene, D. R., Bujnicki, J. M., Hook, M., and Lukomski, S. (2002) Streptococcal Scl1 and Scl2 proteins form collagen-like triple helices. J Biol Chem 277, 27312-27318 
(a)

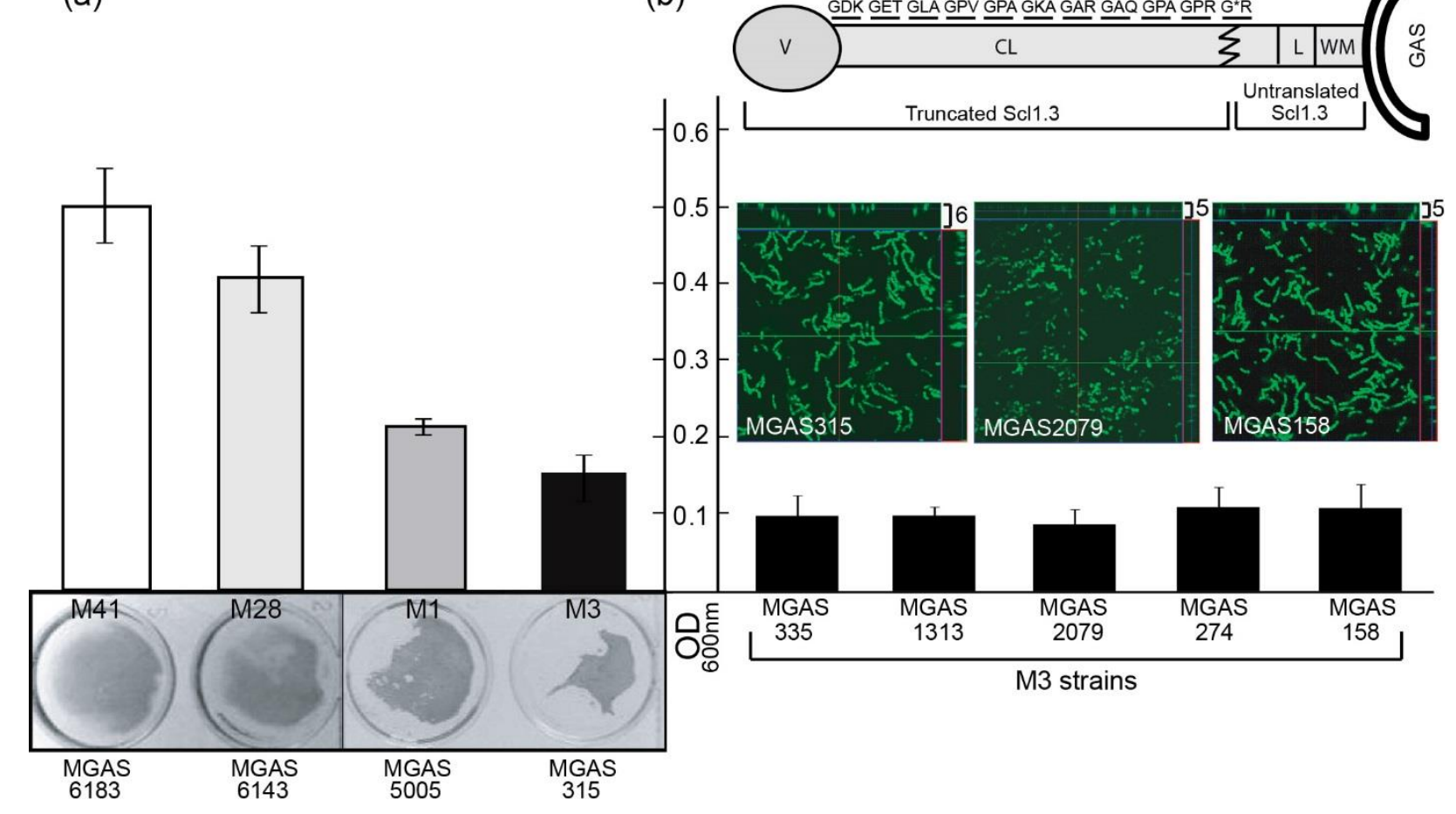

Figure 1

Variation in biofilm formation among GAS strains. (a) Wild type M41-, M28-, M3-, and M1-type GAS strains were grown $24 \mathrm{~h}$ under static conditions and analyzed spectrophotometrically following crystal violet staining (top). Visual representation of corresponding wells is shown below. (b) Schematic representation (not to scale) of Scl1.3 protein of M3-type GAS. Translated GXY repeats within the collagen-like (CL) region are shown with an asterisk representing the location of the premature stop codon resulting in a truncated protein. V, variable region; L, linker region; WM, wall-membrane associated region. Below, spectrophotometric measurements of 24-h biofilms following crystal violet staining are graphed for M3-type GAS strains. Absorbance values $\left(\mathrm{OD}_{600}\right)$ are averages of at least three experiments done in triplicate wells. Corresponding confocal analyses of 24-h biofilms of MGAS315, MGAS2079, and MGAS158 are shown. Images are X-Y orthogonal Z-stack views and average vertical thickness is indicated in micrometers (top right). 
(a)

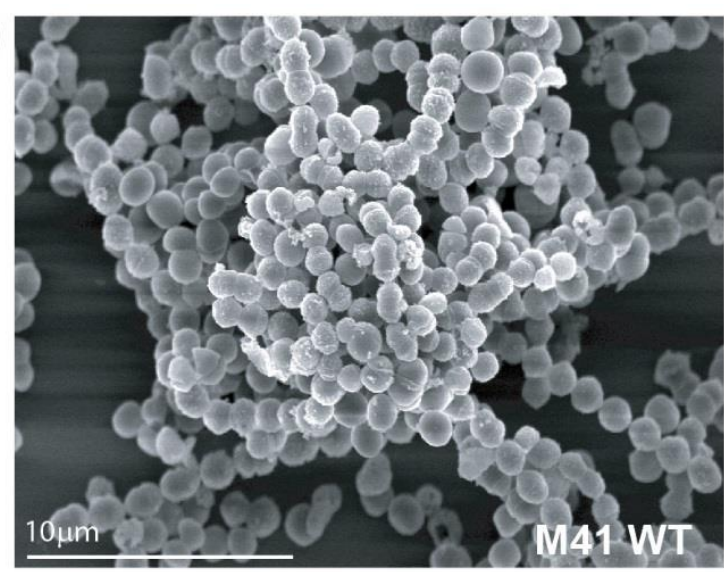

(c)

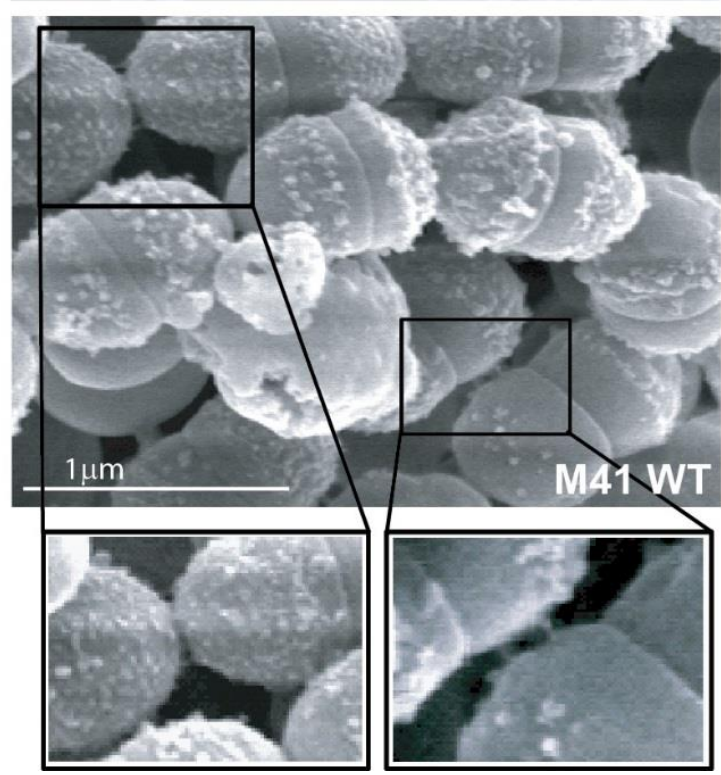

(b)

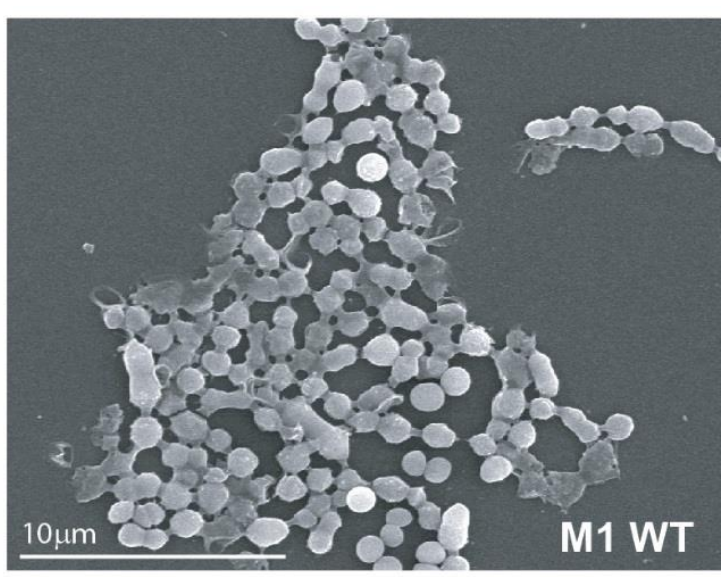

(d)

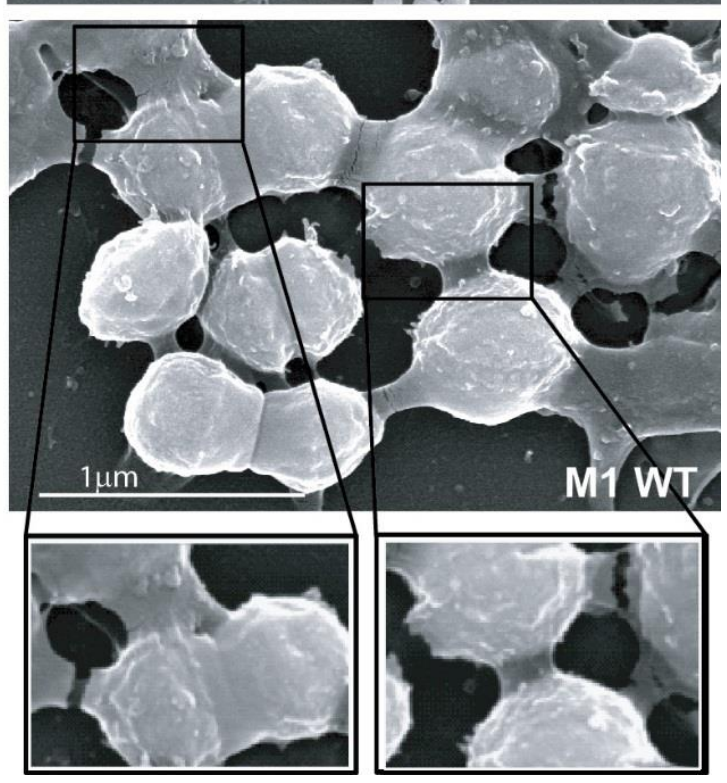

Figure 2

Field emission scanning electron microscopy of GAS biofilms. 24-h biofilms of the M1 - and M41-type GAS strains were grown on glass cover slips and analyzed by FESEM. (a-b) Architecture of GAS microcolonies shown at low magnification. (c-d) Cell surface morphology and cell-to-cell junctions observed at higher magnification. Enlargements of cell-to-cell junctions are shown below. 

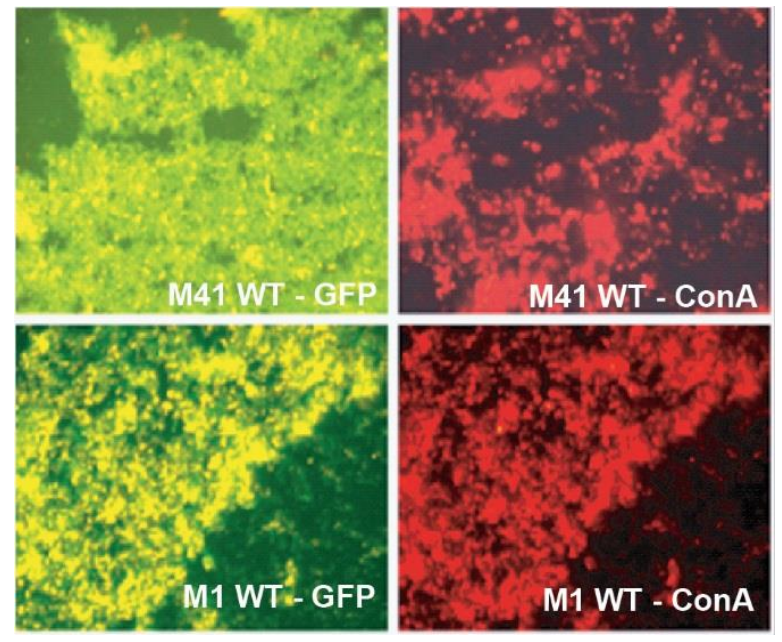

Figure 3

Production of bacterial-associated extracellular matrix. GFP-expressing wild type (WT) M41- and M1-type GAS strains were grown on glass cover slips for $24 \mathrm{~h}$ and stained with TRITC-conjugated concanavalin A (ConA). Confocal laser scanning microscopic (CLSM) images were separated to represent green GFP-expressing GAS cells (left images) and red ConA-TRITC staining (right images) for detection of extracellular matrix associated with each strain. Images are from one representative experiment. 
(a)

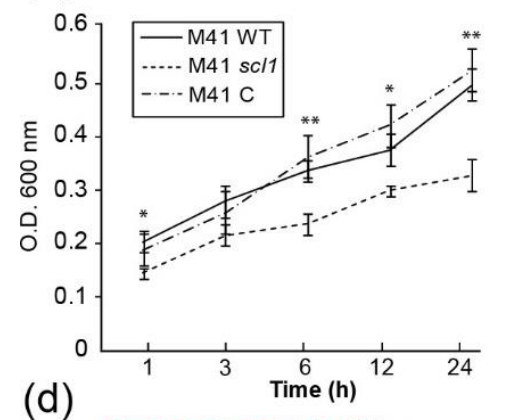

(d)
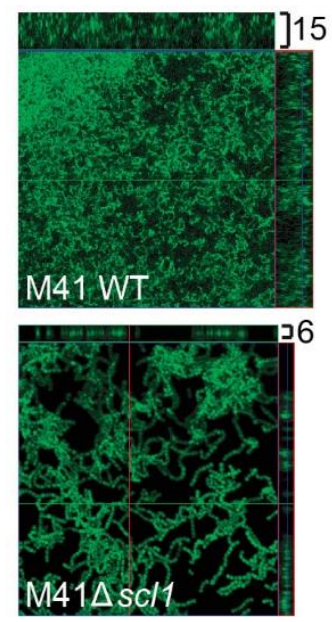

(b)

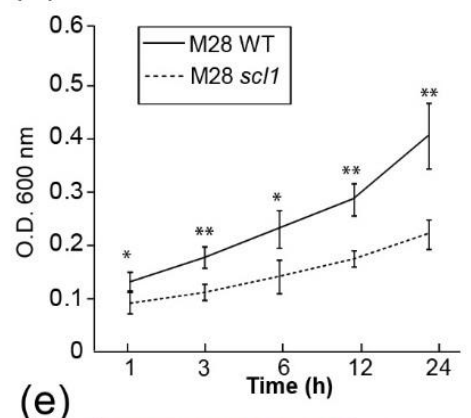

(e)
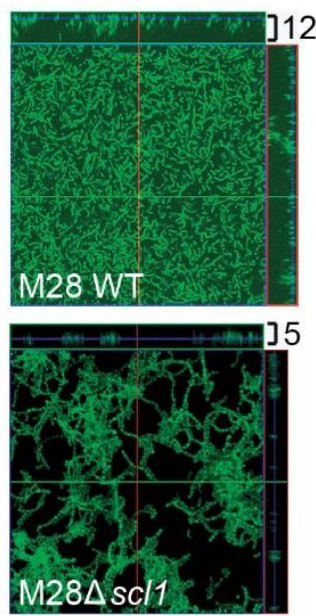

(c)

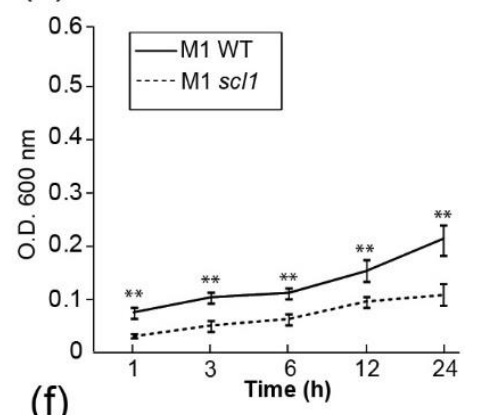

(f)
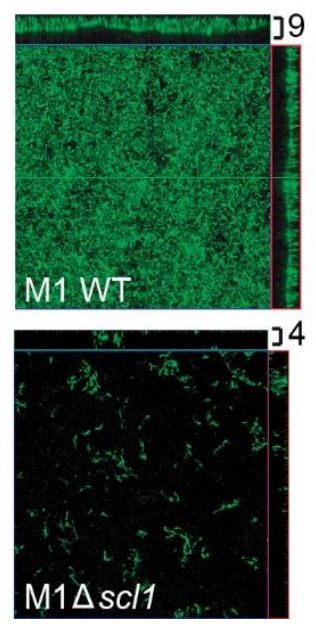

Figure 4

Biofilm formation by wild type and sc/1-inactivated isogenic mutants. Crystal violet staining and confocal laser scanning microscopy (CLSM) of the GFP-expressing GAS were used to compare biofilm formation by GAS strains. Wild type (WT) M41-, M28-, and M1-type strains, sc/1-inactivated mutants (sc/1), and M41 mutant complemented for Scl1.41 expression (M41 C) were used. (a-c) Isogenic GAS strains were grown under static conditions for $24 \mathrm{~h}$ and bacterial biomass was detected spectrophotometrically at indicated time points following crystal violet staining. Absorbance values at $\mathrm{OD}_{600 \mathrm{~nm}}$ are representative of at least three experiments performed in quadruplicate. Statistical significance is denoted as ${ }^{*} P \leq 0.05$ and ${ }^{* *} P \leq 0.001$. (d-f) CLSM analysis of corresponding $24 \mathrm{~h}$ biofilms from same experiment. Images are $\mathrm{X}-\mathrm{Y}$ orthogonal Z-stack views of WT (top) and mutant (bottom) GAS strains. Views are representative of ten images within a single experiment. Average vertical biofilm thickness is indicated in micrometers (top right). 

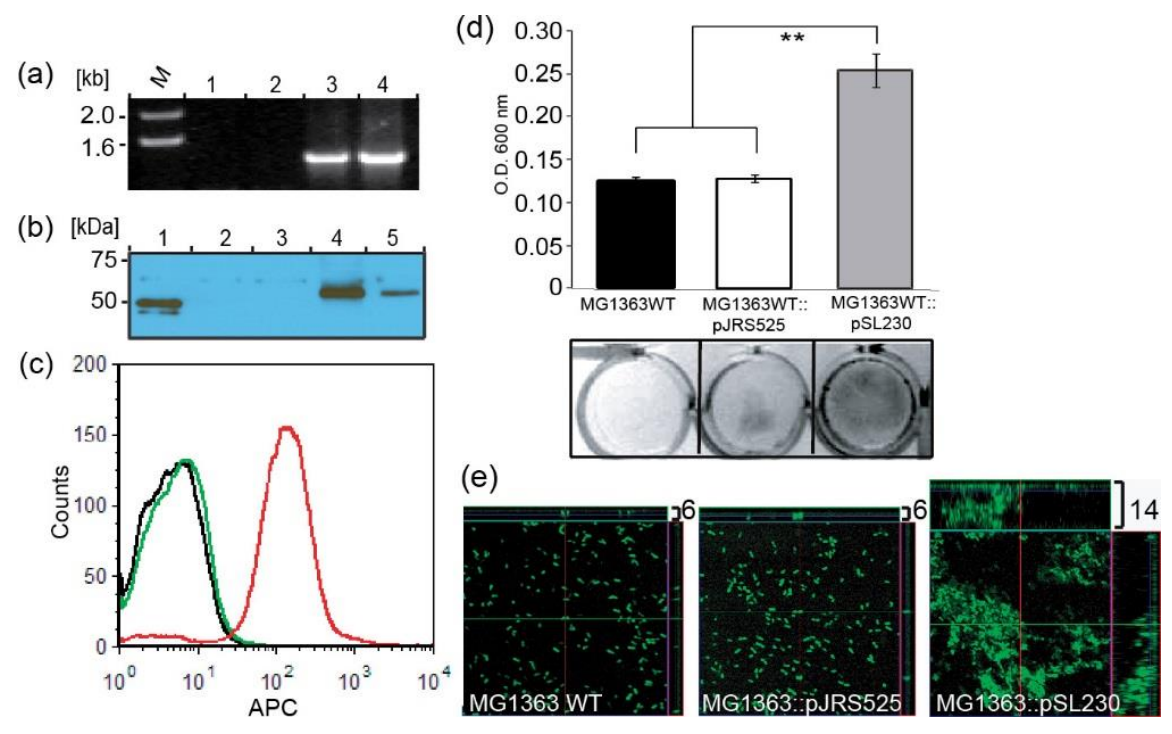

Figure 5

Scl1 expression in L. lactis promotes biofilm formation. L. lactis was transformed with the plasmid construct pSL230 to express Scl1.41 surface protein or with pJRS525 vector. (a) PCR analysis of $L$. lactis transformants using scl1.41-gene-specific primers; lanes: (1) MG1363 wild-type (WT) cells; (2) MG1363::pJRS525 vector-only control; (3) MG1363::pSL230 transformant; (4) control pSL230 plasmid DNA. (b) Scl1.41 expression by western blot analysis of cell-wall extracts prepared from transformed $L$. lactis and control GAS strains using anti- P176 (rScl1.41) antibodies; lanes: (1) purified recombinant P176 protein (truncated Scl1.41); (2) MG1363 WT strain; (3) MG1363::pJRS525 vector; (4) MG1363::pSL230 transformant; (5) MGAS6183 (M41) control. (c) Analysis of Sc1.41 expression by flow cytometry with anti-P176 (rScl1.41) rabbit polyclonal antibodies on the surface of MGAS1363 WT strain (black trace), MGAS1363::pJRS525 vector-only control (green trace) and MG1363:pSL230 transformant (red trace). (d) Crystal violet staining of $24 \mathrm{~h}$ biofilms formed by L. lactis WT strain, MG1363::pJRS525 vector-only control or MG1363::pSL230 transformant (top) with visual representation of the corresponding wells (bottom). Statistical significance is denoted as ${ }^{* *} P \leq 0.001$. (e) CLSM analysis of $24 \mathrm{~h}$ biofilms from same experiment shown in (d). Images are X-Y orthogonal Z-stack views representative of ten images within a single experiment. Average vertical biofilm thickness is indicated in micrometers (top right). 


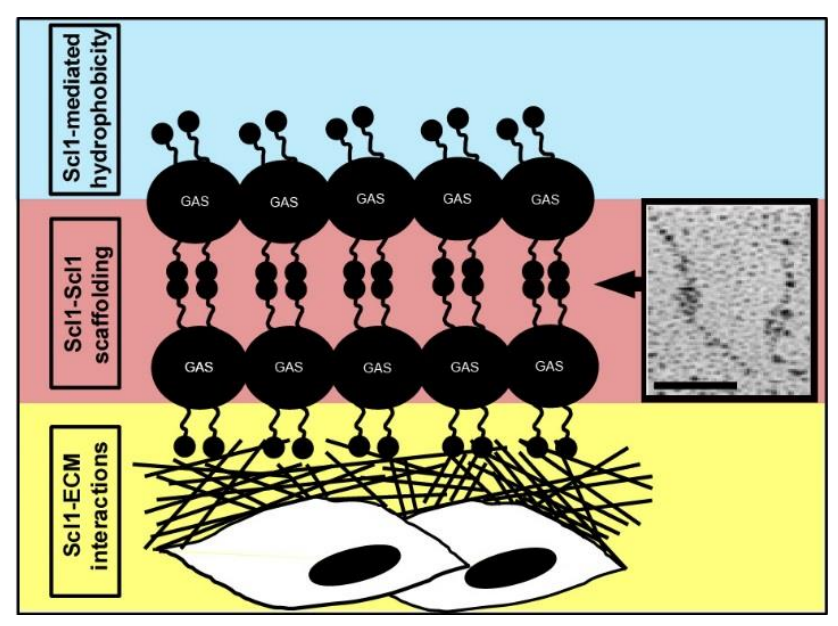

Figure 6

Scl1-mediated model of GAS biofilm (not to scale). Scl1 hydrophobin (current study) initiates bacterial adhesion to animate surfaces (64) within the host (blue field). Scl1 adhesin anchors the growing microcolony by direct binding to tissue extracellular matrix (ECM) components, cellular fibronectin and laminin (19), initiating microcolony formation and anchoring the outside edge of GAS microcolony in tissue (yellow field). Microcolony scaffolding is stabilized by the formation of head-to-head dimers between Scl1 molecules on adjacent chains (pink field). Inset shows Scl1-Scl1 head-to-head dimers formed by rScl1.1 as viewed by electron microscopy after rotary shadowing (68). Bar: $50 \mathrm{~nm}$. 
Table 1. Cell surface hydrophobicity of GAS strains

\begin{tabular}{|c|c|c|c|}
\hline GAS Strain & M-Type & Actual Value $^{\dagger}$ & $\begin{array}{c}\text { Hydrophobicity } \\
\text { Index }\end{array}$ \\
\hline MGAS6183 WT & M41 & $92.6 \pm .86$ & 100 \\
\hline MGAS6183 $\Delta s c / 1$ & M41 & $85.2 \pm 2.2$ & ${ }^{* *} 92$ \\
\hline MGAS6183 $\Delta s c / 1-C$ & M41 & $98.0 \pm .31$ & 105 \\
\hline MGAS5005 WT & M1 & $80.3 \pm .89$ & 100 \\
\hline MGAS5005 $\Delta s c / 1$ & M1 & $63.3 \pm 3.2$ & **79 \\
\hline MGAS6143 WT & M28 & $94.3 \pm .73$ & 100 \\
\hline MGAS6143 $\Delta s c / 1$ & M28 & $72.6 \pm .62$ & **78 \\
\hline \multicolumn{4}{|c|}{$\begin{array}{l}{ }^{\dagger} \text { Actual hydrophobicity values were calculated based on hexadecane binding a } \\
\text { described in Methods. Values are representative of three separate experiments with te } \\
\text { replicates } \pm S D \text {. } \\
\neq \text { Hydrophobicity Index represents the ration of actual hydrophobicity value for each } \\
\text { strain to that of the isogenic wild-type (WT) strain multiplied by } 100 \text {. } \\
* \text { Asterisks denote a statistically significant difference of } \Delta s c / 1 \text { mutants versus WTs at } \\
\leq 0.001 \text {. }\end{array}$} \\
\hline
\end{tabular}


Table 2. Cell surface hydrophobicity of Lactococcus strains

\begin{tabular}{|c|c|c|}
\hline Lactococcus Strain & Actual Value $^{\dagger}$ & $\begin{array}{c}\text { Hydrophobicity } \\
\text { Index }{ }^{\ddagger}\end{array}$ \\
\hline L.lactis $1363 \mathrm{WT}$ & $59.7 \pm 7.2$ & 100 \\
\hline L. lactis 1363::pJRS525 & $56.6 \pm 5.5$ & 98 \\
\hline L. lactis 1363::pSL230 & $82.0 \pm 2.6$ & **137 \\
\hline \multicolumn{3}{|c|}{$\begin{array}{l}{ }^{\dagger} \text { Actual hydrophobicity values were calculated based on hexadecane binding as } \\
\text { described in Methods. Values are representative of three separate experiments with ter } \\
\text { replicates } \pm S D \text {. } \\
\ddagger \text { Hydrophobicity Index represents the ration of actual hydrophobicity value for each } \\
\text { strain to that of the isogenic wild-type (WT) strain multiplied by } 100 \text {. } \\
{ }^{* *} \text { Asterisks denote a statistically significant difference of } \Delta s c / 1 \text { mutants versus WTs at } \\
\leq 0.001 \text {. }\end{array}$} \\
\hline
\end{tabular}




\title{
CHAPTER 2
}

\section{UNIQUE FOOTPRINT IN THE SCL1.3 LOCUS AFFECTS ADHESION AND BIOFILM FORMATION OF THE INVASIVE M3-TYPE GROUP A STREPTOCOCCUS}

\author{
Beth A. Bachert, Soo Jeon Choi, Paul R. LaSala, Tiffany Harper, Dudley H. McNitt,
} Dylan T. Boehm, Clayton C. Caswell, Pawel Ciborowski, Douglas R. Keene, Anthony R. Flores, James M. Musser, Flavia Squeglia, Daniela Marasco, Rita Berisio, and Slawomir

Lukomski

Published in Frontiers in Cellular and Infection Microbiology 2016 6:90

\begin{abstract}
The streptococcal collagen-like proteins 1 and 2 (Scl1 and Scl2) are major surface adhesins that are ubiquitous among group A Streptococcus (GAS). Invasive M3-type strains, however, have evolved two unique conserved features in the scl1 locus: (i) an IS1548 element insertion in the sc/1 promoter region and (ii) a nonsense mutation within the $s c / 1$ coding sequence. The $s c / 1$ transcript is drastically reduced in M3-type GAS, contrasting with a high transcription level of scl1 allele in invasive M1-type GAS. This leads to a lack of Scl1 expression in M3 strains. In contrast, while sc/2 transcription and Scl2 production are elevated in M3 strains, M1 GAS lack Scl2 surface expression. M3type strains were shown to have reduced biofilm formation on inanimate surfaces coated with cellular fibronectin and laminin, and in human skin equivalents. Repair of the nonsense mutation and restoration of Scl1 expression on M3-GAS cells, restores biofilm formation on cellular fibronectin and laminin coatings. Inactivation of scl1 in biofilmcapable M28 and M41 strains results in larger skin lesions in a mouse model, indicating that lack of Scl1 adhesin promotes bacterial spread over localized infection. These studies suggest the uniquely evolved sc/1 locus in the M3-type strains, which prevents surface expression of the major Scl1 adhesin, contributed to the emergence of the invasive M3-type strains. Furthermore these studies provide insight into the molecular mechanisms mediating colonization, biofilm formation, and pathogenesis of group $A$ streptococci.
\end{abstract}




\section{INTRODUCTION}

Group A Streptococcus (GAS) or Streptococcus pyogenes is a human-specific Grampositive pathogen responsible for significant morbidity and mortality worldwide $(1,2)$. The clinical outcomes resulting from GAS infection range from superficial pharyngitis and impetigo to severe life-threatening diseases, such as streptococcal toxic shock syndrome and necrotizing fasciitis, as well as post-infectious sequelae, including rheumatic fever, rheumatic heart disease, and post-streptococcal glomerulonephritis (3). Historically, GAS has been a significant cause of puerperal sepsis, scarlet fever, erysipelas, and pharyngitis (4). GAS strains are epidemiologically subtyped based on nucleotide sequence variation at the 5 '-end of the emm gene, reflecting differences in the hypervariable $\mathrm{N}$-terminal region of the anti-phagocytic surface protein M. Over 240 M-types have been identified (http://www.cdc.gov/abcs/index.html), and certain M-types have been shown to have nonrandom associations with specific disease outcomes $(3,5)$. Since the 1980's there has been resurgence in invasive GAS diseases in the U.S. and other parts of the world. Numerous epidemiology studies conducted in the U.S. (6-11), Canada (12-15), and Europe (16-19) have found associations between infections with M1- and M3-type strains and invasive diseases. Specifically, M3-type strains have been associated with severe invasive disease $(6,19)$ and fatal outcomes $(14,16,17)$.

These epidemiological observations have spurred significant whole genome sequencing efforts aimed at identification of the underlying genetic basis for virulence in M3-type GAS. The complete annotated genomes of invasive M3 strains MGAS315 and SSI-1 have been reported $(20,21)$. These studies have revealed that differing phage elements, insertion sequences, and the large-scale chromosomal inversion identified in SSI-1, contributed to much of the genetic variation between M3 and other M-types. Acquisition of prophages and SNP's drive the expansion of different M3 subclones during epidemic waves of infection $(22,23)$. Additionally, strains causing pharyngitis and those causing invasive infections are derived from the same pool of M3 strains (24). In addition, genetic variation in virulence regulators, including RopB and CovRS, which are "hotspots" for the accumulation of mutations, also contributes to the hypervirulence of M3 strains (23-28). The cause of the hyper-invasive phenotype of $M 3$ strains is multifactorial 
and involves multiple virulence factors controlled by complex regulatory networks that continually undergo remodeling.

The streptococcal collagen-like proteins 1 and 2, or Scl1 and Scl2 (also known as SclA and SclB), are major GAS surface adhesins known to contribute to pathogenesis. Both Scl proteins contain an $\mathrm{N}$-terminal variable region, followed by a collagen-like region containing Gly-X-Y repeats and a cell-wall-anchored C-terminal region (29-33). Transcription of $s c / 1$ is positively regulated by the multiple gene regulator Mga $(30,32,34)$. Scl1 binds cellular fibronectin and laminin (35), and contributes to the formation of biofilm by strains of multiple M-types $(36,37)$. GAS adherence and biofilm formation is enhanced on extracellular matrix (ECM) coatings and on fibroblast-deposited ECM network $(37,38)$, supporting a role for Scl1 in tissue-microcolony formation described during GAS skin infection (39). While the $s c / 1$ gene has been found in every GAS strain tested $(29,32)$, the scl1.3-allele in M3-type strains harbors a null mutation within the coding sequence (29). A rare natural reversion of this polymorphism was identified in a small subset of $M 3$ carrier strains (40).

Scl2-protein expression is regulated during translation by varying numbers of short CAAAA repeats downstream of the start codon that determine whether the protein-coding sequence is in-frame or translation will be prematurely terminated $(30,31)$; genome sequencing indicates majority of the M3-type strain contain in-frame scl2.3 alleles $(18,22)$. Scl2 has been shown in some strains to bind the human thrombin-activatable fibrinolysis inhibitor (41) and contribute to adherence to fibroblasts (31), although its role in GAS pathogenesis is less understood. Recently, the crystal structure of the Scl2.3 globular domain, which is structurally conserved between Scl1 and Scl2, has been reported $(42,43)$ providing insight into the potential binding interactions with host ligands.

In this study, we show that invasive M3-type GAS harbor two unique conserved features of the scl1 locus including the IS1548 insertion in the promoter region and the null mutation in the coding sequence, which results in a secreted instead of cell-attached Scl1 protein. We demonstrate significantly different expression patterns of scl1 and scl2 in M3-type GAS compared to representative strains of M1-, M28- and M41-types. We demonstrate that the expression of the Scl1 adhesin is deficient in serotype M3 strains, as opposed to M1-, M28-, and M41-type strains. However, the Scl2 protein is upregulated 
in M3 strains compared to M1, M28, and M41 GAS. The M3 strains lacked significant biofilms on cellular fibronectin and laminin coatings, compared to M41-type GAS, and did not form tissue microcolonies in a wounded pseudo-organ skin equivalent model of infection. Recombinant Scl1.3 specifically bound cellular fibronectin and laminin, and restoration of surface expression of Scl1.3 conferred significant biofilm formation by M3 strains. Inactivation of Scl1 expression in biofilm-capable M28- and M41-type GAS resulted in larger skin lesions produced by the mutants in a mouse model of subcutaneous infection, supporting a role for Scl1 in maintaining a localized infection. Our model advocates that the lack of surface-associated Scl1 adhesin in M3-type strains causes decreased tissue adherence and decreased capacity for stable microcolony formation, thus, promoting bacterial spread over localized nidus of infection.

\section{MATERIALS AND METHODS}

\section{Bacterial strains and growth}

MGAS315 and MGAS10870 are fully sequenced invasive M3-type strains $(20,23)$. MGAS315 was isolated from a patient with streptococcal toxic syndrome in the 1980's (6) and MGAS10870 was isolated from a patient with soft tissue infection in Ontario in 2002 (23). Additional strains from epidemiologically diverse M-types were used for comparison: MGAS6183 (M41), MGAS5005 (M1), and MGAS6143 (M28). The MGAS6143Ascl1-, MGAS6183 $\Delta s c / 1-$, and MGAS10870 $\Delta s c / 1$-inactivated mutant strains have been previously described $(40,44,45)$. Group A Streptococcus cultures were grown in ToddHewitt broth (Becton Dickinson and Co.) supplemented with $0.2 \%$ yeast extract (THY medium) and on Brain Heart Infusion agar (Becton Dickinson and Co.) at $37^{\circ} \mathrm{C}$ in an atmosphere with $5 \% \mathrm{CO}_{2}$. For antibiotic selection, erythromycin $\left(5 \mu \mathrm{g} \mathrm{mL} \mathrm{m}^{-1}\right)$, chloramphenicol $\left(10 \mu \mathrm{g} \mathrm{mL}^{-1}\right)$, kanamycin $\left(200 \mu \mathrm{g} \mathrm{mL}^{-1}\right)$, and spectinomycin $\left(100 \mu \mathrm{g} \mathrm{mL}^{-}\right.$ 1) were added to the medium.

Cloning experiments were performed in XL-1 Blue and TB1 E. coli cells, while protein expression experiments were performed in BL21 and TB1 E. coli cells grown in Luria-Bertani media (Difco Laboratories) at $37^{\circ} \mathrm{C}$. For antibiotic selection, chloramphenicol $\left(10 \mu \mathrm{g} \mathrm{mL}^{-1}\right)$, kanamycin $\left(50 \mu \mathrm{g} \mathrm{mL}^{-1}\right)$, spectinomycin $\left(100 \mu \mathrm{g} \mathrm{mL}^{-1}\right)$, and ampicillin $\left(100 \mu \mathrm{g} \mathrm{mL}^{-1}\right)$ were added to the medium. 


\section{PCR assays}

\subsection{Analytical PCR}

\subsubsection{Detection of IS element upstream of $s c / 1$ in GAS strains}

The presence of IS1548 upstream of scl1 was determined by PCR with primers IS1548F and Scl1R (Figure 1) using Qiagen Taq DNA polymerase (Qiagen, Germantown, MD) as follows: $95^{\circ} \mathrm{C}, 5 \mathrm{~min}-\left[95^{\circ} \mathrm{C} 1 \mathrm{~min}, 62^{\circ} \mathrm{C} 1 \mathrm{~min}, 72^{\circ} \mathrm{C} 1 \mathrm{~min}\right] \times 30$ cycles- $72^{\circ} \mathrm{C}, 10 \mathrm{~min}$. Sequences of primers used in all PCR assays are listed in Table S1.

\subsubsection{PCR amplification of $s c / 1.3$ and $s c / 2.3$ alleles}

PCR was performed on genomic DNA isolated from M3 strains with primer pairs $232 \mathrm{Up} /$ 232 Rev for scl1.3 amplification and sequencing, and Scl2.3 F/R and SclUp/ SclRev for length polymorphism analysis and sequencing of $s c / 2.3$, respectively. Amplification was performed using Qiagen Taq DNA polymerase as follows: scl1.3: $95^{\circ} \mathrm{C}, 5 \mathrm{~min}-\left[95^{\circ} \mathrm{C} 1\right.$ $\left.\min , 55^{\circ} \mathrm{C} 1 \mathrm{~min}, 72^{\circ} \mathrm{C} 1 \mathrm{~min}\right] \times 30$ cycles- $72^{\circ} \mathrm{C}, 10 \mathrm{~min}$; scl2.3: $95^{\circ} \mathrm{C}, 5 \mathrm{~min}-\left[95^{\circ} \mathrm{C} 1 \mathrm{~min}\right.$, $51^{\circ} \mathrm{C} 1 \mathrm{~min}, 72^{\circ} \mathrm{C} 1 \mathrm{~min} 45 \mathrm{sec}$ ] $\times 30$ cycles- $72^{\circ} \mathrm{C}, 10 \mathrm{~min}$. All PCR products were analyzed in a $1 \%$ agarose gel with $1 \mathrm{~kb}$ Plus DNA ladder as a molecular size marker (Life Technologies, Grand Island, NY).

\subsection{Quantitative reverse transcription PCR (qRT-PCR)}

\subsubsection{RNA isolation}

Total RNA was isolated from the logarithmic phase (OD600 0.5) GAS cultures using RNeasy Protect Bacteria Mini kit (Qiagen), employing an additional pretreatment step with $250 \mu \mathrm{g}$ lysozyme, $100 \mu \mathrm{g}$ proteinase $\mathrm{K}$, and $12.5 \mathrm{U}$ mutanolysin per sample to augment cell wall disruption. Trace genomic DNA was removed by incubation with TurboDNase enzyme (Ambion). RNA quality was assessed in 1\% agarose gel and spectrophotometrically; $\mathrm{A} 260 / 280$ and $\mathrm{A} 260 / 230$ ratios of $>1.8$ were considered acceptable. RNA was used immediately in cDNA synthesis or stored at $-80^{\circ} \mathrm{C}$ for no more than 1 week. 


\subsubsection{Determination of transcription by $q R T-P C R$}

Synthesis of cDNA was performed using iScript Select cDNA synthesis kit (Bio-Rad) with $1 \mu \mathrm{g}$ of RNA per reaction and random primer mix. For each sample, a no reverse transcriptase control was performed, containing only RNA, reaction buffer, and random primer mix, to ensure the absence of genomic DNA. Incubations were carried out on a Bio-Rad C1000 Touch Thermal cycler: $25^{\circ} \mathrm{C} 5 \mathrm{~min}, 42^{\circ} \mathrm{C} 30 \mathrm{~min}, 85^{\circ} \mathrm{C} 5 \mathrm{~min}$, and cDNA was stored at $-20^{\circ} \mathrm{C}$ until used in qRT-PCR. qRT-PCR reactions were performed with 250 nM primers using SsoAdvanced SYBR Green Universal Supermix (Bio-Rad). For each qRT-PCR reaction, template CDNA, diluted 1:20, control genomic DNA, or no reverse transcriptase control were used. PCR was performed using the following conditions on a Bio-Rad CFX96 Thermal cycler: $95^{\circ} \mathrm{C} 2 \mathrm{~min}-\left[95^{\circ} \mathrm{C} 5 \mathrm{sec}, 60^{\circ} \mathrm{C} 30 \mathrm{sec}\right] \times 30-\left[65^{\circ} \mathrm{C}-95^{\circ} \mathrm{C}\right.$, $5 \mathrm{sec}, 0.5^{\circ} \mathrm{C} / \mathrm{step}$. Standard curves were generated for each primer set using cDNA from MGAS315 to determine the linear range and estimate reaction efficiency. Gene expression of $s c / 1, s c / 2, e m m$, and mga was normalized against the expression of tufA gene, which has previously been identified and validated as an appropriate endogenous control (46). The $\Delta \Delta \mathrm{Ct}$ method was utilized to compare gene expression between MGAS315 and other M3 strains, as well as M1, M28, and M41-type strains. Data was averaged from three independent experiments, each containing three technical replicates. Statistical significance was determined using an unpaired $t$-test.

\section{Recombinant Scl ( $\mathrm{rScl}$ ) proteins}

\subsection{Cloning, expression, and purification of M3-derived rScl proteins}

The rScl proteins were generated using the Strep-tag II cloning, expression and purification system (IBA-GmbH, Gottingen, Germany). Proteins were expressed with a Cterminal affinity tag and purified on Strep-Tactin sepharose, as described (47). rScl proteins $\mathrm{rScl1} .3 \mathrm{~V}$ and $\mathrm{rScl1}$.3FL are derived from MGAS315, whereas rScl2.3 protein is derived from the M3 strain MGAS3375. The recombinant Scl2.3V protein has been described previously (42). Construct containing the recombinant full-length Scl1.3FL (rScl1.3FL) protein was previously described (40). Briefly, the scl1.3 coding region from MGAS315 was cloned into the E. coli/GAS shuttle vector pJRS525 (48), generating plasmid pSL501. PCR mutagenesis was used to convert the internal TAA stop codon into 
a GAA glutamate codon, resulting in plasmid pSL502 with continuous full-length sc/1.3FL allele. This sequence was subsequently cloned into the pASK-IBA2 expression vector for recombinant Scl1.3FL protein production; each clone was verified by sequencing. Protein expression constructs are listed in Table 1.

Protein expression was induced by the addition of anhydrotetracycline at $0.2 \mu \mathrm{g}$ $\mathrm{mL}^{-1}$ for 3 hours. Cells were centrifuged and resuspended in high sucrose buffer (100 mM Tris-HCl, $1 \mathrm{mM}$ EDTA, pH 8.0, $500 \mathrm{mM}$ sucrose) or Cell Lytic B Buffer (Sigma), for separation of the periplasmic fraction and subsequent affinity purification. Proteins were dialyzed against $25 \mathrm{mM}$ HEPES, $\mathrm{pH}$ 8.0, and analyzed by SDS-PAGE stained with RAPIDstain $^{\mathrm{TM}}$ (G-Biosciences). Protein sequence was confirmed using mass spectrometry (University of Nebraska Medical Center) and N-terminal Edman Degradation sequencing (lowa State University).

\subsection{Electron microscopy of rotary shadowed rScl proteins}

Electron microscopy visualization of the rotary shadowed preparations of rScl1.3FL and rScl2.3 was used to assess the domain organization of $\mathrm{rScl}$ proteins, as employed previously (49). The rScl proteins were dialyzed against $0.1 \mathrm{M}$ ammonium bicarbonate and mixed with glycerol to a final concentration of $70 \%$ (vol:vol). Samples were nebulized onto mica chips with an airbrush and rotary-shadowed with carbon/platinum using an electron beam gun. Photomicrograph images were acquired using a transmission electron microscope FEI G2 operated at 80KV.

\subsection{Mass spectrometry analysis}

Samples from in-gel trypsin digested proteins were cleaned using a Millipore $\mu$ C18 ZipTip, then resuspended in $0.1 \%$ formic acid. Samples were fractionated on a Eksigent cHiPLC column ( $75 \mu \mathrm{m} \times 15 \mathrm{~cm}$ ChromXP C18-CL $3 \mu \mathrm{m} 120 \AA)$, and resulting peptides were sequenced using 5600 TripleTOF (typical gradient 2-60\% ACN in 60 minutes). Peptides identified were searched against the NCBI protein database with Protein Pilot software employing the following settings: search effort, thorough; taxonomy, none. Positive identification was considered as the identification of two or more unique peptides at high 
confidence $\geq 95 \%$, FDR $=0.05,0.01$, or 0.001 , which matched the same protein entry in the database searched.

\subsection{Binding of rScl proteins to extracellular matrix proteins and synthetic peptide}

\subsubsection{ELISA binding assays}

Binding of $\mathrm{rScl}$ proteins to extracellular matrix proteins, cellular fibronectin (cFn) and laminin (Lm), was tested by ELISA (35). rScl proteins were immobilized onto StrepTactin $\AA$ coated microplate wells (IBA $\mathrm{GmbH})$ at $0.5 \mu \mathrm{M}$ at room temperature for 1.5 hours, then blocked with Tris-buffered saline (TBS) containing 1\% bovine serum albumin (BSA) overnight at $4^{\circ} \mathrm{C}$. The cellular fibronectin from human foreskin fibroblasts (Sigma) and murine laminin (Invitrogen) were added to the wells at $1 \mu \mathrm{g}$ per well and incubated at room temperature for 1 hour. Bound ECM proteins were detected with rabbit anti-laminin at 1:100 (Sigma) and anti- human fibronectin at 1:4,000 (Sigma) polyclonal antibodies. Secondary antibody goat anti-rabbit IgG $(H+L)$ HRP conjugate (Bio-Rad) was next used with 1-step ABTS substrate (2,2'-Azinobis [3-ethylbenzothiazoline-6-sulfonic acid]diammonium salt) (ThermoScientific). Absorption was measured using Spectramax 190 at a wavelength of $415 \mathrm{~nm}$. Statistical analysis is based on three independent experiments each containing three technical replicates, using an unpaired $t$-test.

In antibody inhibition assay, the IST-9 mAb targeting the C-C' loop of EDA domain was utilized (37). CFn was either untreated or pre-treated with increasing concentrations of IST-9 blocking mAb $(0.1 \mu \mathrm{g}-1.0 \mu \mathrm{g})$, and added to Strep-Tactin®-coated microplate wells immobilized with $\mathrm{rScl}$ proteins, then incubated for 1 hour. Bound ECM proteins were detected as above.

\subsubsection{Design and synthesis of the C-C' cyclic peptide}

The C-C' cyclic peptide was designed based on the crystal structure of the EDA domain of cFn (PDB code 1J8K). In particular, the region, which was reported to be involved in Scl1 binding, connecting to two $\beta$-strands $C$ and C' of EDA was elongated to the whole C-C' $\beta$-hairpin by allowing the formation the electrostatic interaction between Arg33 and Glu45. The terminal Tyr32 and Pro48 were mutated to Cys to stabilize the $\beta$-hairpin by introducing a disulfide bond (Figure S4). The obtained sequence, 
CRVTYSSPEDGIHELFC (molecular weight: 1997.1 Da), endowed with a cyclic structure to mimic the structure of this region in EDA, was acetylated and amidated at the $\mathrm{N}$ - and C-terminus, respectively. Synthesis of the designed peptide was performed employing the solid phase method on a $50 \mu \mathrm{mol}$ scale initially following standard Fmoc strategies (50). Due to aspartimide formation during traditional acylation reactions, peptide synthesis was carried out employing microwave technology (51). Cyclization was achieved by treating the peptide at $0.1 \mathrm{mg} / \mathrm{mL}$ (to avoid intermolecular disulphide formation) with buffer carbonate $50 \mathrm{mM}, \mathrm{pH}=9$, overnight. The peptide was purified by RP-HPLC and the identity and purity (> 97\%) was assessed by LC-MS (data not shown).

\subsubsection{Surface plasmon resonance (SPR) experiments}

Real time binding assays were performed at $25^{\circ} \mathrm{C}$ on a Biacore 3000 Surface Plasmon Resonance (SPR) instrument (GE Healthcare). For immobilization, rScl1.3FL protein containing the C-terminal Strep-tag II was injected at a concentration of $40 \mu \mathrm{M}$ on streptavidin-coated sensor chip, SA Biacore, until the desired level of immobilization was achieved (averaged value of $100 \mathrm{RU}$ ). Binding assays were carried out by injecting the C-C' cyclic peptide at concentrations ranging between 10-500 $\mu \mathrm{M}$. Experiments were carried out in HBS buffer (10 mM HEPES, $150 \mathrm{mM} \mathrm{NaCl}, 3 \mathrm{mM}$ EDTA, pH 7.4). The association phase ( $k_{\text {on }}$ ) was followed for $270 \mathrm{~s}$, whereas the dissociation phase ( $k_{\text {off }}$ ) was followed for $300 \mathrm{~s}$. The reference chip sensorgrams were subtracted from sample sensorgrams. Experiments were carried out in duplicates. Kinetic parameters were estimated assuming a 1:1 Langmuir binding model and using version 4.1 Evaluation Software (GE Healthcare).

\subsubsection{Fluorescence binding analysis}

rScl1.3FL protein, at a concentration of $30 \mu \mathrm{M}$, was incubated with increasing concentrations of $\mathrm{C}-\mathrm{C}^{\prime}$ cyclic peptide $(0-300 \mu \mathrm{M})$ at $25.0{ }^{\circ} \mathrm{C}$, using an excitation wavelength of $298.0 \mathrm{~nm}$ and a fluorescence emission wavelength ranging from 300 to $400 \mathrm{~nm}$. The acquisition parameters were set as follows: excitation and emission slits at $5 \mathrm{~nm} ; 120 \mathrm{~nm} / \mathrm{min}$ scan rate; $1.00 \mathrm{~nm}$ data interval averaging time at $0.500 \mathrm{~s}$, PMT voltage at "high". Fluorescence values were recorded at $333 \mathrm{~nm}$, and subtracted from the 
fluorescence intensity of the ligand-free protein, generating $-\Delta$ fluorescence. The $\Delta$ fluorescence values were plotted against the peptide concentration. (52). Experiments were carried out in duplicates. A control assay was carried out employing the buffer as titrant to assess that the dilution effect was under $3 \%$, not affecting the results.

\section{Complementation of GAS strains with full-length Scl1.3FL}

For trans-complementation experiments, plasmid pSL502, encoding full-length cellassociated Scl1.3FL protein, was electroporated into MGAS315 WT and MGAS6183 $\Delta s c / 1$ electrocompetent cells. The pJRS525 vector was electroporated as a control. Transformants were selected on $\mathrm{BHI}$ agar containing $100 \mu \mathrm{g} \mathrm{mL}^{-1}$ spectinomycin, and plasmids were re-sequenced. For MGAS10870 $\Delta$ scl1, which contains a spectinomycin resistance cassette in place of the sc/1.3 allele, the spectinomycin resistance marker in pSL502 was replaced with a kanamycin resistance, generating the plasmid pSL518 and colonies were selected on BHI agar containing $150 \mu \mathrm{g} \mathrm{mL}^{-1}$ kanamycin.

\subsection{Determination of Scl1.3- and Scl2.3-protein expression in wild-type and complemented GAS strains}

\subsubsection{Western blot analysis}

Expression of the Scl1.3 and Scl2.3 proteins was determined by western immunoblotting of the bacterial cell wall and culture supernatant protein fractions, as described before $(29,30)$. Briefly, bacterial cultures were grown to an $\mathrm{OD}_{600}$ of 0.5 and cells were harvested by centrifugation. Culture supernatant proteins were precipitated with trichloroacetic acid (Sigma) to a final concentration of $10 \%$ (vol:vol). The cell wall protein fraction was obtained after cell digestion with lysozyme and mutanolysin in a high sucrose buffer. A total of $10 \mu \mathrm{g}$ of protein samples were separated by SDS-PAGE and transferred to a nitrocellulose membrane. Detection of Scl1.3, Scl2.3, and M3 proteins was performed using the same sample preparations with rabbit polyclonal antibodies generated against the truncated rScl1.3WT protein (anti-Scl1 1:15,000 dilution, reported in (40)) and the rScl2.3V region (anti-rScl2.3V 1:2,500; generated by Proteintech (43)). Horseradish peroxidase-conjugated goat anti-rabbit IgG $(\mathrm{H}+\mathrm{L})$ secondary antibody (Bio-Rad), 
combined with Pierce ${ }^{\mathrm{TM}}$ ECL western blotting substrate (Thermo Scientific) was used for detection. Images were acquired using a ChemiDoc Touch Imaging System (Bio-Rad).

\subsubsection{Flow cytometry analysis}

Surface detection of Scl1.3 and Scl2.3 proteins was determined by flow cytometry. Bacteria grown to an $\mathrm{OD}_{600}$ of 0.5 were harvested by centrifugation, and washed with flow cytometry buffer (sterile phosphate-buffered saline containing 10\% Todd-Hewitt broth supplemented with $0.2 \%$ yeast extract). Cells were incubated with polyclonal antibodies against Scl1.3 and Scl2.3 described above at a dilution of 1:100 for 30 minutes on ice, then washed and incubated with Allophycocyanin (APC)-conjugated donkey anti-rabbit $\lg G(H+L)$ (Jackson ImmunoResearch) for 30 minutes on ice. Cells were washed and fixed in $0.4 \%$ paraformaldehyde, and stored at $4^{\circ} \mathrm{C}$ until analysis. Before analysis, cells were washed twice and resuspended in flow cytometry buffer. Cells were analyzed using a BD LSRFortessa, and 50,000 events were collected per sample. Data was analyzed using the FCS Express Flow 5 software.

\section{Assessment of biofilm formation}

\subsection{Crystal violet staining assay}

Wild-type, mutant, and complemented strains were grown to $\mathrm{OD}_{600}$ of 0.5 and seeded into 24-well culture plates coated with ECM at $2 \mu \mathrm{g}$ per well, then incubated at $37^{\circ} \mathrm{C}$ with $5 \% \mathrm{CO}_{2}$ for 24 hours. Wells were washed with PBS followed by the addition of $0.5 \mathrm{~mL}$ $1 \%$ crystal violet solution (Fisher Scientific) diluted in PBS and incubation at room temperature for 30 minutes. Wells were rinsed twice with PBS and stain was solubilized with $0.5 \mathrm{~mL}$ of $75 \%$ ethanol. Spectrophotometric readings were taken for each sample at $\mathrm{OD}_{600}$. Statistical analysis is shown based on three independent experiments, each containing three technical replicates, using an unpaired $t$-test.

\subsection{Confocal laser scanning microscopy (CLSM)}

To visualize GAS by CLSM, bacterial cells were transformed with a GFP-encoding plasmid pSB027 (53), as before (36). 15-mm glass cover slips were placed into 24-well tissue culture plate wells and coated with $2 \mu \mathrm{g}$ of cFn or Lm per well. Bacterial cultures 
grown to logarithmic-phase were added to the wells and allowed to form biofilms for 24 hours. Wells were rinsed with PBS and bacterial cells were fixed with $3 \%$ paraformaldehyde at room temperature for $30 \mathrm{~min}$. Wells were washed again and coverslips were mounted onto slides using ProLong Gold Antifade Mountant (Thermo Scientific). Confocal images were acquired using a $63 \times / 1.40$ Plan-Apochromat objective and a Zeiss LSM 510 laser scanning confocal microscope.

\section{In vitro and in vivo GAS infection models}

\subsection{GAS infection of in vitro cultured human skin equivalents}

Wounded full-thickness skin equivalents, EpiDerm-FT (MatTek, Boston, MA) were used. The tissues are discs $8 \mathrm{~mm}$ in diameter, which are provided in transwells. A 3-mm wound is generated by performing a punch biopsy to remove the keratinocyte layer. Immediately upon arrival, tissues were equilibrated in antibiotic-free manufacturer's medium overnight at $37^{\circ} \mathrm{C}$ in atmosphere with $5 \% \mathrm{CO}_{2}$. Wounds were infected with $10 \mu \mathrm{L}$ of GFP-expressing log-phase group A streptococcal inocula and incubated in a humid environment at $37^{\circ} \mathrm{C}$ with $5 \% \mathrm{CO}_{2}$ in daily-fresh media; a total of 4 experiments were performed and variables included the inoculum size of $3 \times 10^{6}-1.8 \times 10^{7} \mathrm{CFU}$ and collection time points between 1-5 days. Tissues designated for histopathological evaluation were fixed in $10 \%$ formalin,

whereas tissues for two-photon fluorescence (TPF) microscopy imaging were fixed in $4 \%$ paraformaldehyde for several hours and then transferred to petri dishes containing PBS. For visualization of glycocalyx produced by GAS strains, tissues were permeabilized with $0.1 \%$ Triton X-100 in $1 \times$ PBS and blocked with $0.05 \%$ Triton X-100 in $1 \times$ PBS with $1 \%$ BSA before staining with concanavalin A-tetramethylrhodamine (Molecular Probes). Tissues were then rinsed and stored in PBS at $4^{\circ} \mathrm{C}$ until TPF imaging was performed. For TPF analysis, an Olympus 60x/1.2NA water dipping objective was used. Fixed tissues were imaged by two-photon microscopy with the Ti:sapphire laser (Mira, Coherent) intensity at $60 \mathrm{~mW}$ and input wavelength of $850 \mathrm{~nm}$. Laser scanning images were collected at 0.5- $1 \mu \mathrm{m}$ incremental depths using Scanlmage (Janelia Farms, HHMI). Images were saved in a single TIFF file with 16 bit depth. Deconvolution of the images was performed using AutoQuant $\times 3$ and 3D models of z-stacks were generated using Imaris software. 


\subsection{Mouse model of soft tissue infection}

Animal experiments were conducted in compliance with the regulations and standards under the Animal Welfare Act, the Public Health Service Policy on Humane Care and Use of Laboratory Animals, and the Guide for the Care and Use of Laboratory Animals. The protocol was approved by the West Virginia University Institutional Animal Care and Use Committee (IACUC).

Subcutaneous infections of mice were carried out as described previously (54). Briefly, 5-week-old male, immunocompetent, hairless mice (strain Crl:SKH1-hrBR) were used (Charles River, Wilmington, MA). Groups of 10-15 mice anesthetized with isoflurane were infected subcutaneously at the right flank with $\sim 10^{9}$ GAS CFU of WT or sc/1-mutant strains, and mice were observed for 14 days. The weight and abscess dimensions (length $[L]$ and width [W]) of each mouse were recorded daily during the first week and every other day thereafter. To analyze differences between mice infected with WT and sc/1mutant GAS, the area of each abscess was calculated with the equation for the area $(A)$ of an spherical ellipsoid: $A=\pi(L / 2) \times(W / 2)$; statistical differences were calculated using the student's $t$-test. At the conclusion of the experiments, mice were anesthetized and sacrificed by cervical dislocation.

\section{RESULTS}

M3-type GAS contain unique insertion of IS1548 element and nonsense mutation within the scl1.3 locus

Since the resurgence of invasive GAS infections in the 1980s and an advent of molecular epidemiology fostered by large-scale sequencing, significant efforts have been made to define the molecular basis for the invasive phenotype of M3-type strains. In parallel to these advances, we identified two unique genomic traits in the scl1.3 locus, encoding streptococcal collagen-like protein 1: (i) the presence of an insertional sequence element, IS1548, in the promoter region and (ii) the presence of a nonsense mutation within the coding sequence of $s c / 1.3$ allele that was absent in other M-types.

Studies employing mga-inactivated mutants have indicated that Scl1 expression was positively regulated by the GAS-global transcriptional regulator, Mga $(30,32)$. Two 
putative Mga binding sites were identified upstream of the $s c / 1$ coding sequence in the M1-type strain SF370 and experiments demonstrated that the Mga binding site distal to scl1 was responsible for transcription activation (34). Identical Mga binding sites I and II were also identified upstream of scl1.3 in the sequenced M3-type strain MGAS315 with IS1548 element inserted 38 bp upstream of the distal Mga I-binding site (Figure 1A). To determine if the IS1548 insertion was specific to M3 strains, we BLAST-searched this element in 45 completed GAS genomes representing 21 different M-types. We observed the presence of IS1548 in all strains searched with varying locations and occurrences from one to twelve per genome (Figure 1B). However, the IS1548 insertion upstream of the scl1.3 allele was only found in the sequenced genomes of M3-type strains MGAS315, SSI-1, and M3-b. Interestingly, a complete IS1548 element was not present upstream of sc/1.3 in the recently reported genome of the M3 strain STAB902, which represents a non-invasive isolate (55); instead, a 34-bp remnant of IS1548, including the inverted repeat and additional $14 \mathrm{bp}$, was found. Based on this bioinformatics data, we examined the presence of the IS1548 element upstream of sc/1 by PCR in a panel of 40 M3-type strains, using primers located in the IS1548 and scl1.3 sequences (IS1548F and Scl1R, Table S1). All M3-type strains examined were positive for the IS1548-scl1.3 amplicon, while M1, M41, and M28-type controls were negative (Figure 1C), demonstrating a broad and conserved presence of the IS1548 insertion in this location among M3-type GAS.

The nonsense mutation in the $11^{\text {th }}$ Gly-X-Y repeat in the Scl1.3-CL collagenous region (Figure $1 \mathrm{~A}$, red box), presumably results in a truncated secreted variant, which consists of the Scl1-V variable region and $10 \mathrm{Gly}-\mathrm{X}-\mathrm{Y}$ repeats but lacks the cell wall anchor. This polymorphism was originally identified in five M3-type invasive GAS strains but was absent in 45 other strains analyzed, representing 20 different M-types (29). It was later shown in $98.7 \%$ of 479 sequenced invasive M3-type isolates (40). We performed targeted PCR amplification of scl1.3 from 46 additional M3-type strains and observed identical amplicon sizes in all of them (Figure S1A). Sequencing showed that all tested strains harbored an identical sc/1.3 allele, containing 25 Gly-X-Y repeats in the collagenous domain with the null mutation in the $11^{\text {th }}$ repeat (Data Set S1). A complete lack of genetic variation within $s c / 1.3$ is surprising and differs from the length variation that is commonly observed among $s c l$ alleles from other M-types $(29,32,33)$. These results demonstrate 
that the IS1548 insertion and null mutation we identified in the $s c / 1.3$ locus are unique to and conserved among M3-type GAS. Based on these results, we hypothesized M3 strains produce a truncated, but potentially biologically active Scl1.3 variant, which is secreted instead of being cell-attached.

\section{sc/1.3 expression is diminished in M3-type GAS}

Original reports showed scl1 transcripts in northern blots, as well as full-length Scl1 proteins (both cell-associated and cell-free fractions) in western blots for strains of emm types 1, 28, 52, and $41(29,30,32,45)$. To analyze the expression of the truncated Scl1.3 protein in M3-type GAS, western blot analysis was performed on cell-wall (CW samples) and culture-supernatant (Sup samples) protein fractions of several M3-type strains grown to exponential phase (Figure 2A). The expected truncated Scl1.3 protein was not detected by anti-Scl1 antibodies, whereas the rScl1.3V positive control, corresponding to the $\mathrm{V}$ region of Scl1.3 variant, produced the expected immunoreactive band of $\sim 8.3 \mathrm{kDa}$. In an additional control experiment, the same panel of M3 strains was tested for Scl1.3 on the cell surface using flow cytometry (Figure 2B). No shift in median fluorescence intensity was observed in M3 strains incubated with anti-Scl1 antibody compared to a secondaryonly antibody control, indicating a lack of Scl1 on the cell surface among M3 strains.

Given the unique IS1548-sc/1.3 location and the lack of truncated Scl1.3 products in culture supernatants, we investigated scl1.3 expression by qRT-PCR. Total RNA was isolated from exponential phase cultures of $5 \mathrm{M} 3$-type strains, as well as from previously characterized control strains of emm types 1, 28, and $41(29,30,32,45)$. Expression of scl1 from each strain was compared to scl1.3 transcription in M3 strain MGAS315 (Figure 2C). Each non-M3-type strain tested had significantly higher transcription level of respective sc/1 allele compared to MGAS315. The M1 strain had the most increased expression by 21 -fold, while M28 and M41 strains exhibited 6 -fold and 8-fold higher scl1 expression, respectively. Interestingly, the sc/1.3 transcripts examined in four additional M3-type MGAS strains 158, 335, 1313, and 10870 were significantly reduced as compared to MGAS315, with a range of 25-45-fold decrease. Overall, we observed that M3-type GAS harboring the IS element upstream of sc/1.3 have drastically decreased 
scl1.3 transcript levels and lack the Scl1 protein product, as assessed by western blot and flow cytometry.

Since $s c / 1$ is regulated by the transcriptional activator Mga, we investigated whether the decreased sc/1.3 transcripts in M3 strains were due to lack of mga gene expression in these strains. In parallel, we assessed transcript levels of the emm gene encoding the $\mathrm{M}$ surface protein, a key virulence factor regulated by Mga. For comparison, we included the M1 strain, which had significantly increased scl1 expression compared to the M3 strains. With the exception of MGAS335, which had significantly downregulated mga and emm expression, we found no significant differences in either mga or emm gene expression between MGAS315 and the other M3 strains or M1 strain (Figure 2D). Furthermore, M3 protein was highly expressed, as it was found in both cell wall and supernatant fractions, except for the MGAS335 strain, consistent with transcription data (Figure 2E). These results demonstrate that the striking downregulation of $s c / 1.3$ in M3type GAS is not due to decreased mga expression or non-functional Mga protein, as emm is normally expressed in these strains.

\section{sc/2.3 is expressed in M3-type GAS}

Scl2 shares a similar structure with Scl1 but its biological function is poorly understood. One study demonstrated in a different M-type background that isogenic mutant devoid of Scl2.55 variant had lower adhesion to human skin fibroblasts (Rasmussen and Björck, 2001); however, the Scl2.3 variant present in M3-type GAS has not been investigated for expression and ECM binding. Therefore, we next assessed sc/2.3/Scl2.3 expression in M3 strains. PCR amplification and sequencing showed that the majority of M3 strains contained in-frame scl2.3 allele (Figure S1B, Table S2).

Western blot analysis of cell wall (CW samples) and supernatant (Sup) protein fractions found Scl2.3 protein was expressed by M3 strains MGAS315, 10870, 158, 9517 and 1313, whereas samples obtained from strain MGAS335, which contains an out-offrame scl2.3 allele, generated no immunoreactive band; rScl2.3V control produced the expected $10.1-\mathrm{kDa}$ band (Figure $3 \mathrm{~A}$ ). Mass spectrometry confirmed the identity of the presumed immunoreactive Scl2.3-protein band from MGAS315 (Table S3). Consistently, Scl2.3 was detected on the surface of all five M3 strains containing in-frame scl2.3 alleles 
by flow cytometry, with a positive shift in median fluorescence intensity ranging in 67-131fold change, as compared to the secondary-only antibody control (Figure 3B). We next compared the sc/2-transcription level in M3 strain MGAS315 with scl2-transcription levels in M1-, M28-, and M41-type strains. In striking contrast to the pattern of scl1.3 transcription, scl2 transcripts were significantly decreased in the M1-type strain by 13fold, as well as in the M28 (6-fold) and M41 (3-fold) strains (Figure 3C). Additionally, there was no significant difference between scl2 expression in MGAS315 and the M3 strains MGAS158, 335, 1313, and 10870 (Table S4). These results confirm that the M3 scl2.3 allele is transcribed at high levels, resulting in considerable expression of the Scl2.3 protein. These results suggested that Scl2 has an important biological function in M3 strains and warranted subsequent experiments assessing Scl2.3 function.

\section{GAS infection disseminates through human tissue and inhibits wound healing}

A wounded human skin equivalent, devoid of an inflammatory component, was utilized as a "mechanistic model" of GAS tissue colonization. The epidermal wound of each skin equivalent was infected with GFP-expressing M3-type invasive strain MGAS315 or M41type non-invasive strain MGAS6183, and analyzed after 1-5 days by standard histopathology (H\&E and Gram's stain) and using two-photon fluorescence (TPF) microscopy. H\&E of uninfected tissue controls harvested at day 0 showed the absence of a keratinocyte layer where the punch biopsy was performed (Figure 4A). Complete healing of the wound was observed after 5 days, with a newly-generated intact keratinocyte layer covering the punch biopsy site (Figure 4B). In contrast, tissue infected with either GAS strain exhibited delayed wound closure as late as day 5 post-infection (Figure 4E, H). H\&E staining of skin equivalents infected with either M3 or M41 after 24 hours revealed bacterial colonization of the exposed dermal surface, as well as bacterial invasion into puncture-associated defects extending deep into the dermal layer, largely located at the wound edges (Figure 4D, G). Notably, extensive bacterial growth and spread was largely confined to tissue crevices in the dermis, whereas the presence of large bacterial colonies directly below the wound bed was rarely seen on microscopic examination. By day 5 of infection, bacterial invasion via these dermal defects extended to the bottom of the dermis for both strains, presenting both vertical and lateral spread of 
bacteria (Figure 4E, H). Gram stain of infected tissues showed the formation of superficial colonies near dermal surfaces, as well as biofilm formation on the surface of exposed dermis (Figure 4J-L). Additionally, epidermal tissue neighboring the wound bed exhibited $\sim 60 \%$ decreased thickness of the viable keratinocyte layer in tissues infected with MGAS315 and MGAS6183 by day 5, relative to uninfected tissues (Figure 4C, F, I). This suggests bacterial infection of the wound affects epidermal cells distant from the site of infection in this model.

TPF analysis was then performed on whole infected skin equivalents on day 5 post-infection in order to assess bacterial spread directly below the wound bed. This method allowed us to visualize bacterial structures within tissue that were not apparent in H\&E or Gram-stained sections. Tissue microcolonies were observed in samples infected with M41 GAS (Figure 4N), whereas M3 cells had a scattered appearance (Figure 4P). TRITC-concanavalin A (TRITC-conA) was utilized to visualize glycocalyx associated with bacteria. TRITC-conA stain colocalized with bacterial microcolonies formed by the M41 GAS (Figure 4O, Figure S3), indicating microcolonies were encased in a glycocalyx, much like a classic biofilm. In contrast, TRITC-conA stain was associated with scattered M3-GAS chains located at the bottom cell layer in MGAS315-infected skin equivalents (Figure 4Q). These results indicate that M41 GAS, but not the M3 GAS, forms microcolonies in the tissue during a human skin infection that are encased in a glycocalyx, consistent with the observation that M41, but not M3, GAS forms robust biofilm in vitro on ECM coatings. In addition, M3 GAS disseminates in a form of scattered chains or single cells through the tissue.

\section{M3-type GAS strains form poor biofilms on extracellular matrix coatings}

It was previously reported that M3-type strains have no substantial biofilm formation in vitro on an inanimate surface (36). Here, we tested biofilm formation on cellular fibronectin (cFn) and laminin (Lm) coatings by a panel of representative M3 strains isolated from invasive cases of GAS disease, as compared to the non-invasive biofilm-capable M41type model strain MGAS6183. As expected, wells coated with either cFn or Lm supported robust biofilm formation by the M41 strain, whereas significantly less bacterial biomass was measured for all M3-type GAS on both ECM coatings (Figure 5A). There was also 
no correlation between Scl2 expression and biofilm formation. We hypothesized that M3type GAS, devoid of Scl1 adhesin, have decreased binding to cFn and Lm ECM components, thus, preventing the formation of tissue microcolonies, and that restoration of full-length Scl1.3 (Scl1.3FL) on the GAS cell surface will confer binding to host ECM, as well as biofilm capacity in vivo (Figure 5B).

\section{Full-length recombinant Scl1.3 binds cellular fibronectin and laminin}

To test this hypothesis, we: (i) constructed Scl1.3- and Scl2.3-derived recombinant proteins, (ii) characterized their structural organization, and (iii) assessed their ECMbinding capacities.

First, rScl1.3FL and rScl2.3 proteins were assessed for purity and integrity by SDSPAGE (Figure 6A). The expected 20.6-kDa rScl1.3FL migrated at $\sim 34 \mathrm{kDa}$, which is consistent with previous reports of aberrant migration of rScl proteins $(29,30)$, whereas the rScl2.3 protein migrated according to the expected molecular mass of $16.2 \mathrm{kDa}$; both proteins were verified by mass spectrometry (Table S3). Rotary shadowed rScl1.3FL and rScl2.3 constructs, exhibited the characteristic lollipop-like structural organization (Figure S2), as observed for previously characterized rScl proteins $(47,49)$. Interestingly, rScl1.3FL formed aggregates that were mediated by the intermolecular interactions between the globular domains; such interactions, however, were not observed between rScl2.3 molecules. The appearance of Scl1-Scl1 aggregates implies an attractive hypothesis that V-to-V-region interactions between the Scl1 molecules, but not between the Scl2 molecules, on the surface of neighboring GAS chains may support biofilm structure, as proposed in our model (Figure 5B).

It has been established that Scl1 proteins selectively bind cellular, but not plasma, fibronectin and laminin (35), and that selective cFn binding is achieved by a unique binding mechanism involving the C-C' loop of the extra domain A in cFn (EDA-cFn) (37). Here, we pre-incubated cFn with increasing concentrations of EDA-blocking IST-9 mAb $(0.1,1.0 \mu \mathrm{g})$, then added to wells containing immobilized $\mathrm{rScl}$ proteins and allowed for binding. We observed significant dose-dependent inhibition of cFn binding to rScl1.3FL, with a $36 \%$ inhibition at $0.1 \mu \mathrm{g}$ and a $60 \%$ inhibition at $1.0 \mu \mathrm{g}$ of IST-9 (Figure 6B). In addition, the rScl2.3 protein did not bind $\mathrm{cFn}$, which is consistent with our previous data 
employing several different $\mathrm{rScl} 2$ proteins (35). Surface plasmon resonance (SPR) measurements of binding affinity between rScl1.3FL and EDA-derived C-C' cyclic peptide provided a dissociation constant of $\mathrm{K}_{\mathrm{D}}=63.3 \mu \mathrm{M}$ (Figure $6 \mathrm{C}$ ). To corroborate the rScl1.3FL-EDA binding affinity, an in-solution fluorescence binding assay was performed, in which the variation in tryptophan fluorescence of rScl1.3FL was recorded as a measure of positive binding to the C-C' cyclic peptide. Tryptophan fluorescence emission at 333 $n m$ showed a dose-response quenching upon addition of the C-C' cyclic peptide and $-\Delta$ fluorescence intensity was plotted against peptide concentration (Figure 6D). Data were fitted with a 1:1 model of interaction, providing a $K_{D}=44.54 \pm 9 \mu \mathrm{M}$, in agreement with SPR data (56).

We next assessed binding of rScl1.3FL and $\mathrm{rScl} 2.3$ proteins to laminin $(\mathrm{Lm})$ by ELISA. We determined rScl1.3FL had significant Lm binding, whereas rScl2.3 had not (Figure 6E), consistent with previous findings that Scl1-derived recombinant proteins, but not the Scl2-derived, bind ECM proteins (35). Collectively, these results demonstrate specific binding of Scl1.3FL to the EDA domain of cFn and to Lm and its capacity of being surface adhesin.

\section{Homologous complementation of M3 strains with full-length surface-exposed Scl1.3 adhesin confers biofilm formation on ECM}

To assess the effect of cell-surface Scl1.3FL expression on the capacity to form biofilm, an in-trans complementation of two representative invasive M3 strains was performed, MGAS315 wild-type (WT) strain, naturally lacking Scl1.3 expression, and a previously generated sc/1-inactivated mutant of MGAS10870 (10870 $\Delta s c / 1)$, with plasmids pSL502 $\left(\mathrm{Sp}^{\mathrm{R}}\right)$ and pSL518 $\left(\mathrm{Km}^{\mathrm{R}}\right)$, respectively, both encoding the full-length Scl1.3FL protein. As a control, MGAS315 was complemented with a shuttle vector pJRS525. The cell wallassociated expression of Scl1.3FL in complemented M3-type GAS was first tested by western blot analysis of the cell wall protein fractions and on the GAS-cell surface by flow cytometry. An expected $\sim 35-\mathrm{kDa}$ immunoreactive band in complemented strains was observed, which was absent in the parent strains (Figure 7A). Mass spectrometry analysis of the corresponding bands extracted from the gel confirmed they represented the Scl1.3FL protein, with five unique peptides identified with $17 \%$ sequence coverage for 
complemented MGAS315 and four unique peptides identified with $11 \%$ sequence coverage for complemented MGAS10870 (Table S3). A 6.8-fold increase in median fluorescence intensity of Scl1.3FL-complemented MGAS315 cells was measured by flow cytometry, as compared to the vector-complemented and WT control strains (Figure 7B). The Scl1.3FL-complemented $10870 \Delta s c / 1$ exhibited a 2.2 -fold increase in median fluorescence intensity, as compared to the mutant control (Figure 7B). These results indicate the Scl1.3FL is indeed expressed and surface-exposed in the complemented M3type strains.

Biofilm formation by the complemented and parent strains was then assessed after 24 hours following crystal violet staining and using confocal laser scanning microscopy (CLSM). Scl1.3FL-expressing MGAS315 showed significantly increased biomass on both cFn and Lm coatings compared to the WT parent organism, as well as vectorcomplemented control; 3.6 or 2.5 - and 2.5 or 2.1 -fold $\mathrm{OD}_{600}$ increases on cFn and Lm, respectively, were measured compared to MGAS315 WT or to vector-complemented MGAS315 (Figure 7C). Significantly thicker biofilm formed by Scl1.3FL-complemented MGAS315 was imaged by CLSM. We observed on average a 2.8-fold $(P=0.0002)$ and 2.6-fold ( $P=0.0002$ ) increased biofilm thickness on $\mathrm{cFn}$ and $\mathrm{Lm}$, respectively, compared to MGAS315 WT, and a 2.0-fold $(P=0.0036)$ and 4.8 -fold $\left(P=2.1 \times 10^{-5}\right)$ increased biofilm thickness on $\mathrm{cFn}$ and $\mathrm{Lm}$, respectively, compared to vector-complemented MGAS315 (Figure 7D and E, representative images). Similar results were obtained for the complemented $10870 \Delta s c / 1$ mutant, which had 1.7-fold increase in biomass staining on both cFn and Lm compared to the MGAS10870 WT strain, and 2.2- and 2.6-fold increase in biomass staining on $\mathrm{cFn}$ and $\mathrm{Lm}$, respectively, compared to the $10870 \Delta \mathrm{sc} / 1$ mutant strain (Figure 7C). CLSM data, however, could not be rendered for MGAS10870 strains due to poor GFP expression for unknown reasons. Altogether, it was demonstrated that null mutation in the scl1 gene, which ablates surface Scl1.3 protein and is unique to M3type GAS, is responsible for the decreased biofilm capacity since restoration of the fulllength surface-exposed Scl1.3 adhesin significantly fosters stable biofilm formation. 
Heterologous complementation of M41 $\Delta s c / 1$ mutant strain with full-length surfaceexposed Scl1.3 restores biofilm formation on ECM

In a previous study we showed that scl1.41-inactivation in a non-invasive biofilm-rich M41 strain MGAS6183 resulted in significantly decreased biofilm capacity, which was restored to wild-type level by complementation with surface Scl1.41 (37). Notably, this M41 strain expresses at least one additional major Fn-binding protein, protein F2 (45), which binds both plasma and cellular fibronectin by a mechanism different from Scl1 (57). Here, we hypothesized that expression of rScl1.3FL in the heterologous M41 GAS will confer biofilm formation. Western immunoblotting of the cell wall protein fractions detected the $\sim 35-\mathrm{kDa}$ immunoreactive band, corresponding to full-length Scl1.3, associated with complemented cells, while the M41Ascl1 mutant and WT cells were signal-negative (Figure 8A). Mass spectrometry of the corresponding band extracted from the gel confirmed Scl1.3FL expression, with 3 unique peptides identified, covering $13 \%$ of the amino acid sequence (Table S3). In addition, we showed the expression of the larger Scl1.41 variant in the cell wall of M41 WT, but not in the $\Delta s c / 1$ mutant, by re-probing a portion of the blot with anti-rScl1.41 antibody, using rScl1.41 protein as a positive control. Similarly to complementation with homologous Scl1.41 protein, the heterologous complementation of the M41 $\Delta s c / 1$ mutant with Scl1.3FL also restored biofilm to M41 WT levels on cFn and Lm (Figure 8B-D). Crystal violet staining showed increased bacterial biomass (Figure $8 \mathrm{~B}$ ) and confocal microscopy revealed significantly thicker biofilms, on average 3-fold increased, as compared to the parental M41 $\Delta s c / 1$ mutant strain on both cFn (Figure 8C) and Lm (Figure 8D) coatings (cFn, $P=0.0105$; Lm, $P=0.0011$ ).

\section{Expression of Scl1 adhesin attenuates GAS during subcutaneous infection}

We have previously shown that an $\mathrm{M} 3$ strain harboring a carrier scl1.3 allele, producing a shorter cell-attached Scl1.3 variant, had an attenuated phenotype in a murine model of necrotizing fasciitis (40). Here, we tested our hypothesis that Scl1 adhesin in biofilm-rich M28 and M41 background promotes stable colonization and localized infection, using a murine skin infection model. Hairless, immunocompetent SKH1 mice were subcutaneously infected with $\sim 10^{9}$ GAS CFU of the M28 and M41 wild-type (WT) or their 
isogenic scl1-inactivated mutant $(s c / 1)$ strains $(44,45)$, and mice were assessed for changes in gross pathology of the skin.

Skin lesions caused by both the WT and sc/1 strains were observed as early as 48 hours post-infection, and the lesions began to regress after day 7 with complete resolution of the lesion by the completion of the experiment. The areas of the skin lesions calculated for mice infected with the $s c / 1$ mutant strains were significantly larger than those of WTinfected mice (Figure 9A; 96 hour time-point is shown); images of lesions of representative mice demonstrate differences in lesion severity between WT- and scl1GAS infected mice for both the M28- and M41-infected groups (Figure 9B). Thus, the in vivo data, using GAS strains expressing surface Scl1 proteins and producing rich biofilms, support our hypothesis that decreased adhesion and biofilm formation, due to the absence of Scl1.3 on the surface of M3 strains, bears an inverse correlation to the invasive potential of the infecting GAS strain.

\section{DISCUSSION}

Since the resurgence of invasive GAS disease in the 1980's, and emergence of invasive M3-type isolates, significant efforts have been made to determine the molecular basis for the invasive phenotype of M3-type strains. Numerous whole-genome sequencing projects have identified specific genomic features of $\mathrm{M} 3$ strains that were correlated with their potential to cause invasive infections. In this study, we show that Scl1-negative M3type GAS have reduced adhesion and biofilm formation within host tissue, and therefore are predisposed to invasive spread over superficial infection (Figure 10).

\section{Unique topography and expression patterns of $s c /$ loci}

First, the insertion of IS1548 element in the promoter region of $s c / 1$ exclusively found in the M3-type strains is an intriguing observation, given the abundance of IS1548 insertions across GAS genomes representing a variety of M-types. IS elements are known to cause genomic rearrangements and affect the expression of important genes that contribute to bacterial virulence (58). For example, the insertion of IS1548 element (59) into the $\operatorname{scpB}$ Imb intergenic region in group B Streptococcus has been shown to upregulate Imb-gene transcription and Lmb-adhesin surface expression, resulting in enhanced laminin binding 
(60). The IS1548 insertion in the scl1.3 promoter was conserved in the genomes of sequenced M3 strains MGAS315, SSI-1, and M3-b, as well as in 40 additional M3 strains tested by PCR. However, the recently sequenced STAB902 M3 strain (55), which represents a non-invasive isolate, contained a 34-bp IS1548 remnant, suggesting that M3 circulating strains exist with this polymorphism. We hypothesize that the proximity of the IS1548 insertion to the Mga I binding site in M3 strains affects scl1.3 transcription. Comparative qRT-PCR analysis showed significantly higher levels of scl1 transcription, particularly in M1, as well as in M28 and M41 strains, relative to M3-type strains analyzed.

Variation in the Mga coding sequence and autoregulated mga promoter has been reported to significantly affect Mga-dependent gene expression and virulence properties in GAS $(26,27,61)$. For example, a 12-bp deletion of a single repeat in the VNTR region of the mga promoter is present in M3 carrier strains and absent in the invasive M3 strains, causing a downregulation of Mga expression and Mga-regulated genes in the carrier strains (27). We identified an analogous 12-bp deletion in the VNTR of the mga promoter of the M1-type MGAS5005 and in M3 strains, MGAS335 and MGAS1313, but not in MGAS315, MGAS158 and MGAS10870. It is likely that the variation observed in the VNTR region impacts Mga expression but could not, alone, explain differences in sc/1 transcription patterns observed in this work. Consequently, qRT-PCR analyses showed $m g a$ and emm transcripts (emm is second downstream target of Mga within the Mga regulon) were expressed at similar levels in MGAS5005 and M3 strains, except for the MGAS335, indicating a mechanism of sc/1 downregulation in M3 strains, which is independent from the level of Mga transcription. The presence of emm transcripts and M3-protein products indicate that Mga is present and functional in the majority of M3 strains tested, and therefore the polymorphism we observed in the mga promoter did not affect Mga expression or activity of Mga-controlled sc/1.3 promoter. Altogether, our data hint at the insertion of the IS1548 in the downregulation of $s c / 1.3$ expression, specifically in M3-type GAS, although additional studies are required to firmly establish whether the IS element insertion is responsible.

It is intriguing that $s c / 1$ and $s c / 2$ expression patterns were drastically different between M1 and M3-type GAS, both associated with invasive infections. The sc/1.3 transcript was decreased by 21 -fold in MGAS315, and even further in the remaining M3 
strains studied (additional 20-45 fold), as compared to the M1-type strain MGAS5005. The upregulation of sc/1 in M1-type GAS has previously been shown in invasive M1 isolates, including MGAS5005, as compared to pharyngeal M1 isolates (62). Studies comparing wild-type and isogenic sc/1.1 mutants in M1-type GAS reported that Scl1.1 contributed to immune evasion by inhibiting neutrophil extracellular trap production and by protecting bacteria from the cathelicidin LL-37 (63). We conclude the differences we observed in sc/1 expression are related to different pathogenicity requirements for Scl1 protein in M1- versus M3-type strains. In contrast, Scl2 expression is highly upregulated in M3-type strains, as compared to invasive M1-type GAS. While the majority of M3-type strains contain in-frame scl2.3 alleles, all $21 \mathrm{M} 1$ strains we analyzed contained out-offrame scl2.1 alleles (unpublished data). Although the Scl2.3 human ligands are not known, it may have an unidentified biological function, which is important for pathogenesis of M3, but not M1, strains. Hence, we are reporting striking differences in the expression and features of the Scl1 and Scl2 proteins that evolved in the invasive M1 and M3 strains.

\section{Scl1-mediated adhesion and biofilm formation in vitro}

Scl1 mediates binding to human extracellular matrix components, cellular fibronectin and laminin, as well as biofilm formation (35-37). Previous work identified that Scl1 binds specifically to the C-C' loop of the type III-repeat EDA domain of cellular fibronectin (37). This represents a novel mechanism of fibronectin binding, which is distinct from the mechanism employed by other GAS fibronectin-binding proteins that bind to the $\mathrm{N}$ terminal type I repeats of fibronectin (64). Here, we determined that rScl1.3FL binds to cellular fibronectin via the same C-C' loop-dependent mechanism. It is important for our overall model to acknowledge that the EDA-cFn isoform is specifically produced during embryogenesis and during wound healing in adult tissue (65-67), which indicates Scl1 evolved with a unique function for targeting wounded tissue, a presumed pathogen portal of entry. We also demonstrate significant binding of rScl1.3FL to laminin, a major basement membrane protein at the epidermal-dermal junction; none of those ECM ligands were bound by rScl2.3, consistent with previous knowledge that Scl1, but not Scl2, variants exhibit binding to cFn and Lm (35). These studies show that full-length 
Scl1.3, if expressed on the M3-GAS cell surface, would bind ECM, contributing to tissue colonization.

The diminished Scl1.3 expression and poor biofilm formation, led us to hypothesize that lack of surface-expressed Scl1 in M3-type GAS reduces host ECM binding and stable microcolony formation in tissue, thus, shifting the balance towards invasive spread, augmented by other virulence factors expressed by these strains. Recombinant rScl1.3FL formed aggregates that were mediated by the $\mathrm{V}$-to-V region interactions, which could represent a mechanism of biofilm and microcolony stabilization by Scl1 molecules on adjacent GAS cells. Two representative invasive M3 isolates, MGAS315 and MGAS10870, acquired biofilm formation on $\mathrm{cFn}$ and $\mathrm{Lm}$ when homologous complementation was performed with surface-attached Scl1.3FL. MGAS315 has been shown to contain a missense mutation in the covS gene, causing upregulation of CovRSregulated virulence genes and enhanced virulence during subcutaneous infection of mice, compared to an isogenic strain containing the WT covS allele (68). Additionally, MGAS315 contains a mutation in the regulator of protein $B$ allele, rop $B$, which produces a nonfunctional RopB variant, while MGAS10870 contains a wild-type covR/S and $\operatorname{rop} B$ alleles (28), allowing us to demonstrate the effect of Scl1.3FL function in the presence of differing regulatory networks. Similarly, heterologous complementation with surface Scl1.3FL in a scl1.41-mutant of the non-invasive biofilm-capable strain M41-type MGAS6183, restored its biofilm capacity to the wild-type level. These results indicate that M3-derived Scl1.3FL variant has the full capacity to support biofilm formation to a similar degree as Scl1 from a divergent M-type. The robust biofilm observed in vitro on ECM coatings validates the concept that biofilm capacity combined with adherence to the surrounding host ECM would reinforce the formation of stable tissue microcolonies in vivo.

\section{In vitro skin equivalent model of wound colonization and microcolony formation}

We observed inhibition of wound re-epithelization by GAS infection of wounded skin equivalents, as well as the thinning of the viable epidermal layer at sites distant from the infected wound. In addition to our study, others have reported changes in skin histopathology and wound healing, resulting from bacterial infections. An in vivo study 
has reported epidermal defects as a result of GAS infection in a humanized mouse model with human skin graft (69). Previous study of M3-type GAS infection using a skin equivalent model showed that hyaluronic acid capsule interactions with CD44 receptor on keratinocytes induced intracellular signaling, resulting in cytoskeletal rearrangement and monolayer disruption (70). Infection of an in vitro skin model containing a burn wound with Pseudomonas aeruginosa caused a loss of the keratinocyte layer and basement membrane, while intact epidermis was observed in burned but uninfected tissue (71). Impairment of wound healing has also been demonstrated by staphylococcal infections. Infection of dermal wounds in rabbit ears with Staphylococcus aureus showed the formation of biofilm, production of a persistent, low grade inflammatory response, and significantly delayed wound healing (72). Similarly, delayed wound healing by both $S$. aureus and $S$. epidermidis biofilms was observed in a mouse model of cutaneous wounds (73). The inhibition of wound healing we describe here is by and large consistent with reports by other laboratories generated using in vivo animal and in vitro human skin infection models.

Microcolonies have been identified within human streptococcal impetigo lesions (39) and in tonsils from patients with recurrent GAS pharyngeal tonsillitis (74), and likely represent a superficial or persistent state of GAS colonization. However, streptococcal infections can result in invasive disease due to biofilm disruption and bacterial dissemination $(75,76)$. We observed large rounded microcolonies formed in tissue during infection with M41 strain, while M3 GAS remained scattered throughout the tissue as single cells and chains. Microcolony formation has been previously observed with $S$. aureus infection in organotypic skin model (77). Moreover, wound infection in rabbit ears with $S$. aureus produced mature biofilms encased in exopolysaccharide, as revealed by concanavalin A staining (72). Similarly, we also demonstrated that microcolonies in M41infected tissue were encapsulated in bacterial-associated glycocalyx. However, a lack of glycocalyx-encapsulated microcolonies was associated with infection by M3-type GAS.

These results support our hypothesis that biofilm-poor M3 strains are abolished in stable microcolony formation in vivo, in part due to a lack of the ECM-binding Scl1 protein and an overall lack of surface adhesins, although they likely express the FbaB protein, 
identified in M3 GAS to be involved in the adherence and invasion into epithelial and endothelial cells (78-80).

\section{In vivo mouse model of skin infection}

Recent study reported that a small proportion of non-invasive M3-type strains ( 1.3\%) were found to harbor the sc/1.3 "carrier allele", which resulted from an in-frame deletion in the collagenous region, encompassing the null mutation, producing a shorter cellattached Scl1.3 variant. This MGAS10870 strain containing the sc/1.3 carrier allele was attenuated following intramuscular infection (40). In this study, we utilized the M28- and M41-type strains, representing biofilm-rich producers, for subcutaneous inoculation. We observed that sc/1.28- and sc/1.41-inactivated isogenic mutants produced significantly larger skin lesions as compared to the wild-type parent strains. These results, again, support the hypothesis that lack of Scl1 surface adhesin destabilizes focused nidus of infection, resulting in a shift towards increased tissue spread. However, previous studies performed in a M1 GAS background, utilizing scl1.1-mutants for subcutaneous infection, reported smaller skin lesions in the mutant groups, which likely reflects a differing predominant function of Scl1.1 in M1-type GAS $(29,63)$. Investigations using intranasal and intraperitoneal mouse infection models of Streptococcus pneumoniae have shown that culture-grown bacteria disseminated to the ear and lungs, while biofilm-grown bacteria stably colonized the nasopharynx $(81,82)$. A similar study on Streptococcus pyogenes showed that bacteria grown in biofilms have downregulated virulence genes and tend to colonize the nasal associated lymphoid tissue of mice, while culture-grown bacteria had significantly increased dissemination and were more virulent in a septicemia model (83). Previous studies reported that inactivation of some GAS genes resulted in increased skin pathology produced by the mutants compared to their wild-type organisms, and these genes often encoded surface proteins, including SpyCEP (84), Mrp (85), protein F1 (86), and Spy0128, encoding a major pilus subunit (87). Similarly, the covS mutant of group A streptococcal M1T1 strain with upregulated SpeB-protease activity was hypervirulent and had reduced capacity to bind human epithelial cells and fibronectin, and

also to form biofilm due to increased cleavage of surface proteins (88). Altogether, the concept that expression of a surface adhesin, such as Scl1, involved in biofilm formation 
and host tissue attachment, is inversely related to strain invasiveness has gained support from several studies, including this study.

We show the invasive M1- and M3-type GAS evolved sc/1 and scl2 alleles with opposite expression patterns, with scl1 downregulated and scl2 upregulated in M3 compared to M1 GAS. We show M3-type GAS, devoid of surface-expressed Scl1.3, lacked biofilm formation on ECM coatings and microcolony formation during infection of in vitro wounded skin equivalent. Complementation with surface Scl1.3FL restored biofilm capacity of M3-type GAS on ECM coatings. Mouse infection with the isogenic scl1 mutants of biofilm-rich M28- and M41-type GAS produced larger lesions, supporting the role of Scl1 in a localized tissue infection. Lastly we developed a model for Scl1-mediated microcolony formation (Figure 10), whereby Scl1 expressed on the GAS surface strengthens host colonization by attachment to cFn and Lm expressed within wounded tissue, as well as biofilm formation via Scl1-Scl1 interactions, resulting in a local, stabilized microcolony. Conversely, when Scl1 is absent on the GAS cell surface, as is the case for M3-type GAS, bacteria lack stable anchoring in the surrounding host ECM, as well as structural strength within microcolony, promoting cells to disperse more freely.

\section{ACKNOWLEDGMENTS}

We thank: Mariette Barbier for assistance with qRT-PCR analysis; Meenal Elliott for assistance with some experiments; Karen Martin, Amanda Ammer, and Lingqing Zhang for assistance with imaging experiments; and Jayme Horning for assistance with mass spectrometry. We thank James Dale for providing anti-M3 antibodies. We also thank Mike Federle and Jennifer Franko for a critical reading of the manuscript. This work was supported in part by National Institutes of Health Grants Al50666 and Al083683 (SL); BB and DHM were supported by the NSF-EPSCoR Graduate Fellowship Program under the Research Infrastructure Improvement (RII) Track-1 award, Cooperative agreement 1003907 (BB) and Integrative Graduate Education and Research Training for Research and Education in Nanotoxicology under award number 1144676 (DHM). BB and DHM were also awarded with the Dr. Jennifer Gossling Scholarship in Microbiology. Flow Cytometry experiments were performed in the West Virginia University Flow Cytometry \& Single Cell Core Facility, which is supported by the National Institutes of Health 
equipment grant numbers S10OD016165 and RR020866 and the Institutional Development Awards (IDeA) from the National Institute of General Medical Sciences of the National Institutes of Health under grant numbers P30GM103488 (CoBRE) and P20GM103434 (INBRE). Imaging experiments and image analysis were performed in the West Virginia University Microscope Imaging Facility, which has been supported by the Mary Babb Randolph Cancer Center and NIH grants P20 RR016440, P30 GM103488 and P20 GM103434.

\section{REFERENCES}

1. Carapetis, J. R., Steer, A. C., Mulholland, E. K., and Weber, M. (2005) The global burden of group A streptococcal diseases. Lancet Infect Dis 5, 685-694

2. Sims Sanyahumbi, A., Colquhoun, S., Wyber, R., and Carapetis, J. R. (2016) Global Disease Burden of Group A Streptococcus. in Streptococcus pyogenes: Basic Biology to Clinical Manifestations (Ferretti, J. J., Stevens, D. L., and Fischetti, V. A. eds.), The University of Oklahoma Health Sciences Center, Oklahoma City, OK. pp

3. Cunningham, M. W. (2000) Pathogenesis of group A streptococcal infections. Clin Microbiol Rev 13, 470-511

4. Stevens, D. L., and Kaplan, E. L. (eds). (2000) Streptococcal infections: clinical aspects, microbiology, and molecular pathogenesis, Oxford University Press, New York, N.Y.

5. Shulman, S. T., Tanz, R. R., Kabat, W., Kabat, K., Cederlund, E., Patel, D., Li, Z., Sakota, V., Dale, J. B., and Beall, B. (2004) Group A streptococcal pharyngitis serotype surveillance in North America, 2000-2002. Clin Infect Dis 39, 325-332

6. Musser, J. M., Hauser, A. R., Kim, M. H., Schlievert, P. M., Nelson, K., and Selander, R. K. (1991) Streptococcus pyogenes causing toxic-shock-like syndrome and other invasive diseases: clonal diversity and pyrogenic exotoxin expression. Proc Natl Acad Sci U S A 88, 2668-2672

7. Cleary, P. P., Kaplan, E. L., Handley, J. P., Wlazlo, A., Kim, M. H., Hauser, A. R., and Schlievert, P. M. (1992) Clonal basis for resurgence of serious Streptococcus pyogenes disease in the 1980s. Lancet 339, 518-521

8. Cockerill, F. R., 3rd, MacDonald, K. L., Thompson, R. L., Roberson, F., Kohner, P. C., Besser-Wiek, J., Manahan, J. M., Musser, J. M., Schlievert, P. M., Talbot, J., Frankfort, B., Steckelberg, J. M., Wilson, W. R., and Osterholm, M. T. (1997) An outbreak of invasive group A streptococcal disease associated with high carriage rates of the invasive clone among school-aged children. JAMA 277, 38-43

9. Stevens, D. L., Tanner, M. H., Winship, J., Swarts, R., Ries, K. M., Schlievert, P. M., and Kaplan, E. (1989) Severe group A streptococcal infections associated with a toxic shock-like syndrome and scarlet fever toxin A. N Engl J Med 321, 1-7

10. DiPersio, J. R., File, T. M., Jr., Stevens, D. L., Gardner, W. G., Petropoulos, G., and Dinsa, K. (1996) Spread of serious disease-producing M3 clones of group A 
Streptococcus among family members and health care workers. Clin Infect Dis 22, 490-495

11. Johnson, D. R., Stevens, D. L., and Kaplan, E. L. (1992) Epidemiologic analysis of group A streptococcal serotypes associated with severe systemic infections, rheumatic fever, or uncomplicated pharyngitis. J Infect Dis 166, 374-382

12. Davies, H. D., McGeer, A., Schwartz, B., Green, K., Cann, D., Simor, A. E., Low, D. E., and Group, O. G. A. S. S. (1996) Invasive group A streptococcal infections in Ontario, Canada. N Engl J Med 335, 547-554

13. Kaul, R., McGeer, A., Low, D. E., Green, K., and Schwartz, B. (1997) Populationbased surveillance for group A streptococcal necrotizing fasciitis: Clinical features, prognostic indicators, and microbiologic analysis of seventy-seven cases. Ontario Group A Streptococcal Study. Am J Med 103, 18-24

14. Sharkawy, A., Low, D. E., Saginur, R., Gregson, D., Schwartz, B., Jessamine, P., Green, K., and McGeer, A. (2002) Severe group a streptococcal soft-tissue infections in Ontario: 1992-1996. Clin Infect Dis 34, 454-460

15. Hollm-Delgado, M. G., Allard, R., and Pilon, P. A. (2005) Invasive group A streptococcal infections, clinical manifestations and their predictors, Montreal, 1995-2001. Emerg Infect Dis 11, 77-82

16. Gaworzewska, E., and Colman, G. (1988) Changes in the pattern of infection caused by Streptococcus pyogenes. Epidemiol Infect 100, 257-269

17. Colman, G., Tanna, A., Efstratiou, A., and Gaworzewska, E. T. (1993) The serotypes of Streptococcus pyogenes present in Britain during 1980-1990 and their association with disease. J Med Microbio/ 39, 165-178

18. Meisal, R., Hoiby, E. A., Caugant, D. A., and Musser, J. M. (2010) Molecular characteristics of pharyngeal and invasive emm3 Streptococcus pyogenes strains from Norway, 1988-2003. Eur J Clin Microbiol Infect Dis 29, 31-43

19. Lamagni, T. L., Neal, S., Keshishian, C., Alhaddad, N., George, R., Duckworth, G., Vuopio-Varkila, J., and Efstratiou, A. (2008) Severe Streptococcus pyogenes infections, United Kingdom, 2003-2004. Emerg Infect Dis 14, 202-209

20. Beres, S. B., Sylva, G. L., Barbian, K. D., Lei, B., Hoff, J. S., Mammarella, N. D., Liu, M. Y., Smoot, J. C., Porcella, S. F., Parkins, L. D., Campbell, D. S., Smith, T. M., McCormick, J. K., Leung, D. Y., Schlievert, P. M., and Musser, J. M. (2002) Genome sequence of a serotype M3 strain of group A Streptococcus: phageencoded toxins, the high-virulence phenotype, and clone emergence. Proc Natl Acad Sci U S A 99, 10078-10083

21. Nakagawa, I., Kurokawa, K., Yamashita, A., Nakata, M., Tomiyasu, Y., Okahashi, N., Kawabata, S., Yamazaki, K., Shiba, T., Yasunaga, T., Hayashi, H., Hattori, M., and Hamada, S. (2003) Genome sequence of an M3 strain of Streptococcus pyogenes reveals a large-scale genomic rearrangement in invasive strains and new insights into phage evolution. Genome Res 13, 1042-1055

22. Beres, S. B., Sylva, G. L., Sturdevant, D. E., Granville, C. N., Liu, M., Ricklefs, S. M., Whitney, A. R., Parkins, L. D., Hoe, N. P., Adams, G. J., Low, D. E., DeLeo, F. R., McGeer, A., and Musser, J. M. (2004) Genome-wide molecular dissection of serotype M3 group A Streptococcus strains causing two epidemics of invasive infections. Proc Natl Acad Sci U S A 101, 11833-11838 
23. Beres, S. B., Carroll, R. K., Shea, P. R., Sitkiewicz, I., Martinez-Gutierrez, J. C., Low, D. E., McGeer, A., Willey, B. M., Green, K., Tyrrell, G. J., Goldman, T. D., Feldgarden, M., Birren, B. W., Fofanov, Y., Boos, J., Wheaton, W. D., Honisch, C., and Musser, J. M. (2010) Molecular complexity of successive bacterial epidemics deconvoluted by comparative pathogenomics. Proc Natl Acad Sci U S A 107, 4371-4376

24. Shea, P. R., Beres, S. B., Flores, A. R., Ewbank, A. L., Gonzalez-Lugo, J. H., Martagon-Rosado, A. J., Martinez-Gutierrez, J. C., Rehman, H. A., SerranoGonzalez, M., Fittipaldi, N., Ayers, S. D., Webb, P., Willey, B. M., Low, D. E., and Musser, J. M. (2011) Distinct signatures of diversifying selection revealed by genome analysis of respiratory tract and invasive bacterial populations. Proc Natl Acad Sci U S A 108, 5039-5044

25. Olsen, R. J., Laucirica, D. R., Watkins, M. E., Feske, M. L., Garcia-Bustillos, J. R., Vu, C., Cantu, C., Shelburne, S. A., 3rd, Fittipaldi, N., Kumaraswami, M., Shea, P. R., Flores, A. R., Beres, S. B., Lovgren, M., Tyrrell, G. J., Efstratiou, A., Low, D. E., Van Beneden, C. A., and Musser, J. M. (2012) Polymorphisms in regulator of protease B (RopB) alter disease phenotype and strain virulence of serotype M3 group A Streptococcus. J Infect Dis 205, 1719-1729

26. Cao, T. N., Liu, Z., Cao, T. H., Pflughoeft, K. J., Trevino, J., Danger, J. L., Beres, S. B., Musser, J. M., and Sumby, P. (2014) Natural disruption of two regulatory networks in serotype M3 group A Streptococcus isolates contributes to the virulence factor profile of this hypervirulent serotype. Infect Immun 82, 1744-1754

27. Flores, A. R., Olsen, R. J., Wunsche, A., Kumaraswami, M., Shelburne, S. A., 3rd, Carroll, R. K., and Musser, J. M. (2013) Natural variation in the promoter of the gene encoding the Mga regulator alters host-pathogen interactions in group a Streptococcus carrier strains. Infect Immun 81, 4128-4138

28. Carroll, R. K., Shelburne, S. A., 3rd, Olsen, R. J., Suber, B., Sahasrabhojane, P., Kumaraswami, M., Beres, S. B., Shea, P. R., Flores, A. R., and Musser, J. M. (2011) Naturally occurring single amino acid replacements in a regulatory protein alter streptococcal gene expression and virulence in mice. J Clin Invest 121, 19561968

29. Lukomski, S., Nakashima, K., Abdi, I., Cipriano, V. J., Ireland, R. M., Reid, S. D., Adams, G. G., and Musser, J. M. (2000) Identification and characterization of the $s c l$ gene encoding a group A Streptococcus extracellular protein virulence factor with similarity to human collagen. Infect Immun 68, 6542-6553

30. Lukomski, S., Nakashima, K., Abdi, I., Cipriano, V. J., Shelvin, B. J., Graviss, E. A., and Musser, J. M. (2001) Identification and characterization of a second extracellular collagen-like protein made by group A Streptococcus: control of production at the level of translation. Infect Immun 69, 1729-1738

31. Rasmussen, M., and Bjorck, L. (2001) Unique regulation of ScIB - a novel collagenlike surface protein of Streptococcus pyogenes. Mol Microbiol 40, 1427-1438

32. Rasmussen, M., Eden, A., and Bjorck, L. (2000) SclA, a novel collagen-like surface protein of Streptococcus pyogenes. Infect Immun 68, 6370-6377

33. Whatmore, A. M. (2001) Streptococcus pyogenes $s c / B$ encodes a putative hypervariable surface protein with a collagen-like repetitive structure. Microbiology 147, 419-429 
34. Almengor, A. C., and Mclver, K. S. (2004) Transcriptional activation of $s c / A$ by Mga requires a distal binding site in Streptococcus pyogenes. J Bacteriol 186, 78477857

35. Caswell, C. C., Oliver-Kozup, H., Han, R., Lukomska, E., and Lukomski, S. (2010) Scl1, the multifunctional adhesin of group A Streptococcus, selectively binds cellular fibronectin and laminin, and mediates pathogen internalization by human cells. FEMS Microbiol Lett 303, 61-68

36. Oliver-Kozup, H. A., Elliott, M., Bachert, B. A., Martin, K. H., Reid, S. D., Schwegler-Berry, D. E., Green, B. J., and Lukomski, S. (2011) The streptococcal collagen-like protein-1 (Scl1) is a significant determinant for biofilm formation by group A Streptococcus. BMC Microbiol 11, 262

37. Oliver-Kozup, H., Martin, K. H., Schwegler-Berry, D., Green, B. J., Betts, C., Shinde, A. V., Van De Water, L., and Lukomski, S. (2013) The group A streptococcal collagen-like protein-1, Scl1, mediates biofilm formation by targeting the extra domain A-containing variant of cellular fibronectin expressed in wounded tissue. Mol Microbio/ 87, 672-689

38. Lembke, C., Podbielski, A., Hidalgo-Grass, C., Jonas, L., Hanski, E., and Kreikemeyer, B. (2006) Characterization of biofilm formation by clinically relevant serotypes of group A streptococci. Appl Environ Microbiol 72, 2864-2875

39. Akiyama, H., Morizane, S., Yamasaki, O., Oono, T., and Iwatsuki, K. (2003) Assessment of Streptococcus pyogenes microcolony formation in infected skin by confocal laser scanning microscopy. J Dermatol Sci 32, 193-199

40. Flores, A. R., Jewell, B. E., Versalovic, E. M., Olsen, R. J., Bachert, B. A., Lukomski, S., and Musser, J. M. (2015) Natural variant of collagen-like protein A in serotype M3 Group A Streptococcus increases adherence and decreases invasive potential. Infect. Immun. 83, 1122-1129

41. Pahlman, L. I., Marx, P. F., Morgelin, M., Lukomski, S., Meijers, J. C., and Herwald, $\mathrm{H}$. (2007) Thrombin-activatable fibrinolysis inhibitor binds to Streptococcus pyogenes by interacting with collagen-like proteins A and B. J Biol Chem $\mathbf{2 8 2}$, 24873-24881

42. Squeglia, F., Bachert, B., Romano, M., Lukomski, S., and Berisio, R. (2013) Crystallization and preliminary X-ray crystallographic analysis of the variable domain of Scl2.3, a streptococcal collagen-like protein from invasive M3-type Streptococcus pyogenes. Acta Crystallogr Sect F Struct Biol Cryst Commun 69, 1023-1025

43. Squeglia, F., Bachert, B., De Simone, A., Lukomski, S., and Berisio, R. (2014) The crystal structure of the streptococcal collagen-like protein 2 globular domain from invasive M3-type group A Streptococcus shows significant similarity to immunomodulatory HIV protein gp41. J Biol Chem 289, 5122-5133

44. Han, R., Caswell, C. C., Lukomska, E., Keene, D. R., Pawlowski, M., Bujnicki, J. M., Kim, J. K., and Lukomski, S. (2006) Binding of the low-density lipoprotein by streptococcal collagen-like protein Scl1 of Streptococcus pyogenes. Mol Microbiol 61, 351-367

45. Caswell, C. C., Lukomska, E., Seo, N. S., Hook, M., and Lukomski, S. (2007) Scl1dependent internalization of group A Streptococcus via direct interactions with the 
alpha2beta(1) integrin enhances pathogen survival and re-emergence. $\mathrm{Mol}$ Microbiol 64, 1319-1331

46. Virtaneva, K., Porcella, S. F., Graham, M. R., Ireland, R. M., Johnson, C. A., Ricklefs, S. M., Babar, I., Parkins, L. D., Romero, R. A., Corn, G. J., Gardner, D. J., Bailey, J. R., Parnell, M. J., and Musser, J. M. (2005) Longitudinal analysis of the group A Streptococcus transcriptome in experimental pharyngitis in cynomolgus macaques. Proc Natl Acad Sci U S A 102, 9014-9019

47. Han, R., Zwiefka, A., Caswell, C. C., Xu, Y., Keene, D. R., Lukomska, E., Zhao, Z., Hook, M., and Lukomski, S. (2006) Assessment of prokaryotic collagen-like sequences derived from streptococcal Scl1 and Scl2 proteins as a source of recombinant GXY polymers. Appl Microbiol Biotechnol 72, 109-115

48. Mclver, K. S., and Scott, J. R. (1997) Role of mga in growth phase regulation of virulence genes of the group A Streptococcus. J Bacteriol 179, 5178-5187

49. Xu, Y., Keene, D. R., Bujnicki, J. M., Hook, M., and Lukomski, S. (2002) Streptococcal Scl1 and Scl2 proteins form collagen-like triple helices. J Biol Chem 277, 27312-27318

50. Fields, G. B., and Noble, R. L. (1990) Solid phase peptide synthesis utilizing 9fluorenylmethoxycarbonyl amino acids. Int J Pept Protein Res 35, 161-214

51. Vanier, G. S. (2013) Microwave-assisted solid-phase peptide synthesis based on the Fmoc protecting group strategy (CEM). Methods Mol Biol 1047, 235-249

52. Williamson, M. P. (2013) Using chemical shift perturbation to characterise ligand binding. Progress in nuclear magnetic resonance spectroscopy 73, 1-16

53. Cramer, T., Yamanishi, Y., Clausen, B. E., Forster, I., Pawlinski, R., Mackman, N., Haase, V. H., Jaenisch, R., Corr, M., Nizet, V., Firestein, G. S., Gerber, H. P., Ferrara, N., and Johnson, R. S. (2003) HIF-1alpha is essential for myeloid cellmediated inflammation. Cell 112, 645-657

54. Lukomski, S., Montgomery, C. A., Rurangirwa, J., Geske, R. S., Barrish, J. P., Adams, G. J., and Musser, J. M. (1999) Extracellular cysteine protease produced by Streptococcus pyogenes participates in the pathogenesis of invasive skin infection and dissemination in mice. Infect Immun 67, 1779-1788

55. Soriano, N., Vincent, P., Moullec, S., Meygret, A., Lagente, V., Kayal, S., and Faili, A. (2014) Closed genome sequence of noninvasive Streptococcus pyogenes $\mathrm{M} / \mathrm{emm3}$ strain STAB902. Genome Announc 2

56. Russo, A., Scognamiglio, P. L., Hong Enriquez, R. P., Santambrogio, C., Grandori, R., Marasco, D., Giordano, A., Scoles, G., and Fortuna, S. (2015) In Silico Generation of Peptides by Replica Exchange Monte Carlo: Docking-Based Optimization of Maltose-Binding-Protein Ligands. PLOS ONE 10, e0133571

57. Sela, S., Aviv, A., Tovi, A., Burstein, I., Caparon, M. G., and Hanski, E. (1993) Protein F: an adhesin of Streptococcus pyogenes binds fibronectin via two distinct domains. Mol Microbiol 10, 1049-1055

58. Mahillon, J., Leonard, C., and Chandler, M. (1999) IS elements as constituents of bacterial genomes. Res Microbiol 150, 675-687

59. Granlund, M., Oberg, L., Sellin, M., and Norgren, M. (1998) Identification of a novel insertion element, IS1548, in group B streptococci, predominantly in strains causing endocarditis. J Infect Dis 177, 967-976 
60. Al Safadi, R., Amor, S., Hery-Arnaud, G., Spellerberg, B., Lanotte, P., Mereghetti, L., Gannier, F., Quentin, R., and Rosenau, A. (2010) Enhanced expression of Imb gene encoding laminin-binding protein in Streptococcus agalactiae strains harboring IS1548 in scpB-Imb intergenic region. PLOS ONE 5, e10794

61. Sanson, M., O'Neill, B. E., Kachroo, P., Anderson, J. R., Flores, A. R., Valson, C., Cantu, C. C., Makthal, N., Karmonik, C., Fittipaldi, N., Kumaraswami, M., Musser, J. M., and Olsen, R. J. (2015) A naturally occurring single amino acid replacement in multiple gene regulator of group A Streptococcus significantly increases virulence. Am J Pathol 185, 462-471

62. Sumby, P., Whitney, A. R., Graviss, E. A., DeLeo, F. R., and Musser, J. M. (2006) Genome-wide analysis of group A streptococci reveals a mutation that modulates global phenotype and disease specificity. PLoS Pathog 2, e5

63. Dohrmann, S., Anik, S., Olson, J., Anderson, E. L., Etesami, N., No, H., Snipper, J., Nizet, V., and Okumura, C. Y. (2014) Role for streptococcal collagen-like protein 1 in M1T1 group A Streptococcus resistance to neutrophil extracellular traps. Infect Immun 82, 4011-4020

64. Yamaguchi, M., Terao, Y., and Kawabata, S. (2013) Pleiotropic virulence factor Streptococcus pyogenes fibronectin-binding proteins. Cell Microbiol 15, 503-511

65. Singh, P., Reimer, C. L., Peters, J. H., Stepp, M. A., Hynes, R. O., and Van De Water, L. (2004) The spatial and temporal expression patterns of integrin alpha9beta1 and one of its ligands, the ElllA segment of fibronectin, in cutaneous wound healing. J Invest Dermatol 123, 1176-1181

66. Ffrench-Constant, C., Van de Water, L., Dvorak, H. F., and Hynes, R. O. (1989) Reappearance of an embryonic pattern of fibronectin splicing during wound healing in the adult rat. J Cell Biol 109, 903-914

67. Jarnagin, W. R., Rockey, D. C., Koteliansky, V. E., Wang, S. S., and Bissell, D. M. (1994) Expression of variant fibronectins in wound healing: cellular source and biological activity of the EIIIA segment in rat hepatic fibrogenesis. J Cell Biol 127, 2037-2048

68. Stetzner, Z. W., Li, D., Feng, W., Liu, M., Liu, G., Wiley, J., and Lei, B. (2015) Serotype M3 and M28 group A streptococci have distinct capacities to evade neutrophil and TNF-alpha responses and to invade soft tissues. PLOS ONE 10, e0129417

69. Scaramuzzino, D. A., McNiff, J. M., and Bessen, D. E. (2000) Humanized in vivo model for streptococcal impetigo. Infect Immun 68, 2880-2887

70. Cywes, C., and Wessels, M. R. (2001) Group A Streptococcus tissue invasion by CD44-mediated cell signalling. Nature 414, 648-652

71. Shepherd, J., Douglas, I., Rimmer, S., Swanson, L., and MacNeil, S. (2009) Development of three-dimensional tissue-engineered models of bacterial infected human skin wounds. Tissue Eng Part C Methods 15, 475-484

72. Gurjala, A. N., Geringer, M. R., Seth, A. K., Hong, S. J., Smeltzer, M. S., Galiano, R. D., Leung, K. P., and Mustoe, T. A. (2011) Development of a novel, highly quantitative in vivo model for the study of biofilm-impaired cutaneous wound healing. Wound Repair Regen 19, 400-410 
73. Schierle, C. F., De la Garza, M., Mustoe, T. A., and Galiano, R. D. (2009) Staphylococcal biofilms impair wound healing by delaying reepithelialization in a murine cutaneous wound model. Wound Repair Regen 17, 354-359

74. Roberts, A. L., Connolly, K. L., Kirse, D. J., Evans, A. K., Poehling, K. A., Peters, T. R., and Reid, S. D. (2012) Detection of group A Streptococcus in tonsils from pediatric patients reveals high rate of asymptomatic streptococcal carriage. BMC Pediatr 12, 3

75. Connolly, K. L., Roberts, A. L., Holder, R. C., and Reid, S. D. (2011) Dispersal of Group A streptococcal biofilms by the cysteine protease SpeB leads to increased disease severity in a murine model. PLOS ONE 6, e18984

76. Connolly, K. L., Braden, A. K., Holder, R. C., and Reid, S. D. (2011) Srv mediated dispersal of streptococcal biofilms through SpeB is observed in CovRS+ strains. PLOS ONE 6, e28640

77. Popov, L., Kovalski, J., Grandi, G., Bagnoli, F., and Amieva, M. R. (2014) ThreeDimensional Human Skin Models to Understand Staphylococcus aureus Skin Colonization and Infection. Frontiers in immunology 5, 41

78. Terao, Y., Kawabata, S., Nakata, M., Nakagawa, I., and Hamada, S. (2002) Molecular characterization of a novel fibronectin-binding protein of Streptococcus pyogenes strains isolated from toxic shock-like syndrome patients. J Biol Chem 277, 47428-47435

79. Terao, Y., Kawabata, S., Kunitomo, E., Murakami, J., Nakagawa, I., and Hamada, S. (2001) Fba, a novel fibronectin-binding protein from Streptococcus pyogenes, promotes bacterial entry into epithelial cells, and the fba gene is positively transcribed under the Mga regulator. Mol Microbiol 42, 75-86

80. Amelung, S., Nerlich, A., Rohde, M., Spellerberg, B., Cole, J. N., Nizet, V., Chhatwal, G. S., and Talay, S. R. (2011) The FbaB-type fibronectin-binding protein of Streptococcus pyogenes promotes specific invasion into endothelial cells. Cell Microbiol 13, 1200-1211

81. Marks, L. R., Davidson, B. A., Knight, P. R., and Hakansson, A. P. (2013) Interkingdom signaling induces Streptococcus pneumoniae biofilm dispersion and transition from asymptomatic colonization to disease. MBio 4

82. Blanchette-Cain, K., Hinojosa, C. A., Akula Suresh Babu, R., Lizcano, A., Gonzalez-Juarbe, N., Munoz-Almagro, C., Sanchez, C. J., Bergman, M. A., and Orihuela, C. J. (2013) Streptococcus pneumoniae biofilm formation is strain dependent, multifactorial, and associated with reduced invasiveness and immunoreactivity during colonization. MBio 4, e00745-00713

83. Marks, L. R., Mashburn-Warren, L., Federle, M. J., and Hakansson, A. P. (2014) Streptococcus pyogenes biofilm growth in vitro and in vivo and its role in colonization, virulence, and genetic exchange. J Infect Dis

84. Sumby, P., Zhang, S., Whitney, A. R., Falugi, F., Grandi, G., Graviss, E. A., Deleo, F. R., and Musser, J. M. (2008) A chemokine-degrading extracellular protease made by group A Streptococcus alters pathogenesis by enhancing evasion of the innate immune response. Infect Immun 76, 978-985

85. Boyle, M. D., Raeder, R., Flosdorff, A., and Podbielski, A. (1998) Role of emm and mrp genes in the virulence of group A streptococcal isolate 64/14 in a mouse model of skin infection. J Infect Dis 177, 991-997 
86. Nyberg, P., Sakai, T., Cho, K. H., Caparon, M. G., Fassler, R., and Bjorck, L. (2004) Interactions with fibronectin attenuate the virulence of Streptococcus pyogenes. $E M B O$ J. 23, 2166-2174

87. Crotty Alexander, L. E., Maisey, H. C., Timmer, A. M., Rooijakkers, S. H., Gallo, R. L., von Kockritz-Blickwede, M., and Nizet, V. (2010) M1T1 group A streptococcal pili promote epithelial colonization but diminish systemic virulence through neutrophil extracellular entrapment. J Mol Med (Berl) 88, 371-381

88. Hollands, A., Pence, M. A., Timmer, A. M., Osvath, S. R., Turnbull, L., Whitchurch, C. B., Walker, M. J., and Nizet, V. (2010) Genetic switch to hypervirulence reduces colonization phenotypes of the globally disseminated group A Streptococcus M1T1 clone. J Infect Dis 202, 11-19 


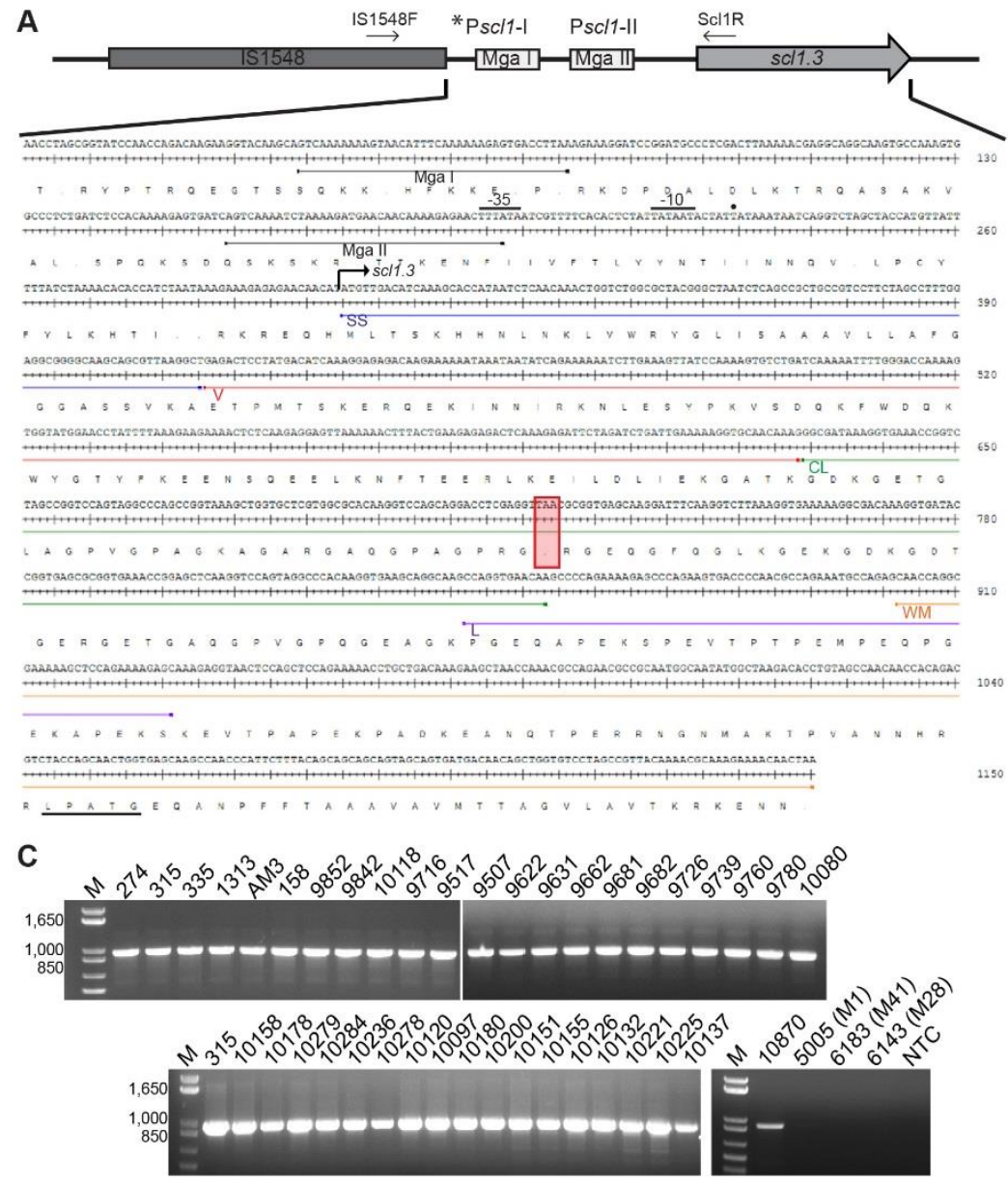

\begin{tabular}{|c|c|c|c|}
\hline Strain & M-type & $\begin{array}{l}\text { No. of } \\
\text { insertions }\end{array}$ & $\begin{array}{l}\text { Upstream of } \\
\text { scl1 allele? }\end{array}$ \\
\hline M1 GAS (SF370) & M1 & 6 & - \\
\hline MGAS476 & M1 & 8 & - \\
\hline MGAS5005 & M1 & 9 & - \\
\hline HKU488 & M1 & 7 & - \\
\hline 5448 & M1 & 7 & - \\
\hline MTB314 & M1 & 7 & - \\
\hline A20 & M1 & 8 & - \\
\hline AP1 & M1 & 5 & - \\
\hline NCTC8198 & M1 & 5 & - \\
\hline MTB313 & M1 & 7 & - \\
\hline MGAS10270 & M2 & 2 & - \\
\hline MGAS315 & M3 & 3 & + \\
\hline SSI-1 & M3 & 3 & + \\
\hline M3-b & M3 & 3 & + \\
\hline STAB902 & M3 & 2 & - \\
\hline MGAS10750 & M4 & 9 & - \\
\hline MEW427 & M4 & 5 & - \\
\hline ATCC19615 & M5/49 & 4 & - \\
\hline MGAS10394 & M6 & 2 & - \\
\hline D471 & M6 & 2 & - \\
\hline JRS4 & M6 & 2 & - \\
\hline MGAS2096 & M12 & 7 & - \\
\hline MGAS9429 & M12 & 8 & - \\
\hline HKU360 & M12 & 7 & - \\
\hline HSC5 & M14 & 3 & - \\
\hline M23ND & M23 & 1 & - \\
\hline FDAARGOS_149 & M24 & 9 & - \\
\hline MGAS6180 & M28 & 4 & - \\
\hline MEW123 & M28 & 4 & - \\
\hline M28PF1 & M28 & 4 & - \\
\hline STAB10015 & M28 & 4 & - \\
\hline 1E1 & M44 & 1 & - \\
\hline STAB901 & M44 & 1 & - \\
\hline NZ131 & M49 & 4 & - \\
\hline Alab49 & M53 & 12 & - \\
\hline MGAS15252 & M59 & 5 & - \\
\hline MGAS1882 & M59 & 6 & - \\
\hline NGAS596 & M82 & 7 & - \\
\hline $7 F 7$ & M83 & 2 & - \\
\hline STAB1102 & M83 & 2 & - \\
\hline NGAS327 & M83 & 2 & - \\
\hline NGAS743 & M87 & 12 & - \\
\hline $\mathrm{H} 293$ & M89 & 6 & - \\
\hline NGAS638 & M101 & 3 & - \\
\hline NGAS322 & M114 & 7 & - \\
\hline
\end{tabular}

Figure 1

M3-type GAS strains harbor unique polymorphisms in the scl1.3 locus. (A) Schematic representation and nucleotide sequence are based on the sc/1.3 locus in the M3-type strain MGAS315 genome. The scl1.3 promoter region contains two putative Mga binding sites, Pscl1-I with Mga I and Psc/1-II with Mga II. IS1548 is inserted 38 bp upstream of the preferred *Pscl1I/Mga I promoter, which was shown to be necessary for scl1 transcription. Transcriptional start site (solid dot), -10 and -35 boxes are shown upstream of scl1.3 coding sequence. Scl1 regions are designated as follows: SS, signal sequence; $\mathrm{V}$, variable region; $\mathrm{CL}$, collagen-like region; $\mathrm{L}$, linker region; WM, wall-membrane region; LPATG, cell-wall anchor. The null mutation in the $11^{\text {th }}$ Gly-X-Y repeat of the CL region is depicted by the red box, presumably resulting in a truncated secreted Scl1.3 protein. Relative location of primers, IS1548F and Scl1R, used to generate amplicons in (C) are shown. (B) IS1548 insertion upstream of scl1.3 is unique to M3 genomes. BLAST search in the NCBI nucleotide ( $\mathrm{nr} / \mathrm{nt}$ ) database using IS1548 (1,317 bp) sequence as query identified insertions in 45 GAS genomes representing 21 different M-types. Only genomes of M3-type strains harbored IS1548 element upstream of the sc/1 allele (bold text). (C) IS1548 insertion upstream of scl1.3 is conserved among M3 strains. Genomic DNA was isolated from a collection of 40 M3-type strains and analyzed by PCR for the presence of IS1548 upstream of scl1 using primers IS1548F and Scl1R (located in conserved scl1 signal sequence). Additional M1-, M41-, and M28-type control strains, and a no template control (NTC) are included. Expected amplicon size, $963 \mathrm{bp} ; \mathrm{M}, 1 \mathrm{~kb}$ Plus DNA Ladder. MGAS designation applies to all strain numbers shown above gel wells, with the exception of strain AM3. 
A

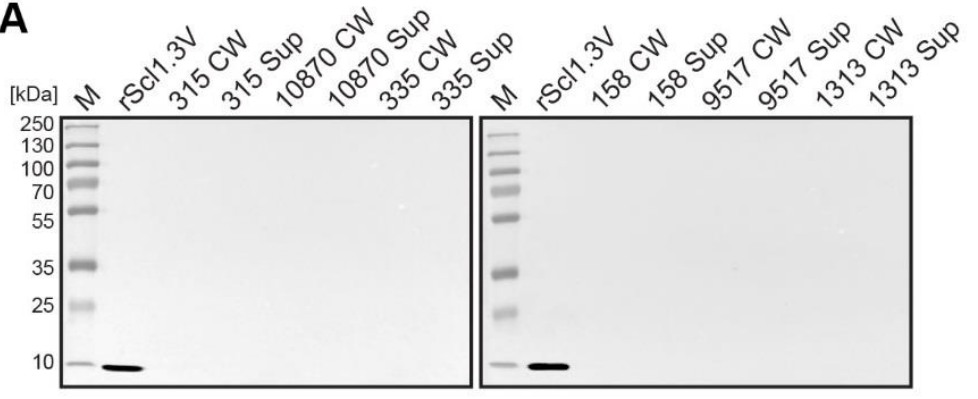

C
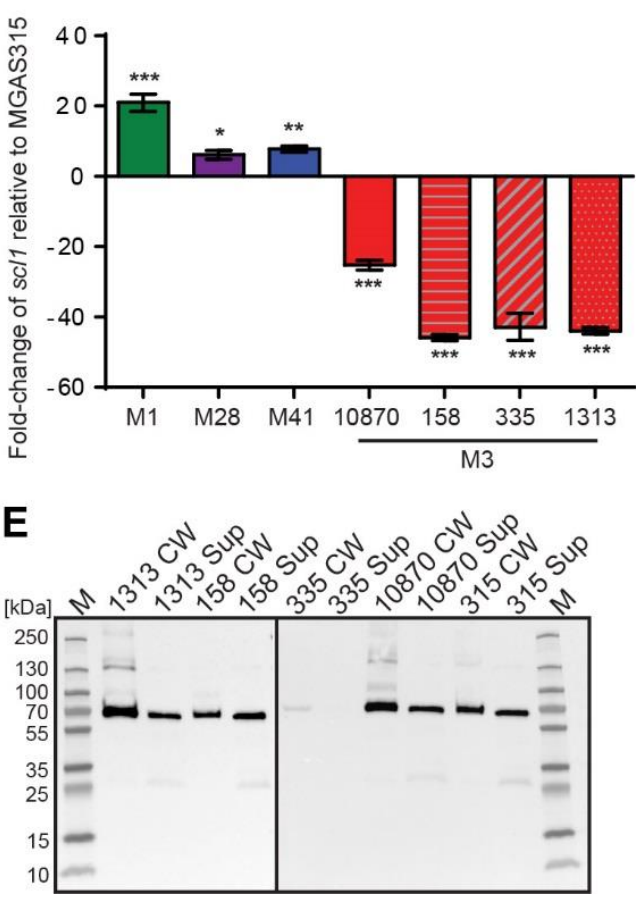

B

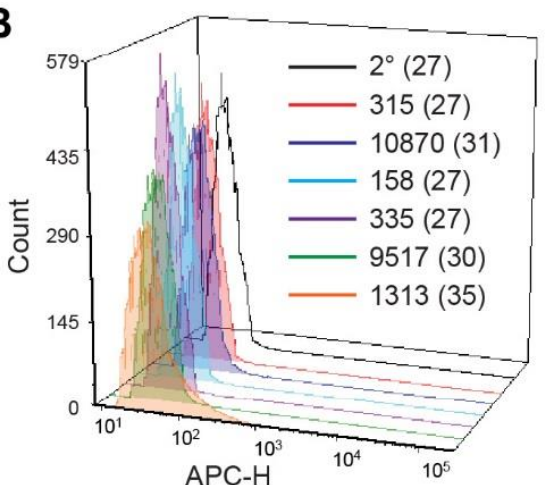

D

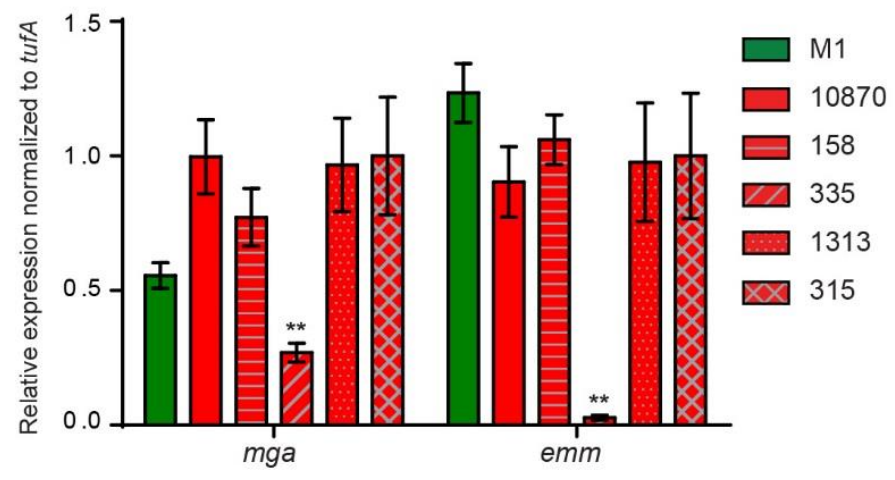

Figure 2

Assessment of Scl1.3 expression. (A) Assessment of Scl1.3 production by M3-type GAS. Cell wall (CW) and culture supernatant (Sup) protein fractions prepared from exponential phase cultures of several M3-type strains were analyzed by western immunoblotting, using anti-Scl1 rabbit polyclonal antibody. Recombinant protein $\mathrm{rScl} 1.3 \mathrm{~V}$, corresponding to the variable region of Scl1.3, was used as a positive control. Expected molecular masses: Scl1.3, $11.4 \mathrm{kDa}$; rScl1.3V, 8.3 kDa. M, PageRuler ${ }^{\mathrm{TM}}$ Plus Prestained Protein Ladder. (B) Detection of Scl1.3 on the surface of M3-type GAS. Flow cytometry analysis of several M3-type strains is shown using anti-Scl1 antibody described in part (A) (color-shaded histograms) or a secondary only control $\left(2^{\circ}\right.$ sample, black outlined histogram). Median fluorescence intensities (MFI) are shown in parentheses for each strain. (C) Assessment of $s c / 1$ transcription. Fold-change of $s c / 1$ transcript levels are shown compared to scl1.3 transcription in M3-type strain MGAS315. qRT-PCR was performed on RNA obtained from exponential phase cultures. Results are shown from three independent experiments, each performed in triplicate wells. Standard errors and statistical analysis were computed from averaged $\Delta \mathrm{Ct}$ values for each biological replicate prior to normalization against the endogenous reference gene tufA; ${ }^{*} P \leq 0.05,{ }^{* \star} P \leq 0.01,{ }^{* \star *} P \leq 0.001$ (student's $t$-test). (D) 
Assessment of $\mathrm{mga}$ and emm transcription. Relative expression levels of $\mathrm{mga}$ and emm genes were compared between MGAS315 and four additional M3 strains or the M1 strain MGAS5005. Results are shown from three independent experiments, each performed in triplicate wells. Standard errors and statistical analysis were computed from averaged $\Delta \mathrm{Ct}$ values for each biological replicate prior to normalization against the endogenous reference gene tufA; ${ }^{* *} P \leq 0.01$. (E) Assessment of M3-protein production by M3-type GAS. The same cell wall (CW) and culture supernatant (Sup) protein samples prepared from exponential phase cultures of M3-type strains (used in panel A) were analyzed by western immunoblotting, using anti-M3 protein rabbit polyclonal antibody. Expected molecular mass: $65 \mathrm{kDa}$. M, PageRuler ${ }^{\mathrm{TM}}$ Plus Prestained Protein Ladder. 

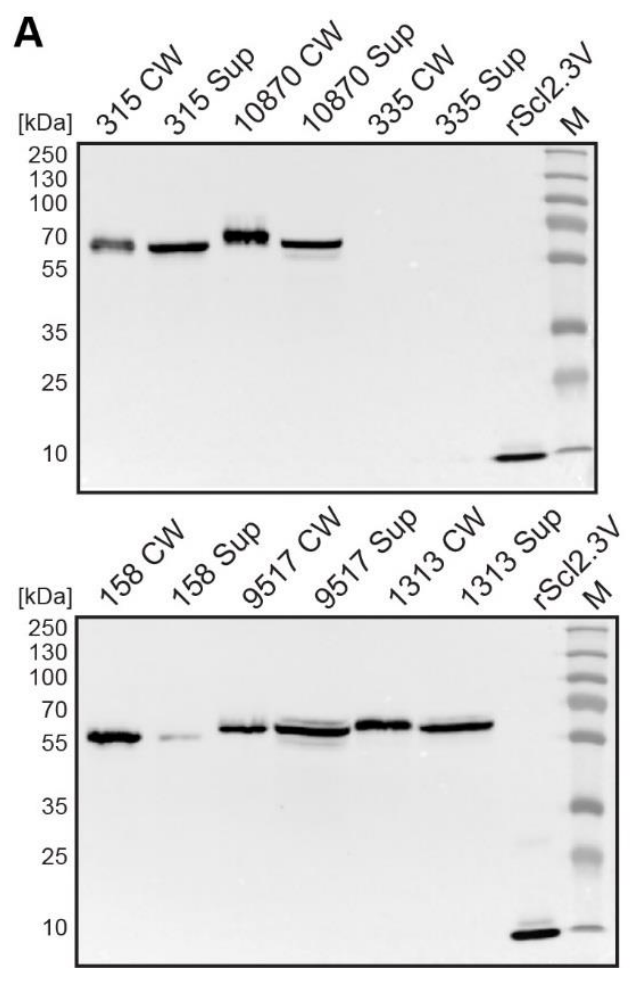

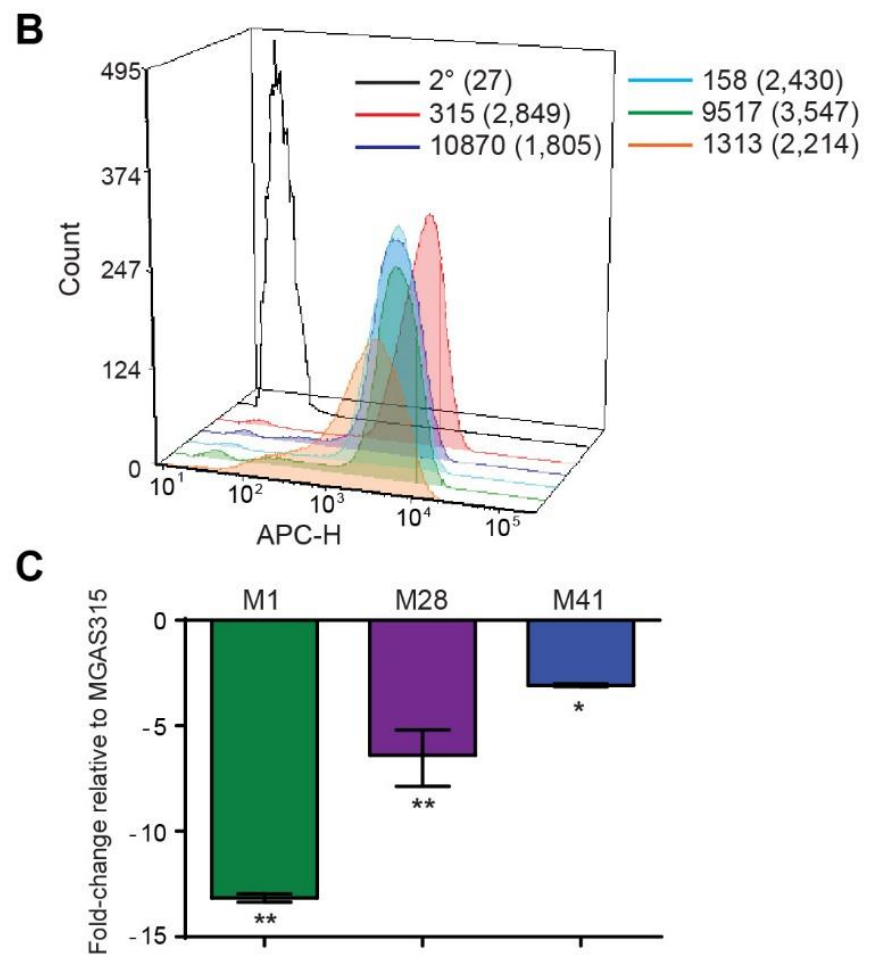

Figure 3

Characterization of the scl2.3 locus in M3-type GAS. (A) Assessment of Scl2.3 production by M3-type GAS. The same cell wall (CW) and culture supernatant (Sup) protein samples prepared from exponential phase cultures of several M3-type strains (used in Fig. 2A, E) were analyzed by western immunoblotting, using anti-rScl2.3V rabbit polyclonal antibody. Recombinant protein rScl2.3V, corresponding to the variable region of Scl2.3 protein, was used as a positive control. Expected molecular masses based on MGAS315: Scl2.3, $52.5 \mathrm{kDa}$; rScl2.3V, $10.1 \mathrm{kDa}$. Aberrant migration of detected Scl2.3 variants is characteristic of Scl proteins. M, PageRuler ${ }^{\mathrm{TM}}$ Plus Prestained Protein Ladder. (B) Detection of Scl2.3 on the surface of M3-type GAS. Flow cytometry analysis of several M3-type strains is shown using anti-rScl2.3V rabbit polyclonal antibody (color-shaded histograms) or a secondary-only control $\left(2^{\circ}\right.$ sample, black outlined histogram). Median fluorescence intensities (MFI) are shown in parentheses for each strain. (C) Assessment of $s c / 2$ transcription. Fold-change of $s c / 2$ transcription levels are shown compared to scl2.3 transcription in M3-type MGAS315. qRT-PCR was performed on reverse-transcribed RNA obtained from exponential phase cultures. Results are shown from three independent experiments, each performed in triplicate wells. Standard errors and statistical analysis were computed from averaged $\triangle \mathrm{Ct}$ values for each biological replicate prior to normalization against the endogenous reference gene tufA; ${ }^{*} P \leq 0.05,{ }^{* *} P \leq 0.01$ (student's $t$-test). 


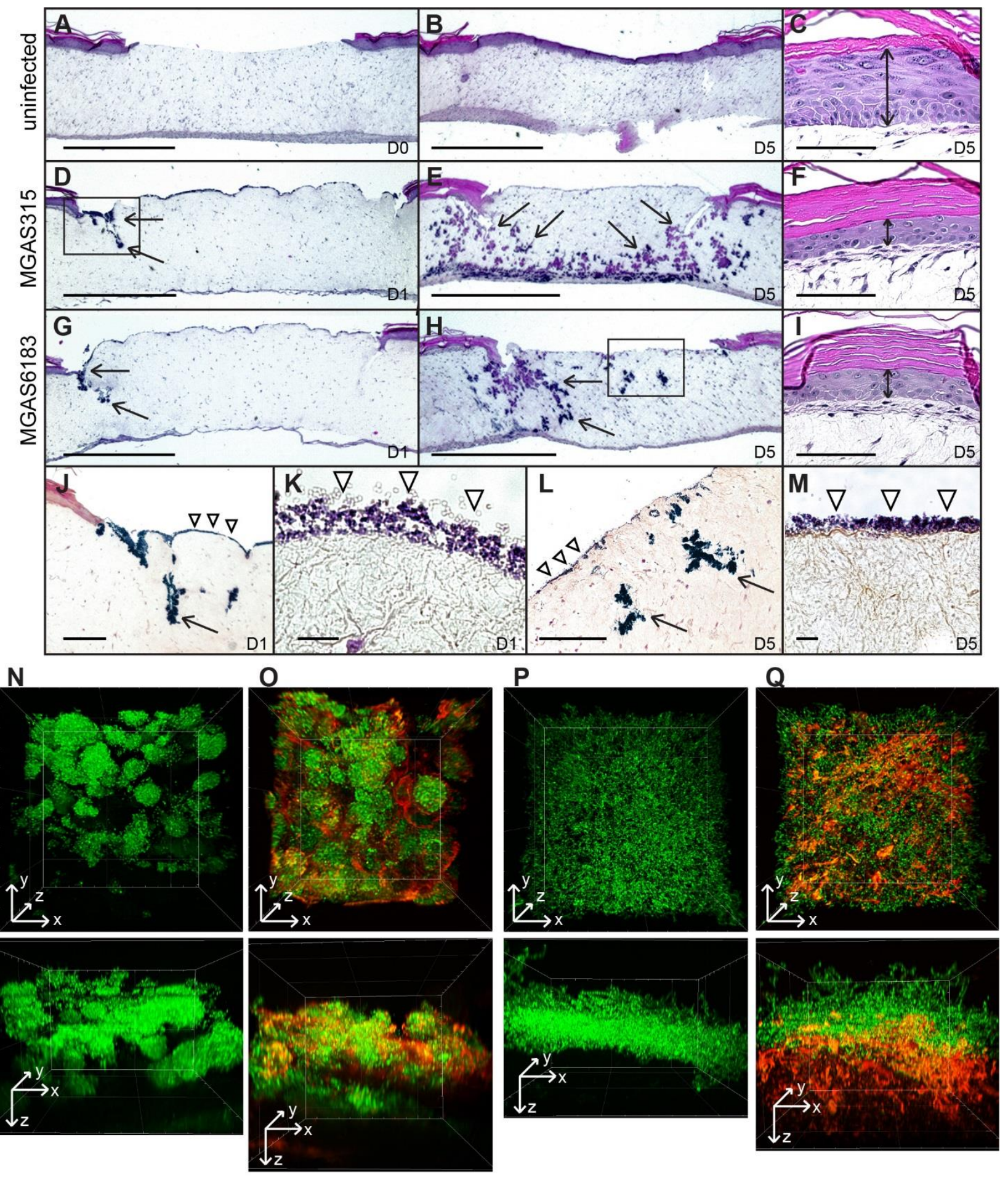

Figure 4

In vitro skin equivalent model of GAS infection. (A-I) H\&E stained sections of uninfected (AC) or infected (D-I) wounded skin equivalents at 40x magnification; scale bar: $1000 \mu \mathrm{m}$. Uninfected wound at day 0 (A) shows a lack of the epidermal layer where biopsy punch was 
performed, which healed by day 5 (B). At day 1, infection of wounded skin equivalents with M3 and M41 GAS revealed superficial colonization of the wound bed, as well as invasion into the defects formed at the wound edge ( $D, G$, arrows). By day 5 , bacteria had disseminated throughout tissue laterally and vertically (arrows), reaching the bottom of the dermis layer $(E, H) .(\mathbf{C}, \mathbf{F}, \mathbf{I})$ H\&E stained sections of the keratinocyte layer of intact skin surrounding the biopsy punch; scale bar: $100 \mu \mathrm{m}$. The thickness of the viable keratinocyte layer outside the wound (C, double arrow) was significantly reduced in tissues infected with $M 3(F)$ and $M 41(\mathrm{I})$ by day 5. (J, L) Gram stained sections of wounded tissue, corresponding to boxed areas in panels $D$ and $H$, show tissue microcolonies (arrows) and superficial bacterial colonization (arrowheads). (J, scale bar: $400 \mu \mathrm{m}$; $\mathrm{L}$, scale bar: $200 \mu \mathrm{m})$. (K, M) 1000x magnification micrographs of surface biofilms shown in $\mathrm{J}$ and $\mathrm{L}$ (scale bar: $10 \mu \mathrm{m}$ ). ( $\mathrm{N}-\mathbf{Q})$ Two-photon fluorescence microscopy analysis of infected skin equivalents. Vertical dissemination through the wound bed and glycocalyx formation by the GFPexpressing M41- (N-O) and M3-type (P-Q) GAS at day 5. 3D projections of z-stacks are shown from the top view of the z-stack (top panels) or the side view (bottom panels). Multi-channel images show GFP fluorescence of GAS cells (N, P) and TRITC-conA stain of glycocalyx (O, $Q)$. All images were acquired at $600 x$ magnification. 


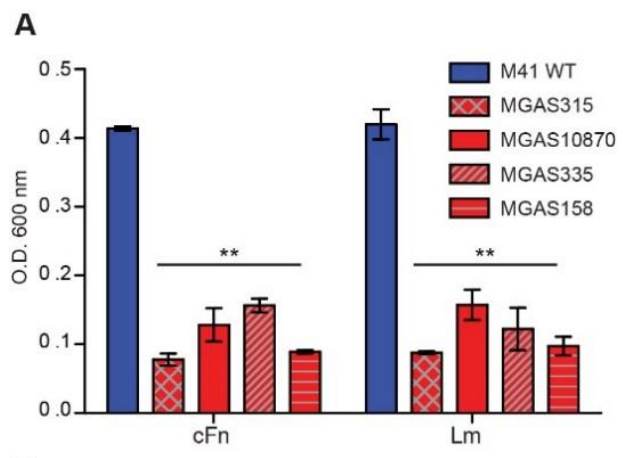

B

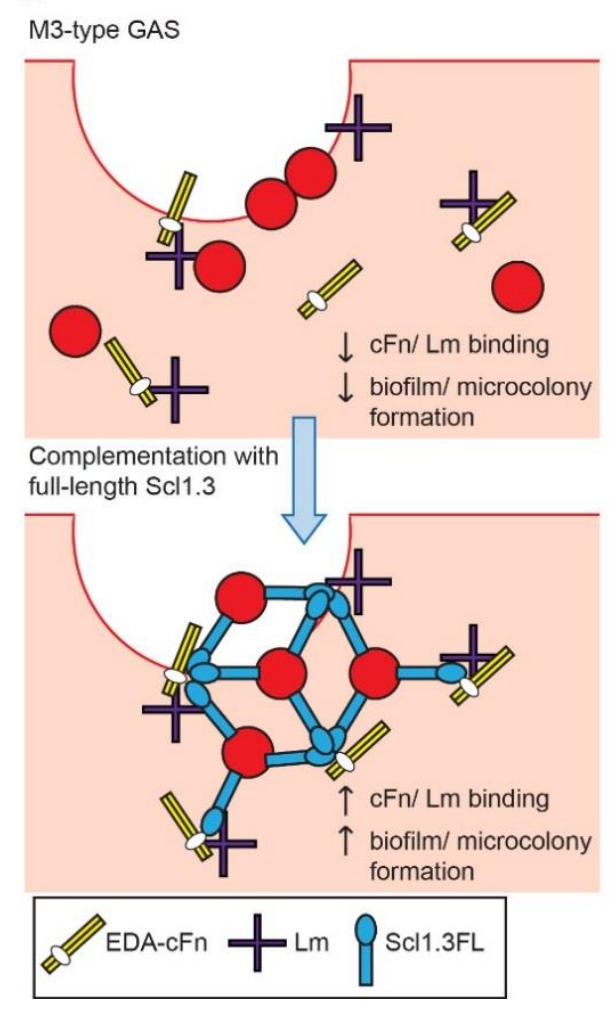

Figure 5

In vitro biofilm formation and hypothetical model of microcolony formation by invasive M3type GAS. (A) Limited in vitro biofilm formation by invasive M3 strains on coatings with cellular fibronectin and laminin. A panel of invasive M3-type strains was compared to a non-invasive biofilm-capable M41-type strain MGAS 6183. Crystal violet staining was used to assess biomass formed after 24 hours of growth in wells coated with cellular fibronectin (cFn) and laminin (Lm); results represent averaged values from at least 3 independent experiments performed in triplicate wells. ${ }^{* *} \mathrm{P} \leq 0.01$ (student's $t$-test). (B) Hypothesis model. Top: Infection of wounded skin with wildtype M3-type GAS. Inherent lack of surface-expressed Scl1.3 causes decreased binding to cFn and Lm expressed in wounded tissue, and reduces biofilm and tissue microcolony formation by M3-type bacteria (red circles). Bottom: In-trans complementation of M3-type GAS with full-length cell-associated Scl1.3, Scl1.3FL, restores binding to cFn and Lm in tissue, which confers biofilm and tissue microcolony formation during infection. 

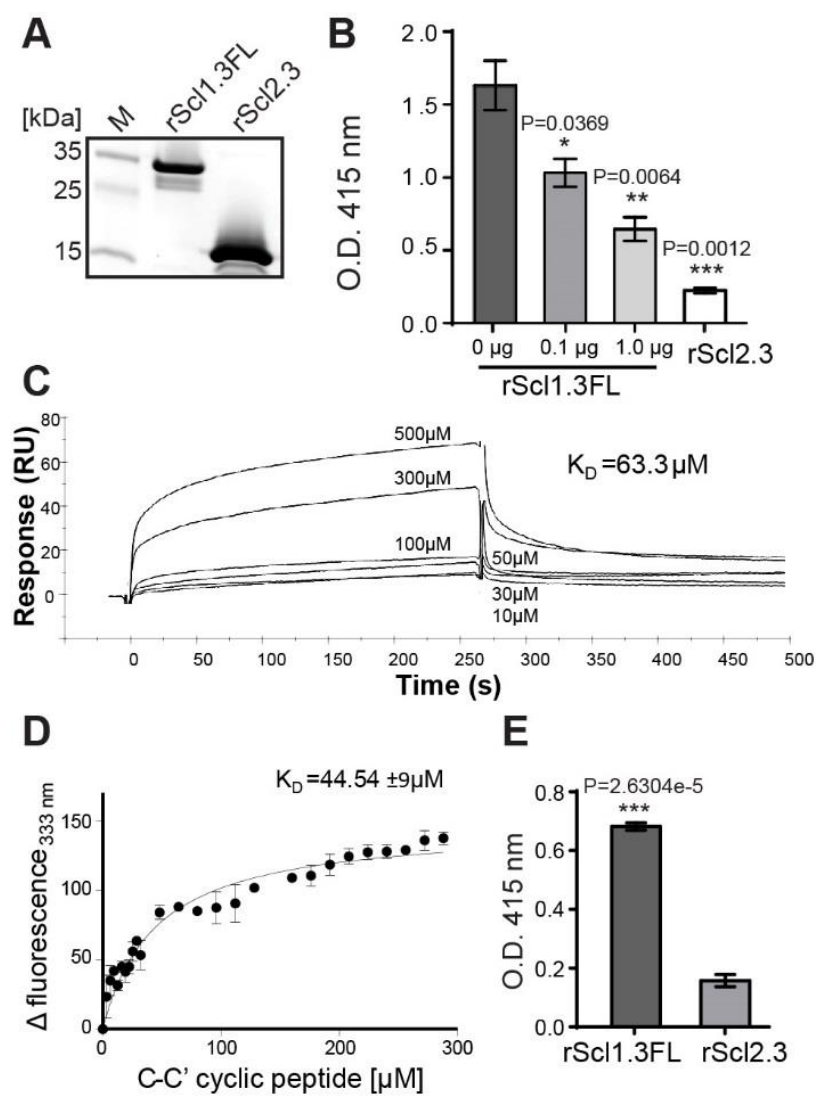

E

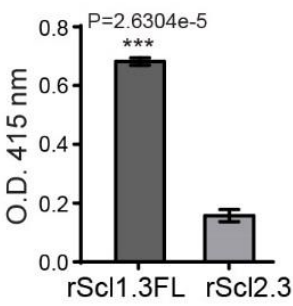

\section{Figure 6}

Construction and binding characterization of recombinant full-length Scl1.3FL and Scl2.3 proteins. (A) Affinity purified $\mathrm{rScl} 1.3 \mathrm{FL}$ and $\mathrm{rScl} 2.3$ proteins were analyzed by $4-20 \%$ gradient SDS-PAGE; expected molecular masses: rScl1.3FL, $20.6 \mathrm{kDa}$ and rScl2.3, $16.2 \mathrm{kDa}$. (B-D-) Binding of rScl1.3FL to extra domain A (EDA) of cellular fibronectin. (B) IST-9 antibody inhibition identifies rScl1.3FL binding to the C-C' loop of EDA-cFN. Inhibition of rScl1.3FL binding to EDAcFn was tested by ELISA following pre-incubation of cFn with blocking IST-9 mAb specific to the C-C' loop. Significance of inhibition by 0.1 and $1.0 \mu \mathrm{g}$ of IST-9 mAb was determined by student's $t$-test as compared to the untreated cFn. A lack of significant cFn binding by rScl2.3 was evident, as compared to rScl1.3FL-cFn binding; student's $t$-test. (C) rScl1.3FL binding to a cyclic peptide mimicking the C-C' loop of EDA using surface plasmon resonance. Overlay of sensorgrams for the interaction between immobilized rScl1.3FL and EDA-derived C-C' cyclic peptide is shown. The experimental curves corresponding to different concentrations of peptide (10-500 $\mu \mathrm{M})$ were fitted according to a single binding model with 1:1 stoichiometry. (D) rScl1.3FL binding to a C-C' cyclic peptide using tryptophan fluorescence assay. Tryptophan fluorescence quenching analysis shows the dose-response curve of the fluorescence values of rScl1.3FL at $333 \mathrm{~nm}$ plotted against the concentration values of C-C' cyclic peptide. (E) Laminin binding to rScl1.3FL and rScl2.3 by ELISA. Recombinant rScl proteins were immobilized onto Strep-Tactin-coated wells and incubated with laminin. Bound laminin was detected with specific primary pAbs and HRPconjugated secondary Abs. Laminin binding was compared between rScl1.3FL and rSc2.3 and evaluated statistically using student's $t$-test. Results for panels $C$ and $F$ represent averaged values from at least 3 independent experiments performed in triplicate wells. ${ }^{*} P \leq 0.05,{ }^{* *} P \leq 0.01,{ }^{* * *} P \leq$ 0.001 . 
A
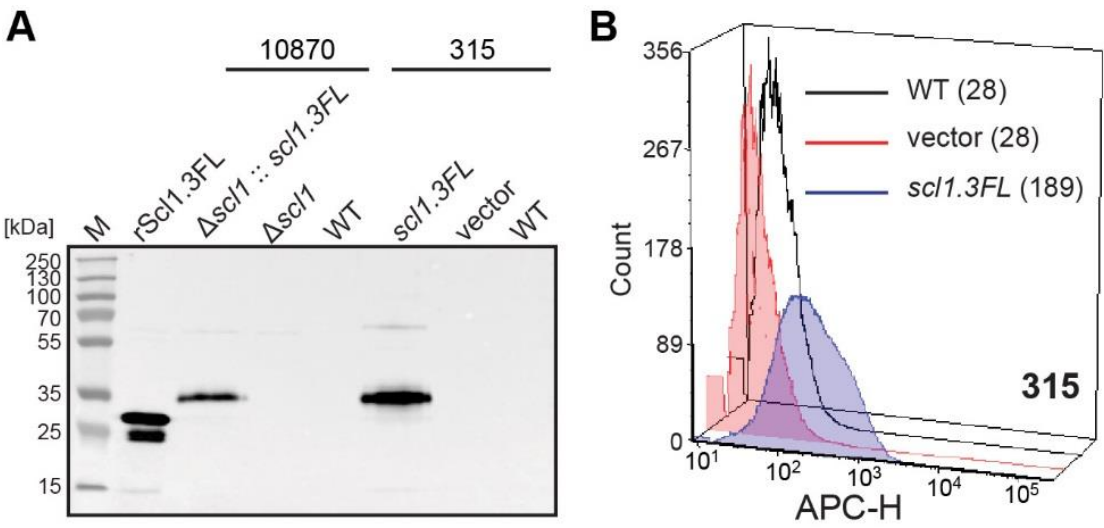

C

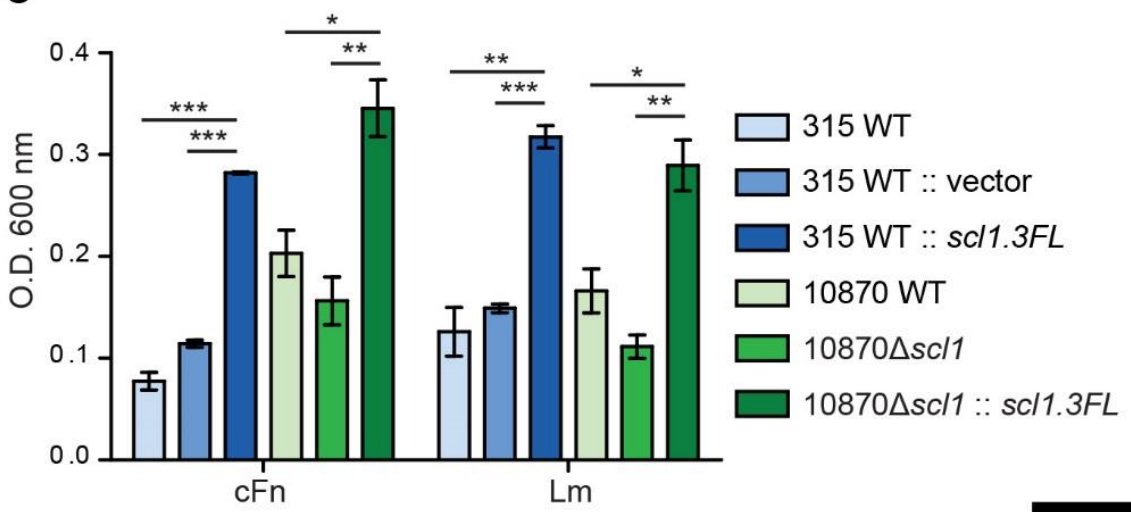

D
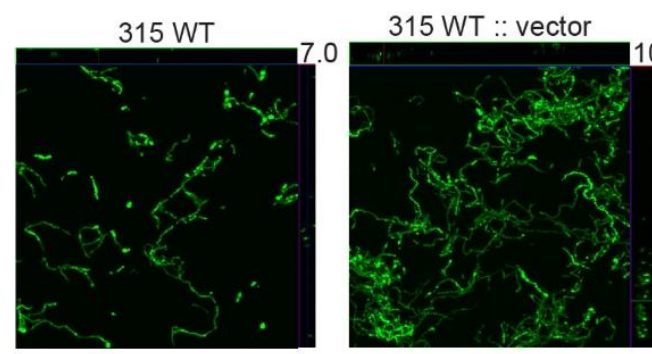

315 WT :: scl1.3FL
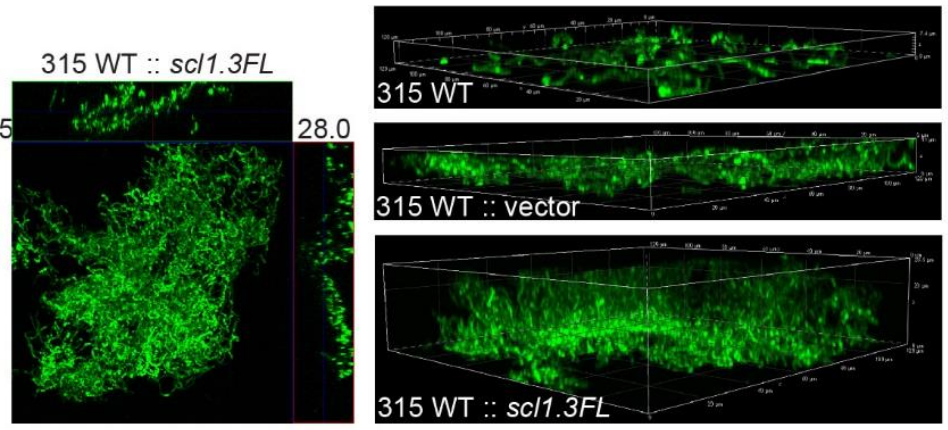

$\mathbf{E}$
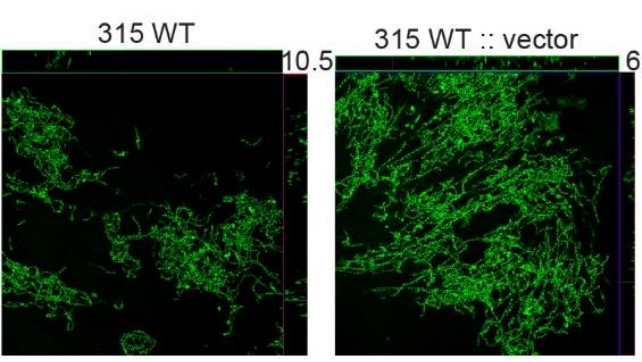

315 WT *. scl1.3FL
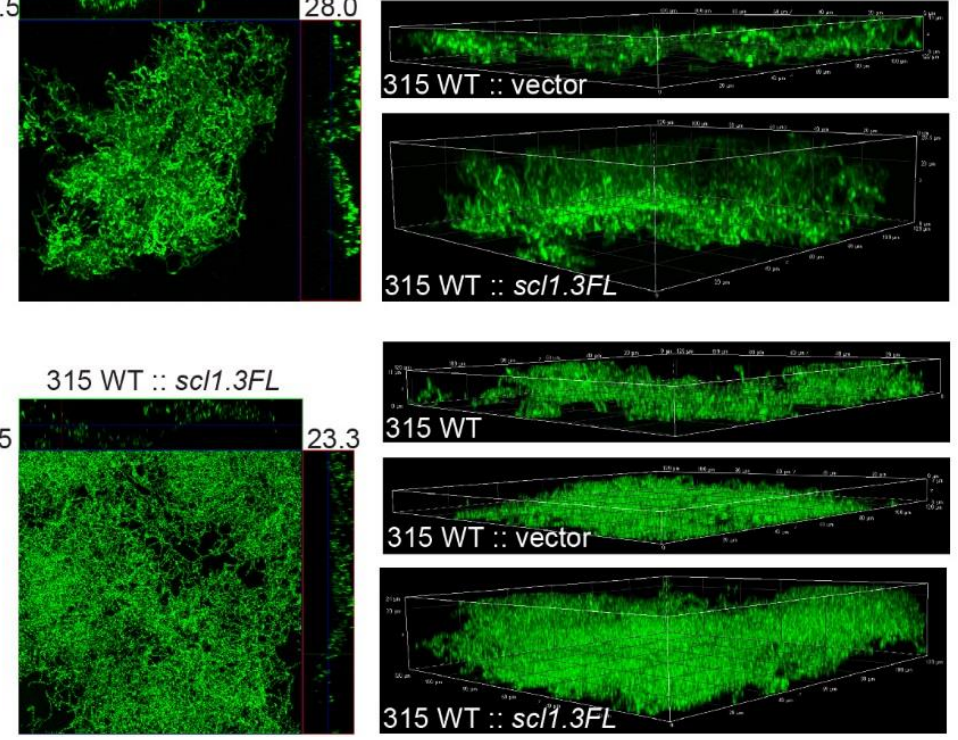

Figure 7
315 WT :: sc/1.3FL

Homologous complementation of M3-type GAS with full-length surface-expressed Scl1.3 protein confers biofilm formation. (A) Western blot detection of full-length Scl1.3 in cell wall fractions of MGAS315 and MGAS10870 $\Delta s c / 1$ complemented in-trans with the scl1.3FL allele. 
Parent strains were included as negative controls and rScl1.3FL was used as a positive control for detection. (B) Flow cytometry detection of Scl1.3FL on the GAS cell surface. Left: Fluorescence intensity of Scl1.3FL-complemented MGAS315 WT strain was compared to vectorcomplemented or WT parent strain. Right: Fluorescence intensity of Scl1.3FL-complemented MGAS10870 $\Delta s c / 1$ was compared to WT and $\Delta s c / 1$ parent strains. Median fluorescence intensities are shown in parentheses for each strain. (C) Crystal violet assessment of bacterial biomass after 24 hours of growth in cFn- or Lm-coated wells. MGAS315 WT complemented in-trans with pJRS525 $\left(\mathrm{Spc}^{\mathrm{R}}\right.$ vector) or pSL502 (Table 1) harboring the scl1.3FL allele was compared to WT parental strain. Spectinomycin resistant MGAS10870 $\Delta$ scl1 mutant was complemented in-trans with $\mathrm{Kan}^{\mathrm{R}}$ pSL518 (Table 1), harboring the scl1.3FL allele. Biofilm biomass of the complemented strain was compared to MGAS10870 WT and sc/1-inactivated parental strains. Results represent averaged values from at least 3 independent experiments performed in triplicate wells. ${ }^{*} \mathrm{P} \leq 0.05$, ${ }^{* *} \mathrm{P} \leq 0.01,{ }^{* * *} \mathrm{P} \leq 0.001$; students $t$-test. (D, E) Confocal laser scanning microscopy analysis of biofilm formation by GFP-expressing MGAS315 vector and scl1.3FL-complemented strains. Biofilms were grown for 24 hours on cFn-coated (D) or Lm-coated (E) coverslips. Maximum intensity projections of GAS biofilms with cross-sectional views (left panels) are representative of $z$-stacks from ten fields within a single experiment. Average vertical thickness is indicated in micrometers. 3D projections of z-stacks (right panels) are shown from the side view. 

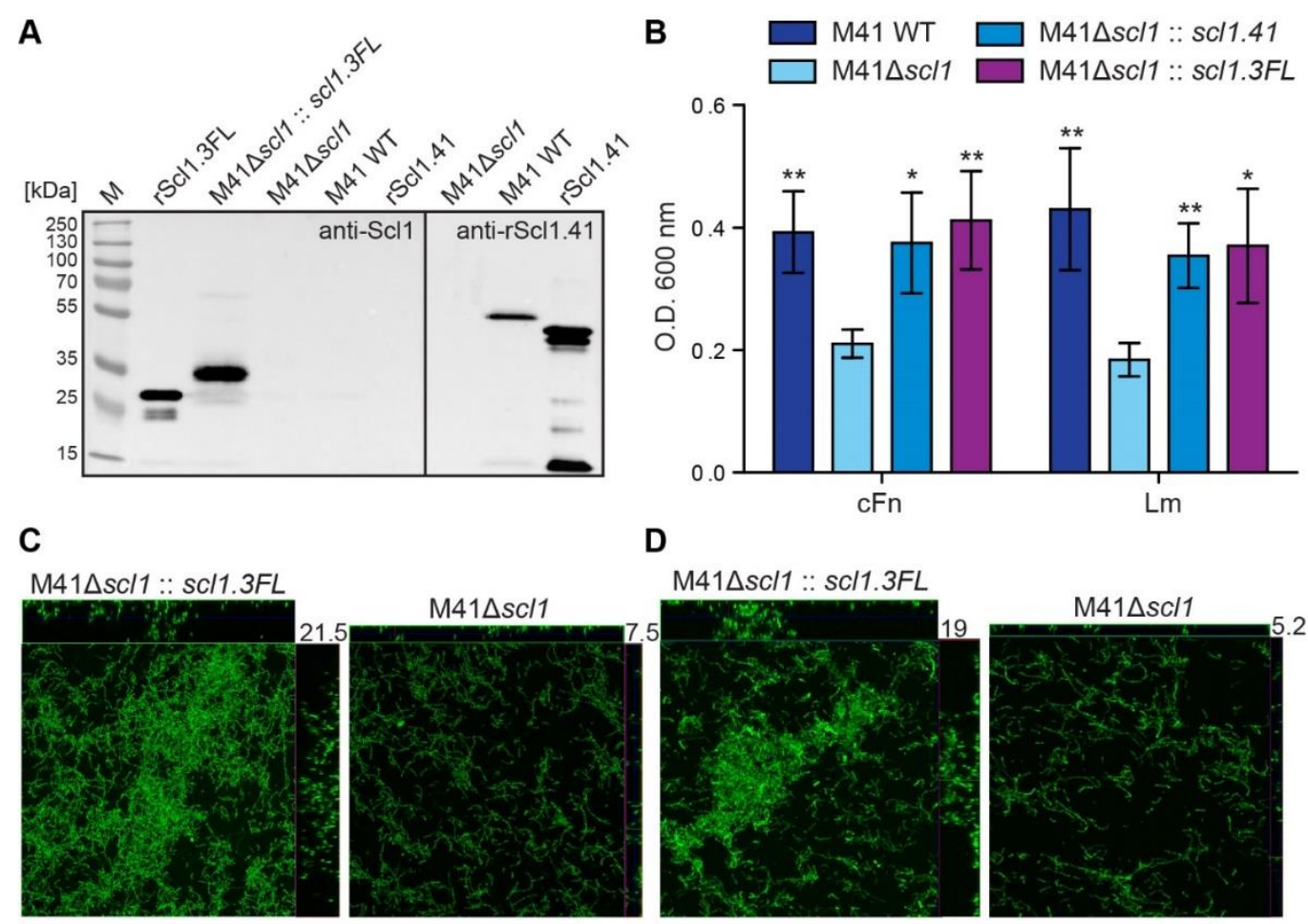

D
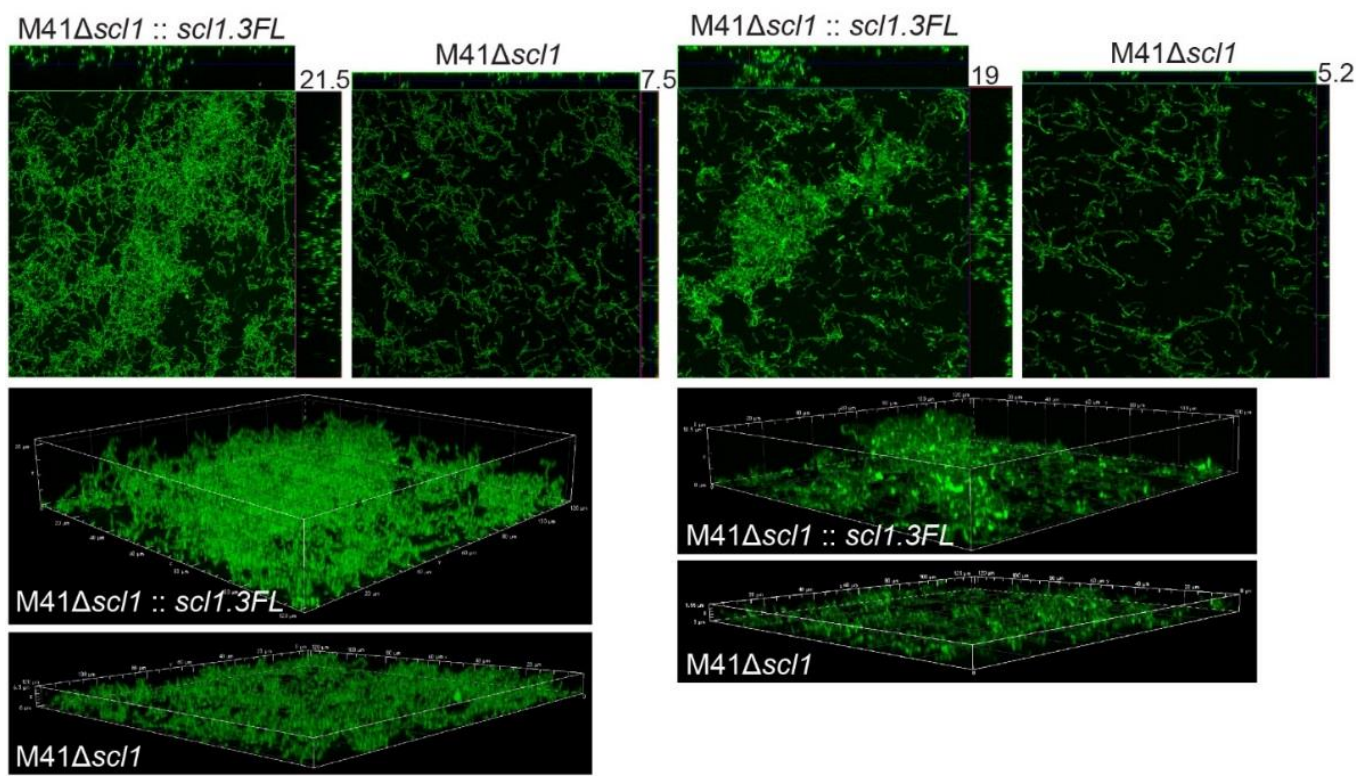

\section{Figure 8}

Heterologous complementation of sc/1-deficient mutant of M41-type GAS with full-length Scl1.3 restores biofilm formation. (A) Left blot: western blot detection of full-length Scl1.3 protein in cell wall fraction of M41 $\Delta s c / 1:: s c / 1.3 F L$ using anti-Scl1 polyclonal antibody. The M41 $\Delta$ scl1 and M41 WT strains were included as negative controls, and rScl1.3FL was included as a positive control. Right blot: western blot detection of Scl1.41 in M41 $\Delta s c / 1$ and M41 WT strains using anti-rScl1.41 antibody and rScl1.41 as a positive control. (B) Crystal violet assessment of biofilm biomass after 24 hours of growth in cFn- or Lm-coated wells. M41 sc/1-inactivated (M41 $\Delta s c / 1)$ mutant was complemented in-trans with either homologous scl1.41 allele harbored on pSL230 or with heterologous sc/1.3FL allele harbored on pSL502 (Table 1). M41 WT, $\Delta s c / 1$ mutant and two complemented mutant strains were assessed for biofilm formation and statistical significance was assessed by students $t$-test as compared to $M 41 \Delta s c / 1$; ${ }^{*} P \leq 0.05,{ }^{* *} P \leq 0.01$. (C, D) Confocal laser scanning microscopy analysis of biofilm formation by GFP-expressing

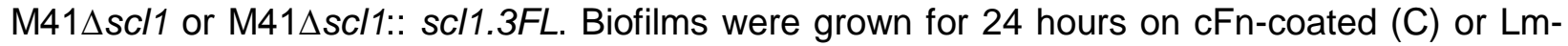
coated (D) coverslips. Maximum intensity projections of GAS biofilms with cross-sectional views (top panels) are representative of $z$-stacks from ten fields within a single experiment. Average vertical thickness is indicated in micrometers. 3D projections of z-stacks (bottom panels) are shown from the side view. 


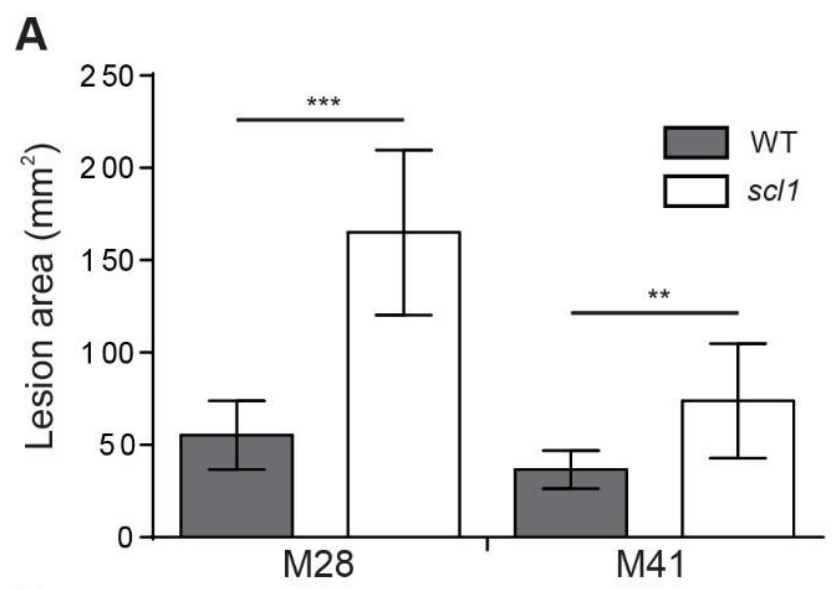

B

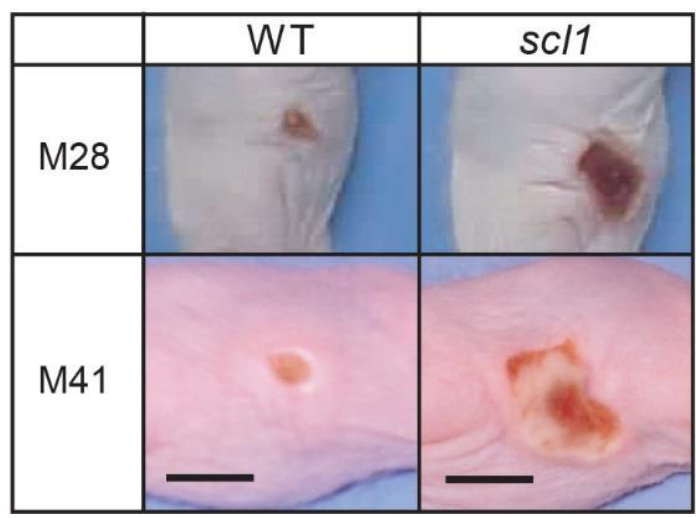

Figure 9

Skin pathology of SKH1 hairless mice infected subcutaneously with wild-type and scl1inactivated mutants of M28- and M41-type GAS. (A) Mean area of skin lesions in mice infected with M28- or M41-type isogenic GAS strains. The severity of skin lesions was compared following infection with wild-type (WT) or sc/1-mutant (sc/1) GAS; 96-hour time-point is shown. The mean lesion area and standard deviation were calculated and compared between experimental groups. Data represents mean lesion size from at least 10 mice per experimental group. Statistical differences were calculated using the student's $t$-test; ${ }^{* *} P \leq 0.01$, ${ }^{* * *} P \leq 0.001$. (B) Gross pathology of the skin lesions. Digital images of the skin lesions of representative animals infected with either WT or sc/1-mutant GAS. The images show skin lesions developed after 96 hours post-infection, and scale bar represents $10 \mathrm{~mm}$. 


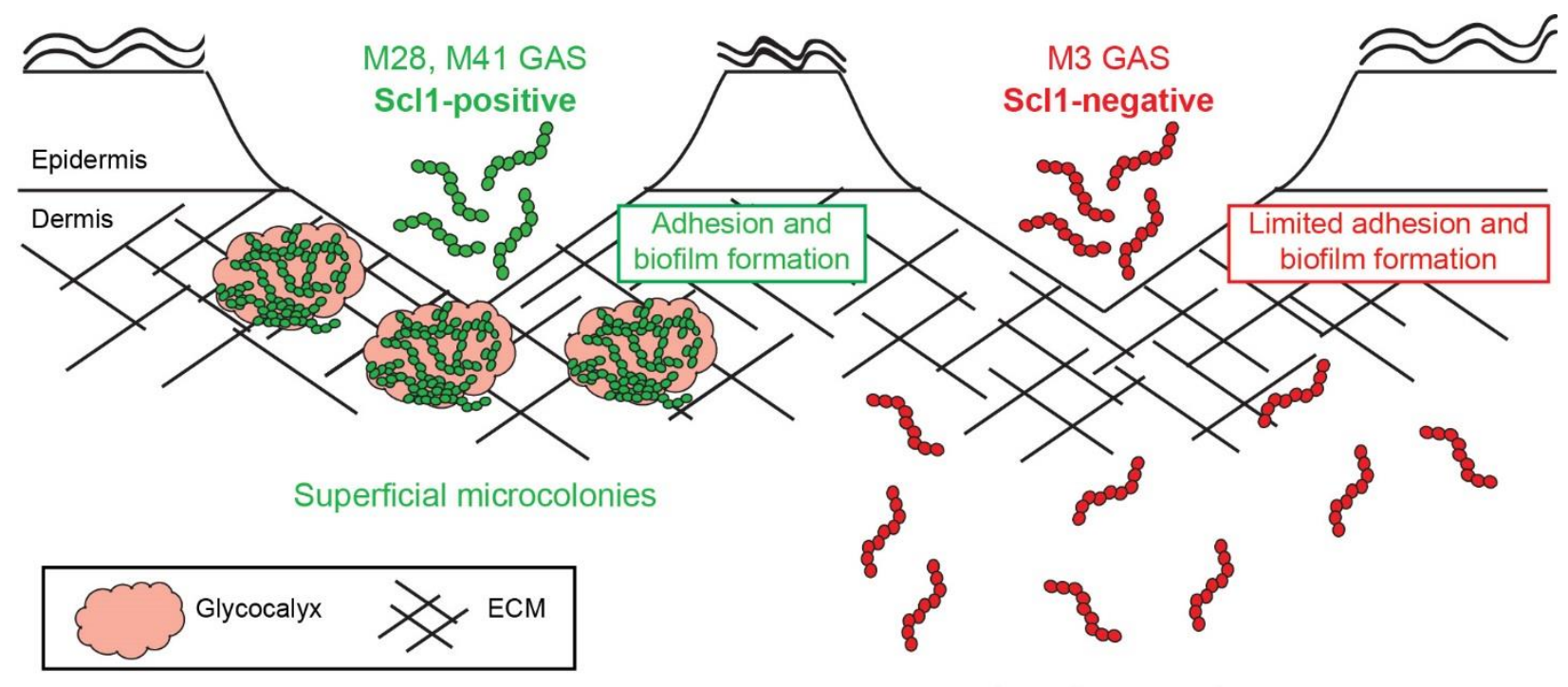

Invasive spread

Figure 10

Model of Scl1-mediated GAS adhesion, biofilm formation, and host colonization. M28- and M41-type GAS express full-length cell-attached Scl1, which mediates adherence to cFn and Lm. ECM binding promotes biofilm formation in wounded tissue and allows the formation of superficial tissue microcolonies resulting in non-invasive colonization. On the contrary, in M3-type GAS, naturally lacking full-length cell-attached Scl1.3 adhesin, bacterial cells have reduced adherence to $\mathrm{CFn}$ and $\mathrm{Lm}$ and reduced capacity for biofilm formation, thereby circumventing the formation of localized tissue microcolonies; infection balance is shifted towards dissemination through tissue, promoting invasive spread. 
Table 1. Constructs used in this study

\begin{tabular}{lll}
\hline Plasmid & Description & Source \\
\hline pJRS525 & E. coli / S. pyogenes shuttle vector & Mclver and Scott. 1997 \\
pSL230 & pJRS525 with scl1.41 allele & Caswell et al. 2007 \\
pSL501 & pJRS525 with scl1.3WT allele & Flores et al. 2015 \\
pSL502 & pSL501 with repaired allele sc/1.3FL & Flores et al. 2015 \\
pSL518 & pSL502 with Kan ${ }^{R}$ in place of Spc ${ }^{R}$ & This study \\
pASK-IBA2 & E. coli expression vector & IBA, Göttingen \\
pSL154 & pASK-IBA2 encoding rScl1.3V & This study \\
pSL503 & pASK-IBA2 encoding rScl1.3FL & Flores et al. 2015 \\
pSL514 & pASK-IBA2 with rScl2.3 & This study \\
pSB027 & GFP-encoding plasmid & Cramer et al. 2003 \\
\hline
\end{tabular}




\title{
CHAPTER 3
}

\section{NATURAL VARIANT OF COLLAGEN-LIKE PROTEIN A IN SEROTYPE M3 GROUP A STREPTOCOCCUS INCREASES ADHERENCE AND DECREASES INVASIVE POTENTIAL}

\author{
Anthony R. Flores, Brittany E. Jewell, Erika M. Versalovic, Randall J. Olsen, Beth A. \\ Bachert, Slawomir Lukomski and James M. Musser \\ Published in Infection and Immunity 2015 83: 1122-1129
}

\begin{abstract}
Group A Streptococcus (GAS) predominantly exists as a colonizer of the human oropharynx that occasionally breaches epithelial barriers to cause invasive diseases. Despite the frequency of GAS carriage, few investigations into the contributory molecular mechanisms exist. To this end, we have identified a naturally occurring polymorphism in the gene encoding the streptococcal collagen-like protein A (SclA) in GAS carrier strains. All previously sequenced invasive serotype M3 GAS possess a premature stop codon in the $s c / A$ gene truncating the protein. The carrier polymorphism is predicted to restore SclA function and was infrequently identified by targeted DNA sequencing in invasive strains of the same serotype. We demonstrate that a strain with the carrier $s c / A$ allele expressed a full-length SclA protein while the strain with the invasive $s c / A$ allele expressed a truncated variant. An isoallelic mutant invasive strain with the carrier $s c / A$ allele exhibited decreased virulence in a mouse model of invasive disease and decreased multiplication in human blood. Further, the isoallelic invasive strain with the carrier $s c / A$ allele persisted in the mouse nasopharynx and had increased adherence to cultured epithelial cells. Repair of the premature stop codon in the invasive $s c / A$ allele restored the ability to bind the extracellular matrix proteins laminin and cellular fibronectin. These data demonstrate that a mutation in GAS carrier strains increases adherence and decreases virulence and suggest selection against increased adherence in GAS invasive isolates.
\end{abstract}




\section{INTRODUCTION}

The bacterial pathogen Streptococcus pyogenes (group A Streptococcus, GAS) causes a wide range of disease in humans. GAS causes severe invasive infections such as toxic shock syndrome and necrotizing fasciitis but is also the cause of milder, more benign infections (e.g. pharyngitis). In addition to causing disease, GAS colonizes the throats of humans in the absence of symptoms. Asymptomatic colonization rates range between 5 $15 \%$ in healthy children (1), a rate that far exceeds that of GAS invasive disease (2). However, despite the high prevalence of GAS carriage, little is known about the molecular factors that contribute to asymptomatic colonization.

Colonization of the host epithelium is a key first step to establishing GAS carriage or disease. GAS elaborates several key surface proteins that contribute to this process including the M protein, fibronectin binding proteins, the GAS pilus, and streptococcal collagen-like (Scl) proteins. The collagen-like protein SclA (also known as Scl1) is found in all GAS serotypes examined to date (3) and is positively regulated by the wellcharacterized regulator Mga (4). The SclA protein extends from the GAS cell surface in a homotrimeric, 'Iollipop-like' fashion (5). The outermost region consists of a globular head that varies considerably between GAS serotypes followed by a repeating Gly-X-Y (GXY) collagen-like sequence that is in turn linked to the cell wall through a proline-rich linker region (Figure $1 \mathrm{~A}$ ). SclA contributes to GAS adherence and colonization through binding cellular fibronectin and laminin via the variable globular head (6) and integrins through the collagen-like region $(7,8)$. Variability in the degree of binding between GAS serotypes to these and other host molecules is predicted based on the differences in the $\mathrm{V}$ and $\mathrm{CL}$ regions of ScIA.

While several studies have begun to unravel the role of SclA as an adhesin, its contribution to phenotypic differences based on source of GAS isolation (e.g. invasive versus carrier) is unknown. Expression of GAS surface proteins may enhance adherence to host surfaces but may also reduce virulence. Expression of pili in serotype M1 GAS promotes adherence but enhances GAS killing through neutrophil extracellular traps (NETs) reducing systemic virulence (9). Similarly, the fibronectin-binding protein PrtF1 increased adherence to human cells but decreased dissemination in a mouse model of GAS infection (10). Similar paradoxical observations exist for surface proteins in 
Staphylococcus aureus (11). In contrast to other GAS serotypes, it has been shown that the $s c / A$ gene in serotype M3 GAS harbors an internal stop codon truncating the predicted protein (Figure 1A) (12). In fact, the truncated $s c / A$ gene was identical in recent whole genome sequencing of $>200$ invasive serotype M3 GAS (13), suggesting selection against a full-length, surface-attached protein. The impact of the truncated $s c / A$ allele on the pathogenesis of invasive serotype M3 GAS remains unknown.

Here, we report that human carrier strains of serotype M3 GAS contain a natural variant of $s c / A$ that restores the full open-reading frame. Expression of the full-length SclA in an invasive strain correlates with increased adherence to epithelial cells in vitro and decreases virulence in invasive disease in mice. The data presented also suggest that the truncated $s c / A$ allele contributes to pathogenesis in invasive serotype M3 GAS. Our findings suggest that mutations negatively affecting virulence and enhancing colonization of mucosal surfaces may contribute to asymptomatic carriage in GAS.

\section{MATERIALS AND METHODS}

\section{Bacterial strains used and culture conditions}

GAS strains are listed in Table S1 and were grown on trypticase soy agar containing 5\% sheep blood agar (SBA) (Becton Dickinson), in Todd-Hewitt broth containing $0.2 \%$ (wt/vol) yeast extract (THY) (Difco Laboratories), or on THY agar. When needed, GAS media was supplemented with chloramphenicol (Sigma-Aldrich) at $10 \mu \mathrm{g} / \mathrm{mL}$ or spectinomycin (Sigma-Aldrich) at $150 \mu \mathrm{g} / \mathrm{ml}$. E. coli DH5a or TOP10 (Invitrogen) grown in Luria-Bertani (LB) broth or on LB agar (Difco Laboratories) were used for cloning and supplemented with ampicillin (Sigma-Aldrich) at $100 \mu \mathrm{g} / \mathrm{mL}$ or chloramphenicol (SigmaAldrich) at $20 \mu \mathrm{g} / \mathrm{mL}$ when appropriate.

\section{PCR amplification and sequencing of sclA}

Primers for PCR and sequencing of $s c / A$ in GAS strains are listed in Table S2. Growth of strains, genomic DNA extraction, PCR amplification and Sanger sequencing were performed as previously described (14). 


\section{Generation of isoallelic and isogenic mutants in MGAS10870}

Plasmids and primers used in this study are listed in Tables S1 and S2. The procedure used in generating the isoallelic mutant MGAS10870sc/A Carrier was as described (15). Briefly, the $s c / A^{\text {Carrier }}$ allele was amplified using the primers $2902 \mathrm{~F}$ and $2487 \mathrm{R}$ from the carrier strain MGAS23440 and subsequently ligated into the temperature sensitive E. coliGram positive shuttle vector pJL1055 using BamHI and Xhol (16) to generate pJSF41. Electrocompetent cells of MGAS10870 were transformed with pJSF41 and allelic replacement carried out as previously described (17). The isoallelic mutant was confirmed using Sanger sequencing (Applied Biosystems).

Insertional inactivation of $S C / A$ in MGAS10870 was performed as previously described (18). Briefly, a three-step process was used to generate a PCR fragment in which 810 bases of the $s c / A$ open reading frame (ORF) were removed. Primer pair 0488R and MSP184 were used to generate a 824-bp fragment including the first 18-bp of the scIA ORF. Likewise, primer pair 2901F and MSP183 were used to generate a 791-bp fragment containing the last 18-bp of the sclA ORF. Primer pair MSP181 and MSP182 were used to amplify aad9 with ends homologous to the first and last 18-bp of the $s c / A$ ORF. The three PCR fragments were then used to generate a 2,581-bp fragment using primer pair 2901F and 0488R in a ligation PCR in which all but 36-bp (12 amino acids) of the $s c / A$ gene were replaced, in-frame, by the spectinomycin cassette aad9. All isoallelic and isogenic mutants were confirmed by Sanger sequencing (Applied Biosystems).

\section{RNA isolation and quantitative real-time PCR analysis}

Transcript levels of $s c / A$ were assayed as previously described (15). Briefly, RNA was isolated and purified using an RNeasy kit according to the manufacturer's instructions (Qiagen). Reverse transcription of RNA to produce cDNA was done with a High Capacity cDNA Reverse Transcription Kit (Applied Biosystems). TaqMan quantitative real-time PCR (qQRT-PCR) was performed with an ABI 7500 Fast Real-Time and tufA used as the endogenous control gene. TaqMan primers and probes used in analyses are listed in Table S2. All reactions were performed in quadruplicate using RNA purified from at least three biologic replicates. 


\section{Generation of anti-SclA rabbit antibodies and western immunoblot analysis of ScIA}

The $s c / A^{\text {Invasive }}$ gene was PCR amplified using primers MSP263 and MSP264 (Table S2) and cloned into the expression vector pET15b (Novagen) to generate pJSF66. The Histagged SclA ${ }^{\text {Invasive }}$ protein was overexpressed in E. coli (BL21) and purified using nickel affinity chromatography. Affinity-purified rabbit polyclonal anti-SclA antibody was generated by LifeTein (Hillsborough, $\mathrm{NJ}$ ).

Complementation (in trans) of the isogenic deletion mutant MGAS10870 $\Delta \mathrm{sclA}$ utilized the Gram-positive expression vector pDC123 (19). The primer pair MSP275 and MSP277 were used to amplify the sclA gene from either MGAS10870 ( $s c / A^{\text {Invasive }}$ ) or MGAS23440 ( $s c / A^{\text {Carrier}}$ ) and ligated into the $E c o R V / H i n d l l l$ sites of $\mathrm{pDC123}$. The resulting plasmids (Table S1) were electroporated into MGAS10870 $\Delta$ sclA to generate the individually transcomplemented strains. Isolation of bacterial proteins and western immunoblot analysis was carried out as described (15).

\section{Cloning, expression, and purification of full-length ScIA (ScIA ${ }^{\mathrm{M} 3-\mathrm{FL}}$ )}

All plasmids and primers are listed in Tables S1 and S2. The sclA coding region of the serotype M3 strain MGAS315 was amplified by PCR using the forward primer $232 \mathrm{Up}$ and the reverse primer ME7, digested with EcoRI and Hpal enzymes, and, cloned into the E. coli/GAS shuttle vector pJRS525. The resulting plasmid, pSL501 served as a template for PCR mutagenesis to obtain the full-length sclA allele (pSL502). The forward PCR primer pJRS525F1 was used with the reverse primer ME6, which contains a single base pair substitution encoding a glutamate residue (GAA) instead of the stop codon (TAA) naturally present in the 11 th GXY repeat of $s c / A$ wild-type allele.

The recombinant full-length SclA protein ( $\mathrm{rScl} \mathrm{A}^{\mathrm{M} 3-\mathrm{FL}}$ ) was generated employing the Strep-tag ${ }^{\circledR}$ II expression and purification system (IBA-GmbH, Goettingen, Germany), as described previously $(5,20)$. PCR amplification was performed using pSL502 DNA template, with the primers Scl1.3 M3VF and Scl1.28WMR. PCR product was digested with Hindll and Eael enzymes and cloned into the pASK-IBA2 E. coli vector designed for periplasmic expression, resulting in pSL503 encoding rSclA ${ }^{\mathrm{M} 3-\mathrm{FL}}$. Each clone, pSL501503 , was confirmed by sequencing. Protein $r S c / A^{M 3-F L}$ was affinity purified on StrepTactin® sepharose, dialyzed against 25 mM HEPES, pH 8.0, and analyzed on SDS- 
PAGE stained with RAPIDstain ${ }^{\top M}$ (G-Biosciences). Protein identity was confirmed by Nterminal Edman degradation (lowa State University, Protein Facility).

\section{Mouse virulence experiments}

Female CD1 mice (Harlan Laboratories) were used for virulence studies as described previously (21). Mice were inoculated in the right hind limb with $1 \times 10^{7} \mathrm{CFU}$ of GAS in $100 \mu \mathrm{l}$ PBS for intramuscular infection or intranasally with $1 \times 10^{7}$ CFU in $50 \mu \mathrm{l}$ PBS for nasopharyngeal colonization. Near-mortality was determined by observation using predefined criteria for mice infected intramuscularly (21). Mouse nasopharyngeal colonization was performed as previously described (22). All mouse experiments were approved by the Institutional Animal Care and Use Committee of Houston Methodist Research Institute.

\section{Growth in human blood}

Experiments assessing the ability to grow in human blood were conducted under a Houston Methodist Research Institute Institutional Review Board human subjects protocol and carried out as described $(22,23)$. A minimum of two healthy, non-immune, adult donors were used for each experiment.

\section{Human epithelial cell adherence assays}

GAS adherence to cultured epithelial cells was carried out as previously described (22). Assays were performed in quadruplicate. Percentage adherence was calculated by dividing the recovered CFU by the original inoculum.

\section{ECM binding assay}

To assess ECM binding by ELISA, recombinant $\mathrm{Scl}(\mathrm{rScl})$ proteins $(0.5 \mu \mathrm{M})$ were immobilized onto Strep-Tactin® coated microplate wells (IBA-GmbH), as described before $(6,24)$. The cellular fibronectin $(\mathrm{cFn})$ from human foreskin fibroblasts ( $F 2518$, Sigma), and laminin (Lm) (23017-015, Invitrogen) were added to wells containing rScls at $1 \mu \mathrm{g}$ per well. Rabbit anti-human fibronectin (F3648, Sigma) and anti-laminin (L9393, Sigma) primary antibodies were used at concentrations of 1:1000 and 1:250, respectively. 
Secondary antibody goat anti-rabbit IgG $(\mathrm{H}+\mathrm{L})$ HRP conjugate (BioRad), was used at a concentration of 1:2000, followed by addition of the HRP substrate 2,20-azino-bis(3ethylbenzthiazoline-6-sulphonic acid) (37615, 1-step ABTS, ThermoScientific); absorption was measured at a wavelength of $415 \mathrm{~nm}$. OD values from BSA-coated control wells were subtracted from the OD values of each test well. Shown are combined results from three independent experiments performed in triplicate.

\section{Statistics}

A two-tailed $t$-test (unequal variance) was used to compare multiplication factors between strains grown in human blood, adherence of strains to epithelial cells, and ECM binding of recombinant $\mathrm{Scl}$ proteins. Kruskal-Wallis analysis of variance was used to compare rates of nasopharyngeal colonization between strains. A $P$-value less than 0.05 was considered significant for all statistical tests.

\section{RESULTS}

The variant scIA allele occurs infrequently in serotype M3 GAS invasive strains and results in expression of a full-length protein

In an effort to identify key molecular genetic differences contributing to asymptomatic carriage of GAS, we performed whole genome sequencing of 37 serotype M3 GAS isolates cultured serially from 9 individuals over time (22). We discovered that 4 strains recovered from one subject contained a variant $s c / A$ allele compared to the reference serotype M3 genome. The variant carrier allele ( $s c / A^{\text {Carrier}}$ ) differed from all previously sequenced serotype M3 $s c / A$ ( $s c / A^{\text {Invasive }}$ ) by a 153-bp deletion removing the internal stop codon within the collagen-like region and restoring the complete open-reading frame (Figure $1 \mathrm{~A}$ ). The predicted SclA ${ }^{\text {Carrier }}$ protein has highest homology ( $>71 \%$ identity) to the SclA proteins from serotypes M6 and M5 GAS.

All invasive serotype M3 GAS strains studied to date possess the sclA allele with an internal stop codon ( $\left.s c / A^{\text {Invasive }}\right)(13)$. The predominance of the truncated $s c l A$ allele in invasive serotype M3 GAS suggests host selective pressure against expression of the full-length SclA protein in invasive disease. However, the possibility exists that variant $s c / A$ alleles restoring the open-reading frame and potentially expressing a complete SclA 
protein are found in more geographically diverse invasive serotype M3 GAS. We hypothesized that the frequency of variation in $s c / A$ would be substantially less in a population of invasive serotype M3 GAS than that found in the carrier population examined. To test our hypothesis we performed targeted DNA sequencing of $s c / A$ using strains obtained from the Centers for Disease Control Active Bacterial Core surveillance spanning the years 1998 to 2008 (http://www.cdc.gov/abcs) (14). Very few (6/479, 1.3\%) of the geographically and temporally diverse invasive serotype M3 GAS isolates harbored the same 153-bp deletion in $s c / A$ as identified in the GAS carrier strains. The $s c / A$ allele in the remaining invasive strains contained the internal stop codon as in the reference serotype M3 genome (MGAS315).

Given that the $s c / A^{\text {Invasive }}$ allele contains an internal stop codon, it is possible invasive strains do not produce $s c / A^{\text {Invasive }}$ transcript or that it is unstable and quickly degraded. We previously showed that peak $s c / A$ transcript coincides with peak mga transcript levels in serotype M3 GAS (15). Thus, we hypothesized that $s c / A$ transcript would be produced in a strain with either $s c / A^{\text {Invasive }}$ or $s c / A^{\text {Carrier. }}$. To test this hypothesis, we generated an isoallelic mutant strain that differed from the invasive parental strain MGAS10870 only by the presence of the $s c / A^{\text {Carrier }}$ gene (MGAS10870sc/ACarrier). We subsequently assayed $s c / A$ transcript in vitro after growth in rich medium (THY). As predicted, we detected $s c / A$ transcript and observed no significant differences between the isoallelic mutant and parental strains (Figure 1B).

We next hypothesized that the full-length SclA Carrier protein is expressed in GAS. To test this hypothesis, we complemented in trans the invasive strain MGAS10870 lacking completely the $s c / A$ gene (MGAS10870 $\Delta s c / A$ ) with a plasmid expressing either $s c / A^{\text {Carrier }}$ or $s c / A^{\text {Invasive }}$ under the native $s c / A$ promoter. Total protein of the knockout and trans-complemented strains were probed using a polyclonal anti-SclA antibody generated using purified SclA ${ }^{\text {Invasive }}$ protein (Figure 1A). Consistent with our hypothesis, we identified the ScIA ${ }^{\text {Carrier }}$ protein at the predicted size $(\sim 21 \mathrm{kDa})$ in MGAS10870pDCsclACarrier (Figure 1C). In addition, we also identified an approximately $12-\mathrm{kDa} S \mathrm{ScI} \mathrm{A}^{\text {Invasive }}$ protein, consistent with the predicted protein size based on the premature stop codon, in MGAS10870pDCsclA Invasive (Figure 1A). Thus, the variant $s c / A$ allele identified in the 
serotype M3 carrier strains restores expression of a full-length protein, however, a shorter, truncated version of SclA is also expressed in invasive serotype M3 GAS.

\section{A serotype M3 invasive strain with $s c / A^{\text {Carrier }}$ has reduced virulence.}

Inasmuch as expression of cell surface adhesion proteins has been shown to reduce virulence in GAS $(9,10)$, we next tested the hypothesis that invasive serotype M3 GAS with $s c / A^{\text {Carrier }}$ will have decreased virulence compared to invasive strains with $s c / A^{\text {lnvasive }}$. To test this hypothesis, we used a mouse model of necrotizing fasciitis to compare the parental MGAS10870 to the isoallelic mutant MGAS10870sclA ${ }^{\text {Carrer. }}$. Compared to the parental invasive strain the isoallelic mutant MGAS10870sclA $A^{\text {Carrier }}$ formed a localized, abscess-like lesion on visual inspection of the infected limb at $48 \mathrm{~h}$ (Figure 2A). Microscopic examination revealed extensive myonecrosis in the parental invasive strain while the isoallelic mutant with $s c / A^{\text {Carrier }}$ showed an abscess-like lesion with surrounding healthy, viable tissue (Figure $2 \mathrm{~A}$ ). This observation is consistent with decreased dissemination of the strain with $s c / A^{\text {Carrier }}$ within the infected tissue. In contrast to the isoallelic carrier mutant, MGAS10870 lacking sclA (MGAS10870 $\Delta s C / A$ ) showed no significant difference following visual and microscopic examination compared to the parental strain.

Given that human antibodies against Scl proteins cross-react with Scl of differing serotypes (25), it is possible that surface expression of SclA enhances phagocytosis of GAS. To further test our hypothesis that the strain harboring $s c / A^{\text {Carrier }}$ reduces GAS virulence we next assessed the ability of the mutants to grow ex vivo in human blood. Consistent with differences in mouse tissue destruction, we observed a significantly decreased ability of MGAS10870 sc/ $A^{\text {Carrier }}$ to grow in human blood but no such difference in MGAS10870 $\Delta s c / A$ compared to MGAS10870 (Figure 2B), suggesting enhanced phagocytosis in the mutant with the carrier allele. Similar to previous studies of GAS surface adhesins reducing virulence $(9,10)$, both in vivo mouse and ex vivo human data confirm a decreased virulence phenotype in an invasive strain expressing SclA ${ }^{\text {Carrier. In }}$ addition, deletion of the $s c / A^{\text {Invasive }}$ allele in an invasive strain had no significant effect on virulence in the models tested. 
Invasive strain harboring $s c / A^{\text {Carrier }}$ persists in mouse nasopharyngeal colonization and demonstrates increased adherence to human epithelial cells.

SclA has previously been shown to contribute to adherence to epithelial cells (8). Furthermore, others have hypothesized that GAS carrier strains are characterized by increased ability to adhere to epithelial surfaces (26). Thus, if our hypothesis is correct and SclACarrier increases adherence, the invasive strain with $s c / A^{\text {Carrier }}$ should have increased ability to colonize epithelial surfaces. We first tested the invasive strain

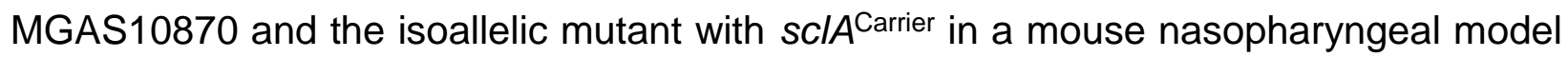
of colonization. We observed a significant decrease in the proportion of mice colonized with the isoallelic mutant MGAS10870sc/A $A^{\text {Carrier }}$ compared to MGAS10870 (Figure 3A). However, mice were stably and persistently colonized by the isoallelic mutant MGAS10870sc/A $A^{\text {Carrier }}$ at a level similar to the parental invasive strain at day 14 (Figure $3 A)$ suggesting an ability to persist on the mouse nasopharyngeal mucosa. We also observed a significant decrease in mice colonized by the deletion mutant MGAS10870 $\Delta s C I A$ (Figure $3 A$ ) compared to the parental strain. In addition to decreased colonization, both the carrier (MGAS10870sc/A Carrier) and deletion (MGAS10870 $\Delta s c / A$ ) mutants had significantly decreased bacterial burden compared to the parental invasive strain (Figure 3B). The finding of decreased bacterial burden in the mouse nasopharyngeal model is strikingly similar to that observed in GAS carrier strains deficient in capsule production (22).

To further characterize adherence in MGAS10870sc/A ${ }^{\text {Carrier }}$ we performed in vitro adherence assays to cultured epithelial cells. Given the known role of SclA in adherence to epithelial surfaces (6), we hypothesized that compared to the parental invasive strain, the strain with $s c / A^{\text {Carrier }}$ would better adhere to cultured epithelial cells. We assayed the in vitro adherence to both a pharyngeal epithelial cell line (HEp-2) and a skin epithelial cell line $(\mathrm{HaCaT})$. Compared to the invasive strain, we observed significantly greater adherence to both HEp-2 and HaCaT cells in the isoallelic strain MGAS10870sc/ACarrier and the deletion mutant MGAS10870 $\Delta s c / A$ (Figure 3C-D). Our data are consistent with

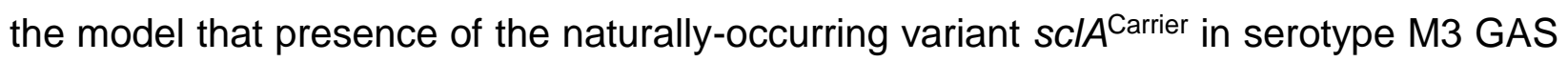
results in decreased virulence, stable and persistent colonization of the mouse nasopharynx, and increased ability to adhere to cultured human epithelial cells. Further, 
while deletion of $s c / A^{\text {Invasive }}$ did not affect virulence in the mouse intramuscular or ex vivo human models of GAS disease, the observed phenotypic differences in colonization and adherence suggest biologic activity of the truncated and secreted ScIA $A^{\text {Invasive }}$ protein.

\section{Full-length serotype M3 SclA protein (SclA ${ }^{\mathrm{M} 3-\mathrm{FL}}$ ) binds extracellular matrix components, cellular fibronectin and laminin}

Several recombinant SclA ( $\mathrm{rScl} A$ ) constructs derived from GAS of different M protein serotypes have been shown to bind human extracellular matrix (ECM) components such as cellular fibronectin (cFn) and laminin ( $\mathrm{Lm})(6,24)$. Since binding is mediated by the SclA variable $(V)$ region, we hypothesized that the observed increase in adherence in MGAS10870sc/A $A^{\text {Carrier }}$ was at least in part due to the expression of a cell-attached, fulllength SclA protein. To test our hypothesis, we cloned the $s c / A$ coding region from invasive serotype $M 3$ GAS and repaired the nonsense mutation, generating a GAA codon encoding a glutamate residue in place of the TAA premature stop codon (Fig. 1A). The resulting full-length $s c / A$ allele $\left(s c / A^{M 3-F L}\right.$ ) was cloned into an expression vector to generate full-length recombinant SclA protein ( $r S c / A^{M 3-F L}$ ), purified (Fig. 4A), and subsequently tested for binding to $\mathrm{CFn}$ and Lm by ELISA. ScIA ${ }^{\mathrm{M} 3-\mathrm{FL}}$ demonstrated significantly greater binding to both $\mathrm{cFn}$ and $\mathrm{Lm}$ compared to $\mathrm{SclB}^{\mathrm{M} 28}(P<0.05)$, an ECMbinding negative control derived from ScIB protein of serotype M28 GAS (Fig. 4B). Binding of $S C l A^{M 3-F L}$ was similar to recombinant $S C l A^{M 1}$ and $S c I A^{M 41}$ proteins, derived from serotype M1 and M41 GAS, respectively, and included as ECM-binding positive controls. Our data indicate that the full-length cell-attached serotype M3 SclA protein and thus the carrier SclA protein function as adhesins able to bind $\mathrm{cFn}$ and $\mathrm{Lm}$ in human tissue. Together, these data suggest that serotype M3 GAS acquired the unique null mutation resulting in secretion of a truncated SclA variant rather than cell-attached protein. Consistent with the mouse intramuscular infection model, lack of a cell-attached ScIA adhesin decreases tissue adherence and thereby promotes dissemination in serotype M3 GAS. 


\section{DISCUSSION}

In contrast to our sophisticated knowledge of bacterial mechanisms contributing to virulence in invasive disease, comparatively little is known regarding the molecular genetic factors contributing to asymptomatic carriage of bacterial pathogens. Several studies exist describing mutations, in both virulence genes and virulence regulators, that contribute to hypervirulence and increased invasive disease caused by GAS. However, despite the much higher prevalence of GAS carriage, few studies have shown that specific mutations contribute to a GAS carrier phenotype. Our data not only suggest that small genetic changes may contribute to GAS carriage but that study of GAS carrier strains may provide greater insight into GAS pathogenesis.

Our data demonstrating that the presence of $s c / A^{\text {Carrier }}$ is associated with decreased virulence and increased adherence are consistent with previous observations in GAS carriage. It has long been suggested that key phenotypic features of GAS carrier strains include decreased virulence and increased ability to adhere to and persist on mucosal surfaces. Further, such a phenotype may contribute to eradication failures in cases of GAS pharyngotonisillitis (27). Sela et al. (26) showed that GAS strains from subjects with eradication failure following treatment for pharyngotonsillitis had higher rates of adherence and internalization compared to strains from subjects with successful eradication. Moreover, previous studies have shown an inverse relationship between expression of bacterial surface adhesins and virulence (9-11). Thus, the mutation identified in $s c / A$ is one of the first specific mutations in a surface protein potentially linking increased adherence and decreased virulence in GAS carrier strains.

In addition to shedding new light on GAS carriage, our data suggest positive selection to maintain the truncated SclA Invasive protein in the invasive serotype M3 GAS population. Invasive strains of serotype M3 have surprisingly few mutations in sclA. Our analysis of geographically and temporally diverse serotype M3 invasive GAS strains demonstrated that $\sim 1 \%$ have the carrier mutation. The remaining invasive strains harbored the same sclA allele - the allele containing the premature stop codon - and virtually no polymorphisms (467 of $473 ; 98.7 \%$ ). The observation that invasive serotype M3 GAS express the truncated protein in vitro (Figure 1B) and the isogenic deletion 
mutant is affected in models of adherence and colonization is intriguing. Recently, it was shown that the homologous protein from serotype M1 GAS enhances survival in neutrophil extracellular traps (NETs) and interferes with myeloperoxidase release (28). Thus, it is possible that expression of a truncated ScIA in serotype M3 GAS acts in a similar manner to enhance survival in vivo and promote invasive disease but, as it is not surface attached, does not directly act as an adhesin and instead facilitates dissemination.

The biologic activity of SclA Invasive appears to be complex and potentially nichespecific based on the differing phenotypes of the deletion mutant in the models tested. The decreased bacterial burden of the deletion mutant in the mouse nasopharynx suggests that, at least in this model, SclA $A^{\text {Invasive }}$ contributes to the ability of invasive serotype M3 GAS to multiply at the mucosal surface. However, as indicated by the increased ability of the same mutant to better adhere to cultured epithelial cells (similar to the SclA ${ }^{\text {Carrier }}$ mutant), the truncated SclA may interfere with bacterial cell binding of host components. The observation that a full-length recombinant ScIA protein binds the ECM components and that the truncated SclA is conserved among invasive serotype M3 GAS and has the identical variable region responsible for ECM binding further supports this model. Further experimentation is needed to test these hypotheses.

The mutation identified in $s c / A$ in GAS carrier strains is likely one of many mutations that may contribute to the carrier phenotype. For instance, it has been demonstrated that a mutation in the promoter of the gene for the virulence regulator Mga contributes to decreased virulence in GAS carrier strains (15). More recently, mutations that eliminate GAS capsule have been shown to arise in vivo during human carriage and result in decreased virulence and increased adherence to epithelial cell surfaces (22). The current study not only suggests that mutations contribute to GAS carriage but enhances understanding of serotype M3 GAS pathogenesis, further emphasizing the importance of carriage research. One interpretation of the data presented is that of a model in which distinct genetic differences between GAS carrier and invasive strains contribute to a carrier phenotype defined by decreased virulence and increased ability to adhere to epithelial surfaces (Figure 5). Further research is needed to fully define genotype-phenotype relationships in GAS carrier strains. 


\section{ACKNOWLEDGMENTS}

We thank Meenal Elliott for her contribution to some experiments. This work was supported by the Pediatric Infectious Disease Society - St. Jude Children's Research Hospital Fellowship Award in Basic Research and the Robert Wood Johnson - Harold Amos Medical Faculty Development Program Award to A. R. F. This work was also supported in part by National Institutes of Health grants Al50666 and Al083683 to S. L. B. A. B. is a recipient of the Gossling Scholarship in Microbiology.

\section{REFERENCES}

1. Shaikh, N., Leonard, E., and Martin, J. M. (2010) Prevalence of streptococcal pharyngitis and streptococcal carriage in children: a meta-analysis. Pediatrics 126, e557-564

2. O'Loughlin, R. E., Roberson, A., Cieslak, P. R., Lynfield, R., Gershman, K., Craig, A., Albanese, B. A., Farley, M. M., Barrett, N. L., Spina, N. L., Beall, B., Harrison, L. H., Reingold, A., and Van Beneden, C. (2007) The epidemiology of invasive group A streptococcal infection and potential vaccine implications: United States, 2000-2004. Clin Infect Dis 45, 853-862

3. Lukomski, S., Nakashima, K., Abdi, I., Cipriano, V. J., Ireland, R. M., Reid, S. D., Adams, G. G., and Musser, J. M. (2000) Identification and characterization of the scl gene encoding a group A Streptococcus extracellular protein virulence factor with similarity to human collagen. Infect Immun 68, 6542-6553

4. Almengor, A. C., and Mclver, K. S. (2004) Transcriptional activation of $s c / A$ by Mga requires a distal binding site in Streptococcus pyogenes. J Bacteriol 186, 78477857

5. Xu, Y., Keene, D. R., Bujnicki, J. M., Hook, M., and Lukomski, S. (2002) Streptococcal Scl1 and Scl2 proteins form collagen-like triple helices. J Biol Chem 277, 27312-27318

6. Caswell, C. C., Oliver-Kozup, H., Han, R., Lukomska, E., and Lukomski, S. (2010) Scl1, the multifunctional adhesin of group A Streptococcus, selectively binds cellular fibronectin and laminin, and mediates pathogen internalization by human cells. FEMS Microbiol Lett 303, 61-68

7. Caswell, C. C., Barczyk, M., Keene, D. R., Lukomska, E., Gullberg, D. E., and Lukomski, S. (2008) Identification of the first prokaryotic collagen-sequence motif that mediates binding to human collagen receptors, integrins alpha 2beta 1 and alpha 11beta 1. J Biol Chem

8. Caswell, C. C., Lukomska, E., Seo, N. S., Hook, M., and Lukomski, S. (2007) Scl1dependent internalization of group A Streptococcus via direct interactions with the alpha2beta(1) integrin enhances pathogen survival and re-emergence. $\mathrm{Mol}$ Microbio/ 64, 1319-1331 
9. Crotty Alexander, L. E., Maisey, H. C., Timmer, A. M., Rooijakkers, S. H., Gallo, R. L., von Kockritz-Blickwede, M., and Nizet, V. (2009) M1T1 group A streptococcal pili promote epithelial colonization but diminish systemic virulence through neutrophil extracellular entrapment. $J$ Mol Med

10. Nyberg, P., Sakai, T., Cho, K. H., Caparon, M. G., Fassler, R., and Bjorck, L. (2004) Interactions with fibronectin attenuate the virulence of Streptococcus pyogenes. EMBO J. 23, 2166-2174

11. McElroy, M. C., Cain, D. J., Tyrrell, C., Foster, T. J., and Haslett, C. (2002) Increased virulence of a fibronectin-binding protein mutant of Staphylococcus aureus in a rat model of pneumonia. Infect Immun 70, 3865-3873

12. Oliver-Kozup, H. A., Elliott, M., Bachert, B. A., Martin, K. H., Reid, S. D., Schwegler-Berry, D. E., Green, B. J., and Lukomski, S. (2011) The streptococcal collagen-like protein-1 (Scl1) is a significant determinant for biofilm formation by group A Streptococcus. BMC Microbiol 11, 262

13. Shea, P. R., Beres, S. B., Flores, A. R., Ewbank, A. L., Gonzalez-Lugo, J. H., Martagon-Rosado, A. J., Martinez-Gutierrez, J. C., Rehman, H. A., SerranoGonzalez, M., Fittipaldi, N., Ayers, S. D., Webb, P., Willey, B. M., Low, D. E., and Musser, J. M. (2011) Distinct signatures of diversifying selection revealed by genome analysis of respiratory tract and invasive bacterial populations. Proc Natl Acad Sci U S A 108, 5039-5044

14. Olsen, R. J., Laucirica, D. R., Watkins, M. E., Feske, M. L., Garcia-Bustillos, J. R., Vu, C., Cantu, C., Shelburne, S. A., 3rd, Fittipaldi, N., Kumaraswami, M., Shea, P. R., Flores, A. R., Beres, S. B., Lovgren, M., Tyrrell, G. J., Efstratiou, A., Low, D. E., Van Beneden, C. A., and Musser, J. M. (2012) Polymorphisms in regulator of protease B (RopB) alter disease phenotype and strain virulence of serotype M3 group A Streptococcus. J Infect Dis 205, 1719-1729

15. Flores, A. R., Olsen, R. J., Wunsche, A., Kumaraswami, M., Shelburne, S. A., 3rd, Carroll, R. K., and Musser, J. M. (2013) Natural variation in the promoter of the gene encoding the Mga regulator alters host-pathogen interactions in group a Streptococcus carrier strains. Infect Immun 81, 4128-4138

16. Gryllos, I., Grifantini, R., Colaprico, A., Cary, M. E., Hakansson, A., Carey, D. W., Suarez-Chavez, M., Kalish, L. A., Mitchell, P. D., White, G. L., and Wessels, M. R. (2008) PerR confers phagocytic killing resistance and allows pharyngeal colonization by group A Streptococcus. PLoS Pathog 4, e1000145

17. Carroll, R. K., Shelburne, S. A., 3rd, Olsen, R. J., Suber, B., Sahasrabhojane, P., Kumaraswami, M., Beres, S. B., Shea, P. R., Flores, A. R., and Musser, J. M. (2011) Naturally occurring single amino acid replacements in a regulatory protein alter streptococcal gene expression and virulence in mice. J Clin Invest 121, 19561968

18. Lukomski, S., Hoe, N. P., Abdi, I., Rurangirwa, J., Kordari, P., Liu, M., Dou, S. J., Adams, G. G., and Musser, J. M. (2000) Nonpolar inactivation of the hypervariable streptococcal inhibitor of complement gene (sic) in serotype M1 Streptococcus pyogenes significantly decreases mouse mucosal colonization. Infect Immun 68, 535-542 
19. Chaffin, D. O., and Rubens, C. E. (1998) Blue/white screening of recombinant plasmids in Gram-positive bacteria by interruption of alkaline phosphatase gene (phoZ) expression. Gene 219, 91-99

20. Han, R., Zwiefka, A., Caswell, C. C., Xu, Y., Keene, D. R., Lukomska, E., Zhao, Z., Hook, M., and Lukomski, S. (2006) Assessment of prokaryotic collagen-like sequences derived from streptococcal Scl1 and Scl2 proteins as a source of recombinant GXY polymers. Appl Microbiol Biotechnol 72, 109-115

21. Olsen, R. J., Sitkiewicz, I., Ayeras, A. A., Gonulal, V. E., Cantu, C., Beres, S. B., Green, N. M., Lei, B., Humbird, T., Greaver, J., Chang, E., Ragasa, W. P., Montgomery, C. A., Cartwright, J., Jr., McGeer, A., Low, D. E., Whitney, A. R., Cagle, P. T., Blasdel, T. L., DeLeo, F. R., and Musser, J. M. (2010) Decreased necrotizing fasciitis capacity caused by a single nucleotide mutation that alters a multiple gene virulence axis. Proc Natl Acad Sci U S A 107, 888-893

22. Flores, A. R., Jewell, B. E., Olsen, R. J., Shelburne, S. A., 3rd, Fittipaldi, N., Beres, S. B., and Musser, J. M. (2014) Asymptomatic carriage of group a streptococcus is associated with elimination of capsule production. Infect Immun 82, 3958-3967

23. Lancefield, R. C. (1959) Persistence of type-specific antibodies in man following infection with group A streptococci. J Exp Med 110, 271-292

24. Oliver-Kozup, H., Martin, K. H., Schwegler-Berry, D., Green, B. J., Betts, C., Shinde, A. V., Van De Water, L., and Lukomski, S. (2013) The group A streptococcal collagen-like protein-1, Scl1, mediates biofilm formation by targeting the extra domain A-containing variant of cellular fibronectin expressed in wounded tissue. Mol Microbiol 87, 672-689

25. Hoe, N. P., Lukomska, E., Musser, J. M., and Lukomski, S. (2007) Characterization of the immune response to collagen-like proteins Scl1 and Scl2 of serotype M1 and M28 group A Streptococcus. FEMS Microbiol Lett 277, 142-149

26. Sela, S., Neeman, R., Keller, N., and Barzilai, A. (2000) Relationship between asymptomatic carriage of Streptococcus pyogenes and the ability of the strains to adhere to and be internalised by cultured epithelial cells. J Med Microbiol 49, 499502

27. Kaplan, E. L., Gastanaduy, A. S., and Huwe, B. B. (1981) The role of the carrier in treatment failures after antibiotic for group A streptococci in the upper respiratory tract. J Lab Clin Med 98, 326-335

28. Dohrmann, S., Anik, S., Olson, J., Anderson, E. L., Etesami, N., No, H., Snipper, J., Nizet, V., and Okumura, C. Y. (2014) Role for streptococcal collagen-like protein 1 in M1T1 group A Streptococcus resistance to neutrophil extracellular traps. Infect Immun 82, 4011-4020 

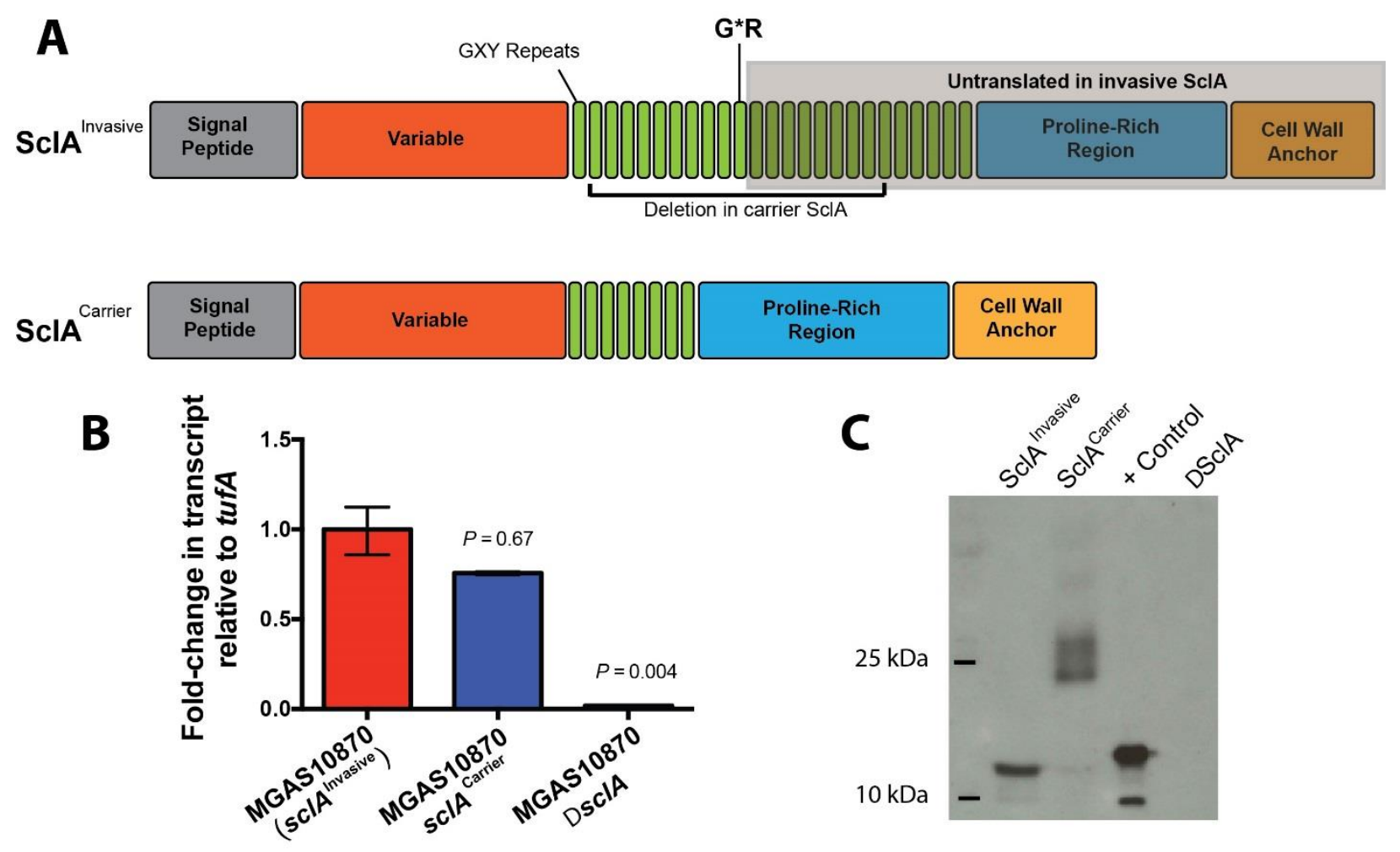

Figure 1

ScIA differs in GAS serotype M3 invasive and carrier strains and is expressed. (A) Invasive strains of serotype M3 GAS are characterized by an sclA allele harboring an internal stop codon truncating the predicted protein after the $11^{\text {th }} \mathrm{GXY}$ repeat $\left(\mathrm{G}^{*} \mathrm{Y}\right)$. The shaded region containing the cell wall anchor is untranslated in invasive SclA, indicating the protein is not cell-attached. The naturally occurring carrier sclA allele is characterized by a deletion removing $17 \mathrm{GXY}$ repeats in the mature protein including the $\mathrm{G}^{*} \mathrm{R}$ (bold) present in the invasive protein. To generate the full-length $S c \mid A^{\text {Invasive }}$ protein, the $G^{*} R$ (Gly-Stop-Arg) was repaired to GER (Gly-Glu-Arg). (B) TaqMan transcript analysis of invasive (MGAS10870), isoallelic (MGAS10870sc/Acarrier), and isogenic (MGAS10870 $\Delta s c / A$ ) mutants. $P$-values determined by $t$-test (unequal variance). (C) Western immunoblot using anti-SclA Invasive antibody and showing SclA expression in MGAS10870 $\triangle s c / A$ transcomplemented with either $s c / A^{\text {Carrier }}$ or $s c / A^{\text {Invasive. The positive }}$ control (+ Control) consisted of purified SclA ${ }^{\text {Invasive }}$ protein used for generation of rabbit anti-SclA antibody. 
A

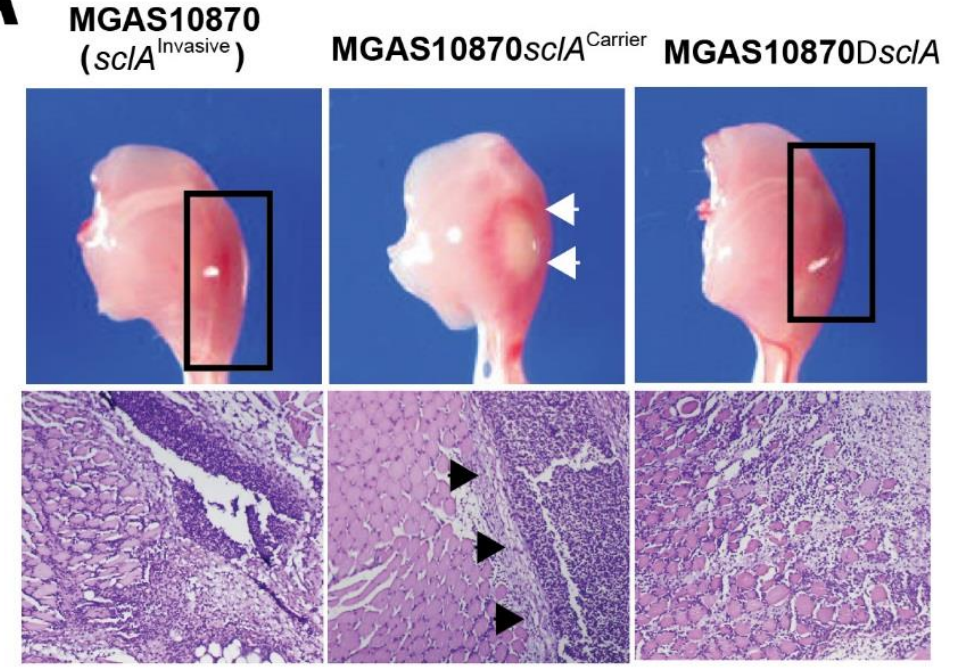

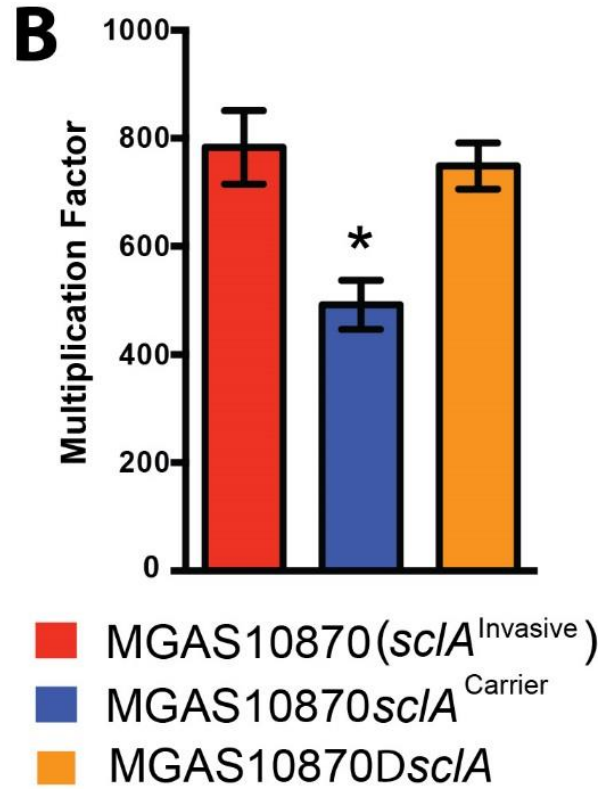

Figure 2

Strain with $s c / A^{\text {Carrier }}$ has reduced virulence compared to the parental invasive strain. (A) Visual (top panels, original magnification 4X) and microscopic (bottom panels, original magnification 20X) examination of mouse hind limb lesions at 48 hours following intramuscular injection of GAS strains. Whereas mice infected with the isoallelic mutant ( $s c / A^{\text {Carrier}}$ ) had a small, circumscribed, abscess-like lesion (white arrowheads), the parental invasive strain and isogenic deletion mutant caused a comparatively larger, grossly necrotic lesion centered at the inoculation site (boxed). Similarly, compared to the extensive spreading myonecrosis observed in the invasive and isogenic deletion strains, the isoallelic mutant showed an abscess-like lesion (black arrowheads) on microscopic examination. (B) Multiplication after growth in human blood. Shown is multiplication after growth in a single donor performed in quadruplicate. Similar results were observed after growth using additional donors. Error bars represent standard deviation and asterisk ( $\left.{ }^{*}\right)$ indicates $P$-value $<0.05$ as determined by $t$-test (unequal variance). 

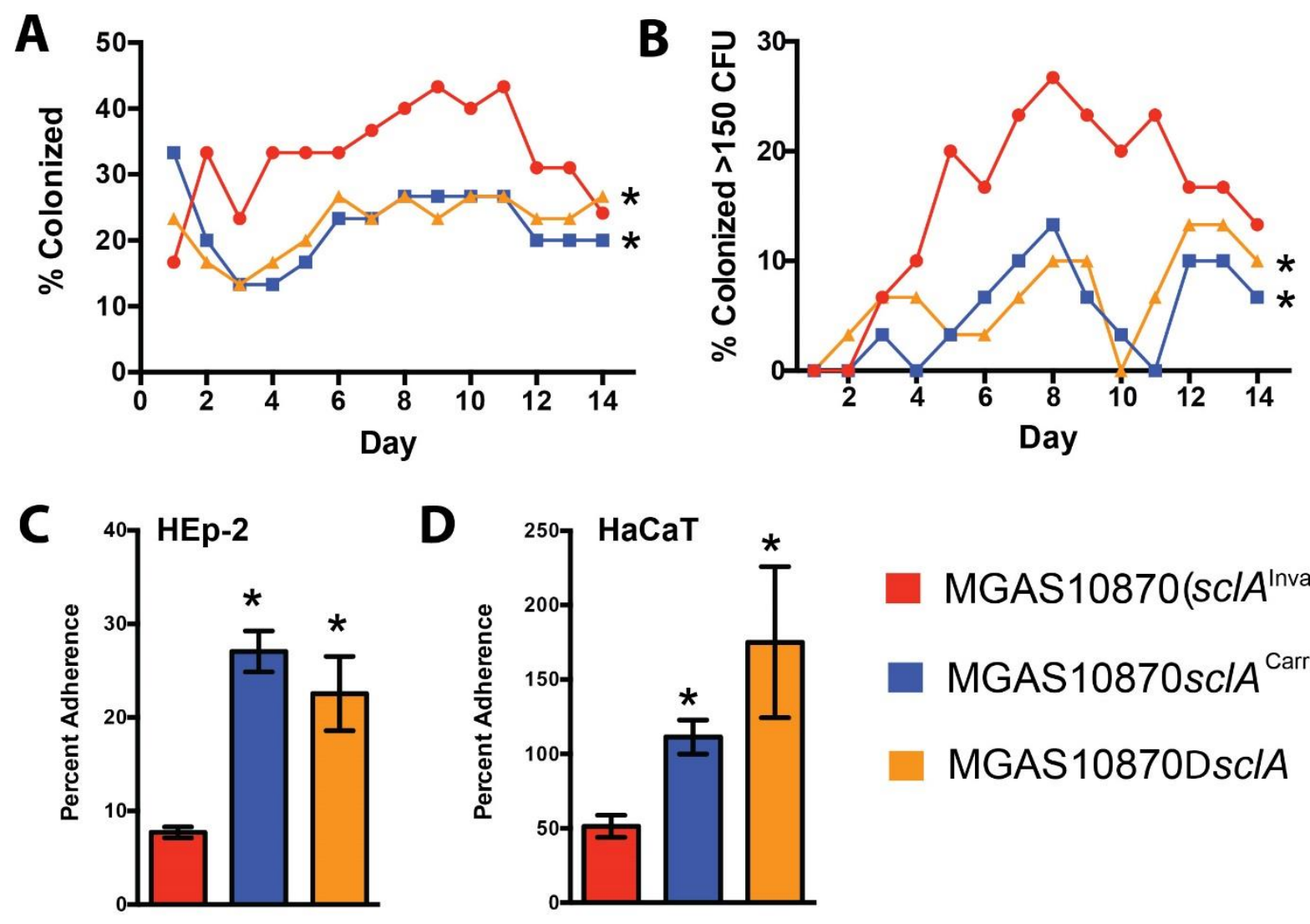
MGAS10870(sclA Invasive $)$
MGAS10870scla ${ }^{\text {Carrier }}$
MGAS10870DsclA

Figure 3

SclA ${ }^{\text {Carrier }}$ affects mouse nasopharyngeal colonization and adherence to cultured epithelial cells. (A) Mouse nasopharyngeal colonization following inoculation of invasive or isoallelic GAS strains. Mice $(n=20)$ were inoculated and swabbed daily for 14 days. (B) Bacterial burden of mice colonized after nasopharyngeal infection. Percent of colonized mice with $>150 \mathrm{CFU}$ after daily swabbing. Asterisk $\left({ }^{*}\right)$ indicates $P<0.05$ compared to the parental invasive strain as determined by Kruskal-Wallis. (C-D) Adherence of GAS strains to cultured epithelial cells. Error bars represent standard deviation and asterisks $\left({ }^{*}\right)$ indicate $P<0.05$ compared to the parental invasive strain as determined by $t$-test (unequal variance). 


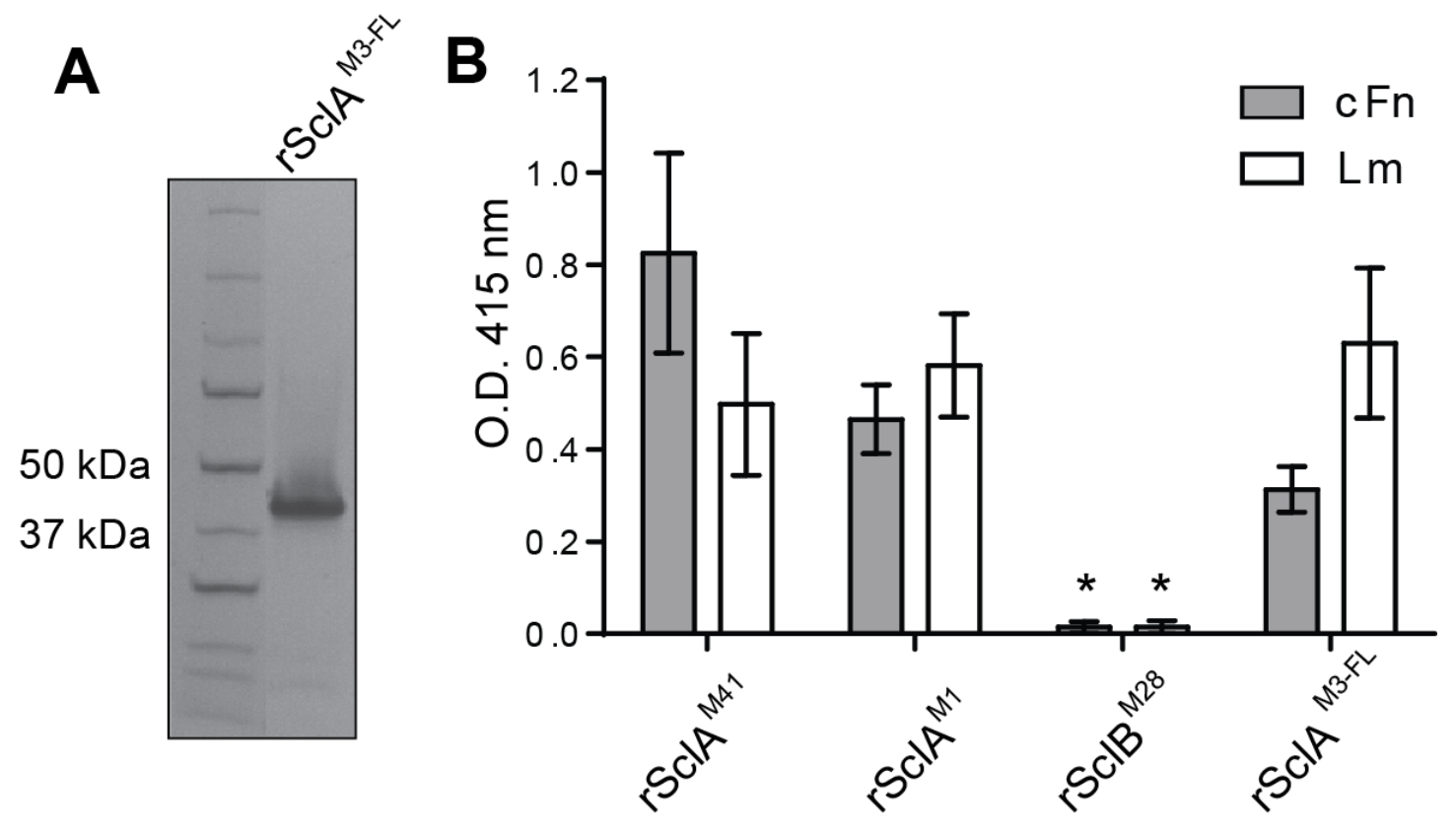

Figure 4

Recombinant full-length ScIA ( $\mathrm{rScl} \mathrm{A}^{\mathrm{M} 3-\mathrm{FL}}$ ) binds the human extracellular matrix proteins cellular fibronectin (cFn) and laminin (Lm). (A) SDS-PAGE gel of purified $\mathrm{rScl}^{\mathrm{M} 3-\mathrm{FL}}$. Expected size of protein is $20.6 \mathrm{kDa}$ and $\mathrm{rScl} \mathrm{A}^{\mathrm{M} 3-\mathrm{FL}}$ migrates at approximately $39 \mathrm{kDa}$; aberrant migration is characteristic of recombinant Scl proteins. (B) ECM binding to $\mathrm{rScl} \mathrm{A}^{\mathrm{M} 3-\mathrm{FL}}$ protein. Test protein $\mathrm{rScl} \mathrm{A}^{\mathrm{M} 3-\mathrm{FL}}$, as well as control ECM-binding positive $\left(r S c l A^{M 41}\right.$ and $\left.r S c l A^{M 1}\right)$ and binding negative $\left(r S B^{M}{ }^{28}\right)$ control proteins, were immobilized onto Strep-Tactin coated wells, and incubated with cFn and Lm. Detection of rScl-bound ECM was carried out using specific primary antibodies and HRP-conjugated secondary antibodies. Bars represent the mean O.D.415nm values normalized for BSA controls. Asterisks represent $P$-values $<0.05$ for $\mathrm{rScl}^{\mathrm{M} 3-\mathrm{FL}}, \mathrm{rScl} \mathrm{A}^{\mathrm{M} 1}$, and $\mathrm{rScl}^{\mathrm{M} 41}$ in comparison to the negative control $\mathrm{rSclB}{ }^{\mathrm{M} 28}$ determined from three independent experiments each performed in triplicate wells, using one-sample $t$-test. 

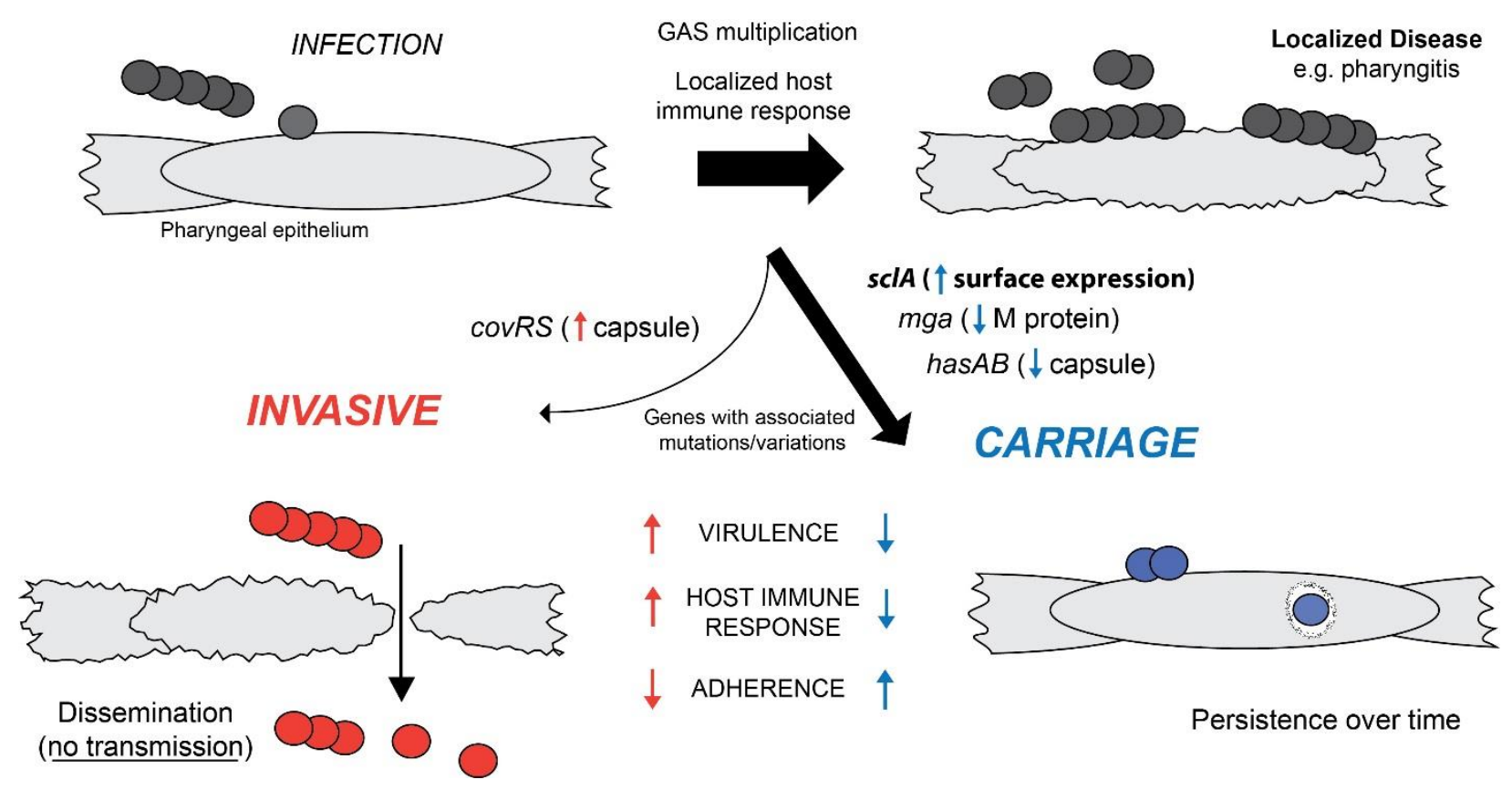

Figure 5

Model summarizing the effect of invasive ( $\left.s c / A^{\text {Invasive }}\right)$ or carrier ( $\left.s c / A^{\text {Carrier }}\right) s c / A$ alleles in serotype M3 GAS. Following establishment of initial infection on an epithelial surface, GAS may progress to invasive disease (indicated by red circles) or carriage (indicated by blue circles). Invasive strains are characterized by the presence of a truncated and secreted SclA protein, decreased adherence to epithelial surfaces, and increased tissue destruction. It is postulated that the truncated SclA ${ }^{\text {Invasive }}$ protein at least partially contributes to decreased adherence to epithelial surfaces and host extracellular matrix, and increased ability to disseminate observed in invasive serotype M3 GAS. Conversely, carrier strains harboring the $s c / A^{\text {Carrier }}$ allele produce a full-length, surfaceattached SclA protein, have decreased tissue destruction, and have increased adherence to host epithelial surfaces. Likewise, it is proposed that the decreased virulence and increased adherence in some carrier strains can be attributed to the naturally occurring SclA Carrier protein. 


\section{PART II \\ STRUCTURAL ANALYSIS OF THE SCL PROTEINS}


CHAPTER 4

\title{
CRYSTALLIZATION AND PRELIMINARY X-RAY CRYSTALLOGRAPHIC ANALYSIS OF THE VARIABLE DOMAIN OF SCL2.3, A STREPTOCOCCAL COLLAGEN-LIKE PROTEIN FROM INVASIVE M3-TYPE STREPTOCOCCUS PYOGENES
}

Flavia Squeglia, Beth Bachert, Maria Romano, Slawomir Lukomski, and Rita Berisio Published in Acta Crystallographica Section F, Structural Biology and Crystallization Communications 2013 69(Pt 9):1023-1025

\begin{abstract}
Streptococcal collagen-like proteins, Scls, are widely expressed by the well-recognized human pathogen Streptococcus pyogenes. These surface proteins contains a signature central collagen-like region, and an amino-terminal globular domain, termed the variable domain, which is protruded away from the cell surface by the collagen-like domain. Despite their recognized importance for bacterial pathogenicity, no structural information is hitherto available on proteins of the Scl class. The variable domain of Scl2 from invasive M3-type S. pyogenes has successfully been crystallized using vapor-diffusion methods. The crystals diffracted to $1.5 \AA$ resolution and belonged to space group $H 32$, with unit-cell parameters $a=44.23, b=44.23, c$ $=227.83 \AA$. The crystal structure was solved by single wavelength anomalous dispersion using anomalous signal from a europium chloride derivative.
\end{abstract}




\section{INTRODUCTION}

Collagen-like proteins that form stable triple-helices have been shown to be present in many bacterial species (1) and to play a role in pathogenicity. Best characterized prokaryotic collagens are the two collagen-like proteins, Scl1 and Scl2, which have been demonstrated to be simultaneously expressed on the cell surface of $S$. pyogenes and to promote bacterial adhesion to the host $(2,3)$. Both Scl1 and Scl2 proteins contain a signal sequence, an $\mathrm{N}$-terminal variable globular domain $(\mathrm{V})$, a highly charged collagen-like triple-helix domain $(\mathrm{CL})$ consisting of (Gly-Xaa-Yaa)n triplet repeats and a C-terminal Gram-positive cell wall attachment domain. The Scl1 and Scl2 proteins form stable triple-helical structures when expressed as recombinant proteins $(4,5)$, and their $\mathrm{N}$-terminal globular $\mathrm{V}$ domain adjacent to the triple-helix domain appears to be important for efficient triple-helix assembly (5-7).

Bacterial adherence to host tissues, an early critical step in the infection process, often involves surface proteins $(8,9)$. Among these, Scl proteins of $S$. pyogenes are crucial to hostpathogen recognition (10). It has been demonstrated that Scl1 can bind selected human extracellular matrix components (11), cellular integrin receptors $(2,12,13)$, and plasma components (14-17). Importantly, human collagen receptors, such as integrin $\alpha_{2} \beta_{1}$, recognize the triple helix $\mathrm{CL}$ domain of $\mathrm{Scl} 1$, and this event results in cell signaling, indicating that collagen-like bacterial proteins display not only structural but also functional similarities to human collagens $(2,12,13)$. Despite the key role of Scl proteins in bacterial pathogenicity, their three-dimensional structure has not been determined. In addition, whereas the triple helical structure of CL domain of Scls can be predicted based on sequence identity to collagen (5), no structural clues regarding the $V$ domain can be obtain from Scl sequences, owing to poor sequence identity with known structures. Here, we report the crystallization and preliminary crystallographic investigations of the $\mathrm{V}$ domain of Scl2 from invasive M3-type $S$. pyogenes (Scl2.3-V). 


\section{MATERIALS AND METHODS}

\section{Cloning, expression and purification of recombinant rScl2.3-V protein}

Recombinant rScl2.3-V protein was produced in the Escherichia coli periplasm using the Streptag II expression and purification system (IBA GmbH, Goettingen, Germany), as reported previously (4). Briefly, the 5'-portion of the scl2.3 gene from strain MGAS315, encoding the amino-terminal Scl2.3-V-region, was PCR-amplified using the forward primer scl2-M3VF (5'GAGATGGCCGATGGTGAAGATGCCCAAAAAAG) and the reverse primer scl2-M3VR (5'CAGCGTCTCAGCGCTATCAAGGACATGATCTTGTATGCC) and was cloned into pASK-IBA2 vector, resulting in plasmid pSL155. Escherichia coli strain DH5a was used for cloning and $E$. coli BL21 was used for protein expression. E. coli harboring plasmid PSL155, which encodes the rScl2.3-V protein, was grown in Luria-Bertani (LB) liquid medium (BD Biosciences) supplemented with ampicillin $\left(100 \mu \mathrm{g} \mathrm{mL}^{-1}\right)$. Plasmid construct $\mathrm{pSL} 155$ was confirmed by DNA sequencing and the identity of purified recombinant protein rScl2.3- $\mathrm{V}$ was confirmed by $\mathrm{N}$ terminal Edman degradation.

\section{Crystallization experiments}

Crystallization trials were performed at $293 \mathrm{~K}$ using the hanging-drop vapor-diffusion method. Preliminary crystallization conditions were set up using a robotic station for high throughput crystallization screening (Hamilton STARlet NanoJet 8+1) and commercially available sparsematrix kits (Crystal Screen, Crystal Screen 2 and Index, Hampton Research). Optimization of the crystallization conditions was performed manually by fine-tuning the protein and precipitant concentrations.

\section{Data collection and processing}

Diffraction data were collected to $1.52 \AA$ in-house from a native crystal at $100 \mathrm{~K}$ using a Rigaku Micromax $007 \mathrm{HF}$ generator producing $\mathrm{Cu} K \alpha$ radiation and equipped with a Saturn944 CCD detector. Cryoprotection of the crystals was achieved without addition of further cryoprotectants, given the composition of the crystallization mother liquor, which contained the cryoprotectant pentaerythritol ethoxylate $(15 / 4 \mathrm{EO} / \mathrm{OH})$ at $30 \%(\mathrm{v} / \mathrm{v})$. For phasing purposes, native crystals were soaked in solutions containing between 2 and $8 \mathrm{mM} \mathrm{EuCl}_{3}$ for different soaking times. Data were collected from several crystals to identify the best single-wavelength 
Anomalous Diffraction (SAD) signal. The data sets were scaled and merged using HKL2000 program package (18). Statistics of data collection are reported in Table 1.

\section{Structure determination and refinement}

Phasing was achieved using in-house SAD data. A preliminary evaluation of the anomalous signal was performed for all tested crystals using the SCALEPACK software, implemented in HKL2000 (18). SHELXD was used to identify europium ion sites (19). Phases were then improved by solvent-flattening density modification and phase extension by RESOLVE (20). The obtained model was further improved using ARP/wARP (21).

\section{RESULTS AND DISCUSSION}

The recombinant $\mathrm{rScl}$ 2.3-V protein corresponds to the N-terminal part (residues 1-77) of Scl2.3 variant from the invasive $S$. pyogenes M3-type strain MGAS315 (22). This protein construct has successfully been purified and crystallized using vapor-diffusion methods. The purified rScl2.3-V showed a single band of approximately $10 \mathrm{kDa}$ on SDS-PAGE, which is in good agreement with the predicted molecular mass of $10,105 \mathrm{Da}$. The initial automated crystallization screening using commercially available solutions provided the first hints of crystallization conditions. Small crystals that were not appropriate for diffractometric experiments were obtained in the presence of pentaerythritol ethoxylate. The quality of these crystals was improved by fine-tuning the concentration of the protein and of the precipitants. Crystals suitable for X-ray diffraction experiments (Figure 1) were obtained in $3 \mathrm{~d}$ using a protein concentration of $5 \mathrm{mg} \mathrm{mL}^{-1}$ and $0.05 \mathrm{M}$ ammonium sulfate, $0.05 \mathrm{M}$ bis-tris $\mathrm{pH} 6.5,30 \%(\mathrm{v} / \mathrm{v})$ pentaerythritol ethoxylate $(15 / 4 \mathrm{EO} / \mathrm{OH})$. These crystals, which diffracted to $1.5 \AA$ resolution (Figure 2), showed three-fold symmetry and belonged to the space group $H 32$, with unit-cell parameters $a=44.23, b=44.23, c=227.83 \AA$ (Table 1). Matthews coefficient calculations (23) suggested the presence of one molecule per asymmetric unit ( $\mathrm{VM}=2.14 \AA^{3} / \mathrm{Da}$, with $42.6 \%$ solvent content).

Lanthanides can yield high-phasing-power derivatives using in house copper sources (24-26). Europium chloride derivative crystals were prepared by soaking the native crystals in stabilizing solutions containing between 2 and $8 \mathrm{mM} \mathrm{EuCl}_{3}$ for increasing soaking times. SAD data were collected for all crystals at $100 \mathrm{~K}$ using a Rigaku Micromax $007 \mathrm{HF}$ generator 
producing $\mathrm{Cu} K \alpha$ radiation. The best SAD data were obtained upon crystal soaking in a solution consisting of $8 \mathrm{mM} \mathrm{EuCl}_{3}, 0.05 \mathrm{M}$ ammonium sulfate, $0.05 \mathrm{M}$ bis-tris, $30 \%(\mathrm{v} / \mathrm{v})$ pentaerythritol ethoxylate $(15 / 4 \mathrm{EO} / \mathrm{OH})$ at $\mathrm{pH} 6.5$ for 3 hours. The data sets were scaled and merged using HKL2000 program package (18) (Table 1). (19), we could identify four Europium sites in the asymmetric unit of the protein. With this substructure, a correlation coefficient of $31.4 \%$ was calculated (CC-all, calculated with all data). The obtained phases were improved by phase extension and density modification, using RESOLVE (27) and ARP/wARP (21). Using this approach, about $80 \%$ of the residues present in the asymmetric unit could be automatically modeled (27). Manual model-building sessions (28) aimed at defining the complete Scl2.3-V structure are in progress.

\section{ACKNOWLEDGMENTS}

Authors thank the Ministero Italiano dell'Istruzione, dell'Università e della Ricerca (PRIN 2009 - prot. 200993WWF9, the COST Action BM1003 (COST-Grants-BM1003-00772) and the Mizutani Foundation for glycoscience for financial support (to R. B.). This work was supported in part by Public Service grant Al50666 from the National Institutes of Health (to S. L.).

\section{REFERENCES}

1. Rasmussen, M., Jacobsson, M., and Bjorck, L. (2003) Genome-based identification and analysis of collagen-related structural motifs in bacterial and viral proteins. J. Biol. Chem. 278, 32313-32316

2. Humtsoe, J. O., Kim, J. K., Xu, Y., Keene, D. R., Hook, M., Lukomski, S., and Wary, K. K. (2005) A streptococcal collagen-like protein interacts with the alpha2beta1 integrin and induces intracellular signaling. The Journal of biological chemistry 280, 1384813857

3. Rasmussen, M., and Bjorck, L. (2001) Unique regulation of SclB - a novel collagen-like surface protein of Streptococcus pyogenes. Mol Microbiol 40, 1427-1438

4. Han, R., Zwiefka, A., Caswell, C. C., Xu, Y., Keene, D. R., Lukomska, E., Zhao, Z., Hook, M., et al. (2006) Assessment of prokaryotic collagen-like sequences derived from streptococcal Scl1 and Scl2 proteins as a source of recombinant GXY polymers. Appl Microbiol Biotechnol 72, 109-115

5. Xu, Y., Keene, D. R., Bujnicki, J. M., Hook, M., and Lukomski, S. (2002) Streptococcal Scl1 and Scl2 proteins form collagen-like triple helices. The Journal of biological chemistry 277, 27312-27318

6. Lukomski, S., Nakashima, K., Abdi, I., Cipriano, V. J., Ireland, R. M., Reid, S. D., Adams, G. G., and Musser, J. M. (2000) Identification and characterization of the scl gene 
encoding a group A Streptococcus extracellular protein virulence factor with similarity to human collagen. Infection and immunity 68, 6542-6553

7. Mohs, A., Silva, T., Yoshida, T., Amin, R., Lukomski, S., Inouye, M., and Brodsky, B. (2007) Mechanism of stabilization of a bacterial collagen triple helix in the absence of hydroxyproline. The Journal of biological chemistry 282, 29757-29765

8. Esposito, C., Pethoukov, M. V., Svergun, D. I., Ruggiero, A., Pedone, C., Pedone, E., and Berisio, R. (2008) Evidence for an elongated dimeric structure of heparin-binding hemagglutinin from Mycobacterium tuberculosis. Journal of bacteriology 190, 4749-4753

9. Chagnot, C., Listrat, A., Astruc, T., and Desvaux, M. (2012) Bacterial adhesion to animal tissues: protein determinants for recognition of extracellular matrix components. Cellular microbiology 14, 1687-1696

10. Berisio, R., and Vitagliano, L. (2012) Polyproline and triple helix motifs in host-pathogen recognition. Curr Protein Pept Sci 13, 855-865

11. Caswell, C. C., Oliver-Kozup, H., Han, R., Lukomska, E., and Lukomski, S. (2010) Scl1, the multifunctional adhesin of group A Streptococcus, selectively binds cellular fibronectin and laminin, and mediates pathogen internalization by human cells. FEMS microbiology letters 303, 61-68

12. Caswell, C. C., Barczyk, M., Keene, D. R., Lukomska, E., Gullberg, D. E., and Lukomski, S. (2008) Identification of the first prokaryotic collagen sequence motif that mediates binding to human collagen receptors, integrins alpha2beta1 and alpha11beta1. The Journal of biological chemistry 283, 36168-36175

13. Caswell, C. C., Lukomska, E., Seo, N. S., Hook, M., and Lukomski, S. (2007) Scl1dependent internalization of group $A$ Streptococcus via direct interactions with the alpha2beta(1) integrin enhances pathogen survival and re-emergence. Molecular microbiology 64, 1319-1331

14. Gao, Y., Liang, C., Zhao, R., Lukomski, S., and Han, R. (2010) The Scl1 of M41-type group A Streptococcus binds the high-density lipoprotein. FEMS microbiology letters 309, 55-61

15. Caswell, C. C., Han, R., Hovis, K. M., Ciborowski, P., Keene, D. R., Marconi, R. T., and Lukomski, S. (2008) The Scl1 protein of M6-type group A Streptococcus binds the human complement regulatory protein, factor $\mathrm{H}$, and inhibits the alternative pathway of complement. Molecular microbiology 67, 584-596

16. Reuter, M., Caswell, C. C., Lukomski, S., and Zipfel, P. F. (2010) Binding of the human complement regulators CFHR1 and factor $\mathrm{H}$ by streptococcal collagen-like protein 1 (Scl1) via their conserved $\mathrm{C}$ termini allows control of the complement cascade at multiple levels. The Journal of biological chemistry 285, 38473-38485

17. Pahlman, L. I., Marx, P. F., Morgelin, M., Lukomski, S., Meijers, J. C., and Herwald, H. (2007) Thrombin-activatable fibrinolysis inhibitor binds to Streptococcus pyogenes by interacting with collagen-like proteins A and B. The Journal of biological chemistry 282 , 24873-24881

18. Otwinowski, Z., and Minor, W. (1997). Methods Enzymol. 276, 307-326

19. Sheldrick, G. M. (2008) A short history of SHELX. Acta Crystallogr A 64, 112-122

20. Terwilliger, T. (2004) SOLVE and RESOLVE: automated structure solution, density modification and model building. J Synchrotron Radiat 11, 49-52

21. Langer, G., Cohen, S. X., Lamzin, V. S., and Perrakis, A. (2008) Automated macromolecular model building for X-ray crystallography using ARP/wARP version 7. Nat Protoc 3, 1171-1179 
22. Beres, S. B., Sylva, G. L., Barbian, K. D., Lei, B., Hoff, J. S., Mammarella, N. D., Liu, M. Y., Smoot, J. C., et al. (2002) Genome sequence of a serotype M3 strain of group A Streptococcus: phage-encoded toxins, the high-virulence phenotype, and clone emergence. Proceedings of the National Academy of Sciences of the United States of America 99, 10078-10083

23. Matthews, B. W. (1968) Solvent content of protein crystals. J Mol Biol 33, 491-497

24. Ruggiero, A., Squeglia, F., Marasco, D., Marchetti, R., Molinaro, A., and Berisio, R. (2011) X-ray structural studies of the entire extracellular region of the serine/threonine kinase PrkC from Staphylococcus aureus. Biochem J 435, 33-41

25. Perez-Dorado, I., Gonzalez, A., Morales, M., Sanles, R., Striker, W., Vollmer, W., Mobashery, S., Garcia, J. L., et al. (2010) Insights into pneumococcal fratricide from the crystal structures of the modular killing factor LytC. Nature structural \& molecular biology $17,576-581$

26. Perez-Dorado, I., Sanles, R., Gonzalez, A., Garcia, P., Garcia, J. L., Martinez-Ripoll, M., and Hermoso, J. A. (2010) Crystallization of the pneumococcal autolysin LytC: in-house phasing using novel lanthanide complexes. Acta crystallographica. Section F, Structural biology and crystallization communications 66, 448-451

27. Terwilliger, T. C. (2003) SOLVE and RESOLVE: automated structure solution and density modification. Methods Enzymo/ 374, 22-37

28. Jones, T. A. (2004) Interactive electron-density map interpretation: from INTER to O. Acta Crystallogr D Biol Crystallogr 60, 2115-2125 


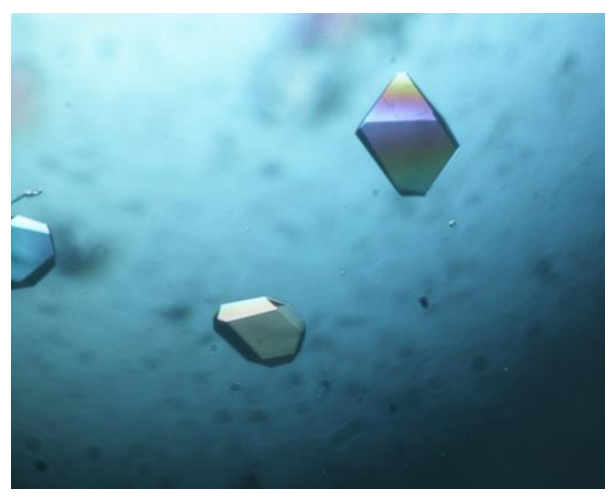

Figure 1

Image of typical rScl2.3-V crystals grown using $5 \mathrm{mg} \mathrm{mL}^{-1}$ protein solution and $0.05 \mathrm{M}$ ammonium sulfate, $0.05 \mathrm{M}$ bis-tris, $30 \%(\mathrm{v} / \mathrm{v})$ pentaerythritol ethoxylate $(15 / 4 \mathrm{EO} / \mathrm{OH}), \mathrm{pH} 6.5$. 


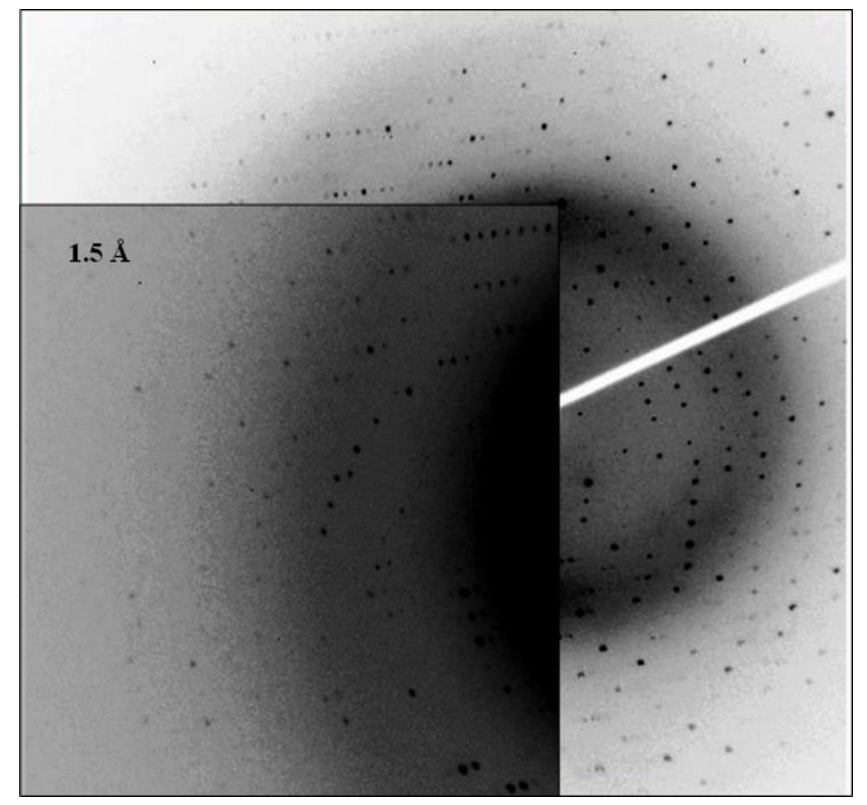

Figure 2

Diffraction pattern of a rScl2.3-V native crystal. Diffraction data are detectable to $1.5 \AA$ resolution. 
Table 1. Data collection statistics. Values in parentheses are for highest resolution shells

\begin{tabular}{|c|c|c|}
\hline & $\mathrm{EuCl}_{3}$ derivative & Native \\
\hline Space group & $H 32$ & H32 \\
\hline Unit cell parameters & $\begin{array}{l}a=b=44.26, c=228.01, y= \\
120\end{array}$ & $\begin{array}{l}a=b=44.23, c=227.83, \gamma= \\
120\end{array}$ \\
\hline Resolution $(\AA ̊)$ & 1.87 & 1.52 \\
\hline $\begin{array}{l}\text { Average } \\
\text { redundancy }\end{array}$ & $9.5(7.5)$ & $5.3(2.6)$ \\
\hline Unique reflections & 7545 & 13802 \\
\hline Completeness (\%) & $100(99.9)$ & $99.2(86.7)$ \\
\hline $\operatorname{Rmerge}^{\dagger}(\%)$ & $0.043(0.419)$ & $0.061(0.345)$ \\
\hline Average $I / \sigma(I)$ & $47.7(5.1)$ & $49.2(3.10)$ \\
\hline
\end{tabular}


CHAPTER 5

\title{
THE CRYSTAL STRUCTURE OF THE STREPTOCOCCAL COLLAGEN-LIKE PROTEIN 2 GLOBULAR DOMAIN FROM INVASIVE M3-TYPE GROUP A STREPTOCOCCUS SHOWS SIGNIFICANT SIMILARITY TO IMMUNOMODULATORY HIV PROTEIN GP41
}

Flavia Squeglia, Beth Bachert, Alfonso De Simone, Slawomir Lukomski and Rita Berisio Published in The Journal of Biological Chemistry 2014 289(8): 5122-5133

\begin{abstract}
The arsenal of virulence factors deployed by streptococci include streptococcal collagenlike (Scl) proteins. These proteins, which are characterized by a globular and a collagenlike domain, play key roles in host-adhesion, host immune defense evasion and biofilm formation. In this work, we demonstrate that the Scl2.3 protein is expressed on the surface of invasive M3-type strain MGAS315 of Streptococcus pyogenes. We report the crystal structure of Scl2.3 globular domain, the first of any Scl. This structure shows a novel fold among collagen trimerization domains, of either bacterial or human origin. Despite there being low sequence identity, we observe that Scl2.3 globular domain structurally resembles the gp41 subunit of the envelope glycoprotein from human immunodeficiency virus type 1 , an essential subunit for viral fusion to human $T$ cells. We combined crystallographic data with modeling and molecular dynamics techniques to gather information on the entire lollipop-like Scl2.3 structure. Molecular dynamics data evidence a high flexibility of Scl2.3, with remarkable inter-domain motions which are likely instrumental to the protein biological function in mediating adhesive or immunemodulatory functions in host-pathogen interactions. Altogether, our results provide molecular tools for the understanding of Scl-mediated streptococcal pathogenesis and important structural insights for the future design of small molecular inhibitors of streptococcal invasion.
\end{abstract}




\section{INTRODUCTION}

Streptococcus pyogenes or group A Streptococcus (GAS) is a human adapted pathogen, causing over 700 million cases worldwide annually (1). GAS infections produce a wide range of clinical outcomes, from superficial throat and skin infections to life-threatening invasive diseases such as streptococcal toxic shock syndrome (STSS) and necrotizing fasciitis $(2,3)$. The mortality resulting from the acquisition of invasive infections is high with 163000 deaths globally each year (4). It is known that M3-type GAS strains are associated with severe infections. In a survey of 108 isolates from the US, $50 \%$ of invasive diseases were caused by $M 1$ and $M 3$ type strains, and $M 3$ type strains contributed to the majority of STSS cases (5). Over the past decade, molecular pathogenomics has facilitated our understanding of the molecular basis for the more severe invasive diseases caused by M3-type strains (6-10). GAS produces cellassociated virulence factors that contribute to host colonization and immune evasion, and include the streptococcal collagen-like proteins Scl1 and Scl2, also known as SclA and SclB (11-15).

The Scl1 and Scl2 proteins share a similar structural organization, including an Nterminal variable globular domain $(\mathrm{V})$, a highly charged collagen-like triple-helix domain $(\mathrm{CL})$ consisting of (Gly-Xaa-Yaa)n triplet repeats and a C-terminal Gram-positive cell wall attachment domain (Figure 1A). Like collagen, an important structural protein in the extracellular matrix of animals, Scl1 and Scl2 form stable triple-helical structures (11,1619). The collagen triple helix is composed of three left-handed polyproline helices twisted into a right-handed supercoiled structure. In mammals, a strong contribution to triple helix stability is given by a high content of hydroxyproline (Hyp) residues at the $\mathrm{Y}$ position of the X-Y-Gly triplets, whereas bacteria lack the prolyl hydroxylase needed for posttranslational modification of proline residues $(17,20,21)$. To explore the basis of bacterial collagen triple-helix stability in the absence of Hyp, biophysical studies were carried out on recombinant Scl2 protein and a set of peptides modeling the Scl2 highly charged repetitive (Gly-X-Y)n sequences (17). These studies showed that bacteria have developed alternative strategies to stabilize the triple helix, involving electrostatic interactions, inter-chain hydrogen bonds, and a hydration-mediated hydrogen bonding network $(17,22)$. 
Similar to that observed for human collagen, the V-domain was hypothesized to be needed for proper folding of the triple helical regions, since their high symmetry constitutes an obstacle for optimal folding. However, the observation that the recombinant CL domain of Scl1 is expressed as a stable triple helix $(16,23)$ contrasts this hypothesis, at least in vivo. Scls have characteristic "lollipop-shaped" domain organization, which seems apt for ligand binding. Indeed, antibody mapping and electron microscopy imaging analyses confirmed that the stalk-forming $C L$ region projects the globular $\mathrm{V}$ region away from the bacterial surface (16), a feature which may facilitate interactions of $V$ regions with their potential targets. Several biologically-relevant V-region ligands have been identified using experimental approaches. Thus, different $\mathrm{Scl}$ variants bind human extracellular matrix proteins, cellular fibronectin and laminin (24), as well as plasma components including the low-density lipoprotein, thrombin-activatable fibrinolysis inhibitor, and complement regulatory proteins factor $\mathrm{H}$ and factor $\mathrm{H}$-related protein-1 $(18,25-28)$. In addition, the CL domain of Scl can bind directly to host cells through cellular receptors, integrins $\alpha_{2} \beta_{1}$ and $\alpha_{11} \beta_{1}$ (29-31). Hence, the two main Scl structural domains bind human ligands and are essential for GAS adhesion, host-cell entry, and immunomodulation of host defenses. Because of the importance of invasive M3-type strains in human morbidity and mortality, the presumed expression of Scl2.3 (Scl2 from M3 strain) was previously used as an epidemiological marker of $S$. pyogenes (7), although its actual expression has not been shown. Here, we demonstrate that Scl2.3 protein is expressed on the cell surface of an invasive M3-type group A Streptococcus. Since no structural clues on Scl2 are available, we have combined x-ray crystallography with molecular modeling and dynamics to obtain information on the structure of the entire molecule. This structure delivers the first atomic description of a Scl protein and opens the field for the understanding of structure-function relationship of key proteins that mediate essential adhesive and immunomodulatory functions of group A Streptococcus. 


\section{MATERIALS AND METHODS}

\section{Bacterial strains and growth}

The group A Streptococcus (GAS) M3-type strain MGAS315 (6) used here was isolated from an invasive case of a streptococcal toxic-like shock syndrome in Texas (5). GAS was routinely grown in Todd-Hewitt broth supplemented with $0.2 \%$ yeast extract (THY medium) or on tryptose agar with $5 \%$ sheep blood (BD Biosciences) at $37^{\circ} \mathrm{C}$ in $5 \% \mathrm{CO}_{2}-$ $20 \% \mathrm{O}_{2}$ atmosphere.

The Escherichia coli strain $\mathrm{DH} 5 \alpha$ was used in cloning experiments and E. coliBL21 was used for protein expression. E. coli strains were grown in Luria-Bertani media (BD Biosciences) supplemented with ampicillin $(100 \mu \mathrm{g} / \mathrm{ml})$.

\section{Protein methods}

Recombinant Scl2.3-V region polypeptide, designated $\mathrm{rScl} 2.3-\mathrm{V}$, was generated using the Strep-tag II expression and purification system (IBA-GmbH), as described previously (16). Briefly, the 5'-portion of the scl2.3 allele from strain MGAS315, encoding the aminoterminal variable $(\mathrm{V})$ region of the presumed mature Scl2.3 protein, was PCR-amplified using scl2-M3VF (5'-GAGATGGCCGATGGTGAAGATGCCCAAAAAAG) forward primer and scl2-M3VR (5'-CAGCGTCTCAGCGCTATCAAGGACATGATCTTGTATGCC) reverse primer, and subsequently cloned into an $E$. coli expression vector pASK-IBA2, generating plasmid pSL155.

The rScl2.3-V polypeptide is fused at the $\mathrm{N}$-terminus to the OmpA signal peptide mediating periplasmic expression of recombinant protein. The OmpA is selectively cleaved off during protein export by an endogenous signal peptidase, thus, releasing the rScl polypeptide; the $\mathrm{N}$-terminal sequence of purified $\mathrm{rScl} 2.3-\mathrm{V}$ was confirmed by Edman Degradation. The rScl2.3-V polypeptide also has a short affinity tag, the Strep-tag II (WSHPQFEK), at the C-terminus, which allowed for affinity chromatography purification on Strep-Tactin Sepharose. Purified rScl2.3-V protein was dialyzed against $25 \mathrm{mM}$ HEPES, pH 8.0 and stored $-20^{\circ} \mathrm{C}$. Recombinant protein $\mathrm{rScl} 2.3-\mathrm{V}$ was tested for purity and integrity on TGX 4-20\% gradient gel (Bio-Rad) and stained with RAPIDstain ${ }^{\mathrm{TM}}$ (GBiosciences). 
The presence of the cell-wall associated Scl2.3 protein was studied using the method described before $(11,15)$. MGAS315 was grown in THY medium until midlogarithmic phase $\left(\mathrm{OD}_{600} \sim 0.5\right)$ before GAS cells were harvested by centrifugation. The cell wall-associated protein fraction was obtained by resuspending the cell pellet in a highsucrose buffer ( $10 \mathrm{mM}$ Tris $\mathrm{pH} 8.0,20 \%$ sucrose), containing $25 \mathrm{U}$ of mutanolysin and 1 $\mathrm{mg} / \mathrm{mL}$ of lysozyme, and incubating at $37^{\circ} \mathrm{C}$ for 1 hour. rScl2.3-V protein and cell-wallassociated fraction of MGAS315 were analyzed by SDS-PAGE and Western immunoblotting, using rabbit polyclonal antibodies raised against rScl2.3-V (Proteintech Group, Inc.). Alkaline phosphatase-conjugated anti-rabbit IgG H\&L goat polyclonal antibodies (Rockland) were used as the secondary antibody, and detection was performed using 1-Step ${ }^{\mathrm{TM}}$ NBT/BCIP substrate (Thermo Scientific). PageRuler Plus Prestained Protein Ladder (Thermo Scientific) was used as a molecular weight marker.

\section{CD spectroscopy}

To analyze the conformational state of rScl2.3-V, far-UV CD spectra were registered at $20^{\circ} \mathrm{C}$. All CD spectra were recorded with a Jasco J-810 spectropolarimeter equipped with a Peltier temperature control system (Model PTC-423-S). Molar ellipticity per mean residue, $[\theta]$ in deg $\mathrm{cm}^{2} \bullet \mathrm{dmol}^{-1}$, was calculated from the equation: $[\theta]=[\theta]$ obs $\bullet \mathrm{mrw} \cdot(10 \bullet \bullet)^{-}$ ${ }^{1}$, where $\left.{ }^{\theta}\right]$ obs is the ellipticity measured in degrees, mrw is the mean residue molecular mass $(116.1 \mathrm{Da}), \mathrm{C}$ is the protein concentration in $\mathrm{g} \cdot \mathrm{L}^{-1}$ and $\mathrm{I}$ is the optical path length of the cell in $\mathrm{cm}$. Far-UV measurements $\left(190-260 \mathrm{~nm}\right.$ ) were carried out at $20^{\circ} \mathrm{C}$ using a 0.1 $\mathrm{cm}$ optical path length cell and a protein concentration of $0.2 \mathrm{mg} \cdot \mathrm{mL}^{-1}$. Thermal denaturation studies were conducted at $222 \mathrm{~nm}$ with increasing temperature from 20 to $70^{\circ} \mathrm{C}$. Proteins were equilibrated at each temperature point for $2 \mathrm{~min}$, and the temperature was increased with an average rate of $0.5^{\circ} \mathrm{C} / \mathrm{min}$. Tm was obtained by taking the peak of the first derivative of the melting curve.

\section{Multiple light scattering}

Purified rScl2.3-V was analysed by size-exclusion chromatography (SEC) coupled to a DAWN MALS instrument (Wyatt Technology) and an OptilabTM rEX (Wyatt Technology). $1 \mathrm{mg}$ of sample was loaded a S75 10/30 column, equilibrated in $25 \mathrm{mM}$ HEPES, $100 \mathrm{mM}$ 
$\mathrm{NaCl}, \mathrm{pH}$ 7.4. A constant flow rate of $0.5 \mathrm{ml} / \mathrm{min}$ was applied. The on-line measurement of the intensity of the Rayleigh scattering as a function of the angle as well as the differential refractive index of the eluting peak in SEC was used to determine the weight average molar mass $(\mathrm{Mw})$ of eluted protein, using the Astra 5.3.4.14 software (Wyatt Technologies).

\section{Crystallization, data collection and processing}

Crystallization trials were performed at $293 \mathrm{~K}$ using the hanging-drop vapor-diffusion method. Preliminary crystallization conditions were set up using a robot station for high throughput crystallization screening (Hamilton STARlet NanoJet 8+1) and commercially available sparse-matrix kits (Crystal Screen kits I and II, Hampton Research, Index). Optimization of the crystallization conditions was performed manually by tuning protein and precipitant concentrations. Best crystals were grown in $0.05 \mathrm{M}$ Ammonium sulfate, $0.05 \mathrm{M}$ Bis-Tris $\mathrm{pH} 6.5,30 \% \mathrm{v} / \mathrm{v}$ Pentaerythritol ethoxylate $(15 / 4 \mathrm{EO} / \mathrm{OH})$ (32). For structure solution, europium chloride derivative crystals were prepared by soaking a native crystal in a solution containing $8 \mathrm{mM} \mathrm{EuCl}_{3}, 0.05 \mathrm{M}$ ammonium sulphate, $0.05 \mathrm{M}$ Bis-Tris, $30 \%$ v/v Pentaerythritol ethoxylate $(15 / 4 \mathrm{EO} / \mathrm{OH})$ for 3 hours at $\mathrm{pH} 6.5$. A singlewavelength anomalous diffraction experiment (SAD) was recorded in-house at $100 \mathrm{~K}$ using a Rigaku Micromax $007 \mathrm{HF}$ generator producing $\mathrm{Cu} \mathrm{K}_{\alpha}$ radiation and equipped with a Saturn944 CCD detector. The data sets were scaled and merged using HKL2000 program package (33) (Table 1).

\section{Structure determination and refinement}

Phasing was achieved using in-house single anomalous dispersion data, using a previously adopted protocol (34). Using these data, both SHELXD (35) and SOLVE (36) identified five europium ions. Phases, improved by phase extension and density modification by RESOLVE (36) and WARP (37), allowed us to trace nearly the entire molecule structure. Crystallographic refinement was carried out against $95 \%$ of the measured data using the CCP4 program suite (38). The remaining $5 \%$ of the observed data, which was randomly selected, was used in Rfree calculations to monitor the 
progress of refinement. The structures was validated using the program PROCHECK (39) and deposited with the PDB (accession code 4nsm).

\section{Sequence and structure alignments}

Alignments of all available Scl2 sequences were performed using the ClustalW program. This sequence alignment was used to obtain phylogenetic relationships. Structure alignments were carried out using the DALI server.

\section{Modeling of the full Scl2.3}

Molecular modeling sessions were carried out to model the collagen like domain and obtain the entire Scl2.3 structure. The collagen-like domain was modeled using the structure of the collagen-like peptide (Pro-Pro-Gly) 10 as a template (40). The full sequence of Scl2.3 was adjusted on the domain structure using ad hoc made routines. The full model was energy minimized using the GROMACS package.

\section{Molecular dynamics simulations}

Molecular dynamics (MD) simulations were performed using the region 7-123 of the Scl2.3 as a starting model, including the crystallographic V-domain (residues 7-77) and part of the modelled region (residues 78-123). MD simulations were carried out with the GROMACS package by using the all-atom AMBER99sb ILDN force field (41) in combination with the TIP4P-ew explicit water model (42). To avoid any bias on the hydration status of the protein derived from the MD analyses, crystallographic water molecules were removed from the starting model. The simulations were carried out in the NPT ensemble with periodic boundary conditions at a constant temperature of $300 \mathrm{~K}$ by using a weak coupling with external bath (V-rescale method) (43) and a constant pressure of $1 \mathrm{~atm}$ (berendesen pressure coupling)(44). A rectangular box was used to accommodate the protein, water molecules, and ions. The system included 28827 water molecules and a total of 120438 atoms.

Bending angles between $\mathrm{CL}$ and Scl2.3-V, or between regions of the collagen triple helix were defined between centre of masses of three group of atoms. For the definition of the global inter-domain angle, these atoms are the $\mathrm{C} \alpha$ atoms of residues 57 and 60 of 
each chain (from Scl2.3-V), residues 75-77 (hinge region) and residues 82-84 (from CL). For the bending angle between three zones of the $\mathrm{CL}$ domain, we selected the $\mathrm{C} \alpha$ atoms of residues 77-78 of each chain (bottom region), residues 94 and 95 (hinge region) and residues 112 and 113 (top region).

\section{RESULTS}

\section{Expression of Scl2.3 protein by M3-type GAS}

Expression of the Scl2 proteins is regulated at the level of translation and depends on a number of pentanucleotide repeats CAAAA found downstream to a GTG start codon $(12,14,15)$. Based on the number of these repeats, the $s c / 2$-coding sequence may be inframe, resulting in expression of the full-length protein, or out-of-frame, leading to early translation termination. We assessed the cell-surface expression of Scl2.3 protein by MGAS315, a strain representative of global invasive M3 organisms.

To generate tools for the detection of Scl2.3 protein, we cloned, expressed, and purified the recombinant Scl2.3- $\mathrm{V}$ protein ( $\mathrm{rScl} 2.3-\mathrm{V})$, corresponding to the $\mathrm{V}$ region of Scl2.3 from MGAS315. SDS-PAGE analysis of purified rScl2.3-V shows a single protein band of the expected size of about $10.1 \mathrm{kDa}$ (Figure 1B), as further confirmed by sequencing. Rabbits were immunized with $\mathrm{rSc} 22.3-\mathrm{V}$ to generate specific anti-Scl2.3 antibodies, which we used to test the presence of the Scl2.3 in the cell wall-associated protein fraction of MGAS315 by western immunoblotting (Figure 1C). In addition to positive control ( $\mathrm{rScl} 2.3-\mathrm{V}$ lane), we detected a prominent immunoreactive band of approximately $65 \mathrm{kDa}$ in the cell-wall fraction (Scl2.3 lane) using post-immune rabbit serum, while probing with control pre-immune serum was negative for the $\mathrm{rScl} 2.3$ and Scl2.3 bands. Based on sequence analysis, the predicted molecular mass of the mature Scl2.3 protein is $\sim 52.5 \mathrm{kDa}$. However, an aberrant migration of $\mathrm{Scl}$ proteins has been well documented $(11,13)$. Altogether, our data show that the Scl2.3 protein is expressed on cell surface of invasive M3-type strain MGAS315 of S. pyogenes.

\section{Structural studies in solution}

Structural features of rScl2.3-V in solution were checked using circular dichroism (CD) and light scattering studies. As previously shown (22), Scl2-V has a typical $\alpha$-helical CD 
spectrum (Figure 2A). Thermal stability curves, determined by monitoring the $C D$ signal at $222 \mathrm{~nm}$, evidences a cooperative unfolding with melting transition at $T_{m}=50^{\circ} \mathrm{C}$. Consistent with previous data, denaturation of $r S c l 2.3-\mathrm{V}$ is fully reversible (Figure 2B). Analytical size-exclusion chromatography (SEC), coupled with multiangle light scattering (MALS) was carried out to investigate the oligomerization state of $\mathrm{rScl} 2.3-\mathrm{V}$ in solution. The on-line measurement of the intensity of the Rayleigh scattering as a function of the angle as well as the differential refractive index of the eluting peak in SEC was used to determine Mw. This analysis produced an Mw value of $26600 \pm 107 \mathrm{Da}$, which corresponds to a trimeric organization of the molecule (Figure $2 \mathrm{C}$ ).

\section{Overall structure of rScI2.3-V}

rScl2.3-V was crystallized in the space group H32. The structure was solved by singlewavelength anomalous dispersion (SAD) analysis of europium-derivatized crystal and refined to a resolution of $1.6 \AA$ (Table 1). Analysis of crystal packing using the software PISA confirms that the biologic unit of $r S c l 2.3-\mathrm{V}$ is a trimer. Consistently, a large surface area is buried ( $32 \%$ of the total surface, $5470 \AA^{2}$ ) upon trimer formation, with a strong gain of free energy of solvation $\left(\Delta^{\mathrm{i}} \mathrm{G}=-42.1 \mathrm{kcal} / \mathrm{mol}\right)$. $\mathrm{rScl} 2.3-\mathrm{V}$ molecules are organized about three-fold crystallographic axes to form a six helical bundle structure (Figure 3A). The inner core of this bundle consists of a parallel, trimeric structure in which helices are wrapped in a gradual left-handed super-helix. Three further helices wrap antiparallel to the internal helices in a left-handed direction around the exterior of the central trimer. The six-helix bundle forms an elongated cylinder measuring about $30 \AA$ in diameter and $60 \AA$ in height. Interestingly, external helices are shifted with respect to internal ones, as a 12residue-long loop, embedding residues from Lys31 to Asp42, connects internal and external helices in each monomer. This region, which contains Pro34 and Pro36, adopts a well-defined polyproline II conformation (Figure 3B).

The V-domain of Scl2 was proposed to be stabilized by coiled coil interactions (23), although prediction servers do not provide a clear answer. We searched rScl2.3-V crystal structure for the typical structural features of coiled coils, named knobs-into-holes, using the software SOCKET (45). In typical coiled coils, hydrophobic side chains at 'a' and 'd' positions on one helix act as knobs and dock into holes formed by diamonds of four 
residues on the partnering helix. This analysis shows that $\mathrm{rScl} 2.3-\mathrm{V}$ does not contain coiled coils.

Interactions between inner helices of the rScl2.3-V six-helix bundle involve different types of contacts along the bundle. Hydrophobic interactions exist at the two poles of the molecule, whereas an intricate pattern of salt bridges is formed in the central part (Figure 4). In this pattern, Arg56 bridges Glu60 of two adjacent protomers and interacts with Asp61 of an adjacent protomer. Further salt bridges exist between the central Glu60 and Arg64 and between Asp61 and more peripheral Lys57 (Figure 4). As a result, as many as 16 salt bridges stabilize the central region of the bundle.

Three outer N-terminal helices (residues 7-38) pack obliquely against the outside of the inner trimer in an antiparallel orientation. As such, they interact through hydrophobic interactions and salt bridges with residues in three grooves on the surface of the central helical trimer, whereas interactions mediated by the PPII strand are mostly hydrophobic (Figure $5 \mathrm{~A}$ ). The analysis of the electrostatic potential surface reveals an uneven distribution of charged patches, with a concentration of negative charges in the region opposite to the origin of the collagen triple helix (Figure 5C, D). The negatively charged patch generated by Asp42 and Asp43 of each chain surrounds a solvent exposed hydrophobic region, generated by Leu41 and Met46. Of these residues, the position of Met46 is occupied by hydrophobic residues in all members of a subgroup of Scl2 sequences, identified by phylogenetic analysis (branch $C$ in Figure 6 ). In the same subgroup, negatively charged residues are often occurring in a region embedding Asp42 and Asp43. Different features characterize the other two subgroups, but all sequences present both charged and hydrophobic residues in loop regions (either experimentally determined or predicted), indicating that these features may be functionally important.

\section{Sequence alignments}

Several sequences of both Scl2 and Scl1, deriving from different S. pyogenes strains, have been identified. Multiple sequence alignment shows that a hallmark of all Scl2 sequences is the occurrence of hydrophobic residues at regular positions, most of which are conserved in all analyzed sequences. An analysis of $\mathrm{rScl} 2.3-\mathrm{V}$ structure shows that these residues constitute the inner core of the 6-helix bundle fold (Figure 4). This finding 
suggests that all Scl2 proteins share the same 6-helix bundle fold we observe in rScl2.3$V$ structure. The same considerations apply to Scl1 sequences, since most conserved hydrophobic residues are also conserved in Scl1 (data not shown). Phylogenetic analysis shows that Scl2 sequences can be subdivided into three main branching groups ( $A, B$ and $C$ in Figure 6). In each branch, specific characteristics are conserved. For example, a striking difference between branches A-B and branch C, which contains Scl2.3, is the presence of a fully conserved Pro residue in A-B in a position corresponding to Scl2.3 Ser26, which belongs to the $\alpha$-helix $\alpha 2$ in Scl2.3 structure. Another almost conserved Pro residue characterizes branch $\mathrm{B}$, in place of Scl2.3 Ser48, which is embedded in $\alpha$-helix a2. These considerations suggest that the structures of proteins in each branch differ in the boundaries of $\alpha$-helices constituting their six-helix bundle fold. Compared to Scl2.3, secondary structure predictions suggest that branch $A$ and $B$ are characterized by shorter $\alpha 1$ and $\alpha 2$ helices, connected by a longer loop in branch $A$, and by a loop-helix-loop motif in branch $B$ (Figure 6).

\section{Scl2.3-V structurally resembles gp41}

A search for similar folds in structural data bases revealed a strong structural relationship between $\mathrm{rScl} 2.3-\mathrm{V}$ and subunit gp41 of the envelope glycoprotein from human immunodeficiency virus 1 (HIV-1) (PDB code 3040, Z=12.1, r.m.s.d. $2.8 \AA$ ), with sequence identity between the two proteins of $9 \%$, after alignment of 165 residues. In addition, the 3-carboxy-cis,cis-muconate lactonizing enzyme from Agrobacterium radiobacter (PDB code 2 fen, $Z=10.0$, r.m.s.d. $3.3 \AA$ ) and the adenylosuccinate lyase from E. coli (PDB code $3 g z h, Z=10.0$, r.m.s.d. $3.4 \AA$ ) also show structural relationship with Scl2.3-V fold, with sequence identities in the range 9-10\%. In the case of the two bacterial proteins identified by DALI (PDB codes 2fen and 3gzh), only five of the six helices of rScl2.3-V six-helix bundle are conserved. On the contrary, the superposition of rScl2.3$\mathrm{V}$ structure on that of gp41 evidences a striking similarity in the helical arrangement of the two six-helix bundles (Figure 7). However, the three inner $\alpha$-helices of gp41 are packed together in the 'knobs-into-hole' arrangement typical of coiled coils whereas coiled coil interactions were not found in rScl2.3-V structure. This feature is likely responsible for a more compact arrangement of inner helices in gp41, compared to 
rScl2.3-V (Figure 7). Also, whereas gp41 fold is a highly regular six-helix bundle, rScl2.3$\mathrm{V}$ presents a polyproline region at the $\mathrm{N}$-terminal side of external helices. Although the position of these proline residues is not conserved among Scl2 sequences (Figure 6), their presence in the region connecting the two main helices forming protomers of the six-helix bundle is a distinctive feature of all Scl2 sequences.

\section{Modeling of triple helical regions and MD simulations}

Good quality electron density maps allowed us to define the conformation of $r S c l 2.3-V$ C-terminal ends, up to Leu76. In Scl2, this is the site of attachment of the collagen-like triple helix. Notably, whereas all three Leu76 residues from rScl2.3-V are in a plane, the triplets of the collagen triple helix are typically staggered by one residue. This poses a question whether the asymmetry of the triple helix is accommodated by the $\mathrm{rScl} 2.3-\mathrm{V}$ structure or if a kink of the two domains is necessary, as previously observed for the engineered foldon-collagen (46). To tackle this question, we modeled the triple helical part of Scl2.3, thus producing the first structural description of a Scl (Figure 8A), and carried out Molecular Dynamics (MD) simulations. To assess the evolution of the structure in the simulation timescale (100 ns), a number of stereo-chemical parameters (gyration radius, secondary structure and RMSD) were monitored along the trajectories. The evaluation of root-mean-square deviations (RMSD) (calculated on the Ca atoms) from the starting structure evidences that large motions characterize the simulated system (Figure 8B). The RMSD values are smaller when they are separately computed for Scl2.3-V and Scl2.3-CL regions. Of these, RMSD values for the Scl2.3-V region are on average smaller than those of the Scl2.3-CL region (Figure 8B). Likely, the difference in the RMSD behavior for the two regions is in its structural characteristics. Indeed, local fluctuations on an elongated structure (i.e. Scl2.3-CL) propagate into larger effects on the RMSD than in globular structures (i.e. Scl2.3-V). The RMSD data are consistent with the presence of a principal motion involving a global inter-domain bending motion between Scl2.3-CL and Scl2.3-V (Figure 8C). Analysis of the MD trajectory structures evidences a continuous evolution from linear conformations, in which the Scl2.3-V and Scl2.3-CL domains are coaxial, to a more 'bent' state. In particular, the bending angle 
between the axis of the Scl2.3-V and Scl2.3-CL domains (See Methods for definition) ranges from $143^{\circ}$ to $180^{\circ}$ in an elastic fashion (Figure $8 C$ ).

A direct indicator of the stability of the triple helix motif is the number of conserved main-chain hydrogen $(\mathrm{H})$-bonds along the trajectory (Figure 9A). These intermolecular $\mathrm{H}$ bonds are distinctive of the triple helix motif and are established between the amide group of the Gly residues and carbonyl groups from complementary peptides that form the triple helix. The analysis confirms that the force field and simulation setup used were able to maintain the initial $\mathrm{H}$-bonding pattern of the structure: on average, $87.5 \%$ of the native main-chain $\mathrm{H}$-bonds were maintained. In addition to the observed inter-domain rearrangements along the trajectory, MD data also evidence a high flexibility of the Scl2.3$\mathrm{CL}$ domain, with a bending angle around the center of the Scl2.3-CL region ranging between 152 and $180^{\circ}$ (Figure 8D). The increased flexibility of amino-acid-rich triple helices, compared to imino-acid rich ones, is in line with previous MD analyses of other collagen-like polypeptides (47-49).

\section{DISCUSSION}

The arsenal of virulence factors deployed by streptococci include streptococcal collagenlike (Scl) proteins, which arm the cell wall of the bacterium and establish multiple functions, like host-adhesion (29-31), evasion of host immune defenses $(27,28)$, or biofilm formation $(50,51)$. There are nearly 300 collagen-like proteins annotated to streptococci (52), including several pathogenic organisms like $S$. pyogenes (11-15), S. pneumoniae (53), and $S$. equi $(54,55)$. In addition to a signature collagen-like domain, Scl proteins contain a globular domain (V domain) and both Gram-positive signal peptide (YSIRK) and cell-wall anchor (LPXTG) domains, predicting that they are all cell-surface proteins. Despite their established importance in bacterial pathogenesis, no three-dimensional structural information is available so far for any of the Scl proteins. In this work, we formally demonstrate that the Scl2 protein is expressed by invasive M3-type strain MGAS315 and is found on the bacterial cell surface (Figure 1). By combining x-ray crystallography with computational techniques, we provide a structural description of the entire Scl2.3 molecule. 
Scl2 is known to be regulated at the level of translation by the varying number of CAAAA pentanucleotide repeats directly downstream of the start codon, which may result in frameshift of the scl2-gene reading frame and early translation termination $(12,14,15)$. Analysis of $s c / 2$ within 50 GAS strains representing 21 different M types showed that the $s c / 2$ allele is present in virtually all strains tested, though the number of the repeats, as well as resulting Scl2 expression, varied among strains. For example, none of the M1type strains, whereas about half of the M28- and M12-type strains were predicted to express the full-length Scl2 variants. Interestingly, all of the M3-type strains initially tested (15) and $84 \%$ of $255 \mathrm{M} 3$ global isolates (7) were found to contain in-frame scl2.3 alleles. This suggests that there may be a selective advantage in M3 strains to express Scl2, and it may have an important role in the pathogenesis of M3-type GAS.

Previous binding studies have delineated roles for Scl1 and Scl2 in both host colonization and immune evasion. Thus, some Scl1 variants may aid host colonization by binding to cellular fibronectin and laminin, which are major components of human extracellular matrix, and integrins $\alpha_{2} \beta_{1}$ and $\alpha_{11} \beta_{1}$, which are present on the host cell surface $(24,31)$. Scl1 has also been shown to bind the plasma lipoproteins and complement regulators of the immune system $(18,27,28)$. Furthermore, both Scl1 and Scl2 proteins have been shown to bind thrombin-activatable fibrinolysis inhibitor, interfering with the normal fibrinolytic breakdown of blood clots (26), which may resemble a role of staphylococcal coagulases that produce clots as protective barrier against the immune response (56). These observations suggest Scl2 is involved in evasion or modulation of the immune response, rather than in host colonization. Though the role of Scl2 during infection is currently unclear, the structural data gained from this study provides very interesting clues into its possible function. The crystal structure of the $\mathrm{V}$ domain of Scl2.3 (rScl2.3-V) unveils a compact trimeric six-helix bundle fold. Consistently, light scattering experiments evidence that $\mathrm{rScl}$ 2.3-V exhibits a trimeric arrangement also in solution (Figure 2). Trimeric six-helix bundle folds are, to date, not observed in bacteria, but typically characterize several glycoproteins involved in viral fusion, including the gp41 subunit of the envelope glycoprotein of human immunodeficiency virus type 1 (HIV-1), the glycoprotein B of Herpes Simplex virus (57) and the GP2 domain of the envelope glycoprotein GP from the Marburg and Ebola viruses (58-61). However, different than 
gp41 and from previously reported data on Scl2 (23), the structure of rScl2.3-V does not contain coiled coils, but it is stabilized by both hydrophobic and ion pair interactions (Figure 4). Alignment of proteins with known structure shows that, even with low sequence identity (9\%), rScl2.3-V structurally resembles the gp41 subunit of HIV-1, a subunit responsible for membrane fusion of the HIV virus $(62,63)$. Since gp41 functions as a viral entry protein into CD4+ $T$ lymphocytes, this might suggest a novel potential role for Scl2 in interacting with T cells and causing hyper-activation of the immune response, which is a hallmark of the STSS infections that are often associated with M3-type strains.

We modeled the triple helical region of Scl2 and performed MD analysis with the aim of investigating structural and dynamic features of Scl2.3. Scl2.3-V is located at the tip of an extremely elongated triple helical structure (about $1030 \AA$, Figure $8 \mathrm{~A}$ ) and exposes highly hydrophobic residues, like Leu41 and Met46 (Figure 5), a feature which may play a role in Scl2.3-mediated interaction of $S$. pyogenes with the hydrophobic milieu in the host. MD data evidenced an extremely flexible nature of Scl2, with a dynamic kink of the inter-domain organization (Figure 8). A kinked structure was previously observed for the an engineered foldon-collagen (46) and reflects the need of the structure to fit the three-fold symmetry of the $V$ domain (which brings the site of attachment of the three collagen-like chains in a plane) with the one-residue stagger of the collagen-like chains. Our data show that the Scl2.3 structure can undertake both kinked and linear conformations, in a rapid equilibrium between them (Figure 8 ). The high structural flexibility we observed in Scl2.3 is likely instrumental to its biological function in mediating adhesive or immune-modulatory functions in host-pathogen interactions.

The $\mathrm{V}$ domain of Scls has been proposed as a trimerization domain which helps collagen folding. Indeed, different than for globular proteins, misfolding of the collagen triple helix is a likely event because of its repeating structure, whose stability is relatively insensitive to lateral shifts by one or more Gly-X-Y repeats. Consistently, trimerization domains have been found in many different proteins containing collagen triple helices. However, it has been shown that the $\mathrm{V}$ domain of Scls is not needed in vivo, since the $\mathrm{CL}$ region of Scl1 can be expressed in a folded triple helical state $(16,23)$. This observation highlights a different folding mechanism of Scl proteins, compared to human collagen, for which trimerization domains are crucial to the correct triple helical 
arrangement (64). Likely, V-domains of Scls display dichotomous functions by acting as triple helix stabilization domains, since they exhibit higher folding temperature than the $C L$ regions (23), and by mediating host-pathogen interaction (29-31).

To date, there are five known atomic structures of trimerization domains of collagen, the NC1 domain of collagen IV (65), the homologous NC1 domain of collagens VIII and X $(66,67)$ and the trimerization domains of collagen XV and XVIII $(68,69)$. A trimerization domain was also characterized for $B c / A$, the major component of the exosporium of the $B$. anthracis spore (70). All of them have a high content of $\beta$-structure but share no structural homology. The structure of rScl2.3-V presents novel features as it is mainly composed of $\alpha$-helices. Multiple sequence alignment suggests that the 6 -helix bundle fold exhibited by Scl2.3 is conserved in all Scl2 sequences, albeit with different $\alpha$-helix boundaries and length of the loop connecting alpha helices $\alpha 1$ and $\alpha 2$ (Figure 6).

Phylogenetic analyses of variation in Scl2 $\mathrm{V}$ region among different $\mathrm{M}$ types revealed several interesting observations. Scl2 sequences from different $M$ types formed three separate clades, referred to as A, B, and C (Figure 6). The invasive M type 3, found in branch $\mathrm{C}$, clustered with $\mathrm{M}$ types 1 and 28 , which are also associated with invasive infections, including streptococcal toxic shock syndrome and necrotizing fasciitis $(5,71,72)$. Our analysis additionally evidenced that cluster $C$ contains $M$ types associated with rheumatic fever, including types $1,3,6$, and $18(71,73)$, thus suggesting a possible role of Scl2.3 in this disease. Altogether, our work delivers the first atomic description of a Scl protein. This structural information, which can be extended to other members of the Scl family, is precious to the understanding the structural basis of Scl-mediated streptococcal infection. 


\section{ACKNOWLEDGMENTS}

Authors thank the Ministero Italiano dell'Istruzione, dell'Università e della Ricerca (PRIN 2009 - prot. 200993WWF9, the COST Action BM1003 (COST-Grants-BM1003-00772) and the Mizutani Foundation for glycoscience for financial support (to R. B.). This work was supported in part by Public Service grant Al50666 from the National Institutes of Health (to S. L.).

\section{REFERENCES}

1. Bisno, A. L., Rubin, F. A., Cleary, P. P., and Dale, J. B. (2005) Prospects for a Group A Streptococcal Vaccine: Rationale, Feasibility, and Obstacles--Report of a National Institute of Allergy and Infectious Diseases Workshop. Clin Infect Dis 41, 1150-1156

2. Kaul, R., McGeer, A., Low, D. E., Green, K., Schwartz, B., Study, O. G. A. S., and Simor, A. E. (1997) Population-based surveillance for group A streptococcal necrotizing fasciitis: clinical features, prognostic indicators, and microbiologic analysis of seventy-seven cases. Am. J. Med. 103, 18-24

3. Sharkawy, A., Low, DonaldÂ E., Saginur, R., Gregson, D., Schwartz, B., Jessamine, P., Green, K., and McGeer, A. (2002) Severe group A streptococcal soft tissue infections in Ontario: 1992-1996. Clin. Infect. Dis. 34, 454-460

4. Carapetis, J., Steer, A., Mulholland, E., and Weber, M. (2005) The global burden of group A streptococcal diseases. Lancet Infect Dis 5, 685-694

5. Musser, J. M., Hauser, A. R., Kim, M. H., Schlievert, P. M., Nelson, K., and Selander, R. K. (1991) Streptococcus pyogenes causing toxic-shock-like syndrome and other invasive diseases: clonal diversity and pyrogenic exotoxin expression. Proc. Natt. Acad. Sci. USA 88, 2668-2672

6. Beres, S. B., Sylva, G. L., Barbian, K. D., Lei, B., Hoff, J. S., Mammarella, N. D., Liu, M.-Y., Smoot, J. C., et al. (2002) Genome sequence of a serotype M3 strain of group A Streptococcus: Phage-encoded toxins, the high-virulence phenotype, and clone emergence. Proc. Natl. Acad. Sci. USA 99, 10078-10083

7. Beres, S. B., Sylva, G. L., Sturdevant, D. E., Granville, C. N., Liu, M., Ricklefs, S. M., Whitney, A. R., Parkins, L. D., et al. (2004) Genome-wide molecular dissection of serotype M3 group A Streptococcus strains causing two epidemics of invasive infections. Proc. Natl. Acad. Sci. USA 101, 11833-11838

8. Beres, S. B., Carroll, R. K., Shea, P. R., Sitkiewicz, I., Martinez-Gutierrez, J. C., Low, D. E., McGeer, A., Willey, B. M., et al. (2010) Molecular complexity of successive bacterial epidemics deconvoluted by comparative pathogenomics. 10.1073/pnas.0911295107 Proceedings of the National Academy of Sciences 107 4371-4376

9. Olsen, R. J., and Musser, J. M. (2010) Molecular pathogenesis of necrotizing fasciitis. Annual Review of Pathology: Mechanisms of Disease 5, 1-31

10. Nakagawa, I., Kurokawa, K., Yamashita, A., Nakata, M., Tomiyasu, Y., Okahashi, N., Kawabata, S., Yamazaki, K., et al. (2003) Genome Sequence of an M3 Strain of Streptococcus pyogenes Reveals a Large-Scale Genomic Rearrangement in 
Invasive Strains and New Insights into Phage Evolution. Genome Res. 13, 10421055

11. Lukomski, S., Nakashima, K., Abdi, I., Cipriano, V. J., Ireland, R. M., Reid, S. D., Adams, G. G., and Musser, J. M. (2000) Identification and characterization of the scl gene encoding a group A Streptococcus extracellular protein virulence factor with similarity to human collagen. Infect. Immun. 68, 6542-6553

12. Rasmussen, M., and Bjorck, L. (2001) Unique regulation of SclB-a novel collagenlike surface protein of Streptococcus pyogenes. Infect. Immun. 40, 1427-1438

13. Rasmussen, M., Eden, A., and Bjorck, L. (2000) SclA, a novel collagen-like surface protein of Streptococcus pyogenes. Infect. Immun. 68, 6370-6377

14. Whatmore, A. M. (2001) Streptococcus pyogenes $s c / B$ encodes a putative hypervariable surface protein with a collagen-like repetitive structure. Microbiology 147, 419-429

15. Lukomski, S., Nakashima, K., Abdi, I., Cipriano, V. J., Shelvin, B. J., Graviss, E. A., and Musser, J. M. (2001) Identification and characterization of a second extracellular collagen-like protein made by group A Streptococcus: control of production at the level of translation. Infect. Immun. 69, 1729-1738

16. Xu, Y., Keene, D. R., Bujnicki, J. M., Hook, M., and Lukomski, S. (2002) Streptococcal Scl1 and Scl2 proteins form collagen-like triple helices. J. Biol. Chem. 277, 27312-27318

17. Mohs, A., Silva, T., Yoshida, T., Amin, R., Lukomski, S., Inouye, M., and Brodsky, B. (2007) Mechanism of stabilization of a bacterial collagen triple helix in the absence of hydroxyproline. The Journal of biological chemistry 282, 29757-29765

18. Han, R., Caswell, C. C., Lukomska, E., Keene, D. R., Pawlowski, M., Bujnicki, J. M., Kim, J. K., and Lukomski, S. (2006) Binding of the low-density lipoprotein by streptococcal collagen-like protein Scl1 of Streptococcus pyogenes. Molecular microbiology 61, 351-367

19. Berisio, R., and Vitagliano, L. (2012) Polyproline and triple helix motifs in hostpathogen recognition. Curr Protein Pept Sci 13, 855-865

20. Berisio, R., De Simone, A., Ruggiero, A., Improta, R., and Vitagliano, L. (2009) Role of side chains in collagen triple helix stabilization and partner recognition. Journal of peptide science : an official publication of the European Peptide Society $15,131-140$

21. Improta, R., Berisio, R., and Vitagliano, L. (2008) Contribution of dipole-dipole interactions to the stability of the collagen triple helix. Protein science : a publication of the Protein Society 17, 955-961

22. Yu, Z., Brodsky, B., and Inouye, M. (2011) Dissecting a bacterial collagen domain from Streptococcus pyogenes: sequence and length-dependent variations in triple helix stability and folding. The Journal of biological chemistry 286, 18960-18968

23. Yu, Z., Mirochnitchenko, O., Xu, C., Yoshizumi, A., Brodsky, B., and Inouye, M. (2010) Noncollagenous region of the streptococcal collagen-like protein is a trimerization domain that supports refolding of adjacent homologous and heterologous collagenous domains. Protein science : a publication of the Protein Society 19, 775-785

24. Caswell, C. C., Oliver-Kozup, H., Han, R., Lukomska, E., and Lukomski, S. (2010) Scl1, the multifunctional adhesin of group A Streptococcus, selectively binds 
cellular fibronectin and laminin, and mediates pathogen internalization by human cells. FEMS Microbiol Lett 303, 61-68

25. Gao, Y., Liang, C., Zhao, R., Lukomski, S., and Han, R. (2010) The Scl1 of M41type group A Streptococcus binds the high-density lipoprotein. FEMS Microbiol. Let. 309, 55-61

26. Pahlman, L. I., Marx, P. F., Morgelin, M., Lukomski, S., Meijers, J. C. M., and Herwald, H. (2007) Thrombin-activatable fibrinolysis inhibitor binds to Streptococcus pyogenes by interacting with collagen-like proteins A and B. J. Biol. Chem. 282, 24873-24881

27. Caswell, C., Han, R., Hovis, K., Ciborowski, P., Keene, D., Marconi, R., and Lukomski, S. (2008) The Scl1 protein of M6-type group A Streptococcus binds the human complement regulatory protein, factor $\mathrm{H}$, and inhibits the alternative pathway of complement. Mol. Microbiol. 67, 584-596

28. Reuter, M., Caswell, C. C., Lukomski, S., and Zipfel, P. F. (2010) Binding of the human complement regulators CFHR1 and factor $\mathrm{H}$ by streptococcal collagen-like protein 1 (Scl1) via their conserved $\mathrm{C}$ termini allows control of the complement cascade at multiple levels. J. Biol. Chem. 285, 38473-38485

29. Humtsoe, J. O., Kim, J. K., Xu, Y., Keene, D. R., Hook, M., Lukomski, S., and Wary, K. K. (2005) A streptococcal collagen-like protein interacts with the a2b1 integrin and induces intracellular signaling. J. Biol. Chem. 280, 13848-13857

30. Caswell, C. C., Lukomska, E., Seo, N. S., Hook, M., and Lukomski, S. (2007) Scl1dependent internalization of group A Streptococcus via direct interactions with the alpha2beta(1) integrin enhances pathogen survival and re-emergence. $\mathrm{Mol}$ Microbio/ 64, 1319-1331

31. Caswell, C. C., Barczyk, M., Keene, D. R., Lukomska, E., Gullberg, D. E., and Lukomski, S. (2008) Identification of the first prokaryotic collagen sequence motif that mediates binding to human collagen receptors, integrins a2b1 and a11b1. $J$ Biol Chem 283, 36168-36175

32. Squeglia, F., Bachert, B., Romano, M., Lukomski, S., and Berisio, R. (2013) Crystallization and preliminary X-ray crystallographic analysis of the variable domain of Scl2.3, a streptococcal collagen-like protein from invasive M3-type Streptococcus pyogenes. Acta crystallographica. Section F, Structural biology and crystallization communications $69,1023-1025$

33. Otwinowski, Z., and Minor, W. (1997). Methods Enzymol. 276, 307-326

34. Ruggiero, A., Squeglia, F., Marasco, D., Marchetti, R., Molinaro, A., and Berisio, R. (2011) X-ray structural studies of the entire extracellular region of the serine/threonine kinase PrkC from Staphylococcus aureus. Biochem J 435, 33-41

35. Sheldrick, G. M. (2008) A short history of SHELX. Acta Crystallogr A 64, 112-122

36. Terwilliger, T. (2004) SOLVE and RESOLVE: automated structure solution, density modification and model building. J Synchrotron Radiat 11, 49-52

37. Langer, G., Cohen, S. X., Lamzin, V. S., and Perrakis, A. (2008) Automated macromolecular model building for $X$-ray crystallography using ARP/wARP version 7. Nat Protoc 3, 1171-1179

38. Potterton, E., Briggs, P., Turkenburg, M., and Dodson, E. (2003) A graphical user interface to the CCP4 program suite. Acta Crystallogr D Biol Crystallogr 59, 11311137 
39. Cheng, T. J., Sung, M. T., Liao, H. Y., Chang, Y. F., Chen, C. W., Huang, C. Y., Chou, L. Y., Wu, Y. D., et al. (2008) Domain requirement of moenomycin binding to bifunctional transglycosylases and development of high-throughput discovery of antibiotics. Proceedings of the National Academy of Sciences of the United States of America 105, 431-436

40. Berisio, R., Vitagliano, L., Mazzarella, L., and Zagari, A. (2002) Crystal structure of the collagen triple helix model [(Pro-Pro-Gly)(10)](3). Protein science : a publication of the Protein Society 11, 262-270

41. Lindorff-Larsen, K., Piana, S., Palmo, K., Maragakis, P., Klepeis, J. L., Dror, R. O., and Shaw, D. E. (2010) Improved side-chain torsion potentials for the Amber ff99SB protein force field. Proteins 78, 1950-1958

42. Horn, H. W., Swope, W. C., Pitera, J. W., Madura, J. D., Dick, T. J., Hura, G. L., and Head-Gordon, T. (2004) Development of an improved four-site water model for biomolecular simulations: TIP4P-Ew. J Chem Phys 120, 9665-9678

43. Bussi, G., Donadio, D., and Parrinello, M. (2007) Canonical sampling through velocity rescaling. J Chem Phys 126, 014101

44. Berendsen, H. J. C., Postma, J. P. M., van Gunsteren, W. F., DiNola, A., and Haak, J. R. (1984) Molecular dynamics with coupling to an external bath. J. Chem. Phys. 81, 3684-3690

45. Walshaw, J., and Woolfson, D. N. (2001) Socket: a program for identifying and analysing coiled-coil motifs within protein structures. J Mol Bio/ 307, 1427-1450

46. Stetefeld, J., Frank, S., Jenny, M., Schulthess, T., Kammerer, R. A., Boudko, S., Landwehr, R., Okuyama, K., et al. (2003) Collagen stabilization at atomic level: crystal structure of designed (GlyProPro)10foldon. Structure 11, 339-346

47. De Simone, A., Vitagliano, L., and Berisio, R. (2008) Role of hydration in collagen triple helix stabilization. Biochem Biophys Res Commun 372, 121-125

48. Raman, S. S., Parthasarathi, R., Subramanian, V., and Ramasami, T. (2006) Role of aspartic acid in collagen structure and stability: A molecular dynamics investigation. J Phys Chem B 110, 20678-20685

49. Vitagliano, L., Berisio, R., and De Simone, A. (2011) Role of hydration in collagen recognition by bacterial adhesins. Biophys $J$ 100, 2253-2261

50. Oliver-Kozup, H., Martin, K. H., Schwegler-Berry, D., Green, B. J., Betts, C., Shinde, A. V., Van De Water, L., and Lukomski, S. (2013) The group A streptococcal collagen-like protein-1, Scl1, mediates biofilm formation by targeting the extra domain A-containing variant of cellular fibronectin expressed in wounded tissue. Molecular microbiology 87, 672-689

51. Oliver-Kozup, H. A., Elliott, M., Bachert, B. A., Martin, K. H., Reid, S. D., Schwegler-Berry, D. E., Green, B. J., and Lukomski, S. (2011) The streptococcal collagen-like protein-1 (Scl1) is a significant determinant for biofilm formation by group A Streptococcus. BMC Microbiol 11, 262

52. Pawlowski, M., Bujnicki, J. M., and Lukomski, S. (2013) Collagen-like proteins of pathogenic streptococci: biology, structure and function. in 7th International Conference on Gram-Positive Microorganisms, Montecatini Terme, Italy

53. Paterson, G. K., Nieminen, L., Jefferies, J. M., and Mitchell, T. J. (2008) PclA, a pneumococcal collagen-like protein with selected strain distribution, contributes to adherence and invasion of host cells. FEMS Microbiol Lett 285, 170-176 
54. Beres, S., Sesso, R., Pinto, S., Hoe, N., Porcella, S., Deleo, F., and Musser, J. (2008) Genome sequence of a lancefield group C Streptococcus zooepidemicus strain causing epidemic nephritis: new information about an old disease. PLoS ONE 3, e3026

55. Karlstrom, A., Jacobsson, K., Flock, M., Flock, J., and Guss, B. (2004) Identification of a novel collagen-like protein, SclC, in Streptococcus equi using signal sequence phage display. Vet Microbiol 104, 179-188

56. Cheng, A. G., McAdow, M., Kim, H. K., Bae, T., Missiakas, D. M., and Schneewind, O. (2010) Contribution of coagulases towards Staphylococcus aureus disease and protective immunity. PLoS Pathog 6, e1001036

57. Cantisani, M., Falanga, A., Incoronato, N., Russo, L., De Simone, A., Morelli, G., Berisio, R., Galdiero, M., et al. (2013) Conformational Modifications of gB from Herpes Simplex Virus Type 1 Analyzed by Synthetic Peptides. J Med Chem

58. Koellhoffer, J. F., Malashkevich, V. N., Harrison, J. S., Toro, R., Bhosle, R. C., Chandran, K., Almo, S. C., and Lai, J. R. (2012) Crystal structure of the Marburg virus GP2 core domain in its postfusion conformation. Biochemistry 51, 7665-7675

59. Lee, J. E., Fusco, M. L., Hessell, A. J., Oswald, W. B., Burton, D. R., and Saphire, E. O. (2008) Structure of the Ebola virus glycoprotein bound to an antibody from a human survivor. Nature 454, 177-182

60. Buzon, V., Natrajan, G., Schibli, D., Campelo, F., Kozlov, M. M., and Weissenhorn, W. (2010) Crystal structure of HIV-1 gp41 including both fusion peptide and membrane proximal external regions. PLOS Pathogens 6, e1000880

61. Heldwein, E. E., Lou, H., Bender, F. C., Cohen, G. H., Eisenberg, R. J., and Harrison, S. C. (2006) Crystal structure of glycoprotein B from herpes simplex virus 1. Science 313, 217-220

62. Tran, E. E., Borgnia, M. J., Kuybeda, O., Schauder, D. M., Bartesaghi, A., Frank, G. A., Sapiro, G., Milne, J. L., et al. (2012) Structural mechanism of trimeric HIV-1 envelope glycoprotein activation. PLOS Pathogens 8, e1002797

63. Weissenhorn, W., Dessen, A., Harrison, S. C., Skehel, J. J., and Wiley, D. C. (1997) Atomic structure of the ectodomain from HIV-1 gp41. Nature 387, 426-430

64. Boudko, S. P., Engel, J., and Bachinger, H. P. (2012) The crucial role of trimerization domains in collagen folding. Int J Biochem Cell Biol 44, 21-32

65. Than, M. E., Henrich, S., Huber, R., Ries, A., Mann, K., Kuhn, K., Timpl, R., Bourenkov, G. P., et al. (2002) The 1.9-A crystal structure of the noncollagenous (NC1) domain of human placenta collagen IV shows stabilization via a novel type of covalent Met-Lys cross-link. Proceedings of the National Academy of Sciences of the United States of America 99, 6607-6612

66. Kvansakul, M., Bogin, O., Hohenester, E., and Yayon, A. (2003) Crystal structure of the collagen alpha1(VIII) NC1 trimer. Matrix Biol 22, 145-152

67. Bogin, O., Kvansakul, M., Rom, E., Singer, J., Yayon, A., and Hohenester, E. (2002) Insight into Schmid metaphyseal chondrodysplasia from the crystal structure of the collagen X NC1 domain trimer. Structure 10, 165-173

68. Wirz, J. A., Boudko, S. P., Lerch, T. F., Chapman, M. S., and Bachinger, H. P. (2011) Crystal structure of the human collagen XV trimerization domain: a potent trimerizing unit common to multiplexin collagens. Matrix Bio/ 30, 9-15 
69. Boudko, S. P., Sasaki, T., Engel, J., Lerch, T. F., Nix, J., Chapman, M. S., and Bachinger, H. P. (2009) Crystal structure of human collagen XVIII trimerization domain: A novel collagen trimerization Fold. Journal of molecular biology 392, 787802

70. Rety, S., Salamitou, S., Garcia-Verdugo, I., Hulmes, D. J., Le Hegarat, F., Chaby, R., and Lewit-Bentley, A. (2005) The crystal structure of the Bacillus anthracis spore surface protein BclA shows remarkable similarity to mammalian proteins. The Journal of biological chemistry 280, 43073-43078

71. Cunningham, M. W. (2000) Pathogenesis of group A streptococcal infections. Clin. Microbiol. Rev. 13, 470-511

72. O'Loughlin, R., Roberson, A., Cieslak, P., Lynfield, R., Gershman, K., Craig, A., Albanese, B., Farley, M., et al. (2007) The epidemiology of invasive group A streptococcal infection and potential vaccine implications: United States, 20002004. Clin Infect Dis 45, 853-862

73. Shulman, S. T., Stollerman, G., Beall, B., Dale, J. B., and Tanz, R. R. (2006) Temporal Changes in Streptococcal M Protein Types and the Near-Disappearance of Acute Rheumatic Fever in the United States. Clin. Infect. Dis. 42, 441-447 

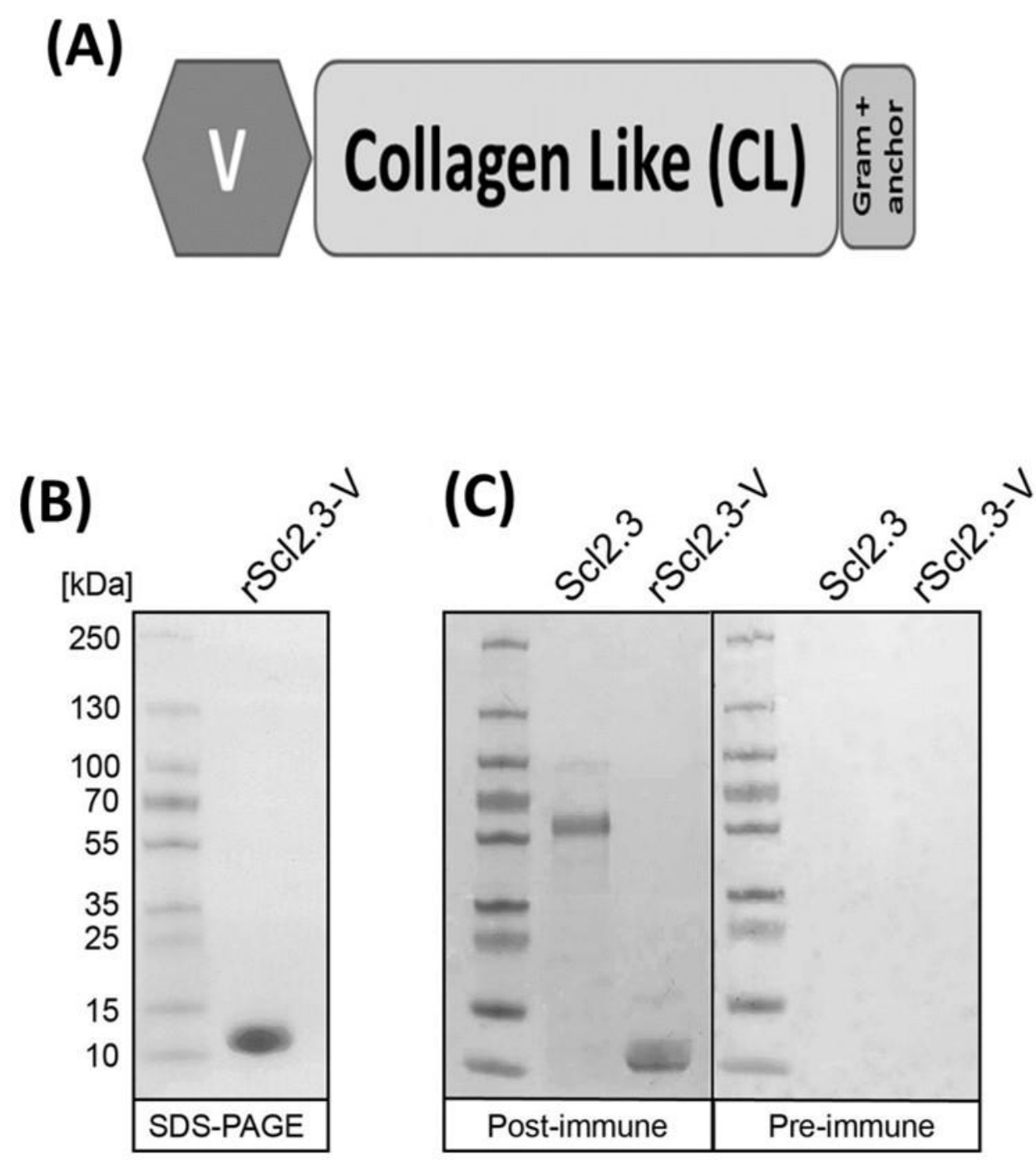

Figure 1

Expression of Scl2.3 surface protein by the invasive M3-type strain MGAS 315. (A) Domain organization of Scl2.3, according to the PFAM database. (B) Characterization of recombinant Scl2.3-V protein (rScl2.3-V). rScl2.3-V polypeptide expressed in E. coli and affinity purified was resolved on $4-20 \%$ SDS-PAGE gradient gel and stained. Purified rScl2.3-V was used to immunize rabbits and the resulting post-immune sera were used to detect native Scl2.3 protein expressed by MGAS315. (C) Surface expression of Scl2.3 protein by GAS. Cell wall-associated protein fraction of MGAS315 was analyzed by Western immunoblotting to detect native Scl2.3 protein using anti-rScl2.3-V post-immune rabbit sera. The same blot developed with pre-immune sera from the same rabbit is shown as a negative control. PageRuler TM Plus Prestained Protein Ladder was used as a molecular weight marker. 
(A)

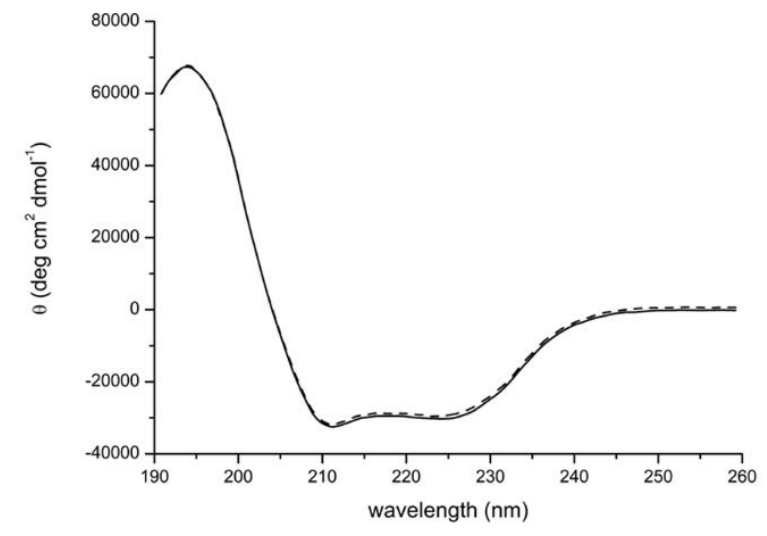

(B)

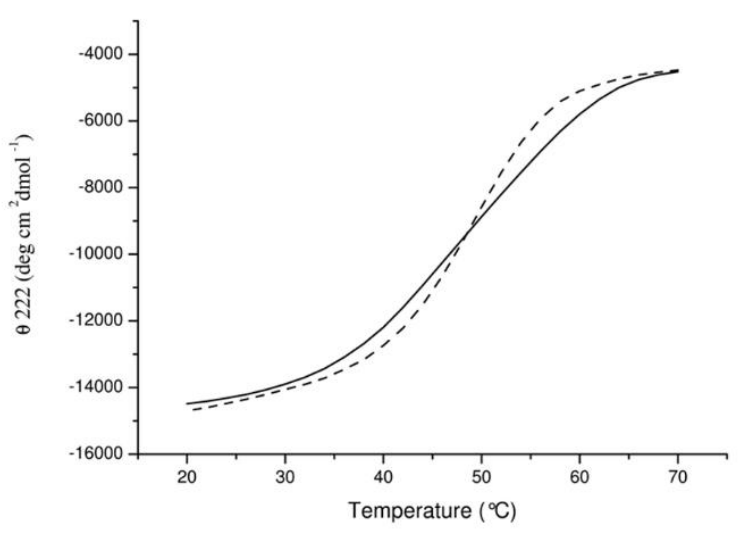

(C)

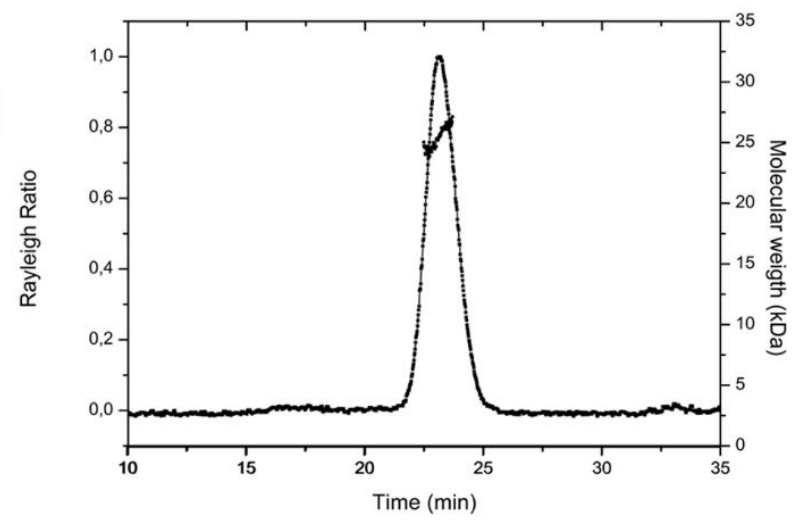

Figure 2

Structural characterization of Scl2.3-V in solution. (A) CD spectra of Scl2.3-V at $20^{\circ} \mathrm{C}$ after refolding are shown as solid and dashed lines, respectively. (B) Denaturation (solid line) and refolding (dashed line) of Scl2.3-V followed at $\lambda=222 \mathrm{~nm}$. (C) Analytical SECMALS; The black curve represents the Rayleigh ratio (left scale) against the retention time. Molecular mass (right scale) values correspond to a trimeric state of Scl2.3-V. 

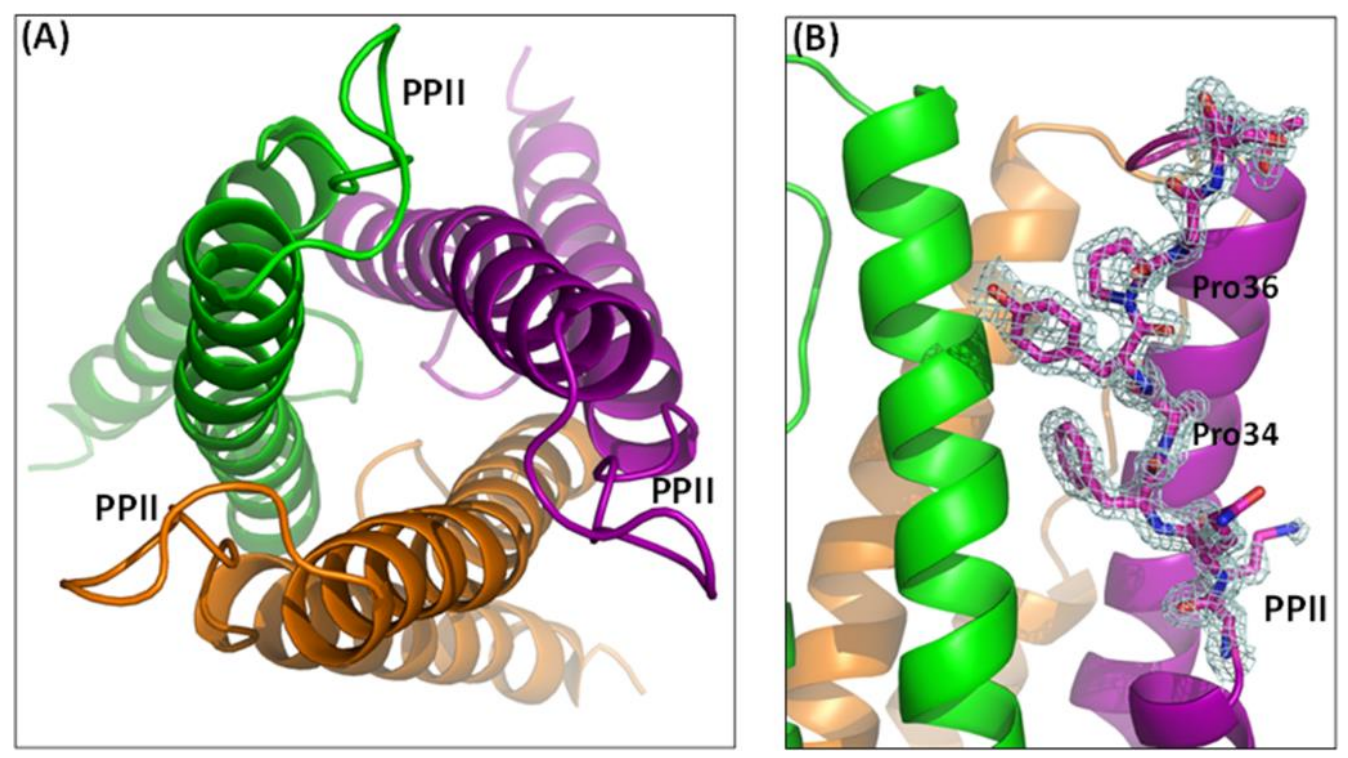

\section{Figure 3}

Cartoon representation of the crystal structure of Scl2.3-V trimer. (A) Top view of Scl2.3-V six-helix bundle. (B) A detail of (2Fo-Fc) Electron density on the polyproline II (PPII) region. 
(A)

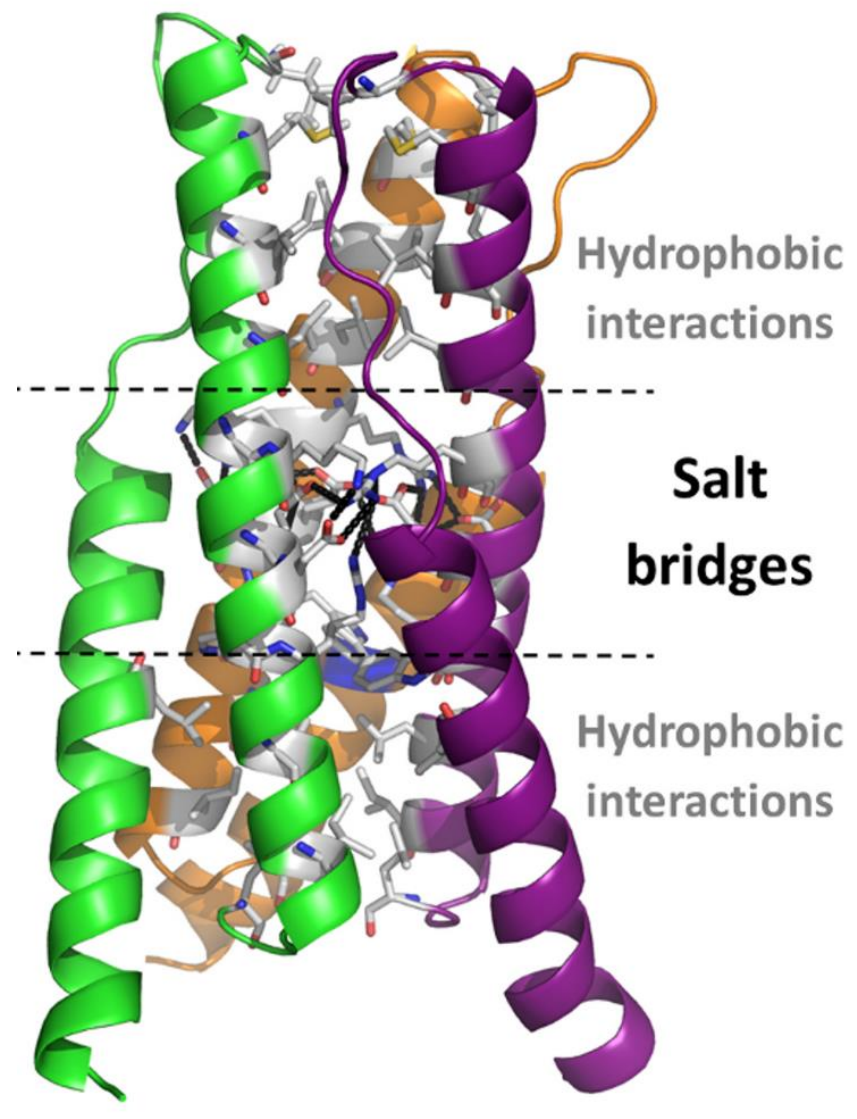

(B)

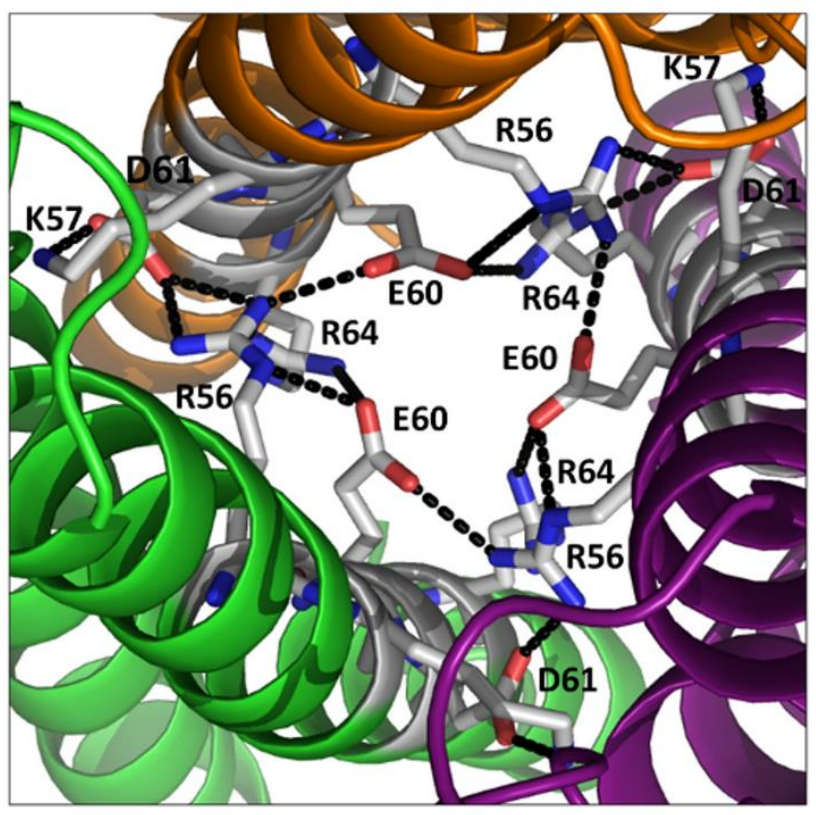

Figure 4

Main interactions stabilizing the six-helix bundle of Scl2.3-V. (A) Three regions are identifiable, with the central one stabilized by salt bridges and the external ones stabilized by hydrophobic interactions. (B) A detail of salt bridge interactions in the central part of the six-helix bundle. 

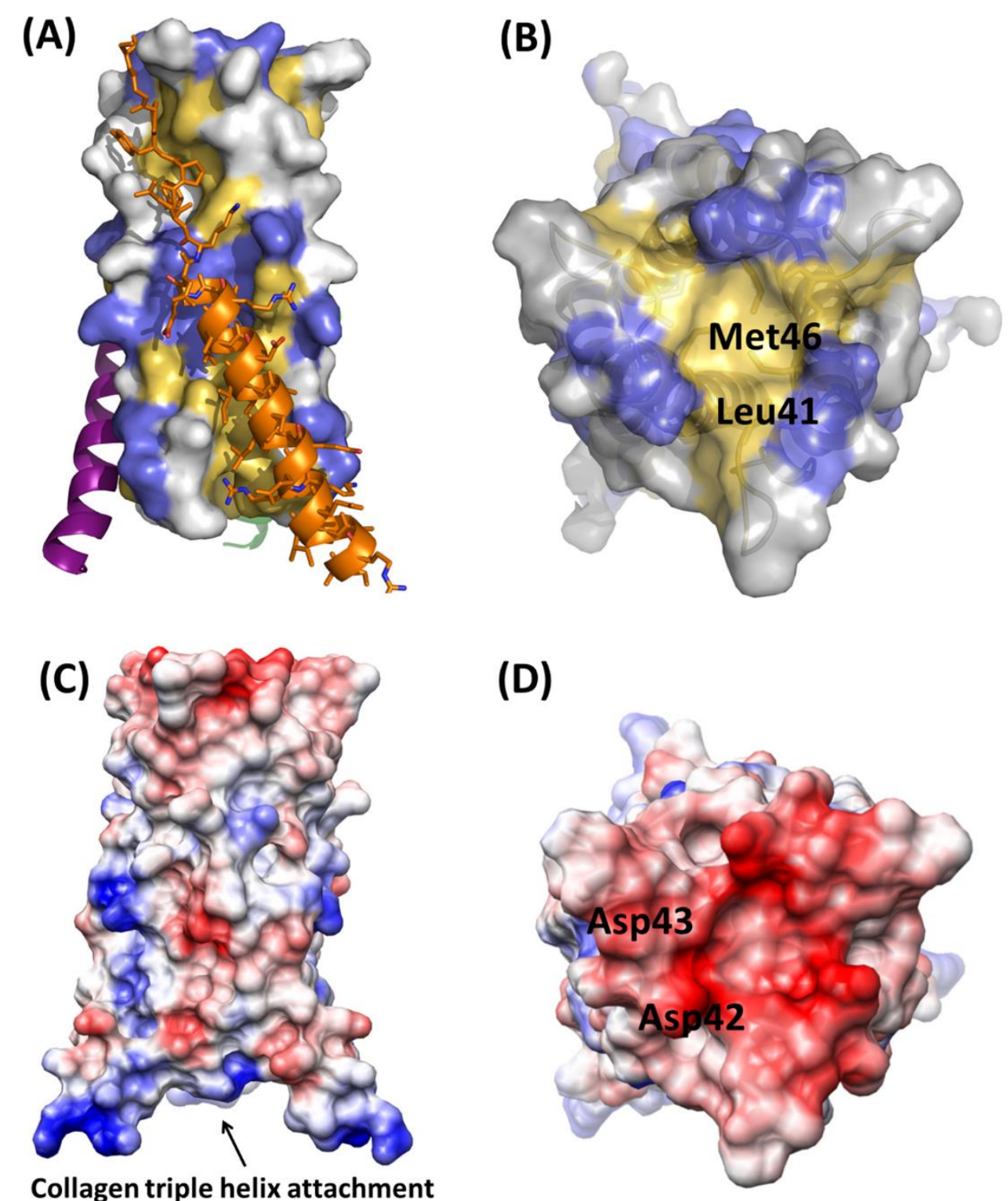

\section{Figure 5}

Surface features of Scl2.3-V. (A) Side and (B) top view of Scl2.3-V surface. Hydrophobic and hydrophilic residues are colored yellow and gray, respectively. Charged residues are colored blue. (C, D) Side and top views of Scl2.3-V electrostatic potential surface. The positive and negative charges are colored blue and red, respectively. 


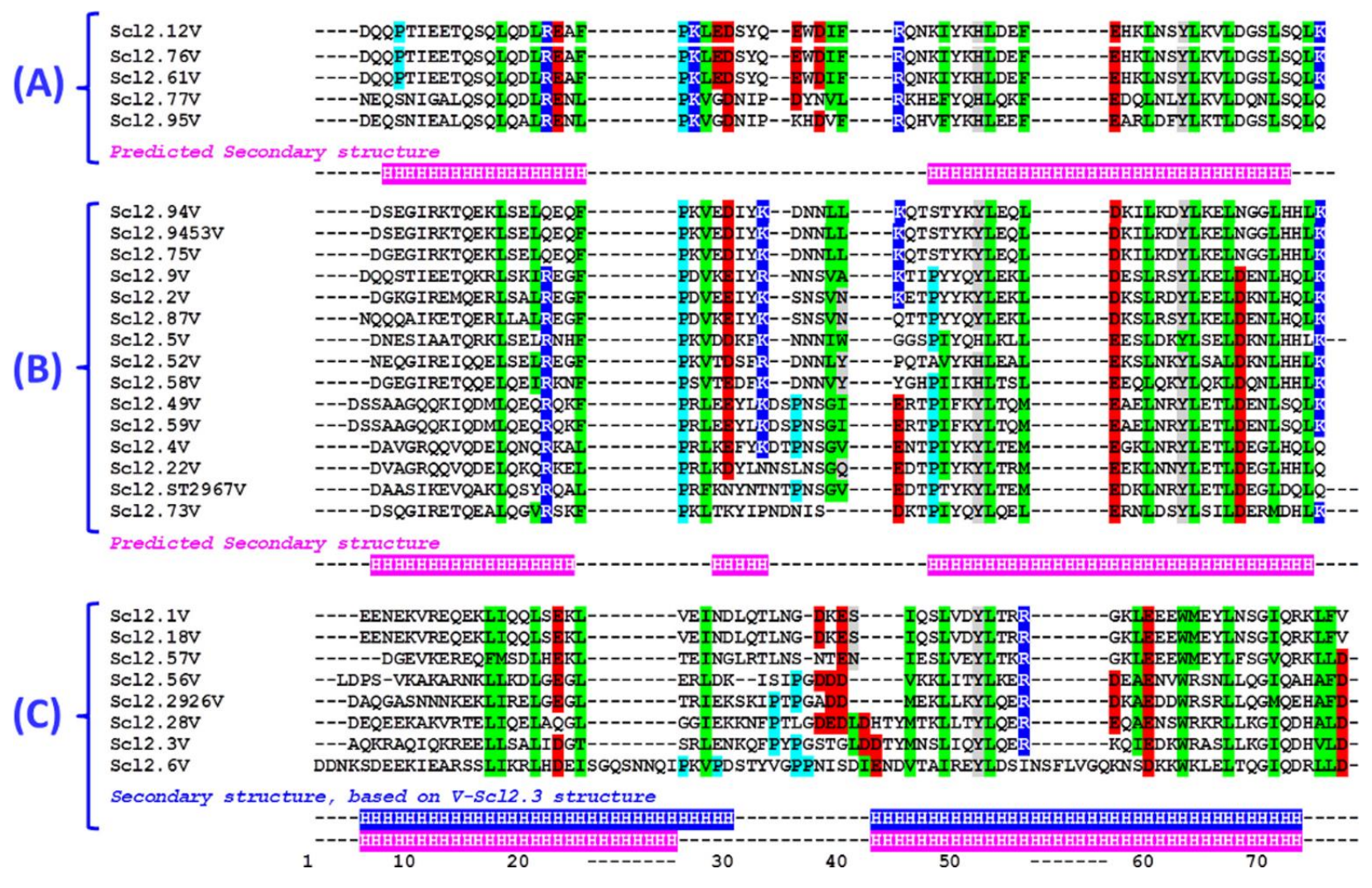

\section{Figure 6}

Multiple sequence alignment analysis of Scl2-V region variants. (A) Sequence alignment was performed using CLUSTALW. Conserved residues are shown in green (hydrophobic), blue (positively charged), red (negatively charged), and grey (polar); Pro residues are shown in cyan. $A, B$ and $C$ refer to the three respective branches calculated by phylogenetic analysis using CLUSTALW. Secondary structure prediction, according to JPRED, is reported in magenta whereas the secondary structure based on Scl2.3-V crystal structure is reported in blue. 


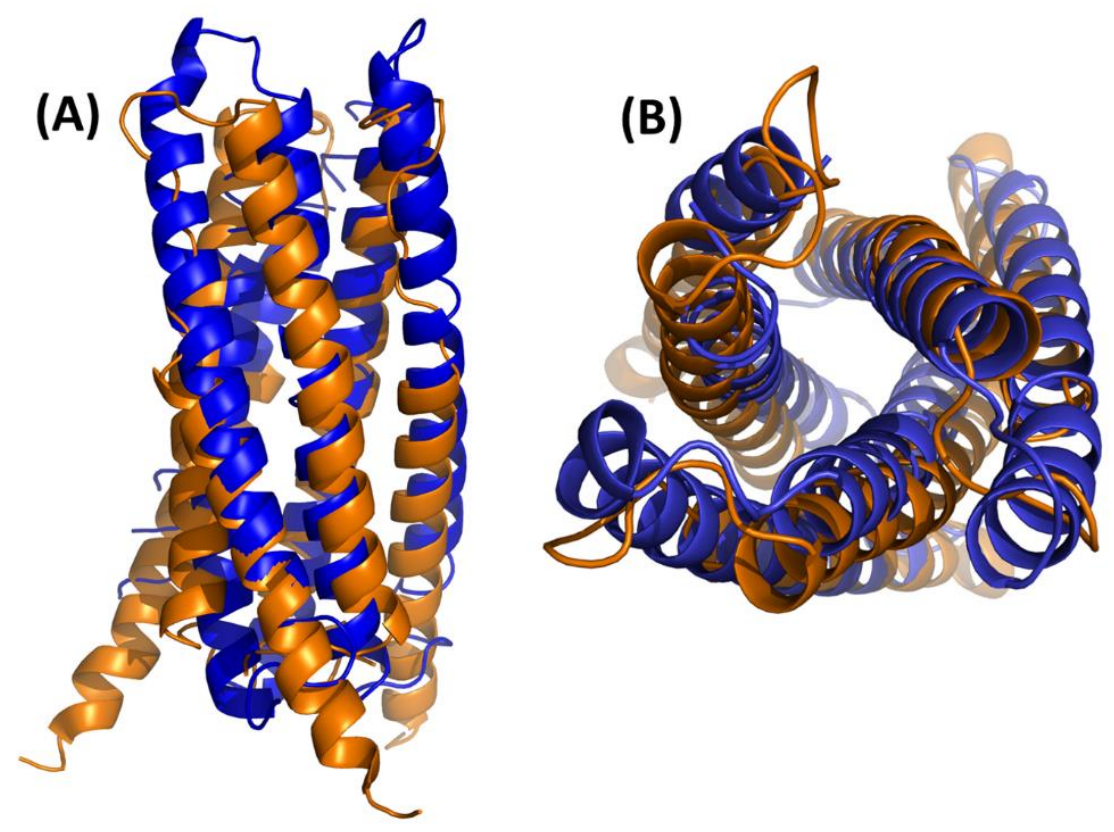

Figure 7

ScI2.3-V structural alignment with the HIV protein gp41. Side $(A)$ and top (B) views of the superposition between Scl2.3-V (orange) with gp41 (blue, PDB code 3040). 


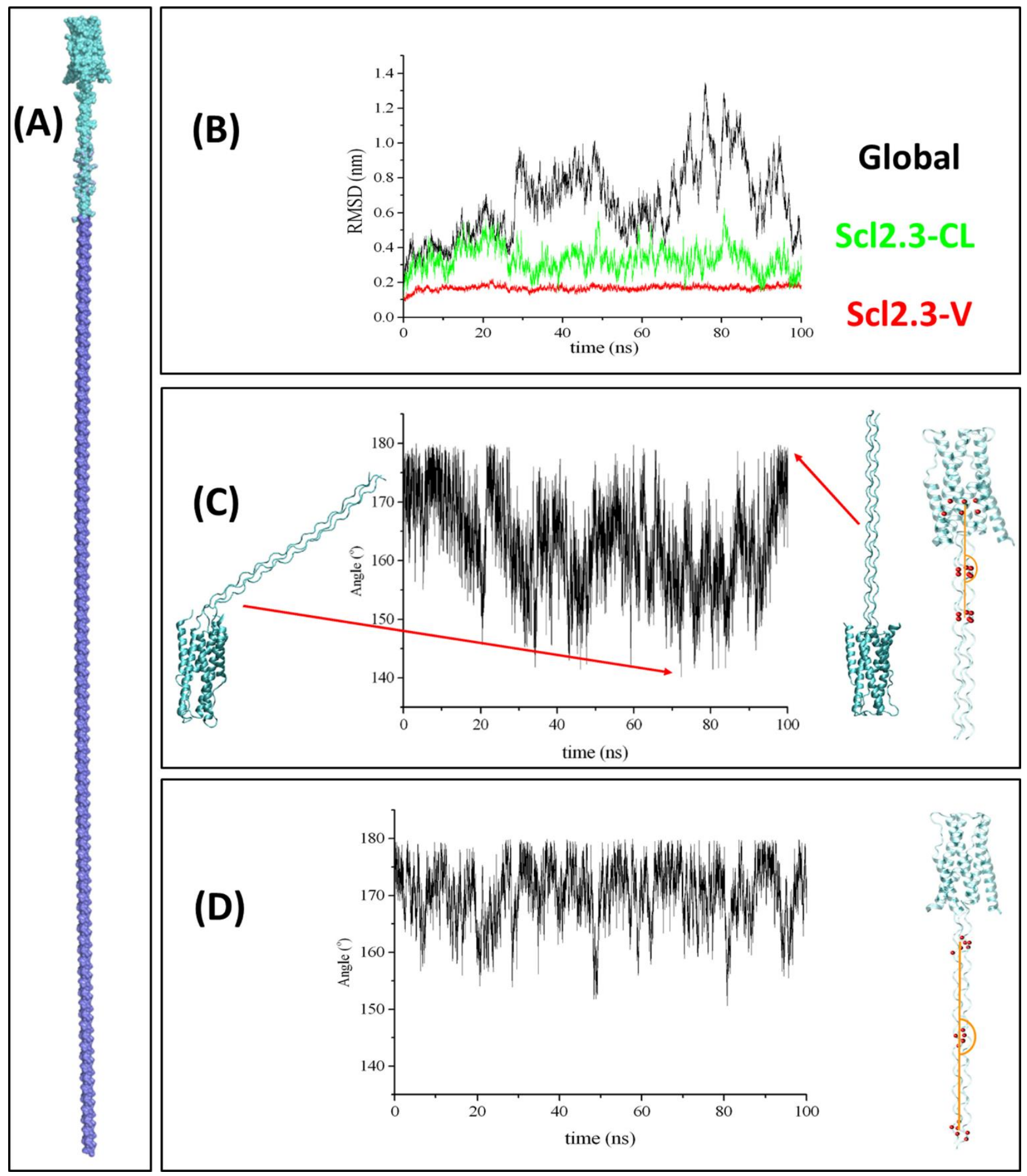




\section{Figure 8}

Modeling and molecular dynamics analyses. (A) Modeling of Scl2.3 structure: a view of the entire Scl2.3 structure; The cyan region (residues 7-123 of each chain of the Scl2.3 trimer) was used for MD studies. (B) Evolution of the RMSD calculated on Ca atoms, throughout the MD trajectory for the entire model (black), the Scl2.3-V (red) and Scl2.3$\mathrm{CL}$ (green). (C) Evolution of the bending angle between the Scl2.3-V and Scl2.3-CL domains throughout the MD trajectory; (D) Evolution of the bending angle between three zones of the Scl2.3-CL domain throughout the MD trajectory; The insets of panels $\mathrm{C}$ and $\mathrm{D}$ show atoms used for the computation of the bending angle as red balls. 
Table 1. Data collection and refinement statistics

\section{A. Data collection ${ }^{\mathrm{a}}$}

Space group

a, b, c $(\AA), \gamma\left({ }^{\circ}\right)$

Resolution $(\AA)$

Average redundancy

Completeness (\%)

Rmerge (\%)

Average $\mathrm{I} / \sigma(\mathrm{I})$

\section{Europium chloride Native derivative}

$44.3,44.3,228.0,120.0 \quad 44.4,44.4,227.8,120.0$

1.87

$9.5(7.5)$

$100(99.9)$

$0.043(0.419)$

$47.7(5.1)$
1.52

$5.3(2.6)$

$99.2(86.7)$

$0.061(0.345)$

$49.2(3.1)$

\section{B. Refinement}

Resolution range $(\AA)$

$15.00-1.52$

Rwork, Rfree (\%)

$18.5,23.3$

No. atoms (protein, ligands,

$623,13,100$ water)

r.m.s. deviations

Bond lengths $(\AA)$

0.02

Bond angles $\left({ }^{\circ}\right)$

1.6

aValues in parentheses are for highest resolution shells: $1.90-1.87 \AA$ for $\mathrm{EuCl}_{3}$ derivative and 1.55-1.52 $\AA$ for the native crystal. 


\section{PART III}

\section{ADDITIONAL PUBLISHED DATA}




\title{
CHAPTER 6
}

\section{REVERSIBLE PHOSPHOLIPID NANOGELS FOR DEOXYRIBONUCLEIC ACID FRAGMENT SIZE DETERMINATIONS UP TO 1,500 BASE PAIRS AND INTEGRATED SAMPLE STACKING}

\author{
Brandon C. Durney, Beth A. Bachert, Hillary S. Sloane, Slawomir Lukomski, James P. \\ Landers, and Lisa A. Holland \\ Published in Analytica Chimica Acta 2015 880: 136-144
}

\begin{abstract}
Phospholipid additives are a cost-effective medium to separate deoxyribonucleic acid (DNA) fragments and possess a thermally-responsive viscosity. This provides a mechanism to easily create and replace a highly viscous nanogel in a narrow bore capillary with only a $10^{\circ} \mathrm{C}$ change in temperature. Preparations composed of dimyristoylsn-glycero-3-phosphocholine (DMPC) and 1,2-dihexanoyl-sn-glycero-3-phosphocholine (DHPC) self-assemble, forming structures such as nanodisks and wormlike micelles. Factors that influence the morphology of a particular DMPC-DHPC preparation include the concentration of lipid in solution, the temperature, and the ratio of DMPC and DHPC. It has previously been established that an aqueous solution containing $10 \%$ phospholipid with a ratio of $[\mathrm{DMPC}] /[\mathrm{DHPC}]=2.5$ separates $\mathrm{DNA}$ fragments with nearly single base resolution for DNA fragments up to 500 base pairs in length, but beyond this size the resolution decreases dramatically. A new DMPC-DHPC medium is developed to effectively separate and size DNA fragments up to 1,500 base pairs by decreasing the total lipid concentration to $2.5 \%$. A $2.5 \%$ phospholipid nanogel generates a resolution of $1 \%$ of the DNA fragment size up to 1,500 base pairs. This increase in the upper size limit is accomplished using commercially available phospholipids at an even lower material cost than is achieved with the $10 \%$ preparation. The separation additive is used to evaluate size markers ranging between 200 and 1,500 base pairs in order to distinguish invasive strains of Streptococcus pyogenes and Aspergillus species by harnessing differences in gene sequences of collagen-like proteins in these organisms. For the first time, a reversible stacking gel is integrated in a capillary sieving separation by utilizing the thermally-responsive viscosity of these self-assembled phospholipid preparations. A
\end{abstract}


discontinuous matrix is created that is composed of a cartridge of highly viscous phospholipid assimilated into a separation matrix of low viscosity. DNA sample stacking is facilitated with longer injection times without sacrificing separation efficiency.

\section{INTRODUCTION}

Estimating the size of DNA is critical in genetic analysis associated with human identification (1), species identification (2,3), detecting personal biomarkers (4), analyzing food (5), and categorizing pathogenicity (6). Moreover, following sample preparation, accurate determination of the size of DNA fragments is a mandatory analytical step in sophisticated high-throughput sequencing techniques. It is also a critical endpoint for methods based on PCR amplification, where the length of the product is used to determine the presence of a specific target sequence in the DNA template. Confirmation of size is achieved by comparing the fragment migration time during electrophoretic sieving using various sieving agents. Slab gel electrophoresis, the workhorse of modern analytical science, is a commonly used tool for DNA sizing due to the simplicity and low cost of the method. Sizing is achieved by comparing the migration time of the targeted DNA with a DNA ladder that contains a mixture of fragments of known size. The DNA ladder standard is run in a separate lane. A serious disadvantage of slab gels is the poor separation efficiency and the reduced throughput even when completed in parallel lanes in the gel $(7,8)$. Although more expensive than slab gel methods, capillary gel electrophoresis provides a size discrimination that is improved substantially, and higher sample throughput. Sequencing of the human genome provides direct proof of this (9).

Capillary gel electrophoresis separations of DNA fragments are accomplished using polymer solutions that provide a size-based separation of DNA fragments by creating networks or matrices that form dynamic pores for sieving (10-12), or separation through DNA entanglement (13). DNA fragments ranging from $50-1,500$ bases are often present in an electropherogram, but the resolution obtained for the larger DNA fragments is poor and accurate sizing is limited to DNA fragments that are shorter than 500 bases in length. Ultra-high resolution separations were achieved with $0.75 \%$ hydroxyethyl cellulose used to size a 256 base pair (bp) DNA fragment (14). Although larger fragments could not be sized, 1,078 bp and 1,353 bp DNA were baseline resolved (14). A 
commercial system utilized hydroxyethyl cellulose to separate $50 \mathrm{bp}$ to $10 \mathrm{kbp}$ DNA and to accurately size 275 to 815 bp DNA (15). In another case, a matrix of $2 \%$ linear polyacrylamide was used to sequence a DNA fragment 1,300 bases long (16). Several polymer properties dictate the utility of the material and the useful size range of the separation (17). Increasing the upper fragment size limit of the separation can be accomplished by increasing the length of the polymer and maintaining the concentration of the gel at or above the entanglement threshold (10). This increase in concentration is accompanied by an increase in viscosity (11), which provides challenges for the introduction to or replacement of, the gel in the separation capillary.

Temporary gels, which rupture and reform during the separation, are an exciting alternative to permanent gels because the upper size limit increases when the rate of gel rupture is optimized (18). Wormlike micelles form large self-assembled structures that dynamically rupture and reform. Aqueous solutions of the long-chain phospholipid dimyristoyl-sn-glycero-3-phosphocholine (DMPC) and the short-chain phospholipid 1,2dihexanoyl-sn-glycero-3-phosphocholine (DHPC) are known to form wormlike micelles $(19,20)$. A dynamic sieving matrix of $10 \%$ phospholipid with [DMPC]/[DHPC] $=2.5$ was optimized for single-base pair resolution that was adequate for detection of short tandem repeats (STR) in selected loci utilized for human identification using a $100 \mathrm{~cm}$ long capillary within a 30 minute run time (21). This is comparable to separations on commercial capillary electrophoresis instruments for forensic STR analyses (22). What makes this particularly attractive, is that the phospholipid nanogel is substantially more cost-effective at roughly one-fourth the cost of commercial gels utilized for human identification (23-25). A significant advantage of the dynamic sieving matrix is the thermally-responsive viscosity. The sieving matrix has low viscosity below the gel-phase transition temperature of the phospholipids and becomes gel-like at higher temperatures. As a result, the sieving agent is easily introduced into or expelled from the narrow bore separation capillary at a temperature of $19^{\circ} \mathrm{C}$. Prior to the separation, the matrix is transformed into a viscous gel in-capillary at a separation temperature of $30^{\circ} \mathrm{C}$.

While a phospholipid additive has been previously described for the separation of DNA fragments below $500 \mathrm{bp}$ (21), we show that the DNA sizing range of the separation can be extended by reducing the concentration of wormlike micelles in solution. The size, 
shape, and stability of the phospholipid structures depend upon the phospholipid concentration, the ratio of DMPC and DHPC, and the temperature. The effect of these parameters on the size limit of DNA separations was evaluated, and the linear range for size separation was extended to 1,500 bp by tuning the concentration of the phospholipid nanogel and the ratio of [DMPC]/[DHPC]. This preparation was used to compare the size of the DNA fragments to internal standards. The effectiveness of the sieving matrix was demonstrated with the analysis of PCR-amplified DNA sequences specific to select pathogens. The difference between the true size of a DNA fragment and the size calculated from internal standards is less than $2.3 \%$ for fragment sizes between 200-500 bp. For fragments that range from $600-1,500$ bp this difference between the true size and measured size is less than or equal to $4 \%$. Different properties of the high and low concentration phospholipids were exploited by integrating both dynamic sieving matrices into a single capillary to create a discontinuous gel in-capillary used to concentrate DNA samples through gel stacking.

\section{MATERIALS AND METHODS}

\section{Chemicals and reagents}

The fluorescently labeled DNA ladder (catalog \# MM-1000-FAM, 50-1,000 bp) was purchased from BioVentures (Murfreesboro, TN). The fluorescent intercalating dye SYBR Green 1 nucleic acid gel stain (catalog \# S-7567), 1 Kb Plus (catalog \# 10787-018, 100$12,000 \mathrm{bp}$ ), and $100 \mathrm{bp}$ (catalog \# 15628-019, 100-2,072 bp) DNA ladders were purchased from Life Technologies (Grand Island, NY). The methanol was purchased through Calbiochem/EMD (Gibbstown, NJ). The DMPC and DHPC phospholipids were purchased through Avanti Polar Lipids (Alabaster, $\mathrm{AL})$, and the 3-(N-morpholino)propanesulfonic acid (MOPS) was purchased from Alfa Aesar (Ward Hill, MA). Sodium hydroxide was obtained from Sigma-Aldrich (St. Louis, MO). All deionized water used was obtained from an ELGA PURELAB ultra water filtration system (Lowell, MA).

\section{Preparation of phospholipid mixture}

The phospholipid preparations were made as described previously (26) to obtain molar ratios of $[\mathrm{DMPC}] /[\mathrm{DHPC}]=0.5$ and $[\mathrm{DMPC}] /[\mathrm{DHPC}]=2.5$. The phospholipids were stored 
at $-20^{\circ} \mathrm{C}$ and thawed before being weighed out. Once they were weighed, an aqueous buffer of $100 \mathrm{mM}$ MOPS ( $\mathrm{pH}$ 7.0) was added to obtain the appropriate concentration of phospholipid per volume of aqueous diluent $(\% \mathrm{w} / \mathrm{v})$. The solution was then mixed using a vortex mixer for several minutes to dissolve the phospholipids. The phospholipid medium was then exposed to three rapid freeze thaw cycles using liquid nitrogen followed by centrifugation $\left(10,000 \mathrm{rpm}\right.$ at $\left.4^{\circ} \mathrm{C}\right)$ for 10 minutes. The matrix was then portioned into working volume aliquots of $200 \mu \mathrm{L}[\mathrm{DMPC}] /[\mathrm{DHPC}]=0.5(5 \% \mathrm{w} / \mathrm{v})$ and $100 \mu \mathrm{L}$ $[\mathrm{DMPC}] /[\mathrm{DHPC}]=2.5(10 \% \mathrm{w} / \mathrm{v})$. The $[\mathrm{DMPC}] /[\mathrm{DHPC}]=2.5$ lipids were then diluted with the same $100 \mathrm{mM}$ MOPS ( $\mathrm{pH} 7.0$ ) buffer to obtain the 7.5, 5.0, and 2.5\% phospholipid used for electrophoresis. No freeze thaws were done after the dilution from the $10 \%$ phospholipid. The additional phospholipid matrices with $[\mathrm{DMPC}] /[\mathrm{DHPC}]=3.0,4.0$, and 5.0 used in the study were prepared the same manner. The MOPS buffer was also used for capillary rinses between runs and served as the solution in the anodic and cathodic reservoirs during electrophoresis separations.

\section{PCR amplification}

Primers were based on scl2 genes with the following gene bank accession numbers and genome locations; M3: AE014074, location 791329-793050; M12: CP000259, location 862738-863880; M28: CP000056, location 781169-782437. Forward primers specific for each scl2 allele, are as follows: M3-Scl2.3_CLflank_2F,

5'-AGGCATACAAGATCATGTCCTTGA-3'; M12-Scl2.12_CLfl_1F,

5'-AAGAGTGGGATATCTTTAGGC-3'; M28-Scl2.28_CLfl_1F,

5'-CTACAGGAACGAGAACAAGC-3'. A single reverse primer Scl2.3_CLflank_2R, 5'-TTTGGTGTATGTGCTGCGGT-3', targeting a conserved 3' region of scl2 gene was paired with each forward primer. Genomic DNA was purified and isolated as described previously (27). PCR reactions were carried out with 1.5 units of Taq DNA polymerase (Qiagen, Germantown, MD) in a final volume of $50 \mu \mathrm{L}$ per reaction and DNA template in the nanogram range using a C1000 Touch Thermal Cycler (BioRad, Hercules, CA). The manufacturer's PCR buffer was supplemented with $0.2 \mu \mathrm{M}$ concentration of each primer and $0.2 \mathrm{mM}$ deoxynucleotide triphosphates (Bioline, Taunton, MA). Amplification conditions consisted of an initial denaturation step at $94{ }^{\circ} \mathrm{C}$ for $3 \mathrm{~min}$, followed by 30 
cycles of $1 \mathrm{~min}$ at $94{ }^{\circ} \mathrm{C}, 1 \mathrm{~min}$ at $51^{\circ} \mathrm{C}$, and $1 \mathrm{~min} 45 \mathrm{~s}$ at $72{ }^{\circ} \mathrm{C}$, followed by a final extension step at $72{ }^{\circ} \mathrm{C}$ for $7 \mathrm{~min}$. The amplicons were analyzed by agarose gel electrophoresis and the true size of each sc/2-PCR product was determined by Sanger DNA sequencing (Eurofins Genomics, Huntsville, $A L$ ). The DNA sequences were assembled and analyzed with the Lasergene 11 Genomics Suite (DNASTAR, Inc., Madison, WI) software, as previously described (28). Isolation of genomic DNA and purification from fungal cultures, PCR primers (Eurofins Genomics, Huntsville, AL), amplification conditions, and verification of conserved amplicon sizes for acl-based detection of Aspergillus species were previously established (29).

\section{Gel extraction of DNA ladder standards}

Fragments from the two unlabeled DNA ladders were separated using a VWR horizontal gel box Midi Plus 15x10cm connected to a Hoefer hsi (San Francisco, CA) power supply using a $1 \%$ agarose (CalbioChem/EMD Chemicals) in 1X TBE buffer (G Biosciences, St. Louis, MO) gel. Slab gel electrophoresis was accomplished using an applied voltage of $110 \mathrm{~V}$ for 3 hours, and alternate gel lanes were stained using ethidium bromide (part \# 15585011, Life Technologies) for UV visualization. Extraction and purification of DNA bands in unstained lanes was done using the illustra ${ }^{\text {TM }}$ GFX ${ }^{\text {TM }}$ PCR DNA and Gel Band Purification Kit manufactured by GE Healthcare (Buckinghamshire, UK) prior to analysis on the P/ACE MDQ Capillary electrophoresis instrument (Beckman Coulter, Fullerton, $\mathrm{CA}$ ). DNA that was isolated and recovered from the agarose gel was then utilized as an internal standard to size PCR amplicons. The $600,1,000,1,500$, and 1,650 bp fragments

were used as internal standards to size $S$. pyogenes and $B$. anthracis amplicons, whereas the 150, 450, and 500 bp were used to size Aspergillus spp. amplicons.

\section{Pre-treating the separation capillary}

Separations were performed using fused silica capillary with an outer diameter of $360 \mu \mathrm{m}$ and an inner diameter of $25 \mu \mathrm{m}$ (Polymicro Technologies, Phoenix, AZ). The separation capillary had a total length of $40 \mathrm{~cm}$, and an effective length of $30.2 \mathrm{~cm}$. New capillary was conditioned with a 4-step rinse procedure performed at $140 \mathrm{kPa}$ with (1) $1 \mathrm{~N} \mathrm{NaOH}$ for $30 \mathrm{~min}$, (2) deionized water for $15 \mathrm{~min}$, (3) methanol for $15 \mathrm{~min}$, (4) deionized water 
for $15 \mathrm{~min}$. The conditioned capillary was then passivated with a semi-permanent phospholipid coating to suppress electroosmotic flow as previously described (30). This was accomplished using a 2-step rinse at $140 \mathrm{kPa}$ with (1) $5 \%$ phospholipid of [DMPC]/[DHPC] $=0.5$ containing $1.25 \mathrm{mM} \mathrm{Ca}^{2+}$ for $20 \mathrm{~min}$ and (2) a 2 min flush with 100 $\mathrm{mM}$ MOPS buffered to $\mathrm{pH}$ 7. This passivation rinse is repeated once (i.e., 4-steps). The capillary was then filled with phospholipid nanogel at an ambient temperature of $19^{\circ} \mathrm{C}$. Once filled with the phospholipid, the capillary temperature was increased to the desired separation temperature. A reverse polarity voltage of $20 \mathrm{kV}$ was applied for 5 min prior to the first run of the day.

\section{Capillary electrophoresis sample introduction and separation}

Sample was injected by first introducing a pre-plug of run buffer at $6.9 \mathrm{kPa}$ for $7 \mathrm{sec}$, then applying a reverse polarity electrokinetic injection of DNA for the specified time, and finally by inserting a post-plug of run buffer at $3.4 \mathrm{kPa}$ for $5 \mathrm{sec}$. The purpose of these pre- and post-plugs was to facilitate stacking and improve the theoretical plate count (31). Separations were performed at $4 \mathrm{kV}$ under reverse polarity conditions. Between each separation the capillary was flushed at $140 \mathrm{kPa}$ for $3 \mathrm{~min}$ with $5 \%$ [DMPC]/[DHPC] $=0.5$, 2 min MOPS ( $\mathrm{pH} 7$ ), and 3 min with the phospholipid preparation used to separate DNA.

\section{Fluorescence detection and analysis of DNA}

The Beckman Coulter P/ACE MDQ was equipped with a laser induced fluorescence detection module and an air cooled argon ion laser $\left(\lambda_{e x}=488 \mathrm{~nm}\right.$ and $\left.\lambda_{e m}=520 \mathrm{~nm}\right)$. The optimization of concentration and composition of phospholipid nanogel was accomplished using a fluorescein end-labeled DNA ladder. The intercalating dye SYBR Green 1 was utilized for separations of PCR amplicons. SYBR dye was diluted to a concentration of 20 $\mu M$ SYBR in phospholipid preparations of [DMPC]/[DHPC] $=2.5$ at a concentration equal to that used for the separation. The concentration of the plug was calculated based on a literature estimate of $20 \mathrm{mM}$ stock concentration from the manufacturer (32). Unless otherwise stated, a 7 second $34.5 \mathrm{kPa}$ plug of phospholipid modified with dye was pushed toward the detection window in the separation capillary prior to sample injection. Under an applied field, the positively-charged dye and negatively-charged DNA migrated toward 
each other and the two bound effectively upon contact as the $K_{d}$ is $3.1 \mathrm{nM}$ (33). Any variation in the ratio of dye and DNA in a separation will change the migration time of the DNA. Reproducible run times are achieved by utilizing a dye concentration compatible with the detector output, in this case an output of approximately 1-5 relative fluorescent units was achievable with a plug of $20 \mu \mathrm{M}$ SYBR Green 1. As summarized in Table S-1, the reproducibility in migration time is less than or equal to $5 \%$ for separations with a 7 second dye plug. The use of internal size standards in each separation further mitigates any bias in migration time due to variation in the ratio of dye to DNA.

The size of the PCR amplicons was calculated using internal standards purified from the DNA ladders listed in the materials section. Sizing of fragments was accomplished using two DNA standards that bracketed the size of the PCR amplicon. All DNA fragments were co-injected and a linear fit was used to relate the migration time to the known size in bp of the DNA standards. Using this fit, the size of each PCR amplicon was calculated based on the migration time. Resolution between fragments was calculated using the expression $R s=0.589^{*}\left(\Delta t / w_{1 / 2 a v}\right)$, where $\Delta t$ is the difference in migration time between the two peaks of interest, and $w_{1 / 2 a v}$ is the average width at half height for the same two peaks. Resolution in base pairs was calculated by dividing the difference in the number of base pairs for the two fragments $(\Delta b p)$ by the calculated resolution. Therefore, base pair resolution can be expressed as $\operatorname{Rsbp}_{\text {bp }}=(\Delta \mathrm{bp}) /\left(0.589^{*}\left(\Delta \mathrm{t} / \mathrm{w}_{1 / 2 \mathrm{av}}\right)\right)$. Data collection and analysis were performed using 32 Karat Software version 7.0 (Beckman Coulter), and peak width at $50 \%$ height as well as theoretical plate count were calculated using the "USP" criterion. 


\section{RESULTS AND DISCUSSION}

\section{Effect of gel concentration on the separation}

The total lipid concentration ( $\% \mathrm{w} / \mathrm{v}$ ) determined the number of 'ribbons' or 'disks' in solution and the degree of long-range interaction, including the extent of lipid entanglement of the nanogel. Using a fluorescently-labeled DNA ladder, the separations were examined in the $500-1,000 \mathrm{bp}$ range to quantify the effect of phospholipid concentration on separation performance. Our previous literature reported the effect of concentrations of phospholipid ranging from $5-15 \%$ for separations based on subtle difference in hydrodynamic volume (26) and from $6-12 \%$ for separations based on sieving (21). For both studies $(21,26)$ a $10 \%$ phospholipid concentration generated the most effective separation efficiency and peak resolution. In the current study concentrations of $2.5,5.0,7.5$, and $10 \%$ were used to evaluate the effect of phospholipid concentration on the useful upper size limit for DNA separations. Preparations below $2.5 \%$ phospholipid did not generate reproducible DNA separations, presumably because wormlike micelles are not stable under these conditions, and consequently, these were not assessed. The peak resolution summarized in Table 1 was measured for a set of DNA fragments at 450 and $475 \mathrm{bp}$ as well as a set at 900 and $950 \mathrm{bp}$. There was no significant improvement in resolution of DNA fragments with a length of 450 and $475 \mathrm{bp}$ for separations accomplished at $23^{\circ} \mathrm{C}$. When the separation was performed at the optimum temperature for each lipid preparation, which was $25^{\circ} \mathrm{C}$ for $5 \%$ nanogel, and $30{ }^{\circ} \mathrm{C}$ for both $7.5 \%$ and $10 \%$ nanogel, the resolution of DNA fragments smaller than $500 \mathrm{bp}$ was similar when comparing a $2.5 \%$ to a $10 \%$ phospholipid nanogel (see Table S-2 in the supporting information). The resolution between the DNA fragments with a length of 900 and $950 \mathrm{bp}$ improved substantially (2.3-fold) when comparing a nanogel of $2.5 \%$ phospholipid to one of $10 \%$ phospholipid. When the separation was performed at the optimum temperature for each lipid preparation (i.e., $25^{\circ} \mathrm{C}$ for $5 \%$ nanogel, and $30{ }^{\circ} \mathrm{C}$ for both $7.5 \%$ and $10 \%$ nanogel), the resolution between fragments of 900 and $950 \mathrm{bp}$ improved 1.8-fold (see Table S-2 in the supporting information). These results indicated that the lipid preparations should be maintained at a concentration of $2.5 \%$ lipid. 
The effect of temperature and composition, factors that impact nanogel morphology, on separation

The ratio of long-to-short-chain lipid determines the shape of the self-assembled aggregate formed in solution. When the relative amount of long chain lipid increases, the aggregates are transformed from a bilayer disk conformation with a small diameter, to disks with larger diameters and 'ribbons' $(19,20)$. The temperature also impacts the transformation from nanodisks to nanoribbons and a substantial change in apparent viscosity is observed over a temperature range specific to the preparation (34-36). The morphologies adopted by the matrix were dependent on the ratio of long-to-short chain lipid as well as the temperature of the preparation. At a total lipid concentration of $2.5 \%$, preparations were examined with a ratio of long-to-short chain lipid of 2.5, 3.0, and 4.0. The results, summarized in Table 2, revealed that the lipid composition had no significant effect on the resolution of DNA fragments under these conditions. When the ratio of longto-short chain lipid was 5.0, the separation performance decreased. The resolution improved marginally for nanogels with different lipid composition when the optimum performance was observed at a temperature different from $23^{\circ} \mathrm{C}$ (see Table S-3 in the supporting information). Although, the performance of the separation matrix was tolerant of changes in lipid composition, the preparation made with a 2.5 ratio of long-to-short chain lipid was used for analytical separations and the effect of temperature on separation performance was tested. The results for a $2.5 \% \mathrm{w} / \mathrm{v}$ preparation composed of [DMPC]/[DHPC] equal to 2.5 are summarized in Table S-4 in the supporting information. The best resolution for both the 450 and 475 bp DNA and the 900 and 950 bp DNA was obtained at $23^{\circ} \mathrm{C}$. At this temperature 450 and 475 bp DNA fragments were separated with 5 bp resolution, and 900 and 950 bp DNA fragments were separated with 8 bp resolution. For both size domains the resolution obtained using a $2.5 \%$ nanogel is $1 \%$ when normalized by dividing the resolution (in bp) by the size of the separated DNA fragments. This is a substantial increase over the $10 \%$ resolution obtained with a commercial system that utilizes a hydroxypropylmethylcellulose matrix for DNA shorter than $1,000 \mathrm{bp}$ (15). This improved sizing obtained with the phospholipid nanogel is an extraordinary advantage for DNA analyses with sieving that require accurate size determinations. 


\section{Expanded size range of sieving using a wormlike nanogel}

These optimization studies confirmed that decreasing the concentration of the lipid preparation separated a wider range of DNA fragments than previously reported. This is demonstrated in Figure 1, which depicts the separation of the 1kilobase DNA ladder. The separation performance obtained with the DNA size ladder improves by successively decreasing the viscosity of the nanogel used for sieving DNA. Figure 1 trace $A$ is of $10 \%$ lipid with [DMPC]/[DHPC] equal to 2.5 at $30{ }^{\circ} \mathrm{C}$, which is the higher viscosity formulation and provides accurate sizing for DNA fragments smaller than $500 \mathrm{bp}$ in length. When the temperature of the $10 \%$ nanogel matrix is decreased the viscosity is decreased as well. The trace in Figure $1 \mathrm{~B}$ obtained using a $10 \%$ nanogel matrix at a separation temperature of $23^{\circ} \mathrm{C}$ demonstrates some improvement in the resolution of larger fragments ranging from 1,000 to $12,000 \mathrm{bp}$ in length. The trace in Figure $1 \mathrm{C}$ is obtained using a $2.5 \%$ nanogel matrix at a separation temperature of $30^{\circ} \mathrm{C}$. This material has a lower viscosity than the $10 \%$ nanogel matrix and relative to the $10 \%$ nanogel the resolution is improved for the fragments ranging from 1,000 to 12,000 base pairs. Figure 1 trace $D$ is of the optimized preparation of $2.5 \%$ lipid with [DMPC]/[DHPC] equal to 2.5 at $23{ }^{\circ} \mathrm{C}$. This nanogel has the lowest viscosity and generates the best resolution for DNA fragments larger than $500 \mathrm{bp}$. For DNA fragments shorter than $500 \mathrm{bp}$, the performance of the optimized preparation was similar. The resolution of 450 and $475 \mathrm{bp}$ fragments decreased from 6.2 , using the $10 \%$ phospholipid preparation with $[\mathrm{DMPC}] /[\mathrm{DHPC}]=2.5$ at $30{ }^{\circ} \mathrm{C}$ (see Table S-2), to 5.0, using the optimized preparation of $2.5 \%$ phospholipid with $[D M P C] /[D H P C]=2.5$ at $23^{\circ} \mathrm{C}$. This slight decrease in resolution performance to $78 \%$ of the previously reported value of 6.2 , did not limit the application of the material to analyze and accurately calculate the size of these PCR amplicons.

\section{Application to assess length polymorphism of PCR amplicons derived from pathogenic bacteria}

PCR amplicons were identified using bioinformatics to predict unique DNA biomarkers of different strains of Streptococcus pyogenes ranging from 634 to 1,332 bp in length. Over 700 million infections annually are attributed to this pathogenic bacterium associated with common throat and skin diseases with more than 0.5 million deaths resulting from 
invasive and autoimmune complications (37). Strains are typically characterized epidemiologically by serological typing of the $\mathrm{M}$ protein or, more recently, by sequencing of the emm gene (38-40). However, epidemiological surveillance during outbreaks of infection requires additional tools to distinguish between strains of the same M-type. The scl2 gene, encoding a common $S$. pyogenes surface protein, exhibits significant length polymorphism between strains of the same M-type, due to the presence of repetitive Gly$X-Y$ motifs in the collagenous domain $(28,41)$. Strain typing by length variation in collagenlike genes has been successfully demonstrated for the bcl genes of Bacillus anthracis (42) and is a viable strategy for typing $S$. pyogenes. The scl2 alleles from strains of Mtypes 3,12 , and 28 were selected here as epidemiological markers since these are among the most prevalent M-types causing infection in the United States and globally $(43,44)$. Importantly, M3-type strains are associated with invasive diseases like necrotizing fasciitis and streptococcal toxic shock syndrome (43,45-47).

Primers were designed to generate PCR amplicons of sc/2 alleles associated with each M-type. The $2.5 \%$ phospholipid preparation was utilized to evaluate the applicability of the method for the analysis of PCR amplicons. Three amplicons were separated, as shown in Figure 2a, and sized with internal standards of 600 and 1,000 bp. The $634 \mathrm{bp}$ and $715 \mathrm{bp}$ amplicons, which were sequenced in the genome of three different M12-type strains, and the 842 bp amplicon, which was sequenced in the M28-type strain, were detected and sized using the phospholipid nanogel. The remaining amplicons $(1,071$, 1,152, and 1,332 bp) sequenced in the M3-type strains were detected and sized (see Fig. $2 \mathrm{~b})$ using internal standards of 1,000 and $1,500 \mathrm{bp}$. The extended sizing range of the phospholipid nanogel enabled detection of larger sc/2-derived amplicons to generate unique DNA markers that distinguish strains of $S$. pyogenes. To further demonstrate the effectiveness of the phospholipid nanogel for size determination, a previously reported amplicon of $b c /$ gene that is 1,446 bp in length (42) was analyzed using internal standards of 1,000 and 1,650 bp as shown in Figure 2c.

\section{Characterization of the difference between true and measured size (bias)}

To demonstrate that the phospholipid preparation optimized for the separation of large DNA fragments remained effective for the analysis of DNA fragments below $500 \mathrm{bp}$ in 
size, unique biomarkers of several Aspergillus species were analyzed (see Figure S-1 in the supporting information). This fungus leads to increased mortality among immunocompromised patients suffering from invasive aspergillosis (48-51) and is also associated with crop contamination (52). Detection of Aspergillus through unique DNA sequences is significant because PCR methods are faster and more conclusive than the traditional means of identifying Aspergillus species based on morphology observed in microbiology laboratory cell cultures. Unique sequences in Aspergillus collagen-like genes ( $a c l$ ) found in five pathogenic species were targeted by PCR resulting in amplicons of distinct lengths: A. nidulans 227 bp; A. terreus 262 bp; A. niger 297 bp; A. flavus 402 bp; and A. fumigatus 338 and $489 \mathrm{bp}$. Using internal standards of 150 and $450 \mathrm{bp}$ to calculate the size, these six biomarkers were resolved in a capillary with $30.2 \mathrm{~cm}$ effective length and a separation additive containing $2.5 \%$ phospholipid preparation of [DMPC]/[DHPC] equal to 2.5 at $23^{\circ} \mathrm{C}$. This separation additive optimized for larger DNA fragments estimated the size with a bias ranging from 0-6 bp (see Table 3), which represented a size accuracy within $2.5 \%$ of the true fragment size. Previously, these biomarkers were separated using a $40 \mathrm{~cm}$ effective length and a 10\% phospholipid preparation of [DMPC]/[DHPC] equal to 2.5 at $30^{\circ} \mathrm{C}(29)$. In that study, the amplicon size was estimated using three size standards of 150, 250, and $350 \mathrm{bp}$ with a bias ranging from 1-7 bp, which represented a size accuracy within $2 \%$ of the true fragment size.

The analysis of DNA biomarkers for strains of $S$. pyogenes revealed that the phospholipid nanogel generated a relative bias in size accuracy less than 1\% (2-4 bp bias) of the total fragment size for 634,715 and $842 \mathrm{bp}$, and increased to $2 \%, 3 \%$, and $4 \%$ (19-48 bp bias) of the total fragment size for 1,071, 1,152, and 1,332 bp, respectively (Table 4). The analysis of the 1,446 bp PCR amplicon for the $b c / C$ gene of Bacillus anthracis shown in Figure 2c had an estimated size of 1,449 \pm 8 bp $(n=5)$, generating a size bias of less than $1 \%$. Each single Gly-X-Y repeating unit in $s c / 2$ or $b c /$ is encoded by $9 \mathrm{bp}$. Thus, the phospholipid nanogel will separate two distinct $s c / 2$ or $b c /$ alleles differing only by one Gly-X-Y repeat at the $\sim 500-850$-bp range and by 1-5 Gly-X-Y repeats at the $\sim 1,000-1,400$-bp range. The phospholipid nanogel performance is similar to the values reported for a commercial system that utilizes hydroxyethyl cellulose, which generated a 
size bias ranging from $0-7 \%$ for 119 to $1270 \mathrm{bp}$ DNA fragments (15). Clearly, the material can be used for DNA fragments approaching $1,500 \mathrm{bp}$.

This is a valuable assay to be used alone or in combination with other epidemiological markers to trace the emergence of new strains with a higher discriminatory power. Accurate size evaluation for any extended repeating sequence will also be an important tool assisting in long-range genomic assemblies produced from short (50-150 bp) DNA sequences obtained using next generation sequencers (53).

\section{Advanced material properties enable in-capillary sample stacking}

Continuous nanogels were used to generate the data in Figures 1 and 2 as well as Tables 1-4; however, the separations in Figure 1 confirmed that DNA had a faster mobility in the $2.5 \%$ phospholipid preparation than in the $10 \%$ preparation, which also has a substantially higher apparent viscosity. The different viscosity of the $10 \%$ vs the $2.5 \%$ phospholipid nanogels was integrated to create a discontinuous nanogel to stack DNA within a small plug of the highly viscous $10 \%$ phospholipid nanogel and then separate the DNA in the low viscosity $2.5 \%$ nanogel that yielded higher separation performance. These two nanogel preparations were introduced serially into the separation capillary. DNA was stacked using a $10 \mathrm{kV}, 30$ second injection in the discontinuous nanogel as shown in Figure $3 \mathrm{~A}$. At a temperature of $19{ }^{\circ} \mathrm{C}$, the capillary was first filled with the $2.5 \%$ nanogel for 3 minutes at $140 \mathrm{kPa}$ (20 psi). This was followed by a small plug of $10 \%$ nanogel introduced for 10 seconds at $69 \mathrm{kPa}(10 \mathrm{psi})$, and finally a pre-plug of aqueous buffer was introduced for 15 seconds at $34 \mathrm{kPa}$ (5 psi). The temperature was raised to $30{ }^{\circ} \mathrm{C}$ to increase the viscosity, which caused the $10 \%$ phospholipid to form a highly viscous nanogel. The DNA was electrokinetically-injected at $30^{\circ} \mathrm{C}$ and stacked. The temperature was then dropped to $23^{\circ} \mathrm{C}$ and the separation proceeded. The enhancement in peak area achieved with stacking is apparent by comparing the electropherograms shown in Figure $3 \mathrm{~A}$ with $3 \mathrm{~B}$ and $3 \mathrm{C}$. The DNA in the electropherogram in Figure $3 \mathrm{~B}$, obtained with a $10 \mathrm{kV}, 2$ second injection in a discontinuous nanogel, was difficult to detect. The peaks were broad when the large $10 \mathrm{kV}, 30$ second injection was performed in the absence of the stacking gel as shown in Figure $3 \mathrm{C}$. The plate count of the $600 \mathrm{bp}$ peak in Figure $3 \mathrm{C}$ $(40,000$ theoretical plates) was 12 -fold lower than that obtained with stacking in Figure 
3A (500,000 theoretical plates). Integrating both materials offered a means to harness advantages of each medium for unique processes of injection and separation of large DNA fragments.

\section{CONCLUSIONS}

A practical alternative to slab gels was developed to separate and size DNA fragments as large as $1,500 \mathrm{bp}$, with a size accuracy ranging from $1-4 \%$. The material was compatible with the higher concentration medium reported previously (21) and was suitable for thermally controlled gel-stacking and separations. By decreasing the concentration of the phospholipid preparation from $10 \%$ to $2.5 \%$, the net cost of the dynamic sieving gel was reduced. The ease with which the dynamic sieving nanogel was introduced into capillary channels was ideal for microscale separations because the low flow resistance required only nominal pressures for loading. This holds potential to benefit microfluidic separations as well because when the separation device can be used repeatedly, the lifetime of the device is increased, which in turn decreases the overall cost of the analytical measurement.

\section{ACKNOWLEDGMENTS}

We thank Soo Jeon Choi for technical assistance on the project. This report is based upon work supported by the National Science Foundation under Grant No. CHE1212537. We acknowledge support from the National Science Foundation, including support for B.A.B. through the WVNano Graduate Fellowship Program (Cooperative Agreement 1003907). 


\section{REFERENCES}

1. Chakraborty, R., Stivers, D. N., Su, B., Zhong, Y., and Budowle, B. (1999) The utility of short tandem repeat loci beyond human identification: Implications for development of new DNA typing systems. Electrophoresis 20, 1682-1696

2. Turenne, C. Y., Sanche, S. E., Hoban, D. J., Karlowsky, J. A., and Kabani, A. M. (1999) Rapid identification of fungi by using the ITS2 genetic region and an automated fluorescent capillary electrophoresis system. J. Clin. Microbiol. 37, 1846-1851

3. Chen, Y.-C., Eisner, J. D., Kattar, M. M., Rassoulian-Barrett, S. L., Lafe, K., Bui, U., Limaye, A. P., and Cookson, B. T. (2001) Polymorphic internal transcribed spacer region 1 DNA sequences identify medically important yeasts. J. Clin.

Microbiol. 39, 4042-4051

4. Klepárník, K., and Boček, P. (2007) DNA diagnostics by capillary electrophoresis. Chem. Rev. (Washington, DC, U. S.) 107, 5279-5317

5. Castro-Puyana, M., García-Cañas, V., Simó, C., and Cifuentes, A. (2012) Recent advances in the application of capillary electromigration methods for food analysis and Foodomics. Electrophoresis 33, 147-167

6. Lagally, E. T., Scherer, J. R., Blazej, R. G., Toriello, N. M., Diep, B. A., Ramchandani, M., Sensabaugh, G. F., Riley, L. W., et al. (2004) Integrated portable genetic analysis microsystem for pathogen/infectious disease detection. Anal. Chem. 76, 3162-3170

7. Swerdlow, H., and Gesteland, R. (1990) Capillary gel electrophoresis for rapid, high resolution DNA sequencing. Nucleic Acids Res. 18, 1415-1419

8. Drossman, H., Luckey, J. A., Kostichka, A. J., D'Cunha, J., and Smith, L. M. (1990) High-speed separations of DNA sequencing reactions by capillary electrophoresis. Anal. Chem. 62, 900-903

9. Venter, J. C., Adams, M. D., Myers, E. W., Li, P. W., Mural, R. J., Sutton, G. G., Smith, H. O., Yandell, M., et al. (2001) The sequence of the human genome. Science 291, 1304-1351

10. Grossman, P. D., and Soane, D. S. (1991) Experimental and theoretical studies of DNA separations by capillary electrophoresis in entangled polymer solutions. Biopolymers 31, 1221-1228

11. Viovy, J.-L., and Duke, T. (1993) DNA electrophoresis in polymer solutions: Ogston sieving, reptation and constraint release. Electrophoresis 14, 322-329

12. Andras, G. (1993) Capillary column containing a dynamically cross-linked composition and method of use. (Patent, U. S. ed.

13. Barron, A. E., Blanch, H. W., and Soane, D. S. (1994) A transient entanglement coupling mechanism for DNA separation by capillary electrophoresis in ultradilute polymer solutions. Electrophoresis 15, 597-615

14. Woolley, A. T., and Mathies, R. A. (1994) Ultra-high-speed DNA fragment separations using microfabricated capillary array electrophoresis chips. Proc. Natl. Acad. Sci. U S A 91, 11348-11352

15. Mueller, O., Hahnenberger, K., Dittmann, M., Yee, H., Dubrow, R., Nagle, R., and Ilsley, D. (2000) A microfluidic system for high-speed reproducible DNA sizing and quantitation. Electrophoresis 21, 128-134 
16. Zhou, H., Miller, A. W., Sosic, Z., Buchholz, B., Barron, A. E., Kotler, L., and Karger, B. L. (2000) DNA sequencing up to 1300 Bases in two hours by capillary electrophoresis with mixed replaceable linear polyacrylamide solutions. Anal. Chem. 72, 1045-1052

17. Barron, A. E., Sunada, W. M., and Blanch, H. W. (1996) The effects of polymer properties on DNA separations by capillary electrophoresis in uncross-linked polymer solutions. Electrophoresis 17, 744-757

18. Duke, T., and Viovy, J. L. (1994) Theory of DNA electrophoresis in physical gels and entangled polymer solutions. Physical Review E 49, 2408-2416

19. Harroun, T. A., Koslowsky, M., Nieh, M.-P., de_Lannoy, C.-F., Raghunathan, V. A., and Katsaras, J. (2005) Comprehensive examination of mesophases formed by DMPC and DHPC mixtures. Langmuir 21, 5356-5361

20. Nieh, M. P., Raghunathan, V. A., Glinka, C. J., Harroun, T. A., Pabst, G., and Katsaras, J. (2004) Magnetically alignable phase of phospholipid "bicelle" mixtures is a chiral nematic made up of wormlike micelles. Langmuir 20, 78937897

21. Durney, B. C., Lounsbury, J. A., Poe, B. L., Landers, J. P., and Holland, L. (2013) A Thermo-responsive Phospholipid Pseudo-gel: Tunable DNA Sieving with Capillary Electrophoresis Anal. Chem. 85, 6617-6625

22. Lounsbury, J. A., Bienvenue, J. M., and Landers, J. P. (2012) Sample-to-result STR genotyping systems: Potential and status. Forensic Sci Rev 24, 123-142

23.

http://avantilipids.com/index.php?option=com_content\&view=article\&id=214\&lte $\mathrm{mid}=206 \&$ catnumber $=850345$. (accessed September 24, 2014) 1,2ditetradecanoyl-sn-glycero-3-phosphocholine (DMPC) PC(14:0/14:0), CAS Number 18194-24-6, catalog number 850345P. Avanti Polar Lipids, Inc

24.

http://avantilipids.com/index.php?option=com_content\&view=article\&id=206\&lte mid=206\&catnumber=850305. (accessed September 24, 2014) 1,2-dihexanoylsn-glycero-3-phosphocholine 06:0 PC (DHPC), CAS number 34506-67-7, catalog number 850305P. Avanti Polar Lipids, Inc

25. http://www.lifetechnologies.com/search/global/searchAction.action?query=pop4+polymer\&resultPage $=1$ \&resultsPerPage $=15$ \&autocomplete $=$ true . (accessed September 24, 2014) POP-4® Polymer for 3130/3130xl Genetic Analyzers, 7000 $\mu \mathrm{l}$ (Applied Biosystems ${ }^{\circledR}$ ), catalog number 4352755. 2012 Life Technologies Corporation

26. Luo, R., Archer-Hartmann, S. A., and Holland, L. A. (2010) Transformable capillary electrophoresis for oligosaccharide separations using phospholipid additives. Anal. Chem. 82, 1228-1233

27. Reda, K. B., Kapur, V., Goela, D., Lamphear, J. G., Musser, J. M., and Rich, R. R. (1996) Phylogenetic distribution of streptococcal superantigen SSA allelic variants provides evidence for horizontal transfer of ssa within Streptococcus pyogenes. Infect. Immun. 64, 1161-1165

28. Lukomski, S., Nakashima, K., Abdi, I., Cipriano, V. J., Shelvin, B. J., Graviss, E. A., and Musser, J. M. (2001) Identification and characterization of a second 
extracellular collagen-like protein made by group A streptococcus: control of production at the level of translation. Infect. Immun. 69, 1729-1738

29. Tuntevski, K., Durney, B. C., Snyder, A. K., LaSala, P. R., Nayak, A. P., Green, B. J., Beezhold, D. H., Rio, R. V. M., et al. (2013) Aspergillus collagen-like (acl) genes: identification, sequence polymorphism and assessment for PCR-based pathogen detection. Appl. Environ. Microbiol. 79, 7882-7895

30. White, C. M., Luo, R., Archer-Hartmann, S. A., and Holland, L. A. (2007) Electrophoretic screening of ligands under suppressed EOF with an inert phospholipid coating. Electrophoresis 28, 3049-3055

31. Archer-Hartmann, S. A., Sargent, L. M., Lowry, D. T., and Holland, L. A. (2011) Microscale exoglycosidase processing and lectin capture of glycans with phospholipid assisted capillary electrophoresis separations. Anal. Chem. 83, 2740-2747

32. Zipper, H., Brunner, H., Bernhagen, J. r., and Vitzthum, F. (2004) Investigations on DNA intercalation and surface binding by SYBR Green I, its structure determination and methodological implications. Nucleic Acids Res. 32, e103

33. Dragan, A. I., Pavlovic, R., McGivney, J. B., Casas-Finet, J. R., Bishop, E. S., Strouse, R. J., Schenerman, M. A., and Geddes, C. D. (2012) SYBR Green I: fluorescence properties and interaction with DNA. Journal of Fluorescence 22, 1189-1199

34. Flynn, A., Ducey, M., Yethiraj, A., and Morrow, M. R. (2012) Dynamic properties of bicellar lipid mixtures observed by rheometry and quadrupole echo decay. Langmuir 28, 2782-2790

35. Wu, X., Langan, T. J., Durney, B. C., and Holland, L. A. (2012) Thermally responsive phospholipid preparations for fluid steering and separation in microfluidics. Electrophoresis 33, 2674-2681

36. Pappas, T., and Holland, L. (2008) Fluid steering in a microfluidic chip by means of thermally responsive phospholipids. Sens. Actuators, B, Chem. 128, 427-434

37. Carapetis, J. R., Steer, A. C., Mulholland, E. K., and Weber, M. (2005) The global burden of group A streptococcal diseases. Lancet Infect. Dis. 5, 685-694

38. Beall, B., Facklam, R., Hoenes, T., and Schwartz, B. (1997) Survey of emm gene sequences and T-antigen types from systemic Streptococcus pyogenes infection isolates collected in San Francisco, California; Atlanta, Georgia; and Connecticut in 1994 and 1995. J. Clin. Microbiol. 35, 1231-1235

39. Courtney, H. S., Hasty, D. L., Li, Y., Chiang, H. C., Thacker, J. L., and Dale, J. B. (1999) Serum opacity factor is a major fibronectin-binding protein and a virulence determinant of M type 2 Streptococcus pyogenes. in Mol. Microbiol., WileyBlackwell

40. Johnson, D. R., and Kaplan, E. L. (1993) A review of the correlation of Tagglutination patterns and M-protein typing and opacity factor production in the identification of group A streptococci. J. Med. Microbiol. 38, 311-315

41. Rasmussen, M., and Björck, L. (2001) Unique regulation of SclB - a novel collagen-like surface protein of Streptococcus pyogenes. Mol. Microbiol. 40, 1427-1438

42. Leski, T. A., Caswell, C. C., Pawlowski, M., Klinke, D. J., Bujnicki, J. M., Hart, S. J., and Lukomski, S. (2009) Identification and classification of bcl genes and 
proteins of bacillus cereus group organisms and their application in bacillus anthracis detection and fingerprinting. Appl. Environ. Microbiol. 75, 7163-7172

43. O'Loughlin, R. E., Roberson, A., Cieslak, P. R., Lynfield, R., Gershman, K., Craig, A., Albanese, B. A., Farley, M. M., et al. (2007) The epidemiology of invasive group A streptococcal infection and potential vaccine implications: United States, 2000-2004. Clin. Infect. Dis. 45, 853-862

44. Steer, A. C., Law, I., Matatolu, L., Beall, B. W., and Carapetis, J. R. (2009) Global emm type distribution of group A streptococci: systematic review and implications for vaccine development. The Lancet Infectious Diseases 9, 611-616

45. O'Brien, K. L., Beall, B., Barrett, N. L., Cieslak, P. R., Reingold, A., Farley, M. M., Danila, R., Zell, E. R., et al. (2002) Epidemiology of invasive group A Streptococcus disease in the United States, 1995-1999. Clin. Infect. Dis. 35, 268276

46. Sharkawy, A., Low, D. E., Saginur, R., Gregson, D., Schwartz, B., Jessamine, P., Green, K., McGeer, A., et al. (2002) Severe group A Streptococcal soft-tissue infections in Ontario: 1992-1996. Clin. Infect. Dis. 34, 454-460

47. Musser, J. M., Hauser, A. R., Kim, M. H., Schlievert, P. M., Nelson, K., and Selander, R. K. (1991) Streptococcus pyogenes causing toxic-shock-like syndrome and other invasive diseases: clonal diversity and pyrogenic exotoxin expression. Proc. Natl. Acad. Sci. U. S. A. 88, 2668-2672

48. Dimopoulos, G., Frantzeskaki, F., Poulakou, G., and Armaganidis, A. (2012) Invasive aspergillosis in the intensive care unit. Ann. N. Y. Acad. Sci. 1272, 3139

49. Groll, A. H., Kurz, M., Schneider, W., Witt, V., Schmidt, H., Schneider, M., and Schwabe, D. (1999) Five-year-survey of invasive aspergillosis in a paediatric cancer centre. Epidemiology, management and long-term survival. Mycoses 42, 431-442

50. Latge, J. P. (1999) Aspergillus fumigatus and aspergillosis. Clin Microbiol Rev 12, 310-350

51. Zmeili, O. S., and Soubani, A. O. (2007) Pulmonary aspergillosis: a clinical update. QJM 100, 317-334

52. Yu, J., Chang, P. K., Cary, J. W., Wright, M., Bhatnagar, D., Cleveland, T. E., Payne, G. A., and Linz, J. E. (1995) Comparative mapping of aflatoxin pathway gene clusters in Aspergillus parasiticus and Aspergillus flavus. Appl. Environ. Microbiol. 61, 2365-2371

53. Salzberg, S. L., Phillippy, A. M., Zimin, A., Puiu, D., Magoc, T., Koren, S., Treangen, T. J., Schatz, M. C., et al. (2012) GAGE: A critical evaluation of genome assemblies and assembly algorithms. Genome Res. 22, 557-567 
A)

I0.25 RFU

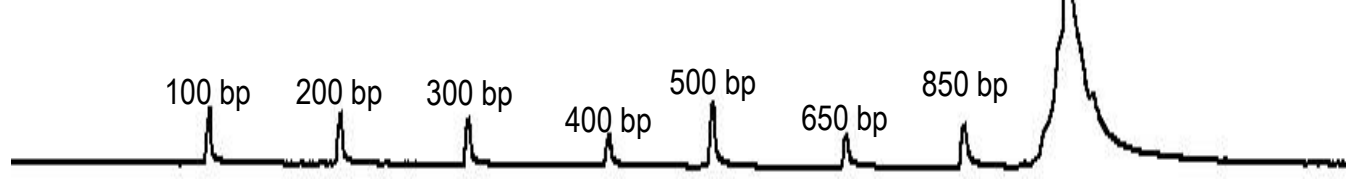

B) 2,000-12,000 bp
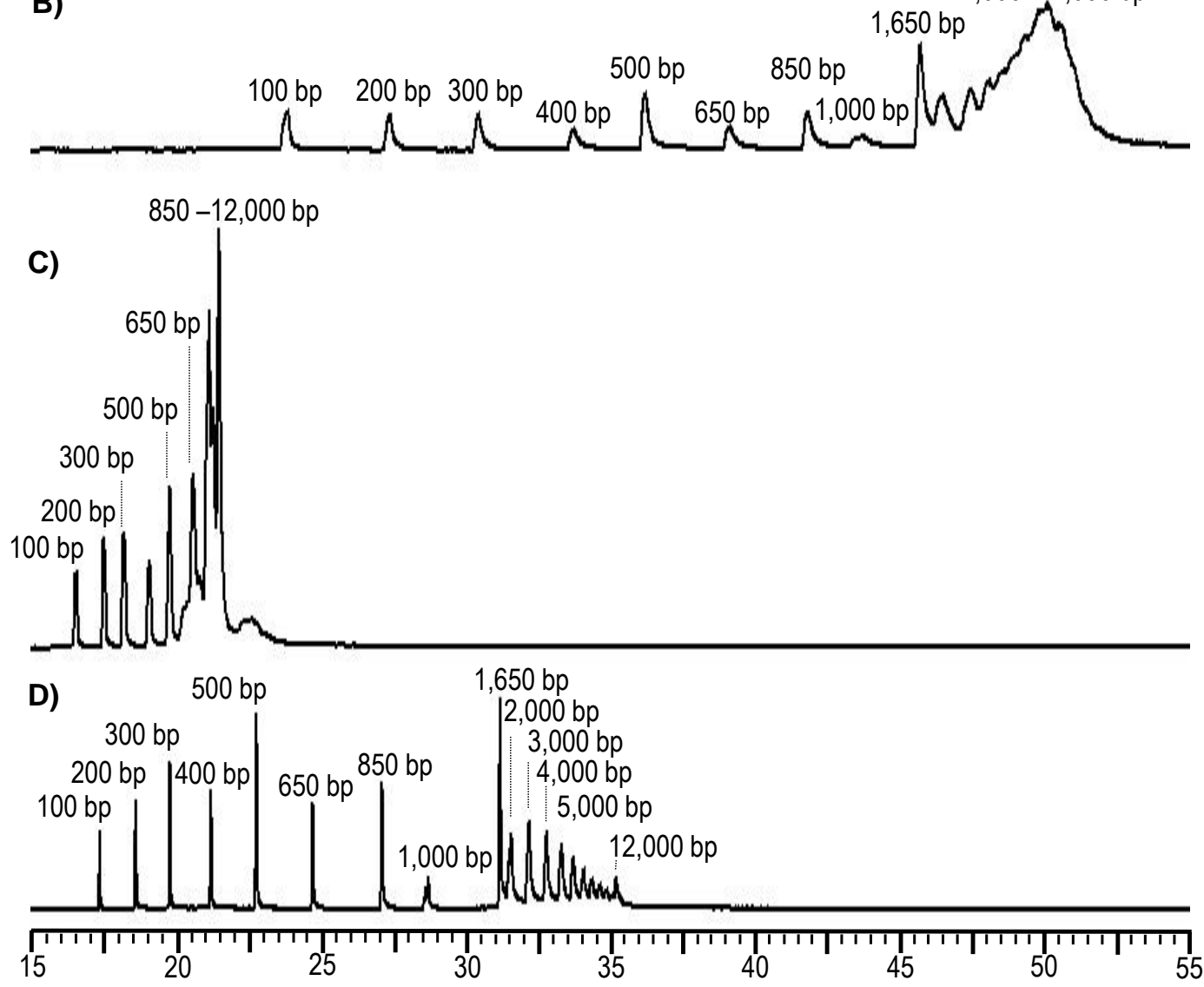

Time (min) 


\section{Figure 1}

Separations obtained at different phospholipid concentration and run temperature. Electropherograms of a 1 kilobase DNA ladder are detected using SYBR Green 1 fluorescent dye using a phospholipid nanogel with $[\mathrm{DMPC}] /[\mathrm{DHPC}]=2.5$ : $(\mathrm{A})$ at a concentration of $10 \%$ and temperature of $30{ }^{\circ} \mathrm{C},(\mathrm{B})$ at a concentration of $10 \%$ and temperature of $23{ }^{\circ} \mathrm{C},(\mathrm{C})$ at a concentration of $2.5 \%$ and temperature of $30{ }^{\circ} \mathrm{C}$, and (d) at a concentration of $2.5 \%$ and temperature of $23{ }^{\circ} \mathrm{C}$. The DNA fragments in the ladder (Life Technologies, \#10787-018) range from 100-12,000 bp and the size in bp specified by the manufacturer is indicated above each corresponding peak. 

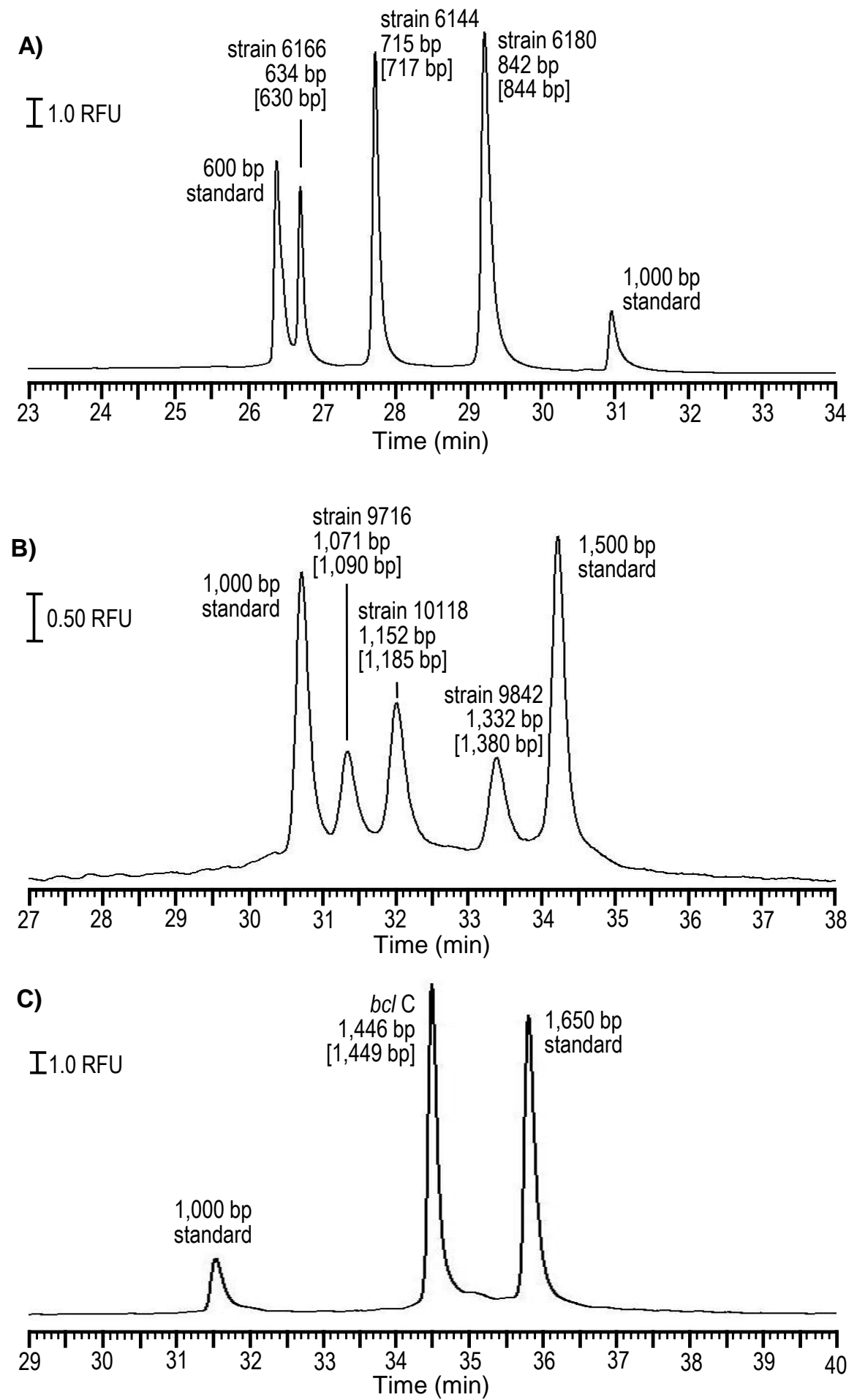

Figure 2

Separation of PCR amplicon obtained with the phospholipid nanogel optimized for DNA up to 1,500 bp in length. Electropherograms detected using SYBR Green 1 fluorescent dye and a phospholipid nanogel with [DMPC]/[DHPC] $=2.5$ at a concentration 
of $2.5 \%$ and temperature of $23{ }^{\circ} \mathrm{C}$. The true sizes are specified above each peak while the measured sizes are denoted in the brackets. The peaks for each marker are labeled to indicate: (A) the three scl2 strains designated 6166, 6140,6180 (B) the three scl2 strains designated $9716,10118,9842$, and (C) bcl C. The separation is accomplished using a capillary that is $40 \mathrm{~cm}$ long, $25 \mu \mathrm{m}$ id, with an effective length of $30.2 \mathrm{~cm}$ and Eapp $=100 \mathrm{~V} / \mathrm{cm}$. 
A) Stack and Separate

Inject at $30^{\circ} \mathrm{C}, 10 \mathrm{kV} 30 \mathrm{sec}$
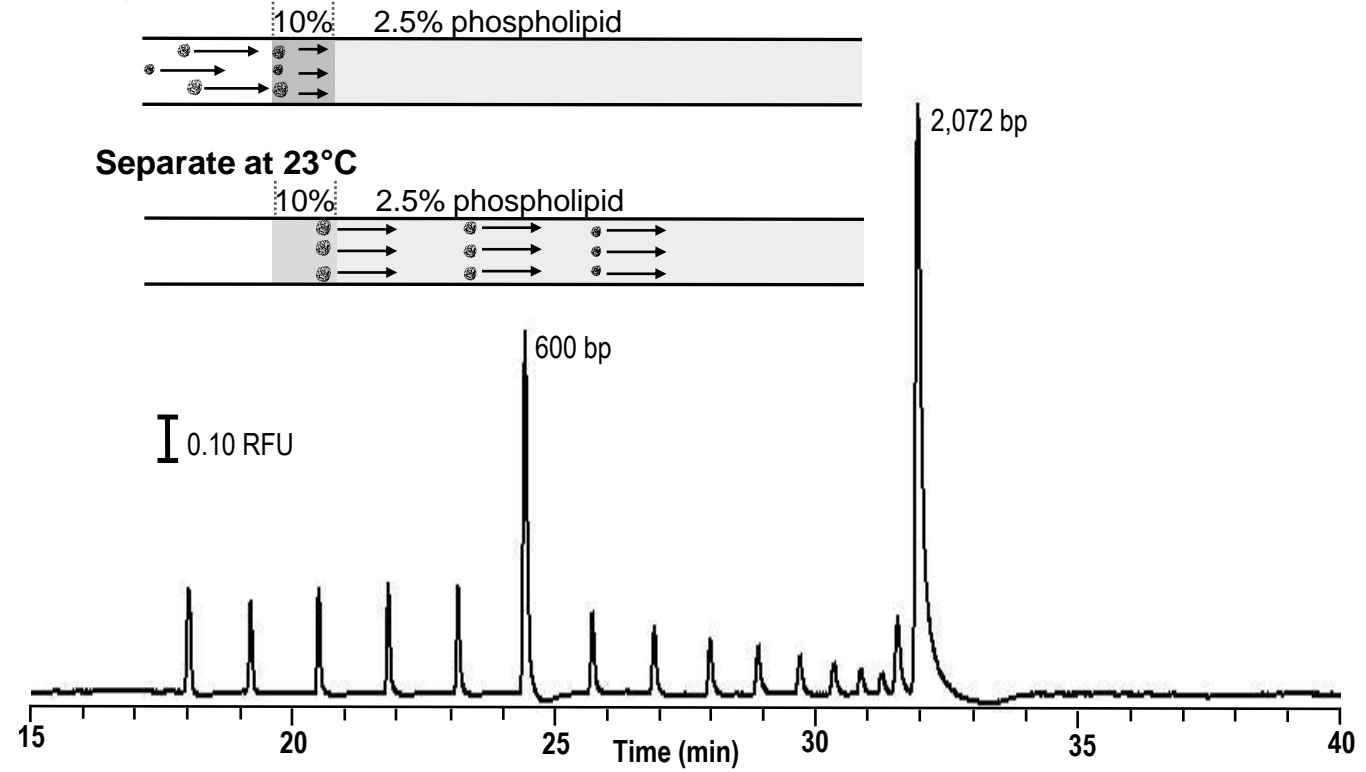

B) Inject (10kV 2 sec), Separate

$10 \% \quad 2.5 \%$

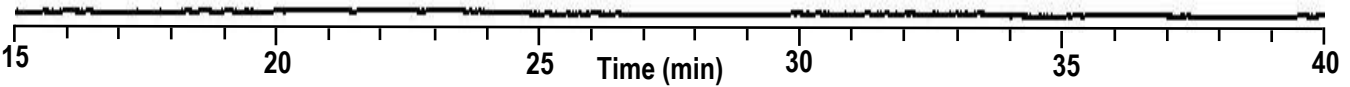

C) No Stacking Gel

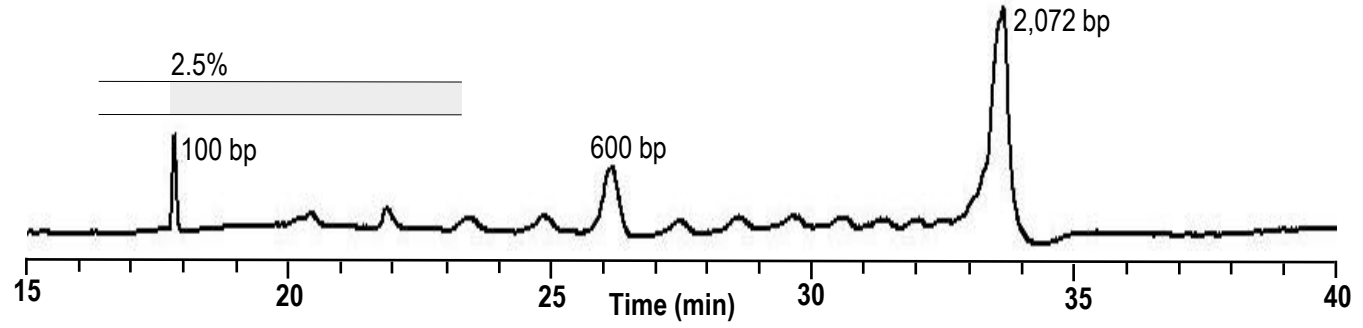

Figure 3

DNA stacking with phospholipid additives. Depicts the process of $(A)$ discontinuous phospholipid nanogels in which the DNA is stacked in the $10 \%$ phospholipid and separated in the $2.5 \%$ phospholipid, (B) discontinuous phospholipid nanogels in which the $10 \mathrm{kV} 2 \mathrm{sec}$ injection is too short to stack DNA, and (C) a continuous nanogel using a $10 \mathrm{kV} 30 \mathrm{sec}$ injection without a stacking plug. The separation is accomplished using a capillary that is $40 \mathrm{~cm}$ long, $25 \mu \mathrm{m}$ id, with an effective length of $30.2 \mathrm{~cm}$ and $E_{\text {app }}=100$ $\mathrm{V} / \mathrm{cm}$. 
Table 1. Effect of concentration on resolution ${ }^{\mathrm{a}}$

\begin{tabular}{|c|c|c|c|c|}
\hline \multirow{2}{*}{$\begin{array}{c}\text { Phospholipid } \\
(\%)\end{array}$} & \multicolumn{2}{|c|}{ Resolution 450/475 bp DNA } & \multicolumn{2}{|c|}{ Resolution 900/950 bp DNA } \\
\hline & Resolution $(\mathrm{CV})^{\mathrm{b}}$ & in $b p^{c}$ & Resolution $(\mathrm{CV})^{\mathrm{b}}$ & in $b p^{c}$ \\
\hline 2.5 & $5.0(6)$ & 5 & $6.4(7)$ & 8 \\
\hline 5.0 & $4.6(10)$ & 5 & $4.3(10)$ & 12 \\
\hline 7.5 & $4.5(9)$ & 6 & $3.4(10)$ & 15 \\
\hline 10.0 & $4.4(10)$ & 6 & $2.8(9)$ & 18 \\
\hline
\end{tabular}


Table 2. Effect of [DMPC]/[DHPC] ratio on resolution at $23^{\circ} \mathrm{C}^{a}$

\begin{tabular}{|c|c|c|c|c|}
\hline \multirow{2}{*}{$\begin{array}{l}\text { [DMPC] } \\
\text { [DHPC] }\end{array}$} & \multicolumn{2}{|c|}{ Resolution 450/475 bp DNA } & \multicolumn{2}{|c|}{ Resolution 900/950 bp DNA } \\
\hline & Resolution (CV) & $\ln \mathrm{bp}$ & Resolution (CV) & $\ln \mathrm{bp}$ \\
\hline 2.5 & $5.0(6)$ & 5 & $6.4(7)$ & 8 \\
\hline 3.0 & $4.4(10)$ & 6 & $5.2(10)$ & 10 \\
\hline 4.0 & $4.8(10)$ & 5 & $5.5(10)$ & 9 \\
\hline 5.0 & $1.1(20)$ & 23 & $1.2(20)$ & 42 \\
\hline
\end{tabular}


Table 3. Performance of 2.5\% phospholipid for Aspergillus biomarkers

\begin{tabular}{lcccc}
\hline Species & True size in bp & Measured size in bp & (CV) & Bias in bp $^{\mathrm{c}}$ \\
\hline A. nidulans & 227 & 227 & $(0.3)$ & 0 \\
A. terreus & 262 & 256 & $(0.4)$ & 6 \\
A. niger & 297 & 301 & $(0.3)$ & 4 \\
A. fumigatus & 338 & 333 & $(0.5)$ & 5 \\
A. flavus & 402 & 402 & $(0.1)$ & 0 \\
A. fumigatus & 489 & 495 & $(0.4)$ & 6 \\
\hline
\end{tabular}

\footnotetext{
aTrue size established as described in [26]. A. fumigatus derived amplicons of gene aclF1-5' and $-3^{\prime}$ were sequenced and represent true sizes of 338, $489 \mathrm{bp}$. Amplicons derived from $A$. terreus (gene ac/T2-5'), A. nidulans (gene aclNI-3'), and A. niger (gene ac/Ni-5') were based on bioinformatics and were also partially sequenced with forward, but not with reverse, primers that were used to generate these amplicons. The amplicon from A. flavus (gene aclFL-3') was sequenced in full and the size of this amplicon is 402 bp.

bSeparation conditions are identical to those reported in Table 1. Size standards of 150 and $450 \mathrm{bp}$ are used.

'Bias is calculated as the difference between the true fragment size and the measured size.
} 
Table 4. Performance of $2.5 \%$ phospholipid for Streptococcus biomarkers

\begin{tabular}{lcccc}
\hline Strain, type & True size in bp $^{\mathrm{a}}$ & Measured size in bp & (CV) & Bias in bp $^{\mathrm{c}}$ \\
\hline $6166, \mathrm{M} 12$ & 634 & 630 & $(0.2)$ & 4 \\
$6144, \mathrm{M} 12$ & 715 & 717 & $(0.4)$ & 2 \\
$6180, \mathrm{M} 28$ & 842 & 844 & $(0.5)$ & 2 \\
$9716, \mathrm{M} 3$ & 1071 & 1090 & $(0.4)$ & 19 \\
10118, M3 & 1152 & 1185 & $(0.3)$ & 33 \\
$9842, \mathrm{M} 3$ & 1332 & 1380 & $(0.2)$ & 48 \\
\hline
\end{tabular}

aTrue size established using DNA sequencing as reported in reference [27].

bSeparations conditions are identical to those reported in Table 1. For strains 6166, 6144, and 6180 the size is determined using 600 and 1,000 bp standards. For strains 9716 , 10118 , and 9842 , the size is determined using 1,000 and 1,500 bp standards

'Bias is calculated as the difference between the true fragment size and the measured size. 


\title{
CHAPTER 7
}

\section{A UNIQUE SET OF THE BURKHOLDERIA COLLAGEN-LIKE PROTEINS PROVIDES INSIGHT INTO PATHOGENESIS, GENOME EVOLUTION AND NICHE ADAPTATION, AND INFECTION DETECTION}

Beth A. Bachert, Soo J. Choi, Anna K. Snyder, Rita V.M. Rio, Brandon C. Durney, Lisa A. Holland, Kei Amemiya, Susan L. Welkos, Joel A. Bozue, Christopher K. Cote, Rita Berisio, and Slawomir Lukomski

Published in PLOS One 2015 10(9)

\begin{abstract}
Burkholderia pseudomallei and Burkholderia mallei, classified as category B priority pathogens, are significant human and animal pathogens that are highly infectious and broad-spectrum antibiotic resistant. Currently, the pathogenicity mechanisms utilized by Burkholderia are not fully understood, and correct diagnosis of $B$. pseudomallei and $B$. mallei infection remains a challenge due to limited detection methods. Here, we provide a comprehensive analysis of a set of 13 novel Burkholderia collagen-like proteins (Bucl) that were identified among $B$. pseudomallei and $B$. mallei select agents. We infer that several Bucl proteins participate in pathogenesis based on their noncollagenous domains that are associated with the components of a type III secretion apparatus and membrane transport systems. Homology modeling of the outer membrane efflux domain of Bucl8 points to a role in multi-drug resistance. We determined that bucl genes are widespread in B. pseudomallei and B. mallei; Fischer's exact test and Cramer's $\mathrm{V}^{2}$ values indicate that the majority of bucl genes are highly associated with these pathogenic species versus nonpathogenic $B$. thailandensis. We designed a bucl-based quantitative PCR assay which was able to detect $B$. pseudomallei infection in a mouse with a detection limit of 50 CFU. Finally, chromosomal mapping and phylogenetic analysis of bucl loci revealed considerable genomic plasticity and adaptation of Burkholderia spp. to host and environmental niches. In this study, we identified a large set of phylogenetically unrelated bucl genes commonly found in Burkholderia select agents, encoding predicted pathogenicity factors, detection targets, and vaccine candidates.
\end{abstract}




\section{INTRODUCTION}

Collagen structure is formed by three polypeptide chains of continuous repetitive GlyXaa-Yaa (GXY) sequence, each adopting left handed polyproline II type helices that combined form a right-handed superhelix (1). It is a universal structure that is broadly found among members of all three domains of life. It is the most abundant protein in mammals where it harbors important structural functions in the extracellular matrix and in support of cell adhesion, differentiation and growth $(2,3)$. The prokaryotic collagen was identified and studied more recently, and has similar GXY sequence and triple helical structure (4-8). In mammalian collagens, proline (Pro) in the $Y$ position is hydroxylated post-translationally and resulting Hyp (hydroxyproline) residues confer the maximum stability to the triple helix. As bacteria lack the prolyl hydroxylase required for these residues, bacterial collagens must be stabilized by other mechanisms, including increased proline content and electrostatic interactions between amino acid side chains (9-12). Several bacterial collagen-like proteins have been shown to form stable triple helices, including streptococcal collagen-like proteins 1 and 2 of Streptococcus pyogenes $(4,13), \mathrm{rCL}_{\mathrm{cp}}$ from Clostridium perfringens (14), and BclA of Bacillus anthracis $(15,16)$. Bacterial collagen-like proteins are found in species that are pathogenic to humans and animals (5-8,16-22). They are often surface-exposed and participate in important pathogenesis processes, including adherence and biofilm formation, host colonization and immune evasion (6,7,18,19,23-30). Several collagen-like genes have been evaluated as biomarkers for pathogen detection by targeting their conserved non-collagenous regions $(31,32)$ and for strain fingerprinting by targeting highly polymorphic repetitive collagen-like sequences (32-35).

The Burkholderia species are ubiquitous in the environment but also include animal and plant pathogens. A group of 17 closely related species, designated $B$. cepacia complex organisms, cause pulmonary infections primarily in patients with cystic fibrosis (36). Two other species, Burkholderia pseudomallei and Burkholderia mallei, are significant human and animal pathogens in endemic regions and also represent biowarfare threats. These bacteria have been classified as category B priority pathogens, in part due to their high infectivity, an intrinsic broad-spectrum antibiotic resistance, and previous use as biological weapons during wartime (37). B. pseudomallei is a soil 
saprophyte endemic to southeastern Asia and northern Australia, which causes melioidosis in humans. Melioidosis has a variety of clinical outcomes, from localized skin infection to pneumonia and acute septicemia, as well as chronic illness with abscess formation in major organs (38). As $50 \%$ of patients with septicemic melioidosis die within 48 hours, rapid diagnosis is crucial to patient survival (39). B. pseudomallei has a large genome of about $7.2 \mathrm{Mb}$, which undergoes frequent horizontal gene transfer as evidenced by multiple genomic islands that differ between strains (40). B. mallei is a closely related bacterium with a smaller genome, $\sim 5.8 \mathrm{Mb}(41)$. It is the causative agent of glanders in horses and other animals that can be transmitted to humans. It has been demonstrated by multi-locus sequence typing analysis that $B$. mallei is a clonal derivative of $B$. pseudomallei (42), which has undergone significant genomic reduction and rearrangement during host-adaptation (41). Consequently, $B$. mallei is unable to survive outside the host. $B$. mallei was one of the first microbes to be weaponized during World War I to infect livestock and humans (37). A third closely related organism, $B$. thailandensis, is considered non-pathogenic for humans (43). $B$. thailandensis is also a soil saprophyte with a large genome of $\sim 6.7 \mathrm{Mb}$, which is endemic to geographical regions coinciding with $B$. pseudomallei $(43,44)$; therefore, it is necessary to differentiate between the two species.

In this study, we identified and characterized an unexpectedly large set of 13 distinct Burkholderia collagen-like (bucl/Bucl) genes and proteins that are conserved in pathogenic $B$. pseudomallei and $B$. mallei species. We report the widespread presence of buclgenes in B. pseudomallei and B. mallei assessed by bioinformatics and analytical $\mathrm{PCR}$, explore their phylogenetic relationships, infer important pathogenicity traits and antibiotic resistance mechanisms associated with Bucl proteins, and demonstrate the use of buclgenes as detection markers for these select agents in an animal model of infection. 


\section{RESULTS}

\section{Identification of Burkholderia collagen-like (bucl) genes}

An increasing number of collagen-like proteins have recently been identified and studied in a variety of bacterial species, including Gram-positive pathogenic group A (5-8,17), B (SL, unpublished data), C $(20,45)$ streptococci and pneumococci $(18)$, bacilli and clostridia $(16,21,32)$, as well as Gram-negative respiratory pathogen Legionella pneumophila ((19); SL, unpublished data). Here, we assessed the presence and distribution of the collagen-like proteins among Burkholderia species in the Pfam collagen family database (PF01391). We identified a total of 85 sequences among the members of the Burkholderiaceae family, with 77 of these sequences designated Burkholderia collagen-like (Bucl) proteins, among various species of the Burkholderia genus. We next focused on 59 protein sequences found in three closely related species of Burkholderia, B. pseudomallei $(\mathrm{Bp})$, B. mallei $(\mathrm{Bm})$, and B. thailandensis (Bt) that we initially categorized into 16 (Bucl1-16) protein types, based on domain organization and GXY-repeat types in their collagen-like $(\mathrm{CL})$ regions; subsequent refinement eliminated three Bucl types, resulting in 13 Bucl proteins $1,2,3,4,5,6,7,8,10,13,14,15$, and 16. To assess their distribution, nucleotide sequences of these $13 \mathrm{bucl}$ genes were used as independent queries to BLASTn-search the NCBI nonredundant database. Though we observed collagen-like sequences in other Burkholderia species, this set of $13 \mathrm{bucl}$ genes and proteins were unique to $\mathrm{Bp}, \mathrm{Bm}$, and $\mathrm{Bt}$ species.

\section{Identification of bucl genes in Bp K96243, proof of principle}

A BLAST search of bucl alleles from various strains against the genome sequence of the reference strain Bp K96243 revealed that all 13 bucl genes were present and were distributed around both chromosomes (Fig. 1A). Six bucl genes were localized on chromosome one and seven bucl genes on chromosome two, and were found on both plus and minus strands (Fig. 1A, B). The presence of each bucl gene in Bp K96243 genome was confirmed by PCR with primers targeting the noncollagenous regions (Fig. 1C). Mapping of bucl genes in additional seven $\mathrm{Bp}$ and four $\mathrm{Bm}$ fully sequenced genomes revealed significant intra- and inter-species genomic rearrangements involving bucl loci (Fig. 2). For example, the region encoding bucl genes 6, 8, and 10 in Bp 668 was inverted 
compared to $\mathrm{Bp} \mathrm{K} 96243$ genome (Fig. 2A). Additionally, we observed both rearrangements (Fig. 2B, C) and deletions of bucl loci (Fig. 2C) in Bm genomes, compared to $\mathrm{Bp}$, which is consistent with $\mathrm{Bm}$-genomic plasticity as well as the evolution of $\mathrm{Bm}$ from $\mathrm{Bp}$ through genome reduction $(41,46)$. To further characterize the genomic organization of these strains, organizational patterns (OP) of bucl biomarkers were assigned according to their position and orientation on each chromosome (Table 1). In aggregate, chromosomal rearrangements occur more frequently on chromosome one (six

distinct organizational patterns were observed for both $\mathrm{Bp}$ and $\mathrm{Bm}$ strains analyzed) compared to chromosome two (three organizational patterns observed). While only one major organizational pattern on chromosome 1, Ch1 OPII, was found exclusively among Bp strains, major organizational pattern on chromosome 2, Ch2 OPI, was found in both $\mathrm{Bp}$ and $\mathrm{Bm}$ genomes. All observed rearrangements were intrachromosomal in both species, indicating no exchange of genetic material involving bucl markers occurred between the chromosomes. In summary, consistent with bioinformatic data, we here confirmed by PCR the presence of all 13 bucl genes in Bp K96243. We also captured significant genomic plasticity of the $\mathrm{Bp}$ and $\mathrm{Bm}$ species by employing bucl markers.

\section{Characterization of Bucl proteins}

Overall characteristics of Bucl proteins were examined in a set of geographically diverse Burkholderia strains sequenced, including $13 \mathrm{Bp}, 11 \mathrm{Bm}$, and $9 \mathrm{Bt}$ strains (Table 2, Table 3). All 13 Bucl proteins identified contained a collagen-like region (CL) flanked by noncollagenous $\mathrm{N}$ - and $\mathrm{C}$-terminal regions. The noncollagenous regions were conserved among all three species within each Bucl with sporadic length variations observed (Table 2). As expected, the $C L$ regions of the same Bucl varied significantly in length between strains due to differing numbers of GXY repeats. For example, Bucl3 varied from 38 repeats to 63 repeats in different strains of $\mathrm{Bp}, \mathrm{Bm}$, and $\mathrm{Bt}$ (Table 2). The triplet usage was unique to each Bucl across species and usually one or two GXY-repeat types dominated each $\mathrm{CL}$ region. For example, Bucl1 and Bucl8 contained exclusively GAN and GAS repeats, respectively, while Bucl3 contained predominantly GTS repeats and Bucl10 had predominantly GIH triplets. 
In order to assess whether the Bucl proteins will form collagen-like triple helices, stability predictions were performed on representative Bucl-CL amino acid sequences. GXY repeat number in Bucl proteins varies from 2 in Bucl14 to 63 in Bucl3 (Table 2). Stability of the predicted collagenous regions of each Bucl was computed using an approach derived from host-guest peptide studies (47). Examination of the stability profiles shows highest stabilities for Bucl2, Bucl5, Bucl13, and Bucl15, with predicted melting temperatures ranging between $35-38^{\circ} \mathrm{C}$, while all other Bucl proteins had melting temperatures between $20-35^{\circ} \mathrm{C}$ (Fig. 3). Transmembrane regions were predicted in $\mathrm{CL}$ domains of Bucl proteins 4, 6, 7, 8, 14, 15, and 16, whose stability ranks low (Fig. 3). Hydrophobic interactions occurring in a membrane environment likely stabilize these triple helices.

\section{Structural Predictions}

In addition to the $\mathrm{CL}$ region, four Bucl proteins were predicted to contain putative domains proven to participate in pathogenesis in other bacterial species (Table 2, Fig. 4A). Bucl3 contains a putative Talin-1 domain; Talin-1 is a cytoskeletal protein that binds and activates integrins in mammals and talin-1-integrin interaction links the cytoskeleton with the extracellular matrix, allowing cell adhesion and migration (48-50). Bucl4 contained a Bac_export_1 domain (Bacterial export proteins, family 1; PF01311) found in members of type III secretion protein family, including the SpaR of Shigella and Salmonella, and the YscT of Yersinia. These proteins form the inner-membrane part of the needle complex, which transports bacterial effector proteins to afflict host cells. Bucl8 contained the OEP domain; the members of outer membrane efflux protein family (PF02321) form channels that allow export of various compounds, including anti-microbial agents, in Gram-negative bacteria across the outer membrane (51). Bucl13 contained a SBP_bac_3 domain (Bacterial extracellular solute-binding proteins, family 3; PF00497), which is found in periplasmic proteins that bind specific solutes within the periplasmic space and are often associated with $A B C$-type transporters (52).

Signal sequences were predicted in Bucl proteins 3, 4, 8, and 15, additionally supporting extracellular location for Bucl4 and Bucl8 (Table 2). Most Bucl proteins had transmembrane regions, interestingly, often associated with the $\mathrm{CL}$ regions (Table 2). 


\section{Modeling of the OEP domains in Bucl8}

The OEP domains found in Bucl8 are inferred in the formation of an efflux pump, thus, contributing to multi-drug resistance of $\mathrm{Bp}$ and $\mathrm{Bm}$ species (53). Two tandem OEP domains were predicted with high confidence (E-values $7 \times 10^{-22}$ and $4.5 \times 10^{-18}$ ). The Bucl8 is also predicted to be a lipoprotein with an amino-terminal lipid-binding cysteine residue and a transmembrane region predicted with TMpred (54).

HMM search in the PDB database using Bucl8-OEP region as a query identified closest similarity (E-value $=6.6 \times 10^{-53}$ ) to the drug discharge outer-membrane lipoprotein OprM of $P$. aeruginosa $(55,56)$. Using OprM structure as a template (pdb code $3 \mathrm{~d} 5 \mathrm{k}$, sequence identity 27\%), the model of Bucl8 was generated with MODELLER 9 v.9 (57).

The OEP domains of Bucl8 form a trimeric structure containing the characteristic $\alpha$-barrel, which spans the periplasmic space, and the $\beta$-barrel, which spans the outer membrane (Fig. 4B). In OprM, the $\beta$-barrel is known to anchor the protein to the outer membrane, and also contains a series of surface exposed loops that are involved in constriction of the $\beta$-barrel pore, thereby preventing influx of xenobiotics at the resting state $(56,58)$. The $\alpha$-barrel contains an arrangement of twelve short helices and six long helices that form a bundle which is constricted at both ends but contains a bulge in the middle that can accommodate antibiotics. Twisting of the helices to loosen the pores forms a funnel-channel structure allowing for the active transport of antibiotics across the outer membrane outside of the bacterial cell (56).

The bucl8 gene was found in all $\mathrm{Bp}$ and $\mathrm{Bm}$ strains tested by PCR and bioinformatics (Table 4), signifying the potential importance of Bucl8-efflux pump in the survival and pathogenesis of these species. Interestingly, all Bt strains analyzed contained DNA sequence homologous to the OEP-domain of Bucl8 in Bp and Bm but lacked the sequence corresponding to the Bucl8-collagenous domain; thus, it could not be recognized as a true Bucl. Additionally, a single nucleotide insertion at position 52, directly preceding the OEP-encoding region, was found, causing a frameshift mutation, which resulted in an altered amino acid downstream sequence. 


\section{Phylogenetic analyses of bucl genes}

To better understand the relationship of bucl genes among Burkholderia spp., parsimony and model-based phylogenetic analyses were performed. All 13 bucl sequences, originally identified in collagen Pfam database, were BLASTn-searched against completed genomes of $\mathrm{Bp}, \mathrm{Bm}$, and $\mathrm{Bt}$, and each bucl sequence was downloaded. The $13 \mathrm{bucl}$ genes demonstrate no sequence similarity, indicating these are non-homologous genes, whereas alleles encoding the same bucl gene were orthologous across species. Nucleotide sequence alignments were generated for each bucl gene present in $13 \mathrm{Bp}$ and $11 \mathrm{Bm}$ strains; analysis of buc/3 and bucl4 also included $9 \mathrm{Bt}$ strains ( $\mathrm{S} 1$ data set). Pairwise alignments of each bucl among the different strains revealed that percent identities ranged from $42 \%-100 \%$, with the average percent identity for each buc/ ranging from $76.5-94.9 \%$ (S2 data set). In general, the non-collagenous regions of bucl genes were conserved, while the CL regions showed significant length polymorphisms. Consequently, the bucl1 phylogeny based on non-CL region sequence produced a star pattern, while the bucl1 phylogeny generated based on the entire bucl1 sequence showed more extensive branching patterns, most of which were supported by Bayesian Posterior Probability values and several of which were also supported by maximum parsimony bootstrap values (S1 Fig). The CL region of bucl1 encodes a single GAN-repeat type, therefore, the only difference between bucl1 alleles from different strains represented in this tree arises from different GAN-repeat numbers. Since this is a common feature of all bucls, and incorporation of these regions would likely lead to long branch attraction, only the non-CL regions were used in further analyses. Multiple sequence alignments of bucl genes $2,5,6,7,10,13,14,15$ and 16 showed highly conserved nucleotide sequence, similar to bucl1, therefore phylogenetic analysis was not performed.

Phylogenetic trees were generated for individual and concatenated buc/3, bucl4, and bucl8, as these genes were present in all three species and contained the most informative characters. We included the OEP-encoding sequence of bucl8 from Bt strains in this analysis, despite the lack of CL-encoding sequence and conserved frameshift mutation, because of significant sequence similarity to buc/8-OEP sequences shared with $\mathrm{Bp}$ and $\mathrm{Bm}$. The phylogeny generated from concatenated sequences showed similar associations as phylogenies for the individual genes, although usually with higher 
statistical support. All analyses showed $\mathrm{Bp}$ and $\mathrm{Bm}$ strains were more closely related to each other than to Bt strains, which formed a main separate branch (Fig. 5, 6, S2). This observation is consistent with the hypothesis that the pathogenic $\mathrm{Bp}$ and $\mathrm{Bm}$ strains diversified from $\mathrm{Bt}(42,59-61)$. On the concatenated tree, Bm strains formed a single clade without further resolution that was strongly supported by both Bayesian posterior probability (PP, 100) and maximum parsimony (MP, 100) bootstrap values (Fig. 5). This observation indicates either inadequate time for the diversification of $\mathrm{Bm}$ strains or purifying selection for the retention of nucleotide identity due to importance in adapting to its host pathogen niche (41). In contrast, Bp strains exhibited higher diversification as shown by the presence of multiple clades. Four supported clusters were observed, two of which, Cluster 1 and Cluster 4, showed geographical associations as these strains were all Australian isolates. Cluster 1 (PP 98, MP 100) contained Bp strains 20B16, MSHR146, MSHR511, and NCTC 13178, all isolates obtained from Australia. Cluster 2 (PP 58, MP 100) contained Bp strains NCTC13179 and 1026b, isolated from human infections in Australia and Thailand, respectively. Cluster 3 (PP 100, MP 100) contained Bp strains 1106a and BPC006, obtained from northeast Thailand and China, respectively. Finally, Cluster 4 (PP 100, MP 100) contained Bp strains MSHR305 and MSHR520, which are both human infection isolates from Australia. Clusters 1 and 4 were also supported by trees based on individual buc/3, bucl4, and bucl8 genes, although strain NCTC 13178 as part of Cluster 1 was only supported by the tree based on bucl4 (Fig. 6, S2). Similar to $\mathrm{Bp}$, Bt strains showed significant diversification as evidenced by the formation of three supported clusters in the concatenated tree. These clusters were numbered consecutively Cluster 5, Cluster 6, and Cluster 7 (Fig. 5). Clusters 5 and 7 were supported by individual buc/3 and bucl4 phylogenies (Fig. 6), while only Cluster 5 was supported by bucl8 phylogeny (S2 Fig). Analysis performed using amino acid sequences of Bucl proteins generated phylogenetic trees with similar patterns, though the support values were lower (S3 Fig), indicating many of the nucleotide changes were synonymous.

Overall, most bucl genes were highly conserved among $\mathrm{Bp}$ and $\mathrm{Bm}$ with most of the variation occurring in the $C L$ region due to differing numbers of GXY repeats. Variation in non-CL regions of bucl 3, bucl 4 , and bucl 8 revealed divergence between $\mathrm{Bt}$ and select agents $\mathrm{Bp}$ and $\mathrm{Bm}$, as well as diversification among Bt strains. Bp and Bm appear more 
closely related, but only Bp strains showed diversification across the bucl loci by the formation of multiple distinct clades with strong statistical support.

\section{Assessment of bucl distribution across Burkholderia spp.}

In order to assess the distribution of bucl genes across Burkholderia, nucleotide BLAST searches were performed using bucl-gene sequences from the reference strain Bp K96243, as queries against completed genomes of $13 \mathrm{Bp}, 11 \mathrm{Bm}$, and $9 \mathrm{Bt}$ strains. All 13 bucl genes were present in all Bp genomes, while the majority of bucl genes were maintained within $\mathrm{Bm}$ genomes (Table 4). Up to three bucl genes were missing in $8 \mathrm{Bm}$ genomes, which is consistent with the reduced genetic material in this species $(41,46)$. In contrast, only complete open reading frames of bucl3 and bucl 4 were present in $\mathrm{Bt}$ genomes, presumably encoding a lipoprotein with a putative Talin-1 domain and a type III secretion inner membrane protein (Table 2, Fig. 4A), respectively.

In addition to bioinformatic data, we tested distribution of bucl genes by standard PCR in a collection of genomic DNA from $25 \mathrm{Bp}$ and $20 \mathrm{Bm}$ strains, as well as the DNA from non-select agent controls $4 \mathrm{Bt}, 3 \mathrm{~B}$. cepacia (Bc), 5 B. cenocepacia (Bce), and $6 B$. multivorans (Bmv) strains (Table 5, Table 6). Consistent with bioinformatic data, virtually all $25 \mathrm{Bp}$ strains were found to contain all $13 \mathrm{bucl}$ genes, with the exception of strain China 3 (BpCh3) which was missing bucl1 and bucl4 (Table 4, Fig. 7, S4 Fig). Almost all Bm strains tested (15 out of 20) were lacking up to three bucl genes, in agreement with bioinformatic results. We calculated bucl frequencies as the proportion of $\mathrm{Bp}$ and $\mathrm{Bm}$ strains positive for each bucl, as tested by both PCR and bioinformatics. High frequencies were observed for buc/3, bucl4, bucl7, and bucl15 (0.90-0.98), while lower frequencies were observed for bucl2 (0.85) and bucl10 (0.82). The bucl2 and bucl10 genes were most frequently absent from Bm strains, missing in about one-third of strains analyzed, indicating these genes are nonessential for Bm survival in mammalian host. Finally, all Bt strains contained only buc/3 and bucl4, while no amplification of these two bucl genes was obtained for other control Burkholderia spp. (Table 4, Fig. 7, S4 Fig).

We next evaluated the association of bucl presence with pathogenicity among Bp, $\mathrm{Bm}$, and Bt strains. The Fisher Exact Probability Test and Cramer's $\mathrm{V}$ analysis were performed on the number of bucl genes present and absent among two groups: 1) 
pathogenic $\mathrm{Bp}$ and $\mathrm{Bm}$ strains and 2) nonpathogenic Bt strains. The Fisher test provides a measure of the statistical significance between two groups, and Cramer's $V$ squared $\left(V^{2}\right)$ is a value, which measures the degree of association between two variables on a scale of zero (no association) to one (perfect association). The Fisher test showed significant differences between group 1 and 2 for all bucl genes, except for bucl3 and bucl4, indicating the presence of collagen-like genes is significantly associated with pathogenic $B$. pseudomallei and $B$. mallei species as compared with non-pathogenic $B$. thailandensis (Table 4). Further calculation of Cramer's $\mathrm{V}^{2}$ showed perfect association $\left(\mathrm{V}^{2}=1\right)$ for bucl genes $5,6,8,13,14$, and 16 that were present in all Bp and Bm strains, while absent in all $\mathrm{Bt}$ strains. High $\mathrm{V}^{2}$ values were calculated for bucl1 $\left(\mathrm{V}^{2}=0.829\right)$, bucl7 $\left(\mathrm{V}^{2}=0.829\right)$, and bucl15 $\left(\mathrm{V}^{2}=0.908\right)$, indicating positive association with these bucl genes with pathogenic $\mathrm{Bp}$ and $\mathrm{Bm}$, as compared with nonpathogenic Bt lacking them. The remaining bucl genes, 2, 3, 4, and 10, had little or no association with pathogenic Bp and $\mathrm{Bm}$ compared to $\mathrm{Bt}\left(\mathrm{V}^{2}<0.5\right)$. Hence, our statistical analyses strongly infer association between the presence of the majority of Bucl proteins and pathogenicity.

\section{Detection of Burkholderia select agents by analytical PCR}

Four conserved amplicons generated from bucl genes that were uniformly found in all Bp and $\mathrm{Bm}$ strains, but were absent in $\mathrm{Bt}, \mathrm{Bc}, \mathrm{Bce}$, and Bmv strains, were assessed for select agent detection by standard agarose gel electrophoresis and capillary gel electrophoresis: bucl5 (216 bp), bucl13 (212 bp), bucl14 (178 bp), and bucl16 (123 bp) (Fig. 7C). Size-identification of bucl-based amplicons by capillary gel electrophoresis was performed in a 10\% phospholipid nanogel, allowing near single base pair resolution (62), including bucl5 and bucl13 amplicons that differ by $4 \mathrm{bp}$. Sizing of the target DNA fragments was accomplished by linear regression analysis for DNA size (in bp) versus migration time. The bucl gene amplicon sizes were calculated using the linear fit obtained for the migration times of internal standards with lengths of $100 \mathrm{bp}$ and $250 \mathrm{bp}$, and the standard deviation calculated from 5 replicate measurements. The bias is calculated as the difference between the true fragment size and the measured size. Sizing results are reported as follows for $n=5$ separations: [gene name (true size): calculated size \pm standard deviation, percent relative size bias defined as bias divided by the true size]; 
bucl5 (216 bp): $218 \pm 2$ bp, 0.9\%; bucl13 (212 bp): $215 \pm 1$ bp, 1\%; bucl14 (178 bp): 181 \pm 1 bp, $2 \%$; bucl16 (123 bp): $120 \pm 1$ bp, $2 \%$.

\section{Detection of Burkholderia select agents by quantitative PCR}

Identification of molecular targets for Burkholderia select agents is challenging due to the high genomic plasticity reported in these organisms that include significant genomic rearrangements and deletions. PCR assays developed for Burkholderia detection include BurkDiff, a dual-probe assay able to detect and differentiate Bp and Bm $(63,64)$, and the TTS1 assay targeting orf2 of type three secretion system I, detecting Bp only (64-66). Here, we developed a qPCR assay for the detection of $\mathrm{Bp}$ and $\mathrm{Bm}$ based on bucl16 target. A locked nucleic acid hydrolysis probe specific for bucl16 gave robust amplification using DNA of $\mathrm{Bp} \mathrm{K96243} \mathrm{(} \mathrm{Cq}=21.85 \pm 1.37)$. This probe was then tested against the genomic DNA collection, providing amplification of all $\mathrm{Bp}$ and all $\mathrm{Bm}$ strains, with no amplification from non-select agent controls including $\mathrm{Bt}$, $\mathrm{Bce}$, and $\mathrm{Bmv}$, as well as a no DNA template control (Fig. 8A). $30 \mathrm{ng}$ of DNA was used for each strain and $\mathrm{Cq}$ values ranged from 23.42- 29.05 .

We next tested the buc/16-based QPCR assay towards detection of an infection with Burkholderia select agents by employing samples spiked with human plasma, and with samples obtained from experimental animals. PCR reactions performed with $30 \mathrm{ng}$ Bp K96243 DNA and spiked with 5\% human plasma produced positive amplification with average $\mathrm{Cq}=24.28 \pm 2.14$ (Fig. 8B), whereas reactions spiked with $10 \%$ and $20 \%$ human plasma produced averaged $\mathrm{Cq}=25.89 \pm 1.76$ and $\mathrm{Cq}=27.96 \pm 1.82$, respectively.

Next, Bp strain HBPUB10134a was used for the detection of Burkholderia infection in vivo. Our recent studies have shown that Bp HBPUB10134a was the most virulent in the intraperitoneal infection model among a panel of $11 \mathrm{Bp}$ strains, with an LD $D_{50}$ of 10 CFU at day 21 post-infection (67). Following the injection, mice presented common clinical manifestations, including abscess and pyrogranuloma formation in the spleen and liver, and in some cases lesions and inflammation in the eyes and tail. A common pathological observation was the loss of rear limb function occurring between 6 and 30 days post-infection, associated with the pyrogranulomatous inflammation in the skin, skeletal muscle, bone, and peripheral nerves in the hind limbs. Here, mice that were 
injected intraperitoneally, were euthanized and sampled after 3,7 , or 14 days postinfection. Homogenized spleen samples were plated on blood agar to assess bacterial loads and $1 \mu \mathrm{L}$ samples of irradiated sterile spleen extracts were used directly in qPCR reactions. Four samples, with original bacterial loads of $5 \times 10^{7}, 5 \times 10^{6}, 2 \times 10^{5}$, and $10^{3} \mathrm{CFU} / \mathrm{mL}$, thus, presumably corresponding to $5 \times 10^{4}, 5 \times 10^{3}, 2 \times 10^{2}$, and $10 \mathrm{CFU}$ per 1 $\mu \mathrm{L}$ added to each qPCR reaction, respectively, were tested using our buc/16-based assay. When crude spleen extracts were used in GPCR, positive detection was obtained for $5 \times 10^{3} \mathrm{CFU}$ and $5 \times 10^{4} \mathrm{CFU}$ samples with averaged $\mathrm{Cq}$ values of $29.49 \pm 1.67$ and $26.39 \pm 1.71$, respectively (Fig. 8B). Importantly, we observed that 1:10 dilution of the sample containing $5 \times 10^{4} \mathrm{CFU} / \mu \mathrm{L}$, resulted in improved amplification, as evidenced by lower $\mathrm{Cq}$ value $(23.32 \pm 0.42)$, while $1: 100$ dilution resulted in similar amplification as undiluted crude sample $(\mathrm{Cq}=27.23 \pm 1.10)$ (Fig. 8B, red curves). Further 1:1000 dilution of spleen extract provided detection level as low as $50 \mathrm{CFU}$ per reaction with a $\mathrm{Cq}$ value of $32.63 \pm 1.57$. On the other hand, $1: 10$ dilution of the sample originally containing $5 \times 10^{3}$ $\mathrm{CFU} / \mu \mathrm{L}$ resulted in poorer amplification $(\mathrm{Cq}=32.66 \pm 2.46)$ than crude undiluted sample (gray curves). We think that crude spleen extracts contained varying levels of inhibitors that differentially affected amplifications in these two samples. Finally, in addition to bucl16, buclgenes 5, 6, 8, 13, and 14 that were found in all Bp and Bm strains are similarly good candidate markers for the development of diagnostic qPCR assays.

\section{DISCUSSION}

Traditionally, collagen has been associated with multicellular animals, although, the number of collagen-like proteins identified in bacterial genomes has recently increased with 2554 sequences currently (search on 04/12/15) deposited in the Pfam collagen data base. The distribution of these collagen-like proteins is not uniform, however; they are absent in some bacteria and are overrepresented in other species. Here, we identified and characterized a group of 13 discrete collagen-like proteins in Burkholderia, referred to as Bucl, which are largely found in the pathogenic $\mathrm{Bp}$ and $\mathrm{Bm}$ species. Furthermore, we found that bucl genes provided important clues on the genomic plasticity and evolution of Burkholderia select agents. We observed Bucl proteins contained domains that are known to be involved in pathogenesis and antibiotic resistance, including an outer 
membrane efflux protein which we modelled. Finally, we utilized bucl genes as detection targets and successfully detected Bp infection in a mouse model.

\section{Characterization of Bucl-CL Region}

Collagen-like sequences, embedding the typical repetition of triplets of the type Gly-X-Y $(2,68-70)$ have been identified in all Bucl sequences. We observed that for each Bucl, one or two GXY types predominated the $C L$ region. This limited variation in GXY content resembles that seen in Bcl proteins of Bacillus anthracis (32) but is in contrast to $\mathrm{Scl}$ proteins of Streptococcus pyogenes, whose GXY sequence varies significantly within the $C L$ region (13). Typical of prokaryotic collagens, these sequences do not contain triplehelix-stabilizing hydroxyprolines, since bacteria lack the prolyl-hydroxylase enzyme necessary for post-translational modification of Pro to Hyp. The highest triple helix stabilities were predicted for Bucl2, Bucl5, Bucl13 and Bucl15, within the range of 35-38 ${ }^{\circ}$, which is similar to that of previously studied bacterial collagens as well as human collagen $(11,13,71,72)$. Similar to the CL regions of other prokaryotic proteins, like Scls from $S$. pyogenes $(7,8)$, the $C L$ regions of these proteins share the common characteristics of possessing charged residues GEX, GLE and GXR triplets, respectively (Fig. 3, Table 2). Indeed, ion pairs play a major role in stabilizing the triple helix, with an enthalpic stabilization, which likely involves interactions of polar groups with an ordered hydration network $(9,10,12)$. Additionally, specific GXY triplets were found to have favorable enthalpy values, corresponding to increased hydrogen bonding potential, including GPE (71), which is a common GXY triplet in the Bucl5 CL region. These regions are likely to be of biological importance in establishing interactions with charged counterparts. Interestingly, bacterial collagens have been shown to have relatively high proline content, $20 \%$ in S. pyogenes and up to $40 \%$ in B. anthracis (11), especially in the X position (73), whereas Bucl proteins lack Pro residues; only Bucl5 contains GPE repeats, likely contributing to its predicted high stability. Other Bucl proteins with lower thermal stability may rely on the hydrophobic membrane environment for triple helix stabilization, as those were predicted to have transmembrane regions, especially within the $C L$ regions. Stability predictions shown here were computed using long CL sequences, whereas some Bucl variants had short $\mathrm{CL}$ regions, which may not form triple helices. This is substantiated by 
the fact that few triplets may also exist in other folds e.g., G5 domain, whose structure presents a pseudo-triple helix (74). In summary, while overall characteristics of the Bucl proteins we identified were similar to previously described bacterial collagen-like proteins, i.e., presence of collagenous and non-collagenous domains and length variation in collagen region, the GXY content observed in Bucls was unique and likely impacts the structural stability of the Bucl-CL triple helix.

\section{Characterization of Bucl non-collagenous domains and their inferred roles in Burkholderia pathogenesis}

It has been observed that collagen-like proteins are often surface associated. Indeed, among 53 bacterial and viral collagen-like proteins analyzed in an initial genome-based study, 16 were annotated as cell-wall attached or membrane associated (73). Additionally, surface expression of collagen-like proteins including Scls of $S$. pyogenes, PclA of S. pneuomonia, and LCl of L. pneumophila, has been demonstrated experimentally $(7,8,18,19)$. Structural predictions performed for Bucl proteins revealed that their majority, 10 out of 13 , have transmembrane regions, supporting the location of Bucl proteins in the inner or outer membrane of Burkholderia spp. Moreover, four of these proteins were predicted to contain both signal sequences and transmembrane domains, further supporting surface association. Further non-collagenous features include wellconserved domains (in Bucl3, Bucl4, Bucl8 and Bucl13), which are inferred in pathogenesis.

Bucl3 was predicted to have a Talin-1 domain. Talin-1 in eukaryotes is known to bind and activate integrins as well as link the cell cytoskeleton to the extracellular matrix (50). Cell-to-cell invasion by Burkholderia is largely achieved by the disruption of the host cytoskeletal network, as well as the fusion of host cells resulting in the formation of multinucleated giant cells, mediated mainly by type III and type VI secretion systems $(75,76)$. The intra- and inter-cellular spread is facilitated by the formation of actin tails which propel the bacterial cells. Thus far, several type III secretion effector proteins are known to be involved with host actin polymerization allowing cell invasion, including BimA, BopE, and BipD (43). The putative Talin-1 domain found in Bucl3 may also be involved 
in interactions with host actin that allow for cell invasion or the formation of actin tails during infection.

The Bucl4 protein is putative inner membrane protein part of the type III secretion T3SS-2 system (77). There are three known type III secretion gene clusters (T3SS-1, T3SS-2, and T3SS-3) distributed among Bp, Bm, and Bt species. T3SS-1 is specific to Bp while T3SS-2 and 3 are found ubiquitously in all three species (78). The T3SS-3 is known to be important for virulence in $\mathrm{Bp}(44,75,79)$, as mutants deficient in the T3SS-3 have reduced replication in host cells, and are unable to escape endocytic vacuoles, and to form membrane protrusions and actin tails (80). The other two secretion systems are less well characterized, and the role of the T3SS-2 secretion system in pathogenesis is not known. The unique association of a collagenous domain with Bac_export_1 domain in this inner membrane protein of T3SS-2 has not been previously acknowledged.

Bucl13 contains the SBP_bac_3 domain, and is predicted to be a bacterial periplasmic solute binding protein. Binding of the solute causes a conformational change, which allows interaction of the solute with inner membrane proteins and subsequent transport of the solute into the cell. Family 3 solute-binding proteins are known to bind polar amino acids and opines (52), therefore Bucl13 is likely associated with amino acid transport; interestingly, Bucl13 is present in all Bp and Bm strains tested, while it is absent in non-pathogenic Bt. Bucl13 was also predicted to have a collagenous domain with a relatively high thermal stability, possibly contributing to its function.

Of particular interest is the Bucl8 protein, which was found to contain two tandem outer membrane efflux protein (OEP) domains that are known to contribute to the multidrug resistant phenotype of $\mathrm{Bp}$ and $\mathrm{Bm}$ species. These organisms are intrinsically resistant to multiple antibiotics including aminoglycosides, macrolides, and $\beta$-lactams $(53,81)$. The outer membrane protein is an integral component of a tripartite ResistanceNodulation-Division (RND) efflux pump that also requires an accessory protein in the periplasm and an inner membrane transport protein. It is known that there are 10 RND efflux pumps annotated in the Bp K96243 strain, many of which have not been explored (40). Currently, only three of these systems, BpeAB-OprB, AmrAB-OprA, and BpeEFOprC, have been investigated for their roles in multidrug resistance (82-84). Interestingly, Bucl8 was found to be present in all Bp and Bm strains and absent in the non-pathogenic 
$\mathrm{Bt}$, suggesting selective pressure for the Bucl8-OEP in human or animal infection. We homology-modeled the Bucl8-OEP region based on OprM protein of Pseudomonas aeruginosa and observed a trimeric arrangement forming an outer membrane-spanning $\beta$-barrel and periplasmic $\alpha$-barrel. The presence of the $\mathrm{CL}$ domain in Bucl8 is an unexpected observation, as collagenous regions have not been reported as part of efflux pump systems. On the other hand, the trimeric arrangement of Bucl8 is consistent with the formation of a collagen triple helix. TMPred predicted a transmembrane region, albeit with lower score, for a part of the collagen-like region (amino acids 539-557) indicating the $C L$ region folds back across the membrane. The $C L$ region is then predicted to extend into the extracellular space, projecting the carboxyl-terminal region from the cell surface. The triple-helical CL region may have a number of functions: i) to project the C-terminal region, which may serve as a surface adhesin, ii) to stabilize the trimeric arrangement of the OEP, and iii) may assist in blocking the $\beta$-barrel pore at the resting state, thus, preventing entry of xenobiotics into the cell. Ongoing studies will determine the potential role of Bucl8-OEP in drug resistance, Bp and Bm pathogenesis, as well as a potential as vaccine candidate.

\section{Bucl phylogeny}

The presence of 13 collagen-like genes in $\mathrm{Bp}$ and $\mathrm{Bm}$ genomes poses the question how have these unique sequences been acquired in Burkholderia? The GXY repeats found in bacterial collagens may have arisen through mechanisms including de novo spontaneous mutation and subsequent triplet repeat expansion independent within each gene, or by horizontal gene transfer. It has been initially suggested that collagen sequences are acquired by horizontal transfer from eukaryotes to prokaryotes based on the lack of collagen sequences in ancestral archaeal genomes and relatively few sequences identified in bacterial genomes (73). However, current collagen Pfam contains 2,554 bacterial collagen sequences, as well as 14 archaeal. A recent study, focused on bacterial molecular mimics of host proteins, proposed that collagen-like sequences found in pathogens evolved independently to mimic human host proteins (85). The uniformity of GXY content within each Bucl indicated they are likely to have evolved from the accumulation of repeats within each gene, resulting in diverse Bucl proteins that share 
the GXY motif but with different GXY composition. Additionally, gene-enrichment analysis showed that collagen-like proteins were related to extracellular matrix mimicry and cell adhesion, supporting the evolution of repetitive sequences in virulence factors. Our phylogenetic analyses show that 13 collagen-like genes observed in numerous $\mathrm{Bp}$ and $\mathrm{Bm}$ genomes are unrelated to each other, which supports their independent acquisition, as well as selective adaptation of their collagen-like sequences in the host environment. This is further supported by the lack of collagen-like proteins (11 out of 13) in the closely related environmental species of $\mathrm{Bt}$, indicating these sequences were acquired after divergence of $\mathrm{Bp}$ and $\mathrm{Bm}$ from Bt. Since Bucl proteins are unrelated and encoded in various locations in the genome, within-gene expansion of GXY-repeat motifs may point to convergent evolution of collagenous sequences to fulfill a similar function.

Phylogenetic trees based on three bucl loci showed Bt strains formed a distinct separate branch from $\mathrm{Bp}$ and $\mathrm{Bm}$ strains. This is consistent with previous studies based on phylogenetic analyses of seven MLST loci (42) and over 11,000 SNPs (60) which showed Bp and Bt isolates were resolved into two groups that were supported in $100 \%$ of bootstrap replicates. We also observed $\mathrm{Bm}$ strains share high sequence similarity, while Bp strains exhibited more intraspecies diversity, forming more extensive clusters that often corresponded to geographical associations. Previous phylogeographic reconstruction of Burkholderia strains based on over 14,000 SNPs showed that Bp and $\mathrm{Bm}$ strains formed separate clusters. The same study also showed Bp strains were significantly divided between those originating from Australia and Asia (60), in agreement with our observation that Australian isolates formed distinct clusters in bucl-based phylogenetic trees.

\section{Bucl distribution}

Both bioinformatic and PCR analyses showed that the majority of bucl genes are unique to $\mathrm{Bp}$ and $\mathrm{Bm}$ strains, with the exception of bucl3 and bucl4. This observation may indicate these two genes are selected for in the environment of $\mathrm{Bp}$ and $\mathrm{Bt}$, as several genomes of host-adapted $\mathrm{Bm}$, lack these genes. The absence of most buc/s from $\mathrm{Bt}$ is a surprising observation since its genome is overall similar to that of $\mathrm{Bp}(59)$, which may suggest either the acquisition of bucls in Bp or the loss of bucls in Bt after divergence of the two species. 
Both $\mathrm{Bp}$ and $\mathrm{Bt}$ species have large genomes of approximately 7.2 and $6.7 \mathrm{Mb}$, respectively, divided between two chromosomes. Comparative genomics showed that Bp and $\mathrm{Bt}$ genomes share a large number of conserved genes involved in both core and accessory functions, while genes associated with virulence in Bp have increased diversity (59). Interestingly, buc/3 and buc/4, encoding proteins potentially involved in pathogenesis, are found in the avirulent Bt. It has been shown that $71 \%$ of virulencerelated genes in Bp are conserved in Bt with similarities of over $80 \%$, including type III secretion gene clusters (59). Amino acid differences in virulence proteins present in both species may confer functional differences impacting virulence in Bp vs. Bt. Lastly, the presence of bucl3 and bucl4 alone in Bt was not sufficient to cause pathogenesis, a new biological trait acquired by $\mathrm{Bp}$ and $\mathrm{Bm}$ after the acquisition of additional virulence factors, including additional Bucls. A prominent feature of Burkholderia genomes is the presence of multiple horizontally acquired genomic islands that differ between Bp and Bt $(40,59)$. These genomic islands are associated with survival in the soil environment and are absent in Bm genomes, possibly explaining why $\mathrm{Bm}$ cannot persist in the environment (46). The presence of most bucl biomarkers in both $\mathrm{Bp}$ and $\mathrm{Bm}$ genomes indicates they are not located within genomic islands but are rather a part of the core genome.

Previously, it has been reported that $\mathrm{Bm}$ is a clonal derivative of $\mathrm{Bp}$, which has evolved to adapt to the host environment. Multilocus sequence typing analyses show that, in contrast to $\mathrm{Bp}, \mathrm{Bm}$ strains are genetically homogenous, while relatively few new genes are being identified, as additional genomes are sequenced (46). However, the variable portion of the genome, though not acquiring new genetic information, is continuing to alter via expansion of IS elements and chromosomal rearrangements. Our phylogenetic analysis of bucl genes within Bm supports the genetic homogeneity among Bm strains, and mapping of bucl markers showed considerable chromosomal rearrangements occurring between $\mathrm{Bm}$ strains.

Different collagen-like proteins, unrelated to 13 Bucls characterized here, were also present in other Burkholderia species. We noticed these collagen-like proteins found in B. cepacia, B. cenocepacia, B. multivorans, B. ambifaria, B. glumae, B. gladioli, and B. xenovorans, contained GTS repeats within the CL region, similar to Bucl3. However, outside of the $C L$ region, sequence identity was very low, therefore these proteins were 
not included in the Bucl3 group. Given the importance of Burkholderia species as human pathogens and part of the Burkholderia cepacia complex (B. cepacia, B. cenocepacia, $B$. multivorans, and B. ambifaria), plant pathogens (B. glumae and B. gladioli), and plant symbionts ( $B$. xenovorans), investigation of these collagen-like proteins is an interesting area for further study.

\section{bucl-based infection detection}

$\mathrm{Bp}$ and $\mathrm{Bm}$ are reported to have fatality rates up to $80 \%$ and $95 \%$, respectively $(86,87)$, making early diagnosis and treatment critical for patient survival. Currently, culture-based identification of Burkholderia select agents remains the gold standard for diagnosis $(86,88)$. Highly variable genomes present a challenge in finding reliable genetic targets that are not subjected to chromosomal deletion, especially for $B$. mallei. Although a few laboratory-developed qPCR tests have been reported, there are no FDA-approved assays for the detection of Burkholderia select agents. The TTS1 assay $(65,66)$ detects specifically Bp, and the BurkDiff assay (63) detects both organisms and differentiates them based on a SNP-associated shift of approximately $1 \Delta \mathrm{Ct}(64)$. Here, we assessed buc/ markers as detection targets. Standard PCR performed on a large collection of gDNA yielded specific amplicons for bucl5, 13, 14, and 16 from Bp and Bm but not from the nonselect agent controls. This PCR test with 4 buc/ targets detected Bp infection in laboratory animals using spleen extracts as a specimen. Separation of these conserved amplicons by capillary electrophoresis in a phospholipid nanogel matrix allowed for size-based identification, similarly to previously described identification of Aspergillus spp. (31) and Streptococcus pyogenes (35). Precise microfluidic separation could be used for strain fingerprinting based on multiplexed amplicons generated with primers flanking the repetitive $C L$ region, as previously tested with $B$. anthracis strains (32). Our achieved resolution $<9$ bp along a wide range of amplicon sizes will allow to differentiate two strains that differ by a single GXY repeat. We further developed a qPCR assay for bucl16, which detects both $\mathrm{Bp}$ and $\mathrm{Bm}$; it was tested with purified genomic DNA templates, gDNA spiked with $5 \%$ human plasma, and spleen extracts from infected mice. The bucl16 assay was able to detect as low as $50 \mathrm{CFU}$ per reaction in diluted spleen samples; however, it should be noted that sample-to-sample variation was observed. The specimen type will also 
affect detection outcome. For example, sputum and pus typically contain high bacterial loads $\left(10^{2}-10^{9} \mathrm{CFU} / \mathrm{mL}\right)(89)$, whereas blood of $45 \%$ of patients with septicemic melioidosis had less than $1 \mathrm{CFU} / \mathrm{mL}$ bacteria in the blood (90), which presents a sensitivity challenge, even for highly performing qPCR assays. In as much as current work was focused on a single assay, which would simultaneously detect both select agents similarly to BurkDiff assay, the ongoing research is focused on the development of a probe-based QPCR assay targeting nucleotide polymorphisms identified in buc/3 and buc/4 genes. In summary, selected bucl genes represent promising detection targets as they are both specific to and ubiquitously found in Bp and Bm strains.

\section{MATERIALS AND METHODS}

\section{Ethics statement}

Animal Studies: Animal research at the United States Army Medical Research Institute of Infectious Diseases (USAMRIID) was conducted under an animal use protocol approved by the USAMRIID Institutional Animal Care and Use Committee (approved by the USAMRIID-IACUC) in compliance with the Animal Welfare Act, PHS Policy, and other Federal statutes and regulations relating to animals and experiments involving animals. The facility where this research was conducted is accredited by the Association for Assessment and Accreditation of Laboratory Animal Care International (AAALAC) and adheres to principles stated in the Guide for the Care and Use of Laboratory Animals, National Research Council, 2011. Tissue samples used in this study were generated in a previously published work (67). Briefly, challenged mice were observed at least daily for 14 days for clinical signs of illness. Humane endpoints were used during all studies, and mice were humanely euthanized when moribund, according to an endpoint score sheet. Animals were scored on a scale of $0-11: 0-2=$ no significant clinical signs; $3-7=$ significant clinical symptoms; such as subdued behavior, hunched appearance, absence of grooming, and impacted hind limb function and hind limb paralysis (increased monitoring was warranted and mice were checked at least twice per day); 8-11 = distress. Those animals receiving a score of 8-11 were humanely euthanized by $\mathrm{CO}_{2}$ exposure using compressed $\mathrm{CO}_{2}$ gas followed by cervical dislocation. The mice that were serially sampled were deeply anesthetized and then euthanized by exsanguination followed by 
cervical dislocation. However, even with multiple observations per day, some animals died as a direct result of the infection in between observation periods.

Human plasma collection: Anonymized human plasma samples were utilized in quantitative PCR experiments. Plasma samples were obtained from an already-existing collection, which was established by the corresponding author (SL; IRB Protocol Number: 1308076685). Collection of human blood of healthy adults was performed in accordance with the Human Research Protections Policy at West Virginia University. This study was approved by the Institutional Review Board at West Virginia University (IORG0000194) and written informed consent was obtained from all participants.

\section{Bioinformatic analyses}

Burkholderia collagen-like proteins, designated Bucl, were identified by searching the Sanger Institute Pfam collagen database (PF01391). Bucl proteins found in $B$. pseudomallei, B. mallei, and B. thailandensis were categorized into 13 Bucl-protein types based on similar domain organization and primary sequence similarity. Next, nucleotide BLASTn search was performed using each of 13 bucl-gene sequence as a query against the NCBI Nucleotide collection (nr/nt) database, as well as whole genome shotgun contigs (wgs) database, to determine bucl distribution in completed Burkholderia spp. genomes. DNA analyses were performed using the Lasergene Core Suite v. 12 (DNASTAR, Inc., Madison, WI).

\section{Protein structure prediction and modeling}

Domain organization of Bucl proteins was adapted from the Pfam collagen database (91) and verified independently using the Fugue 2.0 Server (92), which additionally identified the putative Talin-1 domain within Bucl3. Presence of a signal peptide was predicted with the hidden Markov model component of the SignalP 3.0 Server (http://www.cbs.dtu.dk/services/SignalP-3.0/) (93-95). The presence of transmembrane domains was predicted with TMpred (96).

When possible, as in the case of Bucl8, a 3D model was generated by homology modeling. Best template was identified by employing profile hidden Markov models 
(profile HMMs) and the program HMMer (97). Once the best template was identified (pdb code $3 \mathrm{~d} 5 \mathrm{k}$, sequence identity $27 \%$, residues $51-516$ ), the model of Bucl8 outer membrane efflux protein (OEP) domains was generated using MODELLER 9 V9 (57). Stereochemical quality of the model was improved by energy minimization using GROMACS (98).

Thermal stability along the predicted triple helices of Bucl-collagen domains was assessed with an algorithm developed by Persikov et al. 2005 (47). With this approach, a stability coefficient is assigned for every GXY triplet and averaged over a window of 5 tripeptide units. The averaged relative stability values are plotted against the tripeptide number in the collagen sequence.

\section{Phylogenetic analyses}

Both individual (with and without the collagen-like domains) and concatenated nucleotide sequences were aligned with ClustalV in the Megalign module in DNASTAR® Lasergene software, and verified manually. Maximum parsimony analyses were performed with 1000 bootstrap replicates using MEGA 6.06 (99), with the Tree-Bisection Reconnection heuristic search and 200 max trees saved. The evolutionary models used for each dataset were determined by MrModelTest 2.3 (100) with the Akaike Information Criterion (AIC). Bayesian analyses were performed within MrBayes 3.1.2 (101) implementing six Markov chains, 1000000 generations, with trees sampled every 100 iterations. Posterior probabilities were calculated using the last $20 \%$ of saved trees (burnin=8000). Cutoff values for significance were assigned 95 for Bayesian analysis and 70 for maximum parsimony analysis. All phylogenetic trees were constructed using the majority rule consensus. Trees were viewed in FIGTREE v1.3.1 (http://tree.bio.ed.ac.uk/software/figtree/). Phylogenies were constructed based on single bucl genes as well as concatenated bucl genes.

\section{bucl distribution among Burkholderia species}

bucl distribution was assessed in a broad collection of Burkholderia strains using genomic DNA (Table 2) obtained from: (i) NIH Biodefense and Emerging Infections Research Resources Repository, NIAID, NIH, (ii) Dr. Christopher Cote (The United States Army 
Medical Research Institute of Infectious Disease), and (iii) Dr. Joanna Goldberg (Emory University). The total collection consisted of DNA from 25 B. pseudomallei and $20 B$. mallei strains, and non-select agent control DNA from 4 B. thailandensis, 3 B. cepacia, 5 B. cenocepacia, and 6 B. multivorans strains. Analytical PCR was performed with primers targeting conserved non-collagenous regions of bucl alleles present in the reference strain B. pseudomallei K96243. PCR buffer (10 mM Tris- $\mathrm{HCl}, 1.5 \mathrm{mM} \mathrm{MgCl} 2,50 \mathrm{mM} \mathrm{KCl}$, $\mathrm{pH}$ 8.3) included $0.2 \mu \mathrm{M}$ primers, $0.2 \mathrm{mM}$ dNTP's, and 1.5 M betaine (Sigma-Aldrich, St. Louis, MO) to ameliorate amplification problems associated with high GC content ( 68\%) of Burkholderia genomes (41). A temperature gradient of $50-65^{\circ} \mathrm{C}$ was tested for each primer pair and gDNA of B. pseudomallei K96243 harboring all $13 \mathrm{bucl}$ genes as a template; uniform amplification conditions were established for all bucl genes at an annealing temperature of $64^{\circ} \mathrm{C}$. Amplification was performed with an in-house Taq polymerase as follows: $95^{\circ} \mathrm{C}, 5 \mathrm{~min}-\left[95^{\circ} \mathrm{C} 30 \mathrm{sec}, 64^{\circ} \mathrm{C} 30 \mathrm{sec}, 72^{\circ} \mathrm{C} 45 \mathrm{sec}\right] \times 30$ cycles$72^{\circ} \mathrm{C}, 10 \mathrm{~min} .40 \mathrm{ng}$ of template DNA was used for screening genomic DNA collection and reactions were carried out on a Bio-Rad S1000 thermal cycler. Resultant PCR products were analyzed on a $2 \%$ agarose gel with a 50 -bp ladder DNA standard (New England Biolabs Inc., Boston, MA). Gels were imaged using the Eagle Eye II (Stratagene, La Jolla, CA), and FOTO/ Analyst Investigator/ Eclipse gel documentation workstation (Fotodyne, Harland, WI).

\section{qPCR amplification of bucl targets}

Testing of selected bucl amplicons by real-time PCR with SYBR green intercalating dye was performed to assess potential candidates for probe-based detection of $B$. pseudomallei and B. mallei species. Reactions were carried out with SsoAdvanced ${ }^{\mathrm{TM}}$ SYBR $\circledast$ Green Supermix (Bio-Rad, Hercules, CA), $0.5 \mu \mathrm{M}$ concentration of each primer and $25 \mathrm{ng}$ of gDNA from strain B. pseudomallei K96243 as a template in a total volume of $20 \mu \mathrm{L}$. Amplification curves were obtained with the following program: $95^{\circ} \mathrm{C}, 3 \mathrm{~min}-$ $\left[95^{\circ} \mathrm{C} 5 \mathrm{sec}, 64^{\circ} \mathrm{C} 10 \mathrm{sec}\right] \times 35$ cycles. qPCR was performed using a Bio-Rad CFX96 instrument and data analyzed with the CFX Manager ${ }^{\mathrm{TM}}$ software Version 3.0. PrimeTime ${ }^{\circledR}$ qPCR probe was developed for the bucl16 gene, which yielded robust amplification in 5' nuclease qPCR assays, to detect B. pseudomallei and B. mallei 
species. Locked nucleic acid (LNA) bucl16-based probe (Table 4) contained a 5'-FAM fluorophore and a 3'-lowa Black fluorescent quencher. Reactions were carried out using SsoAdvanced ${ }^{\mathrm{TM}}$ Universal Probes supermix (Bio-Rad), $0.5 \mu \mathrm{M}$ primers, $0.2 \mu \mathrm{M}$ concentration of probe and $25 \mathrm{ng}$ of gDNA template in a total volume of $20 \mu \mathrm{L}$. Amplification curves were obtained with the following program: $95^{\circ} \mathrm{C} 3 \mathrm{~min}-\left[95^{\circ} \mathrm{C} 5 \mathrm{sec}\right.$, $\left.64^{\circ} \mathrm{C} 10 \mathrm{sec}\right] \times 35$ cycles.

\section{Capillary gel electrophoresis}

Reagents for separation of DNA by capillary gel electrophoresis included the nanogel matrix composed of the phospholipids dimyristoyl-sn-glycero-3-phosphocholine (DMPC) and 1,2-dihexanoyl-sn-glycero-3-phosphocholine (DHPC) (Avanti Polar Lipids, Alabaster, $A L)$, 3-(N-morpholino)-propanesulfonic acid (MOPS) (Alfa Aesar, Ward Hill, MA) buffer, and SYBR green 1 (Life Technologies, Grand Island, NY). The phospholipid pseudogel was prepared at a molar ratio of $[\mathrm{DMPC}] /[\mathrm{DHPC}]=2.5$ at $10 \% \mathrm{wt} / \mathrm{vol}$ in an aqueous solution of $100 \mathrm{mM}$ MOPS buffer $(\mathrm{pH} 7)$ in order to generate the nanogel separation matrix. Intercalating dye was incorporated into the nanogel at $1 x$ concentration to enable fluorescent DNA detection. The 50-bp DNA ladder (New England BioLabs, Ipswich, MA) was used as a molecular size marker.

Separations were performed on a Beckman Coulter P/ACE MDQ system equipped with a laser-induced fluorescence detection module and a $3 \mathrm{~mW}$ air-cooled argon ion laser $\left(\lambda_{\mathrm{ex}}=488 \mathrm{~nm}\right.$ and $\lambda_{\mathrm{em}}=520 \mathrm{~nm}$ ). The fused silica capillary was conditioned prior to electrophoresis separation of DNA using previously described rinsing (62) and coating (102) procedures. The capillary was filled with liquid nanogel solution a temperature below $24^{\circ} \mathrm{C}\left(19-21^{\circ} \mathrm{C}\right)$; then the temperature was increased to $30^{\circ} \mathrm{C}$ in order to form the sieving gel for accurate sizing separations of PCR amplicons. DNA samples were electrokinetically injected under reverse polarity as previously described (103). Data collection and analysis were performed with 32 Karat Software version 5.0 (Beckman Coulter). Sizing was accomplished by co-injecting the bucl5, 13, 14, and 16 amplicons with two internal standards of known length that bracketed the size of the DNA targets. Internal standards of $100 \mathrm{bp}$ and $250 \mathrm{bp}$ were used to create a linear fit for DNA size (in bp) versus migration time. The resulting slope and intercept were then used to calculate 
the size (length in bp) of the bucl gene targets based on their migration times. The reported values for the calculated DNA size and standard deviation (in bp) are an average for $n=5$ consecutive separations.

\section{Detection of $B$. pseudomalleigDNA in infected mice and human plasma using bucl markers}

BALB/c mice (female 7-10 weeks of age at time of challenge-National Cancer Institute, $\mathrm{NCl}$-Frederick, MD) were injected by the intraperitoneal (i.p.) route. Mice were infected with a dose equivalent to approximately 6 times the $\mathrm{LD}_{50}$ of $B$. pseudomallei HBPUB10134a (LD50 is $10 \mathrm{CFU}$ ) (67). At various time points after infection mice were euthanized by exsanguination under deep anesthesia and spleens were harvested. Spleens were weighed and homogenized in RPMI 1640 medium (Life Technology, Grand Island, NY). Bacterial load in the freshly prepared spleen extracts was determined by plating serial dilutions on sheep blood agar (ThermoScientific Remel Products, KS). Plates were incubated at $37^{\circ} \mathrm{C}$ for two days before determining CFU counts. The spleen extracts were irradiated and confirmed sterile before use in PCR assays, and were stored at $-70^{\circ} \mathrm{C}$. Standard PCR for bucl genes $5,13,14$, and 16 , and probe-based qPCR for bucl16 were performed as described above using $1 \mu \mathrm{L}$ of DNA-containing spleen specimen. Additionally, qPCR reactions were performed with $30 \mathrm{ng}$ B. pseudomallei K96243 gDNA spiked with 5\% human plasma collected in EDTA tubes to test the feasibility of the assay on clinical samples containing plasma. qPCR experiments were performed in triplicate and $\mathrm{Cq}$ values were averaged.

\section{Statistical analyses}

Statistical significance of bucl presence in pathogenic B. pseudomallei and B. mallei strains vs. nonpathogenic $B$. thailandensis was performed using the Fisher Exact Probability Test, followed by calculation of Cramer's V squared. 


\section{ACKNOWLEDGMENTS}

We acknowledge providers of genomic DNA samples for PCR testing including (i) $\mathrm{NIH}$ Biodefense and Emerging Infections Research Resources Repository, NIAID, NIH, (ii) Dr. Christopher Cote (The United States Army Medical Research Institute of Infectious Disease), and (iii) Dr. Joanna Goldberg (Emory University). We also thank Paul Feustel (Albany Medical College) for consultation on statistical tests.

\section{REFERENCES}

1. Brodsky, B., and Ramshaw, J. A. (1997) The collagen triple-helix structure. Matrix Biol 15, 545-554

2. Brodsky, B., and Persikov, A. V. (2005) Molecular structure of the collagen triple helix. Adv Protein Chem 70, 301-339

3. Ricard-Blum, S. (2011) The collagen family. Cold Spring Harbor perspectives in biology 3, a004978

4. Xu, Y., Keene, D. R., Bujnicki, J. M., Höök, M., and Lukomski, S. (2002) Streptococcal Scl1 and Scl2 Proteins Form Collagen-like Triple Helices. J. Biol. Chem. 277, 27312-27318

5. Rasmussen, M., Eden, A., and Bjorck, L. (2000) SclA, a novel collagen-like surface protein of Streptococcus pyogenes. Infect Immun 68, 6370-6377

6. Rasmussen, M., and Bjorck, L. (2001) Unique regulation of SclB-a novel collagen-like surface protein of Streptococcus pyogenes. Infect Immun 40, 14271438

7. Lukomski, S., Nakashima, K., Abdi, I., Cipriano, V. J., Ireland, R. M., Reid, S. D., Adams, G. G., and Musser, J. M. (2000) Identification and characterization of the scl gene encoding a group A Streptococcus extracellular protein virulence factor with similarity to human collagen. Infect Immun 68, 6542-6553

8. Lukomski, S., Nakashima, K., Abdi, I., Cipriano, V. J., Shelvin, B. J., Graviss, E. A., and Musser, J. M. (2001) Identification and characterization of a second extracellular collagen-like protein made by group A Streptococcus: control of production at the level of translation. Infect Immun 69, 1729-1738

9. De Simone, A., Vitagliano, L., and Berisio, R. (2008) Role of hydration in collagen triple helix stabilization. Biochem Biophys Res Commun 372, 121-125

10. Berisio, R., De Simone, A., Ruggiero, A., Improta, R., and Vitagliano, L. (2009) Role of side chains in collagen triple helix stabilization and partner recognition. $J$ Pept Sci 15, 131-140

11. Yu, Z., An, B., Ramshaw, J. A., and Brodsky, B. (2014) Bacterial collagen-like proteins that form triple-helical structures. $J$ Struct Biol 186, 451-461

12. Mohs, A., Silva, T., Yoshida, T., Amin, R., Lukomski, S., Inouye, M., and Brodsky, B. (2007) Mechanism of stabilization of a bacterial collagen triple helix in the absence of hydroxyproline. J. Biol. Chem. 282, 29757-29765 
13. Han, R., Zwiefka, A., Caswell, C. C., Xu, Y., Keene, D. R., Lukomska, E., Zhao, Z., Hook, M., et al. (2006) Assessment of prokaryotic collagen-like sequences derived from streptococcal Scl1 and Scl2 proteins as a source of recombinant GXY polymers. Appl Microbiol Biotechnol 72, 109-115

14. Xu, C., Yu, Z., Inouye, M., Brodsky, B., and Mirochnitchenko, O. (2010) Expanding the family of collagen proteins: recombinant bacterial collagens of varying composition form triple-helices of similar stability. Biomacromolecules 11, 348-356

15. Boydston, J. A., Chen, P., Steichen, C. T., and Turnbough, C. L., Jr. (2005) Orientation within the exosporium and structural stability of the collagen-like glycoprotein BclA of Bacillus anthracis. J. Bacteriol. 187, 5310-a-5317

16. Sylvestre, P., Couture-Tosi, E., and Mock, M. (2002) A collagen-like surface glycoprotein is a structural component of the Bacillus anthracis exosporium. Mol Microbiol 45, 169-178

17. Whatmore, A. M. (2001) Streptococcus pyogenes sclB encodes a putative hypervariable surface protein with a collagen-like repetitive structure. Microbiol 147, 419-429

18. Paterson, G. K., Nieminen, L., Jefferies, J. M., and Mitchell, T. J. (2008) PclA, a pneumococcal collagen-like protein with selected strain distribution, contributes to adherence and invasion of host cells. FEMS Microbiol Lett 285, 170-176

19. Vandersmissen, L., De Buck, E., Saels, V., Coil, D. A., and Anne, J. (2010) A Legionella pneumophila collagen-like protein encoded by a gene with a variable number of tandem repeats is involved in the adherence and invasion of host cells. FEMS Microbiol Lett 306, 168-176

20. Karlstrom, A., Jacobsson, K., Flock, M., Flock, J., and Guss, B. (2004) Identification of a novel collagen-like protein, ScIC, in Streptococcus equi using signal sequence phage display. Vet Microbiol 104, 179-188

21. Pizarro-Guajardo, M., Olguin-Araneda, V., Barra-Carrasco, J., Brito-Silva, C., Sarker, M. R., and Paredes-Sabja, D. (2014) Characterization of the collagen-like exosporium protein, BclA1, of Clostridium difficile spores. Anaerobe 25, 18-30

22. Beres, S., Sesso, R., Pinto, S., Hoe, N., Porcella, S., Deleo, F., and Musser, J. (2008) Genome sequence of a lancefield group C Streptococcus zooepidemicus strain causing epidemic nephritis: new information about an old disease. PLOS ONE 3, e3026

23. Han, R., Caswell, C. C., Lukomska, E., Keene, D. R., Pawlowski, M., Bujnicki, J. M., Kim, J. K., and Lukomski, S. (2006) Binding of the low-density lipoprotein by streptococcal collagen-like protein Scl1 of Streptococcus pyogenes. Mol Microbiol 61, 351-367

24. Caswell, C., Lukomska, E., Seo, N., Hook, M., and Lukomski, S. (2007) Scl1dependent internalization of group A Streptococcus via direct interactions with the a2b1 integrin enhances pathogen survival and re-emergence. Mol Microbiol 64, 1319-1331

25. Caswell, C. C., Barczyk, M., Keene, D. R., Lukomska, E., Gullberg, D. E., and Lukomski, S. (2008) Identification of the first prokaryotic collagen sequence motif that mediates binding to human collagen receptors, integrins a2b1 and a11b1. $J$ Biol Chem 283, 36168-36175 
26. Caswell, C. C., Oliver-Kozup, H., Han, R., Lukomska, E., and Lukomski, S. (2010) Scl1, the multifunctional adhesin of group A Streptococcus, selectively binds cellular fibronectin and laminin, and mediates pathogen internalization by human cells. FEMS Microbiol Lett 303, 61-68

27. Reuter, M., Caswell, C. C., Lukomski, S., and Zipfel, P. F. (2010) Binding of the human complement regulators CFHR1 and factor $\mathrm{H}$ by streptococcal collagenlike protein 1 (Scl1) via their conserved $\mathrm{C}$ termini allows control of the complement cascade at multiple levels. J Biol Chem 285, 38473-38485

28. Oliver-Kozup, H., Martin, K. H., Schwegler-Berry, D., Green, B. J., Betts, C., Shinde, A. V., Van De Water, L., and Lukomski, S. (2013) The group A streptococcal collagen-like protein-1, Scl1, mediates biofilm formation by targeting the extra domain A-containing variant of cellular fibronectin expressed in wounded tissue. Mol Microbiol 87, 672-689

29. Pahlman, L. I., Marx, P. F., Morgelin, M., Lukomski, S., Meijers, J. C. M., and Herwald, H. (2007) Thrombin-activatable fibrinolysis inhibitor binds to Streptococcus pyogenes by interacting with collagen-like proteins A and B. J. Biol. Chem. 282, 24873-24881

30. Bozue, J., Moody, K., Cote, C., Stiles, B., Friedlander, A., Welkos, S., and Hale, M. (2007) Bacillus anthracis spores of the bclA mutant exhibit increased adherence to epithelial, fibroblast, and endothelial cells but not macrophages. Infect Immun 75, 4498-4505

31. Tuntevski, K., Durney, B. C., Snyder, A. K., Lasala, P. R., Nayak, A. P., Green, B. J., Beezhold, D. H., Rio, R. V., et al. (2013) Aspergillus collagen-like (acl) genes: identification, sequence polymorphism and assessment for PCR-based pathogen detection. Appl Environ Microbiol 79, 7882-7895

32. Leski, T. A., Caswell, C. C., Pawlowski, M., Klinke, D. J., Bujnicki, J. M., Hart, S. J., and Lukomski, S. (2009) Identification and classification of $b c /$ genes and proteins of Bacillus cereus group organisms and their application in Bacillus anthracis detection and fingerprinting. Appl Environ Microbiol 75, 7163-7172

33. Sylvestre, P., Couture-Tosi, E., and Mock, M. (2003) Polymorphism in the collagen-like region of the Bacillus anthracis BclA protein leads to variation in exosporium filament length. J Bacteriol 185, 1555 - 1563

34. Castanha, E. R., Swiger, R. R., Senior, B., Fox, A., Waller, L. N., and Fox, K. F. (2006) Strain discrimination among $B$. anthracis and related organisms by characterization of $b c / A$ polymorphisms using PCR coupled with agarose gel or microchannel fluidics electrophoresis. J Microbiol Methods 64, 27-45

35. Durney, B. C., Bachert, B. A., Sloane, B. S., Lukomski, S., Landers, J. P., and Holland, L. A. (2015) Reversible phospholipid nanogels for deoxyribonucleic acid fragment size determinations up to 1,500 base pairs and integrated sample stacking. Anal Chim Acta

36. Vandamme, P., and Dawyndt, P. (2011) Classification and identification of the Burkholderia cepacia complex: Past, present and future. Systematic and applied microbiology 34, 87-95

37. Wheelis, M. (1998) First shots fired in biological warfare. Nature 395, 213

38. White, N. J. Melioidosis. The Lancet 361, 1715-1722 
39. Anuntagool, N., Naigowit, P., Petkanchanapong, V., Aramsri, P., Panichakul, T., and Sirisinha, S. (2000) Monoclonal antibody-based rapid identification of Burkholderia pseudomallei in blood culture fluid from patients with communityacquired septicaemia. J Med Microbiol 49, 1075-1078

40. Holden, M. T., Titball, R. W., Peacock, S. J., Cerdeno-Tarraga, A. M., Atkins, T., Crossman, L. C., Pitt, T., Churcher, C., et al. (2004) Genomic plasticity of the causative agent of melioidosis, Burkholderia pseudomallei. Proc Natl Acad Sci U $S$ A 101, 14240-14245

41. Nierman, W. C., DeShazer, D., Kim, H. S., Tettelin, H., Nelson, K. E., Feldblyum, T., Ulrich, R. L., Ronning, C. M., et al. (2004) Structural flexibility in the Burkholderia mallei genome. Proc. Natl. Acad. Sci. USA 101, 14246-14251

42. Godoy, D., Randle, G., Simpson, A. J., Aanensen, D. M., Pitt, T. L., Kinoshita, R., and Spratt, B. G. (2003) Multilocus sequence typing and evolutionary relationships among the causative agents of melioidosis and glanders, Burkholderia pseudomallei and Burkholderia mallei. J Clin Microbiol 41, 20682079

43. Lazar Adler, N. R., Govan, B., Cullinane, M., Harper, M., Adler, B., and Boyce, J. D. (2009) The molecular and cellular basis of pathogenesis in melioidosis: how does Burkholderia pseudomallei cause disease? FEMS Microbiol Rev 33, 10791099

44. Galyov, E. E., Brett, P. J., and DeShazer, D. (2010) Molecular insights into Burkholderia pseudomallei and Burkholderia mallei pathogenesis. Annu. Rev. Microbiol. 64, 495-517

45. Karlstrom, A., Jacobsson, K., and Guss, B. (2005) SclC is a member of a novel family of collagen-like proteins in Streptococcus equi subspecies equi that are recognised by antibodies against SclC. Vet Microbiol

46. Losada, L., Ronning, C. M., DeShazer, D., Woods, D., Fedorova, N., Kim, H. S., Shabalina, S. A., Pearson, T. R., et al. (2010) Continuing evolution of Burkholderia mallei through genome reduction and large-scale rearrangements. Genome biology and evolution 2, 102-116

47. Persikov, A. V., Ramshaw, J. A. M., and Brodsky, B. (2005) Prediction of collagen stability from amino acid sequence. J. Biol. Chem. 280, 19343-19349

48. Brown, N. H., Gregory, S. L., Rickoll, W. L., Fessler, L. I., Prout, M., White, R. A., and Fristrom, J. W. (2002) Talin is essential for integrin function in Drosophila. Dev Cell 3, 569-579

49. Tadokoro, S., Shattil, S. J., Eto, K., Tai, V., Liddington, R. C., de Pereda, J. M., Ginsberg, M. H., and Calderwood, D. A. (2003) Talin binding to integrin beta tails: a final common step in integrin activation. Science 302, 103-106

50. Wegener, K. L., Partridge, A. W., Han, J., Pickford, A. R., Liddington, R. C., Ginsberg, M. H., and Campbell, I. D. (2007) Structural basis of integrin activation by talin. Cell 128, 171-182

51. Sun, J., Deng, Z., and Yan, A. (2014) Bacterial multidrug efflux pumps: mechanisms, physiology and pharmacological exploitations. Biochem Biophys Res Commun 453, 254-267 
52. Tam, R., and Saier, M. H., Jr. (1993) Structural, functional, and evolutionary relationships among extracellular solute-binding receptors of bacteria.

Microbiological reviews 57, 320-346

53. Thibault, F. M., Hernandez, E., Vidal, D. R., Girardet, M., and Cavallo, J.-D. (2004) Antibiotic susceptibility of 65 isolates of Burkholderia pseudomallei and Burkholderia mallei to 35 antimicrobial agents. J Antimicrob Chemother 54, 11341138

54. Ikeda, M., Arai, M., Lao, D. M., and Shimizu, T. (2002) Transmembrane topology prediction methods: a re-assessment and improvement by a consensus method using a dataset of experimentally-characterized transmembrane topologies. In Silico Biol 2, 19-33

55. Johnson, J. M., and Church, G. M. (1999) Alignment and structure prediction of divergent protein families: periplasmic and outer membrane proteins of bacterial efflux pumps. J Mol Biol 287, 695-715

56. Phan, G., Benabdelhak, H., Lascombe, M. B., Benas, P., Rety, S., Picard, M., Ducruix, A., Etchebest, C., et al. (2010) Structural and dynamical insights into the opening mechanism of $P$. aeruginosa OprM channel. Structure 18, 507-517

57. Eswar, N., Webb, B., Marti-Renom, M. A., Madhusudhan, M. S., Eramian, D., Shen, M.-y., Pieper, U., and Sali, A. (2002) Comparative Protein Structure Modeling Using Modeller. in Current protocols in bioinformatics / editoral board, Andreas D. Baxevanis ... [et al.], John Wiley \& Sons, Inc. pp

58. Akama, H., Matsuura, T., Kashiwagi, S., Yoneyama, H., Narita, S., Tsukihara, T., Nakagawa, A., and Nakae, T. (2004) Crystal structure of the membrane fusion protein, MexA, of the multidrug transporter in Pseudomonas aeruginosa. J Biol Chem 279, 25939-25942

59. Yu, Y., Kim, H. S., Chua, H. H., Lin, C. H., Sim, S. H., Lin, D., Derr, A., Engels, R., et al. (2006) Genomic patterns of pathogen evolution revealed by comparison of Burkholderia pseudomallei, the causative agent of melioidosis, to avirulent Burkholderia thailandensis. BMC Microbiol 6, 46

60. Pearson, T., Giffard, P., Beckstrom-Sternberg, S., Auerbach, R., Hornstra, H., Tuanyok, A., Price, E. P., Glass, M. B., et al. (2009) Phylogeographic reconstruction of a bacterial species with high levels of lateral gene transfer. $B M C$ Biol 7, 78

61. Brett, P. J., DeShazer, D., and Woods, D. E. (1998) Burkholderia thailandensis sp. nov., a Burkholderia pseudomallei-like species. International journal of systematic bacteriology 48 Pt 1, 317-320

62. Durney, B. C., Lounsbury, J. A., Poe, B. L., Landers, J. P., and Holland, L. A. (2013) A thermally responsive phospholipid pseudogel: tunable DNA sieving with capillary electrophoresis. Anal Chem 85, 6617-6625

63. Bowers, J. R., Engelthaler, D. M., Ginther, J. L., Pearson, T., Peacock, S. J., Tuanyok, A., Wagner, D. M., Currie, B. J., et al. (2010) BurkDiff: a real-time PCR allelic discrimination assay for Burkholderia pseudomallei and B. mallei. PLoS ONE 5, e15413

64. Price, E. P., Dale, J. L., Cook, J. M., Sarovich, D. S., Seymour, M. L., Ginther, J. L., Kaufman, E. L., Beckstrom-Sternberg, S. M., et al. (2012) Development and 
validation of Burkholderia pseudomallei-specific real-time PCR assays for clinical, environmental or forensic detection applications. PLOS ONE 7, e37723

65. Novak, R. T., Glass, M. B., Gee, J. E., Gal, D., Mayo, M. J., Currie, B. J., and Wilkins, P. P. (2006) Development and evaluation of a real-time PCR assay targeting the type III secretion system of Burkholderia pseudomallei. J Clin Microbiol 44, 85-90

66. Meumann, E. M., Novak, R. T., Gal, D., Kaestli, M. E., Mayo, M., Hanson, J. P., Spencer, E., Glass, M. B., et al. (2006) Clinical evaluation of a type III secretion system real-time PCR assay for diagnosing melioidosis. J Clin Microbiol 44, 3028-3030

67. Welkos, S. L., Klimko, C. P., Kern, S., Bearss, J., Bozue, J. A., Bernhards, R. C., Trevino, S., Waag, D., et al. (2015) Characterization of Burkholderia pseudomallei strains using a murine intraperitoneal infection model and in vitro macrophage assays. PLOS ONE

68. Berisio, R., Vitagliano, L., Mazzarella, L., and Zagari, A. (2002) Recent progress on collagen triple helix structure, stability and assembly. Protein Pept Lett 9, 107116

69. Okuyama, K. (2008) Revisiting the molecular structure of collagen. Connect Tissue Res 49, 299-310

70. Shoulders, M. D., and Raines, R. T. (2009) Collagen structure and stability. Annu Rev Biochem 78, 929-958

71. Chan, V. C., Ramshaw, J. A., Kirkpatrick, A., Beck, K., and Brodsky, B. (1997) Positional preferences of ionizable residues in Gly-X-Y triplets of the collagen triple-helix. J Biol Chem 272, 31441-31446

72. Leikina, E., Mertts, M. V., Kuznetsova, N., and Leikin, S. (2002) Type I collagen is thermally unstable at body temperature. Proc Natl Acad Sci U S A 99, 13141318

73. Rasmussen, M., Jacobsson, M., and Bjorck, L. (2003) Genome-based identification and analysis of collagen-related structural motifs in bacterial and viral proteins. J. Biol. Chem. 278, 32313-32316

74. Ruggiero, A., Tizzano, B., Pedone, E., Pedone, C., Wilmanns, M., and Berisio, R. (2009) Crystal structure of the resuscitation-promoting factor (DeltaDUF)RpfB from M. tuberculosis. J Mol Bio/ 385, 153-162

75. Warawa, J., and Woods, D. E. (2005) Type III secretion system cluster 3 is required for maximal virulence of Burkholderia pseudomallei in a hamster infection model. FEMS Microbiol Lett 242, 101-108

76. Schwarz, S., Singh, P., Robertson, J. D., LeRoux, M., Skerrett, S. J., Goodlett, D. R., West, T. E., and Mougous, J. D. (2014) VgrG-5 is a Burkholderia type VI secretion system-exported protein required for multinucleated giant cell formation and virulence. Infect Immun 82, 1445-1452

77. Angus, A. A., Agapakis, C. M., Fong, S., Yerrapragada, S., Estrada-de los Santos, P., Yang, P., Song, N., Kano, S., et al. (2014) Plant-associated symbiotic Burkholderia species lack hallmark strategies required in mammalian pathogenesis. PLOS ONE 9, e83779 
78. Rainbow, L., Hart, C. A., and Winstanley, C. (2002) Distribution of type III secretion gene clusters in Burkholderia pseudomallei, $B$. thailandensis and $B$. mallei. J Med Microbiol 51, 374-384

79. French, C. T., Toesca, I. J., Wu, T. H., Teslaa, T., Beaty, S. M., Wong, W., Liu, M., Schroder, I., et al. (2011) Dissection of the Burkholderia intracellular life cycle using a photothermal nanoblade. Proc Natl Acad Sci U S A 108, 12095-12100

80. Stevens, M. P., Wood, M. W., Taylor, L. A., Monaghan, P., Hawes, P., Jones, P. W., Wallis, T. S., and Galyov, E. E. (2002) An Inv/Mxi-Spa-like type III protein secretion system in Burkholderia pseudomallei modulates intracellular behaviour of the pathogen. Mol Microbiol 46, 649-659

81. Choh, L. C., Ong, G. H., Vellasamy, K. M., Kalaiselvam, K., Kang, W. T., AlMaleki, A. R., Mariappan, V., and Vadivelu, J. (2013) Burkholderia vaccines: are we moving forward? Frontiers in cellular and infection microbiology 3, 5

82. Moore, R. A., DeShazer, D., Reckseidler, S., Weissman, A., and Woods, D. E. (1999) Efflux-mediated aminoglycoside and macrolide resistance in Burkholderia pseudomallei. Antimicrob Agents Chemother 43, 465-470

83. Chan, Y. Y., Tan, T. M., Ong, Y. M., and Chua, K. L. (2004) BpeAB-OprB, a multidrug efflux pump in Burkholderia pseudomallei. Antimicrob Agents Chemother 48, 1128-1135

84. Podnecky, N. L., Wuthiekanun, V., Peacock, S. J., and Schweizer, H. P. (2013) The BpeEF-OprC efflux pump is responsible for widespread trimethoprim resistance in clinical and environmental Burkholderia pseudomallei isolates. Antimicrob Agents Chemother 57, 4381-4386

85. Doxey, A. C., and McConkey, B. J. (2013) Prediction of molecular mimicry candidates in human pathogenic bacteria. Virulence 4, 453-466

86. Hoffmaster, A. R., AuCoin, D., Baccam, P., Baggett, H. C., Baird, R., Bhengsri, S., Blaney, D. D., Brett, P. J., et al. (2015) Melioidosis diagnostic workshop, 2013. Emerg Infect Dis 21

87. Van Zandt, K. E., Greer, M. T., and Gelhaus, H. C. (2013) Glanders: an overview of infection in humans. Orphanet journal of rare diseases 8, 131

88. Currie, B. J. (2015) Melioidosis: evolving concepts in epidemiology, pathogenesis, and treatment. Semin Respir Crit Care Med 36, 111-125

89. Wongsuvan, G., Limmathurotsakul, D., Wannapasni, S., Chierakul, W., Teerawattanasook, N., and Wuthiekanun, V. (2009) Lack of correlation of Burkholderia pseudomallei quantities in blood, urine, sputum and pus. The Southeast Asian journal of tropical medicine and public health 40, 781-784

90. Walsh, A. L., Smith, M. D., Wuthiekanun, V., Suputtamongkol, Y., Chaowagul, W., Dance, D. A., Angus, B., and White, N. J. (1995) Prognostic significance of quantitative bacteremia in septicemic melioidosis. Clin Infect Dis 21, 1498-1500

91. Punta, M., Coggill, P. C., Eberhardt, R. Y., Mistry, J., Tate, J., Boursnell, C., Pang, N., Forslund, K., et al. (2012) The Pfam protein families database. Nucleic Acids Res 40, D290-301

92. Shi, J., Blundell, T. L., and Mizuguchi, K. (2001) FUGUE: sequence-structure homology recognition using environment-specific substitution tables and structure-dependent gap penalties. J. Mol. Biol. 310, 243-257 
93. Bendtsen, J. D., Nielsen, H., von Heijne, G., and Brunak, S. (2004) Improved prediction of signal peptides: SignalP 3.0. J Mol Biol 340, 783-795

94. Nielsen, H., Engelbrecht, J., Brunak, S., and von Heijne, G. (1997) Identification of prokaryotic and eukaryotic signal peptides and prediction of their cleavage sites. Protein Eng 10, 1-6

95. Nielsen, H., and Krogh, A. (1998) Prediction of signal peptides and signal anchors by a hidden Markov model. Proc Int Conf Intell Syst Mol Biol 6, 122-130

96. Hofmann, K., and Stoffel, W. (1993) TMBASE - A database of membrane spanning protein segments. Biological Chemistry Hoppe-Seyler 374

97. Eddy, S. R. (2011) Accelerated Profile HMM Searches. PLoS Comput Biol 7, e1002195

98. Van Der Spoel, D., Lindahl, E., Hess, B., Groenhof, G., Mark, A. E., and Berendsen, H. J. (2005) GROMACS: fast, flexible, and free. J Comput Chem 26, 1701-1718

99. Tamura, K., Stecher, G., Peterson, D., Filipski, A., and Kumar, S. (2013) MEGA6: Molecular Evolutionary Genetics Analysis version 6.0. Mol Biol Evol 30, 27252729

100. Nylander, J. A. A. (2004) MrModeltest v2. Evolutionary Biology Centre, Uppsala University

101. Ronquist, F., and Huelsenbeck, J. P. (2003) MrBayes 3: Bayesian phylogenetic inference under mixed models. Bioinformatics 19, 1572-1574

102. White, C. M., Luo, R., Archer-Hartmann, S. A., and Holland, L. A. (2007) Electrophoretic screening of ligands under suppressed EOF with an inert phospholipid coating. Electrophoresis 28, 3049-3055

103. Luo, R., Archer-Hartmann, S. A., and Holland, L. A. (2010) Transformable capillary electrophoresis for oligosaccharide separations using phospholipid additives. Anal Chem 82, 1228-1233 


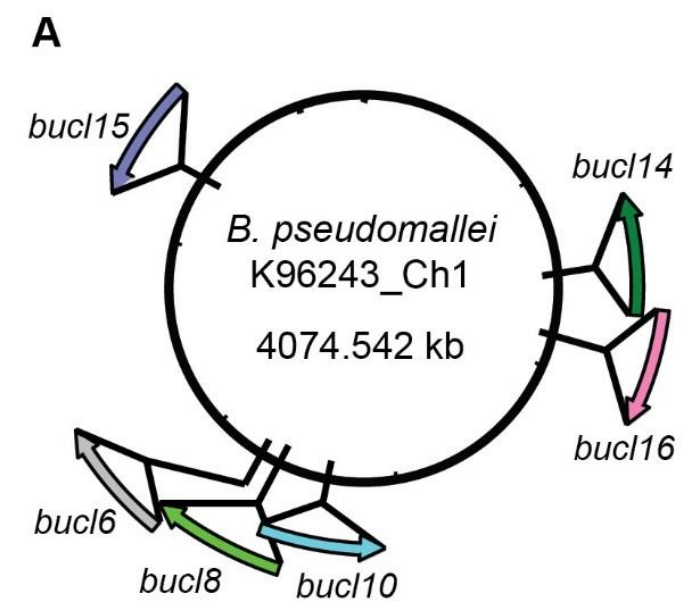

\begin{tabular}{ccccc}
\hline $\begin{array}{c}\text { Bucl } \\
\text { No. }\end{array}$ & $\begin{array}{c}\text { Ch/ } \\
\text { strand }\end{array}$ & Location & $\begin{array}{c}\text { Length } \\
\text { (bp) }\end{array}$ & $\begin{array}{c}\text { Molecular } \\
\text { weight (kDa) }\end{array}$ \\
\hline 1 & $2 /-$ & $2119909-2120421$ & 513 & 17.21 \\
\hline 2 & $2 /+$ & $2647246-2647895$ & 650 & 24.14 \\
\hline 3 & $2 /+$ & $2667859-2669577$ & 1719 & 51.03 \\
\hline 4 & $2 /+$ & $2202787-2203818$ & 1032 & 34.85 \\
\hline 5 & $2 /+$ & $326839-327465$ & 627 & 21.42 \\
\hline 6 & $1 /+$ & $2398535-2398690$ & 156 & 4.80 \\
\hline 7 & $2 /+$ & $1102922-1103488$ & 567 & 20.51 \\
\hline 8 & $1 /+$ & $2346940-2348973$ & 2034 & 68.30 \\
\hline 10 & $1 /-$ & $2171241-2171663$ & 423 & 14.97 \\
\hline 13 & $2 /-$ & $2293634-2294872$ & 1239 & 44.13 \\
\hline 14 & $1 /-$ & $952706-952977$ & 272 & 10.52 \\
\hline 15 & $1 /-$ & $3424165-3424360$ & 196 & 7.08 \\
\hline 16 & $1 /+$ & $1191473-1192156$ & 684 & 22.83 \\
\hline
\end{tabular}
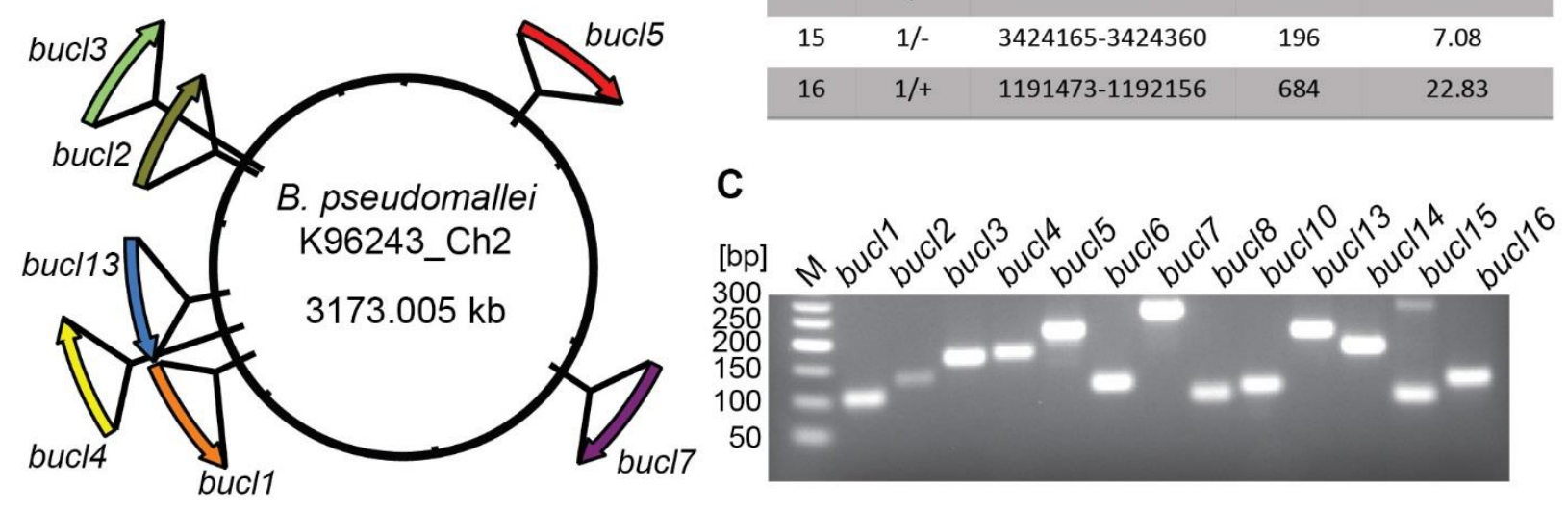

Figure 1

Identification and characterization of bucl genes in $B$. pseudomallei reference strain K96243. (A) Schematic representation of bucl distribution. Relative position and orientation of each bucl gene is shown; six bucl genes are present on chromosome one and seven on chromosome two. (B) Summary table of bucl distribution. bucl location, orientation, and length are mapped to the genome of Bp K96243. Molecular weight of each Bucl protein encoded by each bucl allele is shown. (C) PCR amplification of $13 \mathrm{bucl}$ genes from Bp K96243. Primers were designed targeting the non-collagenous conserved regions, and PCR conditions were established for all bucl amplicons at a uniform annealing temperature of $64^{\circ} \mathrm{C}$. Amplicon sizes; bucl1, $123 \mathrm{bp} ;$ bucl2 $133 \mathrm{bp} ;$ bucl3, 166 bp; bucl4, 176 bp; buc/5, 216 bp; bucl6, 115 bp; bucl7, 264 bp; buc/8, 96 bp; bucl10, 109 bp; bucl13, 212 bp; bucl14, 178 bp; bucl15, 95 bp; and bucl16, 123 bp ; M, 50-bp DNA size marker. 
A
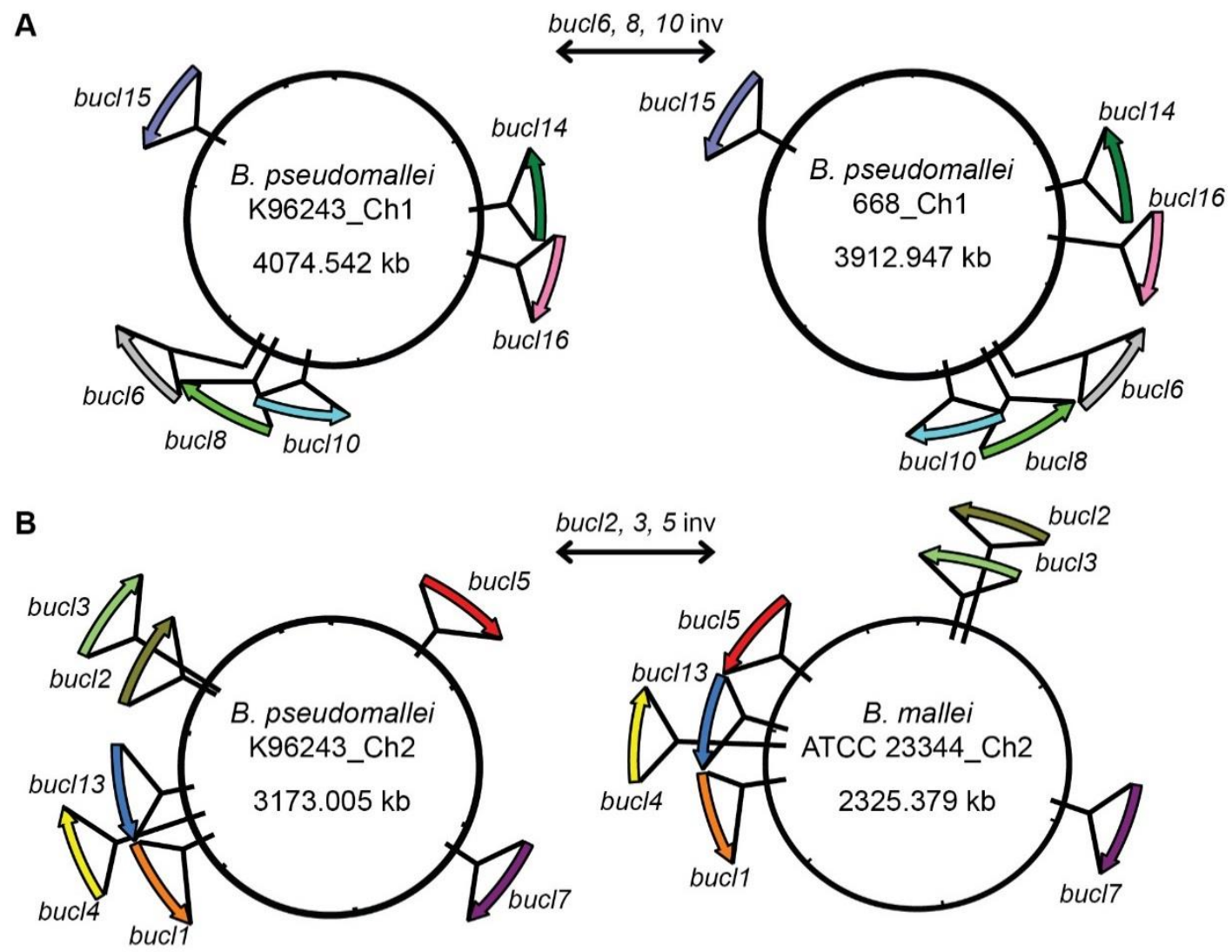

C

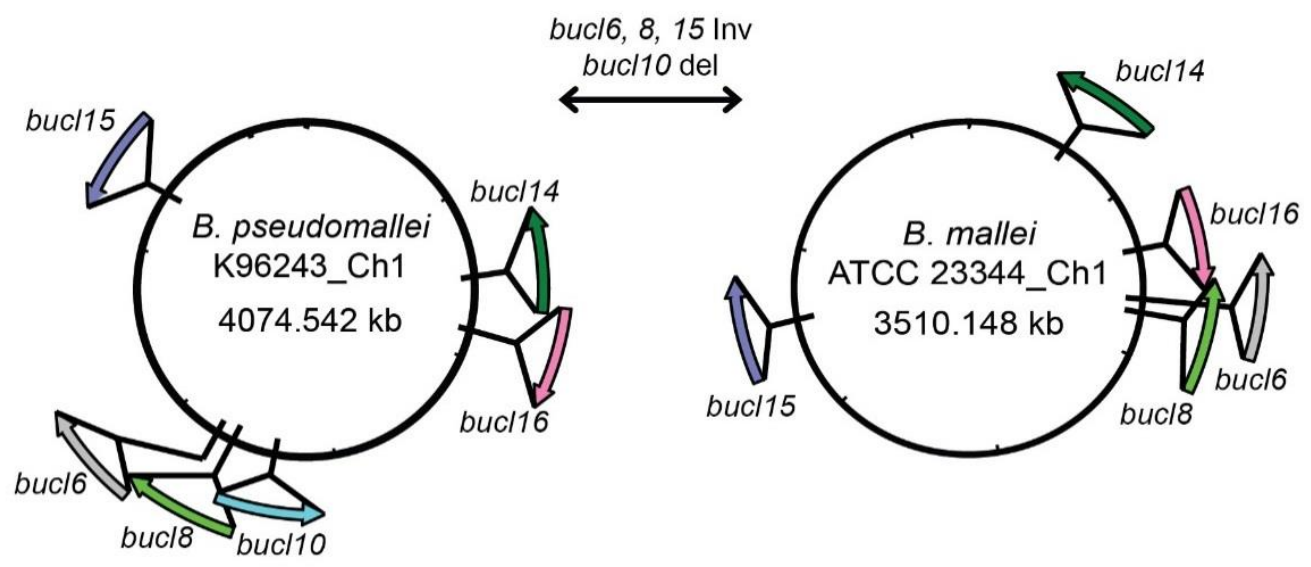

Figure 2

Chromosomal rearrangements and deletions involving bucl loci. Relative positions and orientations of each bucl gene was rendered from the NCBI database, and used for chromosomal mapping. (A) Intraspecies chromosomal inversion (inv) between $B$. pseudomallei strains K96243 and 668 involving the region encoding bucl genes 6, 8, and 10. (B) Interspecies chromosomal inversion between Bp K96243 and Bm ATCC 23344 involving the region encoding bucl genes 2,3 , and 5 on chromosome 2. (C) Interspecies chromosomal inversion involving bucl genes 6,8 , and 15 , and deletion of buc/10 between Bp K96243 and Bm ATCC 23344 on chromosome 1. Ch, chromosome. 
A

\begin{tabular}{|c|c|c|}
\hline $\begin{array}{l}\text { Bucl } \\
\text { No. }\end{array}$ & $\mathrm{CL}$ region sequence & $\begin{array}{l}\text { Average } \\
\text { stability }\end{array}$ \\
\hline 1 & $(\mathrm{GAN})_{16}-(\mathrm{GTN})_{4}-(\mathrm{GAA})-(\mathrm{GAD})$ & $25.6^{\circ} \mathrm{C}$ \\
\hline 2 & $\begin{array}{l}(\mathrm{GEV})_{14-}(\mathrm{GEA})-\mathrm{AEA}-(\mathrm{GEA})_{2}-\mathrm{AEA}-(\mathrm{GEV})- \\
(\mathrm{GEA})\end{array}$ & $35.3^{\circ} \mathrm{C}$ \\
\hline 3 & $(\mathrm{GTS})_{49-}(\mathrm{GTA})$ & $24.0^{\circ} \mathrm{C}$ \\
\hline 4 & $(\mathrm{GVS})_{10-}-(\mathrm{GAS})_{20}-(\mathrm{GTS})$ & $30.7^{\circ} \mathrm{C}$ \\
\hline 5 & $(G L E)_{20}-(G P E)_{2}-(G L D)_{2}-(G F D)$ & $37.2^{\circ} \mathrm{C}$ \\
\hline 6 & $\begin{array}{l}(\mathrm{GAL})-(\mathrm{GAS})_{2^{-}}(\mathrm{GAA})_{10^{-}}(\mathrm{GAS})-(\mathrm{GAA})_{5^{-}} \\
(\mathrm{GAE})\end{array}$ & $32.8^{\circ} \mathrm{C}$ \\
\hline 7 & $(\mathrm{GLS})-(\mathrm{GSS})_{10^{-}}(\mathrm{GAS})_{2}-(\mathrm{GVA})$ & $27.3^{\circ} \mathrm{C}$ \\
\hline 8 & $(\mathrm{GAS})_{21}$ & $33.0^{\circ} \mathrm{C}$ \\
\hline 10 & $(\mathrm{GIH})_{24-(\mathrm{GMR})}$ & $27.1^{\circ} \mathrm{C}$ \\
\hline 13 & $(\mathrm{GIR})_{12}-\mathrm{DIR}-(\mathrm{GIR})_{2}-(\mathrm{GVR})_{8}-(\mathrm{GIR})$ & $36.0^{\circ} \mathrm{C}$ \\
\hline 14 & $\begin{array}{l}(\mathrm{GWC})_{4^{-}}(\mathrm{GRC})_{5}-(\mathrm{GWC})_{2^{-}}-(\mathrm{GRC})_{20^{-}}(\mathrm{GRR})_{5^{-}} \\
(\mathrm{GRA})\end{array}$ & $30.6^{\circ} \mathrm{C}$ \\
\hline 15 & $(\mathrm{GAT})_{2}-(\mathrm{GAl})_{6}-(\mathrm{GAA})$ & $35.3^{\circ} \mathrm{C}$ \\
\hline 16 & $(\mathrm{GFG})_{2}-(\mathrm{GVD})-(\mathrm{GFD})_{26}-(\mathrm{GFG})$ & $20.3^{\circ} \mathrm{C}$ \\
\hline
\end{tabular}

B

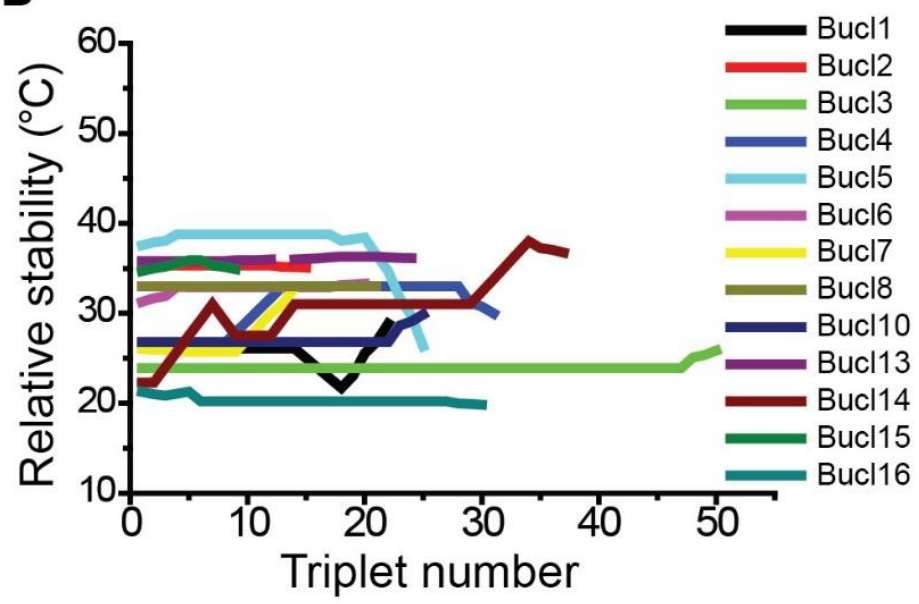

Figure 3

Thermal stability of the Bucl collagen regions. (A) The $C L$ region sequences, representative of all $13 \mathrm{Bucl}$ proteins, plotted in B) are shown with averaged stability values calculated for the entire CL region. (B) Triple helix thermal stability plot. Amino acid sequences for Bucl-CL regions shown in A) were used to model thermal stability with an algorithm developed by Persikov et al. 2005. Relative thermal stability is shown as the melting temperature for each GXY triplet along each Bucl-CL region. 
A

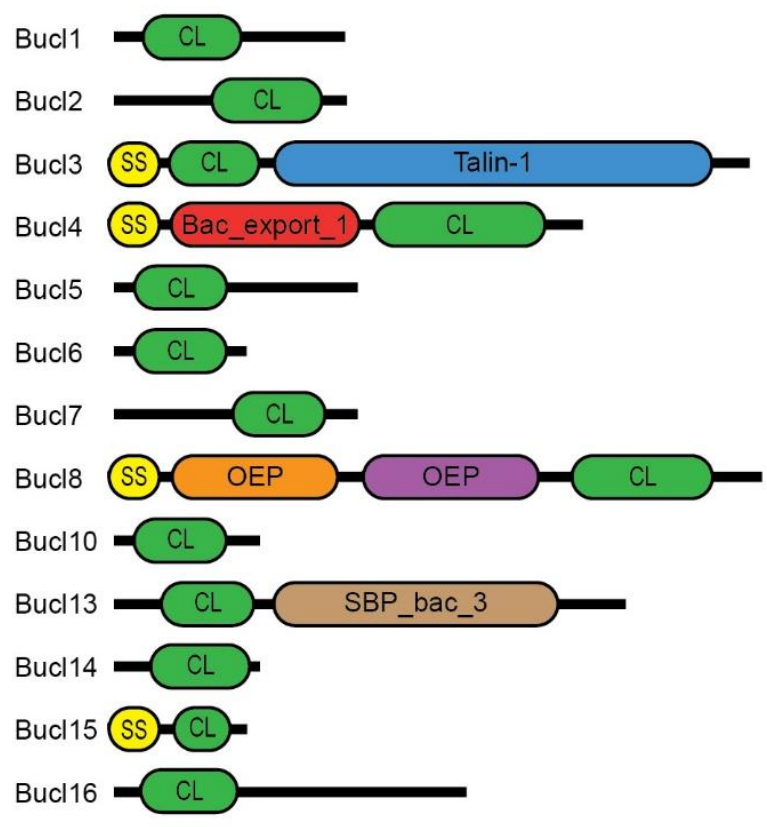

B

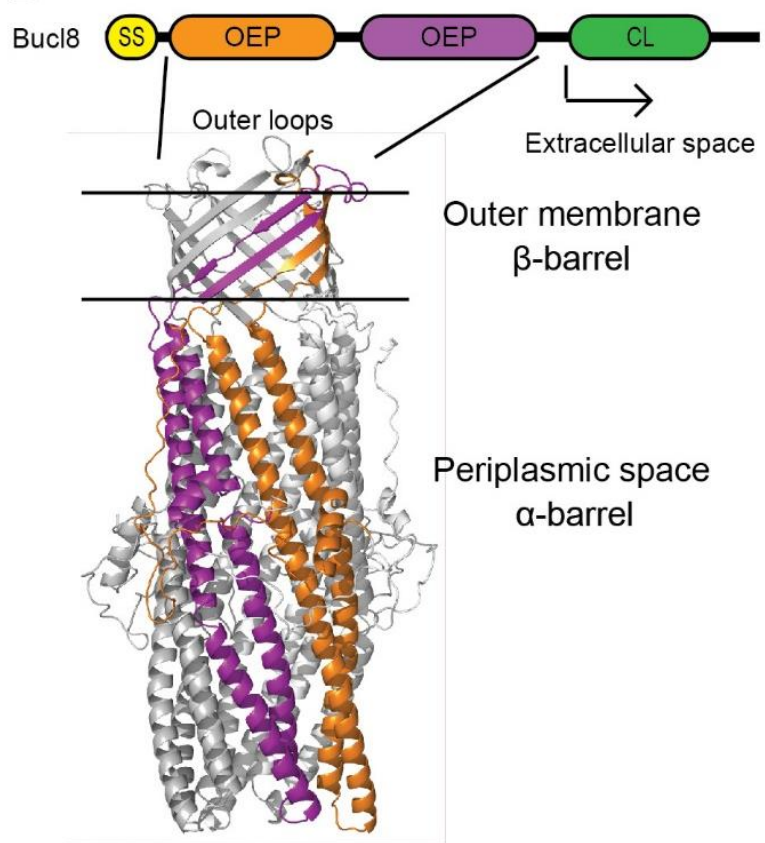

Figure 4

Characterization of Burkholderia collagen-like proteins. (A) Architecture of Bucl proteins identified in collagen Pfam data base (not to scale). Proteins were categorized into 13 distinct Bucl types based on sequence similarities and domain organization. Predicted domains in each Bucl are shown: SS, signal sequence; CL, collagen-like domain; Talin-1 domain; Bac_export_1, bacterial export protein family 1; OEP, Outer Membrane Efflux Protein; and SBP_bac_3, bacterial extracellular solute-binding protein family 3. (B) Cellular organization of Bucl8 and homology modelling of the OEP domains. Bucl8 protein schematic is shown above homology model of OEP domains generated with MODELLER. Three monomers, each containing two OEP domains, assemble to form a homotrimer. Shown from top to bottom are the cell-surface exposed loops, the $\beta$ barrel spanning the outer membrane and the a-barrel spanning the periplasmic space, corresponding to the predicted OEP domains. The two OEP domains from a single monomer are highlighted in orange and purple, and the remaining monomers are colored gray. Following the OEP domains, the $\mathrm{CL}$ region is predicted to be partially extracellular with an additional $\mathrm{C}$-terminal non-collagenous domain. 


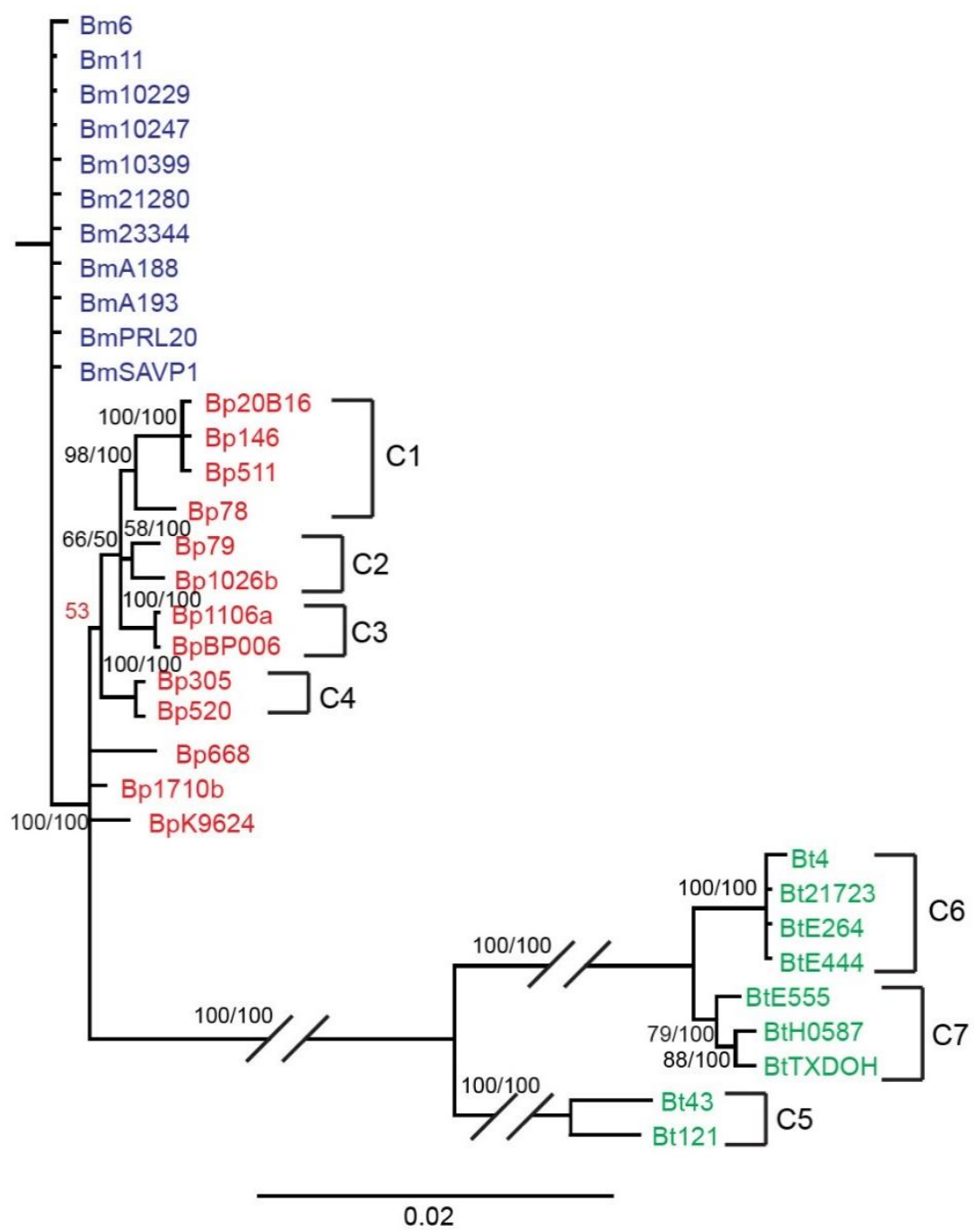

Figure 5

Phylogenetic analysis of B. pseudomallei, B. mallei, and B. thailandensis strains by bucl-locus typing. Bayesian analysis was performed on concatenated nucleotide sequences of the non-collagenous regions of buc/3, bucl4, and bucl/8 present in a set of 13 B. pseudomallei, 11 B. mallei, and 9 B. thailandensis strains (as shown in Table 3). Support values for each branch are shown as posterior probability from Bayesian analysis and bootstrap values from maximum parsimony analysis, respectively (PP/MP). Posterior probability value which was not supported by maximum parsimony analysis is shown in red. Phylogenetic Clusters 1-4 (C1-C4) correlated with geographic location of $B$. pseudomallei strains, whereas Clusters 5-7 (C5-C7) contained $B$. thailandensis strains that made up a separate branch from B. pseudomallei and B. mallei strains. Scale bar is representative of evolutionary distance in substitutions per nucleotide. 

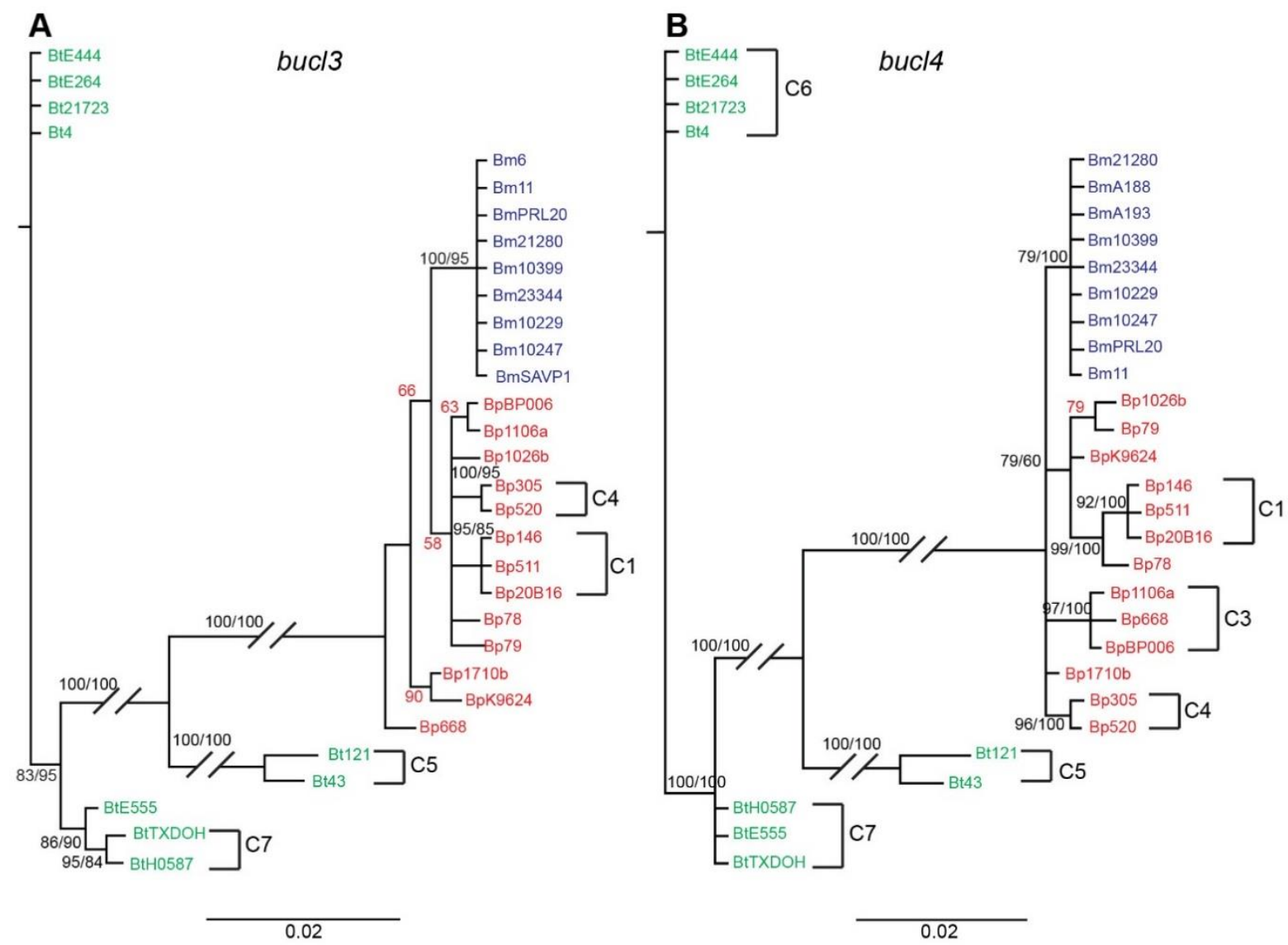

Figure 6

Phylogenetic analysis of B. pseudomallei, B. mallei, and B. thailandensis strains using individual buc/3 and bucl4 genes. Bayesian analysis was performed on nucleotide sequences of non-collagenous regions of a set of Burkholderia strains described in Table 3. Support values for each branch are shown as posterior probability from Bayesian analysis and bootstrap values from maximum parsimony analysis, respectively (PP/MP). Posterior probability values not supported by parsimony analysis are shown in red. Scale bar is representative of evolutionary distance in substitutions per nucleotide. Several clusters of strains corresponding to those observed in the concatenated analysis, C1-C7 in Fig. 5, were also observed in the individual trees. 

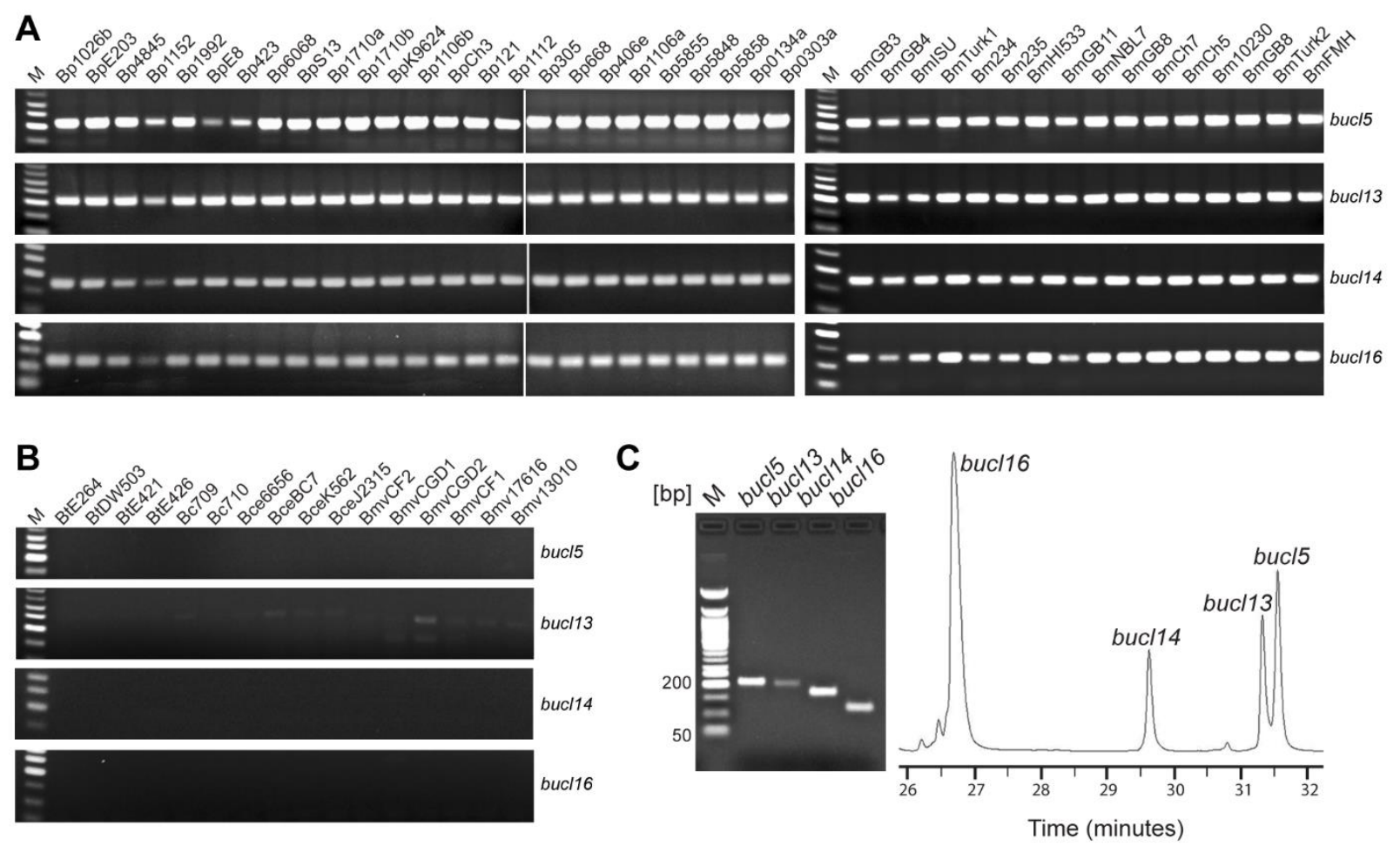

Figure 7

Distribution of bucl genes among Burkholderia spp. select agents by PCR. Presence of bucl genes was assessed by PCR on (A) a collection of genomic DNA from $25 \mathrm{~B}$. pseudomallei and $16 \mathrm{~B}$. mallei strains, as well as (B) in control strains of $B$. thailandensis, B. cepacia, B. cenocepacia, and B. multivorans; selected bucl genes 5,13 , 14 , and 16 are shown. (C) Detection and separation of selected bucl amplicons generated from the B. pseudomallei reference strain K96243 by traditional $2 \%$ agarose gel electrophoresis (left) or by capillary gel electrophoresis (right). Electropherogram generated by capillary gel electrophoresis with phospholipid nanogel matrix shows separation of amplicons over time. Amplicon sizes: bucl5, 216 bp; buc/13, 214 bp; bucl14, $178 \mathrm{bp}$; and bucl16, 123 bp. M, 50-bp DNA ladder. PCR data shown in Panel A for $25 \mathrm{Bp}$ strains come from two merged gel images. 

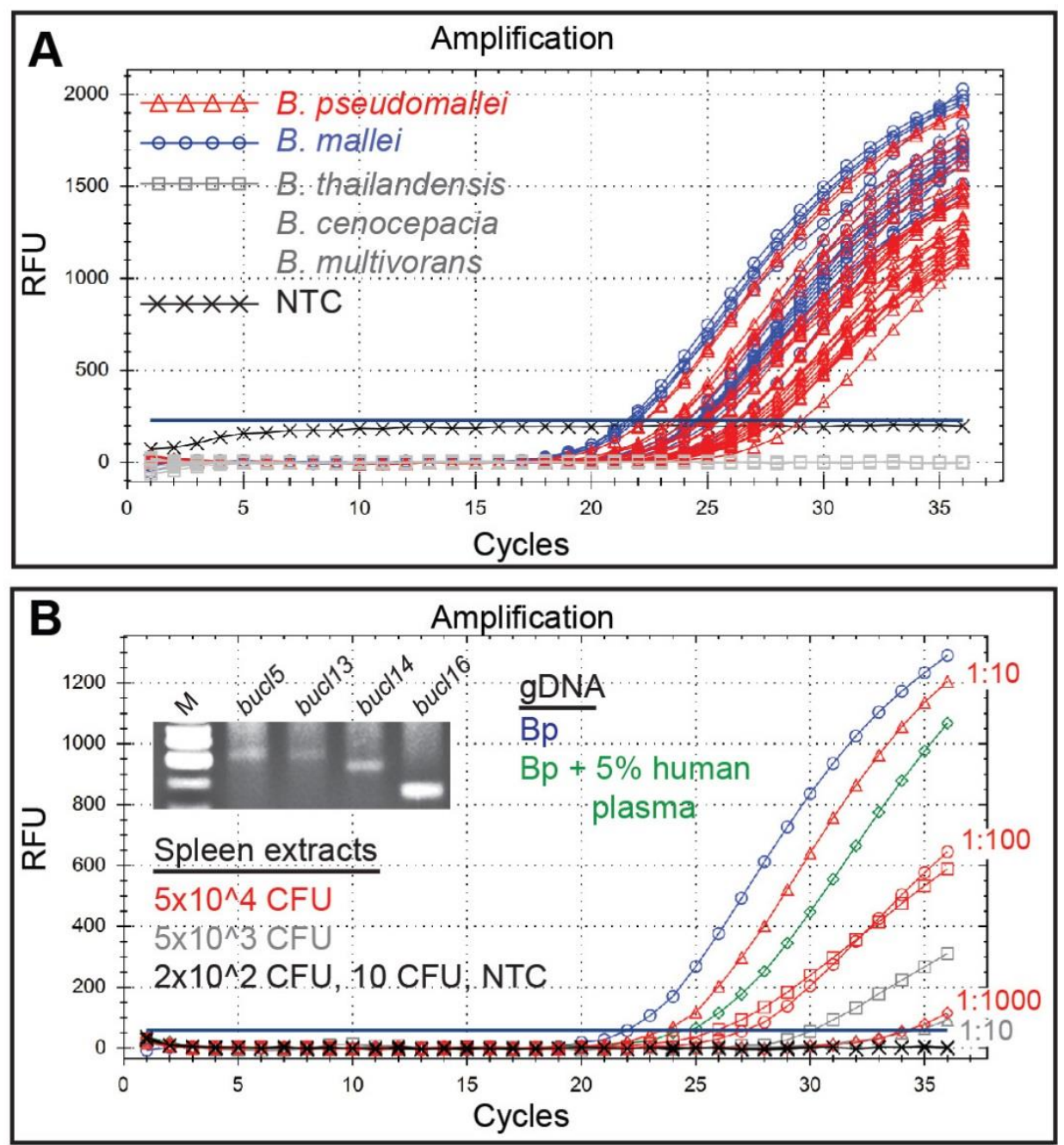

Figure 8

Detection of B. pseudomallei and B. mallei by qPCR. (A) Real-time qPCR detection of bucl16-gene target. Genomic DNA of 25 B. pseudomallei (red) and 15 B. mallei strains (blue), and control DNA from 4 B. thailandensis, 4 B. cenocepacia, and 6 B. multivorans strains (gray). (B) qPCR detection of bucl16 target in the presence of human plasma and in spleen extracts from infected mice. 25 ng of gDNA from Bp K96243 was used as a positive control (blue line). Amplification of bucl16 in qPCR reaction spiked with 5\% human plasma is shown (green line). Mice were infected with Bp HBPUB10134a and CFU counts used in each qPCR reaction were based on plating spleen extracts on blood agar. Positive amplification is shown for spleen samples with $5 \times 10^{4}$ CFU (red lines: square, undiluted; triangle, 1:10 dilution; circle, 1:100 dilution; diamond, 1:1000 dilution) and $5 \times 10^{3}$ CFU (gray lines: square, undiluted; triangle, 1:10 dilution), while no amplification was obtained for crude spleen samples with original $2 \times 10^{2} \mathrm{CFU}$ and $10 \mathrm{CFU}$ per reaction and no template control, NTC (black lines). Inset; amplification of bucl markers $5,13,14$, and 16 by standard PCR using crude spleen samples containing $5 \times 10^{4}$ CFU per reaction. $\mathrm{M}, 50$-bp DNA ladder. 
Table 1. Assessment of genomic plasticity of B. pseudomallei and B. mallei using biomarkers.

\begin{tabular}{|c|c|c|c|c|c|c|c|}
\hline \multirow[b]{2}{*}{ OPa } & \multirow[b]{2}{*}{ Strains } & \multicolumn{2}{|c|}{$\begin{array}{l}\text { bucls on } \\
\text { Chromosome } 1\end{array}$} & \multirow[b]{2}{*}{$\begin{array}{l}\text { Ch2 } \\
\text { OP }\end{array}$} & \multirow[b]{2}{*}{ Strains } & \multicolumn{2}{|c|}{$\begin{array}{l}\text { bucls on } \\
\text { Chromosome } 2\end{array}$} \\
\hline & & $\begin{array}{l}\text { plus } \\
\text { strand }\end{array}$ & $\begin{array}{l}\text { minus } \\
\text { strand }\end{array}$ & & & $\begin{array}{l}\text { plus } \\
\text { strand }\end{array}$ & $\begin{array}{l}\text { minus } \\
\text { strand }\end{array}$ \\
\hline $\begin{array}{l}\text { Ch1 } \\
\text { OPI }\end{array}$ & BpK9624 & $\begin{array}{l}6,8 \\
16\end{array}$ & $\begin{array}{l}10,14 \\
15\end{array}$ & $\begin{array}{l}\text { Ch2 } \\
\text { OPI }\end{array}$ & $\begin{array}{l}\text { BpK9624, Bp668, } \\
\text { Bp1026b, Bp1106a, } \\
\text { Bp1710b, BpBP006, } \\
\text { Bp305, Bp146, Bp511, } \\
\text { Bp520, Bp20B16, } \\
\text { Bp78, Bm10229, } \\
\text { Bm10247, BmSAVP1 }\end{array}$ & $\begin{array}{l}2,3,4 \\
5,7\end{array}$ & 1,13 \\
\hline $\begin{array}{l}\text { Ch1 } \\
\text { OPII }\end{array}$ & $\begin{array}{l}\text { Bp668, Bp1026b, } \\
\text { Bp1106a, Bp1710b, } \\
\text { BpBP006, Bp305, } \\
\text { Bp79, Bp146, Bp511, } \\
\text { Bp520, Bp20B16, Bp78 }\end{array}$ & 10,16 & $\begin{array}{l}6,8 \\
14,15\end{array}$ & $\begin{array}{l}\text { Ch2 } \\
\text { OPII }\end{array}$ & Bp79 & $\begin{array}{l}2,3,4 \\
5\end{array}$ & $\begin{array}{l}1,7 \\
13\end{array}$ \\
\hline $\begin{array}{l}\text { Ch1 } \\
\text { OPIII }\end{array}$ & $\mathrm{Bm} 23344$ & 15,16 & $6,8,14$ & $\begin{array}{l}\text { Ch2 } \\
\text { OPII } \\
\text { I }\end{array}$ & $\mathrm{Bm} 23344$ & 4,7 & $\begin{array}{l}1,2 \\
3,5 \\
13\end{array}$ \\
\hline $\begin{array}{l}\text { Ch1 } \\
\text { OPIV }\end{array}$ & $\mathrm{Bm} 10229$ & $\begin{array}{l}6,8, \\
14, \\
15,16\end{array}$ & 10 & & & & \\
\hline $\begin{array}{l}\text { Ch1 } \\
\text { OPV }\end{array}$ & $\mathrm{Bm} 10247$ & 10,15 & $\begin{array}{l}6,8 \\
14,16\end{array}$ & & & & \\
\hline $\begin{array}{l}\text { Ch1 } \\
\text { OPVI }\end{array}$ & BmSAVP1 & 15 & $\begin{array}{l}6,8 \\
10,14 \\
16\end{array}$ & & & & \\
\hline
\end{tabular}


Table 2. Characterization of Bucl proteins in Burkholderiaa.

\begin{tabular}{|c|c|c|c|c|c|c|c|c|}
\hline \multirow[b]{2}{*}{$\begin{array}{l}\text { Bucl } \\
\text { No. }\end{array}$} & \multicolumn{3}{|c|}{ No. of amino acids } & \multicolumn{2}{|c|}{ Collagen-like region (CL) } & \multicolumn{3}{|c|}{ Structural predictions } \\
\hline & Total & $\begin{array}{l}\mathrm{N}- \\
\text { terminus }\end{array}$ & $\begin{array}{l}\mathrm{C}- \\
\text { terminus }\end{array}$ & $\begin{array}{l}\text { No. of GXY } \\
\text { repeats }\end{array}$ & GXY type & $\begin{array}{l}\text { Putative } \\
\text { domains }\end{array}$ & bSS & ${ }^{\circ}$ TM \\
\hline Bucl1 & $\begin{array}{l}152- \\
197\end{array}$ & 42 & $80-89$ & $7-22$ & GAN & $\mathrm{N} / \mathrm{A}$ & No & Yes \\
\hline Bucl2 & $\begin{array}{l}171- \\
228\end{array}$ & 141 & 21 & $3-19$ & GEV. GEA & $N / A$ & No & No \\
\hline Bucl3 & $\begin{array}{l}551- \\
640\end{array}$ & 44 & $372-405$ & $38-63$ & GTS. GSS & Talin-1 & Yes & Yes \\
\hline Bucl4 & $\begin{array}{l}297- \\
379\end{array}$ & 271 & $9-18$ & $7-30$ & GVS. GAS & Bac_export_1 & Yes & $\begin{array}{l}\text { Yes, CL } \\
\text { region }\end{array}$ \\
\hline Bucl5 & $\begin{array}{l}168- \\
230\end{array}$ & $35-41$ & 114 & $7-25$ & $\begin{array}{l}\text { GLE. GPE. } \\
\text { GLD. GFD }\end{array}$ & $N / A$ & No & No \\
\hline Bucl6 & $\begin{array}{l}40- \\
88\end{array}$ & 1 & 27 & $4-20$ & $\begin{array}{l}\text { GAL. GAS. } \\
\text { GAA. GAE }\end{array}$ & $\mathrm{N} / \mathrm{A}$ & No & $\begin{array}{l}\text { Yes, CL } \\
\text { region }\end{array}$ \\
\hline Bucl7 & $\begin{array}{l}188- \\
212\end{array}$ & 134 & 36 & $5-14$ & $\begin{array}{l}\text { GLS. GSS. } \\
\text { GAS. GVA }\end{array}$ & $\mathrm{N} / \mathrm{A}$ & No & $\begin{array}{l}\text { Yes, CL } \\
\text { region }\end{array}$ \\
\hline Bucl8 & $\begin{array}{l}608- \\
677\end{array}$ & 522 & 74 & $4-24$ & GAS & OEP & Yes & $\begin{array}{l}\text { Yes, CL } \\
\text { region }\end{array}$ \\
\hline Bucl10 & $\begin{array}{l}92- \\
155\end{array}$ & $2-8$ & 63 & $8-25$ & $\begin{array}{l}\text { GIH. GMH. } \\
\text { GMR }\end{array}$ & $N / A$ & No & No \\
\hline Bucl13 & $\begin{array}{l}385- \\
433\end{array}$ & 24 & 318 & $12-25$ & $\begin{array}{l}\text { GIR. GVR. } \\
\text { GSG. GGS. }\end{array}$ & SBP_bac_3 & No & Yes \\
\hline Bucl14 & $\begin{array}{l}83- \\
191\end{array}$ & 11 & 69 & $2-37$ & $\begin{array}{l}\text { GWC. GRC. } \\
\text { GRR. GRH }\end{array}$ & $N / A$ & No & $\begin{array}{l}\text { Yes, CL } \\
\text { region }\end{array}$ \\
\hline Bucl15 & $\begin{array}{l}56- \\
91\end{array}$ & $21-69$ & 4 & $5-14$ & $\begin{array}{l}\text { GVL. GAL. } \\
\text { GML. GAT. } \\
\text { GAI. GAA }\end{array}$ & $N / A$ & Yes & $\begin{array}{l}\text { Yes, CL } \\
\text { region }\end{array}$ \\
\hline Bucl16 & $\begin{array}{l}227- \\
307\end{array}$ & $65-67$ & 148 & $4-30$ & $\begin{array}{l}\text { GFG. GVD. } \\
\text { GFD. GAF }\end{array}$ & $N / A$ & No & $\begin{array}{l}\text { Yes, CL } \\
\text { region }\end{array}$ \\
\hline
\end{tabular}

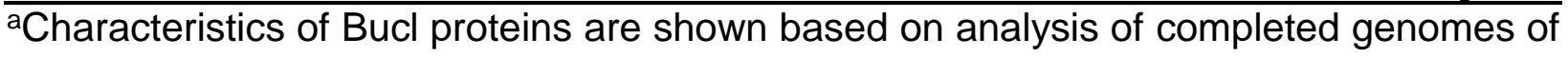
$13 \mathrm{Bp}, 11 \mathrm{Bm}$, and $9 \mathrm{Bt}$ strains (see Table 3). The total protein length and length of protein sequences that are amino- and carboxyl-terminal to $\mathrm{CL}$ regions in each Bucl protein is shown as amino acid number, whereas the length of each $C L$ region, which varies between strains, is expressed as the number of GXY repeats. Predominant GXY repeats are represented in bold text. Putative domains in the noncollagenous regions of each Bucl are shown: Talin-1 domain; Bac_export_1, bacterial export protein family 1; OEP, outer membrane efflux protein; and SBP_bac_3, bacterial extracellular solute-binding protein family 3.

bSS; Signal sequence predictions are based on hidden Markov model predictions in the SignalP 3.0 server.

CTM; Transmembrane domain predictions were made using TMPred. 
Table 3. Burkholderia strains used in this studya

\begin{tabular}{|c|c|c|c|c|}
\hline Species & Abbreviation & Strain & Isolate information & \\
\hline $\begin{array}{l}\text { B. } \\
\text { pseudomallei }\end{array}$ & BpK9624 & K96243 & $\begin{array}{l}\text { female diabetic patient- Khon Kaen hospital, Northeast } \\
\text { Thailand }\end{array}$ & 1996 \\
\hline $\begin{array}{l}\text { B. } \\
\text { pseudomallei }\end{array}$ & Bp1710b & $1710 b$ & $\begin{array}{l}\text { relapse of same patient infected with } 1710 a \text {, blood } \\
\text { culture, Northeast Thailand, Sappasithiprasong } \\
\text { hospital }\end{array}$ & 1999 \\
\hline $\begin{array}{l}\text { B. } \\
\text { pseudomallei }\end{array}$ & Bp305 & MSHR305 & $\begin{array}{l}\text { brain sample, fatal encephalomyelitis, Australia, Royal } \\
\text { Darwin hospital }\end{array}$ & 1994 \\
\hline $\begin{array}{l}\text { B. } \\
\text { pseudomallei }\end{array}$ & Bp1026b & $1026 b$ & $\begin{array}{l}\text { blood culture from } 29 \text {-year old female rice farmer with } \\
\text { diabetes milletus, Northeast Thailand, } \\
\text { Sappasithiprasong hospital }\end{array}$ & 1993 \\
\hline $\begin{array}{l}\text { B. } \\
\text { pseudomallei }\end{array}$ & ВрВР006 & BPC006 & $\begin{array}{l}\text { Blood from patient with Type I diabetes and multiple } \\
\text { abscesses, China, Baoting Town, Hainan }\end{array}$ & 2008 \\
\hline Bseudomallei & $\mathrm{Bp} 1106 \mathrm{a}^{\mathrm{b}}$ & $1106 a$ & $\begin{array}{l}\text { female rice farmer, Northeast Thailand, } \\
\text { Sappasithiprasong hospital }\end{array}$ & 1993 \\
\hline $\begin{array}{l}\text { B. } \\
\text { pseudomallei }\end{array}$ & Bp79 & $\begin{array}{l}\text { NCTC } \\
13179\end{array}$ & skin ulcer, Australia & 2014 \\
\hline pseudomallei & Bp668 & 668 & $\begin{array}{l}\text { blood culture from 53-year old male patient with } \\
\text { severe melioidosis encephalomyelitis, Darwin Australia }\end{array}$ & 1995 \\
\hline $\begin{array}{l}\text { B. } \\
\text { pseudomallei }\end{array}$ & Bp146 & MSHR146 & goat udder, Australia & 1992 \\
\hline $\begin{array}{l}\text { B. } \\
\text { pseudomallei }\end{array}$ & Bp511 & MSHR511 & throat of goat, Australia & 1997 \\
\hline $\begin{array}{l}\text { B. } \\
\text { pseudomallei }\end{array}$ & Bp520 & MSHR520 & human blood culture, Australia & 1998 \\
\hline $\begin{array}{l}\text { B. } \\
\text { pseudomallei }\end{array}$ & Bp20B16 & NAU20B-16 & soil, Australia & 2006 \\
\hline $\begin{array}{l}\text { B. } \\
\text { pseudomallei }\end{array}$ & Bp78 & $\begin{array}{l}\text { NCTC } \\
13178\end{array}$ & human post-mortem brain, Australia & $\mathrm{N} / \mathrm{A}$ \\
\hline B. mallei & BmSAVP1 & SAVP1 & $\begin{array}{l}\text { pathogenic strain which became avirulent after } \\
\text { passage through } 6 \text { equids, originally caused disease in } \\
\text { a mule in India }\end{array}$ & \\
\hline B. mallei & $\mathrm{Bm} 10229$ & $\begin{array}{l}\text { NCTC } \\
10229\end{array}$ & Europe & \\
\hline B. mallei & $\mathrm{Bm} 10247$ & $\begin{array}{l}\text { NCTC } \\
10247\end{array}$ & Europe & \\
\hline B. mallei & $\mathrm{Bm} 23344^{\mathrm{b}}$ & $\begin{array}{l}\text { ATCC } \\
23344\end{array}$ & $\begin{array}{l}\text { human post-mortem knee fluid, skin pustules and } \\
\text { blood, Burma }\end{array}$ & 1944 \\
\hline B. mallei & $\mathrm{Bm} 21280^{\mathrm{b}}$ & 2002721280 & Iran & 1952 \\
\hline B. mallei & BmA188 & A188 & $>8$ passages & \\
\hline B. mallei & $\mathrm{BmA193}$ & A193 & Pasteur Institute, France & 1964 \\
\hline B. mallei & $\mathrm{Bm} 10399^{\mathrm{b}}$ & $\begin{array}{l}\text { ATCC } \\
10399\end{array}$ & horse lung, Southern China & 1949 \\
\hline B. mallei & BmPRL20 & PRL-20 & $\begin{array}{l}\text { blood of a gelding from the Lahore Polo Club, Lahore, } \\
\text { Pakistan }\end{array}$ & 2005 \\
\hline B. mallei & Bm11 & strain_11 & human, Turkey & 1949 \\
\hline B. mallei & $\mathrm{Bm} 6$ & strain_6 & human & 1950 \\
\hline $\begin{array}{l}\text { B. } \\
\text { thailandensis }\end{array}$ & BtE264 & E264 & rice field soil sample, Central Thailand & \\
\hline $\begin{array}{l}\text { B. } \\
\text { thailandensis }\end{array}$ & Bt121 & MSMB121 & soil, Australia & 2007 \\
\hline $\begin{array}{l}\text { B. } \\
\text { thailandensis }\end{array}$ & BtH0587 & H0587 & human pleural wound, LA, United States & 1997 \\
\hline $\begin{array}{l}\text { B. } \\
\text { thailandensis }\end{array}$ & BtE444 & E444 & soil, Thailand & 2002 \\
\hline $\begin{array}{l}\text { B. } \\
\text { thailandensis }\end{array}$ & Bt43 & MSMB43 & $\begin{array}{l}\text { bore water source in Darwin, Australia; first isolate of } \\
B \text {. thailandensis in Australia }\end{array}$ & \\
\hline
\end{tabular}




\begin{tabular}{|c|c|c|c|}
\hline $\begin{array}{l}\text { B. } \\
\text { thailandensis }\end{array}$ & BtTXDOH & TXDOH & United States \\
\hline $\begin{array}{l}\text { B. } \\
\text { thailandensis }\end{array}$ & Bt21723 & 2002721723 & Human, CDC \\
\hline $\begin{array}{l}\text { B. } \\
\text { thailandensis }\end{array}$ & Bt4 & 4 & \\
\hline $\begin{array}{l}\text { B. } \\
\text { thailandensis }\end{array}$ & BtE555 & E555 & \\
\hline
\end{tabular}


Table 4. Distribution of all bucl genes in Burkholderia spp. as assessed by bioinformatics and PCR amplification ${ }^{\mathrm{a}}$.

\begin{tabular}{|c|c|c|c|c|c|c|c|c|c|c|c|c|c|}
\hline \multirow[b]{2}{*}{$\begin{array}{l}\text { Strain } \\
\text { Abbreviatio }\end{array}$} & \multicolumn{13}{|c|}{ bucl No. } \\
\hline & 1 & 2 & 3 & 4 & 5 & 6 & 7 & 8 & 10 & 13 & 14 & 15 & 16 \\
\hline $\mathrm{BpBP006}^{\mathrm{b}}$ & + & + & + & + & + & + & + & + & + & + & + & + & + \\
\hline Bp79b & + & + & + & + & + & + & + & + & + & + & + & + & + \\
\hline Bp146 & + & + & + & + & + & + & + & + & + & + & + & + & + \\
\hline Bp511 b & + & + & + & + & + & + & + & + & + & + & + & + & + \\
\hline Bp520 & + & + & + & + & + & + & + & + & + & + & + & + & + \\
\hline Bp20B16 ${ }^{b}$ & + & + & + & + & + & + & + & + & + & + & + & + & + \\
\hline$B p 78^{b}$ & + & + & + & + & + & + & + & + & + & + & + & + & + \\
\hline Bp1026b & + & + & + & + & + & + & + & + & + & + & + & + & + \\
\hline BpE203 & + & + & + & + & + & + & + & + & + & + & + & + & + \\
\hline Bp4845 & + & + & + & + & + & + & + & + & + & + & + & + & + \\
\hline Bp1152 & + & + & + & + & + & + & + & + & + & + & + & + & + \\
\hline Bp1992 & + & + & + & + & + & + & + & + & + & + & + & + & + \\
\hline BpE8 & + & + & + & + & + & + & + & + & + & + & + & + & + \\
\hline Bp423 & + & + & + & + & + & + & + & + & + & + & + & + & + \\
\hline Bp6068 & + & + & + & + & + & + & + & + & + & + & + & + & + \\
\hline BpS13 & + & + & + & + & + & + & + & + & + & + & + & + & + \\
\hline Bp1710a & + & + & + & + & + & + & + & + & + & + & + & + & + \\
\hline Bp1710b & + & + & + & + & + & + & + & + & + & + & + & + & + \\
\hline BpK9624 & + & + & + & + & + & + & + & + & + & + & + & + & + \\
\hline Bp1106b & + & + & + & + & + & + & + & + & + & + & + & + & + \\
\hline BpCh3 & - & NT & NT & - & + & + & + & + & NT & + & + & + & + \\
\hline Bp121 & + & + & + & + & + & + & + & + & + & + & + & + & + \\
\hline Bp1112 & + & + & + & + & + & + & + & + & + & + & + & + & + \\
\hline Bp305 & + & + & + & + & + & + & + & + & + & + & + & + & + \\
\hline Bp668 & + & + & + & + & + & + & + & + & + & + & + & + & + \\
\hline Bp406e & + & + & + & + & + & + & + & + & + & + & + & + & + \\
\hline${\mathrm{Bp} 1106 a^{b}}^{b}$ & + & + & + & + & + & + & + & + & + & + & + & + & + \\
\hline Bp5855 & + & + & + & + & + & + & + & + & + & + & + & + & + \\
\hline Bp5848 & + & + & + & + & + & + & + & + & + & + & + & + & + \\
\hline Bp5858 & + & + & + & + & + & + & + & + & + & + & + & + & + \\
\hline Bp0134a & + & + & + & + & + & + & + & + & + & + & + & + & + \\
\hline Вp0303a & + & + & + & + & + & + & + & + & + & + & + & + & + \\
\hline $\mathrm{Bm} 10229^{\mathrm{b}}$ & + & - & + & + & + & + & + & + & + & + & + & + & + \\
\hline $\mathrm{Bm} 10247^{\mathrm{b}}$ & + & + & + & + & + & + & + & + & + & + & + & + & + \\
\hline BmA188 & + & - & - & + & + & + & + & + & + & + & + & + & + \\
\hline$B m A 193^{b}$ & + & - & - & + & + & + & - & + & + & + & + & + & + \\
\hline $\mathrm{Bm} 10399^{b}$ & + & + & + & + & + & + & + & + & + & + & + & + & + \\
\hline BmPRL20 ${ }^{b}$ & + & - & + & + & + & + & + & + & + & + & + & + & + \\
\hline$B m 6^{b}$ & + & - & + & - & + & + & + & + & + & + & + & + & + \\
\hline$B m 11^{b}$ & + & + & + & + & + & + & + & + & + & + & + & + & + \\
\hline BmSAVP1 $^{b}$ & - & + & + & - & + & + & - & + & + & + & + & + & + \\
\hline BmGB3 & + & - & - & - & + & + & + & + & + & + & + & + & + \\
\hline BmGB4 & + & + & + & - & + & + & + & + & + & + & + & + & + \\
\hline BmISU & + & + & + & + & + & + & + & + & - & + & + & + & + \\
\hline BmTurk1 $^{c}$ & + & + & + & + & + & + & + & + & + & + & + & + & + \\
\hline Bm234 & + & + & + & + & + & + & + & + & - & + & + & + & + \\
\hline Bm235 & + & + & + & + & + & + & + & + & - & + & + & + & + \\
\hline BmHI533 & + & + & + & + & + & + & + & + & - & + & + & + & + \\
\hline BmGB11 & + & - & + & + & + & + & + & + & + & + & + & + & + \\
\hline BmNBL7 & + & + & + & + & + & + & + & + & - & + & + & + & + \\
\hline BmGB8 & + & + & + & + & + & + & + & + & - & + & + & + & + \\
\hline $\mathrm{Bm} 23344^{\mathrm{b}}$ & + & + & + & + & + & + & + & + & - & + & + & + & + \\
\hline BmTurk2 $^{c}$ & + & + & + & - & + & + & + & + & + & + & + & + & + \\
\hline
\end{tabular}




\begin{tabular}{|c|c|c|c|c|c|c|c|c|c|c|c|c|c|}
\hline BmFMH & + & + & + & + & + & + & + & + & - & + & + & + & + \\
\hline $\mathrm{Bm} 21280^{\mathrm{b}}$ & + & + & + & + & + & + & + & + & - & + & + & - & + \\
\hline Bm85567 & + & + & + & + & + & + & + & + & + & + & + & + & + \\
\hline $\mathrm{Bm} 2700 \mathrm{C}$ & + & - & + & + & + & + & + & + & + & + & + & + & + \\
\hline BmCh7 & + & + & + & + & + & + & + & + & - & + & + & + & + \\
\hline BmCh5 & + & + & + & + & + & + & + & + & + & + & + & + & + \\
\hline Bm10230 & + & - & + & + & + & + & + & + & + & + & + & + & + \\
\hline BmGB8 & + & + & + & + & + & + & + & + & - & + & + & + & + \\
\hline Bt21723 & - & - & + & + & - & - & - & - & - & - & - & - & - \\
\hline BtH0587 & - & - & + & + & - & - & - & - & - & - & - & - & - \\
\hline BtE444 $^{b}$ & - & - & + & + & - & - & - & - & - & - & - & - & - \\
\hline Bt121 b & - & - & + & + & - & - & - & - & - & - & - & - & - \\
\hline BtE555 $5^{b}$ & - & - & + & + & - & - & - & - & - & - & - & - & - \\
\hline$B t 43^{b}$ & - & - & + & + & - & - & - & - & - & - & - & - & - \\
\hline $\mathrm{Bt} 4^{\mathrm{b}}$ & - & - & + & + & - & - & - & - & - & - & - & - & - \\
\hline $\mathrm{BtTXDOH}^{\mathrm{b}}$ & - & - & + & + & - & - & - & - & - & - & - & - & - \\
\hline BtE264 & - & - & + & + & - & - & - & - & - & - & - & - & - \\
\hline BtDW503 & - & - & + & + & - & - & - & - & - & - & - & - & - \\
\hline BtE421 & - & - & + & + & - & - & - & - & - & - & - & - & - \\
\hline BtE426 & - & - & + & + & - & - & - & - & - & - & - & - & - \\
\hline $\begin{array}{l}\text { Fisher } p \text { - } \\
\text { value }\end{array}$ & \multicolumn{2}{|c|}{$<0.0001$} & 0.581 & 0.353 & \multicolumn{9}{|c|}{$<0.0001$} \\
\hline Cramer's V ${ }^{2}$ & 0.829 & 0.486 & 0.009 & 0.018 & 1 & 1 & 0.829 & 1 & 0.426 & 1 & 1 & 0.908 & 1 \\
\hline Bc706 & - & - & - & - & NT & NT & NT & NT & - & NT & NT & NT & NT \\
\hline Bc709 & - & - & - & - & - & - & - & - & - & - & - & - & - \\
\hline Bc710 & - & - & - & - & - & - & - & - & - & - & - & - & - \\
\hline Bce6656 & - & - & - & - & - & - & - & - & - & - & - & - & - \\
\hline BceBC7 & - & - & - & - & - & - & - & - & - & - & - & - & - \\
\hline BceK562 & - & - & - & - & - & - & - & - & - & - & - & - & - \\
\hline BceJ2315 & - & - & - & - & - & - & - & - & - & - & - & - & - \\
\hline Bce103a2 & - & - & - & - & NT & NT & - & NT & - & NT & - & NT & - \\
\hline BmvCF2 & - & - & - & - & - & - & - & - & - & - & - & - & - \\
\hline BmvCGD1 & - & - & - & - & - & - & - & - & - & - & - & - & - \\
\hline BmvCGD2 & - & - & - & - & - & - & - & - & - & - & - & - & - \\
\hline BmvCF1 & - & - & - & - & - & - & - & - & - & - & - & - & - \\
\hline Bmv17616 & - & - & - & - & - & - & - & - & - & - & - & - & - \\
\hline Bmv13010 & - & - & - & - & - & - & - & - & - & - & - & - & - \\
\hline $\begin{array}{l}\text { Dresence } \\
\text { sted (no } \\
\text { athogenic } \\
\text { sing the } \\
\text { Strains fo } \\
\text { Smaller-s }\end{array}$ & $\begin{array}{l}3 p \text { a } \\
\text { sher } \\
\text { whic } \\
\text { d }\end{array}$ & $\begin{array}{l}\mathrm{Bn} \\
\text { xact }\end{array}$ & $\begin{array}{l}f b u \\
\text { nour } \\
\text { spec }\end{array}$ & $\begin{array}{l}\text { am } \\
\text { of } \\
\text { ility } \\
\text { ie w }\end{array}$ & 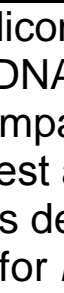 & 98 & $\begin{array}{l}\text { idic } \\
\text { ole). } \\
\text { nonk } \\
\text { ner' } \\
\text { d by }\end{array}$ & $\mathrm{d} \mathrm{b}$ & $\begin{array}{l}\text { ic } \\
\text { alys } \\
\text { ma }\end{array}$ & strá & $5, \mathrm{n}$ & $\begin{array}{l}\text { ely. } 1 \\
\text { esenc } \\
\text { is ass }\end{array}$ & $\begin{array}{l}, r \\
w \\
s s\end{array}$ \\
\hline
\end{tabular}


Table 5. Primers and probe used for bucl amplificationa

\begin{tabular}{|c|c|c|c|}
\hline buc/\# & Primer name & Primer sequence 5'-3' & Amplicon size \\
\hline \multirow[t]{2}{*}{ bucl1 } & Bucl1_4F & GTGGCGCTGGCGCATCGTGAACGGC & $103 \mathrm{bp}$ \\
\hline & Bucl1_4R & CTTCGTCGGTTGCGTGTCGTCCGTTGC & \\
\hline \multirow[t]{2}{*}{ bucl2 } & Bucl2_1F & CGGCGTGCGACGGAA & $133 \mathrm{bp}$ \\
\hline & Bucl2_1R & GCCCACTTCGCGATTCTTC & \\
\hline \multirow[t]{2}{*}{ buc/3 } & Bucl3_2F & CTGCTCGGCGGCCTGTCGGGTTCGG & $166 \mathrm{bp}$ \\
\hline & Bucl3_2R & CGGGCGCGGTCGTCGTCGA & \\
\hline \multirow[t]{2}{*}{ buc/4 } & Bucl4_2F_ext & GACGAATTCATCCGCTTCATCGTG & $176 \mathrm{bp}$ \\
\hline & Bucl4_2R_ext2 & CCGCTGCGCATCGGGCCTTTCA & \\
\hline \multirow[t]{2}{*}{ bucl5 } & Bucl5_2F & AACTCGACGAACTCAACGCGAATCGAC & $216 \mathrm{bp}$ \\
\hline & Bucl5_2R & GCGCGCCGTTCTTTCTAGCGCTGC & \\
\hline \multirow[t]{2}{*}{ bucl6 } & Bucl6_CL flank_F & AGGAGCGGCGCTTGCCGGGCG & $115 \mathrm{bp}$ \\
\hline & Bucl6_Clflank_2R & GAACGGCGACGGTCCGACGCAGC & \\
\hline \multirow[t]{2}{*}{ bucl7 } & Bucl7_2F & ATGGACACGACCACGCAGGACGGG & $264 \mathrm{bp}$ \\
\hline & Bucl7_2R & CCAATGAACGGCCCGCGTCGCTTTC & \\
\hline \multirow[t]{4}{*}{ bucl8 } & Bucl8_2F & GCAGCTCGATTCGTGGAT & $243 \mathrm{bp}$ \\
\hline & Bucl8_2R & AGGTGGTACGACAGGCTCAG & \\
\hline & Bucl8_3F & CTACGCGCTCCTCGACATCGCGC & $96 \mathrm{bp}$ \\
\hline & Bucl8_3R & TGCGTGCCGATGCCCGCGCGCA & \\
\hline \multirow[t]{2}{*}{ bucl10 } & Bucl10_1F & GCATGCGTTGGACACGA & $109 \mathrm{bp}$ \\
\hline & Bucl10_1R & GCAACGTCGTCATCTCGTC & \\
\hline \multirow[t]{2}{*}{ bucl13 } & Bucl13_2F & GTTCGATTTCACGACGTACCGGCTCG & $212 \mathrm{bp}$ \\
\hline & Bucl13_2R & CGTCGTCGTCGAAGTACAGCACGTC & \\
\hline \multirow[t]{2}{*}{ bucl14 } & Bucl14_1F & TCGGCACATCTGTCGCCGCGAACC & $178 \mathrm{bp}$ \\
\hline & Bucl14_1R & CGTATGGCCGCCGTGTCGATCGG & \\
\hline \multirow[t]{2}{*}{ bucl15 } & Bucl15_1F & GATCGCTCGACGCGCCCGRCGTGC & $95 \mathrm{bp}$ \\
\hline & Bucl15_1R & CTAAAACCGCCGGCGYGCCGCGC & \\
\hline \multirow[t]{3}{*}{ bucl16 } & Bucl16_2F & CCGGCAGCACCGACTCGAGCGTGCG & $123 \mathrm{bp}$ \\
\hline & Bucl16_2R & CGTCGTTCGMGCTCGCCGATCGCTCG & \\
\hline & 'Bucl16_5'FAM_3'IBQ & $\mathrm{TCTGCA}+\mathrm{CG}+\mathrm{G}+\mathrm{CG}+\mathrm{GTG}+\mathrm{AGCCGCTTCA}$ & \\
\hline
\end{tabular}

aPrimers were designed to generate conserved amplicons within the non-collagenous region of each bucl gene. Primers for bucl6 and bucl15 were designed flanking the collagenous region, which varies in size among strains (S4 Fig. 4B). Primers Bucl8_3F/3R were used to generate amplicon from Bp K96243 reference strain, whereas primers Bucl8_2F/2R selectively amplify products from $\mathrm{Bp}$ and Bm gDNA and were used in largescale PCR screening (S4 Fig. 4B).

${ }^{\mathrm{b} A m p l i c o n}$ sizes expected for the Bp K96243 reference strain.

'LNA probe for bucl16 detection; + symbols precede LNA bases. 
Table 6. Genomic DNA collection.

\begin{tabular}{|c|c|c|c|c|c|c|}
\hline \multirow[t]{2}{*}{ Species } & \multirow[t]{2}{*}{ Abbreviation } & \multirow[t]{2}{*}{ Strain } & \multicolumn{3}{|l|}{ Isolate information } & \multirow{2}{*}{$\begin{array}{l}\text { Source of } \\
\text { DNA }\end{array}$} \\
\hline & & & Alternative designations & Source of isolate & Year & \\
\hline B. pseudomallei & Bp1026b & 1026B & & $\begin{array}{l}\text { blood culture from 29-year old female rice farmer with } \\
\text { diabetes milletus, Northeast Thailand, } \\
\text { Sappasithiprasong hospital }\end{array}$ & 1993 & USAMRIID \\
\hline B. pseudomallei & BpE203 & E203 & & Soil sample from Roi Et, Thailand & 1997 & USAMRIID \\
\hline B. pseudomallei & Bp4845 & NCTC4845 & $\begin{array}{l}\text { (S. 397, NRRL B-1112, } \\
\text { CCEB 472) }\end{array}$ & Monkey, Singapore & 1935 & USAMRIID \\
\hline B. pseudomallei & Bp1152 & STW-115-2 & & water, Thailand & 1965 & USAMRIID \\
\hline B. pseudomallei & Bp1992 & STW-199-2 & & water, Thailand & 1965 & USAMRIID \\
\hline B. pseudomallei & BpE8 & E8 & & $\begin{array}{l}\text { Soil sample obtained on road to Trakan Phuet Phon } \\
\text { District, Ubon Ratchathani Thailand }\end{array}$ & & USAMRIID \\
\hline B. pseudomallei & Bp423 & 423 & & Blood culture, Cambodia & 2008 & USAMRIID \\
\hline B. pseudomallei & Bp6068 & $\begin{array}{l}\text { Pasteur } \\
6068\end{array}$ & 2002721763 & Vietnam & & $\begin{array}{l}\text { BEI } \\
\text { Resources }^{b}\end{array}$ \\
\hline B. pseudomallei & Bp13 & S13 & & muicodal strain, environmental isolate, Singapore & & BEI Resources \\
\hline B. pseudomallei & Bp1710a & $1710 \mathrm{a}$ & & $\begin{array}{l}\text { blood culture of } 52 \text {-year old male rice farmer with } \\
\text { diabetes milletus, Northeast Thailand }\end{array}$ & 1996 & BEI Resources \\
\hline B. pseudomallei & Bp1710b & $1710 \mathrm{~b}$ & & $\begin{array}{l}\text { relapse of same patient infected with } 1710 a \text {, blood } \\
\text { culture, Northeast Thailand, Sappasithiprasong } \\
\text { hospital }\end{array}$ & 1999 & BEI Resources \\
\hline B. pseudomallei & BpK9624 & K96243 & & $\begin{array}{l}\text { female diabetic patient- Khon Kaen hospital, } \\
\text { Northeast Thailand }\end{array}$ & 1996 & BEI Resources \\
\hline B. pseudomallei & Bp1106b & $1106 b$ & & $\begin{array}{l}\text { relapse of same patient infected with } 1106 a-\text { female } \\
\text { rice farmer, pus aspirated from liver abscess, } \\
\text { Northeast Thailand, Sappasithiprasong hospital }\end{array}$ & 1996 & BEI Resources \\
\hline B. pseudomallei & BpCh3 & China 3 & & septicemia of American soldier, Burma & & BEI Resources \\
\hline B. pseudomallei & Bp121 & NBL 121 & strain 286, MP-S & $\begin{array}{l}\text { chronic melioidosis case, infection acquired while } \\
\text { living in Far East, Louisiana, United States }\end{array}$ & 1953 & BEI Resources \\
\hline B. pseudomallei & Bp1112 & $\begin{array}{l}\text { NRRL B- } \\
1112\end{array}$ & strain S 397, CCEB 472 & naturally infected lab monkey, Singapore & 1935 & BEI Resources \\
\hline B. pseudomallei & Bp305 & MSHR305 & & $\begin{array}{l}\text { brain sample, fatal encephalomyelitis, Australia, Royal } \\
\text { Darwin hospital }\end{array}$ & 1994 & USAMRIID \\
\hline B. pseudomallei & Bp668 & MSHR668 & & $\begin{array}{l}\text { blood culture from } 53 \text {-year old male patient with } \\
\text { severe melioidosis encephalomyelitis, Darwin } \\
\text { Australia }\end{array}$ & 1995 & USAMRIID \\
\hline B. pseudomallei & Bp406e & $406 e$ & & $\begin{array}{l}\text { disseminated melioidosis patient, toe swab, Ubon } \\
\text { Ratchathani province, Northeast Thailand }\end{array}$ & 1988 & USAMRIID \\
\hline B. pseudomallei & Bp1106a & $1106 a$ & & $\begin{array}{l}\text { female rice farmer, Northeast Thailand, } \\
\text { Sappasithiprasong hospital }\end{array}$ & 1993 & USAMRIID \\
\hline B. pseudomallei & Bp5855 & MSHR5855 & & Australia & 2011 & USAMRIID \\
\hline
\end{tabular}




\begin{tabular}{|c|c|c|c|c|c|c|}
\hline B. pseudomallei & Bp5848 & MSHR5848 & & inhalational melioidosis, Australia & 2011 & USAMRIID \\
\hline B. pseudomallei & Bp5858 & MSHR5858 & & & & USAMRIID \\
\hline B. pseudomallei & Bp0134a & $\begin{array}{l}\text { HBPUB } \\
10134 a\end{array}$ & & sputum, Thailand, Mahidol University & 2010 & USAMRIID \\
\hline B. pseudomallei & Вp0303a & $\begin{array}{l}\text { HBPUB } \\
10303 a\end{array}$ & & sputum, Thailand, Mahidol University & 2011 & USAMRIID \\
\hline B. mallei & BmGB3 & GB3 & $\begin{array}{l}\text { 2002734306, } \\
2002734311 \text {, strain A, } \\
\text { NCTC120 }\end{array}$ & Lister Institute, London & 1920 & USAMRIID \\
\hline B. mallei & BmGB4 & GB4 & $\begin{array}{l}\text { M4, 2002734304, strain } \\
6, \text { NCTC10248 }\end{array}$ & human, Ankara, Turkey & 1950 & USAMRIID \\
\hline B. mallei & BmISU & ISU & & lowa State University & & USAMRIID \\
\hline B. mallei & BmTurk1 & Turkey 1 & 2000031065, \#1 Turkey & Turkey, isolated by Dr. Linda Schlater & 2003 & USAMRIID \\
\hline B. mallei & $\mathrm{Bm} 234$ & KC234 & 2002721273,3783 & $\begin{array}{l}\text { human, Burma- isolated via CA Gleisser Army } \\
\text { Medical School }\end{array}$ & 1956 & USAMRIID \\
\hline B. mallei & $\mathrm{Bm} 235$ & KC235 & 2002721274 & Fort Detrick, Maryland, United States & 1956 & USAMRIID \\
\hline B. mallei & $\mathrm{BmHI533}$ & HI533 & $\begin{array}{l}2000031304 \\
2000031281\end{array}$ & $\begin{array}{l}\text { human liver abscess drainage, Maryland, United } \\
\text { States }\end{array}$ & 2000 & USAMRIID \\
\hline B. mallei & BmGB11 & GB11 & $\begin{array}{l}\text { NCTC } 10245 \text {, } \\
2002721275 \text {, China } 5 \\
\text { ATCC } 10399\end{array}$ & horse lung, Southern China & 1949 & USAMRIID \\
\hline B. mallei & BmNBL7 & NBL 7 & China 7 & $\begin{array}{l}\text { Prep of B mallei China } 7 \text { derived from ATCC23344 via } \\
\text { passage through several individuals }\end{array}$ & & BEI Resources \\
\hline B. mallei & BmGB8 & $\begin{array}{l}\text { GB8 horse } \\
4\end{array}$ & & $\begin{array}{l}\text { derivative of ATCC } 23344 \text { passaged through horse } \\
\text { and isolated from the lung as a single colony, } \\
\text { Manitoba, Canada }\end{array}$ & & BEI Resources \\
\hline B. mallei & Bm23344 & $\begin{array}{l}\text { ATCC } \\
23344\end{array}$ & & $\begin{array}{l}\text { human post-mortem knee fluid, skin pustules and } \\
\text { blood, Burma }\end{array}$ & 1944 & BEI Resources \\
\hline B. mallei & BmTurk2 & Turkey 2 & $\mathrm{~T} 2$ & Turkey & & BEI Resources \\
\hline B. mallei & $\mathrm{BmFMH}$ & $\mathrm{FMH}$ & & $\begin{array}{l}\text { derivative of ATCC23344 passaged through human, } \\
\text { laboratory acquired infection- blood }\end{array}$ & 2000 & USAMRIID \\
\hline B. mallei & Bm21280 & 2002721280 & KC1092, 52-236 & Iran & 1952 & BEI Resources \\
\hline B. mallei & Bm86567 & $86-567$ & $\begin{array}{l}\text { India86-567-2, } \\
2000031064\end{array}$ & mule, East India & & BEI Resources \\
\hline B. mallei & $\mathrm{Bm} 2700 \mathrm{C}$ & SR092700C & & & & BEI Resources \\
\hline B. mallei & $\mathrm{BmCh} 7$ & China 7 & NBL7 & preparation produced directly from ATCC 23344 & 1942 & BEI Resources \\
\hline B. mallei & BmCh5 & China 5 & MM-A, NBL4 & lung and nose of infected horse, Kweiyang, China & 1942 & BEI Resources \\
\hline B. mallei & Bm10230 & $\begin{array}{l}\text { NCTC } \\
10230\end{array}$ & strain Ivan & horse with glanders, Hungary & 1961 & BEI Resources \\
\hline B. mallei & $\mathrm{BmGB8}^{* *}$ & $\begin{array}{l}\text { GB8 } \\
\text { (atcc23344) }\end{array}$ & & Laboratory passage of ATCC 23344 in mouse & 1997 & USAMRIID \\
\hline B. thailandensis & BtE264 & E264 & & rice field soil sample, Central Thailand & & BEI Resources \\
\hline B. thailandensis & BtDW503 & DW503 & & $\begin{array}{l}\text { Derived from E264; ( } \Delta \text { amrR-oprA) (Kms Gms Sms); } \\
\text { rpsL (Smr), Central Thailand }\end{array}$ & & BEI Resources \\
\hline
\end{tabular}




\begin{tabular}{|c|c|c|c|c|c|c|}
\hline B. thailandensis & BtE421 & E421 & & $\begin{array}{l}\text { rice field soil sample from Ubon Ratchathani province, } \\
\text { Northeast Thailand }\end{array}$ & 2001 & BEI Resources \\
\hline B. thailandensis & BtE426 & E426 & & $\begin{array}{l}\text { rice field soil sample from Ubon Ratchathani province, } \\
\text { Northeast Thailand }\end{array}$ & 2001 & BEI Resources \\
\hline B. cepacia & $\mathrm{Bc} 706$ & DD-706 & & & & BEI Resources \\
\hline B. cepacia & Bc709 & DD-709 & & & & BEI Resources \\
\hline B. cepacia & $\mathrm{Bc} 710$ & DD-710 & & & & BEI Resources \\
\hline B. cenocepacia & Bce6656 & LMG16656 & & $\begin{array}{l}\text { sputum of cystic fibrosis patient, Edinburgh, United } \\
\text { Kingdom }\end{array}$ & 1989 & BEI Resources \\
\hline B. cenocepacia & BceBC7 & $\mathrm{BC} 7$ & & $\begin{array}{l}\text { sputum from 15-year old patient with "cepacia } \\
\text { syndrome", Canada }\end{array}$ & & Emory Uc \\
\hline B. cenocepacia & BceK562 & $\mathrm{K} 56-2$ & & less antibiotic resistant derivative of $\mathrm{BC} 7$, Canada & & Emory U \\
\hline B. cenocepacia & BceJ2315 & $\mathrm{J} 2315$ & & $\begin{array}{l}\text { sputum from cystic fibrosis patient, Edinburgh, United } \\
\text { Kingdom }\end{array}$ & 1989 & Emory U \\
\hline B. cenocepacia & Bce103a2 & DD-707 & & & & BEI Resources \\
\hline B. multivorans & BmvCF2 & CF2 & & $\begin{array}{l}\text { sputum from cystic fibrosis patient, NIH Clinical } \\
\text { Center }\end{array}$ & & Emory U \\
\hline B. multivorans & BmvCGD1 & CGD1 & & $\begin{array}{l}\text { sputum from chronic granulomatous disease patient, } \\
\text { NIH Clinical Center }\end{array}$ & & Emory U \\
\hline B. multivorans & BmvCGD2 & CGD2 & & $\begin{array}{l}\text { blood from chronic granulomatous disease patient, } \\
\text { NIH Clinical Center }\end{array}$ & & Emory U \\
\hline B. multivorans & BmvCF1 & CF1 & & sputum from cystic fibrosis patient, Belgium & & Emory U \\
\hline B. multivorans & Bmv17616 & $\begin{array}{l}\text { ATCC } \\
17616\end{array}$ & & soil sample, United States & & Emory U \\
\hline B. multivorans & Bmv13010 & LMG13010 & $\begin{array}{l}\text { CCUG 34080, Lauwers } \\
\text { Cepa 002, CIP 105495, } \\
\text { DSM 13243, NCTC } \\
13007\end{array}$ & sputum of cystic fibrosis patient, Belgium & 1992 & BEI Resources \\
\hline
\end{tabular}

aUSAMRIID; United States Army Medical Research Institute of Infectious Disease.

${ }^{b} B E I$ Resources; NIH Biodefense and Emerging Infections Research Resources Repository, NIAID, NIH.

'Emory U; Dr. Joanna Goldberg, Emory University School of Medicine, Atlanta, GA. 


\section{CONCLUSIONS}

\section{Adhesion and biofilm formation: friend or foe?}

The main goal of this work was to investigate the role of the major surface adhesin of GAS, streptococcal collagen-like protein 1 (Scl1), in host tissue attachment, biofilm formation, and virulence. Specifically, our studies focused on the invasive M3-type strains that form poor biofilms in vitro and harbor a unique scl1.3 allele, which encodes a truncated cell-free protein variant. Biofilm formation by bacteria is typically considered disadvantageous to the host because biofilm-embedded bacteria are considerably more resistant to phagocytosis by PMNs and antibiotic treatment (104). However, it has been an emerging concept that adherence and biofilm formation are inversely related to invasiveness and virulence during bacterial infection. Specifically, up to $99.5 \%$ of GAS infections are superficial infections of the throat and skin that are associated with localized tissue microcolonies or biofilms, whereas the remaining $0.5 \%$ are invasive infections that are not biofilm-associated (105). Our unique observations in biofilm-deficient M3-type strains led us to devise the following hypothesis to explain this phenomenon: Invasive M3-type GAS, devoid of Scl1 surface adhesin, have reduced adherence to host ECM proteins, including cellular fibronectin (cFn) and laminin (Lm), and reduced biofilm capacity, thus circumventing the formation of stable tissue microcolonies and shifting the balance towards invasive dissemination instead of localized infection. Our major findings, summarized below in the context of relevant literature, support this model.

Poor biofilm formation and the unique scl1.3 allele of M3-type GAS

Our initial study demonstrated that Scl1 significantly contributes to biofilm formation by GAS on inanimate surfaces (Chapter 1). We showed that deletion of $s c / 1$ in strains of M1, M28 and M41 type resulted in decreased biofilms, with at least $50 \%$ reduced average thickness. Heterologous complementation of the biofilm-deficient Lactococcus lactis with scl1 conferred biofilm formation. Although the mechanism of Scl1-mediated biofilm formation was not fully established, Scl1 expression was correlated with increased surface hydrophobicity in these strains. Notably, several invasive M3 wild-type strains had 
diminished biofilms in comparison to biofilm-capable M1-, M28-, and M41-type strains. We hypothesized that poor biofilm formation was associated with the predicted lack of surface Scl1, based on the presence of a unique null mutation in the sc/1.3 allele of the M3-strains. The nonsense mutation occurred in the $11^{\text {th }}$ Gly-X-Y repeat of the collagenous region, presumably resulting in a truncated secreted Scl1 variant. This unique scl1.3 allele was conserved in $>98 \%$ of invasive M3 isolates (Chapter 3 ). Our successive studies confirmed the lack of Scl1 expression on the surface of M3 strains by flow cytometry, and in cell wall extracts of M3 strains by western blot. Interestingly, the truncated Scl1.3 variant was not detected in supernatant fraction, likely a result of the diminished expression of the scl1.3 transcript that was also perceived for these strains in comparison to other M-types (Chapter 2). Altogether, association between the lack of Scl1 expression, accompanied by limited biofilm formation, prompted further investigations into the invasive traits of M3-type strains.

Scl1-mediated biofilm and ECM binding: implications in tissue microcolony formation Accumulating evidence has supported the importance of Scl1 in mediating the formation of in vivo tissue microcolonies, or biofilms, during infection. First, previous data showed that Scl1-mediated biofilms were enhanced on cFn and Lm coatings, as well as on a complex ECM matrix deposited by human fibroblasts (54). Next, Scl1 specifically binds to extra domain A-containing isoforms of cellular fibronectin (EDA/cFn) and basement membrane laminin $(\mathrm{Lm})$, which are both ECM components found in wounded tissue $(54,60)$. Consequently, both binding functions could foster ligand recognition at the pathogen portal of entry, which supports bacterial adherence and formation of stable tissue microcolonies. Here, we used an in vitro model of wounded human skin equivalents to compare wound colonization and tissue invasion by GAS. The Scl1-lacking biofilmpoor M3-type GAS invaded wound bed in a form of loose chains and cells, whereas Scl1expressing biofilm-capable M41-type GAS formed large rounded microcolonies encased in glycocalyx, as imaged by two-photon microscopy (Chapter 2). These results demonstrated striking phenotypic difference in tissue invasion by M3 and M41 GAS. 


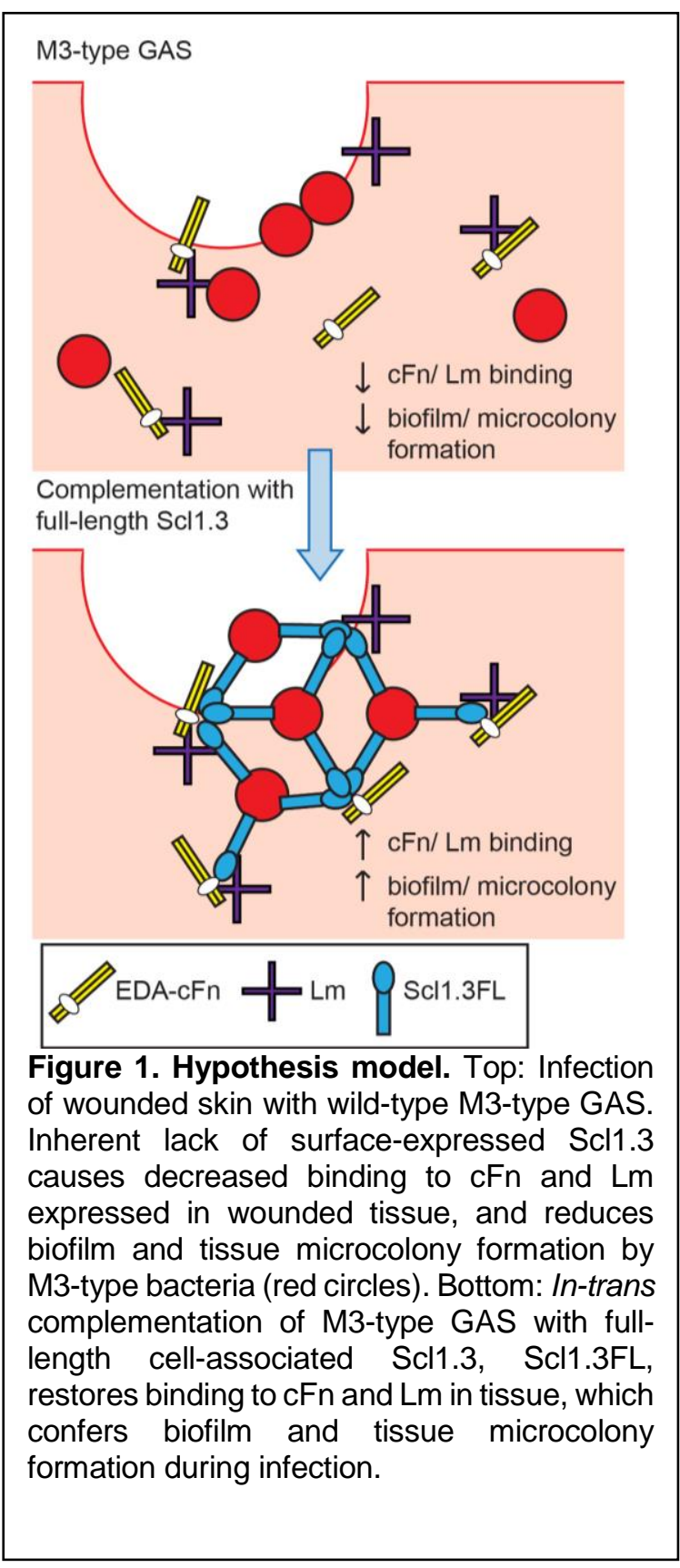

To test our hypothesis shown in Figure 1, we first had to assess whether full-length Scl1.3 variant binds $\mathrm{cFn}$ and $\mathrm{Lm}$, and second, if it restores biofilm formation of M3-type GAS on cFn- and Lm-coated surfaces (Chapter 2). First, recombinant full-length Scl1.3 (rScl1.3FL) protein was generated, following the reversion of the null mutation and restoring the open reading frame, and tested for ECM binding. Positive binding of rScl1.3FL to cFn and Lm was demonstrated by ELISA, surface plasmon resonance, and tryptophan fluorescence binding assays. These results support the concept that Scl1.3FL on the GAS cell surface would behave as an adhesin conferring adherence to EDA/cFn and Lm. Interestingly, rotary shadowing and electron microscopy of $\mathrm{rScl} 1.3$ proteins showed aggregates mediated by $\mathrm{V}$-to- $\mathrm{V}$ domain interactions that could stabilize biofilm structure.

A second set of experiments was designed to test the effect of Scl1.3FL surface expression on GAS biofilm formation. Homologous complementation of two invasive

M3-type strains with Scl1.3FL conferred biofilm formation on cFn- and Lm-coated surfaces. Additionally, heterologous complementation of a $\Delta s c / 1$ mutant of M41-type strain with Scl1.3FL restored biofilm formation to the wild-type level. Biofilms formed by the complemented strains were at least two-fold thicker on average, compared to vectorcomplemented controls. Overall, these results reinforce our hypothesis that M3-type 
strains have reduced adherence and microcolony formation due to a natural lack of surface Scl1 with intrinsic capacity for ECM binding and biofilm support (Figure 1).

Inactivation of Scl1 in GAS results in hypervirulent phenotype

If our hypothesis is correct, the inactivation of $s c / 1$ in biofilm-capable GAS strains should result in increased virulence during infection. We validated this concept by demonstrating that sc/1-inactivated isogenic mutants of the M28- and M41-type strains produced larger skin lesions during subcutaneous infection of mice than the wild-type parental strains (Chapter 2). A similar phenotype was displayed by Scl1-deficient M3-type GAS in comparison to M3 strain containing a rare cell-attached Scl1 variant. While the majority of M3-type strains harbor the scl1.3 allele containing the null mutation, $\sim 1.3 \%$ of $\mathrm{M} 3$ strains harbor a "scl1.3 carrier allele" containing an in-frame deletion encompassing the null mutation, and thus, express a cell-attached variant. An isoallelic replacement strain, harboring the carrier allele, formed a limited abscess compared to the invasive parental M3 strain (Chapter 3). The carrier strain also exhibited increased adherence to human epithelial cells.

Adherence and biofilm formation: an inverse relationship with invasiveness

Overall, our results support the hypothesis that M3-type GAS, naturally lacking surface Scl1, are reduced in adherence and microcolony formation, thus resulting in bacterial dissemination and invasive infection. Figure 2 depicts a model for Scl1-mediated adhesion and microcolony formation during infection, comparing M28- and M41-type GAS expressing Scl1 to M3-type GAS lacking Scl1. 


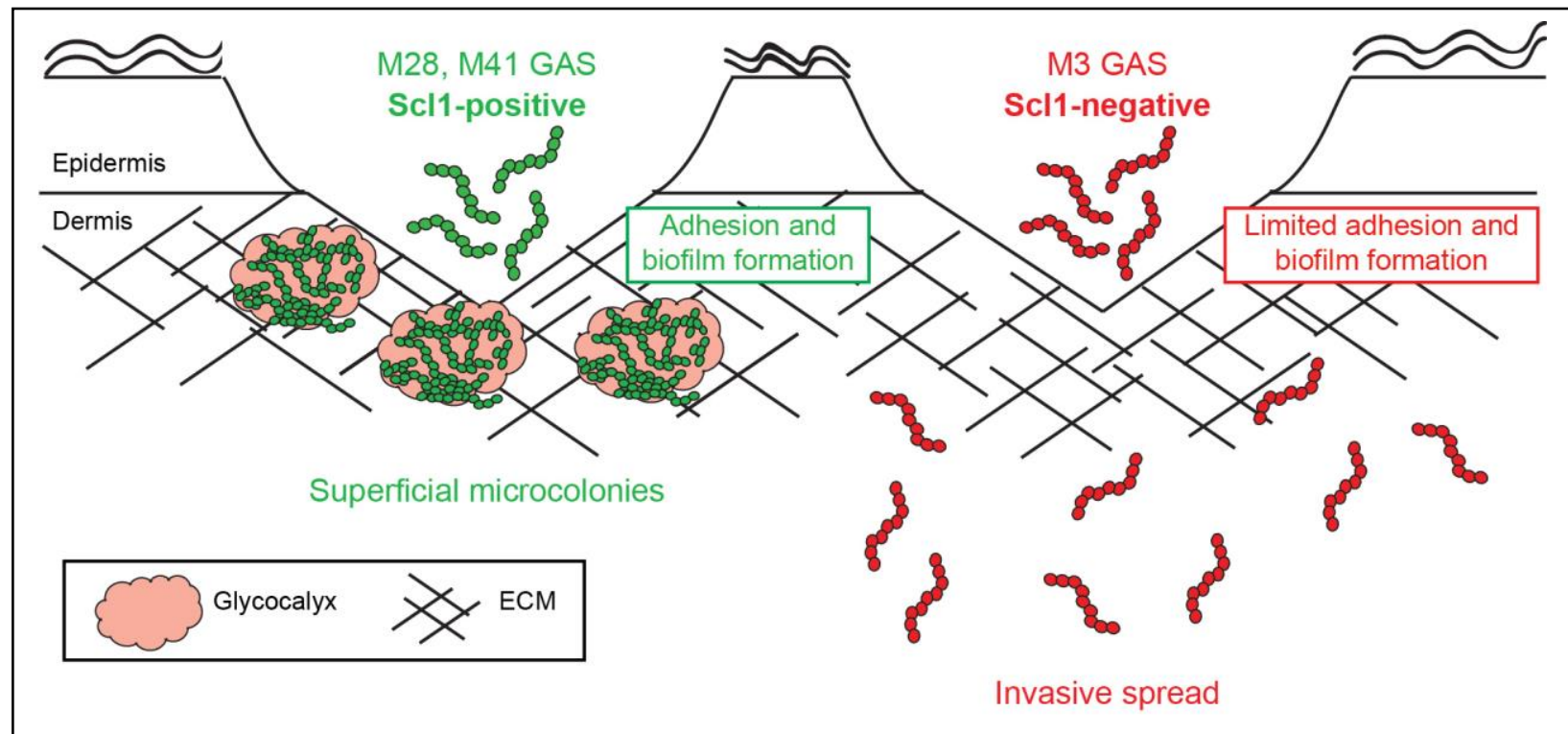

Figure 2. Model of Scl1-mediated GAS adhesion, biofilm formation, and host colonization. M28and M41-type GAS express full-length cell-attached Scl1, which mediates adherence to cFn and Lm. ECM binding promotes biofilm formation in wounded tissue and allows the formation of superficial tissue microcolonies resulting in non-invasive colonization. On the contrary, in M3-type GAS, naturally lacking full-length cell-attached Scl1.3 adhesin, bacterial cells have reduced adherence to cFn and Lm and reduced capacity for biofilm formation, thereby circumventing the formation of localized tissue microcolonies; infection balance is shifted towards dissemination through tissue, promoting invasive spread.

The main hypothesis presented in my thesis, which postulates an inverse relationship between strain invasiveness and its capacity to form biofilm, has gained recent support from several studies. Streptococcus pneumoniae grown in biofilm was less able to disseminate to the blood in mouse models of intranasal and intratracheal infection (106). Biofilm-grown bacteria also had increased adherence to host epithelial cells, as compared to bacteria grown in culture (106). Another study showed that biofilm-deficient mutants of $S$. pneumoniae elicited greater cytokine responses in a mouse model of intranasal infection colonization, and had decreased adherence and increased invasion of epithelial cells in vitro (107). Destabilization of $S$. pneumoniae biofilm and subsequent dissemination was triggered by co-infection with influenza A virus in vivo, as well as febrile temperatures and nutrient availability in vitro. The dispersed bacteria had altered gene expression profile and enhanced dissemination in mice (108). Similarly, GAS cells that formed biofilm in vitro on epithelial cells had downregulated expression of virulence genes, including streptolysins, emm, and hyaluronic acid capsule gene has $A$, and showed enhanced colonization of nasal-associated lymphoid tissue, while dissemination to distant 
organs was decreased (109). Likewise, the cleavage of bacterial surface adhesins by the potent GAS protease SpeB, resulted in dispersal of biofilm, which was associated with increased lesion sizes in mice (110).

\section{Scl variants of GAS- a shared structure with diverse ligand binding properties}

The majority of our work has focused on adherence and biofilm conferred by the Scl1 protein, functions that are lacking for the Scl2 protein; however both Scl proteins are ubiquitous among GAS strains and share a similar overall structure. Early studies based on recombinant Scl proteins of GAS showed they harbor a conserved lollipop-like domain organization with a globular variable domain and stalk-like collagenous domain $(20,21)$. Additionally, multiple sequence alignment and secondary structure analyses of the Scl1and Scl2-variable regions predicted two conserved $\alpha$-helices interspaced by the hypervariable sequence (51). A major contribution that came from current work was the report of the crystal structure for the V domain of the Scl2 protein of M3-type GAS, which provided insights into possible ligand binding sites and function of the Scl proteins (Chapters 4 and 5). The Scl-globular (homotrimeric) domain folds into unique six helical bundle structure, which is predicted to be conserved across all Scl1 and Scl2 variants. The two antiparallel $\alpha$-helices that are conserved in all variants form a structural core for the exposed variable loops that are likely involved in ligand binding. The $\mathrm{V}$ domain was also found to be structurally similar to the gp41 subunit of the envelope glycoprotein of HIV-1, a membrane fusion protein involved in viral entry into CD4+ T cells, although the two proteins had low sequence identity. The six-helix bundle structure is largely absent in bacteria, with the exception of the cholix toxin which was reported to contain a bundle of six helices within the translocation domain (111). Membrane fusion and entry or translocation across the membrane seems to be a function offered by this structural element. Since internalization of GAS by host cells has been reported (112), it would be interesting if the $\mathrm{V}$ domain of $\mathrm{Scl}$ also mediates an interaction with host cell membranes to accomplish host cell entry. 
SUPPLEMENTARY MATERIAL FOR CHAPTER 1

THE STREPTOCOCCAL COLLAGEN-LIKE PROTEIN-1 (SCL1) IS A SIGNIFICANT DETERMINANT FOR BIOFILM FORMATION BY GROUP A STREPTOCOCCUS

Heaven A Oliver-Kozup, Meenal Elliott, Beth A Bachert, Karen H Martin, Sean D Reid, Diane E Schwegler-Berry, Brett J Green, and Slawomir Lukomski

Published in BMC Microbiology 2011 11:262 
Figure S1

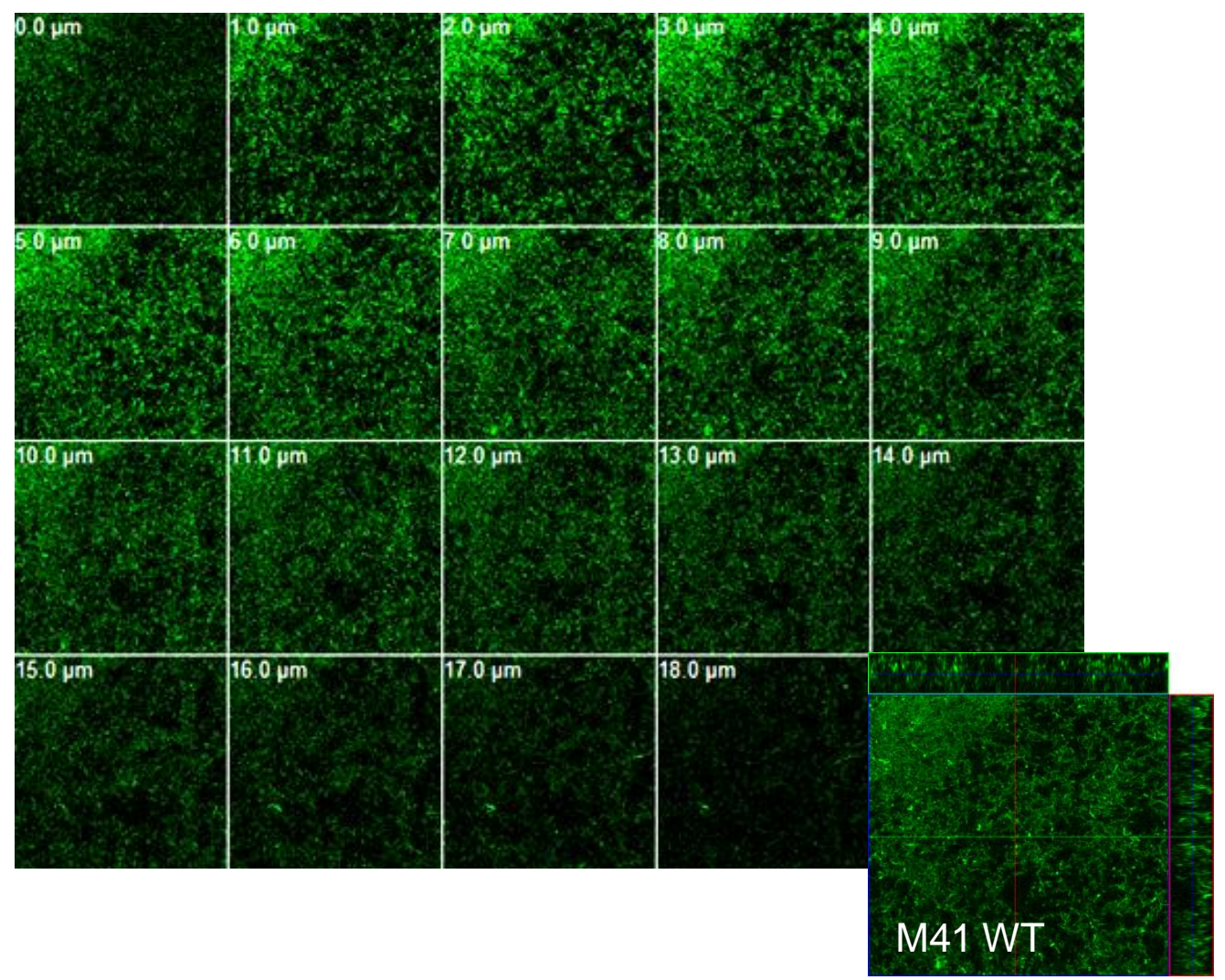

Figure S1 (a). Confocal laser scanning microscopy (CLSM) of GFP-expressing M41 WT GAS biofilm at $24 \mathrm{~h}$. Panels represent a gallery view of consecutive images taken at 1 micrometer increments. Panel shown in lower right corner represents an $\mathrm{X}-\mathrm{Y}$ orthogonal Z-stack view (Fig. 4d). Thickness is indicated in micrometers. 


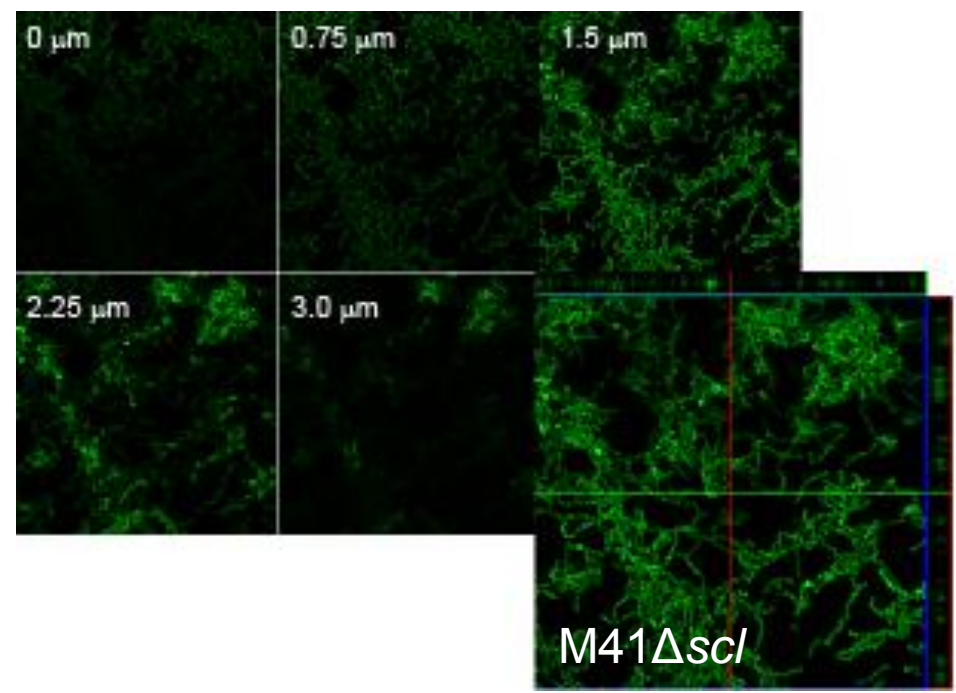

Figure S1 (b). Confocal laser scanning microscopy (CLSM) of GFP-expressing M41 $\Delta s c / 1$ GAS biofilm at $24 \mathrm{~h}$. Panels represent a gallery view of consecutive images taken at 0.75 micrometer increments. Panel shown in lower right corner represents an X-Y orthogonal Z-stack view (Fig. 4d). Thickness is indicated in micrometers. 


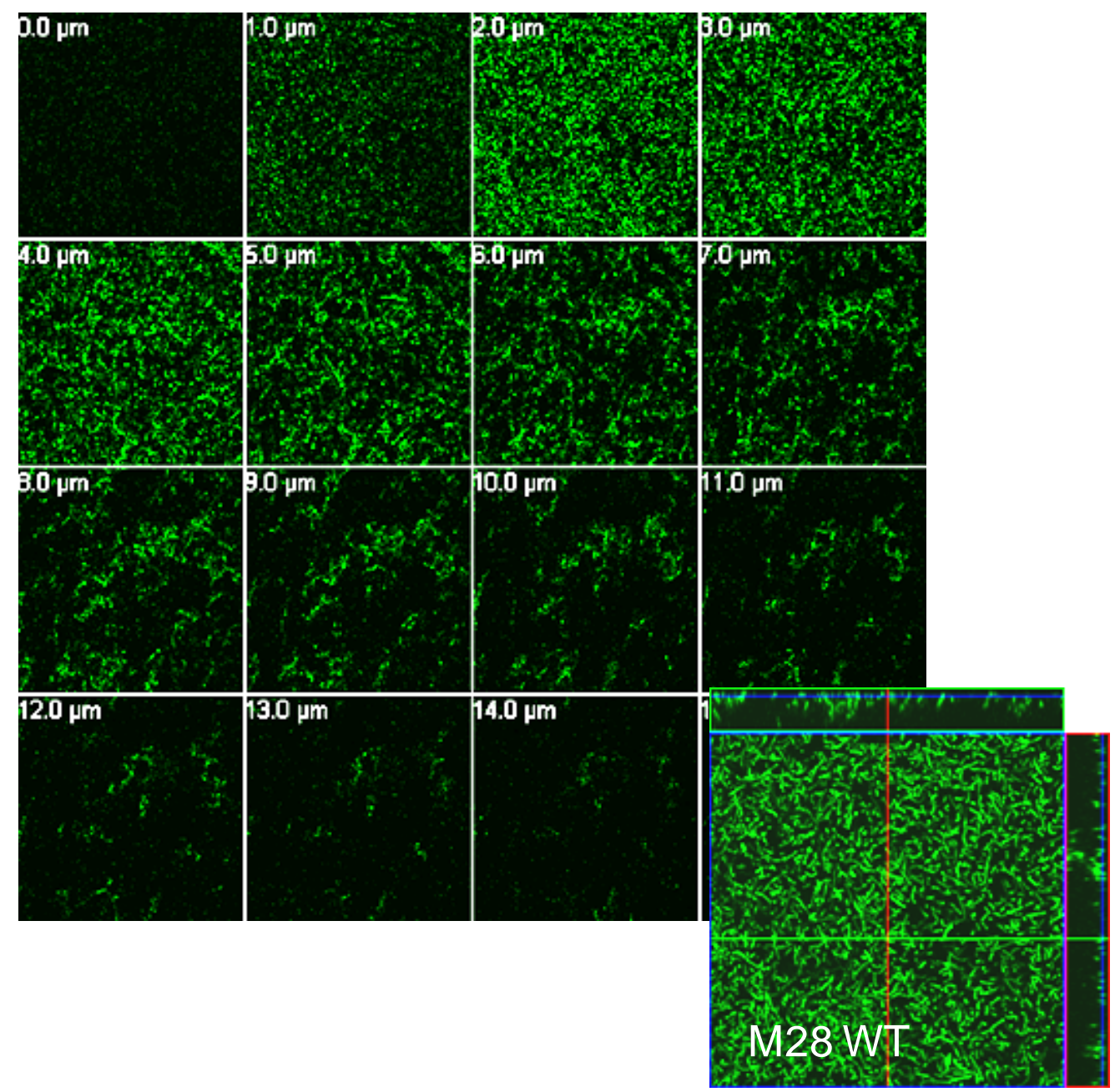

Figure S1 (c). Confocal laser scanning microscopy (CLSM) of GFP-expressing M28 WT GAS biofilm at $24 \mathrm{~h}$. Panels represent a gallery view of consecutive images taken at 1 micrometer increments. Panel shown in lower right corner represents an $\mathrm{X}-\mathrm{Y}$ orthogonal Z-stack view (Fig. 4e). Thickness is indicated in micrometers. 


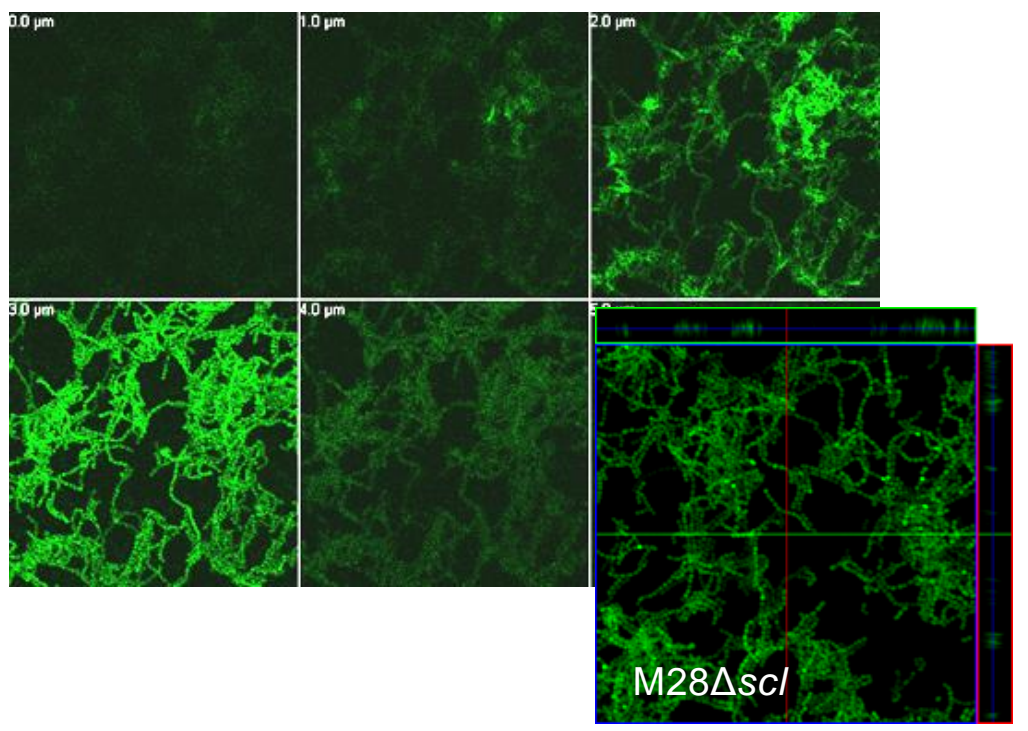

Figure S1 (d). Confocal laser scanning microscopy (CLSM) of GFP-expressing M28 $\Delta s c / 1$ GAS biofilm at $24 \mathrm{~h}$. Panels represent a gallery view of consecutive images taken at 1 micrometer increments. Panel shown in lower right corner represents an $X-Y$ orthogonal Z-stack view (Fig. 4e). Thickness is indicated in micrometers. 


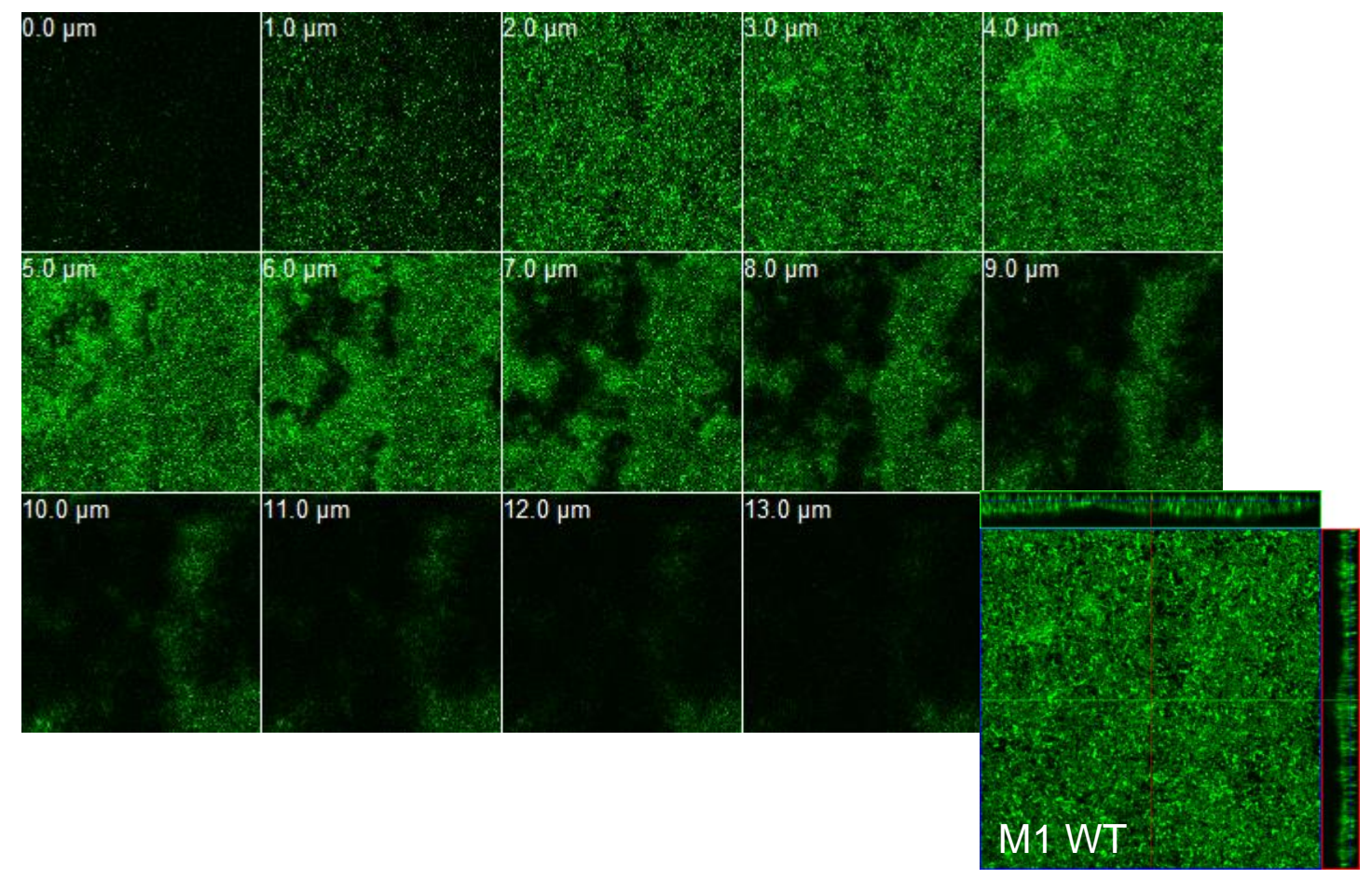

Figure S1 (e). Confocal laser scanning microscopy (CLSM) of GFP-expressing M1 WT GAS biofilm at $24 \mathrm{~h}$. Panels represent a gallery view of consecutive images taken at 1 micrometer increments. Panel shown in lower right corner represents an $\mathrm{X}-\mathrm{Y}$ orthogonal Z-stack view (Fig. 4f). Thickness is indicated in micrometers. 


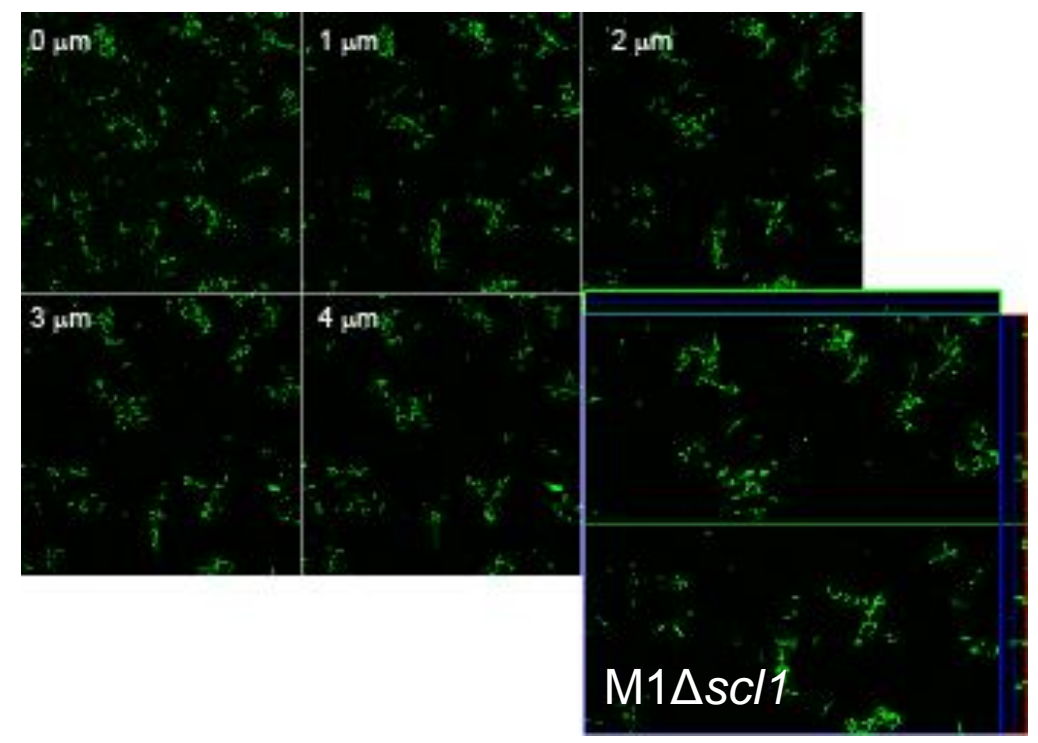

Figure S1 (d). Confocal laser scanning microscopy (CLSM) of GFP-expressing M1 $\Delta s c / 1$ GAS biofilm at $24 \mathrm{~h}$. Panels represent a gallery view of consecutive images taken at 1 micrometer increments. Panel shown in lower right corner represents an X-Y orthogonal Z-stack view (Fig. 4f). Thickness is indicated in micrometers. 


\section{Figure S2}

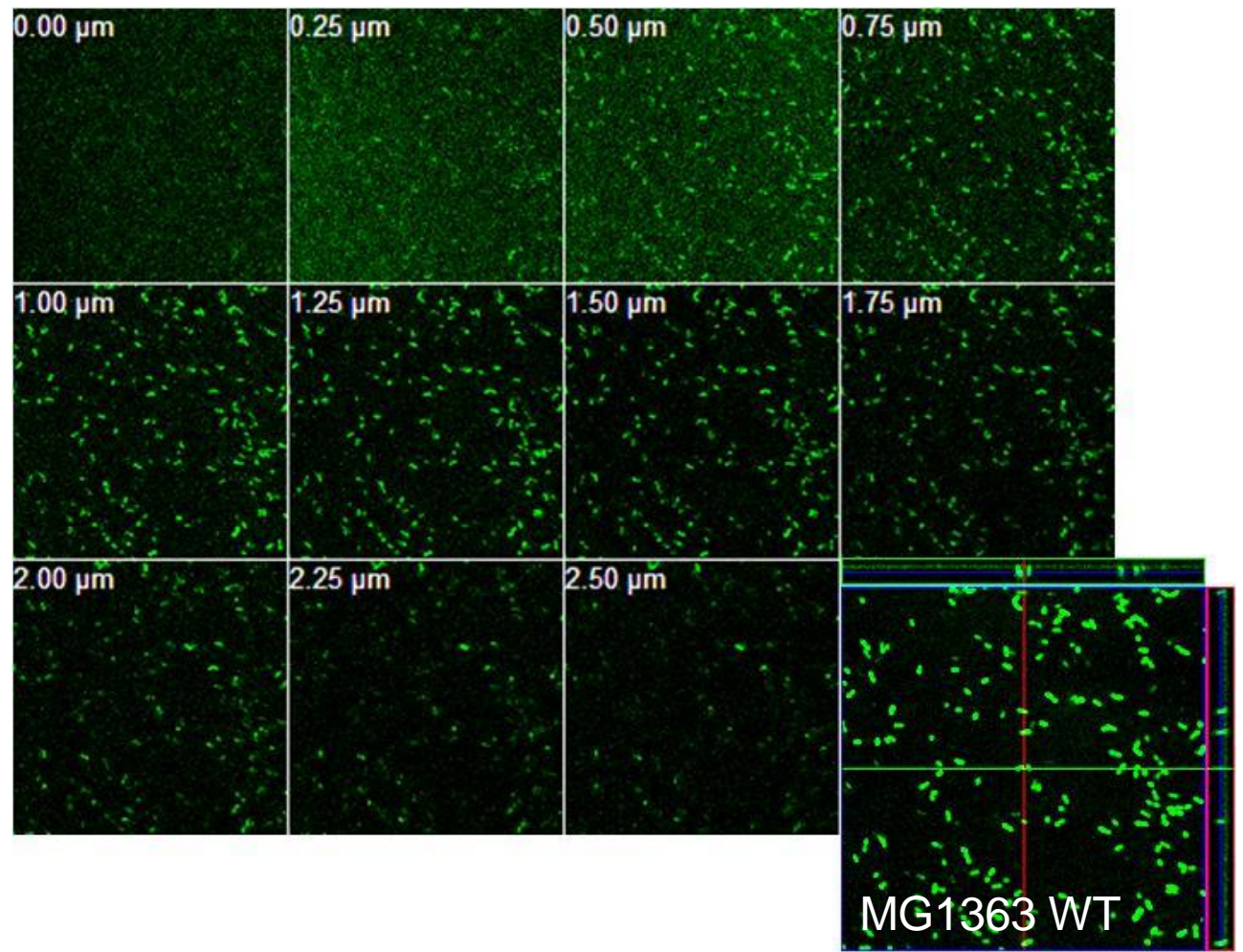

Figure S2 (a). Confocal laser scanning microscopy (CLSM) of GFP-expressing Lactococcus lactis MG1363 WT biofilm at $24 \mathrm{~h}$. Panels represerit a gallery view of consecutive images taken at 0.25 micrometer increments. Panel shown in lower right corner represents an $X-Y$ orthogonal Z-stack view (Fig. 5e). Thickness is indicated in micrometers. 


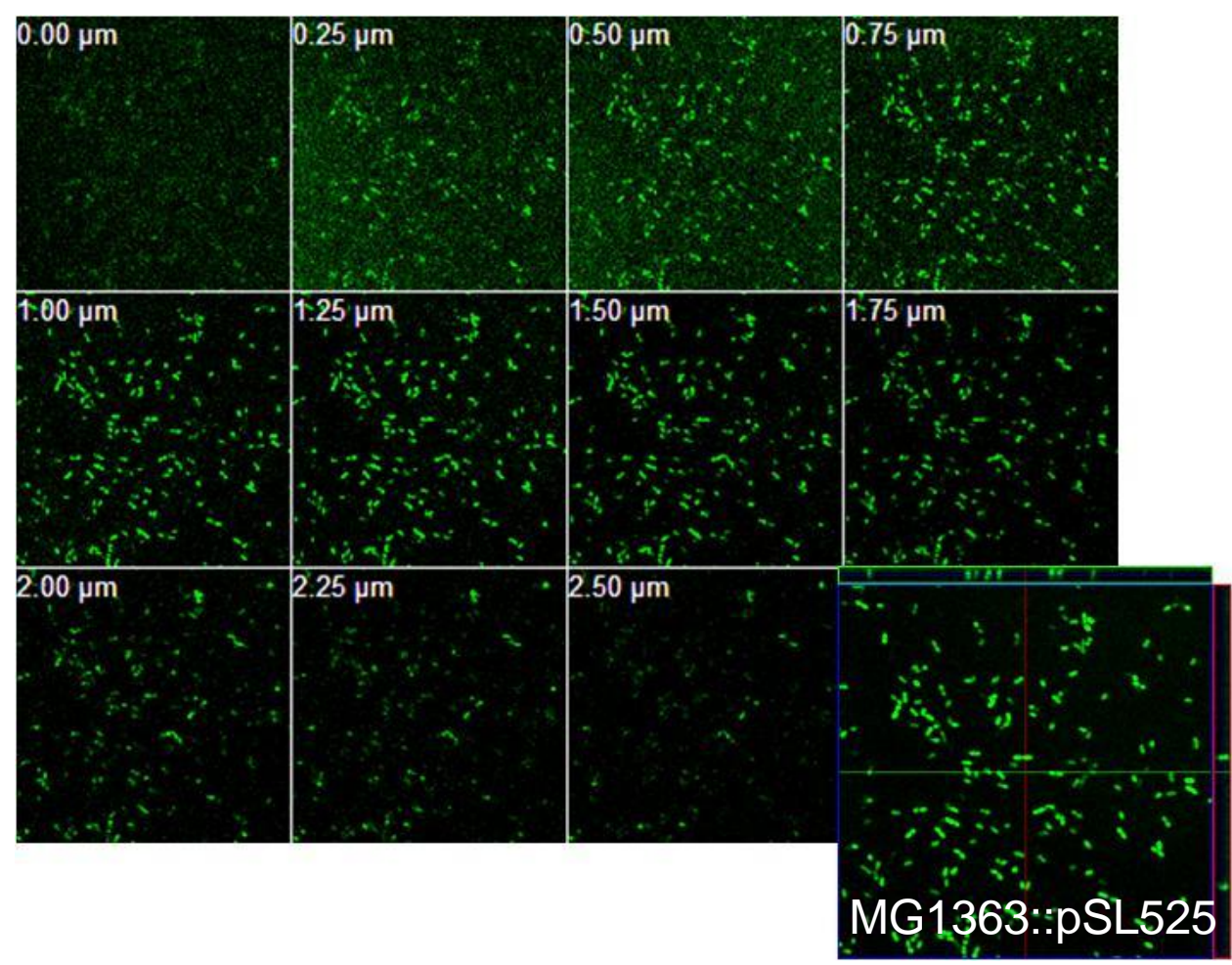

Figure S2 (b). Confocal laser scanning microscopy (CLSM) of GFP-expressing Lactococcus lactis biofilm at $24 \mathrm{~h}$. L. lactis was transformed with the shuttle vector pJRS525 (MG1363::pJRS525). Panels represent a gallery view of consecutive images taken at 0.25 micrometer increments. Panel shown in lower right corner represents an $\mathrm{X}$ $\mathrm{Y}$ orthogonal Z-stack view (Fig. 5e). Thickness is indicated in micrometers. 


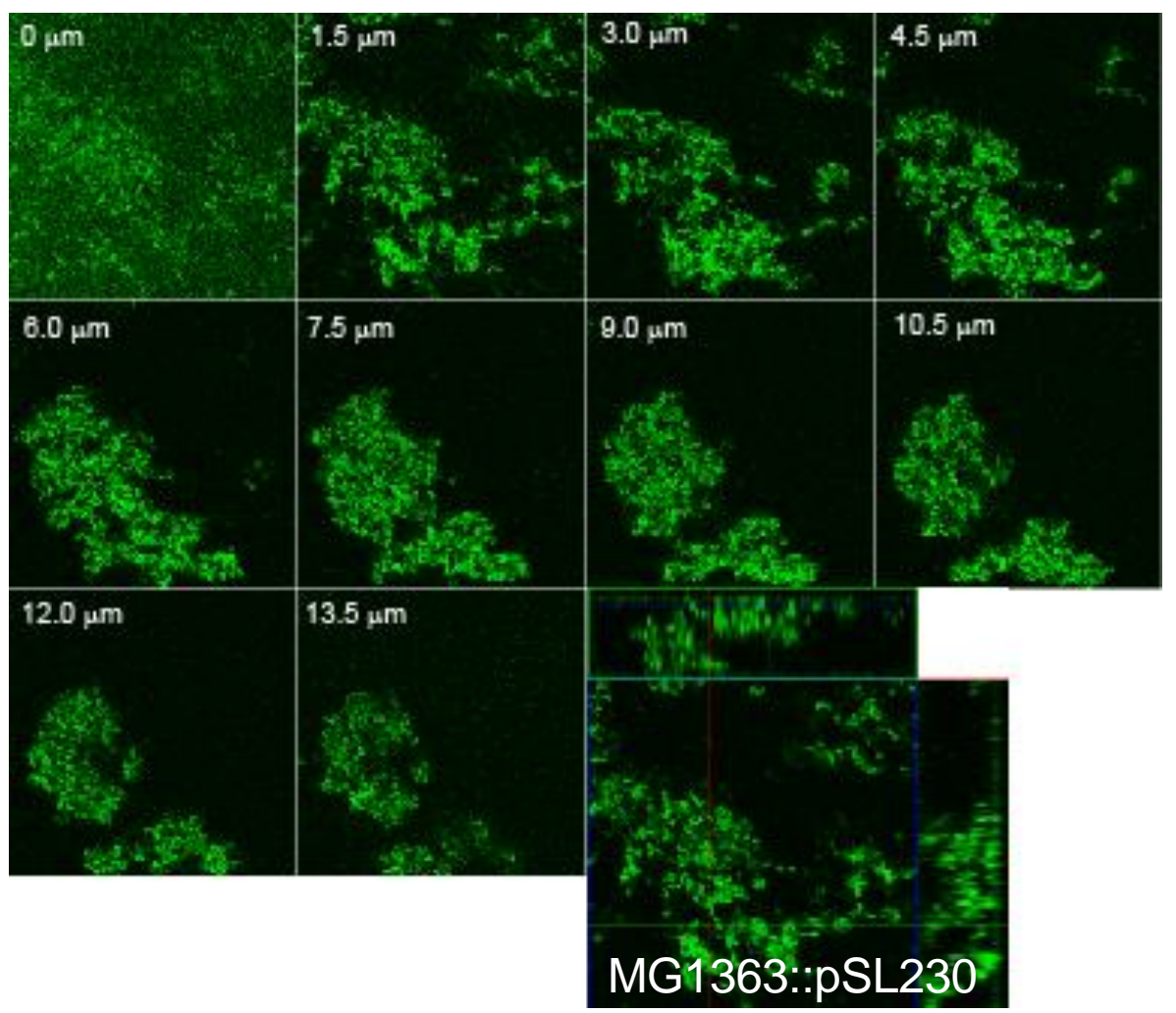

Figure S2 (c). Confocal laser scanning microscopy (CLSM) of GFP-expressing Lactococcus lactis expressing Scl1.41 protein (MG1363::pSL230) biofilm at $24 \mathrm{~h}$. L. lactis was transformed with the plasmid construct pSL230 encoding Scl1.41 protein (MG1363::pSL230). Panels represent a gallery view of consecutive images taken at 0.5 micrometer increments. Panel shown in lower right corner represents an X-Y orthogonal Z-stack view (Fig. 5e). Thickness is indicated in micrometers. 


\section{SUPPLEMENTARY MATERIAL FOR CHAPTER 2}

\section{UNIQUE FOOTPRINT IN THE SCL1.3 LOCUS AFFECTS ADHESION AND BIOFILM FORMATION OF THE INVASIVE M3-TYPE GROUP A STREPTOCOCCUS}

Beth A. Bachert, Soo Jeon Choi, Paul R. LaSala, Tiffany Harper, Dudley H. McNitt, Dylan

T. Boehm, Clayton C. Caswell, Pawel Ciborowski, Douglas R. Keene, Anthony R. Flores, James M. Musser, Flavia Squeglia, Daniela Marasco, Rita Berisio, and Slawomir Lukomski

Published in Frontiers in Cellular and Infection Microbiology 2016 6:90 

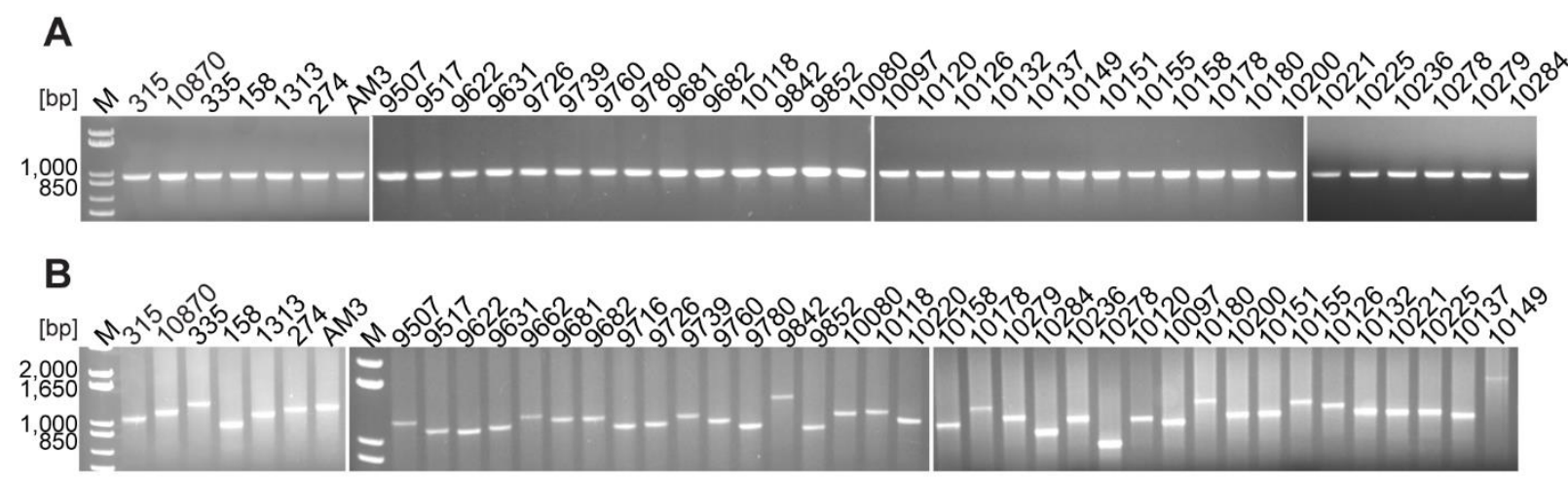

Figure S1. Analytical PCR of scl1.3 and sc/2.3 in M3-type GAS. (A) PCR of scl1.3 in 42 M3-type strains was performed with primers 232Up and 232Rev flanking the sc/1 locus (Table S1). Expected size based on MGAS315: 1,010 bp. M, 1 kb Plus DNA Ladder. (B) PCR of scl2.3 in 42 M3-type strains was performed with primers Scl2.3 F and Scl2.3 R flanking the scl2.3 collagen-like region (Table S1). Expected size based on MGAS315 genome is 1,125 bp. M, $1 \mathrm{~kb}$ Plus DNA Ladder. MGAS designation applies to all strain numbers shown above gel wells, with the exception of strain AM3. 


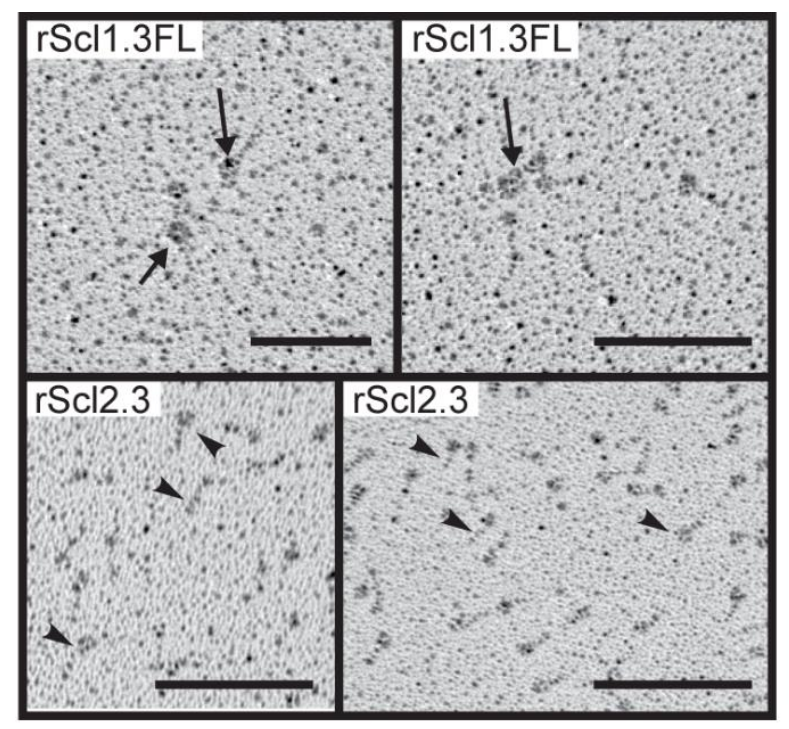

Figure S2. Domain organization of rScl1.3FL and rScl2.3 proteins. Rotary shadowing of rScl proteins demonstrates the conserved lollipop-like domain organization. Aggregates mediated by the interactions between $\mathrm{V}$ regions of $\mathrm{rScl1}$.3FL are seen in the top panels (depicted by arrows). No apparent aggregation was observed in the rScl2.3 preparation (single lollipops are depicted by arrowheads). All scale bars reflect $100 \mathrm{~nm}$. 


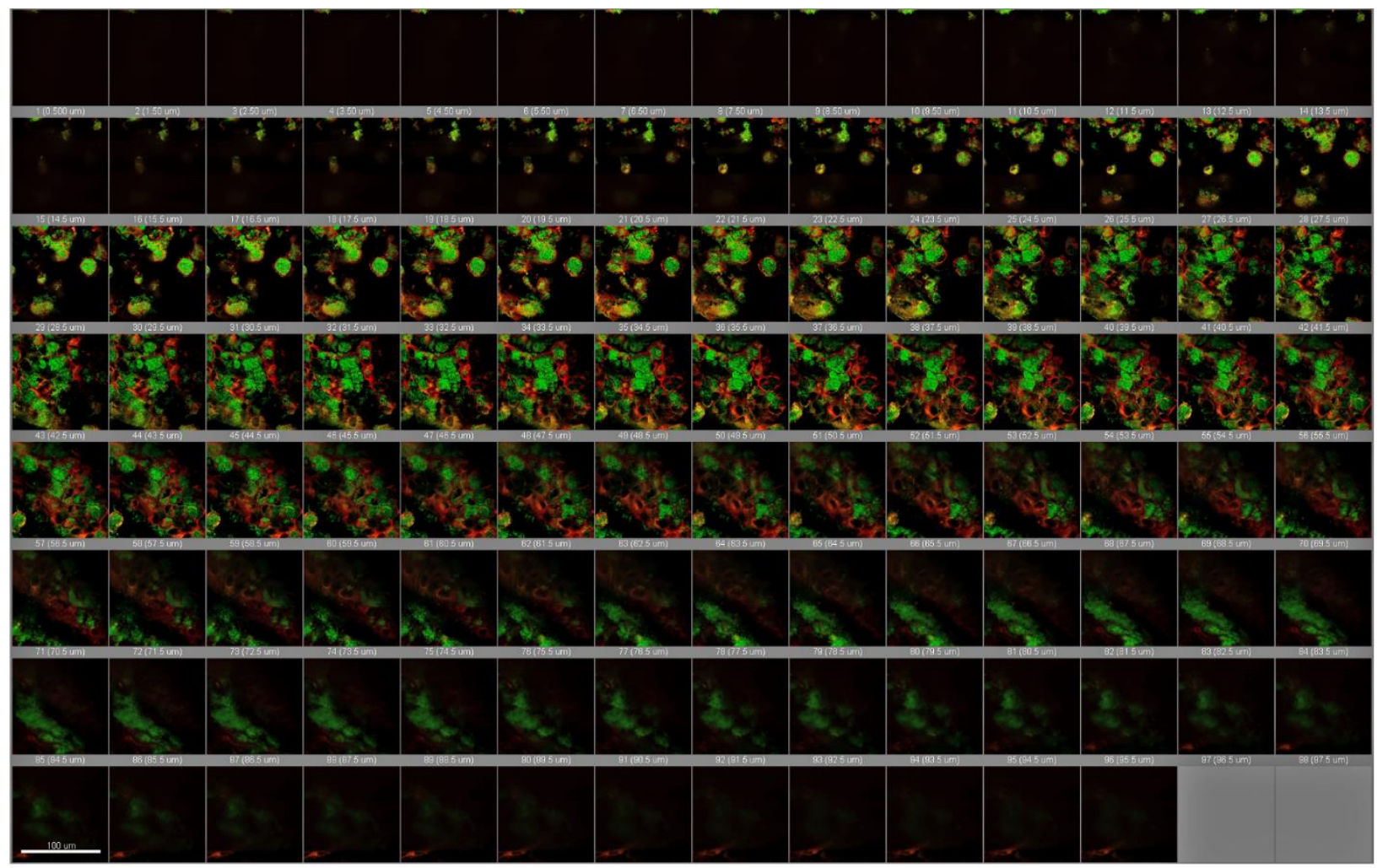

Figure S3. Colocalization of glycocalyx staining with GFP-expressing M41-type GAS during in vitro infection of skin equivalent. Representative TPF gallery image of z-stack layers from 3D projection (Figure $\mathbf{5}$ of manuscript), showing colocalization of TRITCconcanavalin A staining of glycocalyx (red) with GFP-expressing GAS microcolonies (green). Z-stack step size: $1 \mu \mathrm{m} ; 600 x$ magnification. 


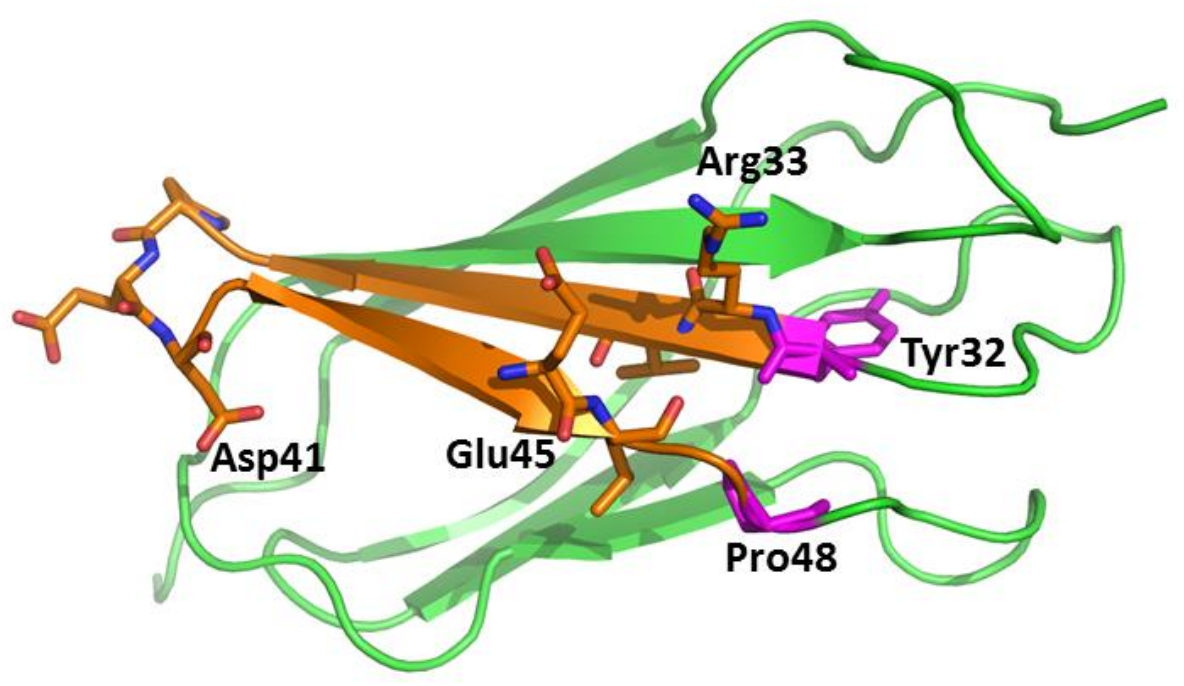

Figure S4. Strategy for C-C' loop peptide design. Cartoon model of the EDA domain of cFn (PDB code 1J8K) used for the C-C' peptide design. The designed peptide includes the C-C' beta-hairpin (orange). The terminal residues of the beta-hairpin (magenta) were mutated to cysteine to induce a disulfide bond and mimic the conformation of the peptide in the protein. The key residue for recognition, Asp41, is reported in ball-and-stick along with the residues Arg33 and Glu45, which form a salt bridge, and the terminal residues mutated to cysteine. 
Table S1. Primers used in this study

\begin{tabular}{|c|c|c|}
\hline Primer name & Sequence $\left(5^{\prime}-3^{\prime}\right)$ & Use \\
\hline \multicolumn{3}{|l|}{ Analytical PCR } \\
\hline $\begin{array}{l}\text { IS1548F } \\
\text { Scl1R }\end{array}$ & $\begin{array}{l}\text { GCCGTCTGCGTGCCCATTGCGTCTA } \\
\text { ACTAGATCTGAGATTATGGTGCTTTGATGTC }\end{array}$ & $\begin{array}{l}\text { Detection of } \\
\text { IS1548 }\end{array}$ \\
\hline $232 \mathrm{U}$ & CTCCACAAAAGAGTGATCAGTC & Amplificatio \\
\hline $232 \mathrm{R}$ & TTAGTTGTTTTCTTTGCGTTT & $\mathrm{n}$ of $s c / 1.3$ \\
\hline Sclá & AGGCATACAAGATCATGTCCTTGA & Amplificatio \\
\hline Scl2.3 R & TTTGGTGTATGTGGTGCGGT & $\mathrm{n}$ of $s \mathrm{cl} 2.3$ \\
\hline & SAATGGATGACGATACC & Amplificatio \\
\hline Scl Rev & ACTTTCCATCAGTTAGGTAGC & $\mathrm{n}$ of $s c l 2.3$ \\
\hline \multicolumn{3}{|l|}{ Cloning } \\
\hline Scl1. & GGCCGAGACTCCTATGA & Cloning \\
\hline Scl1.3 M3VR & $\begin{array}{l}\text { CAGCGTCTCAGCGCTCTTTGTTGCACCTTTTTTC } \\
\text { AATCAG }\end{array}$ & $\begin{array}{l}s c / 1.3 \mathrm{~V} \\
\text { reaion }\end{array}$ \\
\hline 232 Up & AAAAGAGTGATCAGT & Cloning \\
\hline ME7 & TCA & $s c / 1.3 W T$ \\
\hline pJRS525F1 & JACG & Repair of \\
\hline $\begin{array}{l}\text { ME6 }^{\mathrm{a}} \\
\text { Scl13 M3VF }\end{array}$ & TCCAGCAGGACCTCGAGGTGAACGC & $\begin{array}{ll}\text { scl1.3 null } & \text { nuning of } \\
\text { Cloning }\end{array}$ \\
\hline Scl1.28WMR & $\begin{array}{l}\text { TATTATTTTTCGAACTGCGGGTGG } \\
\text { TTTTTCTGGAGCTGGAGTTACC }\end{array}$ & scl1.3FL \\
\hline Scl1.3 M3VF ext & GAGATGGCCGAGACTCCTATGACATCAAAGGA & Cloning of \\
\hline Scl1.3 truncR2 & CAGCGCTACCTCGAGGTCCTGCTGGA & $s$ \\
\hline $\begin{array}{l}\text { Scl2 M3VF } \\
\text { Scl2.28_WMR }\end{array}$ & $\begin{array}{l}\text { GAGATGGCCGATGGTGAAGATGCCCAAAAAAG } \\
\text { GTCAAGCTTATTATTTTTCGAACTGCGGGTGG } \\
\text { CTCCAT }\end{array}$ & $\begin{array}{l}\text { Cloning } \\
\text { scl2.3 }\end{array}$ \\
\hline \multicolumn{3}{|l|}{ qRT-PCR analysis } \\
\hline $\begin{array}{l}\text { tufA } F \\
\text { tufA } R\end{array}$ & $\begin{array}{l}\text { CAACTCGTCACTATGCGCACAT } \\
\text { GAGCGGCACCAGTGATCAT }\end{array}$ & $\begin{array}{l}\text { qRT-PCR of } \\
\text { tufA }\end{array}$ \\
\hline Scl1 WMR exp F & ACAAAGAAGCTAACCAAAC & qRT-PCR of \\
\hline Scl1_WMR_exp_R & TACAGGTGTCT & $s c / 1$ \\
\hline Scl2_WMR_exp_F & COACACOTCO & qRT-PCR of \\
\hline Scl2_WMR_exp_R & TGT & \\
\hline emm3_exp_F2 & AAC & qRT-PCR of \\
\hline & TTCAAGCTCTTTGTTWAGTTTTTCAAG & \\
\hline $\begin{array}{l}\text { Mga_TMF } \\
\text { Maa TMR }\end{array}$ & CAAGTCAACAGTGGAGAGAACTAAAATT & qRT-PCR of \\
\hline $\begin{array}{l}\text { Mga_TM } \\
\text { Mga_F1 }\end{array}$ & & \\
\hline Mga F1 & ATCAGACAAAAACATTAAATTGCATG & Sequencing \\
\hline Mga R1 & TTGCATGTTAGTGAGACAAGTTTGC & 0 \\
\hline
\end{tabular}


Table S2. Variation in scl2.3 gene among M3 strains

\begin{tabular}{lcccll}
\hline Strain & $\begin{array}{l}\text { No. of GXY } \\
\text { repeats }\end{array}$ & $\begin{array}{l}\text { No. of bp in the } \\
\text { CL region }\end{array}$ & $\begin{array}{l}\text { Amplicon size } \\
(\mathrm{bp})^{\mathrm{a}}\end{array}$ & $\begin{array}{l}\text { No. } \\
\text { CAAAA } \\
\text { repeats }\end{array}$ & $\begin{array}{l}\text { of } \\
\begin{array}{l}\text { GTG in } \\
\text { frame }\end{array}\end{array}$ \\
\hline MGAS274 & $\sim 125$ & $\sim 1125$ & $\sim 1206$ & 8 & yes \\
MGAS315 & 116 & 1044 & 1125 & 5 & yes \\
MGAS335 & $\sim 135$ & $\sim 1215$ & $\sim 1296$ & 4 & no \\
MGAS1313 & $\sim 119$ & $\sim 1071$ & $\sim 1152$ & 8 & yes \\
AM3 & $\sim 129$ & $\sim 1161$ & $\sim 1242$ & 10 & no \\
MGAS3375 & 17 & 153 & 234 & 5 & yes \\
MGAS9517 & 113 & 1017 & 1098 & 8 & yes \\
MGAS9622 & 111 & 999 & 1080 & 11 & yes \\
MGAS9631 & 113 & 1017 & 1098 & 18 & no \\
MGAS9716 & 110 & 990 & 1071 & 14 & yes \\
MGAS9726 & 111 & 999 & 1080 & 11 & yes \\
MGAS9739 & 119 & 1071 & 1152 & 5 & yes \\
MGAS9760 & 113 & 1017 & 1098 & 11 & yes \\
MGAS9780 & 106 & 954 & 1035 & 14 & yes \\
MGAS9842 & 139 & 1251 & 1332 & 9 & no \\
MGAS9852 & 103 & 927 & 1008 & 8 & yes \\
MGAS10080 & 117 & 1053 & 1134 & 5 & yes \\
MGAS10118 & 119 & 1071 & 1152 & 5 & yes \\
MGAS10220 & 110 & 990 & 1071 & 12 & no \\
\hline
\end{tabular}

${ }^{a}$ Amplicons were generated using primers Scl2.3 F/ R (Table S1)

b GTG is scl2 start codon 
Table S3. Mass spectrometry identification of $r S c l$ and native Scl proteins ${ }^{a}$

\begin{tabular}{|c|c|c|c|c|c|}
\hline Sample & $\begin{array}{l}\text { No. of } \\
\text { peptides } \\
>95 \% \\
\text { confident }\end{array}$ & Peptide & Confidence & $\begin{array}{l}\text { Sequence } \\
\text { coverage }\end{array}$ & $\begin{array}{l}\text { Confident } \\
\text { ID }\end{array}$ \\
\hline rScl1.3V & 4 & $\begin{array}{l}\text { EENSQEELK } \\
\text { EENSQEELKNFTEER } \\
\text { LKEILDLIEK } \\
\text { WYGTYFKEENSQEELK }\end{array}$ & $\begin{array}{l}99 \% \\
99 \% \\
99 \% \\
99 \%\end{array}$ & $40 \%$ & yes \\
\hline rScl2.3 & 2 & $\begin{array}{l}\text { GIQDHVLDGQDGDR } \\
\text { EELLSALIDGTSR }\end{array}$ & $\begin{array}{l}99 \% \\
98.13 \%\end{array}$ & $18 \%$ & yes \\
\hline \multicolumn{6}{|l|}{ MGAS315 WT } \\
\hline Scl2.3 in Sup & 12 & $\begin{array}{l}\text { DVTPAPQNPSN } \\
\text { DVTPAPQNPSNR } \\
\text { EELLSALIDGTSR } \\
\text { GEAGPAGPR } \\
\text { GIQDHVLDGQDGDR } \\
\text { GIQDHVLDGQDGDRGEAGPA } \\
\text { GPR } \\
\text { GLNKPQTQGGNQL } \\
\text { GLNKPQTQGGNQLAK } \\
\text { NKPQTQGGNQLAK } \\
\text { REELLSALIDGTSR } \\
\text { TPEVPQKPDTAPHTPK } \\
\text { TPQIPGQSK }\end{array}$ & $\begin{array}{l}99 \% \\
99 \% \\
99 \% \\
99 \% \\
99 \% \\
99 \% \\
\\
99 \% \\
99 \% \\
99 \% \\
99 \% \\
99 \% \\
99 \%\end{array}$ & $17 \%$ & yes \\
\hline Scl2.3 in CW & 7 & $\begin{array}{l}\text { DVTPAPQNPSNR } \\
\text { EELLSALIDGTSR } \\
\text { GIQDHVLDGQDGDR } \\
\text { GIQDHVLDGQDGDRGEAGPA } \\
\text { GPR } \\
\text { GLNKPQTQGGNQLAK } \\
\text { REELLSALIDGTSR } \\
\text { TPEVPQKPDTAPHTPK }\end{array}$ & $\begin{array}{l}99 \% \\
99 \% \\
99 \% \\
99 \% \\
99 \% \\
99 \% \\
99 \%\end{array}$ & $15 \%$ & yes \\
\hline \multicolumn{6}{|c|}{ Scl1.3FL- complemented GAS } \\
\hline $\begin{array}{l}\text { 315WT } \\
\text { scl1.3FL }\end{array}$ & 5 & $\begin{array}{l}\text { EENSQEELKNFTEER } \\
\text { EILDLIEK } \\
\text { GDKGETGLAGPVGPAGK } \\
\text { GETGLAGPVGPAGK } \\
\text { LKEILDLIEK }\end{array}$ & $\begin{array}{l}99 \% \\
99 \% \\
99 \% \\
99 \% \\
99 \%\end{array}$ & $17 \%$ & yes \\
\hline $\begin{array}{l}10870 \Delta s c / 1:: \\
\text { scl1.3FL }\end{array}$ & 4 & $\begin{array}{l}\text { EILDLIEK } \\
\text { GDKGETGLAGPVGPAGK } \\
\text { GETGLAGPVGPAGK } \\
\text { LKEILDLIEK }\end{array}$ & $\begin{array}{l}99 \% \\
99 \% \\
99 \% \\
99 \%\end{array}$ & $11 \%$ & yes \\
\hline $\begin{array}{l}\text { M41 } 1 \Delta s c / 1 \quad:: \\
\text { scl1.3FL }\end{array}$ & 3 & $\begin{array}{l}\text { EENSQEELKNFTEER } \\
\text { GDKGETGLAGPVGPAGK } \\
\text { GETGLAGPVGPAGK }\end{array}$ & $\begin{array}{l}99 \% \\
99 \% \\
99 \%\end{array}$ & $13 \%$ & yes \\
\hline
\end{tabular}


Table S4. Summary table of fold-changes in scl1 and scl2 expression ${ }^{\mathrm{a}}$

\begin{tabular}{lllllll}
\hline Strain & Gene & Fold change & + SE & - SE & P value & Significance \\
\hline M1 & $s c / 1$ & 21.05195084 & -2.33583 & -2.62734 & 0.001184755 & $* *$ \\
& $s c / 2$ & -23.6920597 & -1.97551 & -1.82347 & 0.01549836 & $*$ \\
M28 & $s c / 1$ & 6.237987683 & -1.1098 & -1.34997 & 0.01395444 & $*$ \\
& $s c / 2$ & -7.25032516 & 0.960352 & 1.106978 & 0.034822349 & $*$ \\
M41 & $s c / 1$ & 7.787441864 & -0.82018 & -0.91673 & 0.004897482 & $*$ \\
& $s c / 2$ & -3.22615419 & 0.376259 & 0.425935 & 0.141911016 & N.S. \\
M3 MGAS: & & & & & \\
10870 & $s c / 1$ & -25.2160816 & 1.366976 & 1.445328 & $5.48581 E-06$ & $* *$ \\
& $s c / 2$ & -1.84753589 & -0.01174 & -0.01166 & 0.077646106 & N.S. \\
158 & $s c / 1$ & -45.8489636 & -0.86051 & -0.84465 & 0.000121468 & $* * *$ \\
& $s c / 2$ & 1.077129939 & 0.059307 & 0.056212 & 0.753558444 & N.S. \\
335 & $s c / 1$ & -42.9309881 & -4.03211 & -3.68593 & $5.18378 E-05$ & $* * *$ \\
& $s c / 2$ & -2.44408041 & -1.65928 & -0.98832 & 0.283398851 & N.S. \\
1313 & $s c / 1$ & -43.929358 & 0.938527 & 0.959016 & $5.45812 E-06$ & $* * *$ \\
& $s c / 2$ & -3.89390614 & -2.38312 & -1.47835 & 0.117229672 & N.S.
\end{tabular}

aFold-changes in sc/1 and scl2 expression relative to M3 MGAS315 are shown for each strain. Data is based on qRT-PCR analysis of RNA isolated during exponential growth phase and expression is normalized to the expression of tufA gene. +SE, positive standard error; -SE, negative standard error. Significance is calculated based on three independent experiments, each performed in triplicate wells. ${ }^{*} \mathrm{P}<0.05$, ${ }^{* *} \mathrm{P}<0.01,{ }^{* * *} \mathrm{P}<0.001$; student's $t$-test. 


\title{
SUPPLEMENTARY MATERIAL FOR CHAPTER 3
}

\section{NATURAL VARIANT OF COLLAGEN-LIKE PROTEIN A IN SEROTYPE M3 GROUP A STREPTOCOCCUS INCREASES ADHERENCE AND DECREASES INVASIVE POTENTIAL}

\author{
Anthony R. Flores, Brittany E. Jewell, Erika M. Versalovic, Randall J. Olsen, Beth A. \\ Bachert, Slawomir Lukomski and James M. Musser \\ Published in Infection and Immunity 2015 83: 1122-1129
}


Table S1. Strains and plasmids used in this study

\begin{tabular}{|c|c|c|}
\hline Strain & Description & Source \\
\hline MGAS315 & Serotype M3 invasive strain & (1) \\
\hline MGAS10870 & Serotype $M 3$ invasive strain & (2) \\
\hline MGAS23440 & Serotype M3 invasive strain & (3) \\
\hline MGAS10870sclACarrier & Isoallelic mutant with $s c / A^{\text {Carrier }}$ & This study \\
\hline MGAS10870 $\triangle S C I A$ & Isogenic deletion mutant lacking $s c / A$ & This study \\
\hline $\begin{array}{l}\text { MGAS10870 } 1 \text { sclA:: } \\
\text { pDCsclAInvasive }\end{array}$ & $\begin{array}{l}\text { Isogenic deletion mutant complemented in } \\
\text { trans with pDCsclA } A^{\text {Invasive }}\end{array}$ & This study \\
\hline $\begin{array}{l}\text { MGAS10870 } 1 \text { sclA:: } \\
\text { pDCsclA Carrier }\end{array}$ & $\begin{array}{l}\text { Isogenic deletion mutant complemented in } \\
\text { trans with pDCsclA } A_{\text {Carrier }}\end{array}$ & This study \\
\hline Plasmid & Description & Source \\
\hline pDC123 & $\begin{array}{l}\text { E. coli/S. pyogenes shuttle vector used } \\
\text { for trans-complementation and } \\
\text { expression }\end{array}$ & $(4)$ \\
\hline pJL1055 & $\begin{array}{l}\text { E. colilS. pyogenes shuttle vector used } \\
\text { for allelic exchange in GAS }\end{array}$ & \\
\hline pCR2.1 & E. coli cloning vector & Invitrogen \\
\hline $\mathrm{pET15b}$ & E. coli expression vector & Novagen \\
\hline pDCsclA $A^{\text {Invasive }}$ & $\begin{array}{l}\text { pDC123 shuttle vector with } s c / A^{\text {Invasive }} \text { allele } \\
\text { and native promoter from } \\
\text { MGAS10870 }\end{array}$ & This study \\
\hline pDCsclA Carrier & $\begin{array}{l}\text { pDC123 shuttle vector with } s c / A^{\text {Carrier }} \\
\text { allele and native promoter from carrier } \\
\text { strain MGAS23440 }\end{array}$ & This study \\
\hline pJSF41 & pJL1055 with sclA $A^{\text {Carrier from MGAS23431 }}$ & This study \\
\hline pJSF66 & $\begin{array}{l}\text { pET15b containing } s c / A^{\text {Invasive }} \text { from } \\
\text { MGAS10870 used for overexpression and } \\
\text { subsequent antibody generation }\end{array}$ & This study \\
\hline pJRS525 & $\begin{array}{l}\text { E. coli/ S. pyogenes shuttle vector used } \\
\text { for cloning and repair of } s c / A^{\text {Invasive }} \text { allele } \\
\text { from MGAS315 to generate } S c / A^{\mathrm{M} 3-\mathrm{FL}}\end{array}$ & (5) \\
\hline pSL501 & pJRS525 with sclAlnvasive from MGAS315 & This study \\
\hline pSL502 & $\begin{array}{l}\text { pSL501 with repaired allele } s c / A^{\mathrm{M} 3-\mathrm{FL}} \\
\text { generated by PCR mutagenesis }\end{array}$ & This study \\
\hline pASK-IBA2 & $\begin{array}{l}\text { E. coli expression vector used for generation } \\
\text { of } r S c l A^{M 3}-\mathrm{FL}\end{array}$ & IBA, Göttingen \\
\hline pSL503 & pASK-IBA2 with $s c / A^{\mathrm{M} 3-\mathrm{FL}}$ & This study \\
\hline
\end{tabular}


Table S2. Primers used in this study

\begin{tabular}{|c|c|c|}
\hline Primer & Use & Sequence $\left(5^{\prime}-3^{\prime}\right)$ \\
\hline $2902 \mathrm{~F}$ & $\begin{array}{l}3 \text { ' end of } s c / A \text { used to amplify } s c l A \text { from invasive } \\
\text { and carrier strains }\end{array}$ & $\begin{array}{l}\text { ATTTTTGGCATTTCGTT } \\
\text { CCT }\end{array}$ \\
\hline $2487 R$ & $\begin{array}{l}\text { end of } s c / A \text { upstream of promoter used to } \\
\text { pplify } s c / A \text { from invasive and carrier strains }\end{array}$ & $\begin{array}{l}\text { CGAATTTTCCAAGATT } \\
\text { GACGA }\end{array}$ \\
\hline $2901 \mathrm{~F}$ & $\begin{array}{l}\text { ownstream of } s c / A \text { used to generate in-frame } \\
\text { elic replacement of } s c / A \text { with aad } 9\end{array}$ & $\begin{array}{l}\text { GAAATGCGCTCTTGTT } \\
\text { TGTC }\end{array}$ \\
\hline MSP166 & $\begin{array}{l}\text { primer used for PCR amplification and } \\
\text { ng of } s c / A \text { in invasive GAS }\end{array}$ & $\begin{array}{l}\text { TCTTTTGGGATCTCTC } \\
\text { AGGC }\end{array}$ \\
\hline MSP167 & g of $s c / A$ in invasive & $\begin{array}{l}\text { TAAATAATCAGGTCTA } \\
\text { GCTACC }\end{array}$ \\
\hline MSP183 & $\begin{array}{l}\text { flanki } \\
\text { aad9 }\end{array}$ & $\begin{array}{l}\text { AAATAACAGA } \\
\text { AAATTATAAC } \\
\text { AAAAACAACTA } \\
\text { TAAATTGAG }\end{array}$ \\
\hline 81 & ains 18- & $\begin{array}{l}\text { GAGGATT } \\
\text { CTTTGCG } \\
\text { ITTTAATC } \\
\text { AATAG }\end{array}$ \\
\hline MSP182 & ains 18- & $\begin{array}{l}\text { ACAAC } \\
\text { ATCAAA } \\
\text { TGTTAT } \\
\text { AC }\end{array}$ \\
\hline MSP184 & $\begin{array}{l}\text { Used with primer } 0488 \mathrm{R} \text { to generate } 5 \text { '-flanking } \\
\text { region overlapping with aad9 for in-frame } \\
\text { deletion of } s c / A\end{array}$ & $\begin{array}{l}\text { ATTATAAC } \\
\text { GCTTTGA } \\
\text { ATGTTGT } \\
\text { : }\end{array}$ \\
\hline 188R & $\begin{array}{l}\text { of } s c l A \text { us } \\
\text { lacement of }\end{array}$ & CAGGCTTCTG \\
\hline MSP204 & ard TaqMan $\mathrm{p}$ & AGGTGCAACA \\
\hline MSP205 & se TaqMan $r$ & GCTGGACCTT \\
\hline MSP206 & $\begin{array}{l}\text { Probe for } S C l A \text { Tac } \\
\text { Contains 5'- FAM and }\end{array}$ & GCCTACTGGAC \\
\hline MSP263 & $\begin{array}{l}5 \text { 'end of } s c / A \text { and excluded signal sequence } \\
\text { used for cloning of } s c / A \text { for expression; contains } \\
\text { Ndel site }\end{array}$ & $\begin{array}{l}\text { GCAGCGTTAAGCATAT } \\
\text { GACTCCTATG }\end{array}$ \\
\hline N & $\begin{array}{l}\text { 3'-end of } s c / A \text { used for cloning of } s c / A \text { for } \\
\text { expression; contains Xhol site }\end{array}$ & $\begin{array}{l}\text { ICTCCTCGAGTT } \\
\text { ICGTGGTCC }\end{array}$ \\
\hline MSP275 & $\begin{array}{l}\text { 5'-end - including native promoter - of } s c / A \text { and } \\
\text { used with MSP277 to amplify for cloning into } \\
\text { pDC123; contains EcoRV site }\end{array}$ & $\begin{array}{l}\text { TATCCAACGATATCAG } \\
\text { AAGGTACAAG }\end{array}$ \\
\hline
\end{tabular}




\begin{tabular}{|c|c|c|}
\hline MSP277 & $\begin{array}{l}\text { 3'-end of sclA used with MSP275 to amplify for } \\
\text { cloning into pDC123; contains Hindlll site }\end{array}$ & $\begin{array}{l}\text { ACCTTGAAAAGCTTGC } \\
\text { TCACCGCG }\end{array}$ \\
\hline $232 U p$ & $\begin{array}{l}\text { 5' end of } s c / A \text { used for amplification of MGAS315 } \\
s c / A^{\text {Invasive }} \text { allele }\end{array}$ & $\begin{array}{l}\text { CTCCACAAAAGAGTGA } \\
\text { TCAGTC }\end{array}$ \\
\hline ME7 & $\begin{array}{l}\text { Used with } 232 U p \text { to generate } s c / A^{\text {lnvasive }} \text { from } \\
\text { MGAS315 for cloning into pJRS525 }\end{array}$ & $\begin{array}{l}\text { TCAGTGAATTCTCTTT } \\
\text { AGAGGATTAG }\end{array}$ \\
\hline $\begin{array}{l}\text { pJRS525 } \\
\text { F1 }\end{array}$ & $\begin{array}{l}\text { Used with ME6 to generate repaired full-length } \\
\text { sclA fragment from MGAS315 (scl/A3-FL) }\end{array}$ & $\begin{array}{l}\text { GGGTTTTCCCAGTCAC } \\
\text { G }\end{array}$ \\
\hline ME6 & $\begin{array}{l}\text { d for PCR mutagenesis to } \\
\text { n } s c / A^{\text {Invasive }}\end{array}$ & $\begin{array}{l}\text { TCCAGCAGGACCTCG } \\
\text { AGGTGAACGC }\end{array}$ \\
\hline M3VF & $\begin{array}{l}\text { 5' end of sclA for cloning } s c / A^{\mathrm{M} 3-\mathrm{FL}} \text { into } \mathrm{pASK}- \\
\text { IBA2 expression vector }\end{array}$ & $\begin{array}{l}\text { GAGATGGCCGAGACT } \\
\text { CCTATGACATCAAAGG }\end{array}$ \\
\hline $\begin{array}{l}\text { Scl1.28W } \\
\text { MR }\end{array}$ & $\begin{array}{l}\text { 3' end of sclA containing Streptagll } \\
\text { sequence for cloning sclAM3-FL into pASK-IBA2 } \\
\text { expression vector }\end{array}$ & $\begin{array}{l}\text { CTTATTATTTT } \\
\text { TGCGGGTG } \\
\text { AGGTTTTTCT } \\
\text { GGAGTTACC }\end{array}$ \\
\hline
\end{tabular}




\section{References}

1. Musser, J. M., Hauser, A. R., Kim, M. H., Schlievert, P. M., Nelson, K., and Selander, R. K. (1991) Streptococcus pyogenes causing toxic-shock-like syndrome and other invasive diseases: clonal diversity and pyrogenic exotoxin expression. Proc Natl Acad Sci U S A 88, 2668-2672

2. Carroll, R. K., Shelburne, S. A., 3rd, Olsen, R. J., Suber, B., Sahasrabhojane, P., Kumaraswami, M., Beres, S. B., Shea, P. R., et al. (2011) Naturally occurring single amino acid replacements in a regulatory protein alter streptococcal gene expression and virulence in mice. J Clin Invest 121, 1956-1968

3. Flores, A. R., Jewell, B. E., Olsen, R. J., Shelburne, S. A., 3rd, Fittipaldi, N., Beres, S. B., and Musser, J. M. (2014) Asymptomatic carriage of group A streptococcus is associated with elimination of capsule production. Infect Immun 82, 3958-3967

4. Chaffin, D. O., and Rubens, C. E. (1998) Blue/white screening of recombinant plasmids in Gram-positive bacteria by interruption of alkaline phosphatase gene (phoZ) expression. Gene 219, 91-99

5. Mclver, K. S., and Scott, J. R. (1997) Role of mga in growth phase regulation of virulence genes of the group A Streptococcus. J Bacteriol 179, 5178-5187 
SUPPLEMENTARY MATERIAL FOR CHAPTER 6

REVERSIBLE PHOSPHOLIPID NANOGELS FOR DEOXYRIBONUCLEIC ACID FRAGMENT SIZE DETERMINATIONS UP TO 1,500 BASE PAIRS AND INTEGRATED SAMPLE STACKING

Brandon C. Durney, Beth A. Bachert, Hillary S. Sloane, Slawomir Lukomski, James P.

Landers, and Lisa A. Holland

Published in Analytica Chimica Acta 2015 880: 136-144 


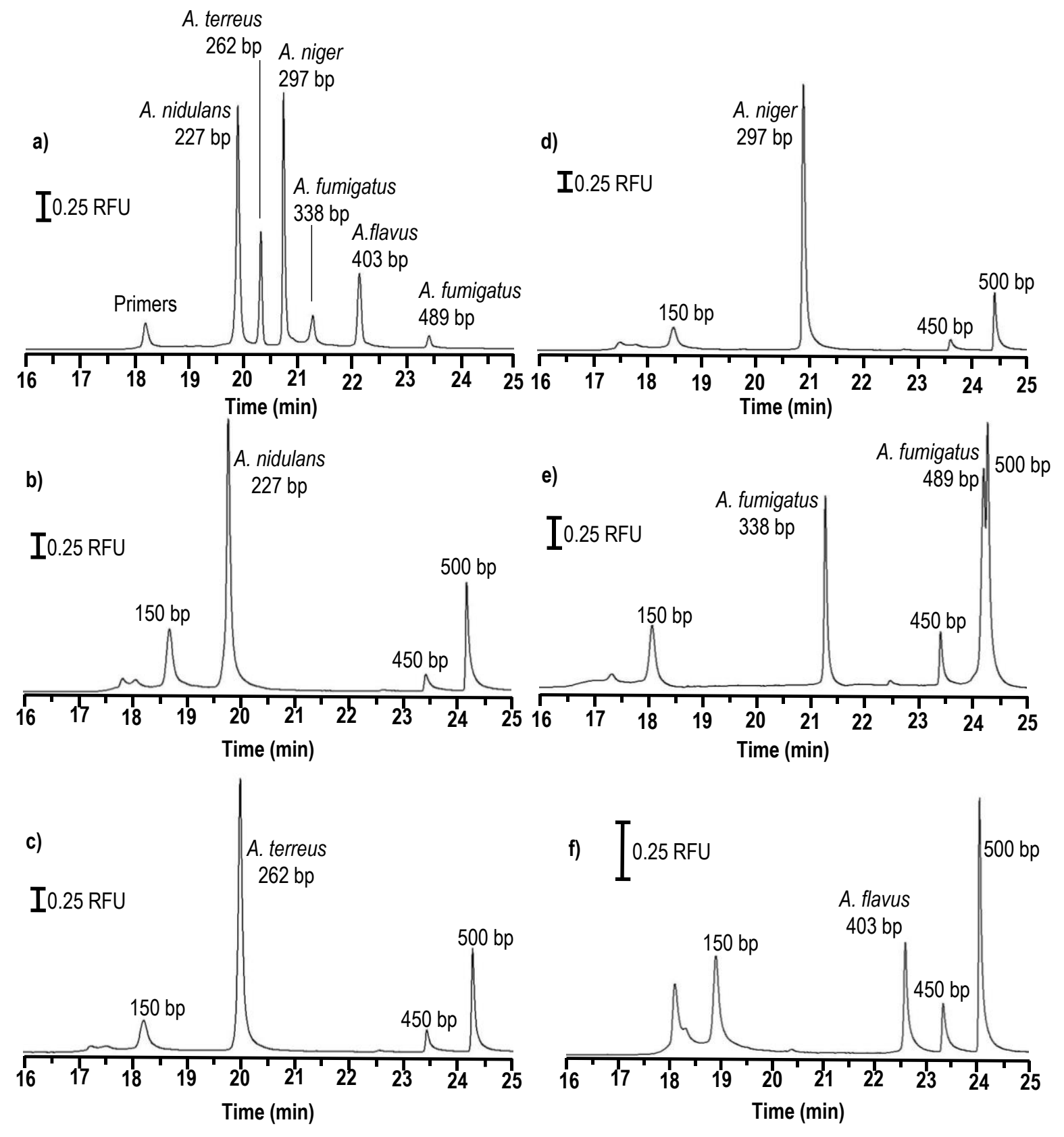

Figure S1. Separation of (a) 5 amplicons unique to different species of Aspergillus as well as electropherograms that contain internal standards of 150,450 , and 500 base pairs as well as a single amplicon for (b) A. nidulans (227 bp); (c) A. terreus (262 bp); (d) A. niger (297 bp); (e) A. fumigatus (338 and 489 bp); (f) A. flavus (403 bp). 
Table S1. Effect of SYBR Green 1 concentration on migration time of $650 \mathrm{bp} \mathrm{DNA}^{\mathrm{a}}$

\begin{tabular}{|c|c|c|c|}
\hline Concentration & Injection size & Migration time in minutes & $\begin{array}{l}\text { Chromatographic }{ }^{2} \\
\text { resolution }(\mathrm{CV})\end{array}$ \\
\hline $2 \mu \mathrm{M}$ & $5 \mathrm{psi} 7 \mathrm{sec}$ & 27.9 & (4) \\
\hline $20 \mu \mathrm{M}$ & $5 \mathrm{psi} 7 \mathrm{sec}$ & 27.8 & (2) \\
\hline $100 \mu \mathrm{M}$ & $5 \mathrm{psi} 7 \mathrm{sec}$ & 26.1 & (5) \\
\hline \multicolumn{4}{|c|}{$\begin{array}{l}\mu[\mathrm{DMPC}] /[\mathrm{DHPC}]=2.5,2.5 \% \text { phospholipid. Separations }(n=5) \text { are accomplished in a } 25 \\
\mu \mathrm{m} \text { id capillary with a total length of } 40 \mathrm{~cm} \text { capillary and effective length of } 30.2 \mathrm{~cm} \text {, with } \\
\text { Eapplied }=100 \mathrm{~V} / \mathrm{cm} \text {. The DNA base ladder ( } 1 \mathrm{~Kb}+\text { DNA ladder, Life Technologies) is } \\
\text { detected with LIF at } 520 \mathrm{~nm} \text { using SYBR green } 1 \text { nucleic acid stain at different } \\
\text { concentrations positioned before the detection window. }\end{array}$} \\
\hline
\end{tabular}


Table S2. Effect of phospholipid concentration on resolution ${ }^{1}$

\begin{tabular}{|c|c|c|c|c|c|}
\hline \multirow[b]{2}{*}{$\begin{array}{l}\text { Hydration } \\
(\%)\end{array}$} & \multirow[b]{2}{*}{$\begin{array}{l}\text { Temp } \\
\left({ }^{\circ} \mathrm{C}\right)\end{array}$} & \multicolumn{2}{|c|}{$\begin{array}{c}\text { Resolution } 450 / 475 \text { base pair } \\
\text { DNA }^{2}\end{array}$} & \multicolumn{2}{|c|}{$\begin{array}{c}\text { Resolution 900/950 base pair } \\
\text { DNA }^{3}\end{array}$} \\
\hline & & $\begin{array}{l}\text { Chromatographic }{ }^{2} \\
\text { resolution }(\mathrm{CV})\end{array}$ & $\begin{array}{l}\text { In base } \\
\text { pairs }^{3}\end{array}$ & $\begin{array}{l}\text { Chromatographic }{ }^{2} \\
\text { resolution }(\mathrm{CV})\end{array}$ & $\begin{array}{l}\text { In base } \\
\text { pairs }^{3}\end{array}$ \\
\hline 2.5 & 23 & $5.0(6)$ & 5 & $6.4(7)$ & 8 \\
\hline 5.0 & 25 & $5.1(10)$ & 5 & $4.5(10)$ & 11 \\
\hline 7.5 & 30 & $5.4(7)$ & 5 & $4.2(20)$ & 12 \\
\hline 10.0 & 30 & $6.2(7)$ & 4 & $3.6(20)$ & 14 \\
\hline
\end{tabular}


Table S3. Effect of [DMPC]/[DHPC] on resolution ${ }^{1}$

Resolution $450 / 475$ base pair

$\mathrm{DNA}^{2}$

Hydration Temp

(\%)

2.5

3.0

4.0

5.0 $\left({ }^{\circ} \mathrm{C}\right)$

23

21

21

21

Chromatographic ${ }^{2}$
resolution $(\mathrm{CV})$

$5.0(6)$

$5.1(8)$

$5.5(10)$

$1.4(20)$

In base
pairs ${ }^{3}$

5

5

5
Resolution 900/950 base pair DNA $^{3}$

Chromatographic $^{2}$ In base
resolution $(\mathrm{CV})$

$6.4(7)$

$5.3(10)$

$5.5(10)$

$2.6(20)$ pairs ${ }^{3}$

8

9

9

36

${ }^{1}$ Separations $(n=5)$ are achieved at the specified temperature with [DMPC]/[DHPC] $=2.5$ in a $40 \mathrm{~cm}$ long, $25 \mu \mathrm{m}$ id capillary. The effective length is $30.2 \mathrm{~cm}$ and $E_{a p p}=100 \mathrm{~V} / \mathrm{cm}$. ${ }^{2}$ Resolution is calculated as $0.589\left(\Delta \mathrm{t} / \mathrm{w}_{1 / 2 \mathrm{av}}\right)$, where $\Delta \mathrm{t}$ is the difference in peak migration times and $w_{1 / 2 a v}$ is the average peak width at half height

${ }^{3}$ Resolution in base pairs is calculated as the difference in base pairs/resolution. 
Table S4. Effect of temperature on resolution ${ }^{1}$

\begin{tabular}{|c|c|c|c|c|}
\hline \multirow[b]{2}{*}{$\begin{array}{l}\text { Temp } \\
\left({ }^{\circ} \mathrm{C}\right)\end{array}$} & \multicolumn{2}{|c|}{$\begin{array}{c}\text { Resolution } 450 / 475 \text { base pair } \\
\text { DNA }^{2}\end{array}$} & \multicolumn{2}{|c|}{ Resolution $900 / 950$ base pair DNA ${ }^{3}$} \\
\hline & $\begin{array}{l}\text { Chromatographic }{ }^{2} \\
\text { resolution }(\mathrm{CV})\end{array}$ & pairs $^{3}$ base & $\begin{array}{l}\text { Chromatographic }^{2} \\
\text { resolution }(\mathrm{CV})\end{array}$ & $\begin{array}{l}\text { In base } \\
\text { pairs }^{3}\end{array}$ \\
\hline 19 & $1.2(20)$ & 22 & $1.8(20)$ & 27 \\
\hline 20 & $3.2(10)$ & 8 & $5.3(10)$ & 9 \\
\hline 21 & $4.5(9)$ & 6 & $5.7(10)$ & 9 \\
\hline 22 & $4.5(8)$ & 6 & $5.8(10)$ & 9 \\
\hline 23 & $5.0(6)$ & 5 & $6.4(7)$ & 8 \\
\hline 24 & $2.7(10)$ & 9 & $4.2(20)$ & 12 \\
\hline 25 & $2.5(10)$ & 10 & $3.3(20)$ & 15 \\
\hline $\begin{array}{l}{ }^{1} \text { Sepa } \\
\text { in a } 40 \\
{ }^{2} \text { Reso } \\
\text { times } \\
{ }^{3} \text { Reso }\end{array}$ & $\begin{array}{l}\text { רs }(n=5) \text { are achi } \\
\text { long, } 25 \mu \mathrm{m} \text { id car } \\
\text { is calculated as }\end{array}$ & $\begin{array}{l}\text { at the specif } \\
\text { The effecti } \\
\left(\Delta t / w_{1 / 2 a v}\right), h \\
\text { k width at he } \\
\text { ted as the } d\end{array}$ & $\begin{array}{l}\text { mperature with [D } \\
\text { igth is } 30.2 \mathrm{~cm} \text { an } \\
\Delta \mathrm{t} \text { is the differenc } \\
\text { ght } \\
\text { nce in base pairs } / \mathrm{r}\end{array}$ & $\begin{array}{l}\text { DHPC] }=2.5 \\
=100 \mathrm{~V} / \mathrm{cm} . \\
\text { ak migration }\end{array}$ \\
\hline
\end{tabular}


SUPPLEMENTARY MATERIAL FOR CHAPTER 7

\begin{abstract}
A UNIQUE SET OF THE BURKHOLDERIA COLLAGEN-LIKE PROTEINS PROVIDES INSIGHT INTO PATHOGENESIS, GENOME EVOLUTION AND NICHE ADAPTATION, AND INFECTION DETECTION

Beth A. Bachert, Soo J. Choi, Anna K. Snyder, Rita V.M. Rio, Brandon C. Durney, Lisa A. Holland, Kei Amemiya, Susan L. Welkos, Joel A. Bozue, Christopher K. Cote, Rita Berisio, and Slawomir Lukomski

Published in PLOS One 2015 10(9)
\end{abstract}



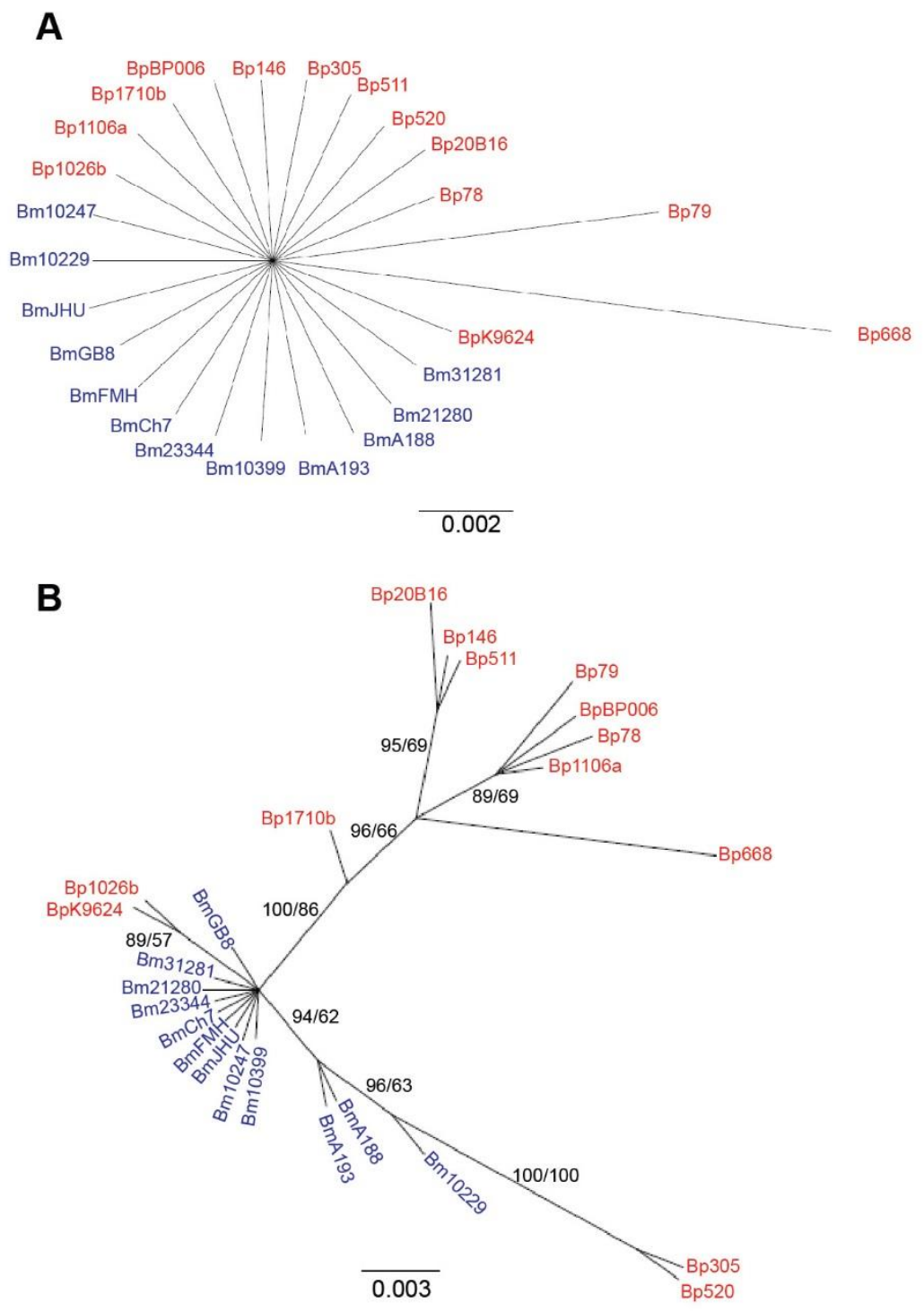

Figure S1. Phylogenetic analyses of bucl1 in B. pseudomallei and B. mallei strains. Nucleotide sequences encoding (A) the noncollagenous domain and (B) entire gene of bucl1 alleles were used. Support values for each branch are shown as posterior probability from Bayesian analysis and bootstrap values from maximum parsimony analysis, respectively (PP/MP). Scale bar is representative of evolutionary distance in substitutions per nucleotide. 


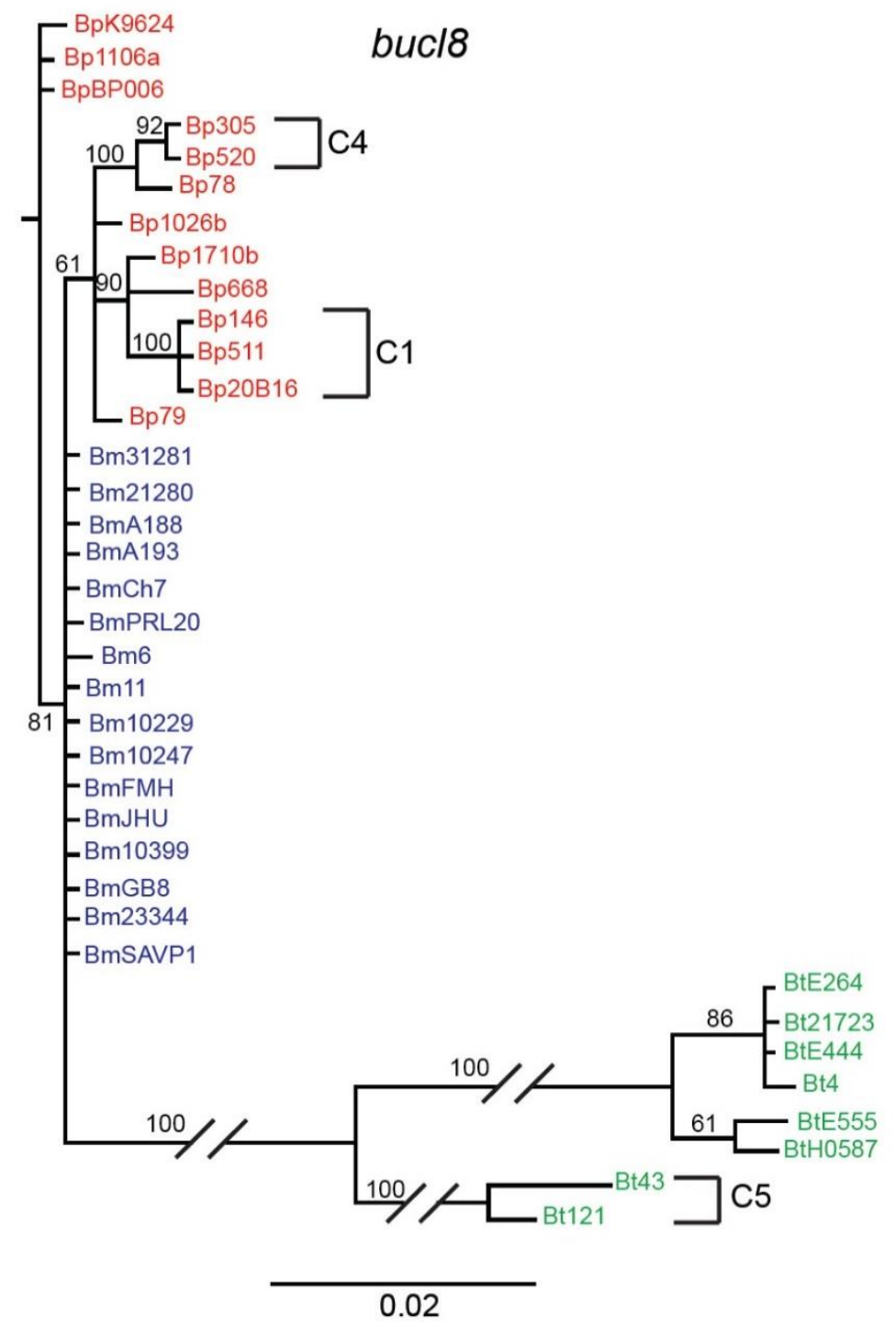

Figure S2. Phylogenetic analysis of bucl8 among Burkholderia strains. Bayesian analysis was performed on nucleotide sequences of bucl8 non-collagenous regions of a set of Burkholderia strains described in Table 3. Support values for each branch are shown as posterior probability from Bayesian analysis. Several clusters of strains, C1, C4, and C5, corresponding to those observed in the concatenated analysis were also observed. Scale bar is representative of evolutionary distance in substitutions per nucleotide. 
A

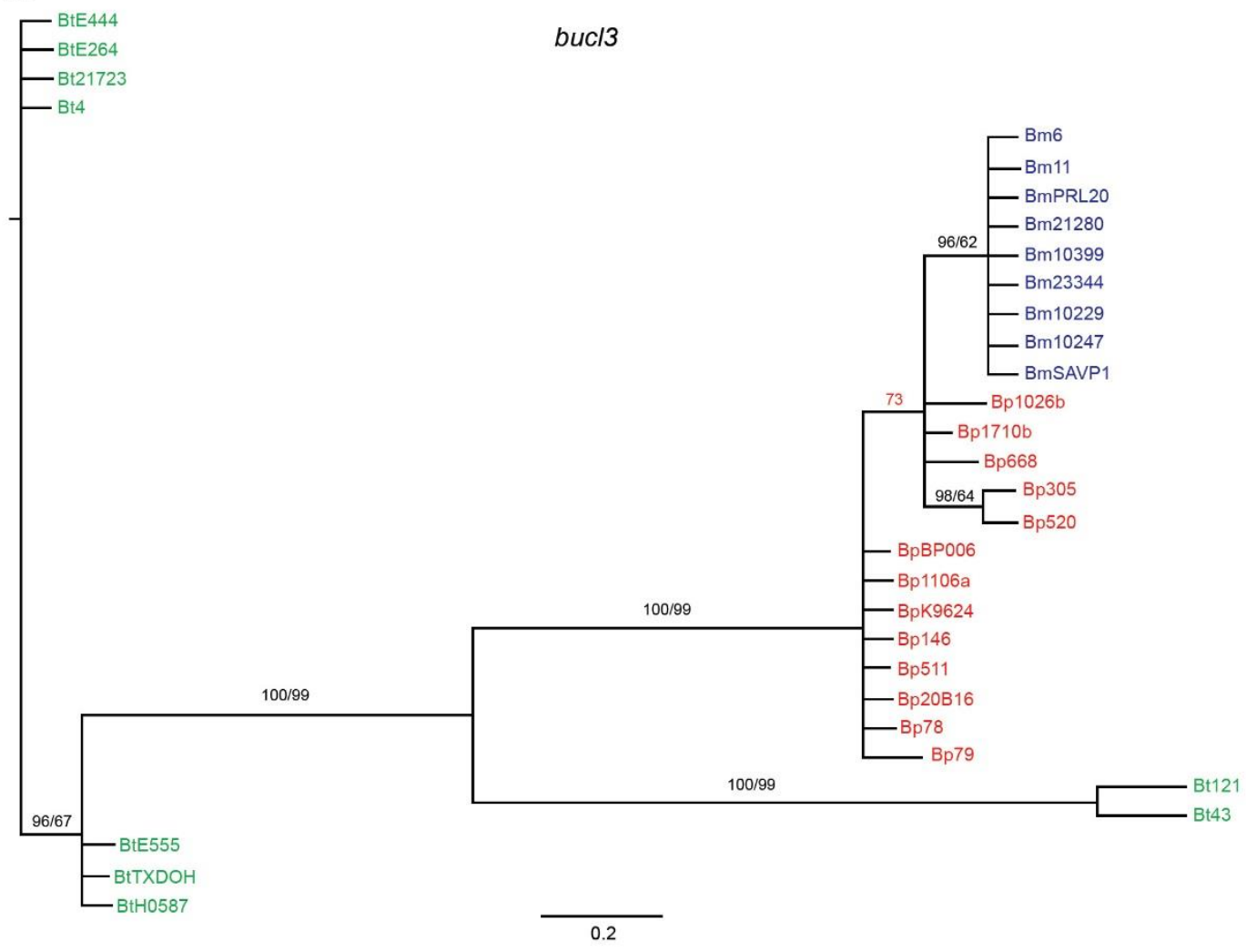

B

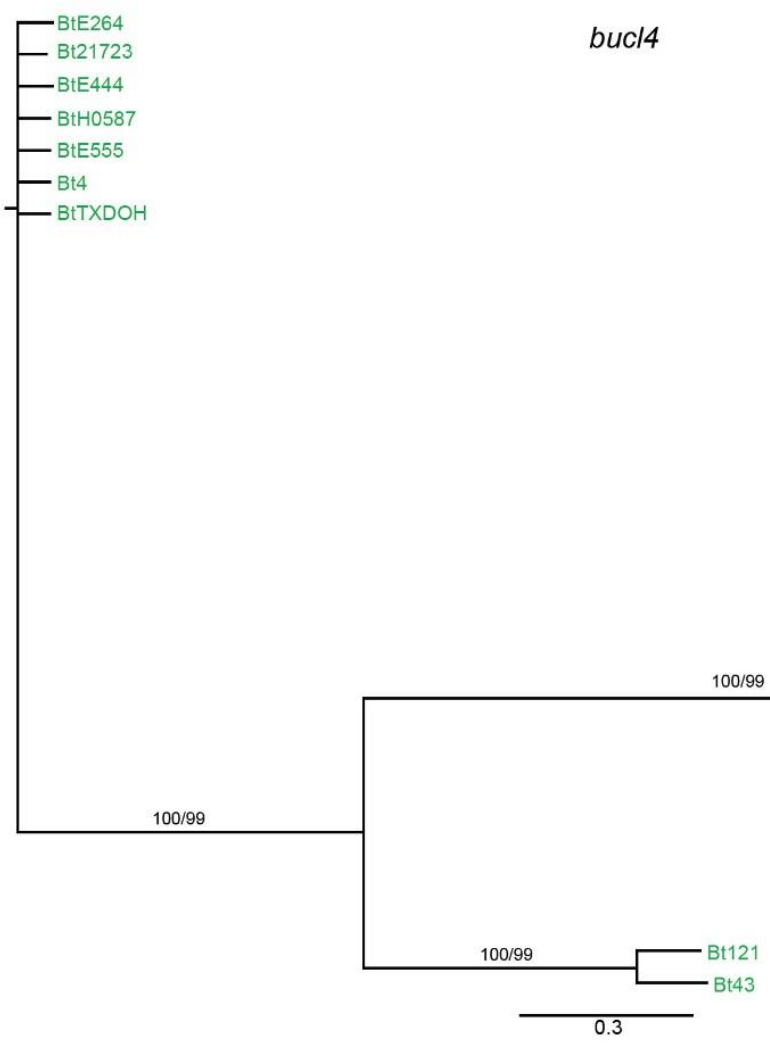


Figure S3. Phylogenetic analysis of Bucl3 and Bucl4 amino acid sequences among Burkholderia strains. Bayesian analysis was performed on amino acid sequences of (A) Bucl3 and (B) Bucl4 non-collagenous regions of a set of Burkholderia strains described in Table 3. Support values for each branch are shown as posterior probability from Bayesian analysis and bootstrap values from maximum parsimony analysis, respectively (PP/MP). Posterior probability value, which was not supported by maximum parsimony analysis is shown in red. Scale bar is representative of evolutionary distance in substitutions per nucleotide. 

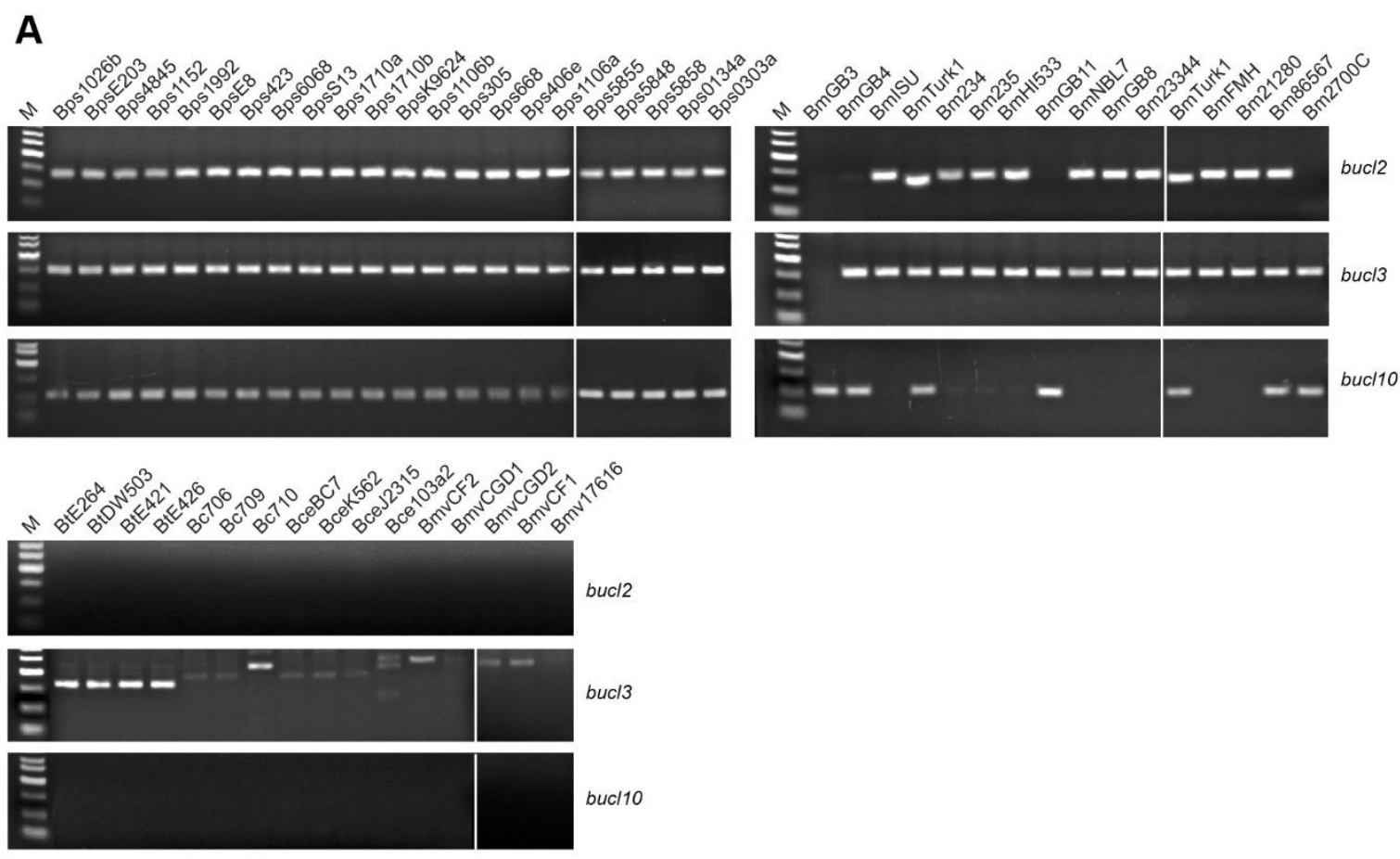

\section{B}

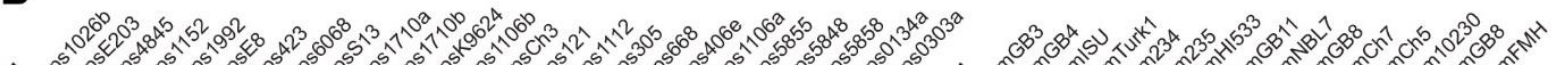

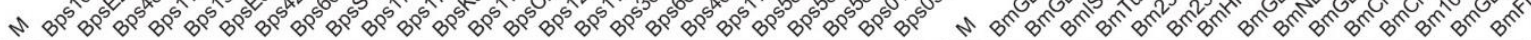
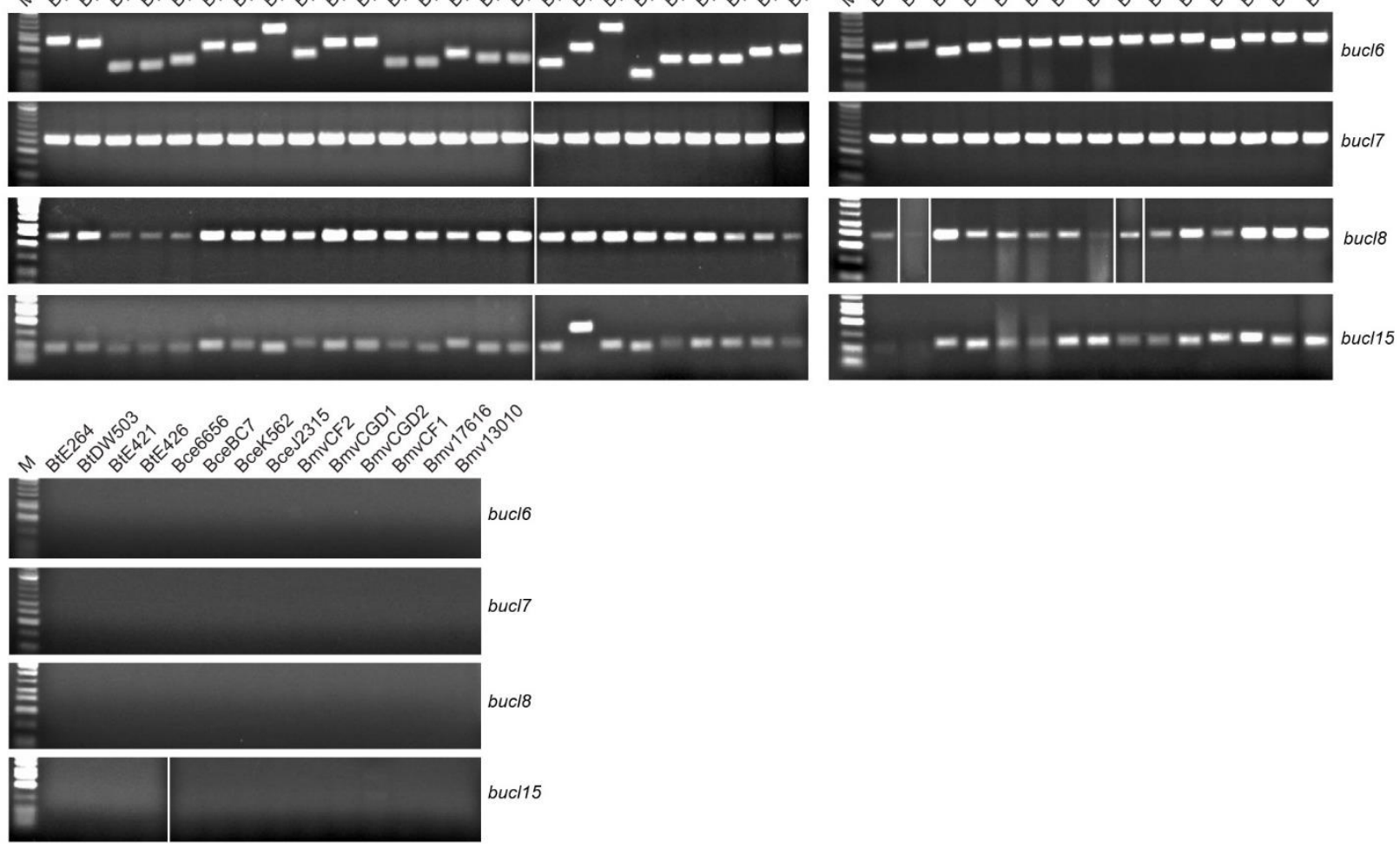
Figure S4. Distribution of bucl genes among Burkholderia spp. select agents by PCR. Presence of (A) bucl genes 2, 3, and 10 and (B) bucl genes 6, 7, 8, and 15, was assessed by PCR on a collection of genomic DNA from B. pseudomallei and B. mallei select agents (top panels), as well as in control strains of B. thailandensis, B. cepacia, B. cenocepacia, and B. multivorans (bottom panels). Amplicon sizes based on Bp K96243: In A) bucl2, 133 bp; buc/3, 166 bp; and bucl10, 109 bp; In B) bucl6, 115 bp; bucl7, 264 bp; bucl8, 243 bp; and bucl15, 95 bp.M, 50-bp DNA ladder. PCR data shown in panels A and B for 25 Bp strains come from two merged gel images. 


\section{GENERAL REFERENCES}

1. Berisio, R., Vitagliano, L., Mazzarella, L., and Zagari, A. (2002) Crystal structure

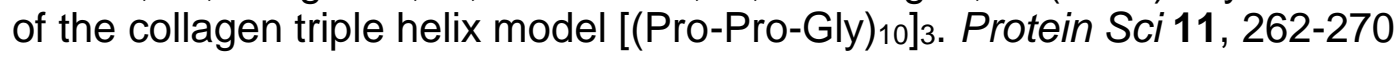

2. Ramachandran, G. N. (1988) Stereochemistry of collagen. Int J Pept Protein Res 31, 1-16

3. Bella, J. (2016) Collagen structure: new tricks from a very old dog. Biochem J 473, 1001-1025

4. Shoulders, M. D., and Raines, R. T. (2009) Collagen structure and stability. Annu Rev Biochem 78, 929-958

5. Brodsky, B., and Persikov, A. V. (2005) Molecular structure of the collagen triple helix. Adv Protein Chem 70, 301-339

6. Exposito, J. Y., Cluzel, C., Garrone, R., and Lethias, C. (2002) Evolution of collagens. Anat Rec 268, 302-316

7. Engel, J. (1997) Versatile collagens in invertebrates. Science 277, 1785-1786

8. Suhre, M. H., Gertz, M., Steegborn, C., and Scheibel, T. (2014) Structural and functional features of a collagen-binding matrix protein from the mussel byssus. Nat Commun 5, 3392

9. Kramer, J. M., Cox, G. N., and Hirsh, D. (1982) Comparisons of the complete sequences of two collagen genes from Caenorhabditis elegans. Cell 30, 599-606

10. Lukomski, S., Nakashima, K., Abdi, I., Cipriano, V. J., Ireland, R. M., Reid, S. D., Adams, G. G., and Musser, J. M. (2000) Identification and characterization of the scl gene encoding a group A Streptococcus extracellular protein virulence factor with similarity to human collagen. Infect Immun 68, 6542-6553

11. Sylvestre, P., Couture-Tosi, E., and Mock, M. (2002) A collagen-like surface glycoprotein is a structural component of the Bacillus anthracis exosporium. Mol Microbiol 45, 169-178

12. Pizarro-Guajardo, M., Olguin-Araneda, V., Barra-Carrasco, J., Brito-Silva, C., Sarker, M. R., and Paredes-Sabja, D. (2014) Characterization of the collagen-like exosporium protein, BclA1, of Clostridium difficile spores. Anaerobe 25, 18-30

13. Paterson, G. K., Nieminen, L., Jefferies, J. M. C., and Mitchell, T. J. (2008) PclA, a pneumococcal collagen-like protein with selected strain distribution, contributes to adherence and invasion of host cells. FEMS Microbiol Lett 285, 170-176

14. Duncan, C., Prashar, A., So, J., Tang, P., Low, D. E., Terebiznik, M., and Guyard, C. (2011) Lcl of Legionella pneumophila is an immunogenic GAG binding adhesin that promotes interactions with lung epithelial cells and plays a crucial role in biofilm formation. Infect Immun 79, 2168-2181

15. Bachert, B. A., Choi, S. J., Snyder, A. K., Rio, R. V. M., Durney, B. C., Holland, L. A., Amemiya, K., Welkos, S. L., et al. (2015) A unique set of the Burkholderia collagen-like proteins provides insight into pathogenesis, genome evolution and niche adaptation, and infection detection. PLOS ONE 10, e0137578

16. Chopra, R. K., and Ananthanarayanan, V. S. (1982) Conformational implications of enzymatic proline hydroxylation in collagen. Proc Natl Acad Sci U S A 79, 7180-7184 
17. Vitagliano, L., Berisio, R., Mazzarella, L., and Zagari, A. (2001) Structural bases of collagen stabilization induced by proline hydroxylation. Biopolymers 58, 459464

18. Berisio, R., and Vitagliano, L. (2012) Polyproline and triple helix motifs in hostpathogen recognition. Curr Protein Pept Sci 13, 855-865

19. Xu, C., Yu, Z., Inouye, M., Brodsky, B., and Mirochnitchenko, O. (2010) Expanding the family of collagen proteins: recombinant bacterial collagens of varying composition form triple-helices of similar stability. Biomacromolecules 11, 348-356

20. Xu, Y., Keene, D. R., Bujnicki, J. M., Hook, M., and Lukomski, S. (2002) Streptococcal Scl1 and Scl2 proteins form collagen-like triple helices. J Biol Chem 277, 27312-27318

21. Han, R., Zwiefka, A., Caswell, C. C., Xu, Y., Keene, D. R., Lukomska, E., Zhao, Z., Hook, M., et al. (2006) Assessment of prokaryotic collagen-like sequences derived from streptococcal Scl1 and Scl2 proteins as a source of recombinant GXY polymers. Appl Microbiol Biotechnol 72, 109-115

22. Chan, V. C., Ramshaw, J. A., Kirkpatrick, A., Beck, K., and Brodsky, B. (1997) Positional preferences of ionizable residues in Gly-X-Y triplets of the collagen triple-helix. J Biol Chem 272, 31441-31446

23. Leikina, E., Mertts, M. V., Kuznetsova, N., and Leikin, S. (2002) Type I collagen is thermally unstable at body temperature. Proc Natl Acad Sci U S A 99, 13141318

24. Mohs, A., Silva, T., Yoshida, T., Amin, R., Lukomski, S., Inouye, M., and Brodsky, B. (2007) Mechanism of stabilization of a bacterial collagen triple helix in the absence of hydroxyproline. J Biol Chem 282, 29757-29765

25. Rasmussen, M., Jacobsson, M., and Bjorck, L. (2003) Genome-based identification and analysis of collagen-related structural motifs in bacterial and viral proteins. J Biol Chem 278, 32313-32316

26. Doxey, A. C., and McConkey, B. J. (2013) Prediction of molecular mimicry candidates in human pathogenic bacteria. Virulence 4, 453-466

27. Frickey, T., and Lupas, A. (2004) CLANS: a Java application for visualizing protein families based on pairwise similarity. Bioinformatics 20, 3702-3704

28. Lukomski, S., Nakashima, K., Abdi, I., Cipriano, V. J., Shelvin, B. J., Graviss, E. A., and Musser, J. M. (2001) Identification and characterization of a second extracellular collagen-like protein made by group A Streptococcus: control of production at the level of translation. Infect Immun 69, 1729-1738

29. Whatmore, A. M. (2001) Streptococcus pyogenes sclB encodes a putative hypervariable surface protein with a collagen-like repetitive structure.

Microbiology 147, 419-429

30. Rasmussen, M., Eden, A., and Bjorck, L. (2000) SclA, a novel collagen-like surface protein of Streptococcus pyogenes. Infect Immun 68, 6370-6377

31. Rasmussen, M., and Bjorck, L. (2001) Unique regulation of SclB - a novel collagen-like surface protein of Streptococcus pyogenes. Mol Microbiol 40, 14271438 
32. Almengor, A. C., and Mclver, K. S. (2004) Transcriptional activation of sclA by Mga requires a distal binding site in Streptococcus pyogenes. J Bacteriol 186, 7847-7857

33. Almengor, A. C., Walters, M. S., and Mclver, K. S. (2006) Mga is sufficient to activate transcription in vitro of sof-sfbX and other Mga-regulated virulence genes in the group A Streptococcus. J Bacteriol 188, 2038-2047

34. Timoney, J. F. (2004) The pathogenic equine streptococci. Vet Res 35, 397-409

35. Karlstrom, A., Jacobsson, K., Flock, M., Flock, J., and Guss, B. (2004) Identification of a novel collagen-like protein, SclC, in Streptococcus equi using signal sequence phage display. Vet Microbiol 104, 179-188

36. Beres, S., Sesso, R., Pinto, S., Hoe, N., Porcella, S., Deleo, F., and Musser, J. (2008) Genome sequence of a lancefield group C Streptococcus zooepidemicus strain causing epidemic nephritis: new information about an old disease. PLoS ONE 3, e3026

37. Karlstrom, A., Jacobsson, K., and Guss, B. (2006) SclC is a member of a novel family of collagen-like proteins in Streptococcus equi subspecies equi that are recognised by antibodies against SclC. Vet Microbiol 114, 72-81

38. Flock, M., Karlström, Å., Lannergård, J., Guss, B., and Flock, J. I. (2006) Protective effect of vaccination with recombinant proteins from Streptococcus equi subspecies equi in a strangles model in the mouse. Vaccine 24, 4144-4151

39. Waller, A., Flock, M., Smith, K., Robinson, C., Mitchell, Z., Karlström, Å., Lannergård, J., Bergman, R., et al. (2007) Vaccination of horses against strangles using recombinant antigens from Streptococcus equi. Vaccine 25, 3629-3635

40. Guss, B., Flock, M., Frykberg, L., Waller, A. S., Robinson, C., Smith, K. C., and Flock, J. I. (2009) Getting to grips with strangles: an effective multi-component recombinant vaccine for the protection of horses from Streptococcus equi infection. PLoS Pathog 5, e1000584

41. Lannergård, J. (2006) Potentially virulence-related extracellular proteins of Streptococcus equi. Doctoral thesis, Swedish University of Agricultural Sciences

42. Bateman, A., Holden, M. T., and Yeats, C. (2005) The G5 domain: a potential Nacetylglucosamine recognition domain involved in biofilm formation.

Bioinformatics 21, 1301-1303

43. Ruggiero, A., Tizzano, B., Pedone, E., Pedone, C., Wilmanns, M., and Berisio, R. (2009) Crystal structure of the resuscitation-promoting factor (DeltaDUF)RpfB from M. tuberculosis. J Mol Bio/ 385, 153-162

44. Ruggiero, A., Squeglia, F., Romano, M., Vitagliano, L., De Simone, A., and Berisio, R. (2016) Structure and dynamics of the multi-domain resuscitation promoting factor RpfB from Mycobacterium tuberculosis. J Biomol Struct Dyn, 19

45. Christner, M., Franke, G. C., Schommer, N. N., Wendt, U., Wegert, K., Pehle, P., Kroll, G., Schulze, C., et al. (2010) The giant extracellular matrix-binding protein of Staphylococcus epidermidis mediates biofilm accumulation and attachment to fibronectin. Mol Microbiol 75, 187-207

46. Schroeder, K., Jularic, M., Horsburgh, S. M., Hirschhausen, N., Neumann, C., Bertling, A., Schulte, A., Foster, S., et al. (2009) Molecular characterization of a 
novel Staphylococcus aureus surface protein (SasC) involved in cell aggregation and biofilm accumulation. PLOS ONE 4, e7567

47. Imai, S., Ito, Y., Ishida, T., Hirai, T., Ito, I., Yoshimura, K., Maekawa, K., Takakura, S., et al. (2011) Distribution and clonal relationship of cell surface virulence genes among Streptococcus pneumoniae isolates in Japan. Clin Microbiol Infect 17, 1409-1414

48. Yu, Z., Mirochnitchenko, O., Xu, C., Yoshizumi, A., Brodsky, B., and Inouye, M. (2010) Noncollagenous region of the streptococcal collagen-like protein is a trimerization domain that supports refolding of adjacent homologous and heterologous collagenous domains. Protein Sci 19, 775-785

49. Squeglia, F., Bachert, B., Romano, M., Lukomski, S., and Berisio, R. (2013) Crystallization and preliminary X-ray crystallographic analysis of the variable domain of Scl2.3, a streptococcal collagen-like protein from invasive M3-type Streptococcus pyogenes. Acta Crystallogr Sect F Struct Biol Cryst Commun 69, 1023-1025

50. Squeglia, F., Bachert, B., De Simone, A., Lukomski, S., and Berisio, R. (2014) The crystal structure of the streptococcal collagen-like protein 2 globular domain from invasive M3-type group A Streptococcus shows significant similarity to immunomodulatory HIV protein gp41. J Biol Chem 289, 5122-5133

51. Han, R., Caswell, C. C., Lukomska, E., Keene, D. R., Pawlowski, M., Bujnicki, J. M., Kim, J. K., and Lukomski, S. (2006) Binding of the low-density lipoprotein by streptococcal collagen-like protein Scl1 of Streptococcus pyogenes. Mol Microbiol 61, 351-367

52. To, W. S., and Midwood, K. S. (2011) Plasma and cellular fibronectin: distinct and independent functions during tissue repair. Fibrogenesis Tissue Repair 4, 21

53. Ffrench-Constant, C. (1995) Alternative splicing of fibronectin--many different proteins but few different functions. Exp Cell Res 221, 261-271

54. Oliver-Kozup, H., Martin, K. H., Schwegler-Berry, D., Green, B. J., Betts, C., Shinde, A. V., Van De Water, L., and Lukomski, S. (2013) The group A streptococcal collagen-like protein-1, Scl1, mediates biofilm formation by targeting the extra domain A-containing variant of cellular fibronectin expressed in wounded tissue. Mol Microbiol 87, 672-689

55. Ffrench-Constant, C., Van de Water, L., Dvorak, H. F., and Hynes, R. O. (1989) Reappearance of an embryonic pattern of fibronectin splicing during wound healing in the adult rat. J Cell Biol 109, 903-914

56. Jarnagin, W. R., Rockey, D. C., Koteliansky, V. E., Wang, S. S., and Bissell, D. M. (1994) Expression of variant fibronectins in wound healing: cellular source and biological activity of the EIIIA segment in rat hepatic fibrogenesis. $\mathrm{J} \mathrm{Cell} \mathrm{Biol}$ 127, 2037-2048

57. Shinde, A. V., Bystroff, C., Wang, C., Vogelezang, M. G., Vincent, P. A., Hynes, R. O., and Van De Water, L. (2008) Identification of the peptide sequences within the EIIIA (EDA) segment of fibronectin that mediate integrin $\alpha 9 \beta 1$-dependent cellular activities. J Biol Chem 283, 2858-2870

58. Singh, P., Reimer, C. L., Peters, J. H., Stepp, M. A., Hynes, R. O., and Van De Water, L. (2004) The spatial and temporal expression patterns of integrin $\alpha 9 \beta 1$ 
and one of its ligands, the EIIIA segment of fibronectin, in cutaneous wound healing. J Invest Dermatol 123, 1176-1181

59. Yamaguchi, M., Terao, Y., and Kawabata, S. (2013) Pleiotropic virulence factor Streptococcus pyogenes fibronectin-binding proteins. Cell Microbiol 15, 503-511

60. Caswell, C. C., Oliver-Kozup, H., Han, R., Lukomska, E., and Lukomski, S. (2010) Scl1, the multifunctional adhesin of group A Streptococcus, selectively binds cellular fibronectin and laminin, and mediates pathogen internalization by human cells. FEMS Microbiol Lett 303, 61-68

61. Fisher, M., Huang, Y.-S., Li, X., Mclver, K. S., Toukoki, C., and Eichenbaum, Z. (2008) Shr is a broad-spectrum surface receptor that contributes to adherence and virulence in group A Streptococcus. Infect Immun 76, 5006-5015

62. Terao, Y., Kawabata, S., Kunitomo, E., Nakagawa, I., and Hamada, S. (2002) Novel laminin-binding protein of Streptococcus pyogenes, Lbp, is involved in adhesion to epithelial cells. Infect Immun 70, 993-997

63. Linke, C., Caradoc-Davies, T. T., Young, P. G., Proft, T., and Baker, E. N. (2009) The laminin-binding protein Lbp from Streptococcus pyogenes is a zinc-receptor. $\checkmark$ Bacteriol

64. Humtsoe, J. O., Kim, J. K., Xu, Y., Keene, D. R., Hook, M., Lukomski, S., and Wary, K. K. (2005) A streptococcal collagen-like protein interacts with the $\alpha 2 \beta 1$ integrin and induces intracellular signaling. $J$ Biol Chem 280, 13848-13857

65. Caswell, C. C., Barczyk, M., Keene, D. R., Lukomska, E., Gullberg, D. E., and Lukomski, S. (2008) Identification of the first prokaryotic collagen sequence motif that mediates binding to human collagen receptors, integrins $\alpha 2 \beta 1$ and $\alpha 11 \beta 1$. J Biol Chem 283, 36168-36175

66. Kim, J., Xu, Y., Xu, X., Keene, D., Gurusiddappa, S., Liang, X., Wary, K., and Hook, M. (2005) A novel binding site in collagen type III for the integrins, $\alpha 1 \beta 1$ and $\alpha 2 \beta 1$. J Biol Chem 280, 32512-32520

67. Akiyama, H., Morizane, S., Yamasaki, O., Oono, T., and Iwatsuki, K. (2003) Assessment of Streptococcus pyogenes microcolony formation in infected skin by confocal laser scanning microscopy. J Dermatol Sci 32, 193-199

68. Roberts, A. L., Connolly, K. L., Kirse, D. J., Evans, A. K., Poehling, K. A., Peters, T. R., and Reid, S. D. (2012) Detection of group A Streptococcus in tonsils from pediatric patients reveals high rate of asymptomatic streptococcal carriage. BMC Pediatr 12, 3

69. Lembke, C., Podbielski, A., Hidalgo-Grass, C., Jonas, L., Hanski, E., and Kreikemeyer, B. (2006) Characterization of biofilm formation by clinically relevant serotypes of group A streptococci. Appl Environ Microbiol 72, 2864-2875

70. Courtney, H. S., Ofek, I., Penfound, T., Nizet, V., Pence, M. A., Kreikemeyer, B., Podbielski, A., Hasty, D. L., et al. (2009) Relationship between expression of the family of $\mathrm{M}$ proteins and lipoteichoic acid to hydrophobicity and biofilm formation in Streptococcus pyogenes. PLOS ONE 4, e4166

71. Oliver-Kozup, H. A., Elliott, M., Bachert, B. A., Martin, K. H., Reid, S. D., Schwegler-Berry, D. E., Green, B. J., and Lukomski, S. (2011) The streptococcal collagen-like protein-1 (Scl1) is a significant determinant for biofilm formation by group A Streptococcus. BMC Microbiol 11, 262 
72. Bachert, B. A., Choi, S. J., LaSala, P. R., Harper, T. I., McNitt, D. H., Boehm, D. T., Caswell, C. C., Ciborowski, P., et al. (2016) Unique footprint in the scl1.3 locus affects adhesion and biofilm formation of the invasive M3-type group A Streptococcus. Front Cell Infect Microbio/ 6, 90

73. Flores, A. R., Jewell, B. E., Versalovic, E. M., Olsen, R. J., Bachert, B. A., Lukomski, S., and Musser, J. M. (2015) Natural variant of collagen-like protein A in serotype M3 Group A Streptococcus increases adherence and decreases invasive potential. Infect Immun 83, 1122-1129

74. Caswell, C. C., Lukomska, E., Seo, N. S., Hook, M., and Lukomski, S. (2007) Scl1-dependent internalization of group A Streptococcus via direct interactions with the $\alpha 2 \beta 1$ integrin enhances pathogen survival and re-emergence. Mol Microbiol 64, 1319-1331

75. Popova, S. N., Lundgren-Akerlund, E., Wiig, H., and Gullberg, D. (2007) Physiology and pathology of collagen receptors. Acta Physiol (Oxf) 190, 179-187

76. Zutter, M. M., and Santoro, S. A. (1990) Widespread histologic distribution of the $\alpha 2 \beta 1$ integrin cell-surface collagen receptor. Am J Pathol 137, 113-120

77. Pfaff, M., Aumailley, M., Specks, U., Knolle, J., Zerwes, H. G., and Timpl, R. (1993) Integrin and Arg-Gly-Asp dependence of cell adhesion to the native and unfolded triple helix of collagen type VI. Exp Cell Res 206, 167-176

78. Dohrmann, S., Anik, S., Olson, J., Anderson, E. L., Etesami, N., No, H., Snipper, J., Nizet, V., et al. (2014) Role for streptococcal collagen-like protein 1 in M1T1 group A Streptococcus resistance to neutrophil extracellular traps. Infect Immun 82, 4011-4020

79. Caswell, C. C., Han, R., Hovis, K. M., Ciborowski, P., Keene, D. R., Marconi, R. T., and Lukomski, S. (2008) The Scl1 protein of M6-type group A Streptococcus binds the human complement regulatory protein, factor $\mathrm{H}$, and inhibits the alternative pathway of complement. Mol Microbio/ 67, 584-596

80. Rodriguez de Cordoba, S., Esparza-Gordillo, J., Goicoechea de Jorge, E., Lopez-Trascasa, M., and Sanchez-Corral, P. (2004) The human complement factor $\mathrm{H}$ : functional roles, genetic variations and disease associations. $\mathrm{Mol}$ Immunol 41, 355-367

81. Jozsi, M., and Zipfel, P. F. (2008) Factor $\mathrm{H}$ family proteins and human diseases. Trends Immunol 29, 380-387

82. Reuter, M., Caswell, C. C., Lukomski, S., and Zipfel, P. F. (2010) Binding of the human complement regulators CFHR1 and factor $\mathrm{H}$ by streptococcal collagenlike protein 1 (Scl1) via their conserved $C$ termini allows control of the complement cascade at multiple levels. J Biol Chem 285, 38473-38485

83. Kraiczy, P., Skerka, C., Kirschfink, M., Brade, V., and Zipfel, P. F. (2001) Immune evasion of Borrelia burgdorferi by acquisition of human complement regulators $\mathrm{FHL}-1 /$ reconectin and Factor $\mathrm{H}$. Eur J Immunol 31, 1674-1684

84. Kraiczy, P., Skerka, C., Brade, V., and Zipfel, P. F. (2001) Further characterization of complement regulator-acquiring surface proteins of Borrelia burgdorferi. Infect Immun 69, 7800-7809

85. Gao, Y., Liang, C., Zhao, R., Lukomski, S., and Han, R. (2010) The Scl1 of M41type group A Streptococcus binds the high-density lipoprotein. FEMS Microbiol Lett 309, 55-61 
86. Pahlman, L. I., Marx, P. F., Morgelin, M., Lukomski, S., Meijers, J. C., and Herwald, H. (2007) Thrombin-activatable fibrinolysis inhibitor binds to Streptococcus pyogenes by interacting with collagen-like proteins A and B. J Biol Chem 282, 24873-24881

87. Valls Seron, M., Plug, T., Marquart, J. A., Marx, P. F., Herwald, H., de Groot, P. G., and Meijers, J. C. (2011) Binding characteristics of thrombin-activatable fibrinolysis inhibitor to streptococcal surface collagen-like proteins A and B. Thromb Haemost 106, 609-616

88. Zipfel, P. F., Skerka, C., Hellwage, J., Jokiranta, S. T., Meri, S., Brade, V., Kraiczy, P., Noris, M., et al. (2002) Factor $\mathrm{H}$ family proteins: on complement, microbes and human diseases. Biochem Soc Trans 30, 971-978

89. Gustafsson, M. C., Lannergard, J., Nilsson, O. R., Kristensen, B. M., Olsen, J. E., Harris, C. L., Ufret-Vincenty, R. L., Stalhammar-Carlemalm, M., et al. (2013) Factor $\mathrm{H}$ binds to the hypervariable region of many Streptococcus pyogenes $\mathrm{M}$ proteins but does not promote phagocytosis resistance or acute virulence. PLoS Pathog 9, e1003323

90. Horstmann, R. D., Sievertsen, H. J., Knobloch, J., and Fischetti, V. A. (1988) Antiphagocytic activity of streptococcal $M$ protein: selective binding of complement control protein factor H. Proc Natl Acad Sci U S A 85, 1657-1661

91. Wurfel, M. M., Kunitake, S. T., Lichenstein, H., Kane, J. P., and Wright, S. D. (1994) Lipopolysaccharide (LPS)-binding protein is carried on lipoproteins and acts as a cofactor in the neutralization of LPS. J Exp Med 180, 1025-1035

92. Bhakdi, S., Tranum-Jensen, J., Utermann, G., and Fussle, R. (1983) Binding and partial inactivation of Staphylococcus aureus alpha-toxin by human plasma low density lipoprotein. J Biol Chem 258, 5899-5904

93. Murch, O., Collin, M., Hinds, C. J., and Thiemermann, C. (2007) Lipoproteins in inflammation and sepsis. I. Basic science. Intensive Care Med 33, 13-24

94. Ravnskov, U. (2003) High cholesterol may protect against infections and atherosclerosis. QJM : monthly journal of the Association of Physicians 96, 927934

95. Netea, M. G., Demacker, P. N., Kullberg, B. J., Boerman, O. C., Verschueren, I., Stalenhoef, A. F., and van der Meer, J. W. (1996) Low-density lipoprotein receptor-deficient mice are protected against lethal endotoxemia and severe gram-negative infections. J Clin Invest 97, 1366-1372

96. Netea, M. G., Demacker, P. N., de Bont, N., Boerman, O. C., Stalenhoef, A. F., van der Meer, J. W., and Kullberg, B. J. (1997) Hyperlipoproteinemia enhances susceptibility to acute disseminated Candida albicans infection in low-densitylipoprotein-receptor-deficient mice. Infect Immun 65, 2663-2667

97. Makoveichuk, E., Cherepanov, P., Lundberg, P., Lundberg, S., Forsberg, A., and Olivecrona, G. (2003) pH6 antigen of Yersinia pestis interacts with plasma lipoproteins and cell membranes. J Lipid Res 44, 320-330

98. Liu, L., Zhou, L., Li, Y., Bai, W., Liu, N., Li, W., Gao, Y., Liu, Z., et al. (2015) Highdensity lipoprotein acts as an opsonin to enhance phagocytosis of group $A$ Streptococcus by U937 cells. Microbiol Immunol 59, 419-425 
99. Zhou, L., Liu, L., Yang, J., Li, Y., Bai, W., Liu, N., Li, W., Gao, Y., et al. (2016) LDL acts as an opsonin enhancing the phagocytosis of group A Streptococcus by monocyte and whole human blood. Med Microbiol Immunol 205, 155-162

100. Courtney, H. S., Zhang, Y. M., Frank, M. W., and Rock, C. O. (2006) Serum opacity factor, a streptococcal virulence factor that binds to apolipoproteins A-I and A-II and disrupts high density lipoprotein structure. J Biol Chem 281, 55155521

101. Bajzar, L. (2000) Thrombin activatable fibrinolysis inhibitor and an antifibrinolytic pathway. Arterioscler Thromb Vasc Biol 20, 2511-2518

102. Plug, T., and Meijers, J. C. (2016) Structure-function relationships in thrombinactivatable fibrinolysis inhibitor. Journal of Thrombosis and Haemostasis 14, 633644

103. Bengtson, S. H., Sanden, C., Morgelin, M., Marx, P. F., Olin, A. I., LeebLundberg, L. M., Meijers, J. C., and Herwald, H. (2009) Activation of TAFI on the surface of Streptococcus pyogenes evokes inflammatory reactions by modulating the kallikrein/kinin system. J Innate Immun 1, 18-28

104. Fiedler, T., Koller, T., and Kreikemeyer, B. (2015) Streptococcus pyogenes biofilms-formation, biology, and clinical relevance. Front Cell Infect Microbiol 5, 15

105. Carapetis, J. R., Steer, A. C., Mulholland, E. K., and Weber, M. (2005) The global burden of group A streptococcal diseases. Lancet Infect Dis 5, 685-694

106. Sanchez, C. J., Kumar, N., Lizcano, A., Shivshankar, P., Dunning Hotopp, J. C., Jorgensen, J. H., Tettelin, H., and Orihuela, C. J. (2011) Streptococcus pneumoniae in biofilms are unable to cause invasive disease due to altered virulence determinant production. PLOS ONE 6, e28738

107. Blanchette-Cain, K., Hinojosa, C. A., Akula Suresh Babu, R., Lizcano, A., Gonzalez-Juarbe, N., Munoz-Almagro, C., Sanchez, C. J., Bergman, M. A., et al. (2013) Streptococcus pneumoniae biofilm formation is strain dependent, multifactorial, and associated with reduced invasiveness and immunoreactivity during colonization. MBio 4, e00745-00713

108. Marks, L. R., Davidson, B. A., Knight, P. R., and Hakansson, A. P. (2013) Interkingdom signaling induces Streptococcus pneumoniae biofilm dispersion and transition from asymptomatic colonization to disease. MBio 4, e00438-00413

109. Marks, L. R., Mashburn-Warren, L., Federle, M. J., and Hakansson, A. P. (2014) Streptococcus pyogenes biofilm growth in vitro and in vivo and its role in colonization, virulence, and genetic exchange. J Infect Dis 210, 25-34

110. Connolly, K. L., Roberts, A. L., Holder, R. C., and Reid, S. D. (2011) Dispersal of Group A streptococcal biofilms by the cysteine protease SpeB leads to increased disease severity in a murine model. PLOS ONE 6, e18984

111. Jorgensen, R., Purdy, A. E., Fieldhouse, R. J., Kimber, M. S., Bartlett, D. H., and Merrill, A. R. (2008) Cholix toxin, a novel ADP-ribosylating factor from Vibrio cholerae. J Biol Chem 283, 10671-10678

112. LaPenta, D., Rubens, C., Chi, E., and Cleary, P. P. (1994) Group A streptococci efficiently invade human respiratory epithelial cells. Proc Natl Acad Sci USA 91, 12115-12119 